
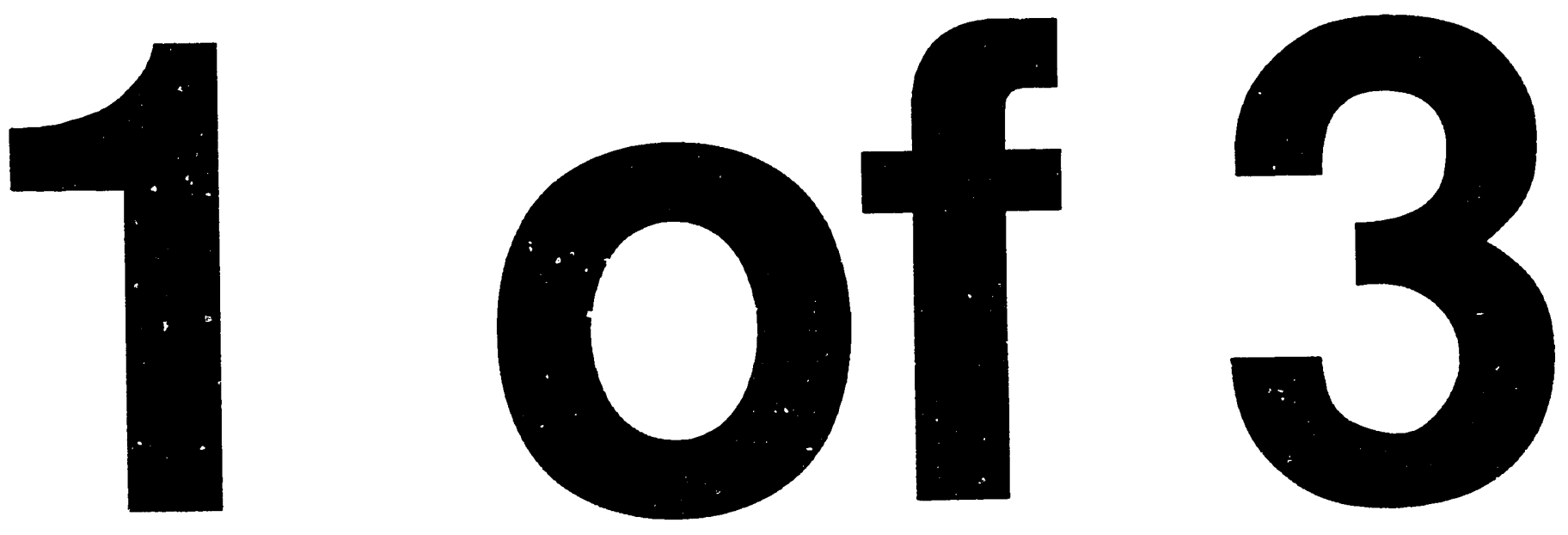
Pion Single Charge Exchange in Three Body Nuclei at Intermediate Energies

Marla L. Dowell* 


\section{ACKNOWLEDGMENTS}

I have had numerous opportunities to interact with many capable scientists while pursuing my graduate degree. Through these interactions and due to the nature of this dissertation, I have accumulated a great number of people to thank.

I would like to express my gratitude to my advisor, June Matthews, for her guidance and forbearance throughout my graduate career at M.I.T. I am indebted to her for her input in the creation of this document-it is a more clear and concise work due to her exertions. Through my research efforts in her group, I have acquired knowledge about the procedures for proposing and performing experiments and have become a more meticulous scientist due to her high standards. I will benefit from her example throughout my career.

Peter Gram has acted as an advisor-in-residence during my stays at the Clinton P. Anderson Meson Physics Facility (LAMPF). Peter ensured that my graduate education was not limited to sitting in front of a computer terminal and much of my hands-on experimental physics knowledge was gained under his helpful tutelage.

I wish to express my gratitude to the other collaborators in my original thesis experiment: Wilson Fong, Ed Kinney, Hojoon Park, Glen Rebka, Don Roberts, Mark Wang, and Mark Yuly. Four people have made many helpful comments on the data analysis of one or another of my experiments; Shalev Gilad, Steinar Høibråten, Stephen Pate, and Bob Redwine.

I would like to thank both Bill Gibbs and Lothar Tiator for providing me with calculations for my exclusive single charge exchange results. Andi Klein deserves my thanks for supplying me with his quasielastic scattering on ${ }^{3} \mathrm{He}$ results on very short notice.

There are a number of people at LAMPF who have been of assistance at one time or another: Ron Richardson of the LAMPF Electronics Pool, Cy Høffman, and Vern Sandberg. Several people in the MP-6 and MP-10 groups were helpful with many computer and electronics questions over the years, most notably Jim Amann, Will Foreman, Steve Greene, Jim Knudson, Tom Kozlowski, Elvira Martinez, and Mike Oothoudt. I would also like to thank Karen Poelakker of MP-9 for her help and inierest in my progress. 


\section{Table of Contents}

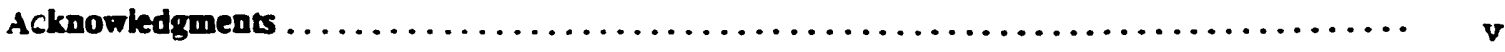

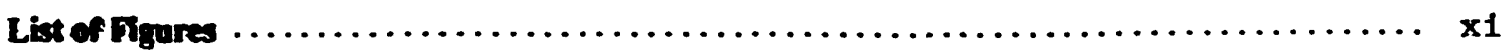

List of Tables ............................................

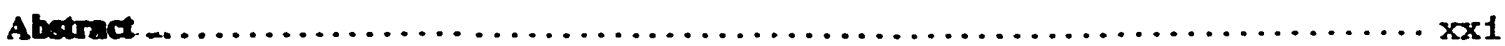

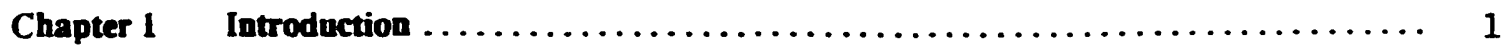

Overview of Pion Interactions at Intermediate Energies................ 2

Pion-nucleon Interactions and the $\Delta$-resonance $\ldots \ldots \ldots \ldots \ldots \ldots \ldots \ldots, 5$

Pion-nucleus interactions and the $\Delta$-resonance $\ldots \ldots \ldots \ldots \ldots \ldots \ldots \ldots, 8$

Elastic Single Charge Exchange Measurements: ${ }^{3} \mathrm{H}\left(\pi^{*}, \pi^{9}\right)^{3} \mathrm{He} \ldots \ldots \ldots \ldots \ldots . \ldots$

Review of Previous Theoretical Work ....................... 10

Review of Previous Experimental Work $\ldots \ldots \ldots \ldots \ldots \ldots \ldots \ldots \ldots \ldots, 17$

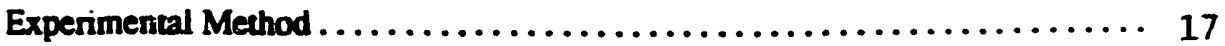

Inclusive Pion Single Charge Exchange Measurements on ${ }^{3} \mathrm{He}:{ }^{3} \mathrm{He}\left(\pi^{\circ}, \pi^{9}\right)$ and

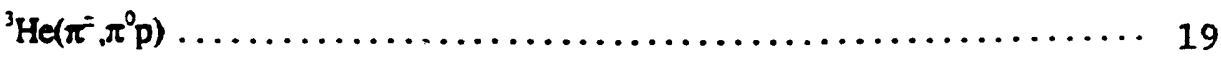

Review of Previous Work. $\ldots \ldots \ldots \ldots \ldots \ldots \ldots \ldots \ldots \ldots \ldots \ldots, 21$

Quasi-free Pion Single Charge Exchange on ${ }^{16} \mathrm{O} \ldots \ldots \ldots \ldots \ldots \ldots \ldots . \ldots \ldots$

Pion-induced Proton Knockout on ${ }^{3} \mathrm{He}:{ }^{3} \mathrm{He}(\pi ;, \pi \mathrm{p}) \ldots \ldots \ldots \ldots \ldots \ldots, 21$

Experimental Method $. \ldots \ldots \ldots \ldots \ldots \ldots \ldots \ldots \ldots \ldots \ldots \ldots \ldots, 27$

\section{Elastic Single Charge Exchange Measurements: ${ }^{3} \mathrm{H}\left(\pi^{+}, \mathrm{Hi}\right) \pi^{0}$}

Chapter 2 Experimental Apparatus and Data Acquisition System for the ${ }^{3} \mathrm{hi}\left(\pi^{\circ},{ }^{3} H e\right) \pi^{0}$

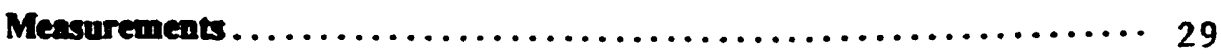

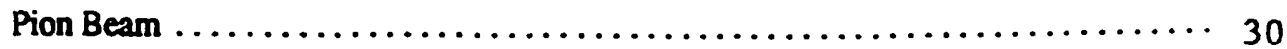

Beam Monitoring and Operation $\ldots \ldots \ldots \ldots \ldots \ldots \ldots \ldots \ldots \ldots \ldots \ldots \ldots \ldots \ldots \ldots \ldots$

Recoil Detection Scheme................................. 34

Little Yellow Spectrometer. . . . . . . . . . . . . . . . . . . . 34

oii 
Detectors

Electronics and Data Acquisiton System................... 42

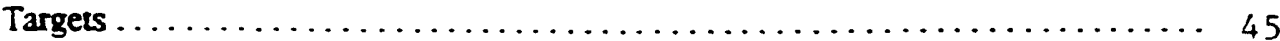

Chapter 3 Experimental Procedures. Data Analysis, and Resuits for the ' $H\left(\pi^{-}\right.$, 'He $\pi^{3}$

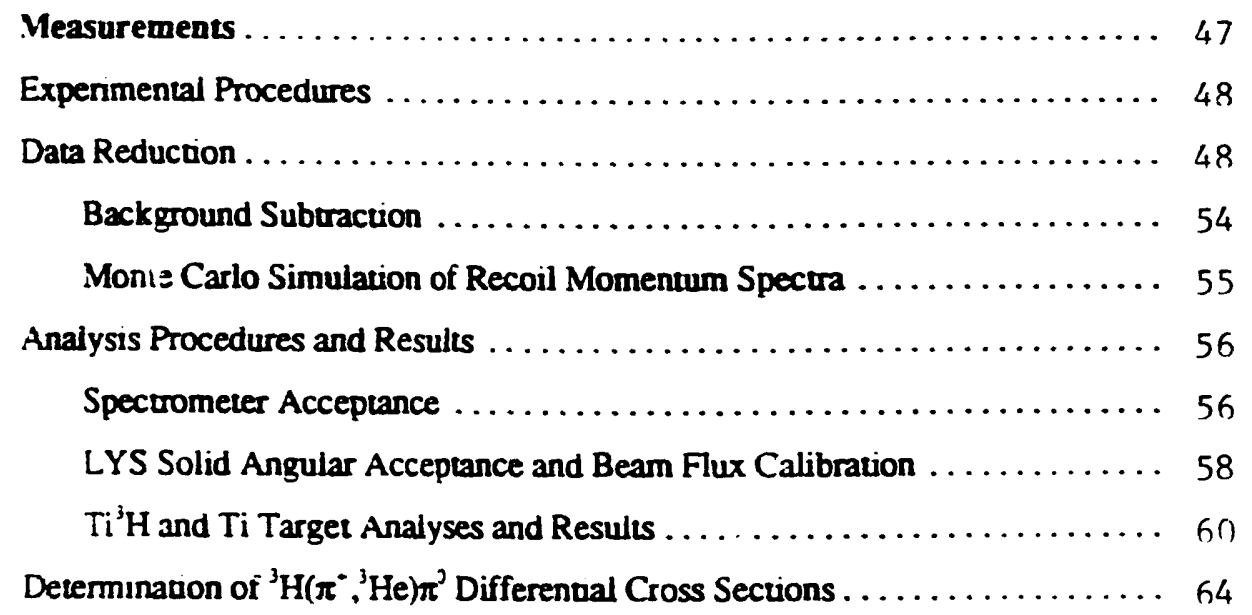

Chapter 4 Presentation of ${ }^{3} \mathbf{H}\left(\pi^{*},{ }^{3} H e\right) \pi^{0}$ Differential Cross Sections................ 69

\section{Inclusive Pion Single Charge Exchange Measurements on 'He}

Chapter 5 Experimental Apparatus and Data Acquisition System for Inciusive Pion Single

Charge Exchange Measurements on ${ }^{3} \mathrm{He:}{ }^{3} \mathrm{He}\left(\pi^{\circ}, \pi^{0}\right)$ and ${ }^{3} \mathrm{He}\left(\pi^{\circ}, \pi^{0} \mathrm{p}\right) \ldots \ldots 73$

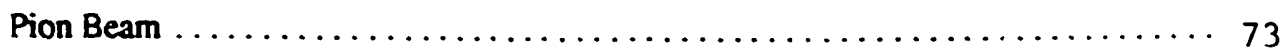

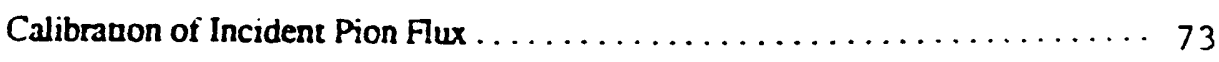

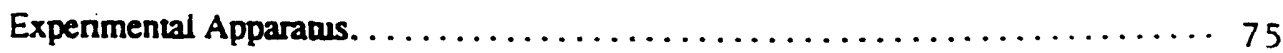

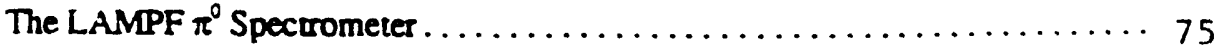

Prown Detector Array................................ 78

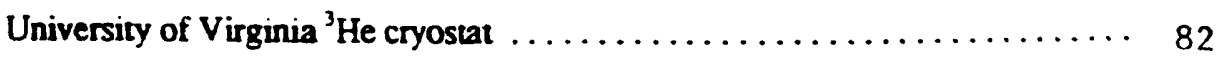

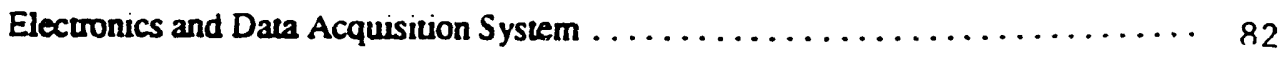

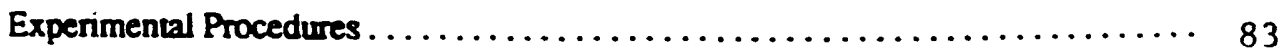

Chapter 6 Data Analysis and Results for Inclusive Pion Single Charge Exchange

Measurements on 'He: 'He( $\left.\pi^{\prime}, \pi^{9}\right)$ and ${ }^{3} H e\left(\pi^{\prime}, \pi^{0} p\right) \ldots \ldots \ldots \ldots \ldots \ldots 85$

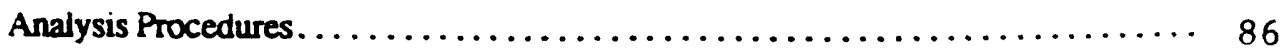

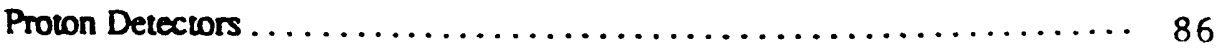

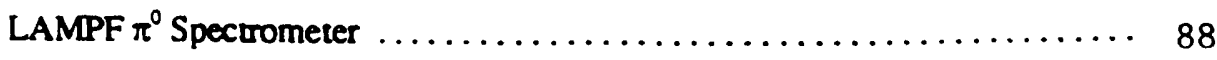


Timing and Pulse Height Constraints $\ldots \ldots \ldots \ldots \ldots \ldots \ldots \ldots \ldots . \quad 89$

Wire Chamber Analysis. ......................... 92

$\pi^{0}$ Spectrometer Acceptance and Conversion Efficiency .............. 95

Solid Angular Acceptance of the $\pi^{0}$ Spectrometer .............. 95

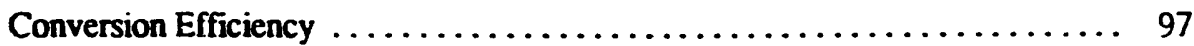

Determination of ${ }^{3} \mathrm{He}\left(\pi^{*}, \pi^{9}\right)$ and ${ }^{3} \mathrm{He}\left(\pi^{*} \pi^{0} \mathrm{p}\right)$ Differential Cross Sections........ 102

Procedure for Determining $\pi^{0}$ Energy Spectra .................. 102

Background Subtraction . . . . . . . . . . . . . . . . . . . . . 104

Statistical and Systematic Uncertainties..................... 105

Chapter 7 Presentation of Results from Inclusive Pion Single Charge Exchange Measurements

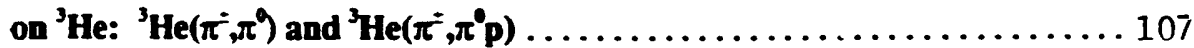

Quasi-free Pion Single Charge Exchange on ${ }^{3} \mathrm{He}:{ }^{3} \mathrm{He}\left(\pi^{ \pm}, \pi^{9}\right) \ldots \ldots \ldots \ldots \ldots . \ldots 108$

Doubly Differential Cross Sections for the ${ }^{3} \mathrm{He}\left(\pi^{2}, \pi^{9}\right)$ Reactions . . . . . . . 108

Angular Distribution for the ${ }^{3} \mathrm{He}\left(\pi^{*}, \pi^{9}\right)$ Reactions................ 118

Ratios of Doubly Differential Cross Sections from Single-Arm Measurements of

Pion Charge Exchange and Inelastic Scattering $\ldots \ldots \ldots \ldots \ldots \ldots \ldots \ldots \ldots \ldots \ldots$

Isospin Scaling Predictions for Single and Double Scattering Ratios. . . . . 122

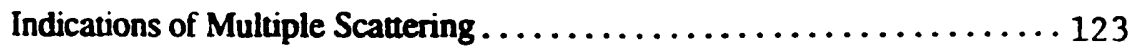

Coincidence Measurements of Quasi-free Pion Single Charge Exchange on ${ }^{3} \mathrm{He}$ :

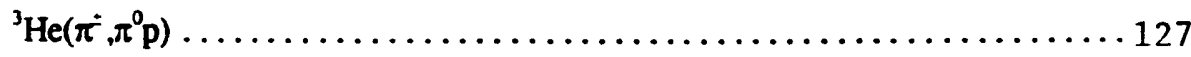

Triply Differential Cross Sections for the ${ }^{3} \mathrm{He}\left(\pi^{ \pm}, \pi^{0} \mathrm{p}\right)$ Reactions . . . . . . . 127

Proton Angular Distributions for the ${ }^{3} \mathrm{He}\left(\pi^{*}, \pi^{0} \mathrm{p}\right)$ Reactions . . . . . . . . 141

$\pi^{0}$ Angular Distribution in the ${ }^{3} \mathrm{He}\left(\pi^{+}, \pi^{0} \mathrm{p}\right)$ Reaction . . . . . . . . . . . . 146

Comparisons with Previous Coincidence Measurements of Pion Quasi-elastic

Scattering on ${ }^{3} \mathrm{He}:{ }^{3} \mathrm{He}\left(\pi^{*}, \pi^{\dagger} \mathrm{p}\right) \ldots \ldots \ldots \ldots \ldots \ldots \ldots \ldots \ldots \ldots \ldots$

Chapter 8 Comparison of Inclusive Pion Single Charge Exchange Measurements with

Theoretical Calculations. . . . . . . . . . . . . . . . . . . . 153

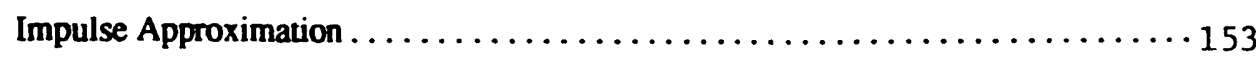

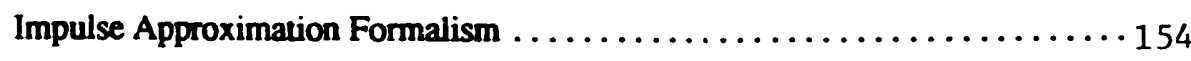

Initial Energy and Final Energy Prescriptions. . . . . . . $\ldots \ldots \ldots \ldots$

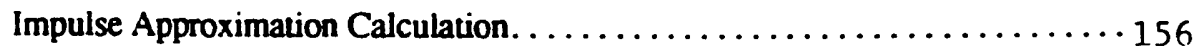

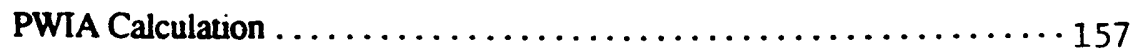

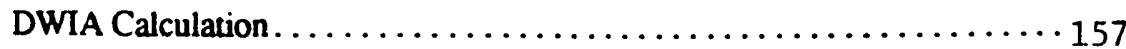


Comparisons of Impulse Approximatioa Calculations with Experimental Resuits . . 158 Comparisons of PWLA and DWIA Calculations with Inclusive Pion Single Charge Exchange Results. . . . . . . . . . . . . . . . . . . . . . 158

$\pi^{0}$ Angular Distributions from the ${ }^{3} \mathrm{He}\left(\pi^{ \pm} \pi^{9}\right)$ and ${ }^{3} \mathrm{He}\left(\pi^{*} \pi^{0} \mathrm{p}\right)$ Reactions. ... 159 Comparisons of DWIA Calculations with Results from Pion Single Charge

Exchange and Inelastic Scattering Measurements on ${ }^{3} \mathrm{He} . \ldots \ldots \ldots \ldots 166$

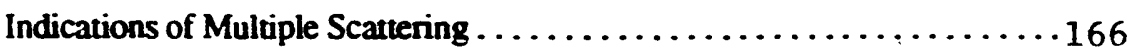

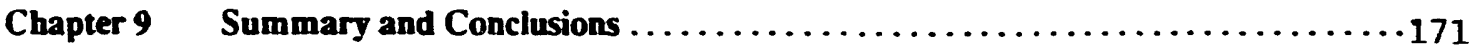

Appendix A Outline of Elastic Single Charge Exchange Analysis . . . . . . . . . . . 177

Appendix B Monte Carlo Simulations of the Little Yellow Spectrometer Performance ......181

Overview of Monte Carlo Simulation $\ldots \ldots \ldots \ldots \ldots \ldots \ldots \ldots \ldots \ldots \ldots \ldots \ldots \ldots \ldots \ldots$

Monte Carlo Peak Positions . . . . . . . . . . . . . . . . . . . . . . 183

Monte Carlo Dispersion Constant and Acceptance Function ..............183

Monte Carlo Angular Distributions for Elastic Scattering and Charge Exchange

Reactions.......................................

Monte Carlo Event Generator for Complicated Angular Distributions . . . . . 188

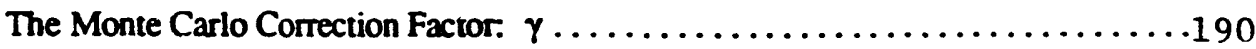

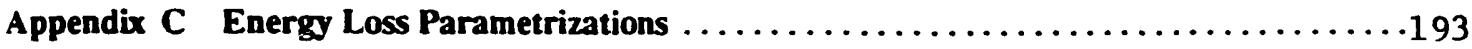

Appendix D Proton Detector Calibration .............................. 195

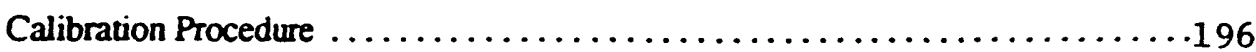

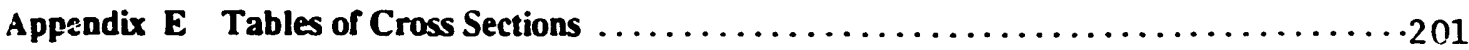

Doubly Differential Cross Sections for the ${ }^{3} \mathrm{He}\left(\pi^{ \pm} \pi^{9}\right)$ Reactions .............202

Triply Differential Cross Sections for the ${ }^{3} \mathrm{He}\left(\pi^{*} \pi^{0} \mathrm{p}\right)$ Reactions .............. 208

Doubly Differential Cross Sections for the ${ }^{3} \mathrm{He}\left(\pi^{ \pm} \pi^{0} \mathrm{p}\right)$ Reactions ...........220

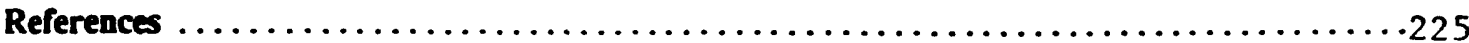




\section{List of Figures}

Figure 1.1 Feynman diagrams for a) virtual pion exchange between two nucleons and b) virual photon exchange between two electrons $\ldots \ldots \ldots \ldots \ldots \ldots \ldots \ldots \ldots \ldots \ldots$

Figure 1.2 Schematic diagram of the nucleon-nucleon potential . . . . . . . . . . . . . . . 4

Figure 1.3 Diagrams for pn a) scattering and b) charge exchange. . . . . . . . . . . . . . 5

Figure 1.4 Total cross sections for $\pi$ p scattering as a function of incident pion energy $\ldots \ldots \ldots \ldots 7$

Figure 1.5 Total cross sections for $\pi$ p elastic scattering and charge exchange in the $\Delta$-resonance region from $\pi \mathrm{N}$ phase shift calculations $\ldots \ldots \ldots \ldots \ldots \ldots \ldots \ldots \ldots \ldots \ldots \ldots$

Figure 1.6 Diagrams for pion double charge erchange on ${ }^{3} \mathrm{He}$ and the ${ }^{3} \mathrm{He}\left(\pi^{-}, \pi^{0} \mathrm{p}\right)$ reaction ..... 9

Figure 1.7 Angular distributions for ${ }^{3} \mathrm{He}\left(\pi^{4}, \pi\right)^{-}$ite $(\mathrm{left})$ and ${ }^{3} \mathrm{He}\left(\pi^{-}, \pi^{9}\right)^{3} \mathrm{H} \ldots \ldots \ldots \ldots$

Figure 1.8 Total cross section for ${ }^{3} \mathrm{H}\left(\pi^{-}, \pi^{9}\right)^{3} \mathrm{He}$ with a measurement from Cooper et. al... . . . . . 12

Figure 1.9 Angular distribution for ${ }^{3} \mathrm{He}\left(\pi^{-}, \pi^{2}\right)^{3} \mathrm{H}$ at $\mathrm{T}_{n}=200 \mathrm{MeV} \ldots \ldots \ldots \ldots \ldots \ldots \ldots \ldots \ldots$

Figure 1.10 Angular distribution for ${ }^{3} \mathrm{H}\left(\pi^{+}, \pi^{0}\right)^{3} \mathrm{He}$ at $\mathrm{T}_{\mathrm{\pi}}=148 \mathrm{MeV} \ldots \ldots \ldots \ldots \ldots \ldots \ldots$

Figure 1.11 Existing data on the isobaric analog transition in $A=3$ nuclei below $400 \mathrm{MeV} \ldots \ldots \ldots 16$

Figure 1.12 Pion energy späira from ${ }^{3} \mathrm{He}\left(\pi^{-}, \pi^{9}\right)$ at $200 \mathrm{MeV} \ldots \ldots \ldots \ldots \ldots \ldots \ldots \ldots \ldots \ldots \ldots$

Figure 1.13 Diagrams for a) pion single charge exchange and b) pion scattering on a $\mathrm{T}=0$ pair . . . 20

Figure 1.14 Outgoing pion spectra from ${ }^{16} \mathrm{O}\left(\pi^{*}, \pi^{\dagger}\right)$ at $163 \mathrm{MeV}$ and ${ }^{16} \mathrm{O}\left(\pi^{+}, \pi^{9}\right)$ at $160 \mathrm{MeV} \ldots \ldots .22$

Figure 1.15 Pion energy spectra from ${ }^{16} \mathrm{O}\left(\pi^{+}, \pi^{0} \mathrm{p}\right)$ at $245 \mathrm{MeV} \ldots \ldots \ldots \ldots \ldots \ldots \ldots \ldots \ldots \ldots$

Figure 1.16 Comparison of ${ }^{3} \mathrm{He}\left(\pi^{+}, \pi^{+}\right)$and ${ }^{3} \mathrm{He}\left(\pi^{+}, \pi^{+} \mathrm{p}\right)$ coincidence spectrum at $270 \mathrm{MeV}, \theta_{\pi^{0}}=75^{\circ}$

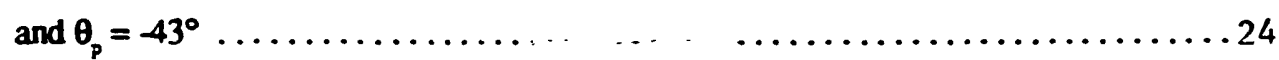

Figure 1.17 Diagram for quasi-free single charge exchange on ${ }^{3} \mathrm{He}:{ }^{3} \mathrm{He}\left(\pi^{\star}, \pi^{9}\right) \ldots \ldots \ldots \ldots \ldots 24$

Figure 1.18 Feynman diagrams for a) and b) ${ }^{3} \mathrm{He}\left(\pi^{-}, \pi^{0} \mathrm{p}\right)$ and $\left.c\right)^{3} \mathrm{He}\left(\pi^{-}, \pi^{\dagger}\right)$ through a sequential

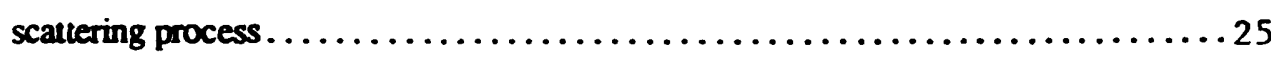

Figure 1.19 Feynman diagrams for a) and b) ${ }^{3} \mathrm{He}\left(\pi^{-}, \pi^{0} \mathrm{p}\right)$ and $\left.\mathrm{c}\right){ }^{3} \mathrm{He}\left(\pi^{-}, \pi^{0}\right)$ through a direct $\Delta$-nucleu:

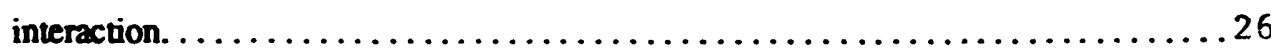

Figure 1.20 Feynman diagrams for a) and b) ${ }^{3} \mathrm{He}\left(\pi^{-}, \pi^{0} \mathrm{p}\right)$ and c) ${ }^{3} \mathrm{He}\left(\pi^{-}, \pi^{0}\right)$ through final state

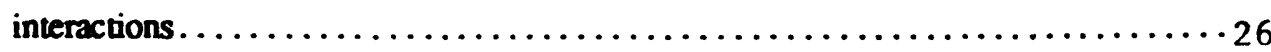

Figure 1.21 Feynman diagrams for a) and b) ${ }^{3} \mathrm{He}\left(\pi^{-}, \pi^{\circ} \mathrm{p}\right)$ and $\left.\mathrm{c}\right){ }^{3} \mathrm{He}\left(\pi^{-}, \pi^{2}\right)$ initial state interactions .26

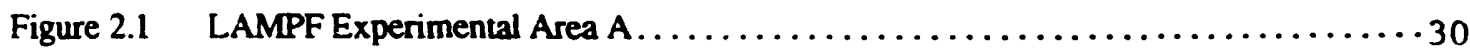

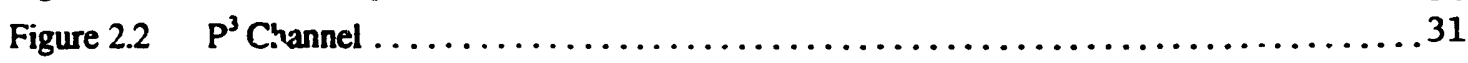

Figure 2.3 Top view of the Little Yellow Spectrometer and beam monitoring system . . . . . . . 32

Figure 2.4 Side view of the Little Yellow Spectrometer. . . . . . . . . . . . . . . . . 36 
Figure 2.5 Side view of the Liule Yellow Spectrometer focmi plane region. . . . . . . . . 38

Figure 2.6 Detailed view of focal plane scintillation counter . . . . . . . . . . . . . . . 39

Figure 2.7 Typical silicon detecto pulse height spectrum $\ldots \ldots \ldots \ldots \ldots \ldots \ldots \ldots \ldots \ldots \ldots, 40$

Figure 2.8 Energy loss in $\mathbf{4 0 0} \boldsymbol{\mu m}$ silicon surface barrier detector as a function of spectrometer

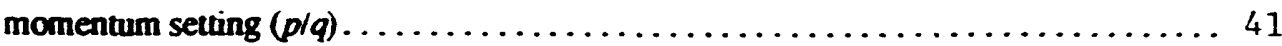

Figure $2.9 \quad$ E1026 electronics diagrem. . . . . . . . . . . . . . . . . . . . . . . . . 43

Figure 2.10 Electronics logic diagram for a pion scattering monitor telescope $\ldots \ldots \ldots \ldots \ldots \ldots 44$

Figure 2.11 Ionization chamber and "FIDO" electronics diagram .................... 44

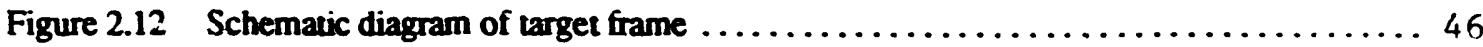

Figure 3.1: Typical silicon detector pulse height spectra....................... 49

Figure 3.2 Typicai fit to a silicon surface barrier detector pulse height spectrum from $\pi^{+3} \mathrm{H}$ elastic scattering using a $\mathrm{Ti}^{3} \mathrm{H}$ target $\ldots \ldots \ldots \ldots \ldots \ldots \ldots \ldots \ldots \ldots \ldots \ldots \ldots \ldots \ldots$

Figure 3.3 Silicon detector pulse height spectrum with and without a scintillator coincidence

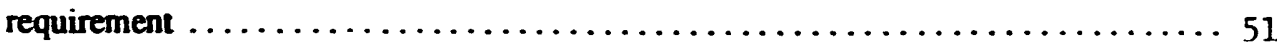

Figure 3.4 MINUIT and Brute results for ${ }^{3} \mathrm{He}$ yields from a $\mathrm{Ti}^{3} \mathrm{H}$ target.............. 53

Figure 3.5 MINUIT and Brute results for raw ${ }^{3} \mathrm{He}$ Yields from $\mathrm{Ti}^{3} \mathrm{H}$ and $\mathrm{Ti}$ Targets $\ldots \ldots \ldots \ldots 53$

Figure 3.6 Example of re-binned and normalized momentum spectrum from $\pi$ p elastic scautering

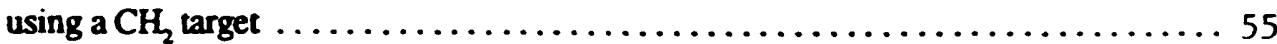

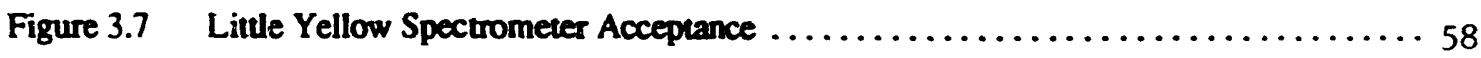

Figure $3.8 \quad$ Net proton yields from $\pi^{*}$ p elastic scattering at 142 and $180 \mathrm{MeV} \ldots \ldots \ldots \ldots \ldots . \ldots 59$

Figure 3.9 Ratios of a particle yields from tritiated $\left(\mathrm{Ti}^{3} \mathrm{H}\right)$ and untritiated (Ti) titanium foils as a

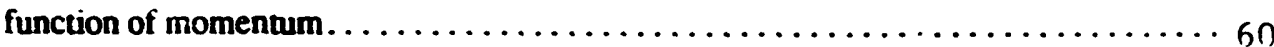

Figure 3.10 ${ }^{3} \mathrm{H}$ elastic peak from $\pi^{+3} \mathrm{H}$ scattering in comparison with Litule Yellow Spectrometer

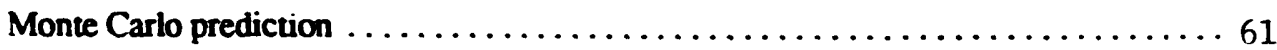

Figure 3.11 Differential cross section for the ${ }^{3} \mathrm{H}\left(\pi^{*}, \pi^{*}\right)^{3} \mathrm{H}$ reaction at $\mathrm{T}_{\pi}=180 \mathrm{MeV} \ldots \ldots \ldots \ldots 62$

Figure 3.12 Doubly differential cross sections of ${ }^{3} \mathrm{He}$ production from $\mathrm{Ti}$ and $\mathrm{Ag} \ldots \ldots \ldots \ldots 63$

Figure 3.13 Momentum spectra of ${ }^{3} \mathrm{He}$ particles from ${ }^{3} \mathrm{H}\left(\pi^{*}{ }^{3} \mathrm{He}\right) \pi^{0}$ at $\mathrm{T}_{\boldsymbol{n}}=142 \mathrm{MeV} \ldots \ldots \ldots \ldots 67$

Figure 3.14 Momenum spectra of ${ }^{3} \mathrm{He}$ narticles from ${ }^{3} \mathrm{H}\left(\pi^{+},{ }^{3} \mathrm{He}\right) \pi^{0}$ at $\mathrm{T}_{\pi}=180 \mathrm{MeV} \ldots \ldots \ldots \ldots 68$

Figure 4.1 Center-of-mass differential cross sections for the ${ }^{3} \mathrm{H}\left(\pi^{+},{ }^{3} \mathrm{He}\right) \pi^{0}$ reaction at $142 \mathrm{MeV} \ldots 71$

Figure 4.2 Center-of-mass differential cross sections for the ${ }^{3} \mathrm{H}\left(\pi^{+},{ }^{3} \mathrm{He}\right) \pi^{0}$ reaction at $180 \mathrm{MeV} \ldots 72$

Figure 5.1 Schematic drawing of Low Energy Pion Channel..................... 74

Figure 5.2 Top view of experimental set-up .............................. 76

Figure 5.3 Schematic diagram of $\pi^{0}$ Spectrometer photon arms in one-post mode $\ldots \ldots \ldots \ldots 77$

Figure 5.4 Schematic diagram of a proton telescope.......................... 79

Figure 5.5 Proton detector configurations: a) ventical and b) horizontal. . . . . . . . . . . . . 79

Figure 5.6 Side view of proton detector array in the vertical configuration $\ldots \ldots \ldots \ldots \ldots . .20$ 
Figure 5.7 Schematuc diagram of the University of Virginia ${ }^{3} \mathrm{He}$ cryostat.............. 81

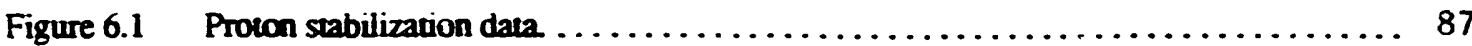

Figure 6.2 Example of a proton polygon gate on a two dimensional plot of pulse height spectra from the $\Delta E$ and $E$ scintillation counters of the central proton detector. . . . . . . . . 88

Figure 6.3 Relative timing between the two photon arms of the $\pi^{0}$ Spectrometer .......... 89

Figure 6.4 Pulse height spectrim trom a photon arm veto scintillation counter........... 90

Figure 6.5 Reconstructed distribution of the fractional energy difference between two detected

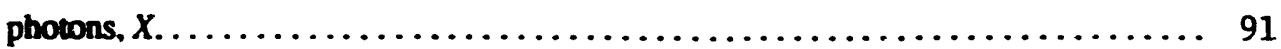

Figure 6.6 Effect of XCUT of on the statistical uncentainty and energy resolution of $\pi^{0}$ energy spectra from the $p\left(\pi^{-}, \pi^{0}\right)$ n and ${ }^{3} \mathrm{He}\left(\pi^{*}, \pi^{9}\right)$ reactions. ...................... 91

Figure 6.7 Effect of XCUT on statistical uncertainty for ${ }^{3} \mathrm{He}\left(\pi^{\circ}, \pi^{\circ} \mathrm{p}\right)$ measurements at $\theta_{\pi} \mathrm{D}=90^{\circ}$ and $\theta_{p}$

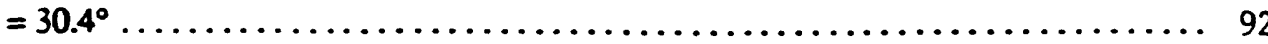

Figure 6.8 Wire chamber efficiencies from replay hot wire tables, tracking efficiencies, and on-line MWPC efficiencies ..................................... 94

Figure 6.9 Solid angular acceptance of $\pi^{0}$ Spectrometer as a function of $\pi^{0}$ kiretic energy for each

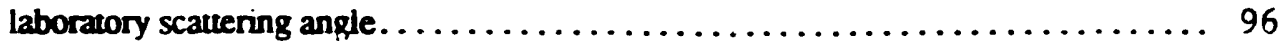

Figure 6.10 Normalized $\pi^{0}$ energy spectra from $\mathrm{CH}_{2}$ and ${ }^{12} \mathrm{C}$ targets $\ldots \ldots \ldots \ldots \ldots \ldots \ldots . . \ldots 9$

Figure 6.11 Normalized $\pi^{0}$ energy spectra from a $\mathrm{CH}_{2}$ target with ${ }^{12} \mathrm{C}$ background subtracted at $\theta_{8} 0=$

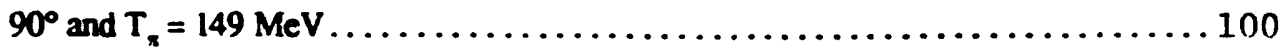

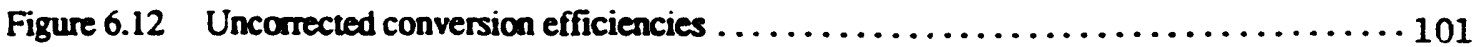

Figure 6.13 Corrected conversion efficiencies ................................. 101

Figure 6.14 Raw $\pi^{0}$ energy spectra for real and accidental $\pi^{0}$ events deriveo from a ${ }^{3} \mathrm{He}\left(\pi^{-}, \pi^{0}\right)$

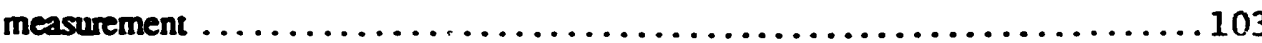

Figure 6.15 Normalized $\pi^{0}$ energy spec $r a$ from ${ }^{3} \mathrm{He}\left(\pi^{-}, \pi^{9}\right)$ measurements $\ldots \ldots \ldots \ldots \ldots \ldots 104$

Figure 7.1 Doubly differential cross sections for the ${ }^{3} \mathrm{He}\left(\pi^{*}, \pi^{9}\right)$ reaction at $T_{8}=245 \mathrm{MeV}$ and $\theta_{x^{0}}=$

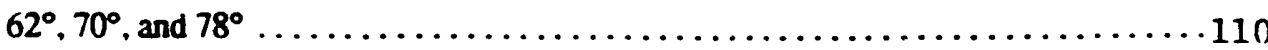

Figure 7.2 Doubly differential cross sections for the ${ }^{3} \mathrm{He}\left(\pi^{*}, \pi^{9}\right)$ reaction at $T_{n}=245 \mathrm{MeV}$ and $\theta_{n^{0}}=$

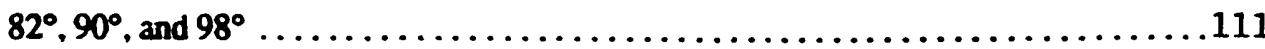

Figure 7.3 Doubly differential cross sections for the ${ }^{3} \mathrm{He}\left(\pi^{*}, \pi^{9}\right)$ reaction at $T_{x}=245 \mathrm{MeV}$ and $\theta_{\pi^{0}}=$

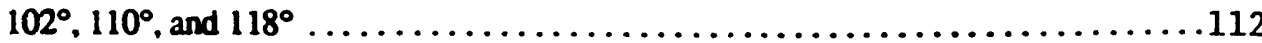

Figure 7.4 Doubly differential cross sections for the ${ }^{3} \mathrm{He}\left(\pi^{*}, \pi^{9}\right)$ reaction at $T_{\pi}=245 \mathrm{MeV}$ and $\theta_{x^{\circ}}=$

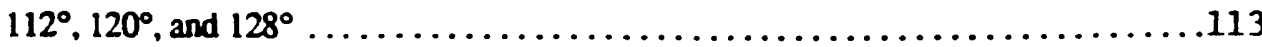

Figure 7.5 Doubly differential cross sections for the ${ }^{3} \mathrm{He}\left(\pi^{-}, \pi^{2}\right)$ reaction at $T_{\mathrm{x}}=245 \mathrm{MeV}$ and $\theta_{\mathrm{z}} \mathrm{O}=$

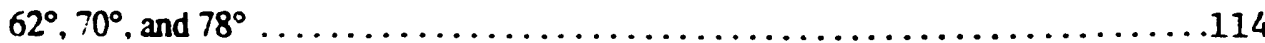

Figure 7.6 Doubly differential cross sections for the ${ }^{3} \mathrm{He}\left(\pi^{-}, \pi^{2}\right)$ reaction at $T_{x}=245 \mathrm{MeV}$ and $\theta_{\mathrm{x}} \mathrm{O}=$

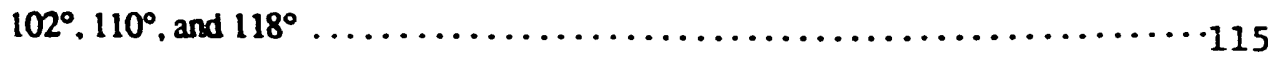


Figure 7.7 Compansons of the doubly differentual cross sections for the ${ }^{3} \mathrm{He}\left(\pi^{\circ}, \pi^{3}\right)$ reacuons at $T_{8}=$

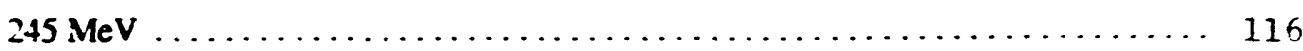

Figure 7.8 Compansons of the doubly differentual cross secuons for the ${ }^{3} \mathrm{He}\left(\pi^{2}, \pi^{2}\right)$ reactoons at $T_{\tau}=$

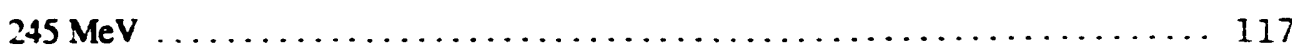

Figure 7.9 Compansons of $\pi^{0}$ energy spectra from the ${ }^{3} \mathrm{He}\left(\pi^{*} \pi^{3}\right)$ measurements which employe different geometrical set-ups of the $\pi^{3}$ Spectrometer................. 118

Figure 7.10 Doubly differenual cross section for ${ }^{3} \mathrm{He}\left(\pi^{\circ}, \pi^{0}\right)$ at $245 \mathrm{MeV}$ and $\theta_{\mathrm{x}^{\circ}}=110^{\circ} \ldots \ldots \ldots 119$

Figure 7.11 Differential cross sections for ${ }^{3} \mathrm{He}\left(\pi^{*}, \pi^{9}\right)$ at $T_{2}=245 \mathrm{MeV} \ldots \ldots \ldots \ldots \ldots \ldots \ldots 121$

Figure 7.12 Ratio of the doubly differenual cross sections ior ${ }^{3} \mathrm{He}\left(\pi^{2}, \pi^{3}\right)$ to ${ }^{3} \mathrm{He}\left(\pi^{\circ}, \pi^{9}\right)$ at $62^{\circ}, 70^{\circ}$, and

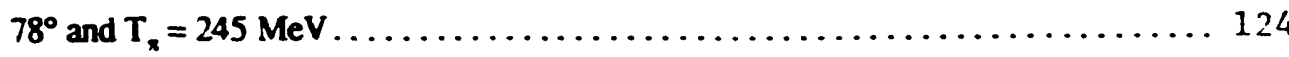

Figure 7.13 Ratio of the doubly differential cross sections for ${ }^{3} \mathrm{He}\left(\pi^{-} \pi^{9}\right)$ to ${ }^{3} \mathrm{He}\left(\pi^{*}, \pi^{9}\right)$ at $102^{\circ}, 110^{\circ}$.

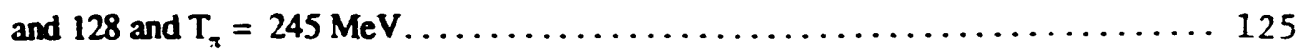

Figure 7.14 Comparisons of doubly differential cross suctions for pion non-charge exchange, single charge exchange, and double charge exchange on ${ }^{3} \mathrm{He} \ldots \ldots \ldots \ldots \ldots \ldots \ldots \ldots$

Figure 7.15 Triply differenual cross sections for the ${ }^{3} \mathrm{He}\left(\pi^{*}, \pi^{0} \mathrm{p}\right)$ reaccion at an incident pion energy of $245 \mathrm{MeV}$ and $\theta_{\pi^{\circ}}=70^{\circ}$

Figure 7.16 Triply differenual cross sections for the ${ }^{3} \mathrm{He}\left(\pi^{\circ}, \pi^{0} \mathrm{p}\right)$ reacuon at an incident pion energy of

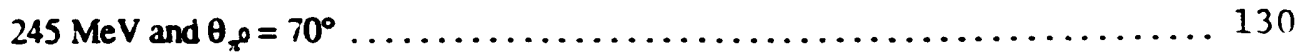

Figure 7.17 Triply differential cross sections for the ${ }^{3} \mathrm{He}\left(\pi^{*}, \pi^{0} \mathrm{p}\right)$ reaction at an incident pion energy of

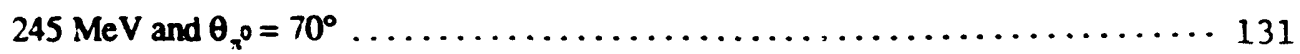

Figure 7.18 Triply differenual cross sections for the ${ }^{3} \mathrm{He}\left(\pi^{\circ}, \pi^{\circ} \mathrm{p}\right)$ reaction at an incident pion energy of

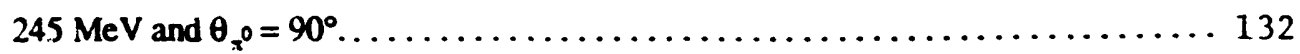

Figure 7.19 Triply differential cross sections for the ${ }^{3} \mathrm{He}\left(\pi^{*}, \pi^{0} \mathrm{p}\right)$ reaction at an incident pion energy of

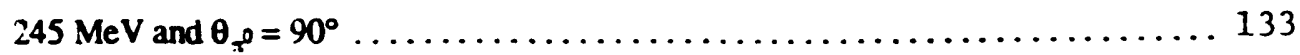

Figure 7.20 Triply differential cross sections for the ${ }^{3} \mathrm{He}\left(\pi^{\circ}, \pi^{0} \mathrm{p}\right)$ reaction at an incident pion energy of

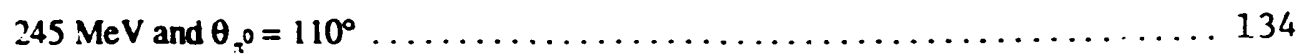

Figure 7.21 Triply differentual cross sections for the ${ }^{3} \mathrm{He}\left(\pi^{0}, \pi^{0} \mathrm{p}\right)$ reaction at an incident pion energy of

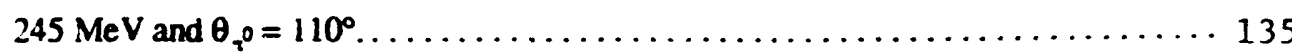

Figure 7.22 Triply differenual cross sections for the ${ }^{3} \mathrm{He}\left(\pi^{*}, \pi^{3} \mathrm{p}\right)$ re: $z$ tion at an incident pion energy of

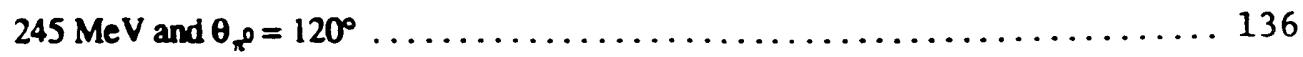

Figure 7.23 Triply differential cross sections for ine ${ }^{3} \mathrm{He}\left(\pi^{*}, \pi^{0} \mathrm{p}\right)$ reaction at an incident pion energy of $245 \mathrm{MeV}$ and $\mathrm{e}_{\pi^{0}}=120^{\circ}$.

Figure 7.24 Triply differential cross sections for the ${ }^{3} \mathrm{He}\left(\pi^{-}, \pi^{3} \mathrm{p}\right)$ reaction at an incident pion energy of

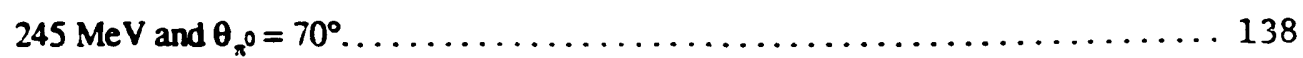

Figure 7.25 Triply differential cross sections for the ${ }^{3} \mathrm{He}\left(\pi^{-}, \pi^{0} \mathrm{p}\right)$ reaction at an incident pion energy of

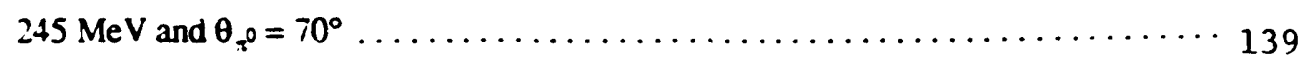

Figure 7.26 Triply differential cross sections for the ${ }^{3} \mathrm{He}(\pi, \pi \mathrm{p})$ reaction at an incident pion energy of

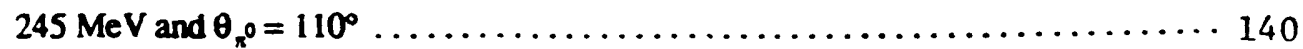

Figure 7.27 Proton angular distributions in the ${ }^{3} \mathrm{He}\left(\pi^{\circ}, \pi^{\circ} \mathrm{p}\right)$ reaction at $245 \mathrm{MeV}$ and $\theta_{\pi^{\circ}}=70^{\circ} \ldots 142$ 
Figure 7.28 Proton angular distributions in the ${ }^{5} \mathrm{He}\left(\pi^{\circ}, \pi^{\circ} \mathrm{p}\right)$ reaction at $245 \mathrm{MeV}$ and $\theta_{x^{0}}=90^{\circ} \ldots 142$

Figure 7.29 Proton angular distributions in the ${ }^{3} \mathrm{He}\left(\pi^{*}, \pi^{0} \mathrm{p}\right)$ reaction at $245 \mathrm{VeV}$ and $\theta_{\mathrm{x}^{0}}=110^{\circ} \ldots 143$

Figure 7.30 Proton angular distributions in the ${ }^{3} \mathrm{He}\left(\pi^{\circ}, \pi^{\circ} \mathrm{p}\right)$ reaction at $245 \mathrm{MeV}$ and $\theta_{x^{\circ}}=120^{\circ} \ldots 143$

Figure 7.31 Proton angular distributions in the ${ }^{3} \mathrm{He}\left(\pi^{\circ}, \pi^{\circ} \mathrm{p}\right)$ reaction at $245 \mathrm{MeV}$ and $\theta_{x^{0}}=70^{\circ} \ldots 144$

Figure 7.32 Proton angular distributions in the ${ }^{3} \mathrm{He}\left(\pi^{-}, \pi^{\circ} \mathrm{p}\right)$ reaction at $245 \mathrm{MeV}$ and $\theta_{x^{0}}=110^{\circ} \ldots 144$

Figure 7.33 Comparison of Proton angular distributions in the ${ }^{3} \mathrm{He}\left(\pi^{*}, \pi^{0} \mathrm{p}\right)$ and ${ }^{3} \mathrm{He}\left(\pi^{-}, \pi^{0} \mathrm{p}\right)$ reactions at

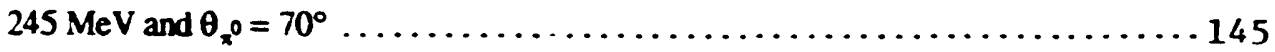

Figure 7.34 Comparison of Proton angular distributions in the ${ }^{3} \mathrm{He}\left(\pi^{*}, \pi^{0} \mathrm{p}\right)$ and ${ }^{3} \mathrm{He}\left(\pi^{-}, \pi^{0} \mathrm{p}\right)$ reactions at

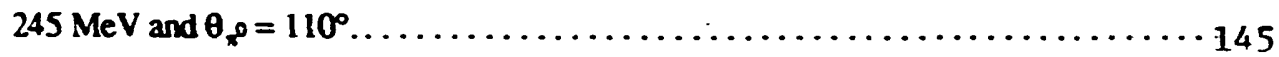

Figure 7.35 Comparison of two dimensional gaussian fit with the measured proton angular distribution

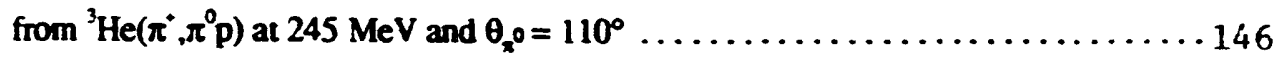

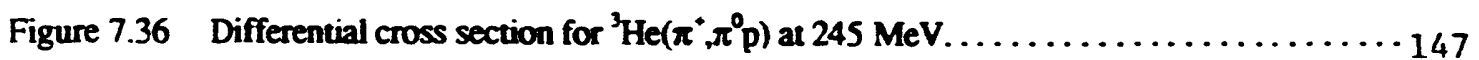

Figure 7.37 Comparison of quasi-free pion single charge exchange and inelastic scattering on ${ }^{3} \mathrm{He} .15 n$

Figure 7.38 Comparisons of single-arm and coincidence measurements of quasi-free pion single charge

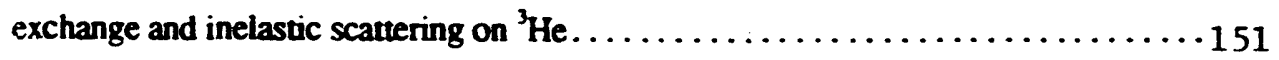

Figure 8.1 Schematic diagram of quasi-free pion-induced nucleon knockout reaction . . . . . . . 154

Figure 8.2 Comparison of PWIA and DWIA calculations for $\pi^{\circ}$ energy spectra from the $\mathrm{He}\left(\pi^{ \pm}, \pi^{9}\right)$ reactions at $\pi^{\circ}$ taboratory scattering angles of $62^{\circ}, 70^{\circ}$, and $78^{\circ}$ and an incident pion

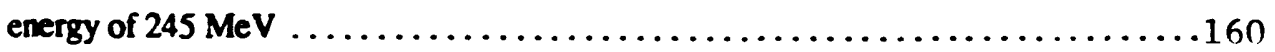

Figure 8.3 Comparison of PWIA and DWIA calculations for $\pi^{\circ}$ energy spectra from the ${ }^{3} \mathrm{He}\left(\pi^{*}, \pi\right)$ reactions at $\pi^{\circ}$ laboratory scattering angles of $102^{\circ}$ and $110^{\circ}$ and an incident pion energy of

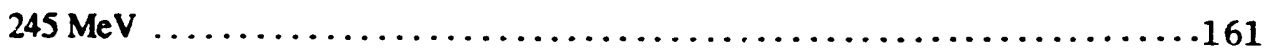

Figure 8.4 Comparison of PWIA and DWIA calculations for $\pi^{0}$ energy spectra from the ${ }^{3} \mathrm{He}\left(\pi^{ \pm}, \pi^{9}\right)$ reactions at $\pi^{\circ}$ laboratory scattering angles of $90^{\circ}, 120^{\circ}$, and $128^{\circ}$ and an incident pion

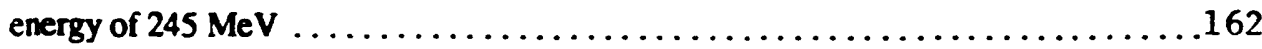

Figure 8.5 Differential crass sections for ${ }^{3} \mathrm{He}\left(\pi^{*}, \pi^{0}\right)$ and ${ }^{3} \mathrm{He}\left(\pi^{-}, \pi^{0}\right)$ at an pion incident energy of 245 $\mathrm{MeV}$ in comparison with DWIA predictions. . . . . . . . . . . . . . . . . . . 164

Figure 8.6 Differential cross sections for ${ }^{3} \mathrm{He}\left(\pi^{\circ}, \pi^{0} \mathrm{p}\right)$ at an pion incident energy of $245 \mathrm{MeV}$ in

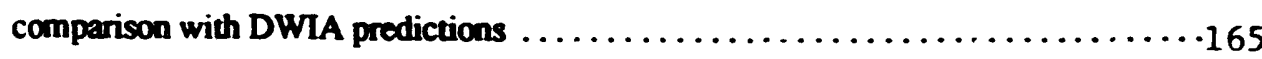

Figure 8.7 Comparisons of doubly differential cross sections for pion inelastic scattering and single charge exchange on ${ }^{3} \mathrm{He}$ with DWIA calculations. . . . . . . . . . . . . . . . 168

Figure 8.8 Domparisons of doubly differential cross sections for pion inelastic scattering and single charge exchange on ${ }^{3} \mathrm{He}$ with DWIA calculations. . . . . . . . . . . . . . . . . . . . 169

Figure 8.9 Comparisons of doubly differential cross sections for pion inelastic scatuering and charge exchange on ${ }^{3} \mathrm{He}$ with DWIA calculations. ........................ 170

Figure B.1 Litule Yellow Spectrometer Monte Carlo Flow Chart.................... 182 
Figure B.2 Comparison of Mionte Carlo resulti with measured acceptance function of the Litle Yellow Spectrometer ................................ 184

Figure B.3 Deviation from the central momentum setting of the spectrometer of a monoenergetic point-like source of particles as a function of Monte Carlo peak position in the dispersive direction at the focal plane.

Figure B.4 Reci il ${ }^{3} \mathrm{H}$ momentum distribution from $\pi^{\circ}{ }^{3} \mathrm{H}$ elastic scattering at $180 \mathrm{MeV}$ and $\theta_{3}=60^{\circ}$ in cor parison with Monte Carlo predictions. . . . . . . . . . . . . . . . 186

Figure B.5 Laboratory differential cross secticns for $p\left(\pi^{*}, p\right) \pi^{*}$ elastic scattering at $142 \mathrm{MeV}$ from $\pi \mathrm{N}$

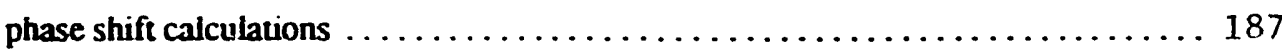

F:gure B.6 Empirical functions to generate angular coordinates using the rejection method for Monte

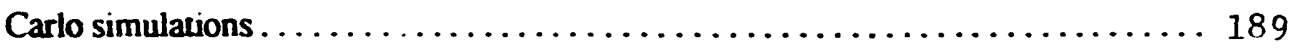

Fir,ure B.7 Monte Carlo distribution of proton laboratory scattering angles in comparison with the polynomial fit to the laboratory differential cross section for $\pi^{*} p$ elastic scattering at 142

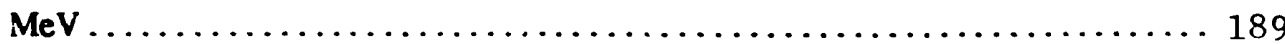

Figure B.8 Measured ${ }^{3} \mathrm{He}$ yield from ${ }^{3} \mathrm{H}\left(\pi^{+}{ }^{3} \mathrm{He}\right) \pi^{0}$ at $142 \mathrm{MeV}$ and $\theta_{3}=65^{\circ} \ldots \ldots \ldots \ldots \ldots 190$

Figure B.9 Calculation of the correction iactor, $\gamma \ldots \ldots \ldots \ldots \ldots \ldots \ldots \ldots \ldots \ldots \ldots \ldots \ldots \ldots \ldots \ldots \ldots$

Figure C. 1 Typical power law fit to data from proton range tables................. 194

Figure D.1 Pulse height spectra from an E scintillation counter $\ldots \ldots \ldots \ldots \ldots \ldots \ldots \ldots \ldots$

Figure D.2 Pulse height spectra from a $\Delta E$ sintillation counter.................... 198

Figure D.3 E scintillation counter calibration data......................... i99

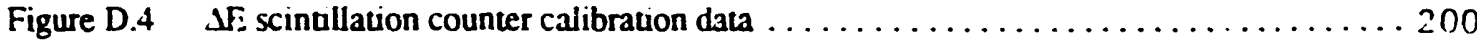




\section{List of Tables}

Table $1.1 \quad$ Properties of the $\Delta$-resonance. ................................ 5

Table 1.2 A selection of theoretical work on elastic single charge exchange in the three nucleon

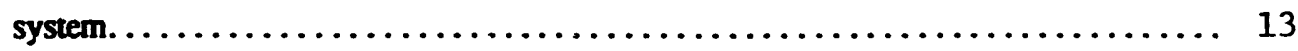

Table 1.3 Selection rules for $\pi \mathrm{NN} \leftrightarrow \mathrm{NN}$ near threshold $\ldots \ldots \ldots \ldots \ldots \ldots \ldots \ldots \ldots \ldots, 20$

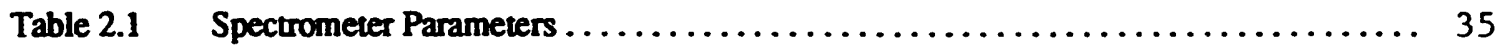

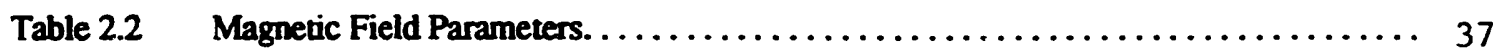

Table $2.3 \quad$ Legend for E1026 Electronics Diagram........................... 42

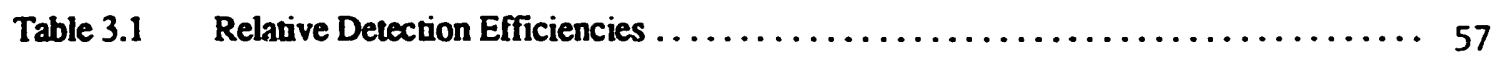

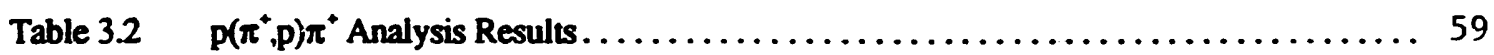

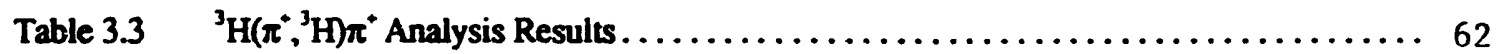

Table 3.4 Doubly Differential Cross Sections for Ti $\left(\pi^{+}{ }^{3}{ }^{3} \mathrm{He}\right) \mathrm{X} \ldots \ldots \ldots \ldots \ldots \ldots \ldots \ldots \ldots \ldots$

Table 3.5 Net ${ }^{3} \mathrm{He}$ yields and correction factors for ${ }^{3} \mathrm{H}\left(\pi^{+},{ }^{3} \mathrm{He}\right) \pi^{0}$ at $\mathrm{T}_{\mathrm{x}}=142 \mathrm{MeV} \ldots \ldots \ldots \ldots 66$

Table 3.6 Net ${ }^{3} \mathrm{He}$ yields and correction factors for ${ }^{3} \mathrm{H}\left(\pi^{+}{ }^{+}, \mathrm{He}\right) \pi^{0}$ at $\mathrm{T}_{x}=180 \mathrm{MeV} \ldots \ldots \ldots \ldots 66$

Table 4.1 Diffesential cross sections for the ${ }^{3} \mathrm{H}\left(\pi^{+},{ }^{3} \mathrm{He}\right) \pi^{0}$ reaction at 142 and $180 \mathrm{MeV} \ldots \ldots \ldots 70$

Table 6.1 List of $\mathrm{CH}_{2}$ target thickness for conversion efficiency measurements $\ldots \ldots \ldots \ldots .99$

Table 7.1 Experimental parameters for the single-arm inclusive single charge exchange

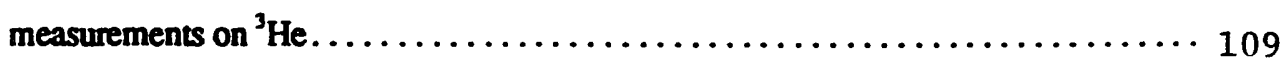

Table 7.2 Differential cross sections for the ${ }^{3} \mathrm{He}\left(\pi^{-}, \pi^{9}\right)$ reaction at $245 \mathrm{MeV} \ldots \ldots \ldots \ldots \ldots \ldots 119$

Table 7.3 Differential cross sections for the ${ }^{3} \mathrm{He}\left(\pi^{+}, \pi^{9}\right)$ reaction at $245 \mathrm{MeV} \ldots \ldots \ldots \ldots \ldots \ldots$

Table 7.4 Experimental parameters for the $\pi^{\circ} \mathrm{p}$ coincidence measurements of single charge exchangc

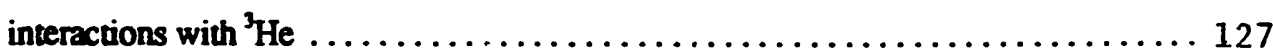

Table 7.5 List of kinematic values for the free single charge exchange reaction, $n \pi^{*} \rightarrow p \pi^{\circ}$, at 245

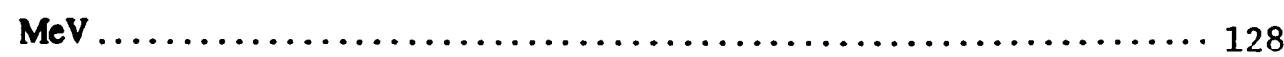

Table 7.6 Differential cross sections for the ${ }^{3} \mathrm{He}\left(\pi^{*}, \pi^{0} \mathrm{p}\right)$ reaction at $245 \mathrm{MeV} \ldots \ldots \ldots \ldots \ldots 147$

iable 8.1 DWLA predictions for differential cross sections for ${ }^{3} \mathrm{He}\left(\pi^{*}, \pi^{9}\right)$ at $245 \mathrm{MeV} \ldots \ldots \ldots 163$

Table 8.2 DWLA predictions for differential cross sections for ${ }^{3} \mathrm{He}\left(\pi^{-}, \pi^{9}\right)$ at $245 \mathrm{MeV} \ldots \ldots \ldots 164$ xoii 
Table 8.3 DWLA predictions for differential cross sections for ${ }^{3} \mathrm{He}\left(\pi^{*}, \pi^{0} \mathrm{p}\right)$ at $245 \mathrm{MeV} \ldots \ldots . .165$

Table A.1 HPL markers for particle identification. ......................... 177

Table B.1 Comparison of measured peak positions with Monte Carlo results for elastic scantering measurements. . . . . . . . . . . . . . . . . . . . . . . . . . . . . . 183

Table B.2 Monte Carlo parameters for recoil angular distributions. . . . . . . . . . . . . 187

Table C.1 Range parameters for energy loss calculations $\ldots \ldots \ldots \ldots \ldots \ldots \ldots \ldots \ldots \ldots$

Table D.1 Proton detector calibration measurements .............................. 195

Table D.2 Energy calibration for E scintillation counters...................... 199

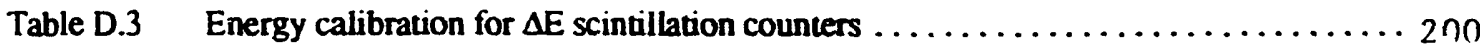

Table E.1 Doubly differential cross sections for the ${ }^{3} \mathrm{He}\left(\pi^{*}, \pi^{9}\right)$ reaction at $T_{\pi}=245 \mathrm{MeV}$ and $\theta_{\pi^{0}}=$

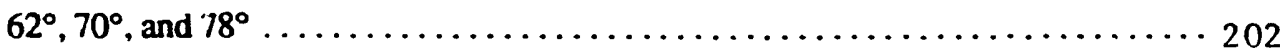

Table E. 2 Doubly differential cross sections for the ${ }^{3} \mathrm{He}\left(\pi^{*}, \pi^{9}\right)$ reaction at $\mathrm{T}_{n}=245 \mathrm{MeV}$ and $\theta_{\pi^{0}}=$

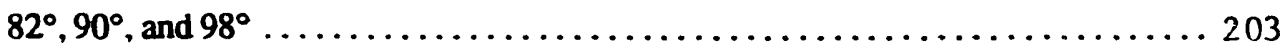

Table E.3 Doubly differential cross sections for the ${ }^{3} \mathrm{He}\left(\pi^{+}, \pi^{9}\right)$ reaction at $T_{x}=245 \mathrm{MeV}$ and $\theta_{\pi^{0}}=$

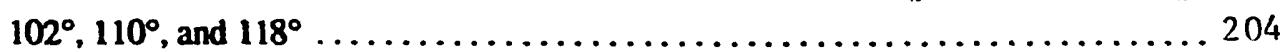

Table E.4 Doubly differential cross sections for the ${ }^{3} \mathrm{He}\left(\pi^{*}, \pi^{9}\right)$ reaction at $\mathrm{T}_{n}=245 \mathrm{MeV}$ and $\theta_{\pi^{0}}=$

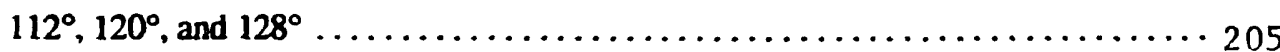

Table E.5 Doubly differential cross sections for the ${ }^{3} \mathrm{He}\left(\pi^{-}, \pi^{9}\right)$ reaction at $\mathrm{T}_{n}=245 \mathrm{MeV}$ and $\theta_{\pi^{0}}=$

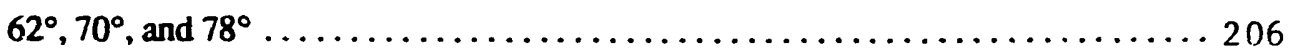

Table E.6 Doubly differential cross sections for the ${ }^{3} \mathrm{He}\left(\pi^{-}, \pi^{0}\right)$ reaction at $T_{n}=245 \mathrm{MeV}$ and $\theta_{\pi^{0}}=$

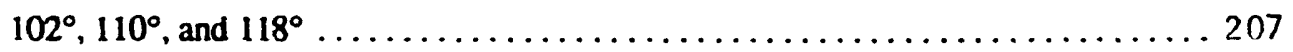

Table E.7 Triply differential cross sections for the ${ }^{3} \mathrm{He}\left(\pi^{*}, \pi^{9}\right)$ reaction at $T_{\pi}=245 \mathrm{MeV}$ and

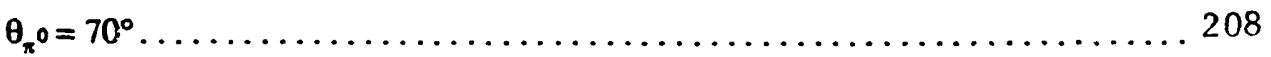

Table E.8 Triply differential cross sections for the ${ }^{3} \mathrm{He}\left(\pi^{*}, \pi^{9}\right)$ reaction at $T_{n}=245 \mathrm{MeV}$ and

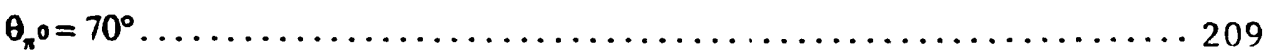

Table E.9 Triply differential cross sections for the ${ }^{3} \mathrm{He}\left(\pi^{*}, \pi^{9}\right)$ reaction at $T_{\pi}=245 \mathrm{MeV}$ and

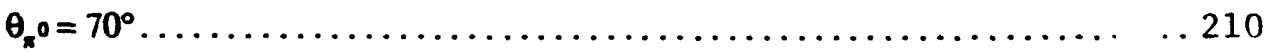

Table E.10 Triply differential cross sections for the ${ }^{3} \mathrm{He}\left(\pi^{*}, \pi^{9}\right)$ reaction at $T_{\pi}=245 \mathrm{MeV}$ and

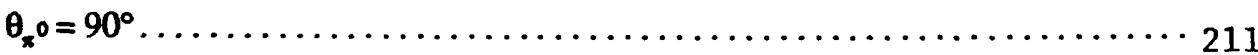

Table E.11 Triply differential cross sections for the ${ }^{3} \mathrm{He}\left(\pi^{*}, \pi^{9}\right)$ reaction at $T_{n}=245 \mathrm{MeV}$ and

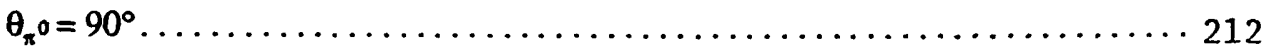

Table E.12 Triply differential cross sections for the ${ }^{3} \mathrm{He}\left(\pi^{*}, \pi^{9}\right)$ reaction at $T_{\pi}=245 \mathrm{MeV}$ and

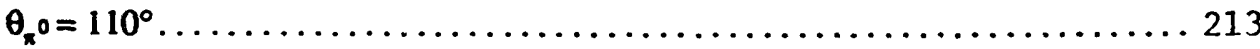


Table E.13 Triply differential cross sections for the ${ }^{3} \mathrm{He}\left(\pi^{*}, \pi^{9}\right)$ reaction at $\mathrm{T}_{\mathrm{x}}=245 \mathrm{MeV}$ and

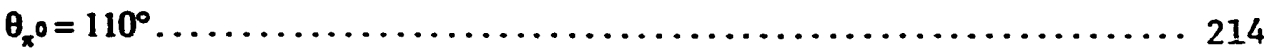

Table E.14 Triply differential cross sections for the ${ }^{3} \mathrm{He}\left(\pi^{+}, \pi^{9}\right)$ reaction at $\mathrm{T}_{\mathrm{x}}=245 \mathrm{MeV}$ and

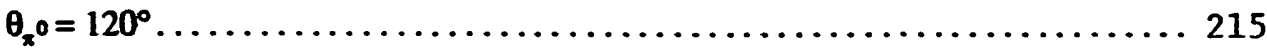

Table E.15 Triply differential cross sections for the ${ }^{3} \mathrm{He}\left(\pi^{+}, \pi^{9}\right)$ reaction at $\mathrm{T}_{\mathrm{n}}=245 \mathrm{MeV}$ and

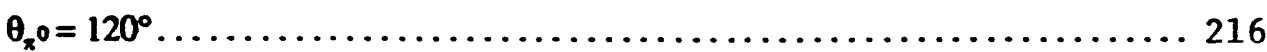

Table E.16 Triply differential cross sections for the ${ }^{3} \mathrm{He}\left(\pi^{-}, \pi^{2}\right)$ reaction at $T_{\pi}=245 \mathrm{MeV}$ and

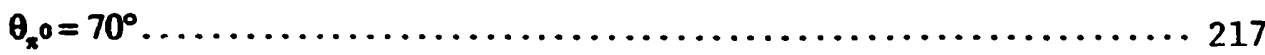

Table E.17 Triply differential cross sections for the ${ }^{3} \mathrm{He}\left(\pi^{-}, \pi^{9}\right)$ reaction at $\mathrm{T}_{\mathrm{z}}=245 \mathrm{MeV}$ and

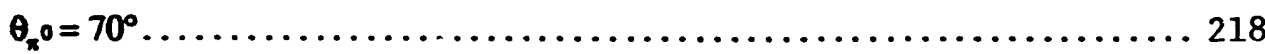

Table E.18 Triply differential cross sections for the ${ }^{3} \mathrm{He}\left(\pi^{-}, \pi^{\circ}\right)$ reaction at $\mathrm{T}_{\mathrm{z}}=245 \mathrm{MeV}$ and

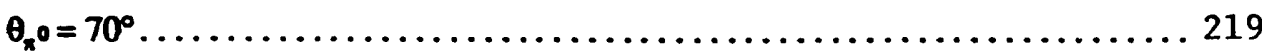

Table E.19 Doubly differential cross sections for the ${ }^{3} \mathrm{He}\left(\pi^{*}, \pi^{9}\right)$ reaction at $T_{x}=245 \mathrm{MeV}$ and

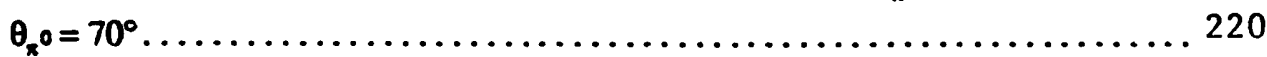

Table E.20 Doubly differential cross sections for the ${ }^{3} \mathrm{He}\left(\pi^{*}, \pi^{9}\right)$ reaction at $\mathrm{T}_{\mathrm{x}}=245 \mathrm{MeV}$ and

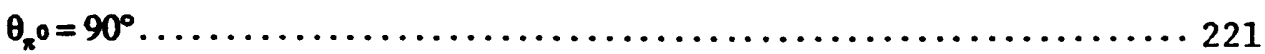

Table E.21 Doubly differential cross sections for the ${ }^{3} \mathrm{He}\left(\pi^{*}, \pi^{9}\right)$ reaction at $\mathrm{T}_{\mathrm{x}}=245 \mathrm{MeV}$ and

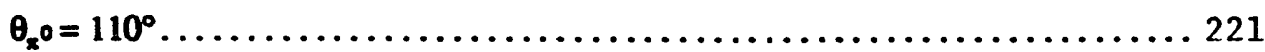

Table E.22 Doubly differential cross sections for the ${ }^{3} \mathrm{He}\left(\pi^{*}, \pi^{9}\right)$ reaction at $\mathrm{T}_{\mathrm{x}}=245 \mathrm{MeV}$ and

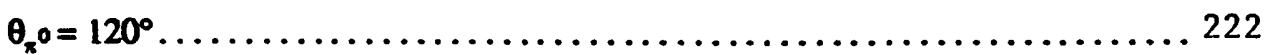

Table E.23 Doubly differential cross sections for the ${ }^{3} \mathrm{He}\left(\pi^{-}, \pi^{0}\right)$ reaction at $\mathrm{T}_{\mathrm{z}}=245 \mathrm{MeV}$ and

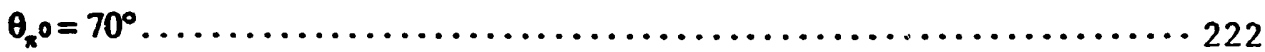

Table E.24 Doubly differential cross sections for the ${ }^{3} \mathrm{He}\left(\pi^{-}, \pi^{0}\right)$ reaction at $\mathrm{T}_{\pi}=245 \mathrm{MeV}$ and

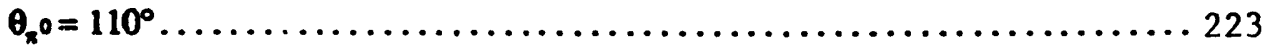




\title{
PION SINGLE CHARGE EXCHANGE \\ IN THREE BODY NUCLEI AT INTERMEDIATE ENERGIES
}

by

\author{
Marla L. Dowell
}

\begin{abstract}
The purpose of this thesis is to present new experimental information about modifications to the pionnucleon single charge exchange interaction, $\pi^{+} n \rightarrow \pi^{0} p$ or $\pi \mathbf{p} \rightarrow \pi^{\mathbf{l}} \mathbf{n}$, due to the presence of other nucleons. The results of two experimental studies of pion single charge exchange in the three nucleon system near the $\Delta$-resonance are presented. Both of these experiments were performed at the Clinton P. Anderson Meson Physics Facility (LAMPF), a division of the Los Alamos National Laboratory. Each explored different aspects of pion single charge exchange in three body nuclei- ${ }^{3} \mathrm{He}$ and ${ }^{3} \mathrm{H}$. Since the nuclear wavefunctions of the three nucleon systems are believed to be well understood, it should be possible to perform theoretical calculations of pion interactions with this system and compare their predictions with the experimental results.

The first study examined the pion-induced isobaric analog transition in the $A=3$ system, ${ }^{3} \mathrm{H}\left(\pi^{+}, \pi^{0}\right)^{3} \mathrm{He}$, at incident pion energies of 142 and $180 \mathrm{MeV}$. The three nucleon system is the simplest system in which this exclusive single charge exchange process may be observed. Although several experiments have observed the ${ }^{3} \mathrm{H}\left(\pi^{+}, \pi^{0}\right)^{3} \mathrm{He}$ or ${ }^{3} \mathrm{He}\left(\pi, \pi^{0}\right)^{3} \mathrm{He}$ process from incident pion energies of 131 to $525 \mathrm{MeV}$, the data are limited to intermediate and large $\pi^{0}$ center of mass angles with the exception of one forward angle measurement at $200 \mathrm{MeV}$. The incident energies and $\pi^{0}$ angular ranges for this set of measurements were chosen to overlap with existing exclusive pion single charge exchange and elastic scattering data on ${ }^{3} \mathbf{H}$. A magnetic spectrometer, equipped with an array of nineteen $400 \mu \mathrm{m}$ silicon surface barrier Jetectors, was used to detect the recoil ${ }^{3} \mathrm{He}$ particle. The tritium content of the tritiated titanium foils, the material used as targets in this measurement, was determined from ${ }^{3} \mathrm{H}\left(\pi^{+},{ }^{3} \mathrm{H}\right) \pi^{+}$elastic scattering measurements.

The exclusive single charge exchange measurements presented in this thesis are the first of their kind at forward $\pi^{0}$ angles below $200 \mathrm{MeV}$. These results are a better test of the spin-flip contribution to the differential cross section. Previous measurements at large $\pi^{0}$ angles are not as sensitive to the spin-flip contribution which is expected to have a strong influence on the angular distribution.

The second set of measurements observed two types of inclusive single charge exchange processes on ${ }^{3} H e$. Both of these measurements were performed at LAMPF using the LAMPF $\pi$ spectrometer, an array of $\Delta E$-E plastic scintillation counters, and a liquid ${ }^{3} \mathrm{He}$ target. In one measurement, the outgoing $\pi^{0}$ array was detected: ${ }^{3} \mathrm{He}\left(\pi^{ \pm}, \pi^{0}\right)$. Doubly differential cross sections were measured at $\pi^{0}$ laboratory scattering angles between $62^{\circ}$ and $128^{\circ}$ and an incident pion energy of $245 \mathrm{MeV}$. The outgoing $\pi$ energy spectrum was measured over the quasi-free scattering region. These measurements were used as a test of isospin scaling predictions based on the relative strengths of the free pion-nucleon cross sections in the $\Delta$-resonance region. Comparisons with recent measurements of inelastic $\pi^{t}$ scattering from ${ }^{3} \mathrm{He}$ and distorted wave impulse approximation (DWIA) calculations are presented. In the second measurement, neutral pions and protons were detected in coincidence: ${ }^{3} \mathrm{He}\left(\pi^{ \pm}, \pi^{0} \mathrm{p}\right)$. The energy spectra of scattered neutral pions were computed by integrating over outgoing proton kinetic energy. These measurements were performed at an incident pion energy of $245 \mathrm{MeV}$ and $\pi^{0}$ laboratory scattering angles between $70^{\circ}$ and $120^{\circ}$. In the $3 \mathrm{He}\left(\pi^{+}, \pi^{0} \mathrm{p}\right)$ process, single scattering processes should dominate in the quasi-free scattering region. In ${ }^{3} \mathrm{He}\left(\pi, \pi^{0} \mathrm{p}\right)$, at least two nucleons must be involved in the interaction so that a proton may be detected in coincidence.
\end{abstract}




\section{Chapter 1: Introduction}

This thesis presents recent results from two measurements of pion single charge exchange on three body nuclei at intermediate energies. These experiments were performed at the Clinton P. Anderson Meson Physics Facility (LAMPF). The first experiment examined an exclusive single charge exchange channel in the three nucleon system: ${ }^{3} \mathrm{H}\left(\pi^{*}, \pi^{9}\right)^{3} \mathrm{He}$. The second experiment focused on features of quasi-free pion single charge exchange interactions with ${ }^{3} \mathrm{He}$ through a series of singlo-arm and coincidence measurements: ${ }^{3} \mathrm{He}\left(\pi^{+}, \pi^{9}\right)$ and ${ }^{3} \mathrm{He}\left(\pi^{+}, \pi^{0} \mathrm{p}\right)$. Both experiments were performed at incident pion energies near the $\Delta$-resonance. While extensive data on pion-nucleon interactions exist' and are believed to be well understood $^{23}$ in this energy regime, the theoretical interpretation of pion-nucleus data is more complicated. In a multi-nucleon system, additional pion reaction channels become accessible and multiple scattering cannot be neglected due to the strong nature of the pion-nucieon interaction. Theoretical calculations of these interactions often involve phenomenological potentials to model the strong interaction between pions and nuclei. Theoretical efforts to extend free pion-nucleon scattering amplitudes to off-shell behavior have successfully reproduced the general features of pion elastic and quasi-elastic scattering data on $A=3$ nuclei. These models have not been as successful with existing pion single charge exchange data on the same nuclei. ${ }^{4.56}$

After a brief discussion of the general features of the pion-nucleon interaction, the motivations for the specific single charge exchange studies on three body nuclei will be reviewed. Previous measurements ${ }^{78.9}$ of the pion-induced isobaric analog transition, $\quad{ }^{3} \mathrm{H}\left(\pi^{+}, \pi^{9}\right)^{3} \mathrm{He}$ and ${ }^{3} \mathrm{He}\left(\pi^{-}, \pi^{9}\right)^{3} \mathrm{H}$ reactions, will be presented. Then, the top:c of quasi-free pion scattering from ${ }^{3} \mathrm{He}$ will be developed and previous quasi-elastic measurements ${ }^{6}$ on ${ }^{3} \mathrm{He}$ will be presented. The remainder of this thesis may be divided into 
two parts. The first part, Chapters 2 through 4, describes the experimental apparatus, data analysis, and results of the exclusive single charge exchange measurement. Recent calculations ${ }^{10.11}$ of differential cross sections for the pion-induced isobaric analog transition will be compared with this measurement. In the second part, Chapters 5 and $6 \mathrm{f}$ resent a similar outline of the experimental set-up and data analysis procedures for the inclusive single charge exchange measurements on ${ }^{3} \mathrm{He}$. The results of the single-arm and coincidence measurements are presented in Chapter 7 and compared with recent Distorted Wave Impuise Approximation (DWIA) calculations in Chapter 8. Finally, the results of both measurements will be summarized in Chapter 9.

\section{Overview of Pion Interactions at Intermediate Energies}

Research efforts in nuclear physics have focused on the study of nuclear structure and reactions, of which the simplest is the nucleon-nucleon (NN) interaction. The nucleon, the neutron or proton, is believed to be made up of three quarks bound together by the strong or "color" force. In current models of the fundamental forces in nature, elementary particles may be classified as either quarks or leptons and quantum chromodynamics (QCD) is the relativistic quantum field theory which describes the fundamental interactions between quarks. Although the nucleon itself is a color neutral object, the nucleon-nucleon interaction is a direct result of the strong interaction between quarks. ${ }^{12}$ At this time, no quantitative connection between the strong interaction and the nucleon-nucleon interaction exists. By gathering experimental information about the nucleon-nucleon interaction, it may be possible to elicit a relationship between the nucleon-nucleon interaction and the underlying quark structure of nucleons.

The meson exchange model is a useful starting point for discussion of the nucleon-nucleon interaction. In this model, introduced by Yukawa in 1934, two nucleons interact via the exchange of a virtual meson. This is analogous to the Coulomb interaction between two electrons via the excitange of a virtual photon. Examples of Feynman diagrams for these two processes are shown in Figure 1.1. In Yukawa's original model, the mass of this virtual meson was deduced from the range of the nucleonnucleon interaction and is approximately $140 \mathrm{MeV} / \mathrm{c}^{2}$. This meson, the pion or $\pi$, was first observed 
experimentally in 1948. As it turns out, the pion is the lightest member of the meson family, and thus is responsible for the long range part of the interaction, i.e. distances greater than $\sim 2 \mathrm{fm} .{ }^{12}$ One can construct a phenomenological potential based on the meson exchange model that is consistent with the radial dependence of the nucleon-nucleon interaction as shown in Figure 1.2.

a)

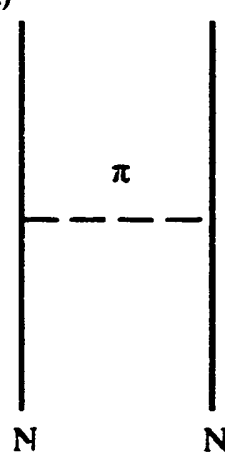

b)

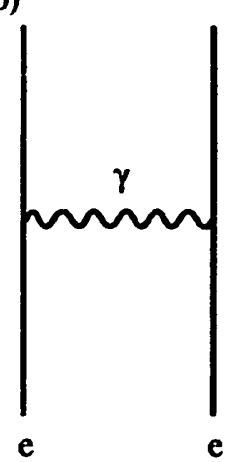

Figure 1.1: Feynman diagrams for a) virual pion exchange between two nucleons and b) virtual photon exchange between two electrons.

In the strong interaction picture, protons and neutrons may be thought of as different states of the same particle: the nucleon. Both are spin 1/2 particles and the mass difference between them is less than 1\%. Similarly, the three charge states of the pion may be thought of as different states of the same particle: $\pi^{+}, \pi^{0}$, and $\pi^{-}$. All three are spin 0 bosons with negative intrinsic parity. The mass difference between the charged and neutral pions is less than $3 \%$. Isospin is a quantum number which can be used to distinguish between these different charge states of the nucleon and pion. The rules for the addition of isospin are the same as those for the addition of angular momenta. The value assigned to the total isospin of a nucleon or pion is $1 / 2$ or 1 respectively. The third component of isospin, $T_{z}$, is related to the charge, $Q$, of a nucleon or pion through the following relationship,

$$
Q=T_{z}+\frac{1}{2} B
$$

where $B$ is the baryon number of the particle. 
Figtire 1.2: Schematic diagram ${ }^{12}$ of the nucleonnucleon potential as a function of the radial distance between two nucleons, $r$. In this diagram, the one pion exchange picture, responsible for the long range part of the nucleon-nucleon interaction, has been generalized to include the exchange of other bosons. In this model, a potential, with the appropriate radial dependence at shor $(r<1 \mathrm{fm})$, intermediate $(1 \mathrm{fm} \leq$ $r \leq 2 \mathrm{fm})$, and long distances $(r>2 \mathrm{fm})$, can be constructed by including the exchanges of heavier mesons or the exchange of more than one pion. The strength of the potential at intermediate ranges is ascribed to the exchange of two pions or a heavier meson, e.g. a p-meson. The short range, or hard core, part of the interaction is attributed to the exchange of heavy mesons, multi-pion exchanges, and may also inciude QCD effects. A phenomenological potential, known as the one boson exchange potential, has been developed to simplify the nucleon-nucleon interaction picture. In this model, only the exchange of a single boson is allowed. The range of each meson in this model is an adjustable parameter which has been determined from fitting experimental data, i.e. nucleon-nucleon scattering data. More than $1 \mathrm{GeV}$ of kinetic energy must be added to the two nucleon system to shorten the radial separation between two nucleons to less than $1 \mathrm{fm}$ and overcome the hard core repulsion. ${ }^{12}$

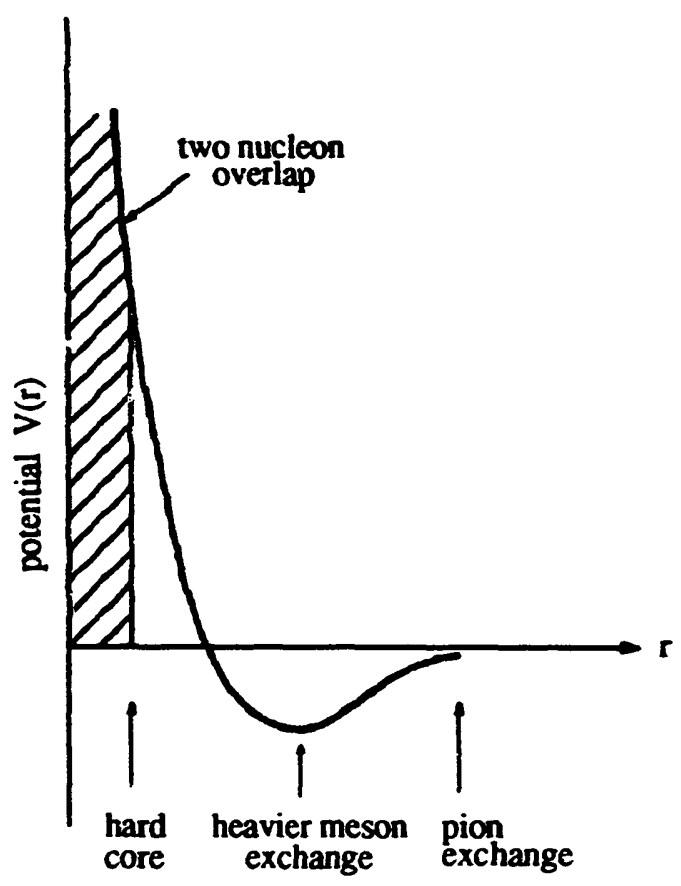

Several important properties of the pion make it a unique probe of the nucleon-nucleon interac-

tion. Since the pion is a boson it may either be created or absorbed: $\pi N N \rightarrow N N$ or $\pi N \rightarrow \pi \pi N$, which is an essential feature of the pion in its role as mediator of the nucleon-nucleon interaction. As an isovector particle, the pion is also responsible for the charge exchange part of the NN interaction as shown in Figure 1.3. Since the pion is a strongly interacting particle, it may be used as a probe of nuclear matter distributions in a manner similar to the use of electrons as probes of the electromagnetic properties of nuclei. 

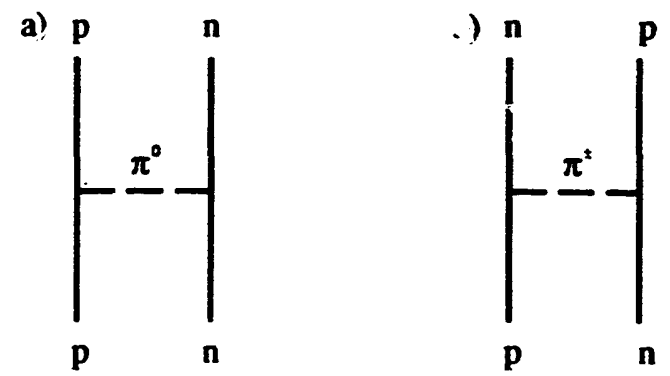

Figure 1.3: Diagrams of pn a) scattering and b) charge exchange.

\begin{tabular}{|c|l|}
\hline mass & $1232 \mathrm{MeV}$ \\
\hline $\mathrm{J}^{\mathrm{p}}$ & $3 / 2^{+}$ \\
\hline FWHM $\Gamma$ & $-115 \mathrm{MeV}$ \\
\hline Isospin & $3 / 2$ \\
\hline Charge states & $\Delta^{*+}, \Delta^{*}, \Delta^{\circ}, \Delta^{-}$ \\
\hline Decay Mode & $\pi \mathrm{N}(99.4 \%)$ \\
\hline
\end{tabular}

Table 1.1: Properties of the $\Delta$-resonance. ${ }^{13}$

\section{Pion-nucleon Interactions and the $\Delta$-resonance}

To first approximation, the pion interacts with each nucleon in a nucleus individually. Therefore, in order to understand pion-nucleus reactions it is crucial that the free pion-nucleon $(\pi N)$ interaction be well understood. Near incident pion energies of $200 \mathrm{MeV}$, the total cross section for $\pi \mathrm{N}$ scattering is dominated by the $\Delta$-resonance as shown in Figure 1.4. The properties of the $\Delta$-resonance are summarized in Table 1.1. 
The $\Delta$-resonance has several important features. It has well-defined quantum numbers $(\mathrm{J}=3 / 2$, $T=3 / 2$ ) and is well separated from any other resonances observert in $\pi N$ reactions. The $\Delta$, which is sometimes thought of as an excited state of the nucleon, is excited primarily through a $p$-wave $(\ell=1)$ coupling between the pion and nucieon. Therefore, the $\pi \mathrm{N}$ differential cross sections exhibit a characteristic angular dependence in the $\Delta$-resonance region of the form:

$$
F\left(\theta_{a x}\right)=1+3 \cos ^{2} \theta_{c u}
$$

The dominance of the $\Delta$-resonance in $\pi \mathrm{N}$ interactions at intermediate energies also leads to simple isospin scaling relationships between cross sections for different $\pi \mathrm{N}$ reaction channels below incident pion energies of $350 \mathrm{MeV}$. The isospin wavefunctions, $\left|T T_{z}\right\rangle$, of a $\pi \mathrm{N}$ system may be decomposed into $\mathrm{T}=3 / 2$ and $T=1 / 2$ components as îllows,

$$
\begin{gathered}
\left|\pi^{+} p\right\rangle=\left|\frac{3}{2} \frac{3}{2}\right\rangle \\
\left|\pi^{0} p\right\rangle=\sqrt{\frac{2}{3}}\left|\frac{3}{2} \frac{1}{2}\right\rangle-\frac{1}{\sqrt{3}}\left|\frac{1}{2} \frac{1}{2}\right\rangle \\
\left|\pi^{+} n\right\rangle=\frac{1}{\sqrt{3}}\left|\frac{3}{2} \frac{1}{2}\right\rangle+\sqrt{\frac{2}{3}}\left|\frac{1}{2} \frac{1}{2}\right\rangle \\
\left|\pi^{-} p\right\rangle=\frac{1}{\sqrt{3}}\left|\frac{3}{2}-\frac{1}{2}\right\rangle-\sqrt{\frac{2}{3}}\left|\frac{1}{2}-\frac{1}{2}\right\rangle \\
\left|\pi^{0} n\right\rangle=\sqrt{\frac{2}{3}}\left|\frac{3}{2}-\frac{1}{2}\right\rangle+\frac{1}{\sqrt{3}}\left|\frac{1}{2}-\frac{1}{2}\right\rangle \\
\left|\pi^{-} n\right\rangle=\left|\frac{3}{2}-\frac{3}{2}\right\rangle
\end{gathered}
$$

where $T$ and $T_{z}$ are the total isospin and its third component respectively. The total sections for $\pi \mathrm{N}$ reactions may be expressed in terms of $T=3 / 2$ and $T=1 / 2$ scattering amplitudes, e.g.,

$$
\begin{aligned}
\sigma\left(\pi^{-} p \rightarrow \pi^{-} p\right) & \propto\left\langle\pi^{-} p|A| \pi^{-} p\right\rangle^{2} \\
& =\frac{1}{9}\left|A_{3 / 2}\right|^{2}+\frac{4}{9}\left|A_{1 / 2}\right|^{2}
\end{aligned}
$$

Comparisons of measurements of the cross sections for $\pi^{*} p$ scattering, shown in Figures 1.4 and 1.5 , indicate that the contribution from the $T=1 / 2$ channel may be ignored in the $\Delta$-resonance regime.

Predictions for the relative strengths of each $\pi \mathrm{N}$ channel may be computed using Equations 1.3 through 1.7. Neglecting the contributions of the $T=1 / 2$ scattering amplitude leads to the following scaling relationship near incident pion energies of $200 \mathrm{MeV}$, 


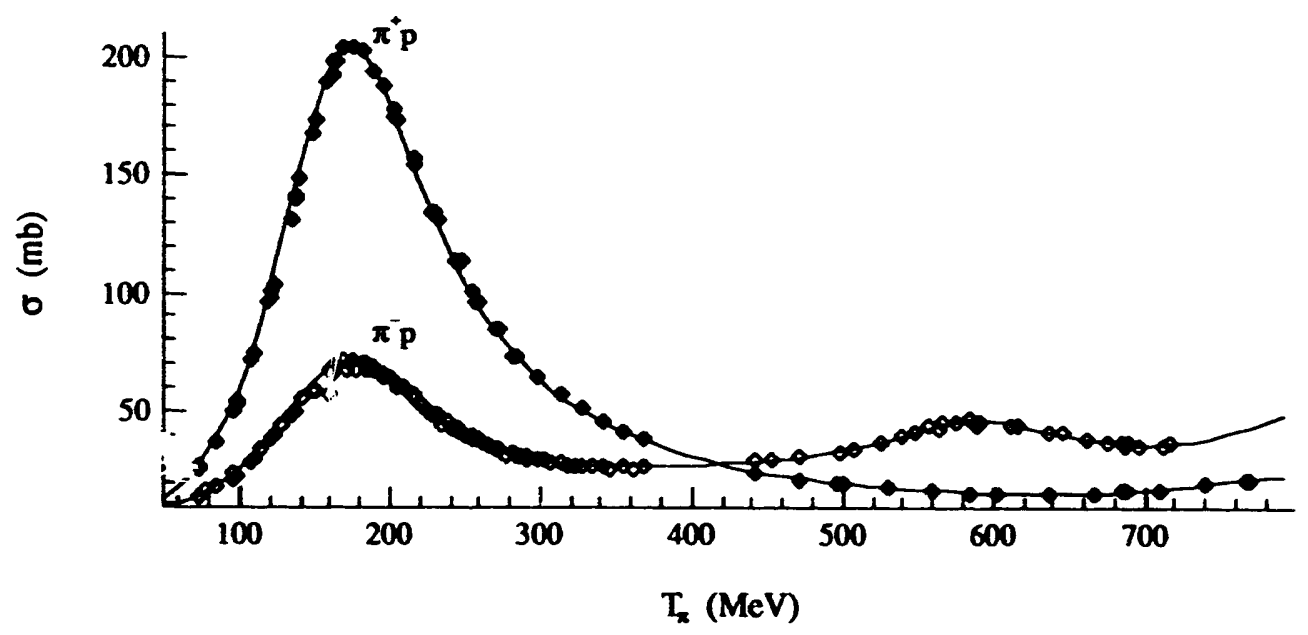

Figure 1.4: Total cross sections for $\pi^{+} p$ (solid points) and $\pi$ p (open points) reactirins as a function of incident pion energy. The $\pi$ p result includes tharge exchange and non-charge exchange contributions to the total cross section. The curves are from $\pi \mathrm{N}$ phase shift calculations; ${ }^{14}$ the data points represent a selection of experimental results. ${ }^{15,1617,18,99}$

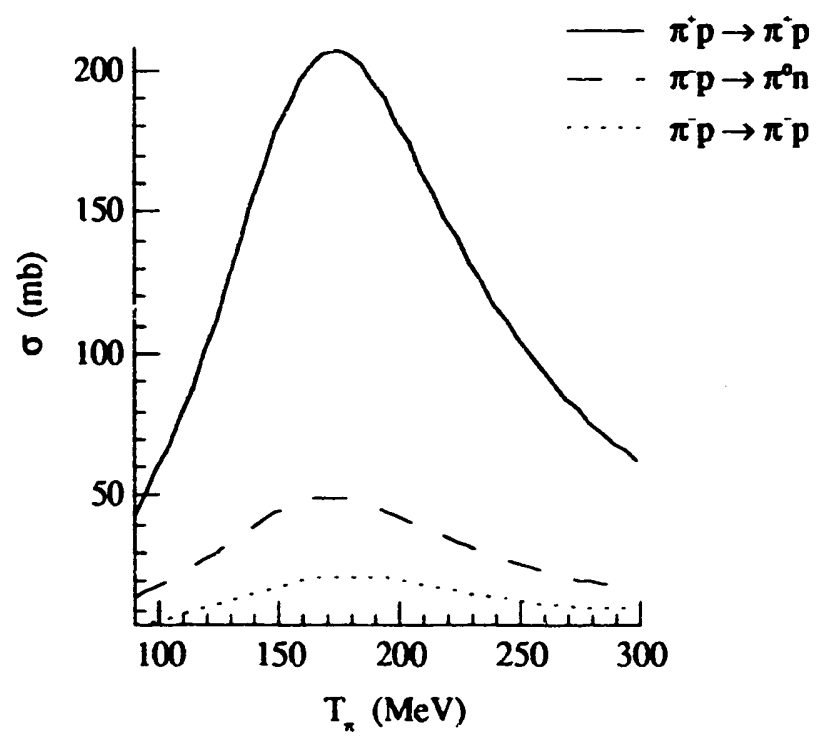

Figure 1.5: Total cross sections for $\pi$ p elastic scattering and charge exchange in the $\Delta$-resonance region from $\pi \mathrm{N}$ phase shift calculations. ${ }^{20}$ These calculations reproduce $\pi^{+} \mathrm{p}$ data quite well as shown in Figure 1.4. In the $\Delta$-resonance region, these total cross sections scale as the contributions of the $T=3 / 2$ scattering amplitudes as expressed in Equation 1.10. 


$$
\sigma\left(\pi^{+} p \rightarrow \pi^{+} p\right): \sigma\left(\pi^{-} p \rightarrow \pi^{0} n\right): \sigma\left(\pi^{-} p \rightarrow \pi^{-} p\right)=9: 2: 1
$$

A compari:on of $\pi \mathrm{N}$ phase shift predictions for charge exchange and non-charge exchange cross sections is shown in Figure 1.5. The relative strengths of these cross sections agree with the simple isospin prediction from Equation 1.10.

\section{Pion-Luclo, as Interactions and the $\Delta$-resonance}

To understand the role of the pion in the nucleon-nuck:on interaction, one must examine pion interactions with nuclei in addition to the free $\pi \mathrm{N}$ interaction. Experimental information on pion absorption and pion production interactions is necessary to characterize the pion's role as mediator of the nucleonnucleon interaction. Siudies of pion absorption processes must be performed with a nuclear system since at least two nucleons must be involved in the interaction to satisfy energy and momentum conservation constraints. Moreover, multiple scattering and other multi-nucleon processes can play an important role in pion-nucleus interactions. Theoretical calculations of pion-nucleus interactions can be difficult to perform due to the strong coupling between the pion and the nuclear system and the abundance of available reaction channels, e.g. scattering, absorption, and three-body interactions. Fortunately, the dominance of the $\Delta$-resonance and its set of well-defined quantum numbers can be exploited to simplify the interpretation of pion-nucleus interactions at intermediate energies. This $\Delta$-dominance leads to a natural representation of these interactions: the $\Delta$-hole model. In this model, a pion can couple to a nucleon and excite a $\Delta$ leaving a 'hole' in an energy shell. The $\Delta$ may in turn decay $(\Delta \rightarrow \pi N)$, be absorbed $(\Delta N \rightarrow N N)$, or scatter from another nucleon ( $\Delta N \rightarrow \Delta N)$ as it propagates through the nucleus. Since the $\Delta$ is distinguishable from a nucleon, the Pauli exclusion principle does not apply as it would to a nucleon propagating through a nucleus. In this manner, the $\Delta$ acts as a 'doorway' to a variety of other reaction channels."

Although information from multi-nucleon processes may be difficult to interpret, it is necessary to complete the theoretical picture of the nucleon-nucleon interaction. Studies of pion-nucleus interactions may lead to a better understanding of modifications to the nucleon-nucleon interaction in the nuclear medium and of nucleon-nucleon correlations in nuclei. Pions and other strongly interacting particies are better probes of multi-nucleon processes than electrons since the ratio of the coupling constants for the 
strong and electromagnetic interactions is roughly

$$
\alpha_{s}: \alpha=1: \frac{1}{137} .
$$

A series of experiments, which expliciuly probe multi-nucleon processes with pions, has been devised. In one type of experiment, studies of pion double charge exchange (DCX) reactions, an inc.dent piun exchanges two units of charge with a nucleus. At least two nucleons must be involved in the DCX reaction for it to proceed. An example of pion double charge exchange on ${ }^{3} \mathrm{He}$ is shown in Figure 1.6 . The results of an experiment to observe the ${ }^{3} \mathrm{He}\left(\pi^{-}, \pi^{0} \mathrm{p}\right)$ reaction are presented in this thesis. As in DCX, at least two nucleons must be involved in this reaction for a proton with sufficient kinetic energy to be detected in coincidence. Diagrams of some two nucleon mechanisms for the ${ }^{3} \mathrm{He}\left(\pi^{-}, \pi^{0} \mathrm{p}\right)$ reaction are shown in Figures 1.6, and 1.18 through 1.21. This process will be discussed in more detail later in this Chapter.

a)

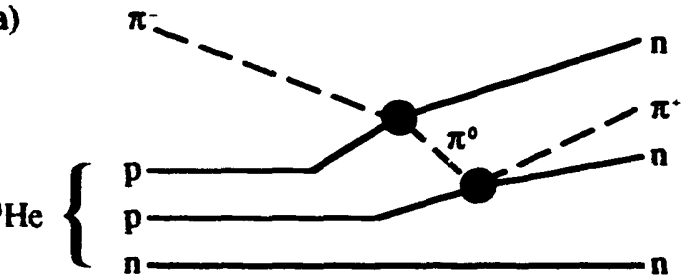

b)

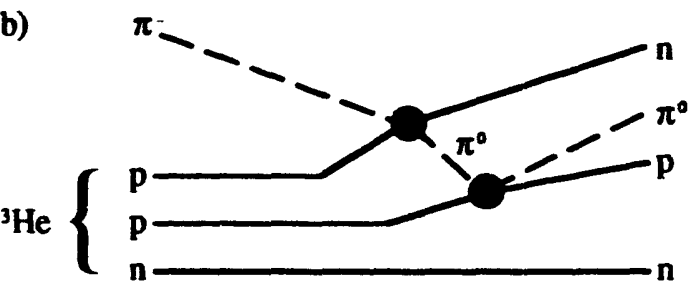

Figure 1.6: Diagrams for a) pion double charge exchange on ${ }^{3} \mathrm{He}$ and b) the ${ }^{3} \mathrm{He}\left(\pi^{-}, \pi^{0} \mathrm{p}\right)$ reaction. In both of these examples, the reaction proceeds through two sequential pion interactions, either single charge exchange or scattering, with the two protons in ${ }^{3} \mathrm{He}$.

The general structure of the three nucleon system is believed to be well understood. The momentum distribution and separation energies of protons in ${ }^{3} \mathrm{He}$ have been deduced from ${ }^{3} \mathrm{He}\left(\mathrm{e}, \mathrm{e}^{\prime} \mathrm{p}\right)$ measurements. ${ }^{22.23}$ In addition, the longitudinal and transverse structure functions of ${ }^{3} \mathrm{He}$ and ${ }^{3} \mathrm{H}$ have been extracted from inclusive electron scattering measurements. ${ }^{20.25}$ Since the nuclear structure is well known, calculations of pion interactions with either ${ }^{3} \mathrm{H}$ or ${ }^{3} \mathrm{He}$ may elicit modifications to the pion-nucleon 
interaction due to the presence of other nucleons. Furthermore, a number of interesting comparisons can be made between the inclusive pion single charge exchange measurements from this work and the existing pion scautering and double charge exchange data on ${ }^{3} \mathrm{He}$ and ${ }^{3} \mathrm{H}$.

\section{Elastic Single Charge Exchange Measurements: ${ }^{3} \mathrm{H}\left(\pi^{+}, \pi^{0}\right)^{3} \mathrm{He}$}

The excitation of an isobaric analog state through pion charge exchange is an important reaction channel in pion-nucleus interactions. The isobaric analog state of a nucleus can be arrived at through the application of an isospin raising or lowering operator to the original nuclear wavefunction, i.e. the isobaric analog will have the same mass number, $A$, but different atomic number, $Z \pm 1$, from the initial nucleus. The isobaric analog state is nearly an eigenstate of the initial nuclear wavefunction and shares many common nuclear properties with the original nucleus, e.g. mass and total iscspin. Experimentally, an isobaric analog state may be excited through a $(p, n)$ reaction as well as a pion single charge exchange reaction. The pion-induced isobaric analog transition is often referred to as an elastic charge exchange process since the initial and final nuclear states are nearly identical if the third component of isospin is neglected. The three nucleon system is the simplest system in which a pion-induced isobaric analog transition may be observed, i.e. ${ }^{3} \mathrm{He}\left(\pi, \pi^{9}\right)^{3} \mathrm{H}$ or ${ }^{3} \mathrm{H}\left(\pi^{*}, \pi^{9}\right)^{3} \mathrm{He}$.

\section{Review of Theoretical Work}

In 1975, Sparrow predicted that the spin flip $(\Delta S=1)$ contribution to elastic charge exchange in the three nucleon system should be quite large for incident pion energies between 100 and $200 \mathrm{MeV}$. Until this time, the spin flip contribution was expected to have the same negligible effect on elastic charge exchange as in pion-nucleus elastic scattering. The results of Sparrow's calculation are shown in Figure 1.7. In the region of the nor:-spin flip $(\Delta S=0)$ minimum, the spin flip contribution can produce as large as a factor of five enhancement in the differential cross section. Wakamatsul ${ }^{27}$ performed a full analysis of second order contributions to the elastic scattering and elastic single charge exchange process using an optical potential model. This model included spin and isospin degrees of freedom. According to his calculations, second order effects are more important for elastic charge exchange than for elastic scauering. 

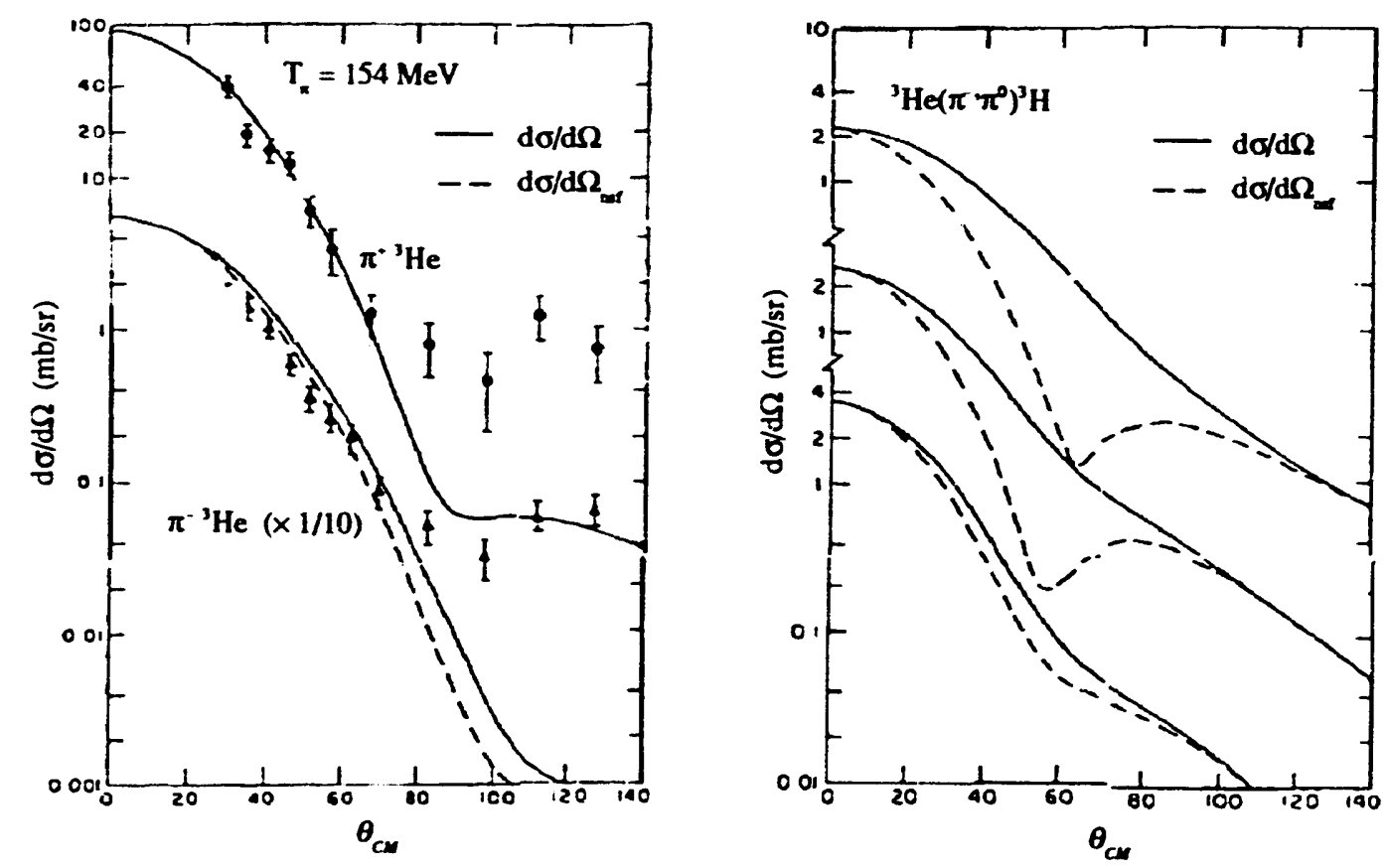

Figure 1.7: Angular distributions ${ }^{26}$ for ${ }^{3} \mathrm{He}\left(\pi^{+}, \pi^{ \pm}\right)^{3} \mathrm{He}$ (left) and ${ }^{3} \mathrm{He}\left(\pi^{-}, \pi^{9}\right)^{3} \mathrm{H}$ (right) with (solid) and without (dashed) spin flip contributions. The angular distribution of the charge exchange reaction is dranatically affected by the spin flip contribution. The charge exchange cross section is plotted for incident pion energies from top to bottom of 160,200 , and $240 \mathrm{MeV}$ respectively.

This is perhaps not surprising since the ratio of the total cross sections for ${ }^{3} \mathrm{He}\left(\pi^{*}, \pi\right)^{3} \mathrm{He}$ and ${ }^{3} \mathrm{H}\left(\pi^{*}, \pi^{9}\right)^{3} \mathrm{He}$ near incident pion energies of $180 \mathrm{MeV}$ is roughly

$$
\sigma_{E}: \sigma_{s c x} \approx 20: 1 \text {. }
$$

Since Sparrow's work in 1975, several authors have calculated angular distributions for elastic scattering and single charge exchange reactions in the three nucleon system. The important features of these charge exchange calculations are a relatively featureless angular dependence and a total cross section whose energy dependence is comparatively flat in the $\Delta$-resonance region. This deviation, from the energy dependence of the total cross section for charge exchange on a free nucleon (see Figure 1.5) and from the energy dependence of the total cross section for pion elastic scattering on $A=3$ nuclei, which also exhibits a 
strong resonance peak, is perhaps surprising in such a simple nucleus. An example of the expected energy dependence for the total elastic single charge exchange cross section as predicted by Hess and Gibson ${ }^{23}$ is shown in Figure 1.8. Examples of the angular distributions are shown in Figures 1.9 through 1.11.

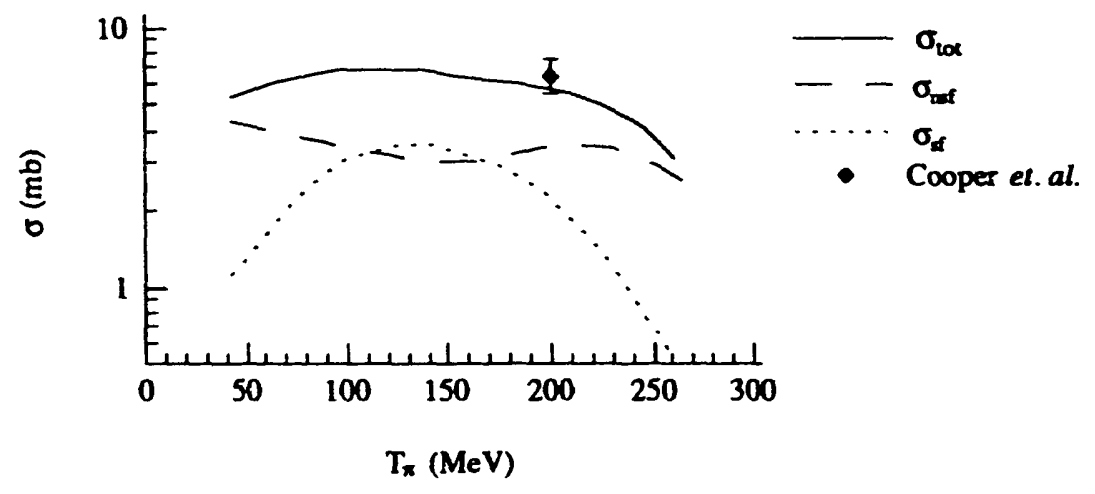

Figure 1.8: Total cross section ${ }^{23}$ (solid line) for ${ }^{3} \mathrm{H}\left(\pi^{-}, \pi^{9}\right)^{3} \mathrm{He}$ with a measurement from Cooper et al. ${ }^{7}$ The non-spin flip (dashed line) and spin flip (dot-dashed line) contributions to the total cross section are shown separately.

The types of theoretical calculations which have been performed on the pion-induced isobaric analog transition in $A=3$ nuclei fall into four categories: 1) Glauber multiple scattering, 2) Gibbs multiple scattering, 3) optical potential, and 4) coupled channel approach. A selection of the theoretical work which is representative of these types of calculations is presented in Table 1.2 Examples of the first three types of calculations are shown in Figure 1.9 with measurements by Cooper et al. ${ }^{7}$ and Kallne et al., 9 and in Figure 1.10 with a measurement by Glodis $e t$ al. None of these calculations is in good agreement with all of the existing measurements.

In the Glauber model, it is assumed that the pion scatters from individual nucleons within a nucleus using a small angle approximation. This prescription is exact to first order as it includes rescattering from other nucleons within the nucleus but ignores nucleon-nucleon correlation effects. Two 
assumptions of the Glauber model are violated in the $\Delta$-resonance regime: 1) that each partial wave contributes equally to the scattering amplitude, and 2) that small angle scatterings are dominant. Because of these violations, the validity of the Glauber method has been questioned by some authors. ${ }^{27}$ Therefore, the reasonable agreement of the Glauber calculation with the Cooper results in Figure 1.9 is perhaps fortuitous. The validity of the optical potential approach is also questionable in such a simple nuclear system as $\mathrm{A}=3$ nuclei.

The Gibbs multiple scattering approach is a fixed nucleon approximation which requires fewer assumptions than either the optical potential or Glauber models. Unlike the Glauber multiple scattering method, the Gibbs approach does not assume equal contributions from each partial wave or employ a small angle scattering approximation. Uncertainties in this approach arise from the extension of the free $\pi \mathrm{N}$ scattering amplitudes off of the mass shell. The binding energy of a nucleon inside the nucleus must also be taken into account. Calculations by Hess and Gibson, ${ }^{28}$ which employ this approach and are shown in Figures 1.9 and 1.10, are not in very good agreement with previous measurements. However, more recent efforts $^{11}$ using the Gibbs multiple scattering approach, which reproduce measurements by Glodis et al., will be presented in Chapter 4 .

\begin{tabular}{|c|l|}
\hline \multicolumn{1}{|c|}{ Formalism } & \multicolumn{1}{|c|}{ Authors } \\
\hline Glauber Multiple Scattering & $\begin{array}{l}\text { Lohs and Mandelzweig } \\
\text { Gerace } \text { el }^{30}\end{array}$ \\
\hline Gibbs Multiple Scattering & $\begin{array}{l}\text { Gibbs and Gibson } \\
\text { Gibbs }^{\text {11 }} \\
\text { Hess and Gihson }\end{array}$ \\
\hline Optical Potential & $\begin{array}{l}\text { Wakamatsu } \\
\text { Landau }^{29}\end{array}$ \\
\hline Coupled Channel & $\begin{array}{l}\text { Kamalov, Tiator, and } \\
\text { Bennhold }\end{array}$ \\
\hline
\end{tabular}

Table 1.2: A selection of theoretical work on elastic single charge exchange in the three nucleon system. 


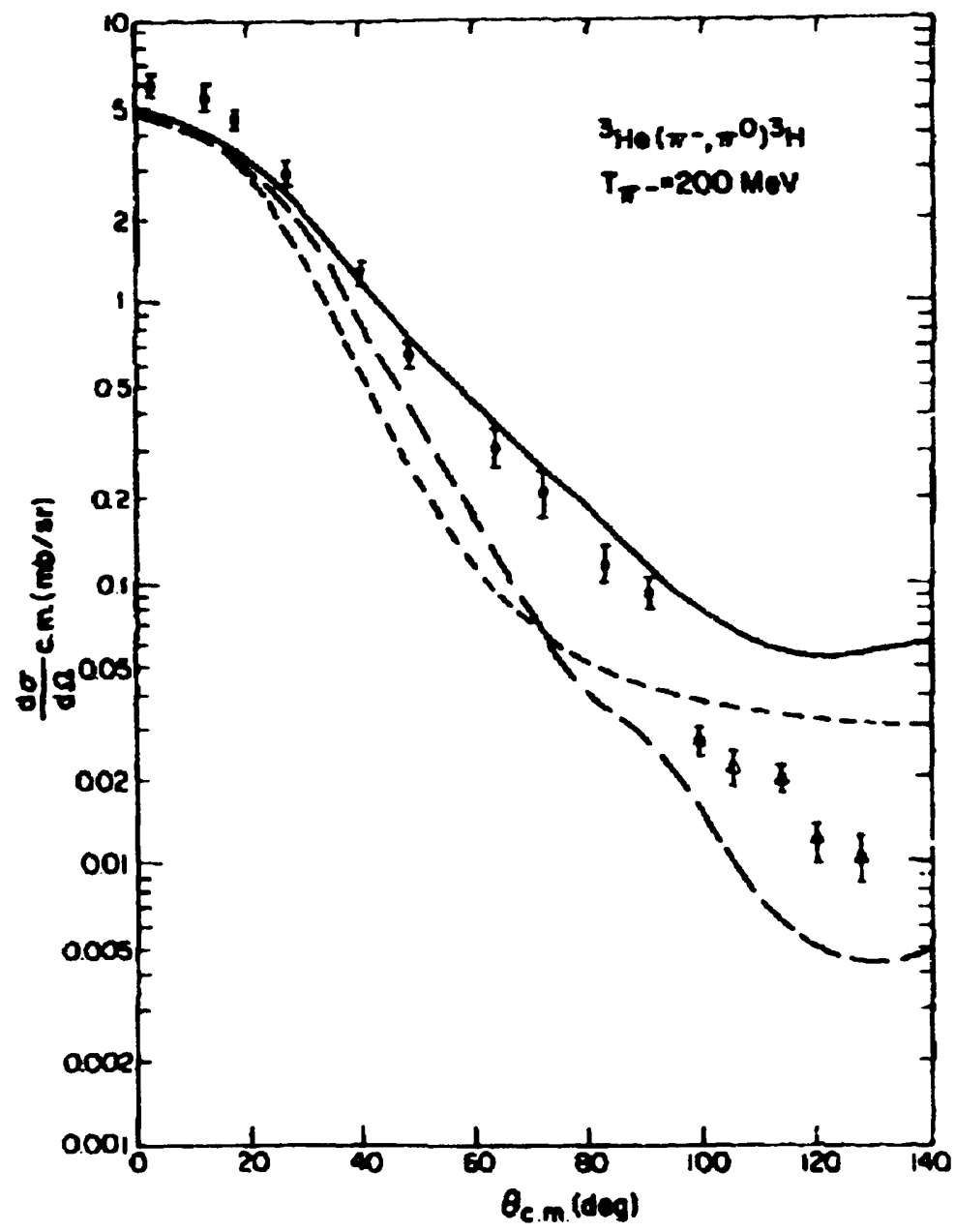

Figure 1.9: Angular distribution for ${ }^{3} \mathrm{He}\left(\pi^{-}, \pi^{9}\right)^{3} \mathrm{H}$ at $\mathrm{T}_{n}=200 \mathrm{MeV}$ from measurements by Cooper et al. (circles) and Källne et al. (triangles). The three calculations are representative of the theoretical work on this reaction: a Glauber multiple scattering calculation by Gerace et al..$^{33}$ (solid line); an optical potential calculation by Landau ${ }^{29}$ (long dash); and a Gibbs multiple scattering calculation by Hess and Gibson ${ }^{23}$ (short dash). 


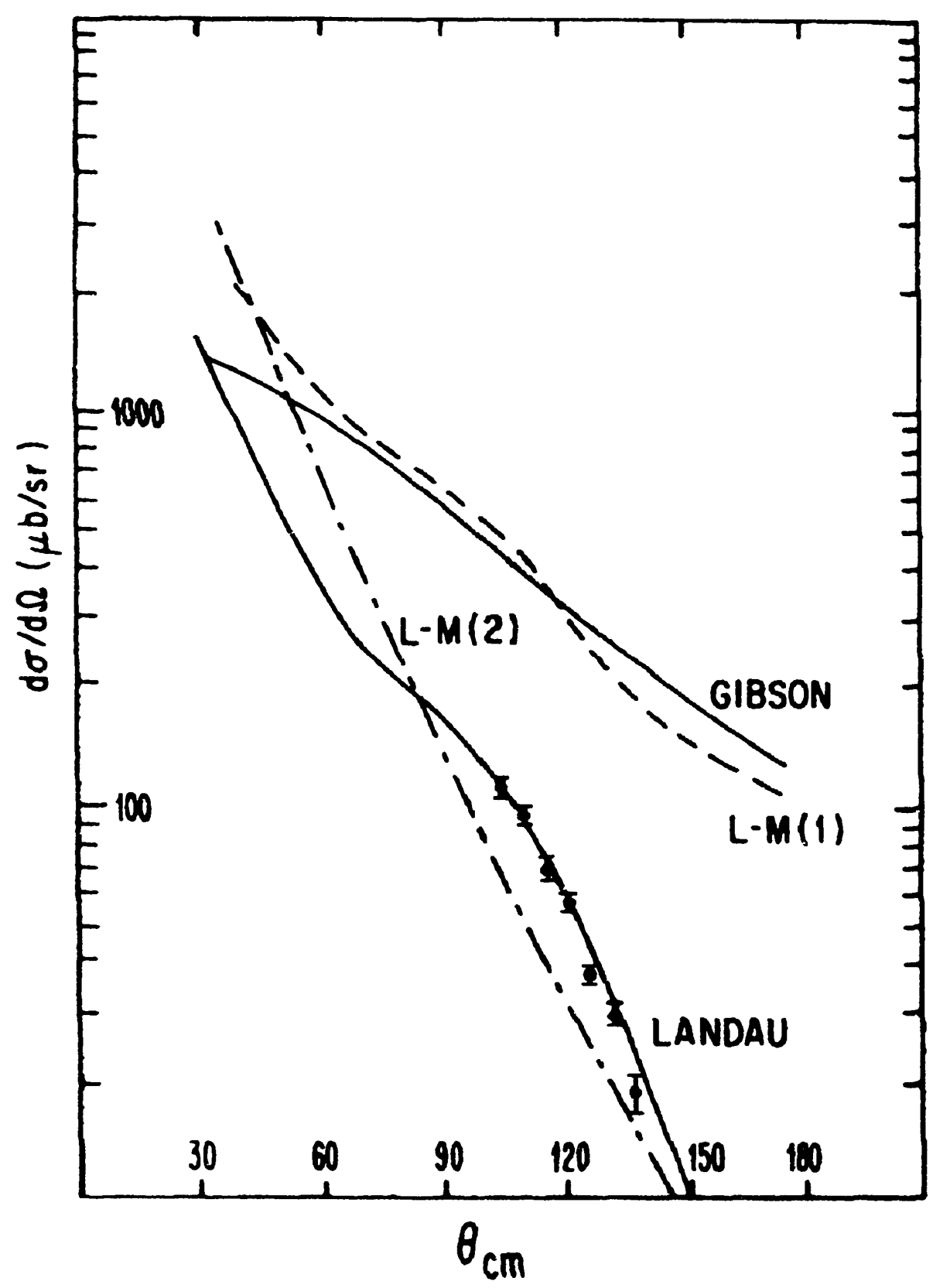

Figure 1.10: Angular distribution for ${ }^{3} \mathrm{H}\left(\pi^{+}, \pi^{0}\right)^{3} \mathrm{He}$ at $\mathrm{T}_{n}=148 \mathrm{MeV}$ from measurements by Glodis et al. ${ }^{8}$ The three calculations are representative of the types of theoretical work on this reaction: two Glauber multiple scattering calculations ${ }^{3031}(\mathrm{~L}-\mathrm{M}(1)$ and $\mathrm{L}-\mathrm{M}(2))$; an optical potential calculation (Landau $\left.{ }^{29}\right)$; and a Gibbs multiple scattering calculation (Gibson ${ }^{29}$. 

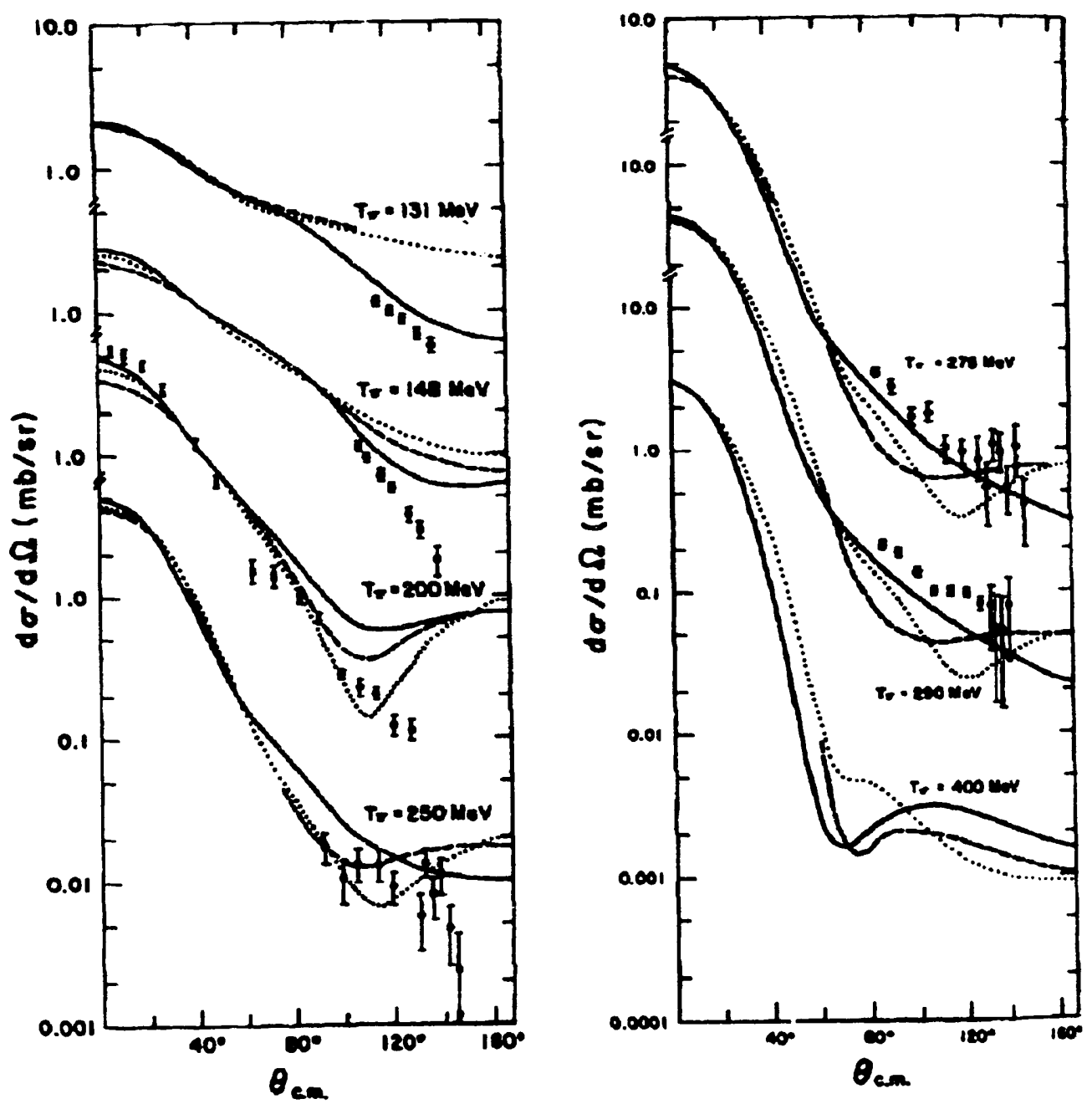

Figure 1.11: Existing data ${ }^{78.932}$ on the pion-induced isobaric analog transition in $A=3$ nuclei below $400 \mathrm{MeV}$. The curves correspond to Glauber multiple scattering calculations ${ }^{33}$ which include different assumptions about the nuclear wave functions and form factors. The douted and dashed lines correspond to calculations which use a Schiff wave function with and without mixed symmetry respectively. The solid line corresponds to a calculation which assumes realistic ${ }^{3} \mathrm{He}$ magnetic form factors. 
The coupled channel approach eliminates particular reaction channels which do not satisfy the constraints placed on the reaction mechanism by the initial and final nuclear states, thus forming a reduced set of coupled equations to describe the pion-nucleus interaction. The results of a recent coupled channel calculation ${ }^{10}$ in comparison with the measurements from this work will be shown in Chapter 4 .

\section{Review of Previous Experimental Work}

A summary of the experimental work prior to this work is shown in Figure 1.11. Previous measurements of the elastic charge exchange reaction in $A=3$ nuclei are limited to large $\pi^{0}$ center-of-mass angles with the exception of one forward angle measurement at $200 \mathrm{MeV}^{7}$ Except for this one measurement which detected the neutral pion, all other measurements employed the recoil detection technique. The recoil measurements were limited to large $\pi^{0}$ center of mass angles by their inability to detect low energy recoil particles. The Cooper measurement was incapable of distinguishing between neutral pions from the pion-induced isobaric analog transition, ${ }^{3} \mathrm{He}\left(\pi^{-}, \pi^{0}\right)^{3} \mathrm{H}$, and those from the continuum reactions, ${ }^{3} \mathrm{He}\left(\pi^{-}, \pi^{0}\right) \mathrm{X}$ at large $\pi^{0}$ center-of-mass angles as the differential cross section for the isobaric analog transition falls off steeply with increasing $\pi^{0}$ scattering angles. Examples of $\pi^{0}$ energy spectra from the Cooper measurement as a function of $\pi^{0}$ laboratory scattering angle are shown in Figure 1.12. Therefore, predictions of the influence of the spin flip contribution on the angular distribution as well as the energy dependence of the total cross section remain untested since a complete angular distribution has been measured at only one energy.

\section{Experimental Method}

The original goal of the experiment, which is described in this work was to measure as complete an angular distribution as possible over a range of incident energies so as to determine the energy dependence of the total cross section as well as the effect of the spin flip contribution on the angular distribution. The pion-induced isobaric ana.og transition between ${ }^{3} \mathrm{H}$ and ${ }^{3} \mathrm{He}$ may be observed in four different ways. One may observe either the ${ }^{3} \mathrm{He}\left(\pi^{-}, \pi^{9}\right)^{3} \mathrm{H}$ or the ${ }^{3} \mathrm{H}\left(\pi^{+}, \pi^{9}\right)^{3} \mathrm{He}$ reaction. In each case, either the recoil nucleus or the neutral pion may be detected. The detection of a recoil nucleus in the final state is a definitive indication that the isobaric analng transit , has taken place. In the present measurement, the 
recoil detection technique was employed and thin tritiated titanium foils were used as targets so as to minimize the energy loss of recoil ${ }^{3} \mathrm{He}$ particles from the ${ }^{3} \mathrm{H}\left(\pi^{+},{ }^{3} \mathrm{He}\right) \pi^{0}$ reaction. The experimental set-up consisted of a magnetic spectrometer equipped with a ladder of silicon surface barrier detectors. This system was capable of detecting ${ }^{3} \mathrm{He}$ particles with kinetic energies of as litule as $1.4 \mathrm{MeV}$ and as much as $80 \mathrm{MeV}$, which corresponded to an observable $\pi^{\circ}$ angular range of $35^{\circ} \leq \theta_{c u} \leq 130^{\circ}$ at an incident $\pi^{*}$ energy of $180 \mathrm{MeV}$.

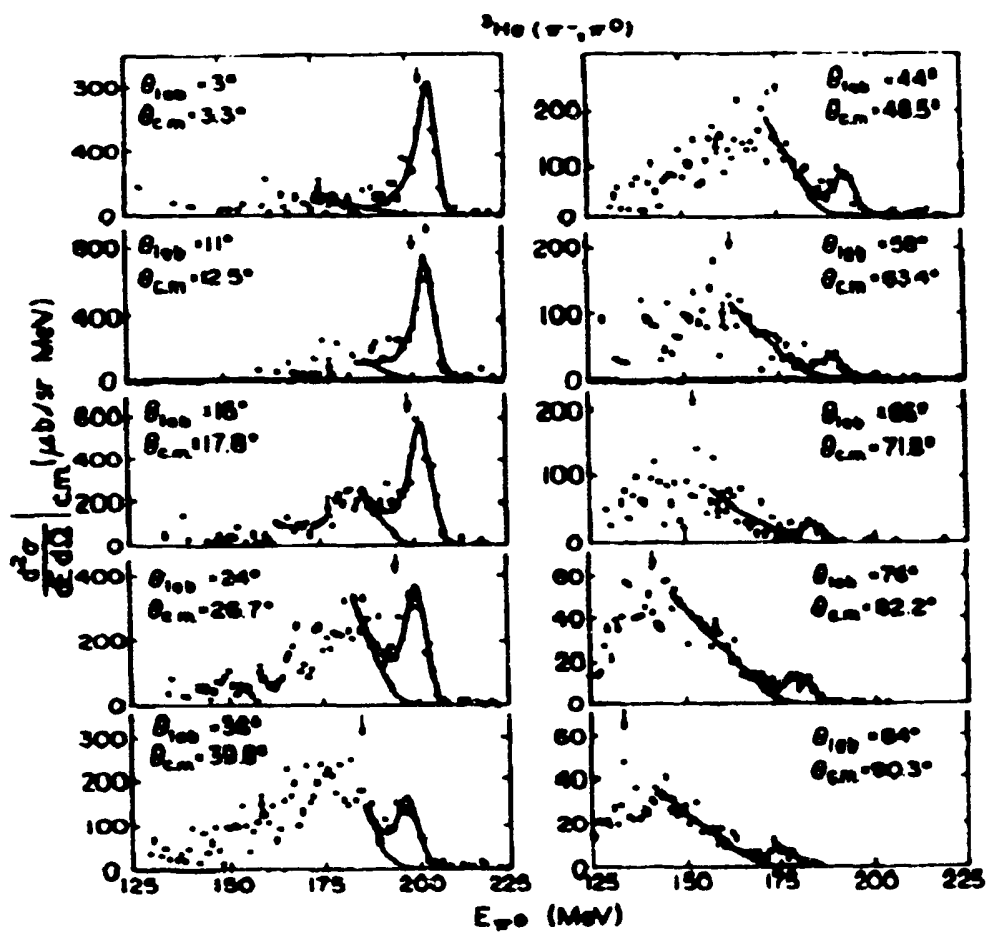

Figure 1.12: Pion energy spectra ${ }^{7}$ from ${ }^{3} \mathrm{He}\left(\pi^{-}, \pi^{0}\right)$. Arrows correspond to $\pi^{0}$ energy for free single charge exchange on a proton: $p\left(\pi^{2}, \pi^{9}\right)$ n. Solid lines correspond to empirical fits for extracting the elastic charge exchange differential cross section. 


\section{Inclusive Pion Single Charge Exchange Measurements on ${ }^{3} \mathrm{He}$ : ${ }^{3} \mathrm{He}\left(\pi^{+}, \pi^{0}\right)$ and ${ }^{3} \mathrm{He}\left(\pi^{ \pm}, \pi^{0} \mathbf{p}\right)$}

A goal of this thesis was to elucidate modifications to the pion-nucleon interaction due to the presence of other nucleons. Inclusive measurements of pion interactions with nuclei can provide information about these modifications. This thesis presents the first experimental results of inclusive single-arm and coincidence measurements of quasi-free pion charge exchänge on ${ }^{3} \mathrm{He}$. These results can be compared with previous experimental results of quasi-free pion scattering on ${ }^{3} \mathrm{He}$ to investigate isospin scaling predictions for the single charge exchange and non-charge exchange channels in ${ }^{3} \mathrm{He}$ based on the isospin dependence of the free $\pi \mathrm{N}$ interactions. The theoretical interpretations of the quasi-free single charge exchange results from this work should be relatively simple since the nuclear structure of ${ }^{3} \mathrm{He}$ is well known.

Multiple scattering processes are expected to play a more important role in pion single charge exchange than in charged pion scattering because of a weaker coupling to the two nucleon absorption channel. ${ }^{34}$ Unlike charged pion scattering, single charge exchange on a $\mathrm{T}=0$ pair must lead to a $\mathrm{T}=1$ pair as shown in Figure 1.13. However, the dominant absorption channel for a $\mathrm{T}=1$ pair, an intermediate ${ }^{5} \mathrm{~S}_{2}$ $\left({ }^{2 s+1} \mathrm{~L}_{\mathrm{J}}\right) \Delta \mathrm{N}$ state, is forbidden by angular momentum, parity, and isospin conservation rules. ${ }^{34}$ The selection rules for pion absorption and pion production near threshold are presented in Table 1.3. In the $\Delta$-resonance regime, the dominant pion absorption channel is an intermediate $\Delta \mathrm{N}$ state which is excited by a $p$-wave resonance $\left(\ell_{n}=1\right)$. The cross section for absorption on a $T=1$ pair, which is allowed in single

charge exchange, is approximately an order of magnitude smaller than absorption on a $\mathrm{T}=0$ pair. ${ }^{35,36}$ These arguments have been invoked to account for the enhancement at low pion energies which is observed in pion energy spectra ${ }^{37}$ from single charge exchange, ${ }^{16} \mathrm{O}\left(\pi^{+}, \pi^{9}\right)$, over charged pion scattering, ${ }^{16} \mathrm{O}\left(\pi^{+}, \pi^{+}\right)$, on ${ }^{16} \mathrm{O}$. A comparison of these spectra is shown in Figure 1.14. 

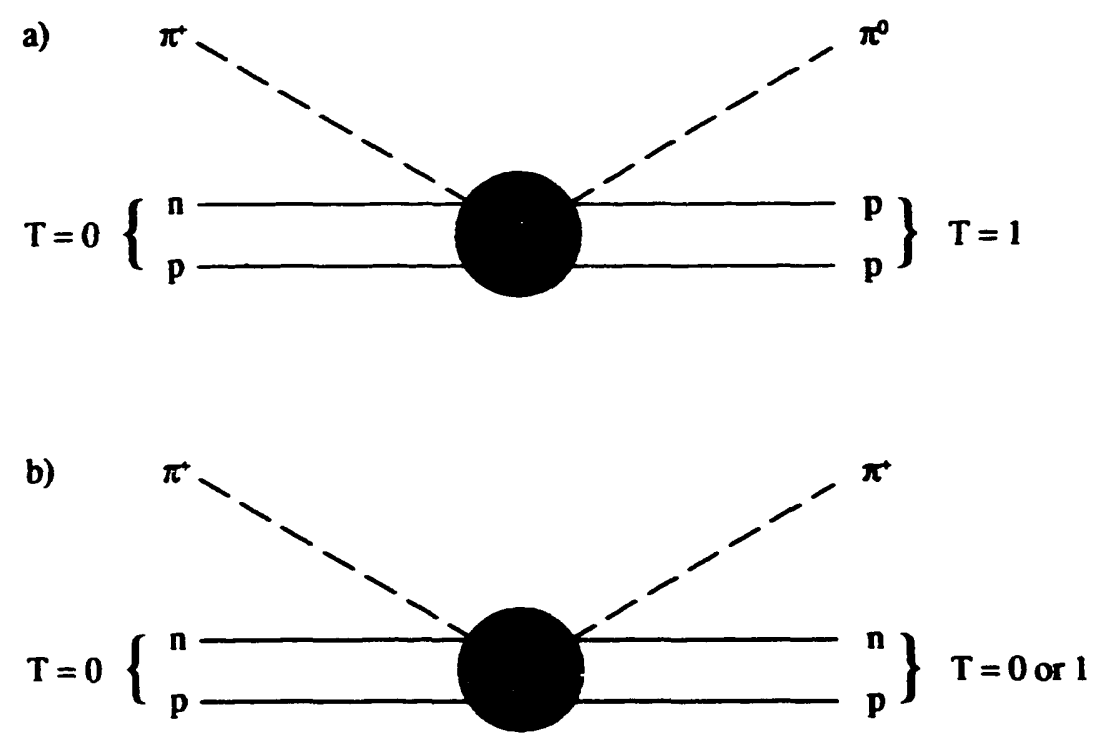

Figure 1.13: Diagrams for a) pion single charge exchange and b) pion scattering on a $T=0$ pair.

\begin{tabular}{|l|l|l|l|}
\hline \multicolumn{3}{|c|}{$\begin{array}{l}\text { Selection Rules for } 2 \mathrm{~N} \leftrightarrow 2 \mathrm{~N}+\pi \\
\text { Threshold }\end{array}$} \\
\hline \hline$\ell(\pi)$ & $\Delta L$ (2N) & $(\mathrm{TS})_{\mathrm{i}} \leftrightarrow(\mathrm{TS})_{\ell}$ & $\Delta J$ \\
\hline 0 & 1 & $\begin{array}{l}(1,0) \leftrightarrow(1,1) \\
(0,1) \leftrightarrow(1,1)\end{array}$ & 0 \\
\hline 1 & 0,2 & $\begin{array}{l}(1,1) \leftrightarrow(1,1) \\
(1,1) \leftrightarrow(0,0)\end{array}$ & 0,1 \\
& $(1,0) \leftrightarrow(0,1)$ & \\
\hline
\end{tabular}

Table 1.3: Selection rules for $\pi N N \leftrightarrow N N$ near threshold. ${ }^{38}$ The change in relative angular momentum for the nucleon pair is expressed as " $\Delta L$ " and (TS) $)_{i}$ and (TS) $)_{f}$ refer to the total isospin and spin of the nucleon pair in the initial and final state respectively. 


\section{Review of Previous Work}

Although this work represents the only existing experimental information on inclusive pion single charge exchange in ${ }^{3} \mathrm{He}$, there are several previous experimental efforts with which to compare and contrast these results. An earlier experimental study of quasi-free single charge exchange on ${ }^{16} \mathrm{O}$ supplied pan of the motivation for those measurements on ${ }^{3} \mathrm{He}$.

\section{Quasi-free Pion Single Charge Exchange on ${ }^{16} \mathrm{O}$}

A selection of results from a previous measurement of the $\pi^{0}$ energy distribution in the ${ }^{16} O\left(\pi^{+}, \pi^{0} p\right)$ reaction is shown in Figure 1.15. This measurement employed the LAMPF $\pi^{0}$ Spectrometer and an array of plastic scintillation detectors in an experimental set-up similar to the one employed in the coincidence measurements which are presented in this work. This earlier experiment was the first coincidence measurement to study the $A$-dependence of the $\left(\pi^{+} \pi^{0}\right)$ and $\left(\pi^{*}, \pi^{0} \mathrm{p}\right)$ reactions using ${ }^{16} \mathrm{O},{ }^{56} \mathrm{Fe},{ }^{120} \mathrm{Sn}$, and ${ }^{208} \mathrm{~Pb}$ targets at incident pion energies of 165 and $245 \mathrm{MeV}$. Theoretical calculations ${ }^{34}{ }^{39}$ which reproduce the general shape of pion energy spectra from ${ }^{16} \mathrm{O}\left(\pi^{+}, \pi^{\dagger}\right)$ (see Figure 1.14) are not successful in describing the proton knockout data, ${ }^{16} \mathrm{O}\left(\pi^{+}, \pi^{0} \mathrm{p}\right),{ }^{40}$ shown in Figure 1.15 . The authors of this $\Delta$-hole calculation have concluded that additional information about the interaction of the $\Delta$ with the nuclear system is needed to understand this mechanism. It has been suggested that these calculations should be less difficult to perform on a simpler system such as ${ }^{3} \mathrm{He}$.

Pion-induced Proton Knockout on ${ }^{3} \mathrm{He}:{ }^{3} \mathrm{He}\left(\pi^{t}, \pi^{t} p\right)$

A number of interesting comparisons may be drawn between previous quasi-elastic inclusive ${ }^{6}$ and knockout measurements ${ }^{41,2}$ on ${ }^{3} \mathrm{He}$ and the quasi-free charge exchange measurements presented in this work. Quasi-free pion scattering on ${ }^{3} \mathrm{He}$ may provide imporant information about $\Delta$-nucleus interactions since it is a relatively simple nucleus. In the $\Delta$-resonance region, a pion can excite a $\Delta$ within the nucleus which in turn may propagate and interact with other nucleons. A comparison of outgoing pion energy spectra from the ${ }^{3} \mathrm{He}\left(\pi^{+}, \pi^{+} \mathrm{p}\right)$ and ${ }^{3} \mathrm{He}\left(\pi^{+}, \pi^{+}\right)$reactions is shown in Figure 1.16. In this figure, the coincidence measurement is in better agreement with the general shape of a DWIA prediction than is the single-arm quasi-elastic measurement. The enhancement of the low energy tail in the single-arm spectrum 


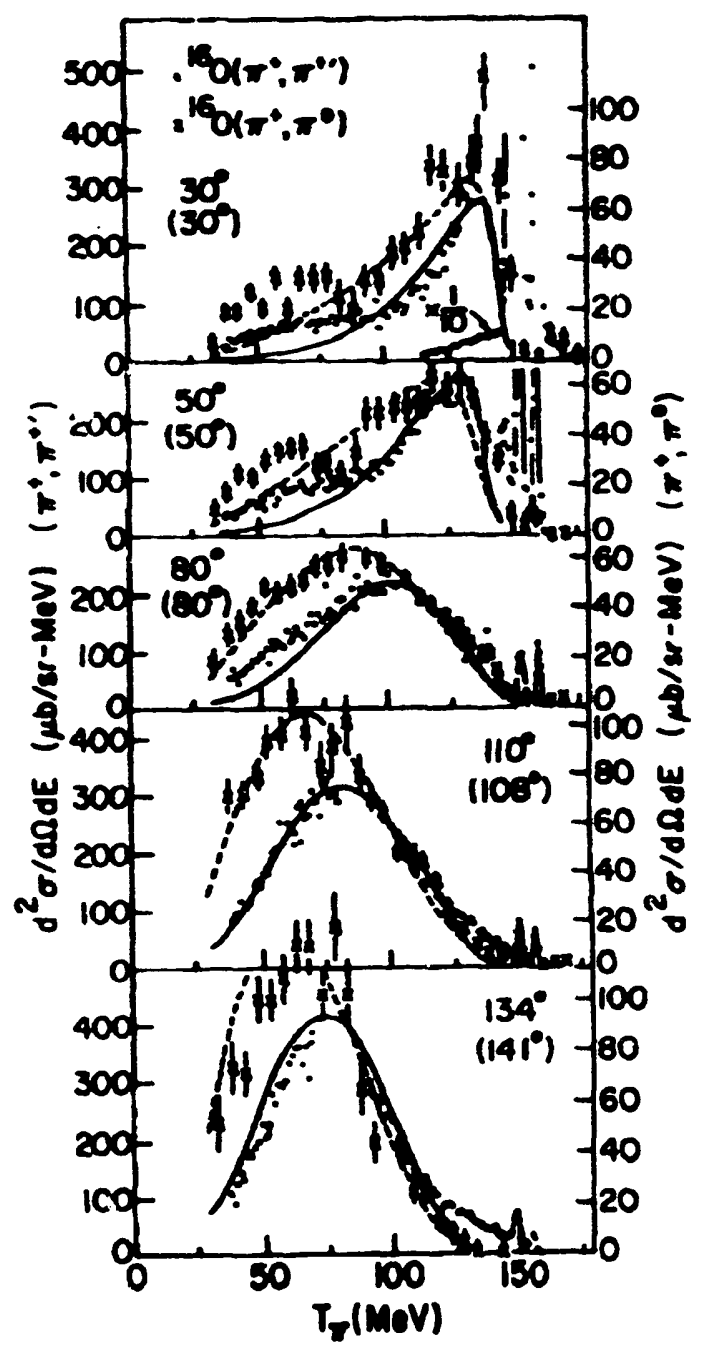

Figure 1.14: Outgoing pion spectra ${ }^{37}$ from ${ }^{16} \mathrm{O}\left(\pi^{+}, \pi^{3}\right)$ at $163 \mathrm{MeV}$ and ${ }^{16} \mathrm{O}\left(\pi^{+}, \pi^{9}\right)$ at $160 \mathrm{MeV}$. The $y$-axes have been adjusted according to the isospin dependence of the free $\pi \mathrm{N}$ cross sections. Angles in parentheses are for the charge exchange data. The results of a $\Delta$-hole calculation ${ }^{34}$ for the inelastic scattering results are shown (solid line). The dashed line corresponds to a modified DWIA calculation which employed a closure approximation. This closure approximation ignores contributions from true pion absorption. Therefore, the reasonable agreement with the charge exchange results may be an indication of a weaker coupling to the absorption channel for the charge exchange relative to the non-charge exchange reaction in ${ }^{16} \mathrm{O}$. 

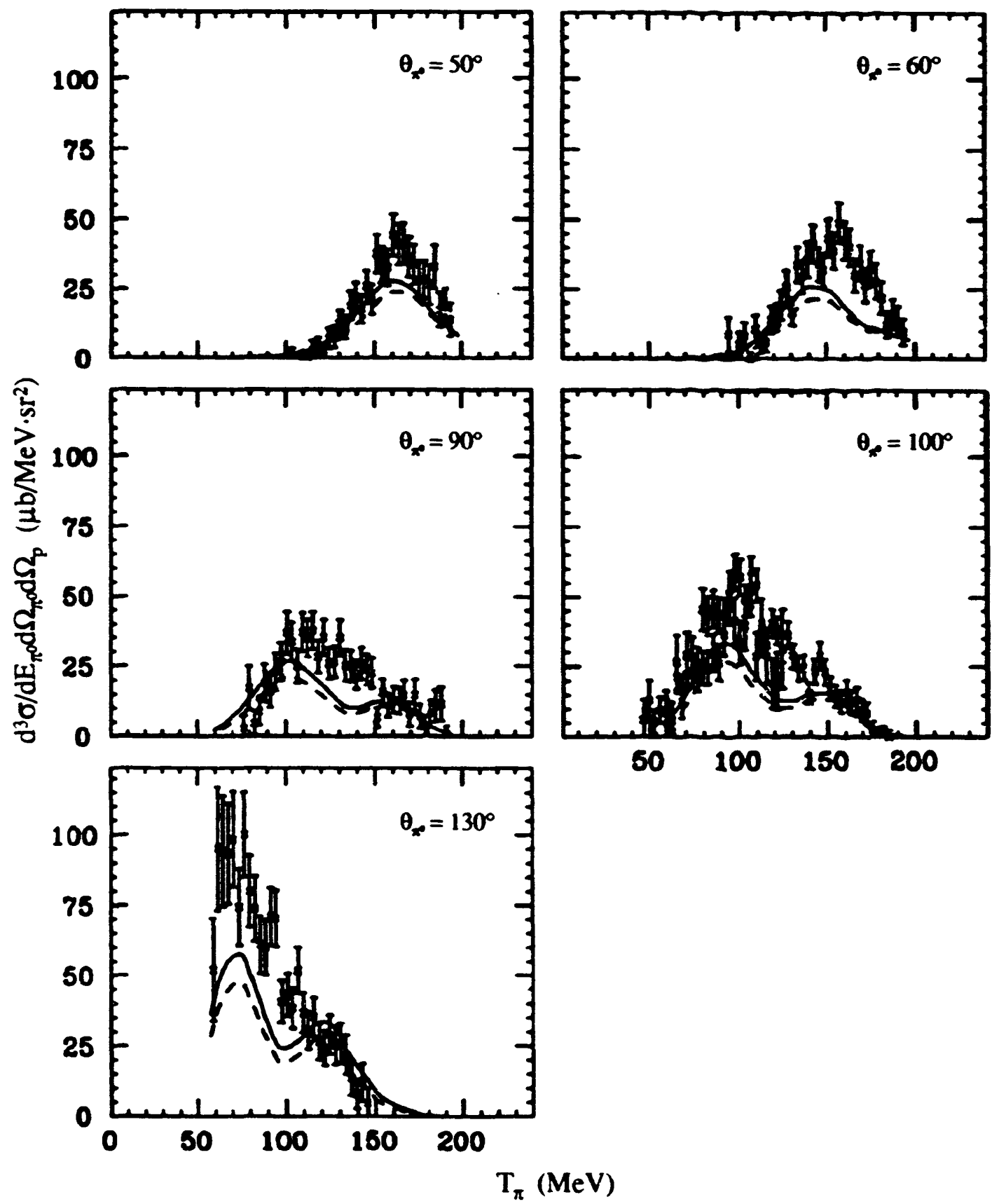

Figure 1.15: Pion energy spectra ${ }^{40} \mathrm{fmm}{ }^{16} \mathrm{O}\left(\pi^{*}, \pi^{0} \mathrm{p}\right)$ at $245 \mathrm{MeV}$. Modified DWIA (dashed line) and $\Delta$-hole zalculations ${ }^{39}$ (solid line) are shown. 


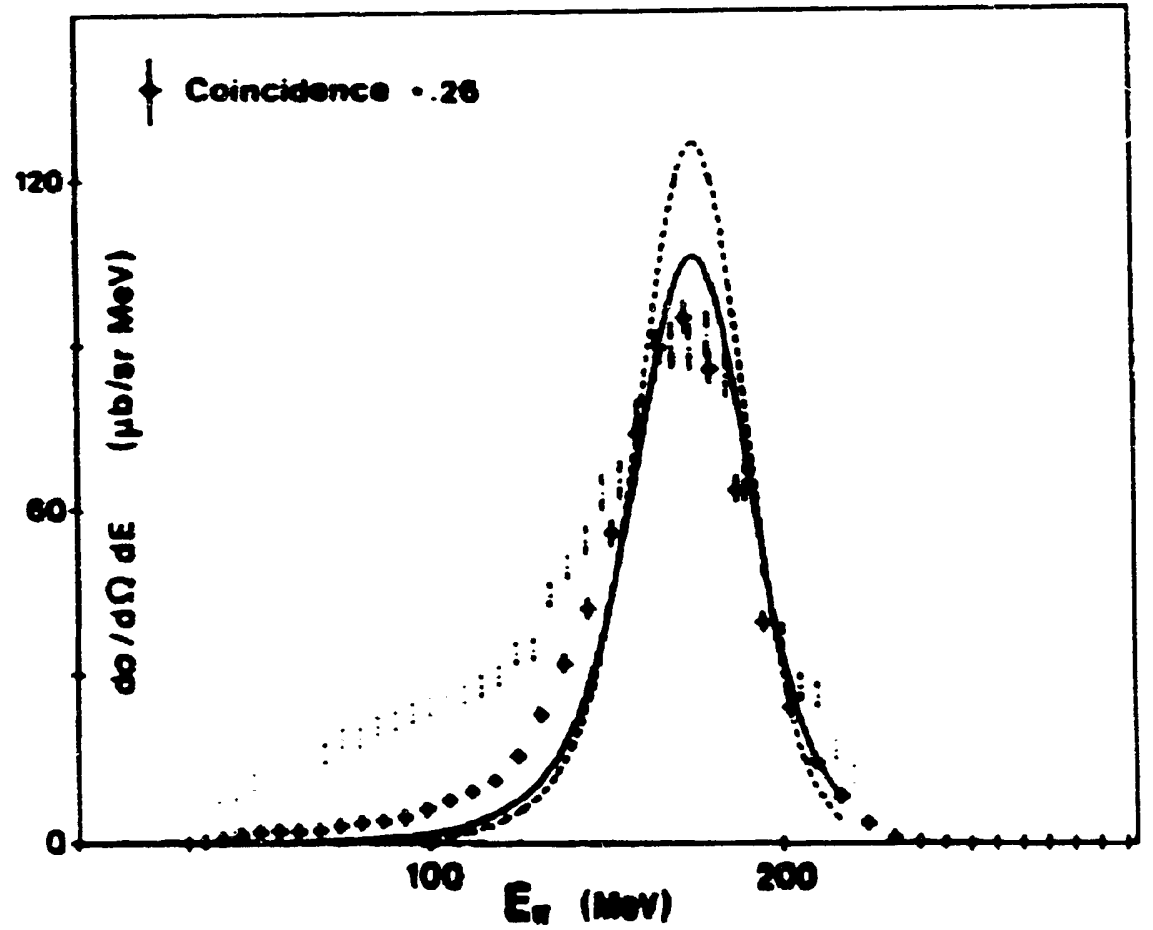

Figure 1.16: Comparison of ${ }^{3} \mathrm{He}\left(\pi^{*}, \pi^{*}\right)$ and ${ }^{3} \mathrm{He}\left(\pi^{+}, \pi^{*} \mathrm{p}\right)$ coincidence spec $3 \mathrm{~m}^{41}$ at $270 \mathrm{MeV}, \theta_{x^{0}}=75^{\circ}$ and $\theta_{p}=-43^{\circ}$. The coincidence data are integrated over recoil proton energy and scaled to have the same peak height as the single arm data. The curve corresponds to a DWIA calculation.

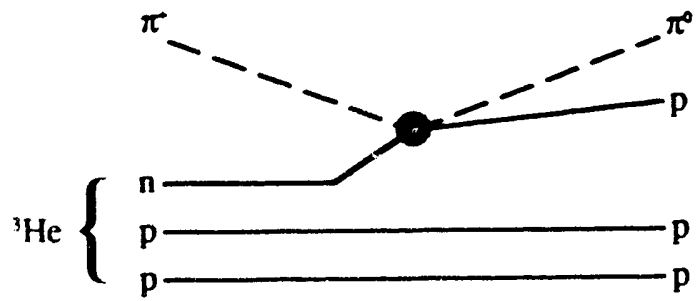

Figure 1.17: Diagram for quasi-free single charge exchange on ${ }^{3} \mathrm{He}:{ }^{3} \mathrm{He}\left(\pi^{+}, \pi^{9}\right)$ 
may be altributed to multiple scattering processes.

The quasi-free charge exchange channel, $\left(\pi^{*}, \pi^{0} \mathrm{p}\right)$, is expected to account for a large fraction of the Strength which is observed in single-arm measurements, $\left(\pi^{*}, \pi^{0}\right)$. A diagram of this mechanism for the ${ }^{3} \mathrm{He}$ nucleus is shown in Figure 1.17. Comparisons between single-arm and coincidence measurements for the charge exchange channel might show an enhancement in the single-arm $\pi^{0}$ energy spectra at low outgoing kinetic energies. A similar comparison of pion energy spectra from the single-arm measurements of this work with previous non-charge exchange measurements on ${ }^{3} \mathrm{He}$ might confirm the enhancement seen in earlier comparisons between charge exchange and non-charge exchange measurements on ${ }^{16} \mathrm{O}$. (See Figure 1.14.) These enhancements could be interpreted as indications of multiple scattering or other more complex processes.

The three nucleon system is also the simplest system for which experiments may be designed to distinguish between single scattering and multi-nucleon interactions: ${ }^{3} \mathrm{He}\left(\pi^{*}, \pi^{0} \mathrm{p}\right)$. The ${ }^{3} \mathrm{He}\left(\pi^{+}, \pi^{0} \mathrm{p}\right)$ reaction is expected to be primarily a single scattering process. In ${ }^{3} \mathrm{He}\left(\pi^{-}, \pi^{0} \mathrm{p}\right)$, the reaction must take place on two nucleons for a proton of sufficient energy to be detected in coincidence. The $\pi^{0}$ energy dependence and proton angular distributions may provide information about the dominant mechanisms for these reactions. Diagrams of several two-step mechanisms for the ${ }^{3} \mathrm{He}\left(\pi, \pi^{0} \mathrm{p}\right)$ reaction are shown in comparı: with similar mechanisms for double charge exchange on ${ }^{3} \mathrm{He},{ }^{3} \mathrm{He}\left(\pi^{-}, \pi\right)$, in Figures 1.18 through 1.21.
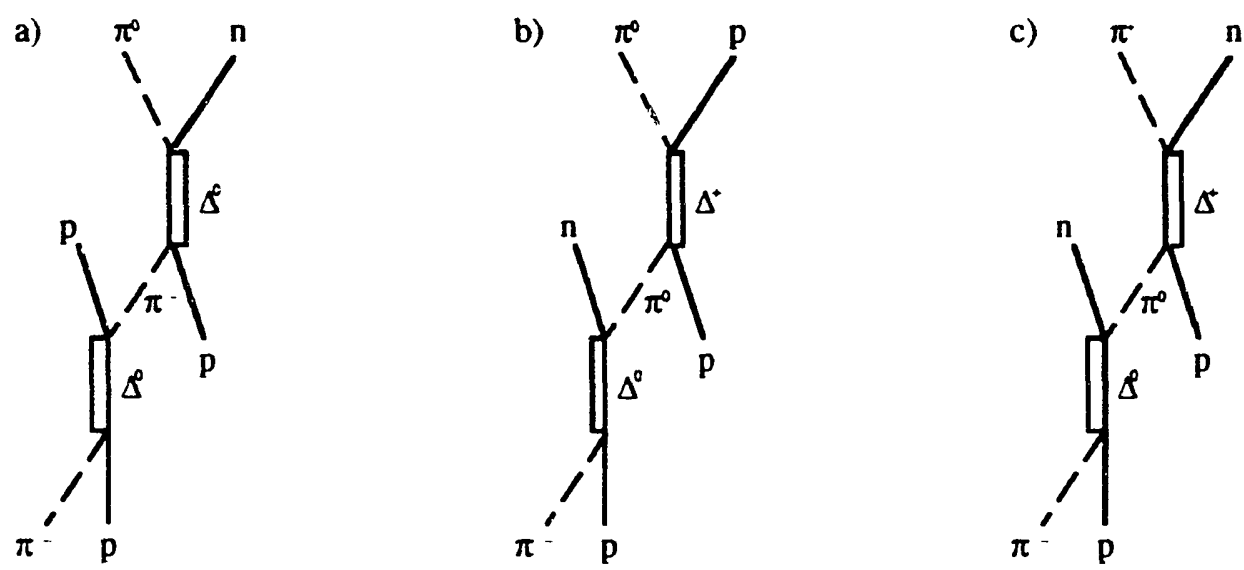

Figure 1.18: Feynman diagrams for a) and b) ${ }^{3} \mathrm{He}\left(\pi^{-}, \pi^{0} \mathrm{p}\right)$ and $\left.c\right){ }^{3} \mathrm{He}\left(\pi^{-}, \pi^{*}\right)$ through a sequential scattering process. 

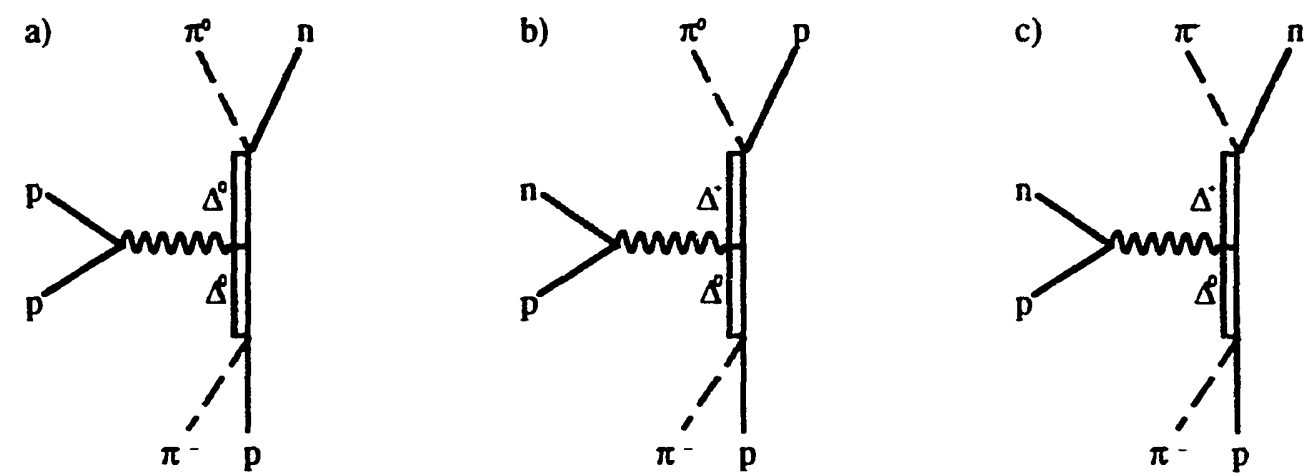

Figure 1.19: Feynman diagrams for a) and b) ${ }^{3} \mathrm{He}\left(\pi^{-}, \pi^{0} \mathrm{p}\right)$ and c) ${ }^{3} \mathrm{He}\left(\pi^{-}, \pi^{3}\right)$ through a direct $\Delta$-nucleus interaction.

a)

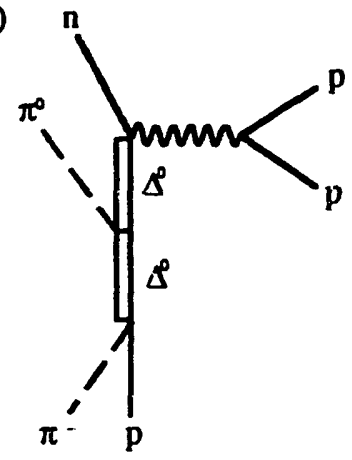

b)

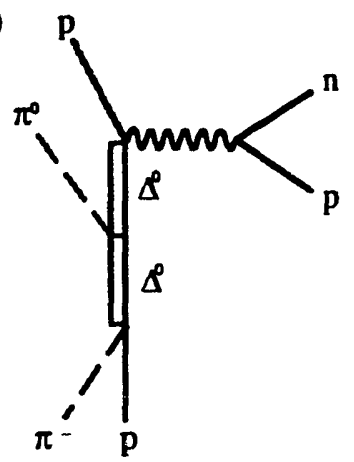

c)

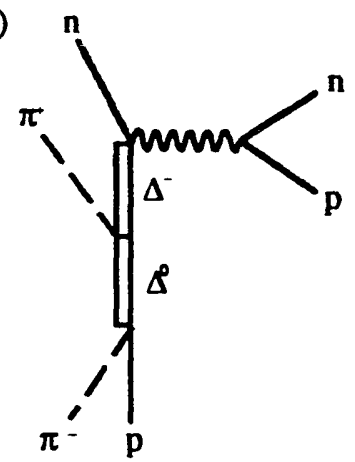

Figure 1.20: Feynman diagrams for $a)$ and $b)^{3} \mathrm{He}\left(\pi^{-}, \pi^{0} \mathrm{p}\right)$ and $\left.c\right){ }^{3} \mathrm{He}\left(\pi^{-}, \pi^{+}\right)$through final state interactions.

a)

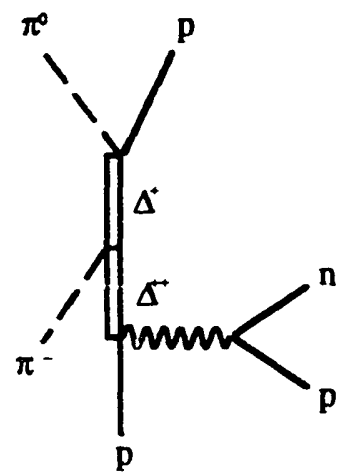

b)

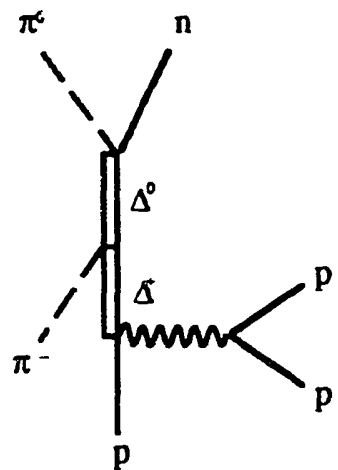

c)

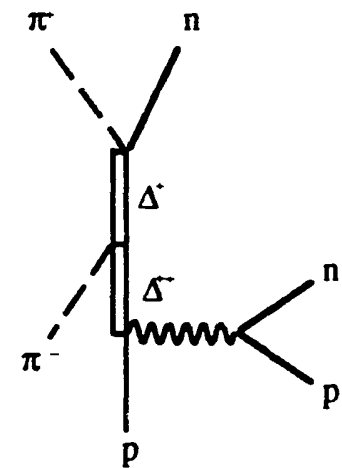

Figure 1.21: Feynman diagrams for a) and $b)^{3} \mathrm{He}\left(\pi^{-}, \pi^{0} p\right)$ and c) ${ }^{3} \mathrm{He}\left(\pi^{-}, \pi^{+}\right)$initial state interactions. 


\section{Experimental Method}

The inclusive single charge exchange measurements presented in this thesis were performed at LAMPF using the LAMPF $\pi^{\circ}$ Spectrometer, an array of eight $\triangle E-E$ plastic scintillation counters, and a liquid ${ }^{3} \mathrm{He}$ target. Measurements were performed at an incident pion energy of $245 \mathrm{MeV}$ and $\pi^{0}$ laboratory scattering angles ranging from $62^{\circ}$ to $128^{\circ}$. The proton detector array covered an angular range of nearly $60^{\circ}$ in the laboratory scattering plane. Two of the counters were placed out of the scattering plane so that the in-plane and out-of-plane prown angular distributions could be measured. The detector array was positioned at laboratory scattering angles near the proton scattering angle for the free charge exchange process, $\pi^{+} n \rightarrow \pi^{0} \mathrm{p}$. In both the single-arm and coincidence measurements, neutral pions were detected over an energy range which corresponded to quasi-free kinematics using the $\pi^{0}$ Spectrometer. 


\section{Chapter 2: Experimental Apparatus and Data Acquisition System for the ${ }^{3} \mathrm{H}\left(\pi^{+},{ }^{3} \mathrm{He}\right) \pi^{0}$ Measurements}

The goal of this experiment was to observe the elastic single charge exchange reaction, ${ }^{3} \mathrm{H}\left(\pi^{4}, \pi^{9}\right)^{3} \mathrm{He}$, over as wide an angular and incident energy range as possible. The pion-induced isobaric analog transition between ${ }^{3} \mathrm{He}$ and ${ }^{3} \mathrm{H}$ may be observed in four different ways. Either the ${ }^{3} \mathrm{He}\left(\pi, \pi^{9}\right)^{3} \mathrm{H}$ or ${ }^{3} \mathrm{H}\left(\pi^{*}, \pi^{9}\right)^{3} \mathrm{He}$ reaction may be observed by detecting either a neutral pion or a recoil nucleus. As the differential cross section for the isobaric analog transition decreases by roughly two orders of magnitude from forward to backward $\pi^{0}$ center of mass angles, the peak associated with this transition becomes quite small. (See Figures 1.9 and 1.12.) Therefore, it becomes increasingly difficult to distinguish between neutral pions from the isobaric analog transition and those from the continuum at large $\pi^{\circ}$ angles as can be seen in Figure 1.13. The detection of a recoil nucleus in the final state is a unique indication that the isobaric analog transition has taken place because the ground states of ${ }^{3} \mathrm{H}$ and ${ }^{3} \mathrm{He}$ are the only bound states of these nuclei. Previous experimental studies, employing the recoil detection technique, have been limited to large $\pi^{0}$ center of mass angles by their inability to detect very low energy recoil nuclei. Charged particles with as litte as $1.4 \mathrm{MeV}$ were detected using this experimental set-up and therefore measurements of the differential cross section could in principle be performed at $\pi^{\circ}$ center of mass angles as small as $30^{\circ}$. In addition, this method allowed measurements of the differential cross section at large $\pi^{0}$ angles since recoil nuclei were detected rather than neutral pions. 


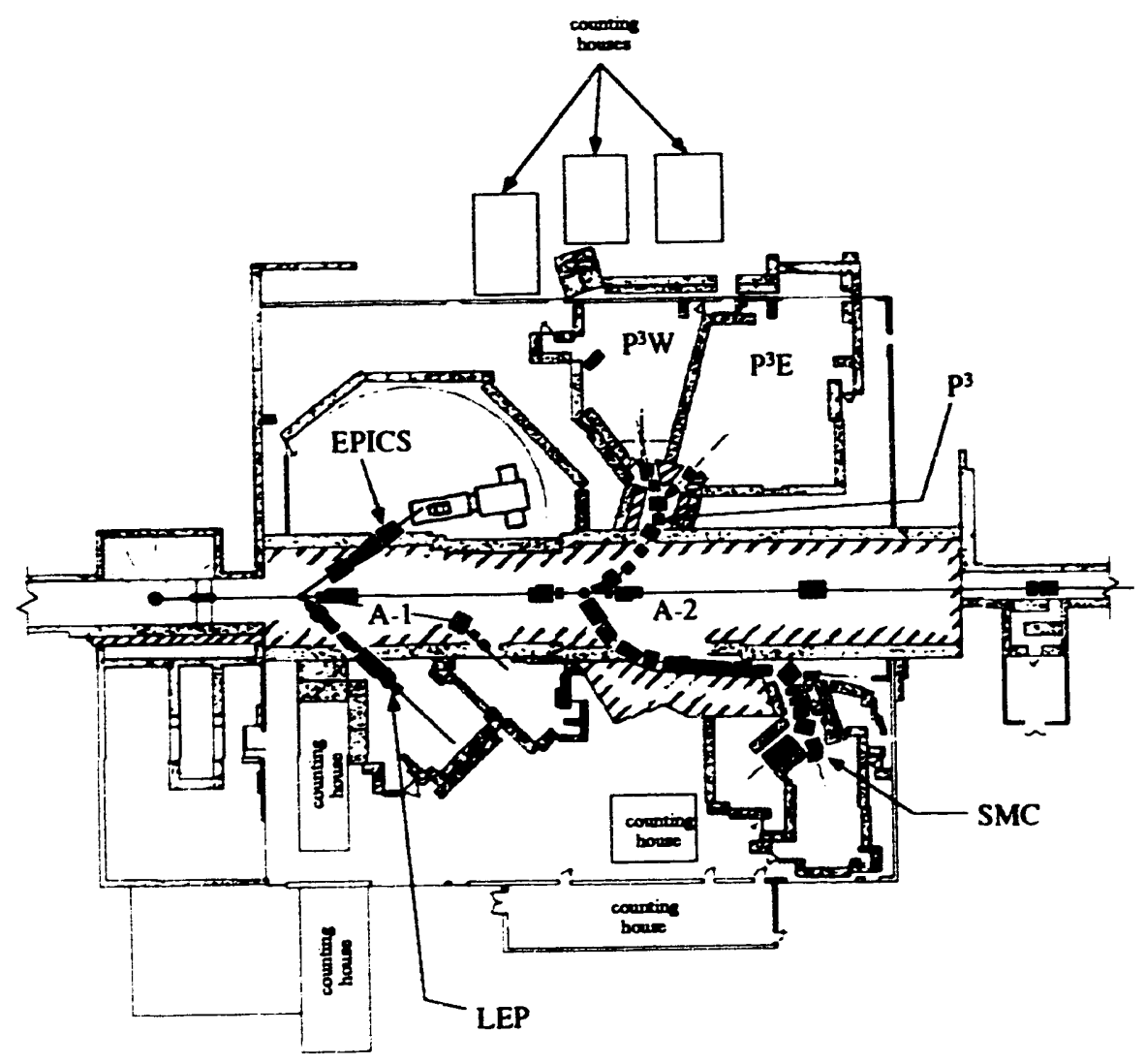

Figure 2.1: LAMPF Experimental Area A

\section{Pion Beam}

This experiment was performed at the Clinton P. Anderson Meson Physics Facility (LAMPF) which is located at the Los Alamos National Laboratory in Los Alamos, New Mexico. LAMPF is a half mile long linear accelerator capable of accelerating protons to an energy of up $10800 \mathrm{MeV}$ at an average intensity of $1 \mathrm{~mA}$ Pion beams are manufactured through proton-induced pion production on graphite targets. The meson physics experimental halls are located in Area A which is shown in Figure 2.1. In this figure, the two graphite production targets are designated A-1 and A-2. There are three pion beam lines located in Area A. These channels are labeled EPICS, $\mathrm{P}^{3}$, and LEP in Figure 2.1. The momentum and momentum dispersion of the pion beam is selected by a series of magnetic elements and collimating slits located in each channel. These elements focus and transport the pion beam from the production target to the 


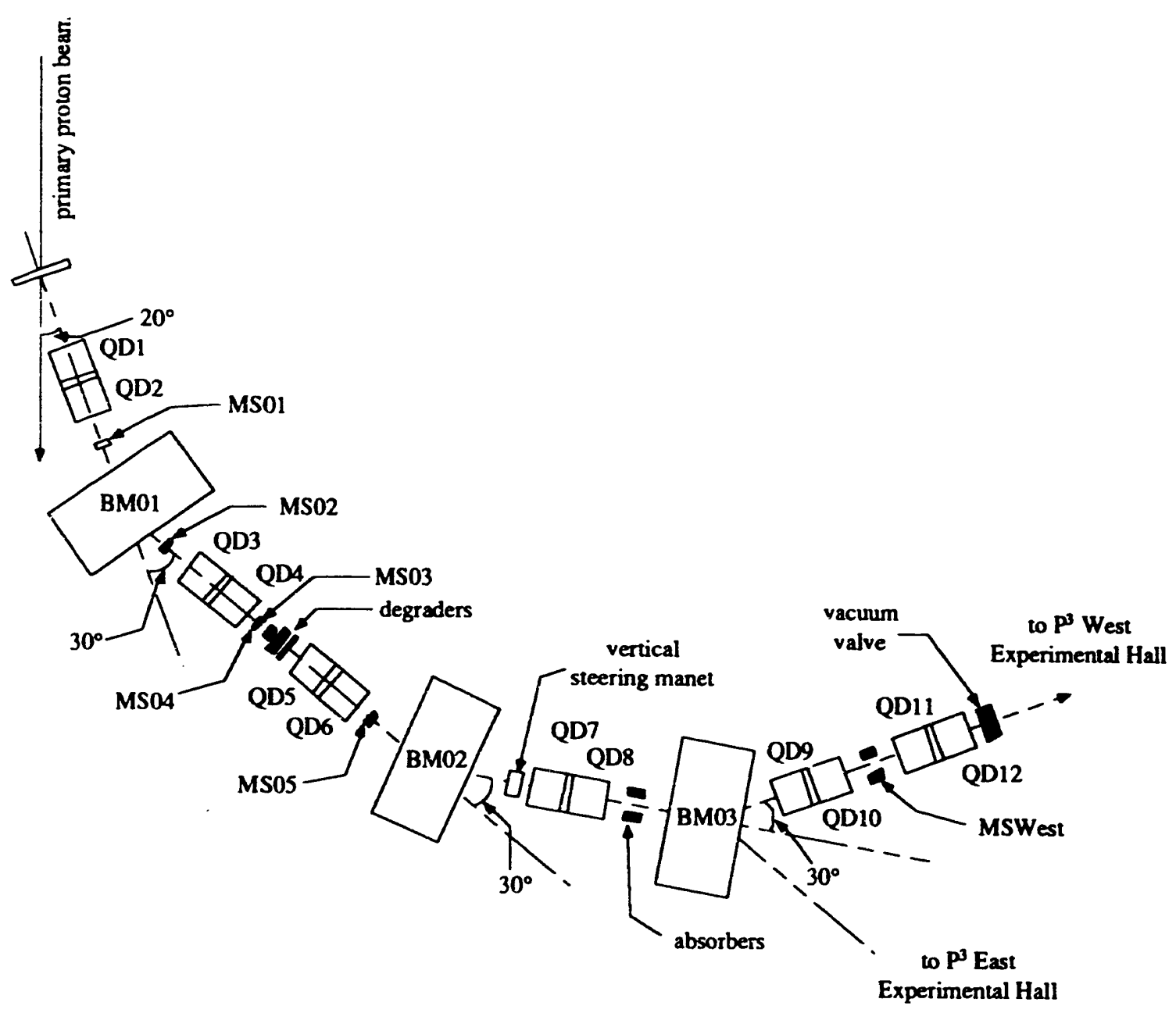

Figure 2.2: $\mathrm{P}^{3}$ Channel

experimental halls. A schematic view of the High Energy Pion Channel $\left(\mathrm{P}^{3}\right)$ is shown in Figure 2.2. The pion beam from the target region may be steered through the $\mathrm{P}^{3}$ channel into one of two experimental halls $-P^{3}$ East or $P^{3}$ West. This experiment was set up in the smaller of the two experimental halls, $P^{3}$ West. A variable amount of degrader and absorber material may be used to separate positive pions from protons and positrons, and negative pions from electmns, of the same magnetic rigidity. The proton and electron degraders in the $\mathrm{P}^{3}$ channel are located just after the momentum defining slits, MS03 East and MS03 West. Additional collimating slits, MS04, MS05, and MS West, may be used to define the spatial extent of the beam. The field settings of the dipole and quadrupole magnets were determined by a computer program 
which was designed to simulate the $\mathrm{P}^{3}$ channel optics. The values of the field settings were calculated for each incident beam energy. The field settings of the last two quadrupoles, QD11 and QD12, were fine tuned so that the beam waist was centered at the target. For a more detailed description of the $\mathrm{P}^{3}$ chanrel optics, operation of the secondary pion channels, and performance characteristics of the LAMPF facility see the LAMPF Users Handbook. ${ }^{43}$

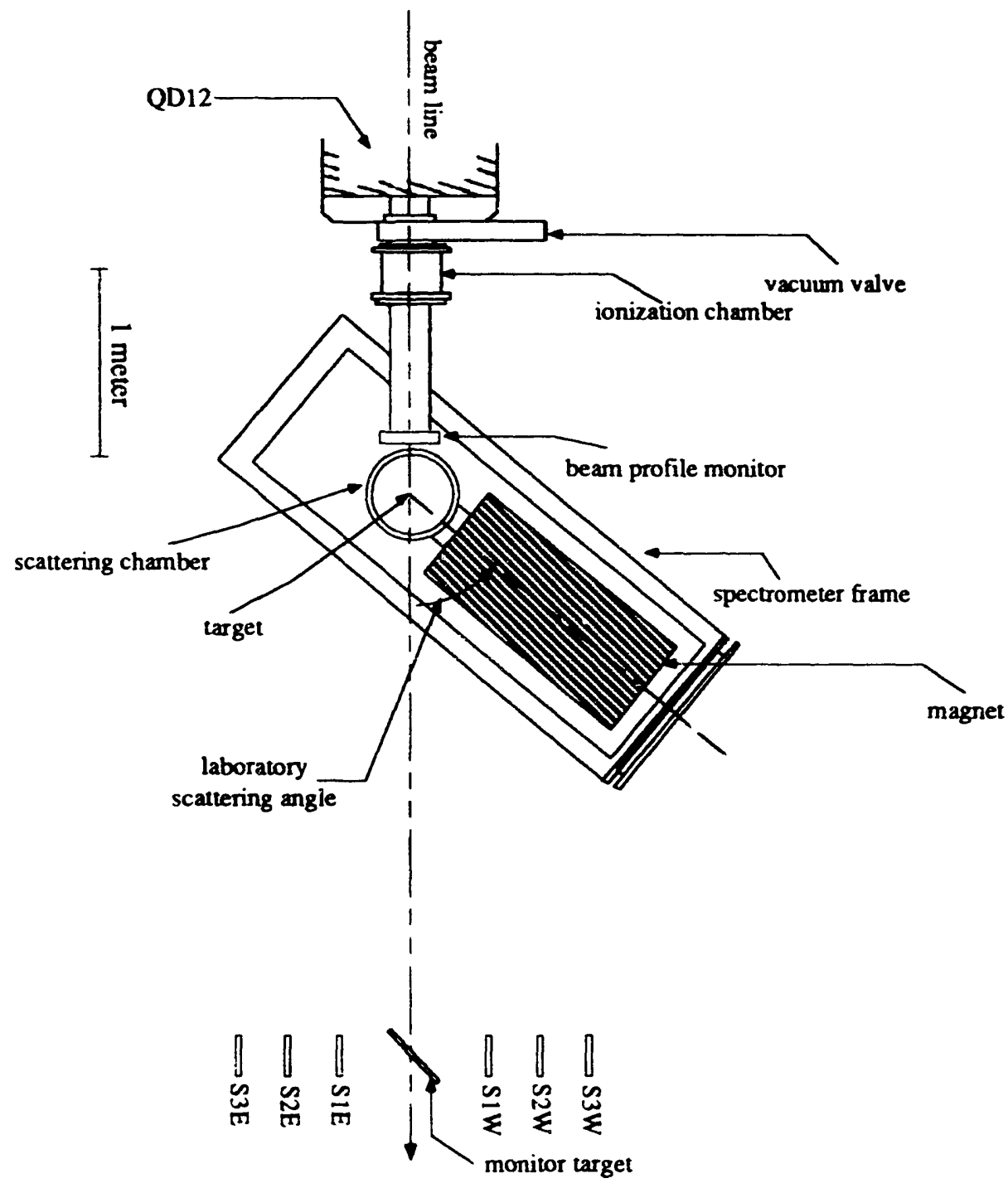

Figure 2.3: Top view of the Litule Yellow Spectrometer and beam monitoring system. 


\section{Beam Monitoring and Operation}

The beam position and flux were monitored continuously throughout the experiment using a beam profile monitor, an ionization chamber and a pion scattering monitor. For adjusting the last two quadrupoles, a second beam profile monitor was placed next to the scattering chamber downstream of the target. The position of the beam waist and the transverse profile of the beam were adjusted by observing the focal point of the beam shift back and forth between the upstream and downstream monitors. The magnetic field settings of the quadrupoles were then tuned so that the waist of the beam intersected the target and the transverse divergence of the beam spot was minimized as it passed through the interaction region. The downstream monitor was removed during data acquisition and is not shown in Figure 2.3.

The beam profile monitor was also used as a diagnostic tool for monitoring the spatial position and extent of the pion beam. The incident pion beam flux is too high for conventional multiwire proportional counter (MWPC) read out systems to tolerate. The beam profile monitor design by Krausse and Gram, ${ }^{\mu}$ based on a modified version of a MWPC, uses an analog data-processing system to read out the position information from two multiwire proportional chambers with orthogonal planes of $1 \mathrm{~mm}$ spaced wires. The bias voltages on the chambers may be adjusted to cover a range of incident beam fluxes from $10^{5}$ to $10^{10}$ particles/s. Typical beam fluxes during this experiment were $10^{8} \pi / \mathrm{s}$.

An ionization chamber was employed for monitoring the incident beam flux during the experiment. The ionization chamber detected ion pairs produced by incident pions and other charged particles as they passed through an argon filled cylindrical chamber which was $25.4 \mathrm{~cm}$ long and $31.1 \mathrm{~cm}$ in diameter. Four $6 \mu \mathrm{m}$ thick aluminized mylar plates, kept near ground, collected the ions and generated a current signal. A $150 \mathrm{MeV}$ pion would typically lose $50 \mathrm{KeV}$ in kinetic energy as it passed through the ionization chamber. An additional five $6 \mu \mathrm{m}$ thick aluminized mylar plates, interspersed between the ground plates and kept at $900 \mathrm{~V}$, established an electric field for collecting the ions. The current signal from the ionization chamber was integrated and digitized. Since the pion beam was contaminated by protons, positrons, and muons, the ionization chamber response was calibrated at each incident beam energy. In this experiment, $\pi^{+} p$ elastic 
scattering measurements were performed to provide a simultaneous calibration of the ionization chamber response and the solid angular acceptance of the spectrometer. This calibration procedure is discussed in Chapter 3.

The pion scattering monitor was used to monitor the incident beam stability. The scattering monitor consisted of an 0.125 in thick polyethylene $\left(\mathrm{CH}_{2}\right)$ target placed at an angle of $45^{\circ}$ with respect to the incident beam and two sets of three scintillation counters located at $90^{\circ}$ with respect to the monitor target (see Figure 2.3). This monitor detected $\pi^{*}$ scattering from hydrogen in the polyethylene target. A threefold coincidence from either set of scintillation counters formed the trigger for a good pion scattering event. Fluctuations in the coincidence rate for a particular beam energy were indicators of possible beam intensity or position variations.

\section{Recoil Detection Scheme}

In this experiment, lzavy charged particles, such as protons, deuterons, tritons, ${ }^{3} \mathrm{He}$ particles and $\alpha$ particles, were detected with a magnetic spectrometer. This spectrometer was equipped with an array of nineteen silicon surface barrier detectors and a thin plastic scintillator at the focal plane. A schematic diagram of the magnetic spectrometer and detection system is shown in Figure 2.4. Although this detection system has been successfully used in a number of experiments to detect charged pions, this experiment represents the first time that it has been used to detect heavier charged particles. The design and operation of the Litule Yellow Spectrometer has been discussed in great detail by several authors. ${ }^{6,45} \times 6,47 \times 3.49$

\section{Little Yellow Spectrometer}

The Liule Yellow Spectrometer (LYS) is a double focusing, $180^{\circ}$ vertical bend spectrometer. The important spectrometer parameters are summarized in Table 2.1. The focal plane of the spectrometer lies approximately one and one half meters above the beam line. Charged particles are transported through the 
spectrometer by a pair of $90^{\circ}$ bending magnets. The fringe fields of the magnets minimize the spatial dispersion of particles as they travel through the spectrometer in the horizontal plane while the principal field disperses the particles in the vertical plane according to their deviation from the central momentum setting of the spectrometer. The full-width-at-half-maximum momentum bite, $\Delta \mathrm{p} / \mathrm{p}$, at the focal plane of the spectrometer is approximately $8.5 \%$. The $3.5 \mathrm{~m}$ flight path from target to focal plane scintillator is in continuous vacuum to minimize energy loss and multiple scattering. The spectrometer may rotate about the target axis over an angular range of $25^{\circ} 10130^{\circ}$.

The central momentum setting of the spectrometer was determined by the magnetic field settings of the bending magnets. The magnetic fields were monitored by measuring a shunt voltage - a voltage drop across a metal alloy resistor in series with the coils energizing the two bending magnets which was in tum proportional to the current that was supplied to the two bending magnets.

\begin{tabular}{|c|l|}
\hline \multicolumn{2}{|c|}{$\begin{array}{c}\text { Litule Yellow Spectrometer } \\
\text { Parameters }\end{array}$} \\
\hline$\Delta \Omega$ & $=15 \mathrm{msr}$ \\
\hline$\Delta \mathrm{p} / \mathrm{p}(\mathrm{FWHM})$ & $8.5 \%$ \\
\hline Maximum Momentum & $325 \mathrm{MeV} / \mathrm{c}$ \\
\hline Dispersion ( $\delta \mathrm{p} / \mathrm{p} / \mathrm{cm})$ & $0.44 \% / \mathrm{cm}$ \\
\hline Radius of Curvature & $60 \mathrm{~cm}$ \\
\hline Flight Path & $3.5 \mathrm{~m}$ \\
\hline Bend Angle & $180^{\circ}$ \\
\hline
\end{tabular}

Table 2.1: Spectrometer Parameters 


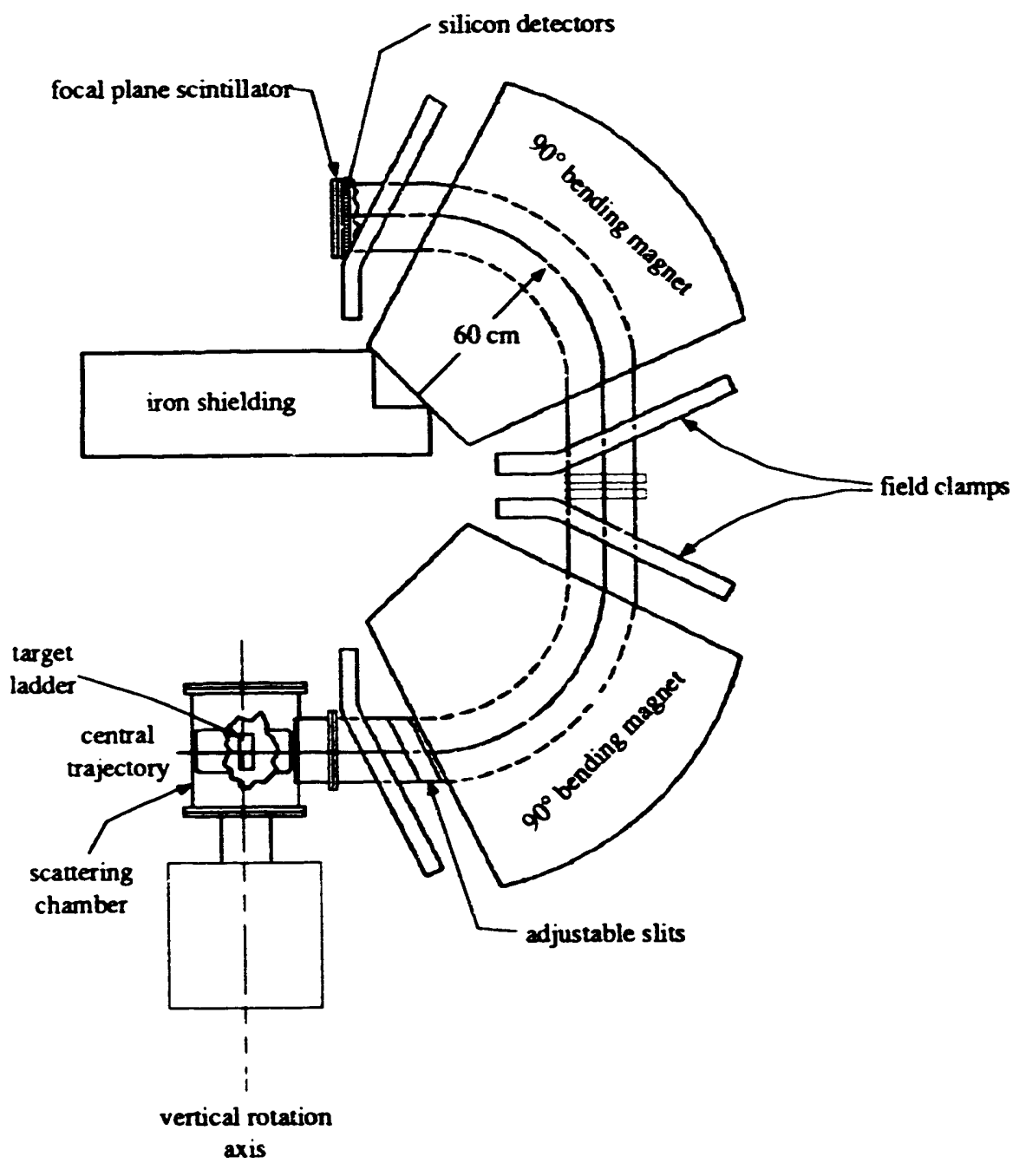

Figure 2.4: Side View of the Little Yellow Spectrometer

The magnetic field of the spectrometer, $H$, can be expressed in terms of the shunt voltage $x$ as

$$
H(x)=A+B \sigma[1+\exp (-\mu / \sigma)] \cdot \ln \left(\frac{1+\exp (\mu / \sigma)}{1+\exp [(\mu-x) / \sigma]}\right),
$$

where $\mu, \sigma, A$, and $B$ are calibration constants previously determined by measurements using a NMR probe. ${ }^{49}$ The central momentum of the spectrometer for a particle with charge $q$ is related to the magnetic fields of the two bending magnets $\left(H_{1}\right.$ and $\left.H_{2}\right)$ by the expression 


$$
p=\frac{a}{2}\left[H_{1}(x)+H_{2}(x)\right] \cdot q,
$$

where $a$ is $18.119 \pm 0.012 \mathrm{MeV} / \mathrm{kG} \cdot \mathrm{c}$. The parameters in the magnetic field calibrat.un are given in Table 2.2. Typical field setuings during this experiment ranged from approximately 1 to $20 \mathrm{kGauss.}$

\begin{tabular}{|c|c|c|c|c|}
\hline \multicolumn{5}{|c|}{ Liule Yellow Spectrometer Magnetic Field Parameters } \\
\hline $\mathrm{i}$ & A (Gauss) & B (Gauss/mV) & $\mu$ (mV) & $\sigma(M V)$ \\
\hline 1 & $12.55 \pm 8.98$ & $278.9 \pm 0.42$ & $74.02 \pm 1.13$ & $7.436 \pm 0.54$ \\
\hline 2 & $19.65 \pm 3.45$ & $277.8 \pm 0.15$ & $72.34 \pm 0.39$ & $6.484 \pm 0.19$ \\
\hline
\end{tabular}

Table 2.2: Magnetic Field Parameters

The geometrical acreptance of the spectrometer is determined for the most pan by the size of the vacuum box within the spectrometer. This acceptance can affect the relative detection efficiency differently for each silicon surface barrier detector at the focal plane of the spectrometer. The geometrical effect on the spectrometer acceptance has been calculated by a Monte Carlo simulation of the Liule Yellow Spectrometer system. However, there may also be differences due to variations in detector surface areas and depletion depths as well as other possible mechanical or electrical differences between the detectors, and thus the Monte Carlo acceptance must be checked with experimental measurements. This procedure is described in Chapter 3.

In order to deduce the momentum of a particular particle from the focal plane position information, one must know the momentum dispersion of the spectrometer. Although the dispersion was not measured in this experiment, it has been previously measured in several other experiments which have utilized the Litule Yellow Spectrometer in the same configuration. 


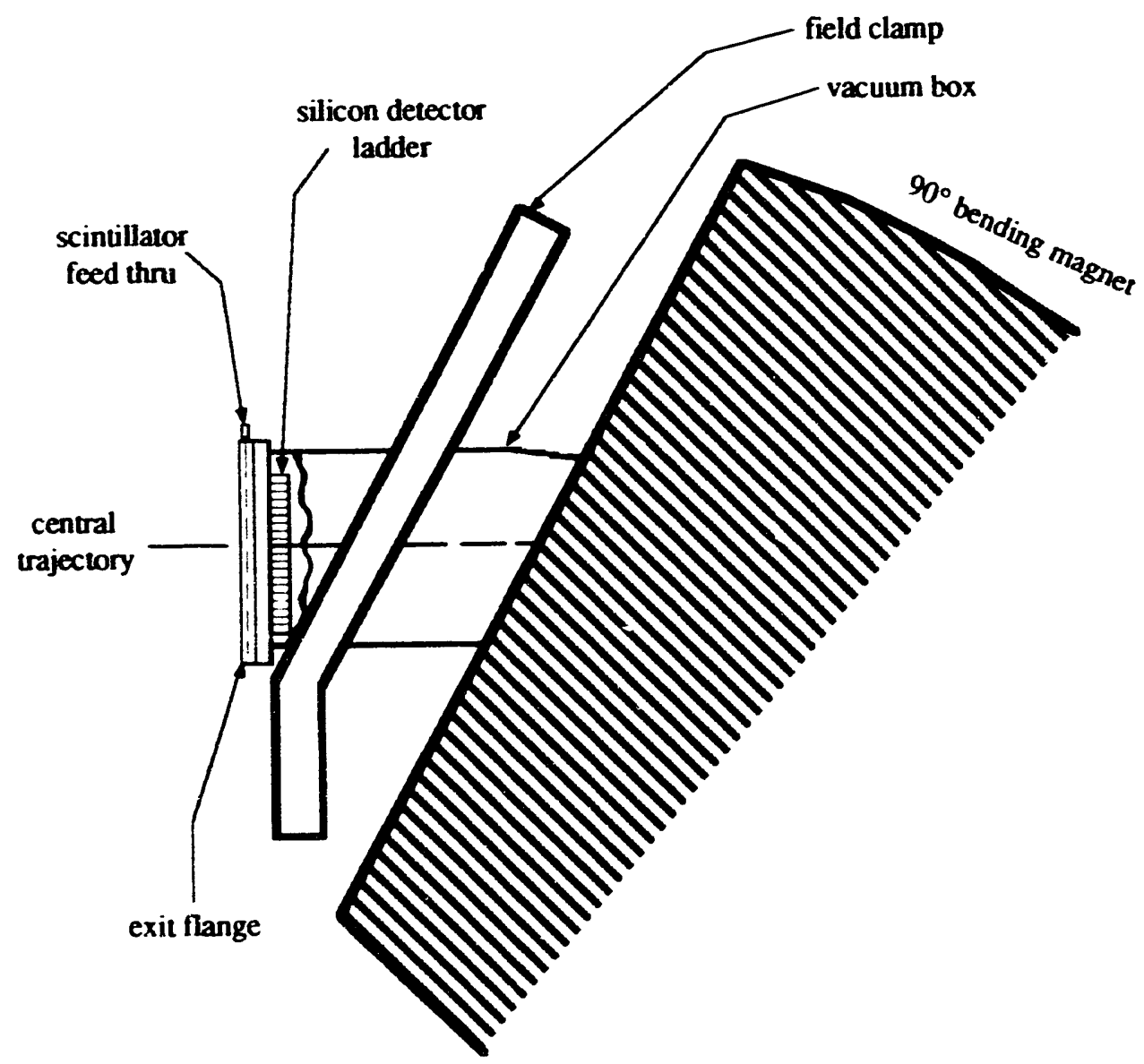

Figure 2.5: Side view of the Litule Yellow Spectrometer focal plane region. The scintillator is located inside the exit flange.

\section{Detectors}

The detector package for this experiment consisted of two elements-an array of nineteen silicon surface barrier detectors and a thin plastic scintillation counter. A schematic drawing of the focal plane region of the spectrometer is shown in Figures 2.5 and 2.6. The nineteen silicon detectors spanned a 9.5\% momentum bite at the focal plane of the spectrometer. The $1 \mathrm{~cm} \times 3 \mathrm{~cm} \times 0.04 \mathrm{~cm}$ silicon surface barrier ( stectors were ceated with a $10 \mu \mathrm{g} / \mathrm{cm}^{2}$ layer of aluminum on one side and a $40 \mu \mathrm{g} / \mathrm{cm}^{2}$ layer of gold on the other side forming a $p-n$ junction. The bias voltages on the detectors were adjusted so that each detector was fully depleted. These detectors were placed in a ladder with alternate sides of aluminum and gold 
facing the entrance of the spectrometer to attain $89 \%$ coverage at the focal plane. ${ }^{49}$ The design of the Litule Yellow Spectrometer provided a relatively large depth of focus of approximately $4 \mathrm{~cm}$. This allowed the centroids of the detectors, which were placed $1.125 \mathrm{~cm}$ apart in the dispersive direction, to be staggered $\pm 0.2 \mathrm{~cm}$ longitudinally about the focal plane of the spectrometer. This detector arrangement provideci a total coverage of $21.25 \mathrm{~cm}$ in the dispersive direction and $3 \mathrm{~cm}$ in the non-dispersive direction at the focal plane. Charged particles with as little as $1.4 \mathrm{MeV}$ kinetic energy could be detected with this silicon detector arrangement (see Figure 2.7).

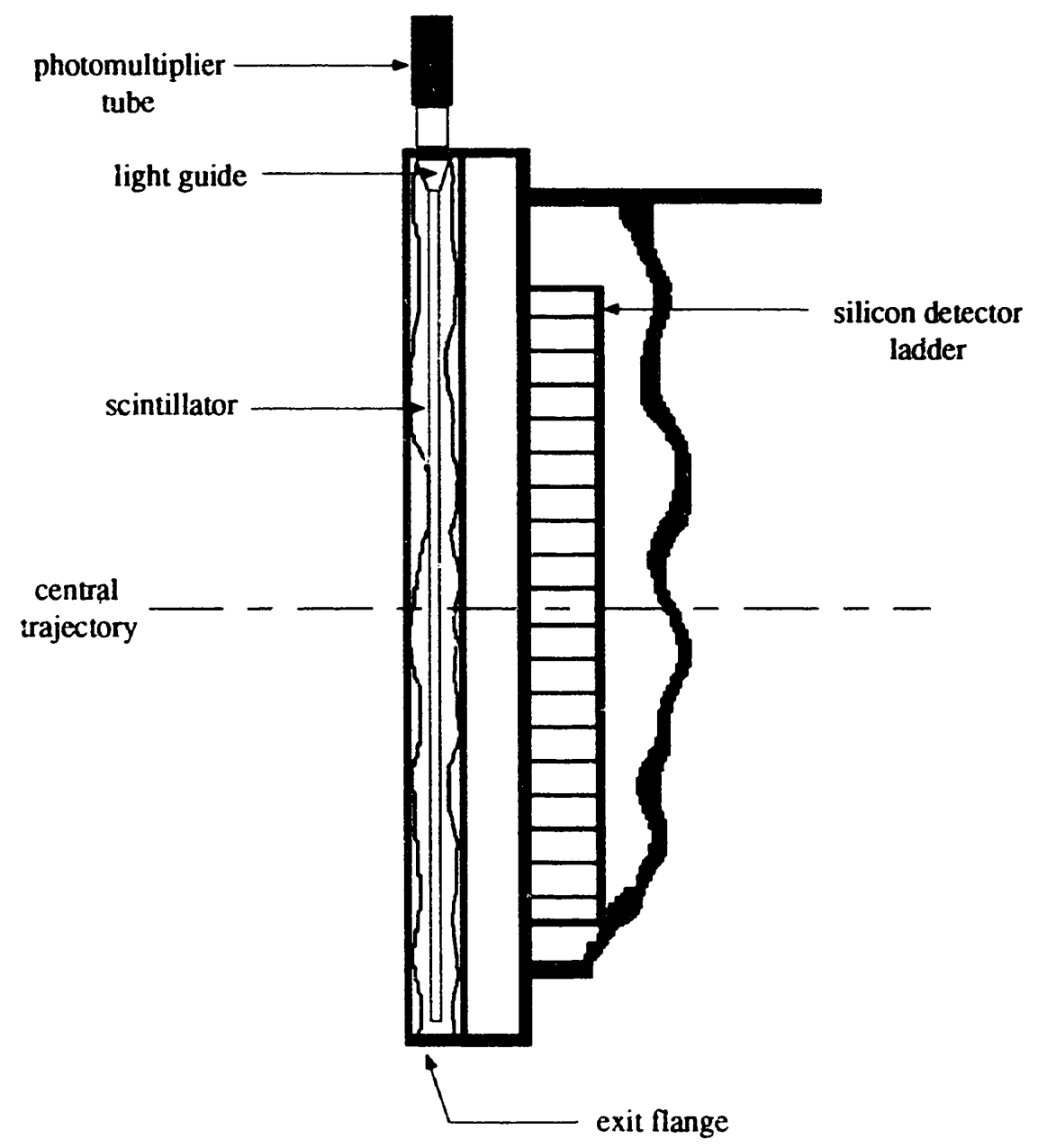

Figure 2.6: Detailed view of focal plane scintillation counter. The 0.125 " scintillation counter was located inside the spectrometer vacuum box. The light from the scintillator was directed through the top of the exit flange by a small light guide and collected by a 0.5 " diameter Hamamatsu phototube. 


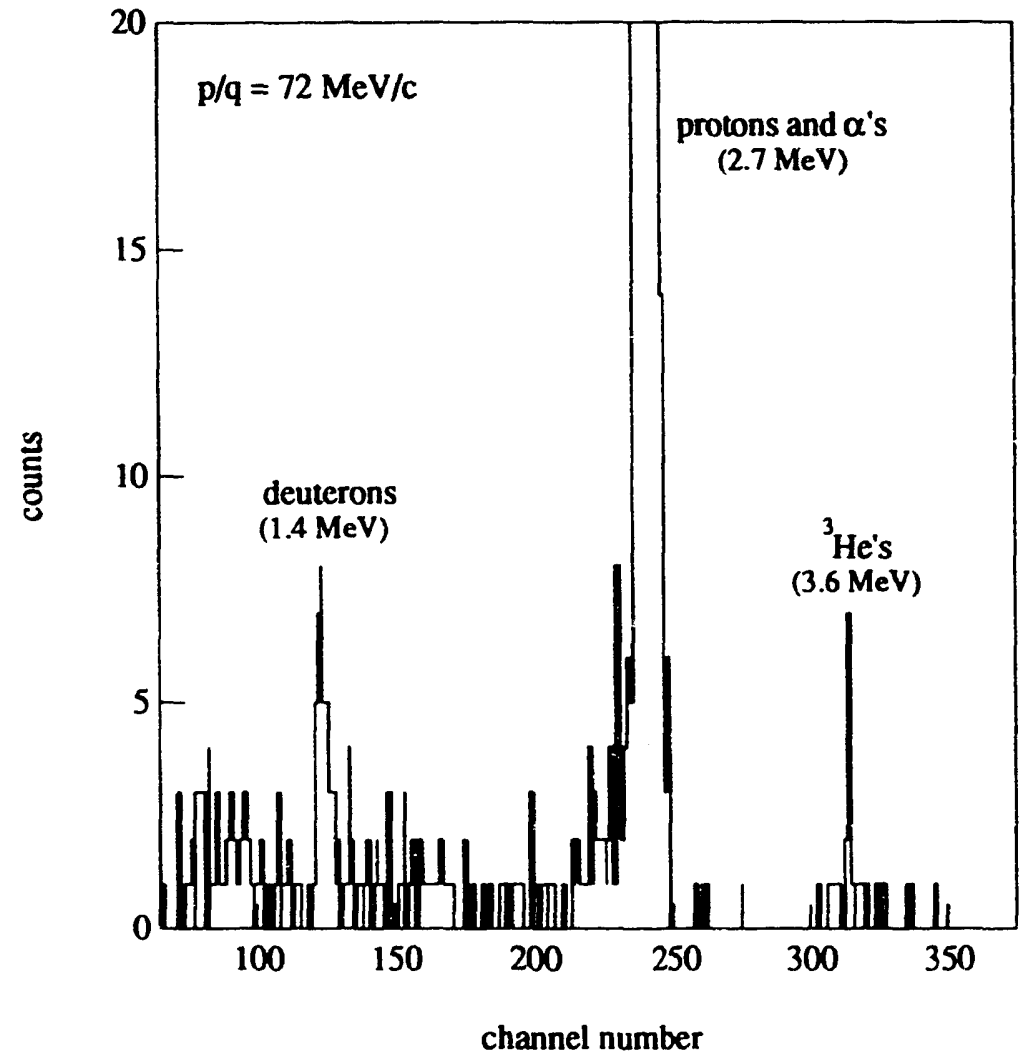

Figure 2.7: Typical silicon detector pulse height spectrum. Heavy charged particles with as little as 1.4 $\mathrm{MeV}$ kinetic energy could be detected by the silicon surface barrier detectors.

The design of the thin scintillation counter, its light collection and mounting schemes, was constrained by the limited available space between the silicon detectors and the exit window of the spectrometer vacuum box. A view of the silicon detector and scintillator arrangement is shown in Figure 2.6. The scintillator was placed inside the spectrometer vacuum chamber so as to maximize the detection efficiency for heavy charged particles, such as ${ }^{3} \mathrm{He}$ particles, which did not stop in the silicon detectors. The exit flange of the spectrometer was re-designed to accommodate both the exit window and the scintillator. The 
$0.125^{n}$ thick scintillator covered a $25.4 \mathrm{~cm}$ by $9 \mathrm{~cm}$ region and could be used as either a coincidence or veto with the silicon detectors for improved particle identification. The scintillator pulse height information was important since at some spectrometer momentum settings the pulse heights from the silicon surface barrier detectors for different types of particles overlapped. In Figure 2.8, the energy loss in the silicon detectors is shown as a function of the magnetic rigidity, p/q, for protons, deuterons, tritons, ${ }^{3} \mathrm{He}$ particles, and $\alpha$ particles. In instances, where two different types of particles generated similar pulse heights in a particular silicon detector, the particles which did not stop in the silicon detector generated a signal in the scintillator. The light from the scintillator, directed through the top of the exit flange by a small light guide, was collected by a 0.5 " diameter Hamamatsu phototube.

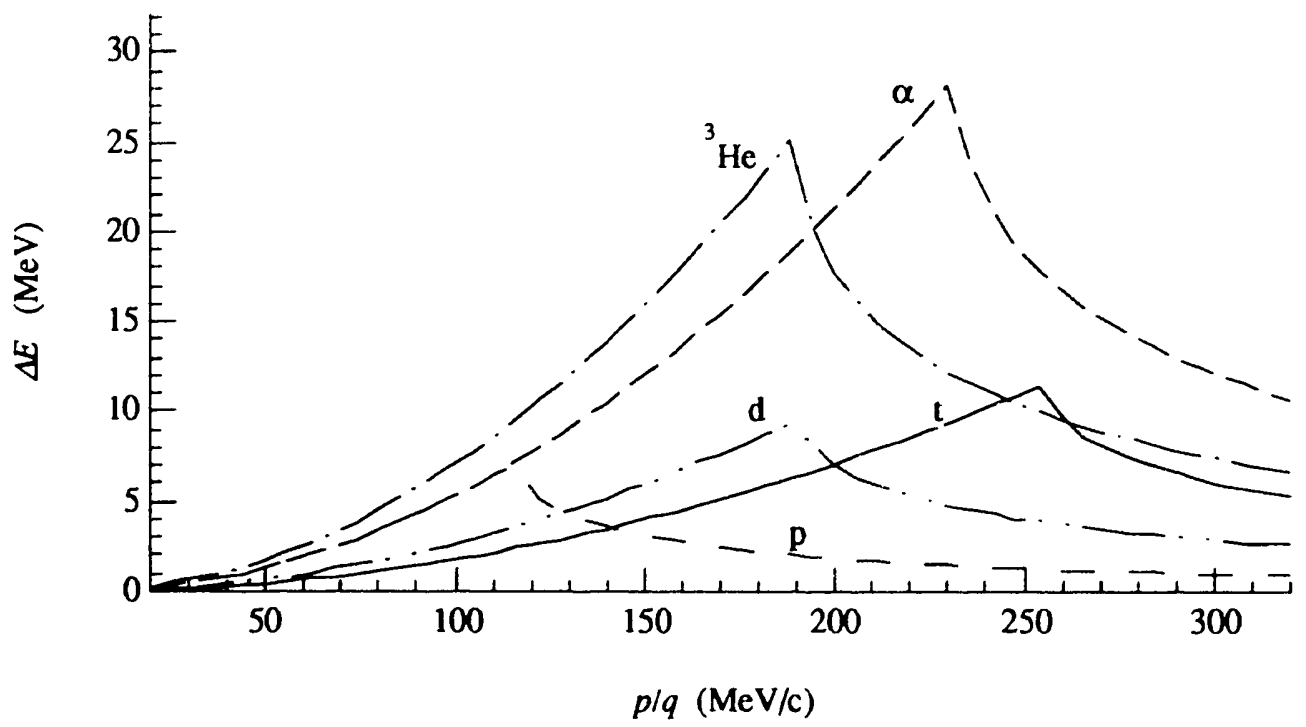

Figure 2.8: Energy loss in $400 \mu \mathrm{m}$ silicon surface barrier detector as a function of spectrometer momentum setting $(p / q)$. In instances where the pulse height from two different particles overlapped, the pulse height information from the focal plane scintillation counter could be used distinguish between them, e.g. energy deposited by deuterons and tritons with $\sim 200 \cdot q \mathrm{MeV} / \mathrm{c}$ momenta, where $q$ is the charge of the recoil particle. 


\section{Electronics and Data Acquisition System}

Schematic diagrams of the electronics for this experiment are shown in Figure 2.9 through 2.11. A charged particle passing through a silicon detector generated a pulse whose total charge was proportional to the energy loss of the particle in the active region of the silicon detector. The signal from a silicon detector was fed into a charge sensitive preamplifier which was located near the focal plane of the spectrometer outside the vacuum box. Pulses from each of the silicon detector preampl..iers were fed into amplifiers located in the counting house. An unipolar output pulse, with amplitude proportional to the energy lost in the silicon detector, was sent to a peak-sensing ADC and digitized. A bipolar output pulse was sent to a timing single channel analyzer which generaied a logic signal for every zero crossing. An inclusive OR of the nineteen logic signals formed the event trigger for a silicon detector event. These nineteen logic signals were also fed into a pattern unit which recorded the hit pattern of charged particles in the silicon detectors for an event. A logic pulse from a "dead" preamplifier was used to generate a "spike" veto so that events which were the result of electrical noise in the detectors were ignored.

The number of triggers was also recorded by two scalers, one of which was gated by the BUSY OR circuit. The BUSY OR circuit included a run gate, a computer busy, and a beam gate. The run gate was constructed using a computer signal, which indicated that data acquisition was in progress, and a mechanical switch. This mechanical switch allowed a user to stop or start the data acquisition process manually. The beam gate was derived from a signal which was keyed to the pulse structure of the primary accelerator and allowed data acquisition only during the beam pulses. Additional signals, registering that either the computer was busy processing events or that the electronics were busy, were also included in the BUSY

\begin{tabular}{|c|c|c|c|c|c|c|c|c|c|}
\hline \multicolumn{10}{|c|}{ E1026 Electronics Diagram Legend } \\
\hline symbol & AMP & ADC & D & DEL & FIFO & GG & LAM & LTM & TSCA \\
\hline description & amplifier & $\begin{array}{l}\text { analog-10- } \\
\text { digital } \\
\text { converter }\end{array}$ & $\begin{array}{l}\text { discrim- } \\
\text { inator }\end{array}$ & $\begin{array}{l}\text { delay } \\
\text { fan-in } \\
\text { fan-out }\end{array}$ & $\begin{array}{l}\text { gate } \\
\text { generator }\end{array}$ & $\begin{array}{l}\text { look-at-me } \\
\text { (computer } \\
\text { busy) }\end{array}$ & $\begin{array}{l}\text { LAMPF } \\
\text { trigger } \\
\text { module }\end{array}$ & $\begin{array}{l}\text { timing single } \\
\text { channel } \\
\text { analyzer }\end{array}$ \\
\hline
\end{tabular}

Table 2.3: Legend for E1026 Electronics Diagram 


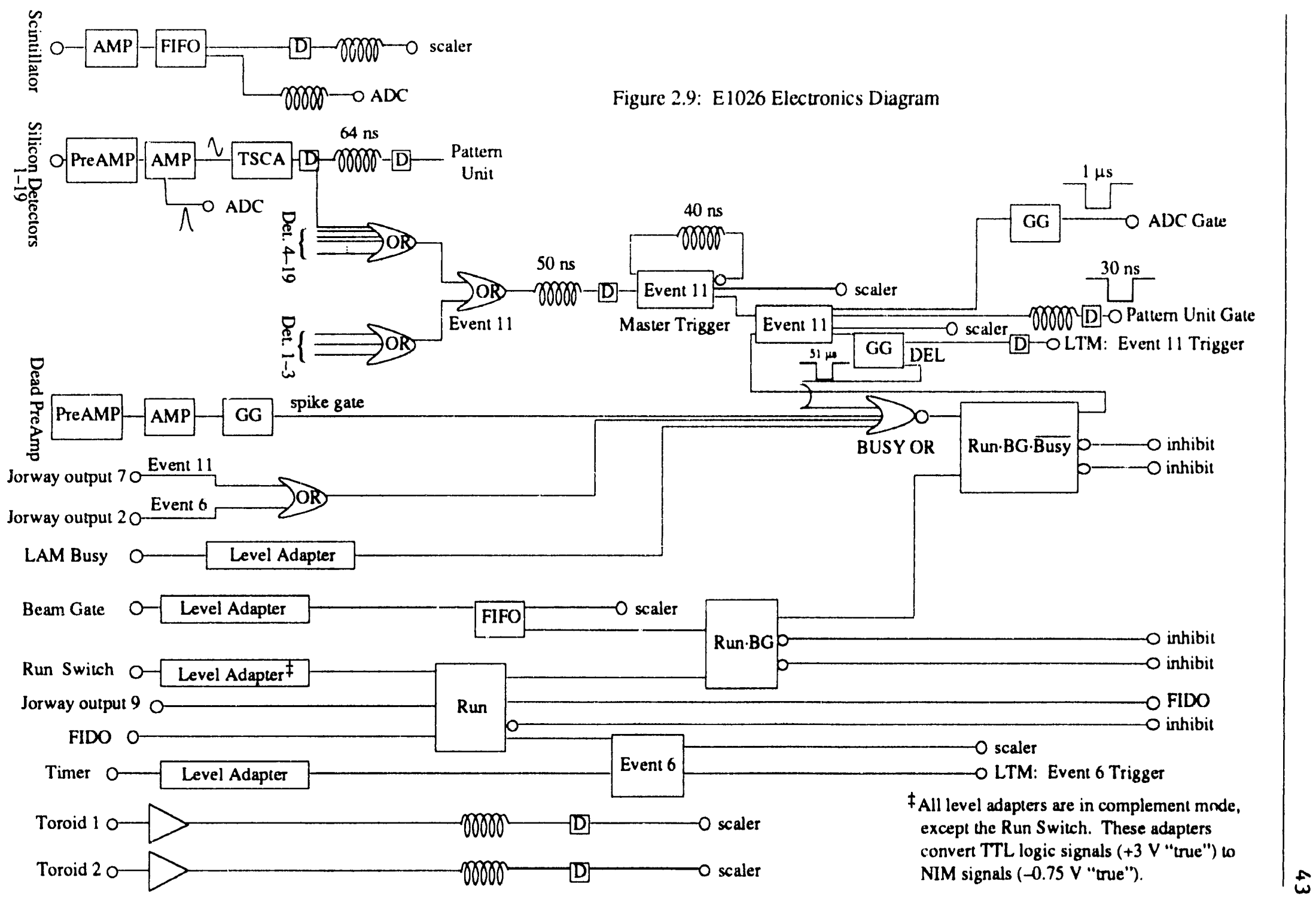




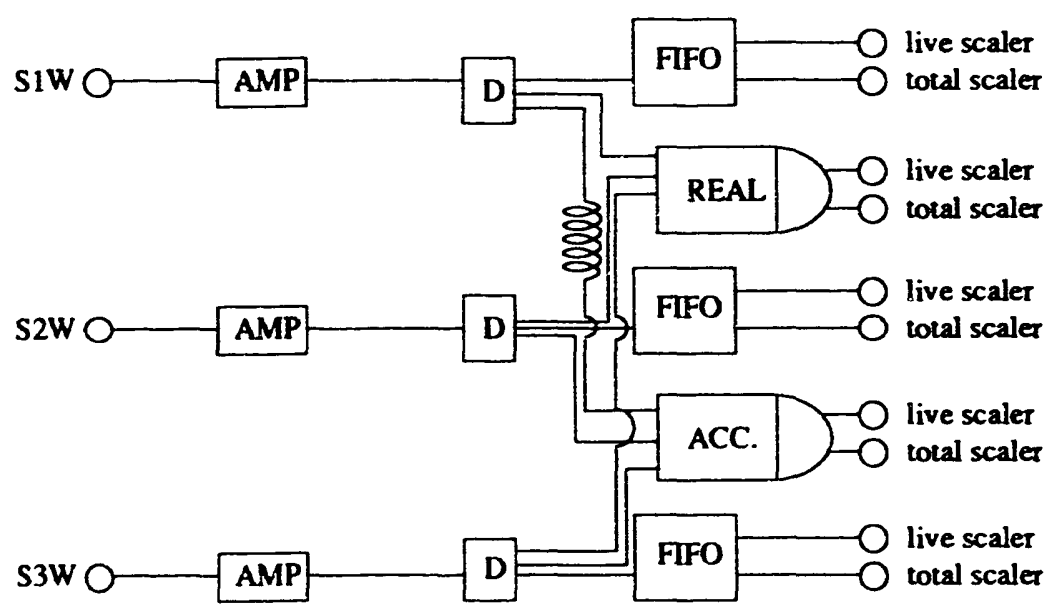

Figure 2.10: Electronics logic diagram for a pion scattering monitor telescope. The logic for the East telescope is identical to that shown above for the West counters. The live and total scalers refer to scaler modules which were gated by RUN.BG.BUSY and RUN-BG respectively. The symbols "REAL" and "ACC." refer to the real and accidental coincidences between the three counters respectively.

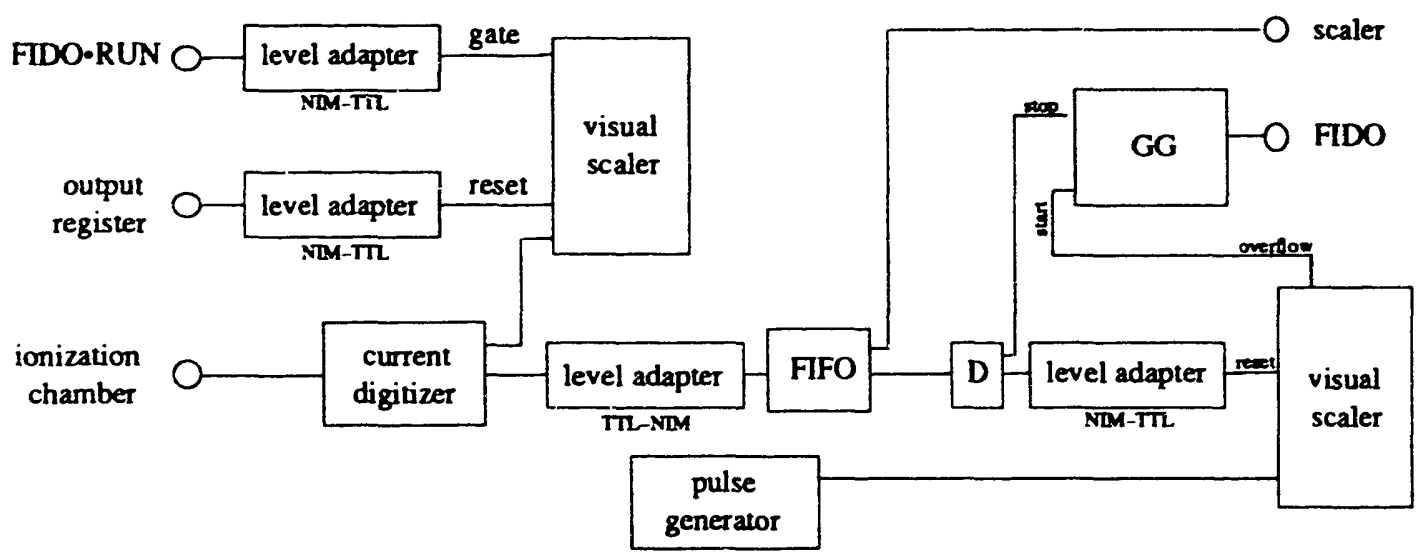

Figure 2.11: Ionization chamber and "FIDO" electronics diagram. The FIDO circuit causes data acquisition to cease if there is no beam present.

OR circuit. Events were not recorded when the BUSY OR output was "true." The BUSY OR circuit logic and event trigger processing logic were very similar to the logic used in previous Litule Yellow Spectrometer experiments which has been described by several authors. ${ }^{6.45 .46}$ 
A Microprogrammable Branch Driver (MBD) controlled the CAMAC electronics and acted as a buffer for the digitized data between the electronics and the data acquisition computer, a DEC microV ax. The LAMPF data acquisition package, $Q,{ }^{50}$ was used to control, monitor, and display the accumulated data. Experiment specific Q Analyzer I_anguage (QAL) code was written to execute CAMAC functions and manipulate the CAMAC devices through the MBD. This code contained the information about what types of CAMAC functions to perform for a specific event trigger. A LAMPF trigger module notified the MBD of the type of event to process. In this experiment there were three kinds of events: a silicon detector event (Event 11), a scaler event (Event 6), and a spectrometer shunt read event (Event 106). The spectrometer angle and the target ladder position were also recorded during an Event 6. Events 6 and 106 were triggered at uniform intervals by an external timer and at the end of every run by the computer. A Jorway model 41 output register inhibited event triggers when the MBD was busy and generated the computer and electronic signals for the BUSY OR circuit. The output register also reset the ionization chamber visual scaler at the beginning of each run.

A new method for recording the spectrometer shunt voltage during data acquisition was developed for this experiment. In past years, the CAMAC read out device for the shunt voltage was too slow relative to the other electronic read out devices and would occasionally cause the MBD to malfunction. The read out method devised for this experiment bypassed the CAMAC electronics and recorded the shunt voltage through a terminal port on the microvax using a GPIB-RS232 connector. Once in place, this GPIB read out system worked continuously without incident for a period of longer than six weeks.

\section{Targets}

Several different targets were employed during these measurements. Thin polyethylene (0.024"

$\left.\mathrm{CH}_{2}\right)$ and graphite $\left(0.010^{12} \mathrm{C}\right)$ targets were used in the $\pi^{+}$pelastic scattering measurements for calibration of the spectrometer acceptance and ionization chamber response. A 0.120 " polyethylene target was used to calibrate the silicon detector pulse height spectra at each spectrometer momentum setting for the single 
charge exchange and $\pi^{+3} \mathrm{H}$ elastic scattering measurements. Thin tritiated titanium foils $\left(2 \mu \mathrm{g} / \mathrm{cm}^{2}\right)$ were used in the single charge exchange and $\pi^{+3} \mathrm{H}$ elastic scattering measurements. The tritium content of the tritiated foils was determined from $\pi^{+3} \mathrm{H}$ elastic scattering. Untritiated titanium foils were used to measure the background from pion interactions with titanium. These thin $4 \mathrm{~cm} \times 8 \mathrm{~cm}$ foils were seated in aluminum frames and mounted in a target ladder along with an empty aluminum frame, the polyethylene targets and a graphite target. This target ladder mounting scheme minimized the exposure of the extremely britte tritiated foils to unnecessary handling. A schematic diagram of a target frame is shown in Figure 2.12. A new target ladder controller was developed for this experiment. This controller supplied the power to the two small AC motors which were used to adjust the target position from the counting house and read out the target height and angle position with respect to the incident beam.

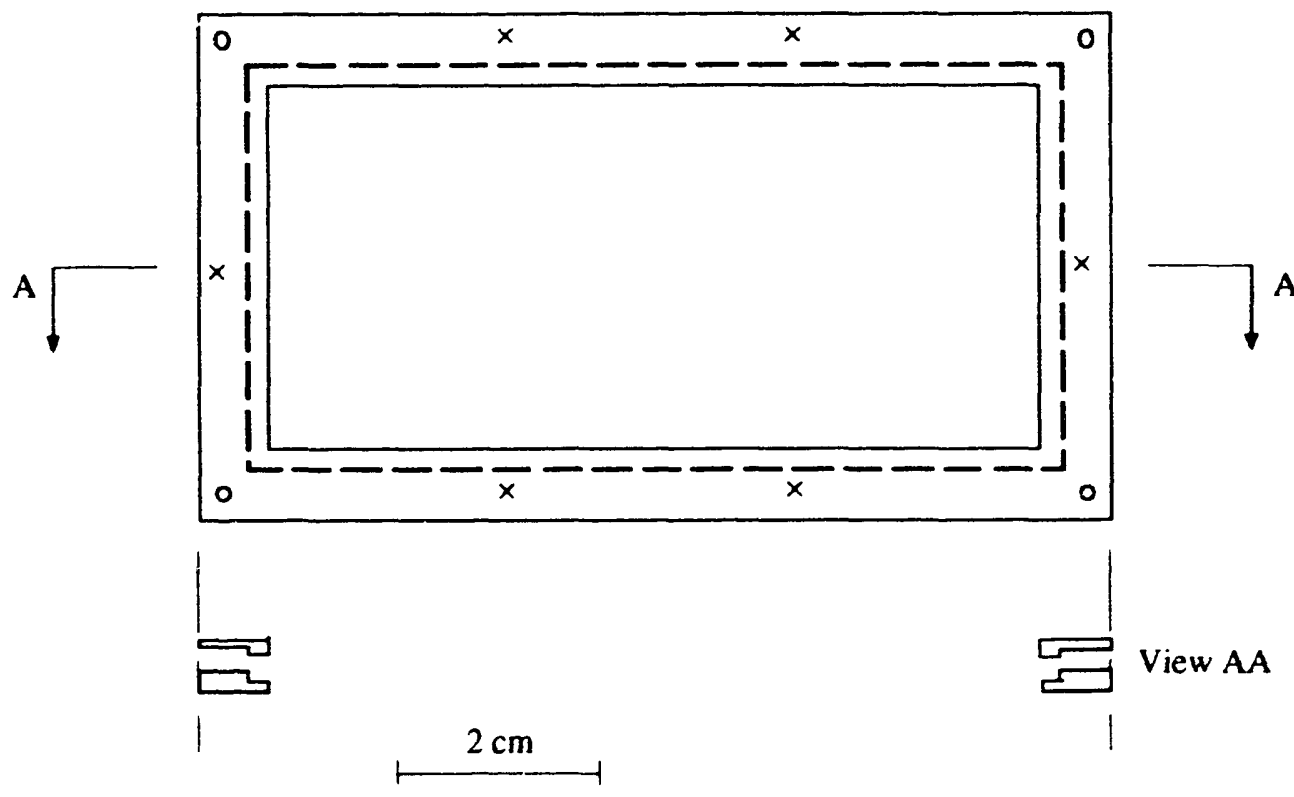

Figure 2.12: Schematic diagram of a target frame. A target frame consisted of two pieces of $1 / 16$ " thick aluminum. The $\mathrm{CH}_{2},{ }^{12} \mathrm{C}$, tritiated and untritiated titanium foils were sandwiched between the two aluminum frames. These frames were held together by six 2-56 screws ( $\times$ 's) and mounted on a target ladder using roll pins (circles). Seven of these target frames could be mounted on the target ladder simultaneously. 


\section{Chapter 3: Experimental Procedures, Data Analysis, and Results for the ${ }^{3} \mathrm{H}\left(\pi^{+},{ }^{3} \mathrm{He}\right) \pi^{0}$ Measurements}

Upon completion of the measurements described in the previous chapter, the raw data were analyzed to extract differential cross sections. It is necessary to know several parameters of the experimental apparatus, such as the solid angle acceptance of the spectrometer, the efficiencies of the individual detectors, the incident beam flux, and the target thicknesses in order to compute a cross section.

The analysis procedures and their results which were developed to extract differential cross sections for the ${ }^{3} \mathrm{H}\left(\pi^{+},{ }^{3} \mathrm{He}\right) \pi^{0}$ reaction are described in this Chapter. Momentum spectra of recoil ${ }^{3} \mathrm{He}$ nuclei were generated following these procedures. These spectra were then integrated in order to determine differential cross sections. First, the experimental procedures for accumulating the calibration data are outlined. Next, the analysis method to extract and correct yields of specific particles from the raw event data for quantities which varied on a run by run basis, such as the number of incident pions, will be discussed. Then, the analysis steps for the calibration data will be described and the results will be presented. Finally, the procedures for combining the calibration information and the Little Yellow Spectrometer Monte Carlo calculations to compute differential cross sections from the ${ }^{3} \mathrm{H}\left(\pi^{+},{ }^{3} \mathrm{He}\right) \pi^{0}$ data are discussed. 


\section{Experimental Procedures}

Four types of measurements were performed during this experiment. In addition to measurements of the ${ }^{3} \mathrm{H}\left(\pi^{+}{ }^{3} \mathrm{He}\right) \pi^{0}$ differential cross section, a differential cross section for $\pi^{+} \mathrm{p}$ elastic scattering was measured at each incident beam energy to determine the ionization chamber calibration and the solid angular acceptance of the spectrometer. A differential cross section for $\pi^{+3} \mathrm{H}$ elastic scattering was measured to ascertain the tritium content of the tritiated titanium foils. The uritium content of the target and the ionization chamber calibration were determined by comparing the measured cross sections with

previous results. ${ }^{20,51}$ Proton momentum spectra from the ${ }^{12} \mathrm{C}\left(\pi^{*}, \mathrm{p}\right) \mathrm{X}$, reaction were measured at several spectrometer momentum settings to determine the relative efficiency of the silicon surface barrier detectors. The proton momentum spectrum was relatively flat over the $8 \%$ momentum bite of the spectrometer for the incident pion energies and proton recoil angles which were used in these measurements.

\section{Data Reduction}

The first step in this analysis was to conven the event-by-event data into a histogram format. Data were replayed using the on-line data acquisition package which was developed for this experiment, and pulse height spectra from the nineteen silicon surface barrier detectors in each data run were constructed. At the same time, quantities which depended on the experimental conditions were calculated or retrieved from the raw data. The pulse height spectra were then corrected for these quantities, such as the spectrometer momentum setting, target angle, and live time.

For each measurement, it was necessary to identify the particle of interest in a pulse height spectrum so that a raw yield could be extracted. The pulse height spectra from $\mathrm{CH}_{2}$ calibration data were examined and the peaks from different types of particles were identified at each momentum setting. Typical pulse height spectra from measurements with the tritiated $\left(\mathrm{Ti}^{3} \mathrm{H}\right)$ and untritiated (Ti) titanium foils are shown in comparison with a $\mathrm{CH}_{2}$ calibration spectrum in Figure 3.1. In these spectra, the proton, 

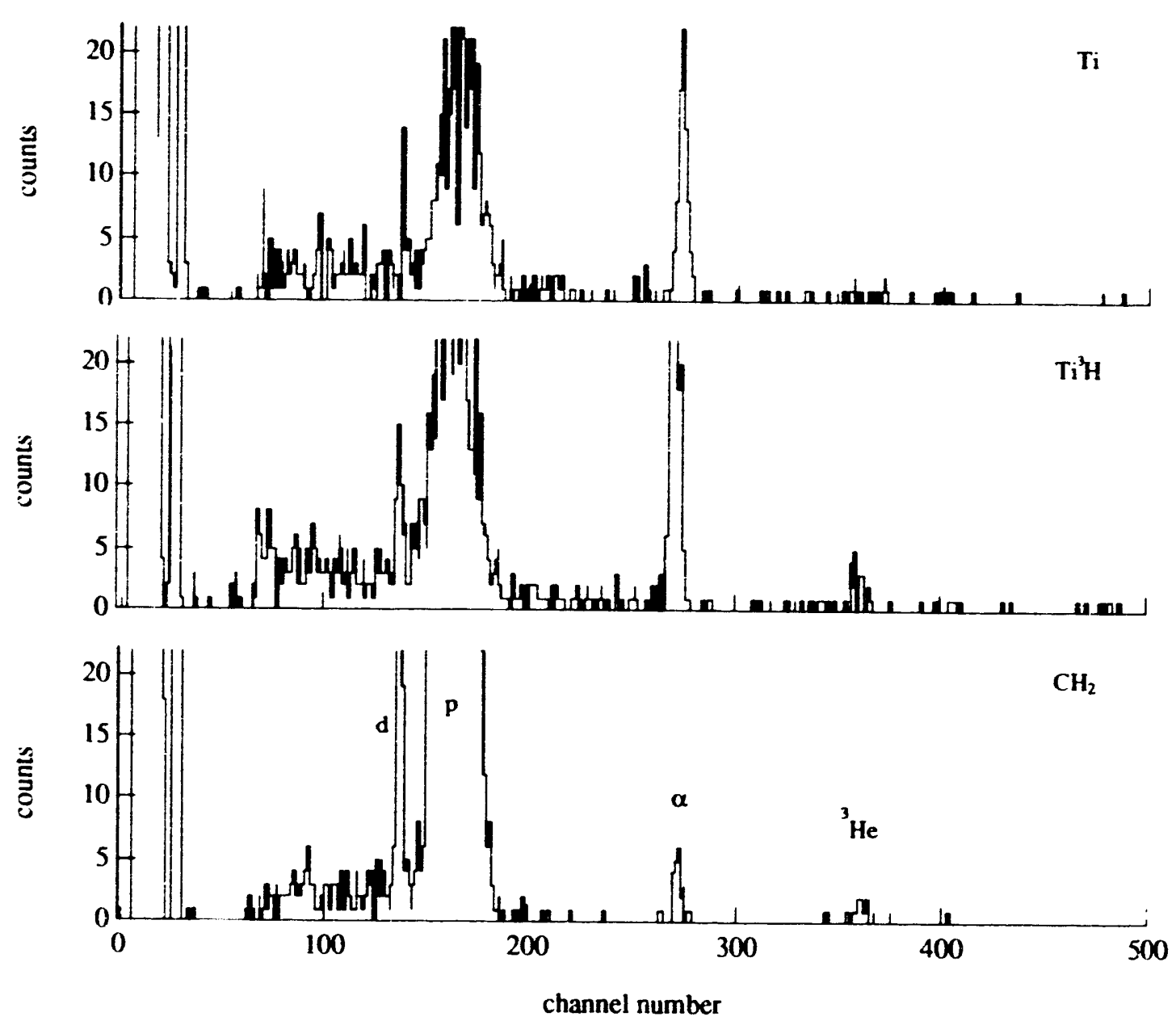

Figure 3.1: Typical silicon detector pulse height spectra. The $\mathrm{CH}_{2}$ data could be obtained relatively quickly and thus had less background. In addition, the relative positions of the peaks could be used to determine the position of the ${ }^{3} \mathrm{He}$ peak based on the energy deposited in the silicon detectors (see Figure 2.8). In this example, the relative positions of the proton, deuteron, and $\alpha$-particle peaks were used to locate the position of the ${ }^{3} \mathrm{He}$ peak. Therefore, good statistical accuracy was unnecessary for determining the position of the ${ }^{3} \mathrm{He}$ peak from the $\mathrm{CH}_{2}$ data.

deuteron, ${ }^{3} \mathrm{He}$, and $\alpha$ particle peaks are cleanly separated. A detailed step by step description of the analysis procedures and software can be found in Appendix $A$.

Once the peak for the particle of interest had been identified, the raw yield could be extracted using one of two methods. In the first method, referred to as the MINUIT analysis method, the pulse height for channel $x, Y(x)$, was fitted by a sum of skewed gaussians and a polynomial background of the form 


$$
\begin{gathered}
Y(x)=a+b \cdot z+c \cdot z^{2}+\sum S\left(z_{0}\right) \cdot A_{i} \cdot g\left(z_{0}\right) \\
S(z)=1+s_{i} \cdot\left(1-\frac{1}{3} z^{2}\right) \cdot z \\
g(z)=\frac{1}{\sqrt{2 \pi}} \cdot \exp \left(-\frac{z^{2}}{2}\right) \\
\text { and } z(x)=\frac{(x-\bar{x})}{\sigma}
\end{gathered}
$$

Each peak in a pulse height spectrum, identified by markers derived from the $\mathrm{CH}_{2}$ calibration spectnum, was fit by a skewed gaussian with variable coefficients $s$, the skewness parameter, $A$, the peak area, $\sigma$, the peak widh, and $\bar{x}$, the peak position. The background was described by a polynomial function with variable coefficients $a, b$, and $c$. An additional centroid, $\bar{x}$, and width, $\sigma$, were also used to parameterize the polynomial background. A kast-squares minimization was performed using subroutines from the MINUIT analysis package." Due to the limited tatistics of these measurements, the Poisson statistic, $\Xi^{2}$, was minimized to determine the best fit to a pulse height spectrum. The Poisson statistic has the form,

$$
\Xi^{2}=2 \sum\left[Y\left(x_{i}\right)-N_{i}-N_{i} \cdot \ln \frac{Y\left(x_{i}\right)}{N_{i}}\right],
$$

where $N_{i}$ is the number of counts in channel $x_{i}$ and $Y\left(x_{i}\right)$ is the value of the fitting function for channel $x_{i}$ In the limit of large $N, \Xi^{2}$ converges to the gaussian statistic, $\chi^{2}$.

The MINUTT analysis method was performed as follows. First, the $\mathrm{CH}_{2}$ calibration data were fitted to determine the peak positions, widths, and skewness parameters for each peak in a particular pulse height spectrum. Then, these peak parameters were held constant and only the peak areas and background parameters allowed to vary. The peak area corresponded to the net yield of a particular type of particle in a given silicon detector. This method was used in the $\pi^{+} p$ and $\pi^{+3} \mathrm{H}$ elastic scattering analysis. An example of a typical fit to a pulse height spectrum is shown in Figure 3.2 which compares the MINUIT fiuing result with $\pi^{+}{ }^{3} \mathrm{H}$ elastic scattering data taken with a tritiated titanium foil. 


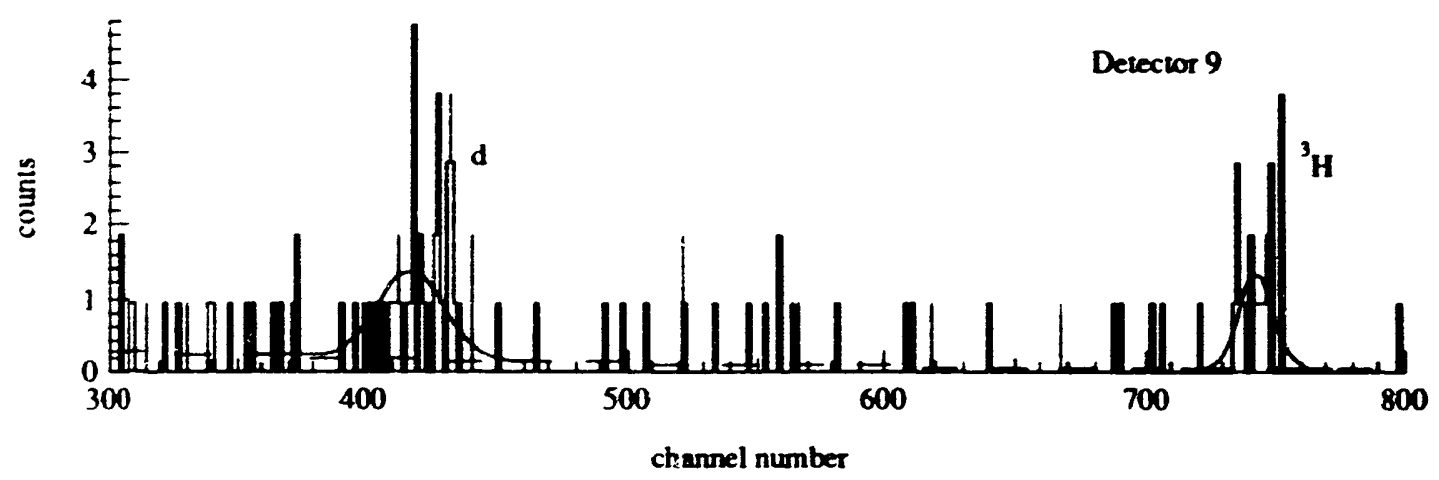

Figure 3.2: Typical fit to a silicon surface barrier detector pulse height spectrum from $\pi^{*}$ 'H elastic scattering using a $\mathrm{Ti}^{3} \mathrm{H}$ target. The fit to the background is shown as a dashed line.

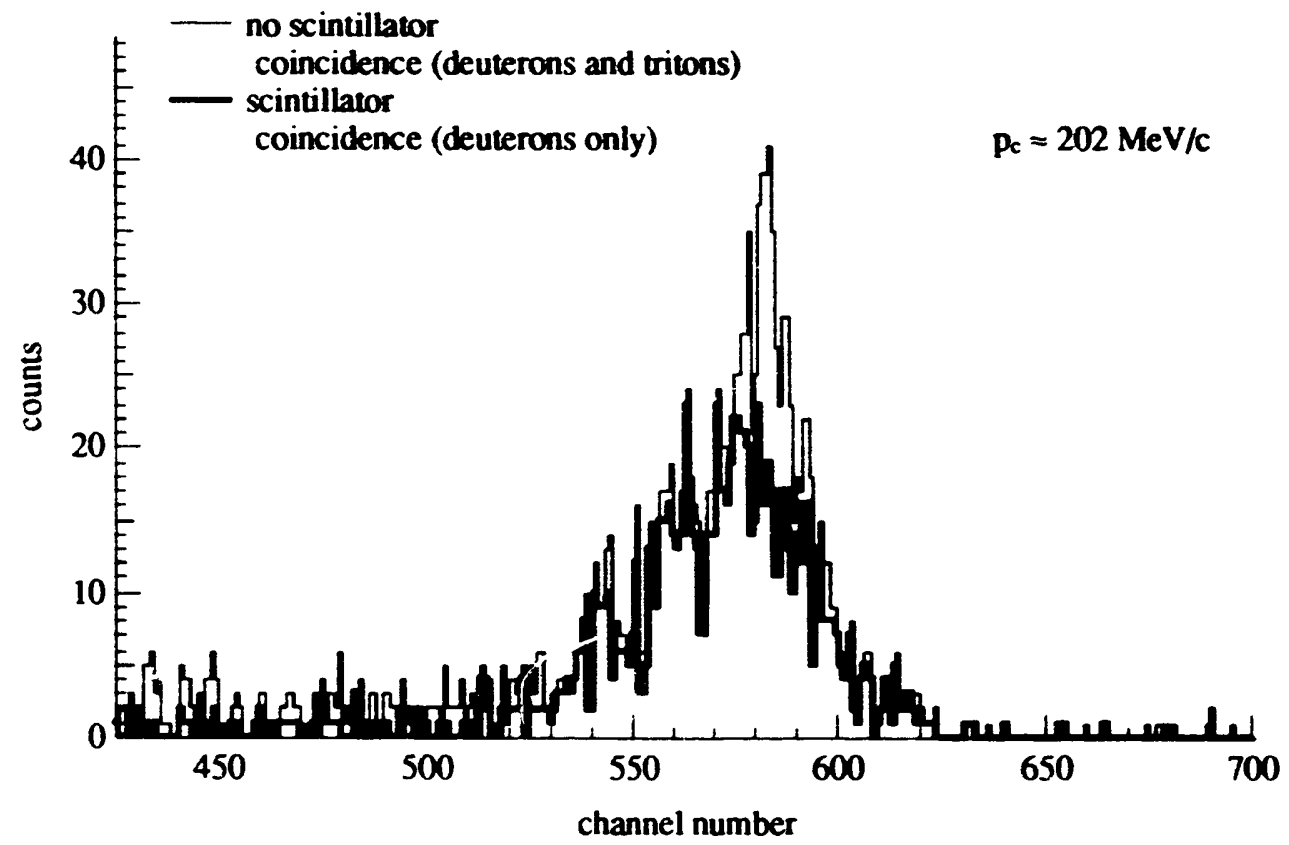

Figure 3.3: Silicon detector pulse height spectrum with (thick line) and without (thin line) a scintillator coincidence requirement. The central momentum setting of the spectrometer in this example was 202 $\mathrm{MeV} / \mathrm{c}$. 
The MINUIT analysis procedures were repeated for each silicon detector pulse height spectrum for a particular spectrometer momentum setting. In some instances, where the pulse heights from different paricles overlapped, the requirement of a coircidence with the focal plane scintillator could be used to distinguish between them. An example of this is shown in Figure 3.3, where a scintillator coincidence was used to distinguish between overlapping triton and deuteron peaks in the $\pi^{+}{ }^{3} \mathrm{H}$ elastic scattering measurements.

The analysis of the single chargc exchange data was more diffi:ult due to the poor statistical quality of the measurement. The MINUIT fitting method failed to converge on consistent results for instances in which a peak contained 10 or fewer counts. The large background, sometimes accounting for as much as $85 \%$ of the net ${ }^{3} \mathrm{He}$ yield, was an additional complication. In these cases, the $\Xi^{2}$ per degree of freedom was typically much less than one and the peak distribution could be as well described by a flat distribution as by a gaussian distribution. In the end, ${ }^{3} \mathrm{He}$ yields from the single charge exchange data were extracted using a "Brute" analysis method, where the number of counts within a specified range of a pulse height spectrum, determined from the $\mathrm{CH}_{2}$ calibration data, were summed up and a flat background was subtracted from this yield. The flat background was determined by calculating the average number of counts per chasinici from equal sized regions, typically 75 channels wide, on either side of the range in a pulse height spectrum assigned to the ${ }^{3} \mathrm{He}$ peak. The average background per channel was multiplied by the number of channels covered by the ${ }^{3} \mathrm{He}$ region. This method worked quite well since the peaks associated with ${ }^{3} \mathrm{He}$ particles were well separated from any other features in the pulse height spectra. A comparison of the Brute and MINUIT methods is shown in Figure 3.4.

The results of the MINUIT method for the single charge exchange data proved consistent with the Brute analysis method in the instances where the fitting program converged properly, as can be seen in Figure 3.4 and 3.5. A comparison of the raw ${ }^{3} \mathrm{He}$ yields from the single charge exchange data using the Brute and MINUT methods is shown in Figure 3.5. 

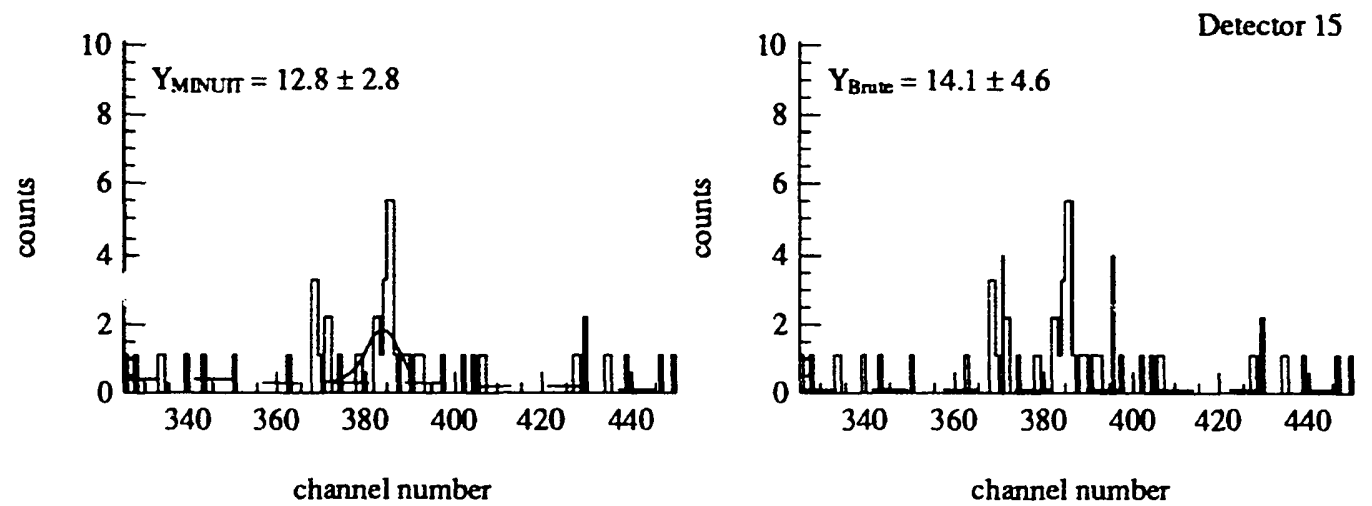

Figure 3.4: MINUIT and Brute results for ${ }^{3} \mathrm{He}$ yields $(\mathrm{Y})$ from a $\mathrm{Ti}^{3} \mathrm{H}$ target. The dashed lines correspond to the background contribution. The markers on either side of the Brute spectrum correspond to the software gates determined from the $\mathrm{CH}_{2}$ calibration data.
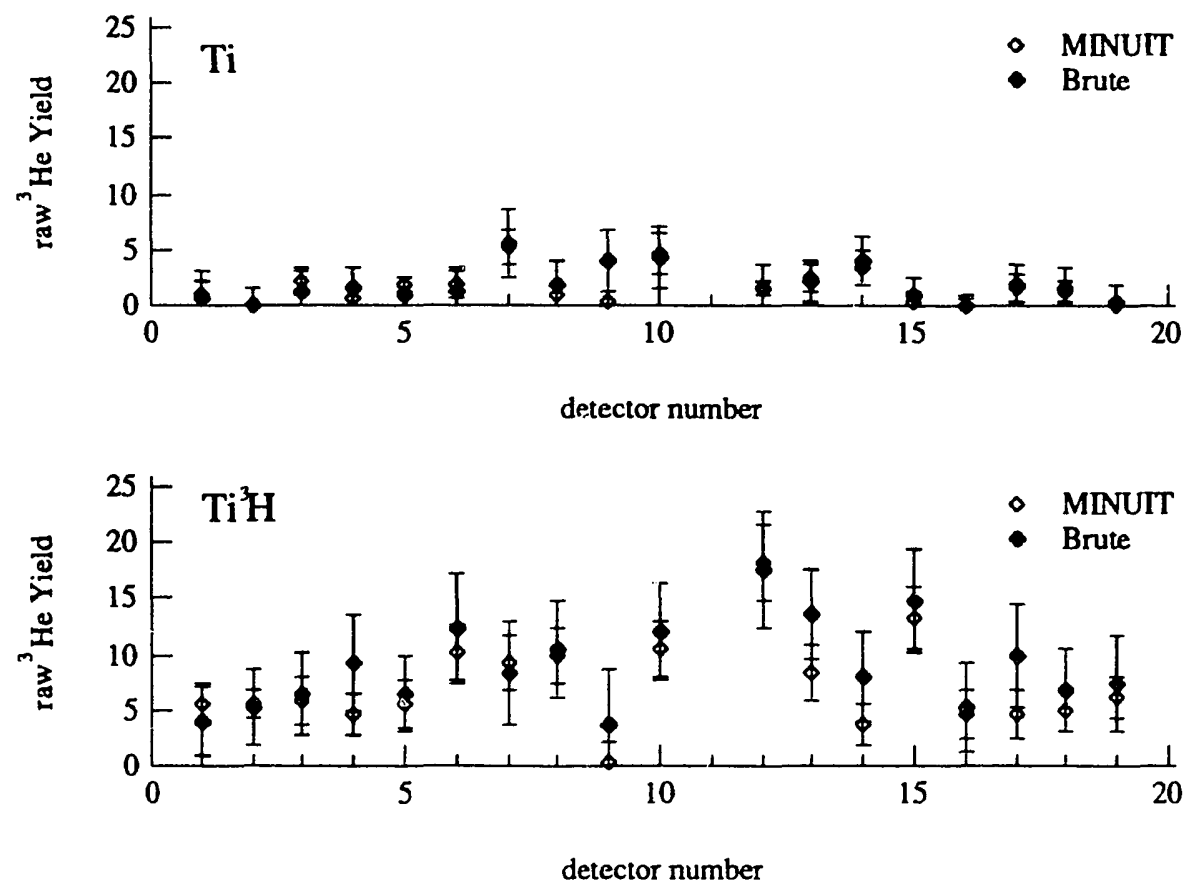

Figure 3.5: MINUIT and Brute results for raw ${ }^{3} \mathrm{He}$ yields from $\mathrm{Ti}^{3} \mathrm{H}$ and $\mathrm{Ti}$ Targets The spectrometer momentum setting in this example is $122.4 \mathrm{MeV} / \mathrm{c}$. 
Next, the yields from each detector for each run were corrected for live time, $\ell$, relative detciiór efficiency, $\varepsilon_{i}$, target angle, $\theta_{t}$, and the momentum bite of a detector, $\Delta p_{i}$. In addition, the yields were normalized to the total number of ionization chamber counts, IC, in the following manner,

$$
\begin{gathered}
\bar{Y}_{i}=\frac{Y_{i} \cdot \cos \theta_{i}}{\varepsilon_{i} \cdot \Delta p_{i} \cdot \ell \cdot I C}, \\
\Delta p_{i}=p_{c} \cdot \delta p
\end{gathered}
$$

where $p_{c}$ is the central momentum setting of the spectrometer and $\Phi$ is the momentum dispersion per detector at the focal plane. The raw and normalized particle yields from detector $i$ are denoted by $Y_{i}$ and $\tilde{Y}_{i}$ respectively. The relative detector efficiencies were determined from measurements of proton momentum spectra from the ${ }^{12} \mathrm{C}\left(\pi^{+}, \mathrm{p}\right) \mathrm{X}$ reaction and will be discussed later in this Chapter. The momentum assigned to detector $i$ was determined from the central momentum setting of the spectrometer using the following relationship,

$$
p_{i}=p_{c} \cdot[1+(i-10) \cdot \delta p]
$$

Finally, the normalized yields from all runs at a particular scattering angle were added together and re-binned into equal spaced momentum bins. Each run was treated as an independent measurement. Data were accumulated at several spectrometer momentum settings for the single charge exchange as well as the $\pi^{+} \mathrm{p}$ and $\pi^{+} \cdot \mathrm{H}$ elastic scattering measurements since the momentum spectra of the recoil nuclei were quite broad, due to energy loss of the recoil particles in the targets and kinematic broadening due to the finite angular acceptance of the spectrometer. An example of a re-binned momentum spectrum from $\pi^{*} p$ elastic scattering measurements using a 0.024" $\mathrm{CH}_{2}$ target is shown in Figure 3.6. This spectrum represents data accumulated at five separate spectrometer settings.

\section{Background Subtraction}

After the single charge exchange and elastic scattering data were normalized and re-binned, the background was subtracted. Possible sources of background in the single charge exchange measurement included room background and target related background, such as ${ }^{3} \mathrm{He}$ production from titanium in the tritiated titanium foils or from the aluminum frames which held the foils. Untritiated titanium foils and thin 
graphite targets were used to measure the target background for the single charge exchange and $\pi^{+{ }^{3}} \mathrm{H}$, and $\pi^{+} p$ elastic scattering measurements respectively. Empty aluminum target frames were used to sample the room and target frame background.

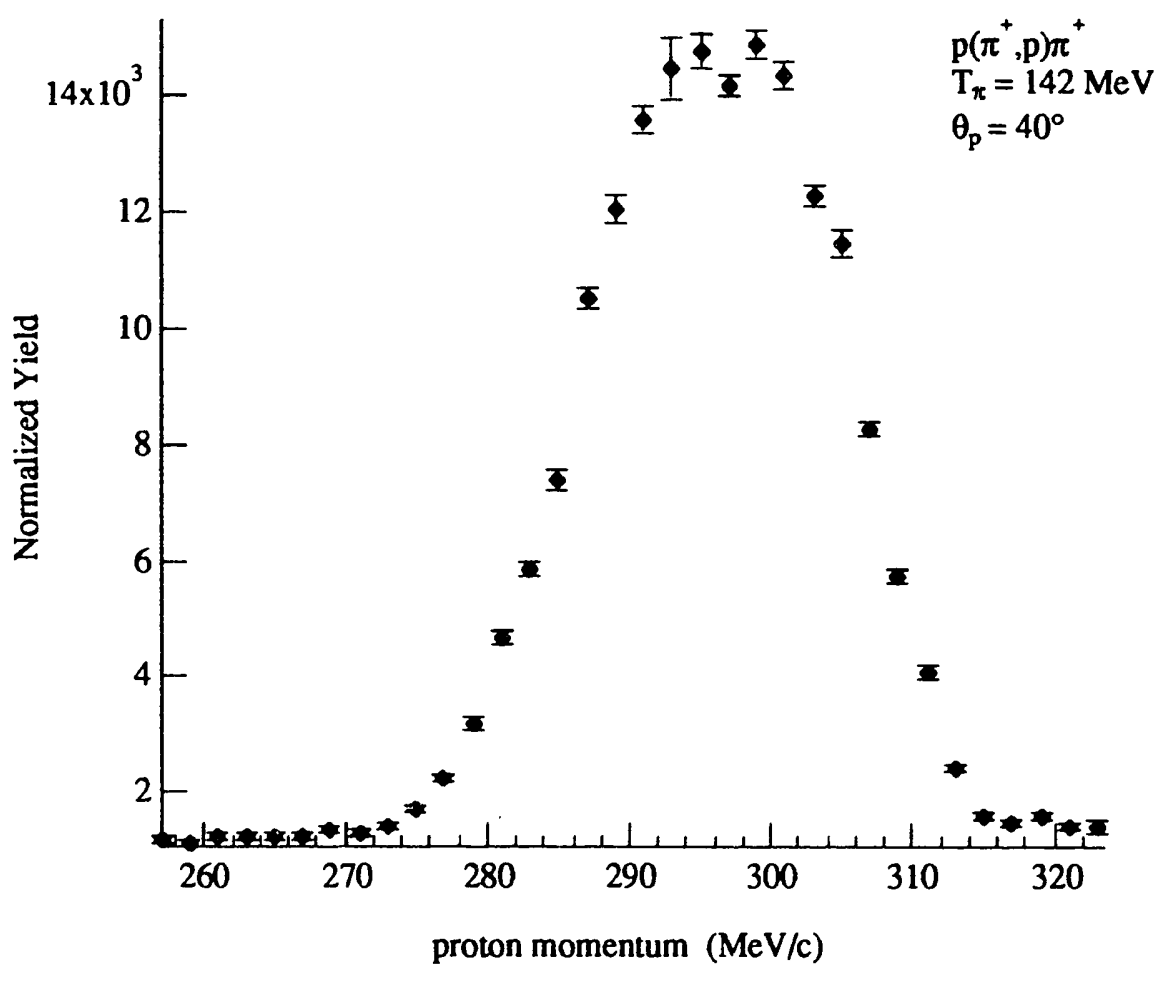

Figure 3.6: Example of re-binned and normalized momentum spectrum from $\pi^{+} \mathrm{p}$ elartic scattering using a $\mathrm{CH}_{2}$ target.

\section{Monte Carlo Simulation of Recoil Momentum Spectra}

After the background had been subtracted, the momentum spectra of the scattered particles were integrated to determine the net yield at a particular scattering angle. In some instances only a portion of the momentum spectrum from the single charge exchange or elastic scattering reactions was sampled. The results of a Monte Carlo simulation were used to predict the shape of the momentum spectrum for each reaction channel and to estimate the fraction of unmeasured yield. The code simulated the magnetic 
transport properties and detector response of the Little Yellow Spectrometer and its focal plane instrumentation. It included the following assumptions about the experimental conditions: a finite beam spot size, a finite momentum spread of the incident beam $(\Delta \mathrm{p} / \mathrm{p})$, appropriate target thicknesses, and a 400um thick active region in each silicon surface barrier detector. Elements of the LAMPF data analysis package, $Q$, were used to display and evaluate the Monte Carlo simulations. See Appendix B for a more detailed description of the Monte Carlo simulation and comparisons with experimental results.

\section{Analysis Procedures and Results}

Once the raw data had been reduced to momentum spectra, a number of calibration constants had to be determined before a differential cross section could be extracted. These constants included the spectrometer acceptance, the ionization chamber calibration, the solid angular acceptance of the spectrometer, and the target thickness. In this section, the procedures for determining these quantities and the results are presented.

\section{Spectrometer Acceptance}

Variation in the detection efficiencies of the silicon surface barrier detectors was primarily due to the geometrical acceptance of the spectrometer, but also due to differences in the physical characteristics of the individual silicon surface barrier detectors, such as the depletion thickness. The relative detection efficiencies, presented in Table 3.1, were determined by measuring a relatively flat proton momentum distribution from the ${ }^{12} \mathrm{C}\left(\pi^{+}, \mathrm{p}\right) \mathrm{X}$ reaction at several spectrometer momentum settings. The relative detection efficiency, $\varepsilon_{i}$, for detector $i$ can be expressed as,

$$
\varepsilon_{1}=\frac{Y_{1}}{Y_{10}},
$$

where $Y_{i}$ is the net proson yield in detector $i$ and the net proton yield from detector 10, $Y_{10}$ was used as a reference with which to compare the other detector efficiencies. The spectrometer acceptance functions measured at 142 and $180 \mathrm{MeV}$ are shown in comparison with a Monte Carlo simulation in Figure 3.7. 


\begin{tabular}{|c|c|c|c|c|c|}
\hline \multirow[b]{2}{*}{ Detector \# } & \multicolumn{2}{|c|}{$142 \mathrm{MeV}$} & \multicolumn{2}{|c|}{$180 \mathrm{MeV}^{\dagger}$} & \multirow{2}{*}{$\frac{\mathrm{MC}}{\varepsilon_{\mathrm{i}}}$} \\
\hline & $\varepsilon_{i}$ & $\Delta \varepsilon_{i}$ & $\varepsilon_{\mathrm{i}}$ & $\Delta \varepsilon_{\mathrm{i}}$ & \\
\hline 1 & 0.530 & 0.016 & 0.479 & 0.013 & 0.619 \\
\hline 2 & 0.639 & 0.018 & 0.609 & 0.015 & $0.7 m 2$ \\
\hline 3 & 0.685 & 0.019 & 0.648 & 0.016 & 0.776 \\
\hline 4 & 0.785 & 0.018 & 0.785 & 0.018 & 0.842 \\
\hline 5 & 0.935 & 0.020 & 0.855 & 0.019 & 0.909 \\
\hline 6 & 0.905 & 0.020 & 0.919 & 0.020 & 0.973 \\
\hline 7 & 0.987 & 0.021 & 1.025 & 0.022 & 0.997 \\
\hline 8 & 0.989 & 0.023 & 0.965 & 0.021 & 0.995 \\
\hline 9 & 1.057 & 0.022 & 0.974 & 0.021 & 1.002 \\
\hline 10 & 1.000 & 0.021 & 1.000 & 0.022 & 1.000 \\
\hline 12 & 1.020 & 0.022 & 0.985 & 0.021 & 0.997 \\
\hline 13 & 1.057 & 0.022 & - & - & 0.995 \\
\hline 14 & 1.039 & 0.022 & 0.974 & 0.021 & 0.971 \\
\hline 15 & 0.908 & 0.022 & 0.913 & 0.020 & 0.907 \\
\hline 16 & 0.878 & 0.021 & 0.888 & 0.020 & 0.839 \\
\hline 17 & 0.821 & 0.018 & 0.841 & 0.019 & 0.776 \\
\hline 18 & 0.722 & 0.017 & 0.734 & 0.017 & 0.698 \\
\hline 19 & 0.633 & 0.016 & 0.680 & 0.016 & 0.618 \\
\hline
\end{tabular}

'Detector 13 was inoperable during the measurements at $180 \mathrm{MeV}$

Table 3.1: Relative Detection Efficiencies. These efficiencies were determined from measurements of proton momentum distributions from the ${ }^{12} \mathrm{C}\left(\pi^{+}, p\right) \mathrm{X}$ reactions at several spectrometer momentum settings. 


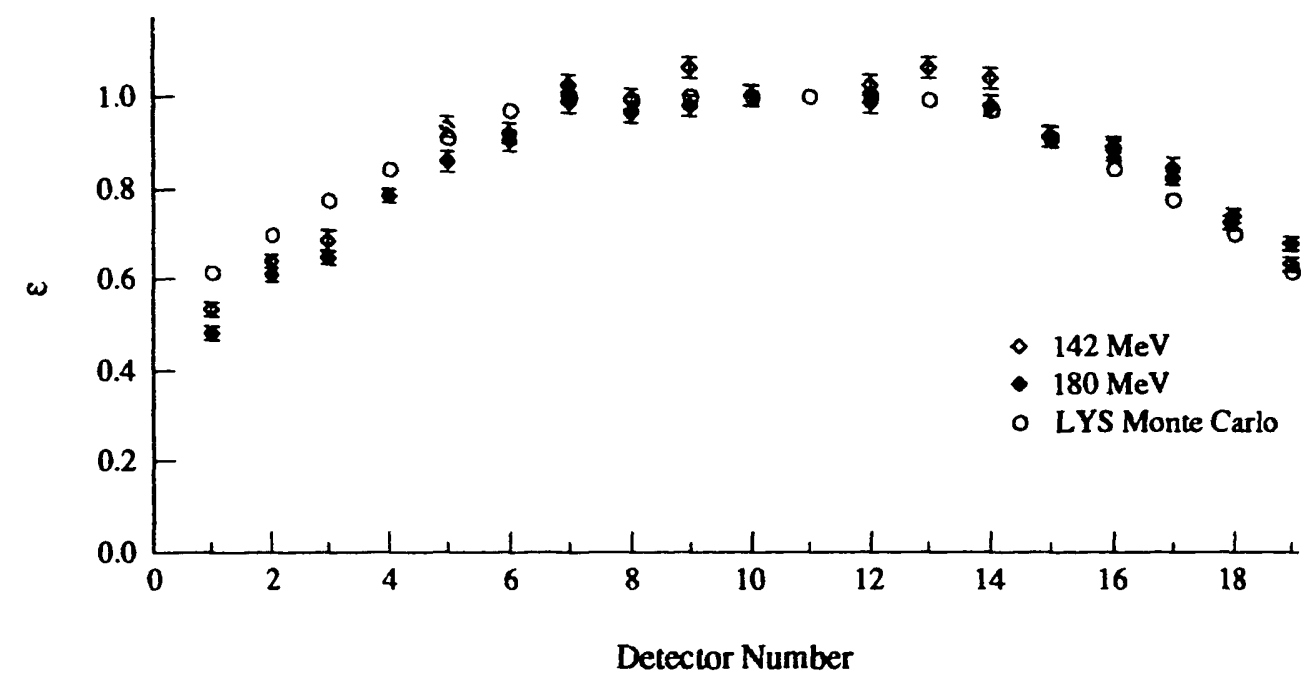

Figure 3.7: Little Yellow Spectrometer Acceptance

\section{LYS Solid Angular Acceptance and Beam Flux Calibration}

The product of the solid angle acceptance of the spectrometer $\Delta \Omega$ and the ionization chamber calibration constant $x$ was determined by observing $\pi^{+} p$ elastic scattering from a $\mathrm{CH}_{2}$ target and comparing the measured yield with the cross section obtained from $\pi \mathrm{N}$ phase shift calculations ${ }^{20}$ in the following manner:

$$
\kappa \Delta \Omega=\frac{Y_{p}}{\rho \tau_{H} \cdot \frac{d \sigma}{d \Omega_{L}}},
$$

where $Y_{p}$ is the normalized proton yield. The hydrogen areal thickness, $\rho \tau_{H}$ is $5.190 \pm 0.035 \times 10^{-6}$ $\mathrm{H} \cdot \mathrm{mb}^{-1}$, as determined from measurements of the $\mathrm{CH}_{2}$ target surface area and weight. The differential cross section in the laboratory frame, $\frac{d \sigma}{d \Omega_{L}}$, was calculated for each $\pi^{+} p$ experimental set-up using the $\pi \mathrm{N}$ phase shift code, SCATPI. ${ }^{20}$ The proton momentum spectra and total yields from $\pi^{+} \mathrm{p}$ elastic scattering at 142 and $180 \mathrm{MeV}$ are shown in Figure 3.8. The results of the $\pi^{+} p$ elastic scattering analysis, in units of (IC click) $\cdot(\pi \cdot \text { sr })^{-1}$, are presented in Table 3.2. 


\begin{tabular}{|l|c|c|}
\hline$T_{n}(\mathrm{MeV})$ & 142 & 180 \\
\hline $\begin{array}{c}\text { net proton yield }\left(10^{5}\right) \\
\text { (protons/1C click) }\end{array}$ & $2.999 \pm 0.021$ & $2.451 \pm 0.049$ \\
\hline$\frac{d \sigma}{d \Omega}(\mathrm{mb} / \mathrm{sr})$ & $21.4 \pm 0.8$ & $27.7 \pm 0.3$ \\
\hline$\frac{1}{\kappa \Delta \Omega}\left(\frac{\mathrm{IC} \text { click }}{\pi \cdot \mathrm{sr}}\right)\left(10^{10}\right)$ & $3.71 \pm 0.14$ & $5.86 \pm 0.14$ \\
\hline
\end{tabular}

Table 3.2: $\mathrm{p}\left(\pi^{+}, \mathrm{p}\right) \boldsymbol{\pi}^{+}$Analysis Results
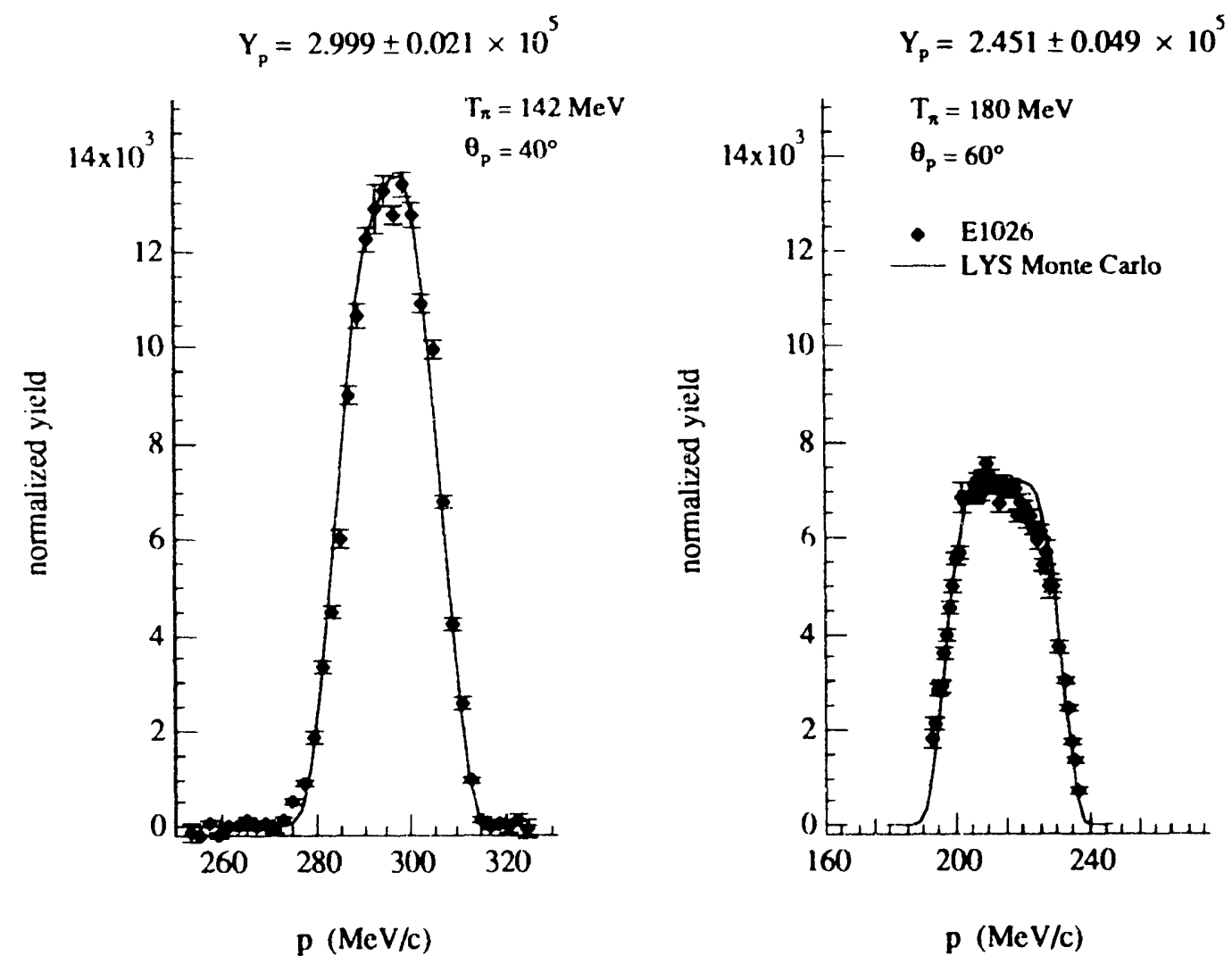

Figure 3.8: Net proton yields from $\pi^{*}$ p elastic scattering at 142 and $180 \mathrm{MeV}$. 


\section{TîH and Ti Target Thickness Analyses and Results}

Based on the manufacturer's specifications, the nominal thickness of the tritiated and untritiated titanium foils was $2 \mu \mathrm{g} / \mathrm{cm}^{2}$ and the expected ratio of $\mathrm{Ti}:{ }^{3} \mathrm{H}$ nuclei in the tritiated foil was $2: 1$. The relative thicknesses of both foils needed to be known accurately in order to subtract the titanium related background from the measurements with the tritiated titanium foil. The relative thickness was obtained from

comparisons of the $\alpha$ particle yields from the tritiated and untritiated foils, as any $\alpha$ particles must be due to pion interactions in titanium. The results of this comparison are shown in Figure 3.9.

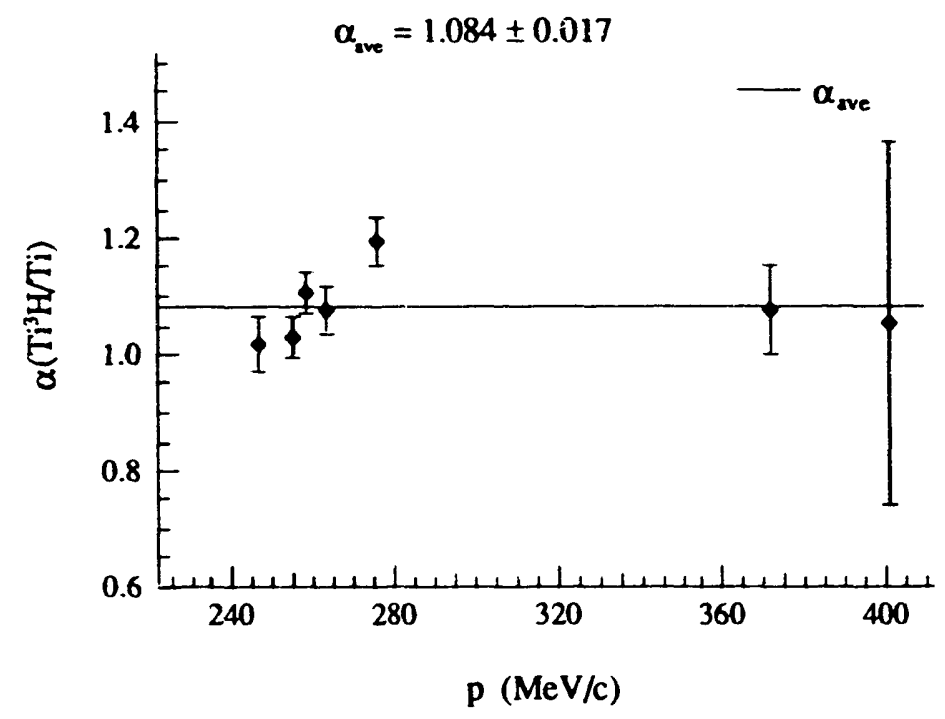

Figure 3.9: Ratios of $\alpha$ particle yields from tritiated $\left(\mathrm{Ti}^{3} \mathrm{H}\right)$ and untritiated $(\mathrm{Ti})$ titanium foils as a function of momentum. The solid line corresponds to the weighted average, $\alpha_{\text {ave }}$, of these measurements.

Once the relative titanitm thickness of the tritiated and untritiated foils had been determined, the normalized ${ }^{3} \mathrm{He}\left(\right.$ or ${ }^{3} \mathrm{H}$ ) yield, $\mathrm{Y}_{3}$, for a particular momentum bin from the ${ }^{3} \mathrm{H}\left(\pi^{+},{ }^{3} \mathrm{He}\right) \pi^{0}$ (or ${ }^{3} \mathrm{H}\left(\pi^{+},{ }^{3} \mathrm{H}\right) \pi^{+}$) reaction was extracted using the weighted average, $\alpha_{\text {ave }}$, of the $\alpha$ particle yield ratios as follows,

$$
Y_{3}=Y_{r+1}-\alpha_{m e n} \cdot Y_{T_{1}}
$$

where $Y_{T H}$ and $Y_{T_{i}}$ are the normalized ${ }^{3} \mathrm{He}\left({ }^{3} \mathrm{H}\right)$ yields for a specific momentum bin from the tritiated and 
untritiated foils respectively. The measured ${ }^{3} \mathrm{H}$ momentum spectrum from $\pi^{+}{ }^{3} \mathrm{H}$ elastic scatering at 180 $\mathrm{MeV}$ and a triton laboratory scattering angle of $60^{\circ}$ is shown in Figure 3.10 in comparison with the LYS Monte Carlo prediction. The total yield of ${ }^{3} \mathrm{He}\left({ }^{3} \mathrm{H}\right)$ particles, $\tilde{Y}_{3}$, was determined by integrating over the measured ${ }^{3} \mathrm{He}\left({ }^{3} \mathrm{H}\right)$ momentum spectrum,

$$
\bar{Y}_{3}=\sum Y_{3} \cdot \Delta p
$$

where $Y_{3}$ is the net yield for momentum bin $i$ of bin width $\Delta p$ (in units of $\mathrm{MeV} / \mathrm{c}$ ). The tritium areal thickness of the tritiated titanium foils was deduced from the following relationship,

$$
\rho \tau_{3}=\frac{\tilde{Y}_{3}}{d \sigma / d \Omega_{x 1} \cdot \Omega \Omega}
$$

where $\kappa \Omega$ is the calibration constant discussed in the previous section, and $d \sigma / d \Omega_{x}$ is the differential cross section for $\pi^{+3} \mathrm{H}$ elastic scattering as interpolated from measurements of the differential cross section by Pillai et al., ${ }^{51}$ which are shown in Figure 3.11. The results of the $\pi^{+3} \mathrm{H}$ elastic scattering analysis are presented in Table 3.3 .

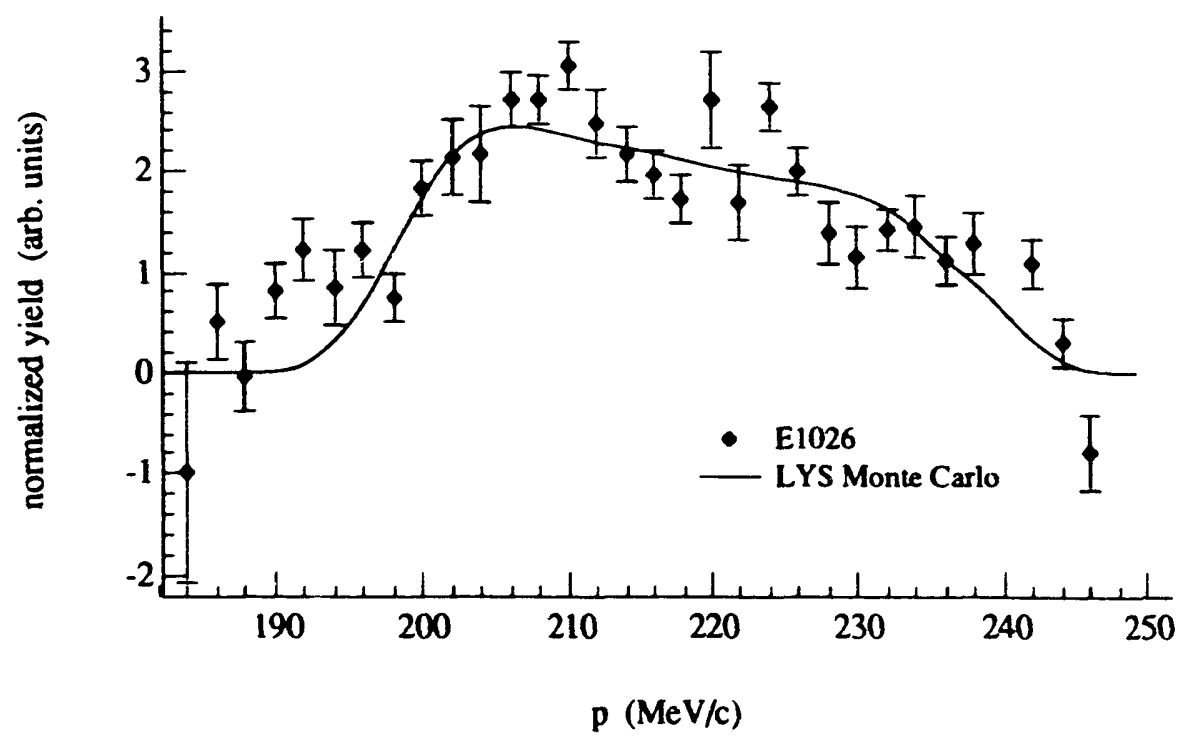

Figure 3.10: ${ }^{3} \mathrm{H}$ elastic peak from $\pi^{+3} \mathrm{H}$ scattering in comparison with Little Yellow Spectrometer Monte Carlo prediction. 


\begin{tabular}{|l|c|}
\hline $\begin{array}{l}\text { net }{ }^{3} \mathrm{H} \text { yield }\left(10^{5}\right) \\
\left({ }^{3} / / \mathrm{C} \text { click }\right)\end{array}$ & $89.9 \pm 4.0$ \\
\hline$\frac{d \sigma}{d \Omega}\left({ }^{3} \mathrm{H}\left(\pi^{+},{ }^{3} \mathrm{H}\right) \pi^{*}\right)(\mathrm{mb} / \mathrm{sr})$ & $11.2 \pm 0.6$ \\
\hline$\frac{1}{\kappa \Delta \Omega}\left(\frac{\mathrm{IC} \text { click }}{\pi \cdot \mathrm{sr}}\right)\left(10^{10}\right)$ & $5.86 \pm 0.14$ \\
\hline$\rho \tau_{3}\left({ }^{3} \mathrm{H} / \mathrm{mb}\right)\left(10^{-9}\right)$ & $4.70 \pm 0.35$ \\
\hline $\mathrm{Ti}:{ }^{3} \mathrm{H}$ & $5.3 \pm 0.4$ \\
\hline
\end{tabular}

Table 3.3: ${ }^{3} \mathrm{H}\left(\boldsymbol{\pi}^{+}, \mathrm{H}\right) \boldsymbol{\pi}^{+}$Analy $\mathrm{sis}$ Results

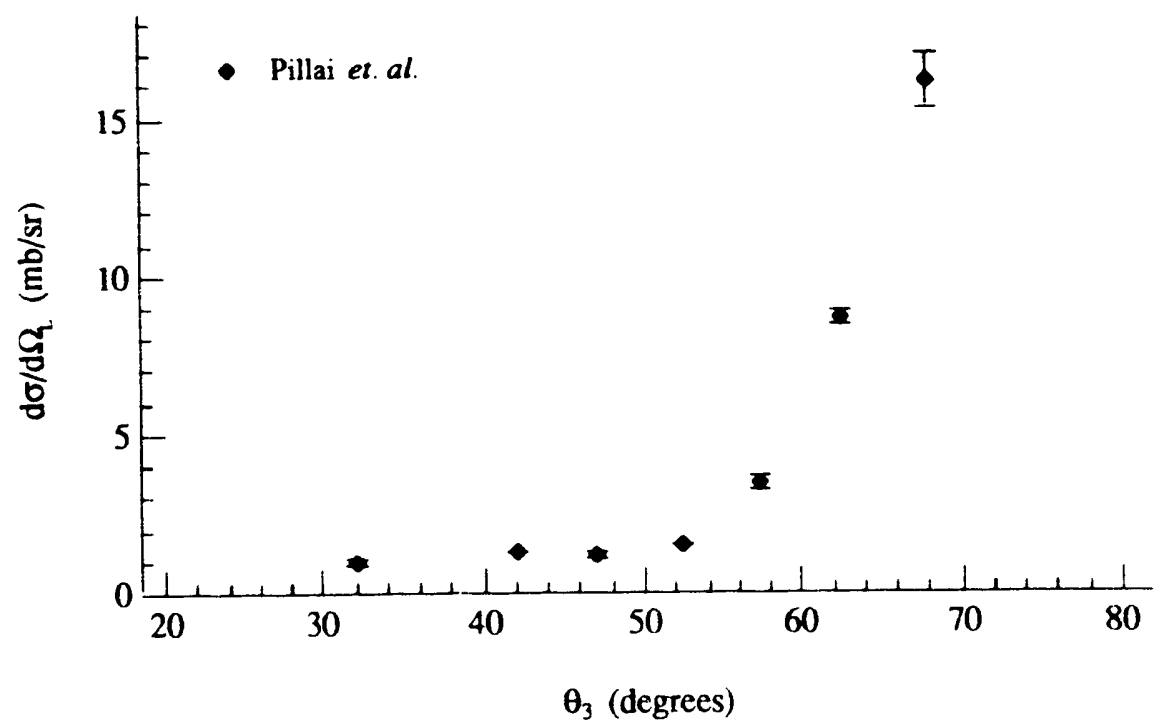

Figure 3.11: Differential cross section for the ${ }^{3} \mathrm{H}\left(\pi^{+}, \pi^{+}\right)^{3} \mathrm{H}$ reaction at $\mathrm{T}_{x}=180 \mathrm{MeV}$, where $\theta_{3}$ is the laboratory angle of the recoil ${ }^{3} \mathrm{H}$ particle. 


\begin{tabular}{|l|l|l|}
\hline \multicolumn{3}{|c|}{$T_{x}=142 \mathrm{MeV}$} \\
\hline $\begin{array}{c}\theta_{3} \\
(\text { deg. })\end{array}$ & $\begin{array}{c}\mathrm{T}_{3} \\
(\mathrm{MeV})\end{array}$ & $\begin{array}{c}\mathrm{d}^{2} \sigma / \mathrm{dEd} \Omega \\
(\mu \mathrm{b} / \mathrm{MeV} \cdot \mathrm{sr})\end{array}$ \\
\hline 55 & 11.5 & $125 \pm 19$ \\
\hline 35 & 6.2 & $81 \pm 37$ \\
\hline 70 & 4.4 & $93 \pm 192$ \\
\hline
\end{tabular}

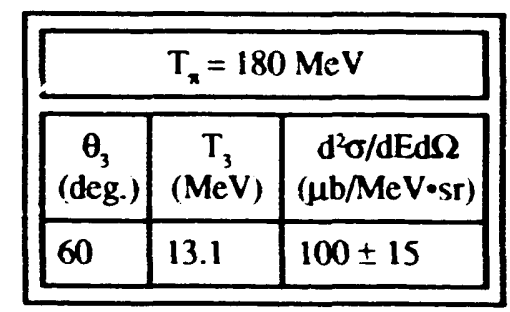

Table 3.4: Doubly Differential Cross Sections for Ti $\left(\pi^{+},{ }^{3} \mathrm{He}\right) \mathrm{X}$

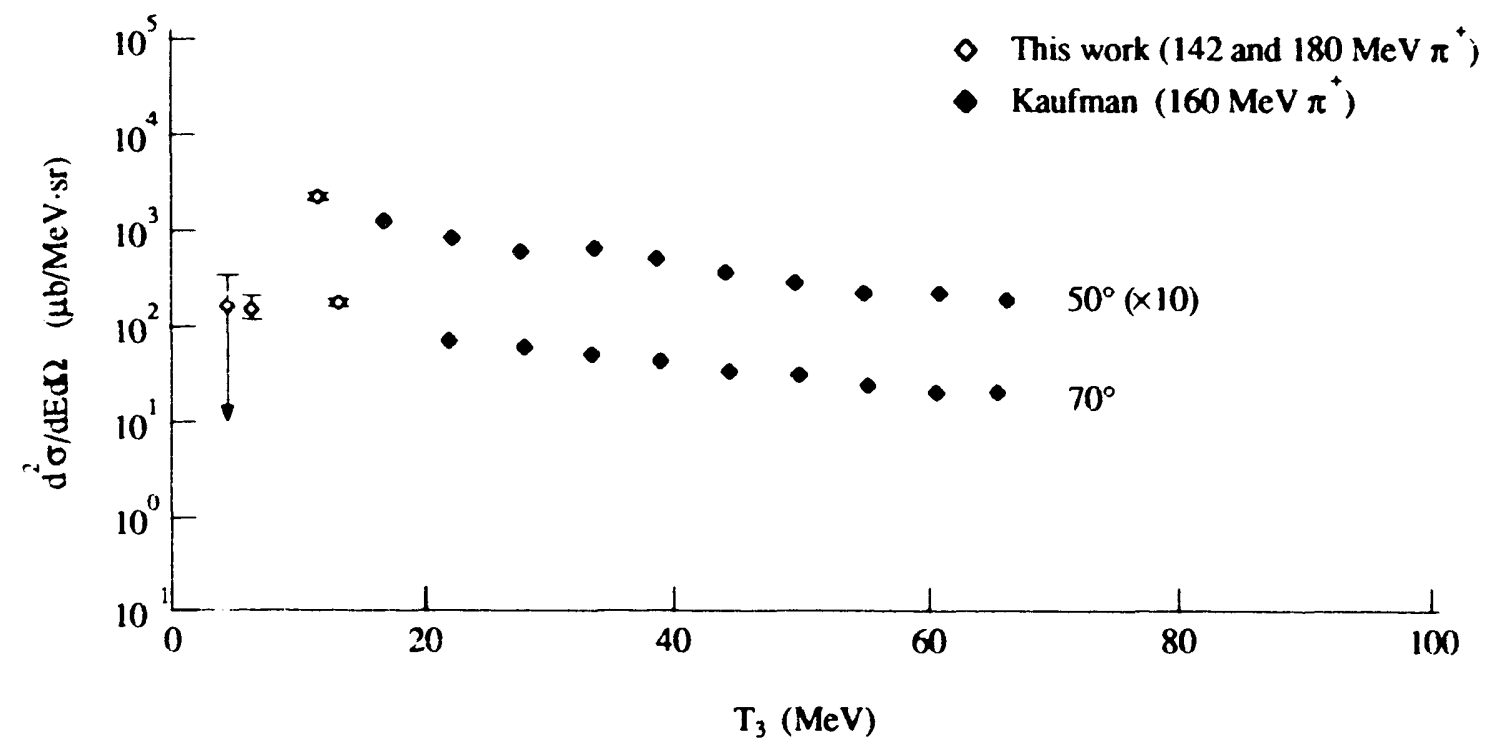

Figure 3.12: Doubly differential cross sections of ${ }^{3} \mathrm{He}$ production from $\mathrm{Ti}$ and $\mathrm{Ag}$ as a function of ${ }^{3} \mathrm{He}$ kinetic energy. The Ti measurements have been scaled by $\mathrm{A}^{2 / 3}$. The $50^{\circ}$ measurements have been multiplied by a factor of 10 .

Doubly differential cross sections for ${ }^{3} \mathrm{He}$ production from titanium, determined in the course of the titanium background analysis, are presented in Table 3.4 and shown in Figure 3.12 , scaled by ${ }^{2 / 3}$, in comparison with measurements of doubly differential cross sections for the $\operatorname{Ag}\left(\pi^{*},{ }^{3} \mathrm{He}\right) \mathrm{X}$ reaction by Kaufman et al. ${ }^{53}$ at incident energy of $160 \mathrm{MeV}$. The titanium results are consistent with the Kaufmann data when an $\mathrm{A}^{2 / 3}$ dependence for the production reaction is assumed. This assumes that the production reaction is confined to the surface of the nucleus, which is reasonable in the $\Delta$-resonance region. 


\section{Determination of ${ }^{3} \mathrm{H}\left(\pi^{+},{ }^{3} \mathrm{He}\right) \pi^{0}$ Differential Cross Sections}

After the calibration constants had been determined, as discussed in the previous section and presented in Tables 3.2 and 3.3, differential cross sections for the ${ }^{3} \mathrm{H}\left(\pi^{+}, \mathrm{He}\right) \pi^{0}$ reaction were calculated. The differential cross section may be expressed in terms of experimentally measured q. antities as follows,

$$
\frac{d \sigma}{d \Omega_{s \alpha}}=\tilde{Y}_{3} \cdot \frac{1}{\cos \Omega} \cdot \frac{1}{\rho \tau_{3}}
$$

where $\frac{1}{\kappa \wedge \Omega}$ and $\rho \tau_{3}$ are the calibration constants and $\bar{Y}_{3}$ is the number of detected ${ }^{3} \mathrm{He}$ particles. Ideally, the momentum spectrum of ${ }^{3} \mathrm{He}$ particles from the ${ }^{3} \mathrm{H}\left(\pi^{+},{ }^{3} \mathrm{He}\right) \pi^{0}$ reaction for a fixed laboratory scattering angle and incident beam energy would be a delta function. However, several contributing factors led to very broad ${ }^{3} \mathrm{He}$ momentum spectra. These factors included the energy loss of the low energy ${ }^{3} \mathrm{He}$ particles in the targets, the finite angular acceptance of the Litule Yellow Spectrometer, and the momentum spread of the incident beam. Because of the breadth of the spectra and the unexpectedly large backgrounds, it was difficult to determine during the course of the experiment whether the entire ${ }^{3} \mathrm{He}$ peak had been sampled. When only a portion of the total ${ }^{3} \mathrm{He}$ yield had been measured, a correction factor, $\gamma$, was defined as follows,

$$
\tilde{Y}_{3}=\frac{Y_{E}}{\gamma}
$$

where $Y_{E}$ and $\tilde{Y}_{3}$ are the experimentally measured and anticipated ${ }^{3} \mathrm{He}$ yields from the ${ }^{3} \mathrm{H}\left(\pi^{+},{ }^{3} \mathrm{He}\right) \pi^{0}$ reaction respectively.

This correction factor was determined by first calculating the momentum spectrum of ${ }^{3} \mathrm{He}$ particles from the exclusive charge exchange process using a Monte Carlo simulation. This momentum distribution, $F(p)$, which is a function of the experimental conditions - scattering angle, incident beam energy and momentum spread - as well as the differential cross section for the ${ }^{3} \mathrm{H}\left(\pi^{+},{ }^{3} \mathrm{He}\right) \pi^{0}$ reaction, was normalized so that its integral was unity,

$$
\int_{0}^{\overline{0}} F(p) d p=1
$$

Then, $\gamma$ may be expressed as 


$$
\gamma=\int_{D}^{p} F(p) d p,
$$

where $p_{\max }$ and $p_{\min }$ are the upper and lcwer limits on the experimentally measured ${ }^{3} \mathrm{He}$ momentum distribution.

Even though some of the corrections to the measured ${ }^{3} \mathrm{He}$ yields were large, this method is accurate for the following reasons. First, the Monte Carlo simulation reproduces the shapes and positions of proton and triton elastic peaks quite well, as shown in Figures 3.8 and 3.10 respectively. In addition, the Monte Carlo acceptance is in agreement with the measured spectrometer acceptance shown in Figure 3.7. Although the simulation included some implicit assumptions about the angular distribution of the ${ }^{3} \mathrm{H}\left(\pi^{+},{ }^{3} \mathrm{He}\right) \pi^{0}$ reaction, several different calculations were tested and produced consistent results.

The correction factors and normalized ${ }^{3} \mathrm{He}$ yields are presented in Tables 3.5 and 3.6. The momentum spectra of the recoil ${ }^{3} \mathrm{He}$ particles from the ${ }^{3} \mathrm{H}\left(\pi^{+},{ }^{3} \mathrm{He}\right) \pi^{0}$ reaction are shown in comparison with the Monte Carlo predictions in Figures 3.13 and 3.14. The uncertainties in the net yields and momentum spectra shown in Figures 3.8, 3.10,3.13, and 3.14 and presented in Tables 3.2, 3.3,3.5, and 3.6 include uncertainties in the relative detector efficiencies as well as the statistical uncertainties. 


\begin{tabular}{|l|l|l|l|}
\hline \multicolumn{4}{|c|}{$\mathrm{T}_{x}=142 \mathrm{MeV}$} \\
\hline $\begin{array}{c}\theta_{3} \\
\text { (deg.) }\end{array}$ & $\gamma$ & $\begin{array}{c}Y_{E} \\
\text { ('He/IC click) }\end{array}$ & $\begin{array}{c}\tilde{Y}_{3} \\
\text { (He/IC click) }\end{array}$ \\
\hline $55^{\circ}$ & 0.97 & $9.8 \pm 4.2$ & $10.1 \pm 4.4$ \\
\hline $65^{\circ}$ & 0.75 & $13.5 \pm 3.3$ & $18.0 \pm 4.4$ \\
\hline $70^{\circ}$ & 0.51 & $8.7 \pm 2.6$ & $17.1 \pm 5.1$ \\
\hline
\end{tabular}

Table 3.5: Net ${ }^{3} \mathrm{He}$ yields and correction factors for ${ }^{3} \mathrm{H}\left(\pi^{+},{ }^{3} \mathrm{He}\right) \pi^{0}$ at $\mathrm{T}_{\mathrm{x}}=142 \mathrm{MeV}$.

\begin{tabular}{|l|l|l|l|}
\hline \multicolumn{4}{|c|}{$\mathrm{T}_{n}=180 \mathrm{MeV}$} \\
\hline $\begin{array}{c}\theta_{3} \\
(\text { deg. })\end{array}$ & $\gamma$ & $\begin{array}{c}Y_{E} \\
\text { ('He/C click) }\end{array}$ & $\begin{array}{c}\bar{Y}_{3} \\
\text { ('He/IC click) }\end{array}$ \\
\hline $60^{\circ}$ & 0.67 & $1.0 \pm 1.6$ & $1.5 \pm 2.4$ \\
\hline $65^{\circ}$ & 0.46 & $3.3 \pm 3.6$ & $7.2 \pm 7.9$ \\
\hline $70^{\circ}$ & 0.36 & $1.1 \pm 4.1$ & $3.1 \pm 11.4$ \\
\hline $73.5^{\circ}$ & 0.28 & $5.1 \pm 6.9$ & $18.2 \pm 24.6$ \\
\hline
\end{tabular}

Table 3.6: Net ${ }^{3} \mathrm{He}$ yields and correction factors for ${ }^{3} \mathrm{H}\left(\pi^{+}{ }^{3} \mathrm{He}\right) \pi^{0}$ at $\mathrm{T}_{n}=180 \mathrm{MeV}$. 

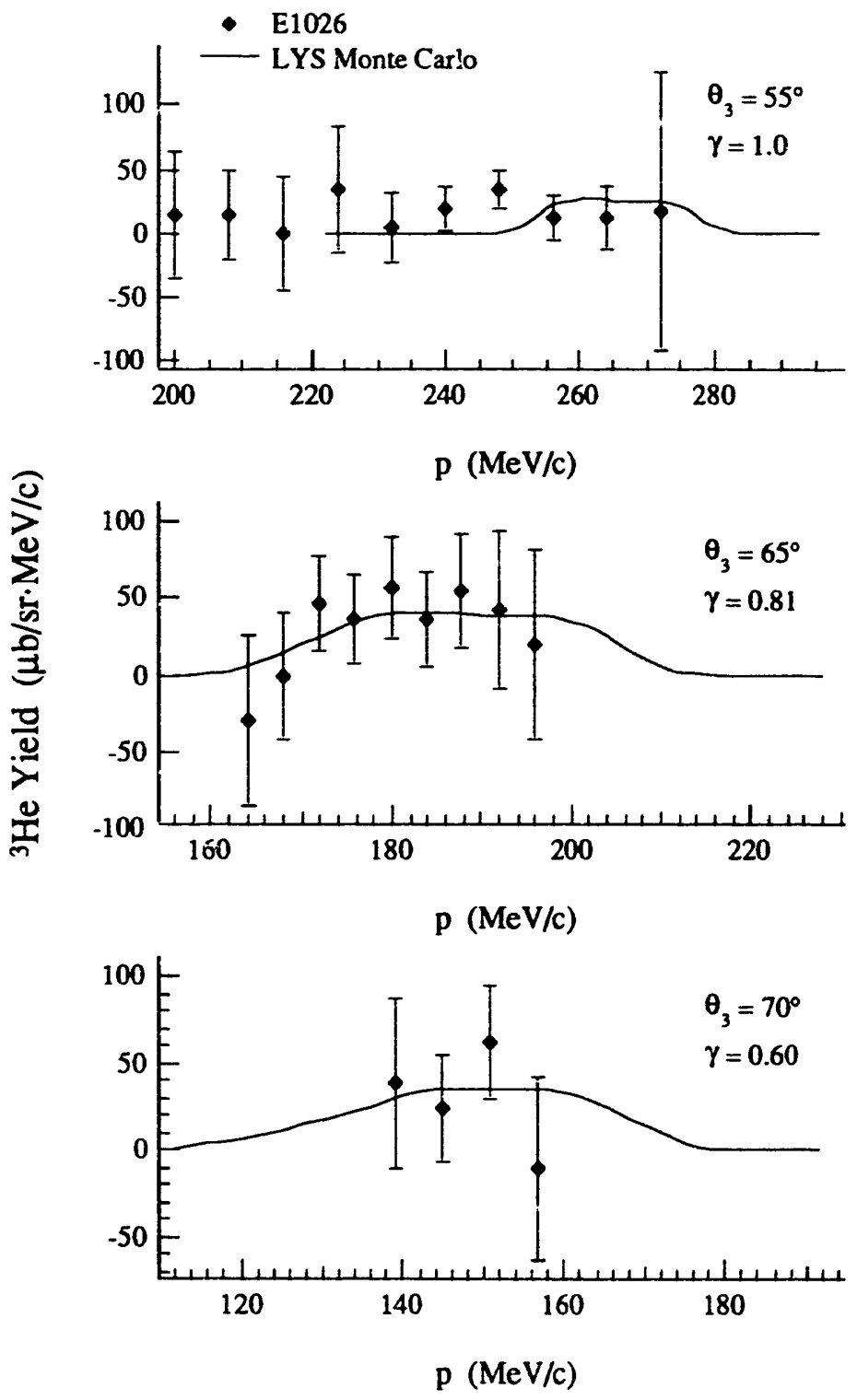

Figure 3.13: Momentum spectra of ${ }^{3} \mathrm{He}$ particles from ${ }^{3} \mathrm{H}\left(\pi^{+},{ }^{3} \mathrm{He}\right) \pi^{0}$ at $\mathrm{T}_{\pi}=142 \mathrm{MeV}$ and recoil ${ }^{3} \mathrm{He}$ laboratory angle, $\theta_{3}$. The correction factor, $\gamma$, is also given for each experimental set-up. The solid line corresponds to the Monte Carlo prediction for the peak shape and net yield of recoil ${ }^{3} \mathrm{He}$ particles from the ${ }^{3} \mathrm{H}\left(\pi^{+},{ }^{3} \mathrm{He}\right) \pi^{0}$ reaction based on the measured yield of ${ }^{3} \mathrm{He}$ particles and experimental conditions. 

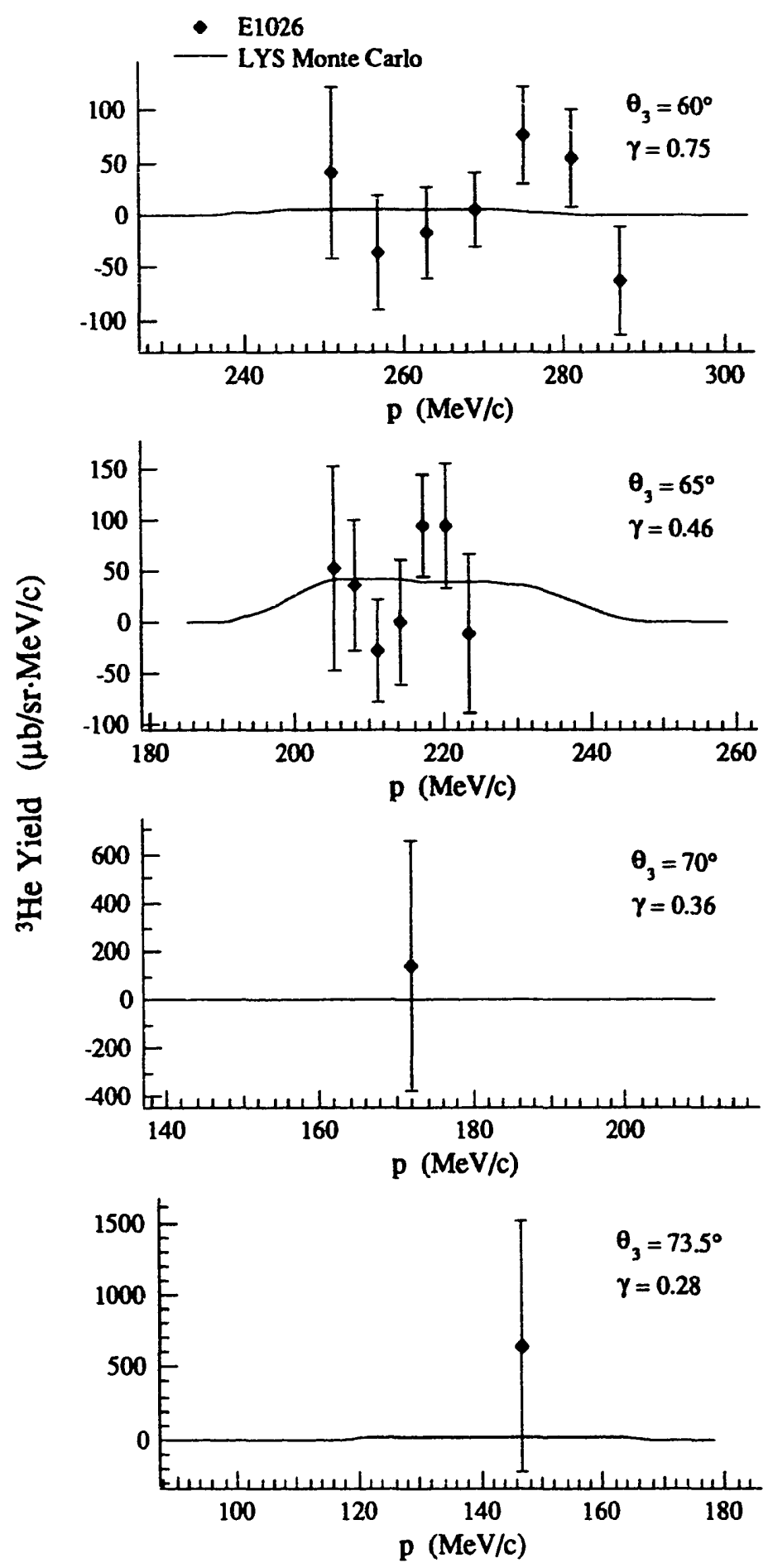

Figure 3.14: Momentum spectra of ${ }^{3} \mathrm{He}$ particles from ${ }^{3} \mathrm{H}\left(\pi^{+},{ }^{3} \mathrm{He}\right) \pi^{0}$ at $\mathrm{T}_{\pi}=180 \mathrm{MeV}$ and recoil ${ }^{3} \mathrm{He}$ laboratory angle, $\theta_{3}$. The correction factor, $\gamma$, is also given for each experimental set-up. The solid line corresponds to the Monte Carlo prediction for the peak shape and net yield of recoil ${ }^{3} \mathrm{He}$ particles from the ${ }^{3} \mathrm{H}\left(\pi^{+}, \mathrm{He}\right) \pi^{0}$ reaction based on the measured yield of ${ }^{3} \mathrm{He}$ particles and experimental conditions. 


\section{Chapter 4: Presentation of ${ }^{3} \mathrm{H}\left(\pi^{+},{ }^{3} \mathrm{He}\right) \pi^{0}$ Differential Cross Sections}

The original intent of this experiment was to perform a systematic study of the pion-induced isobaric analog transition in the three nucleon system over a range of incident energies and to measure as complete an angular distribution as possible. Due to unexpected experimental difficulties - insufficient tritium content of the targets, a large ${ }^{3} \mathrm{He}$ background from titanium, and a large room background - the results of this experiment are two limited measurements at 142 and $180 \mathrm{MeV}$ shown in Figures 4.1 and 4.2 and presented in Table 4.1. The uncertainties in these measurements include systematic as well as statistical uncertainties although statistics were the dominant contribution. The total contribution of the various normalization effects - target thicknesses, solid angular acceptance of the spectrometer, etc. - to the overall experimental uncertainty is less than $10 \%$.

Despite the poor statistical quality of the data, these measurements mimic the general features of several theoretical predictions for the ${ }^{3} \mathrm{H}\left(\pi^{+}, \mathrm{He}\right) \pi^{0}$ differential cross section, shown in Figures 4. la and 4.2a, and provide evidence for the spin flip contribution at $142 \mathrm{MeV}$, shown in Figure 4.1b.

The important features of these calculations are a relatively smooth angular dependence and a total cross section whose energy dependence is flat in the $\Delta$-resonance region, perhaps surprising behavior in such a simple nucleus. The results of this experiment at $142 \mathrm{MeV}$ are compared with several theoretical predictions and a previous measurement by Glodis et al. ${ }^{8}$ in Figure 4.1 . While the measurements at 142 $\mathrm{MeV}$ do not appear to favor any one model, the data are cunsistent with the smooth angular dependence of the various theoretical calculations. The angular distributions shown in Figure 4.1 a represent three types of calculations of the elastic single charge exchange cross section in the three-nucleon system. The Gibbs ${ }^{11}$ result is a separable $\pi \mathrm{N} T$-matrix calculation using Gibbs multiple scattering theory. This calculation 
includes assumptions about the off-shell behavior of the $\pi \mathrm{N}$ scattering amplitudes and the nuclear binding energy. The optical potential calculation by Landaust ${ }^{\text {st }}$ reproduces the differential cross section at energies below $200 \mathrm{MeV}$ and large $\pi^{0}$ center-of-mass angles, ${ }^{8}$ but disagrees with existing measurements at energies above the $\Delta$-resonance. ${ }^{7.932}$ A more recent coupled channel approach by Kamalov, Tiator, and Bennhold ${ }^{4}$ also fails to reproduce the differential cross sections above $200 \mathrm{MeV}^{79.32}$ although it is in good agreement with $\pi^{ \pm}{ }^{3} \mathrm{H}$ and $\pi^{ \pm} \mathrm{He}$ elastic scattering data at incident pion energies of up $5400 \mathrm{MeV} .^{51}$ The results of this study at $180 \mathrm{MeV}$, shown in Figure 4.2 with calculations by Gibbs ${ }^{11}$ and Tiator, ${ }^{10}$ are merely upper limits and can provide only an estimate of the magnitude and general shape of the differential cross section.

\begin{tabular}{|c|c|}
\hline \multicolumn{2}{|c|}{$T_{x}=142 \mathrm{MeV}$} \\
\hline $\begin{array}{c}\theta_{\mathrm{CM}} \\
\text { (deg.) }\end{array}$ & $\begin{array}{c}\mathrm{d} \sigma / d \Omega_{\mathrm{cm}} \\
(\mu \mathrm{b} / \mathrm{sr})\end{array}$ \\
\hline 42.0 & $854 \pm 264$ \\
\hline 51.5 & $794 \pm 203$ \\
\hline 71.0 & $342 \pm 150$ \\
\hline
\end{tabular}

\begin{tabular}{|c|c|}
\hline \multicolumn{2}{|c|}{$T_{\pi}=180 \mathrm{MeV}$} \\
\hline $\begin{array}{c}\theta_{\mathrm{cM}} \\
(\mathrm{deg} .)\end{array}$ & $\begin{array}{c}\mathrm{d} \sigma / \mathrm{d} \Omega_{\mathrm{cM}} \\
(\mu \mathrm{b} / \mathrm{sr})\end{array}$ \\
\hline 35.4 & $2023 \pm 2738$ \\
\hline 42.0 & $287 \pm 1056$ \\
\hline 51.1 & $341: ? 6$ \\
\hline 60.8 & $85 \pm 136$ \\
\hline
\end{tabular}

Table 4.1: Differential cross sections for ${ }^{3} \mathrm{H}\left(\pi^{+},{ }^{3} \mathrm{He}\right) \pi^{0}$ at 142 and $180 \mathrm{MeV}$. 

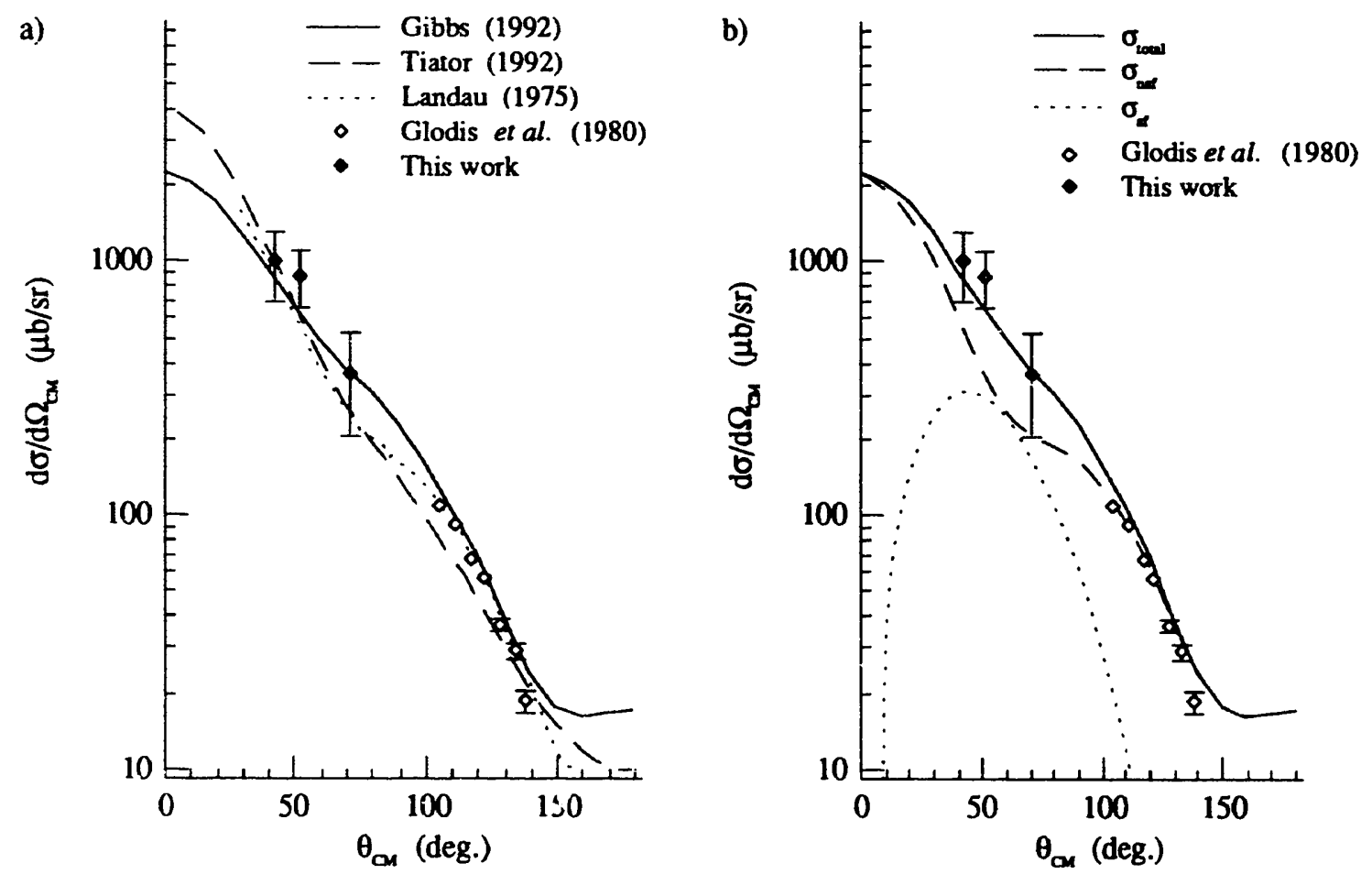

Figure 4.1: Center-of-mass differential cross sections for the ${ }^{3} \mathrm{H}\left(\pi^{+},{ }^{3} \mathrm{He}\right) \pi^{0}$ reaction at $142 \mathrm{MeV}$. a) Solid points are this work; open points are measurements by Glodis et al. at $148 \mathrm{MeV}$; solid line corresponds to a $\pi N$ T-matrix calculation by Gibbs; dashed line corresponds to a coupled channel calculation by Tiator, dotted line corresponds to an optical potential calculacion by Landau. b) Solid line corresponds to the full Gibbs' calculation; dashed line corresponds to non-spin flip contribution; dotted line corresponds to spin flip contribution. 

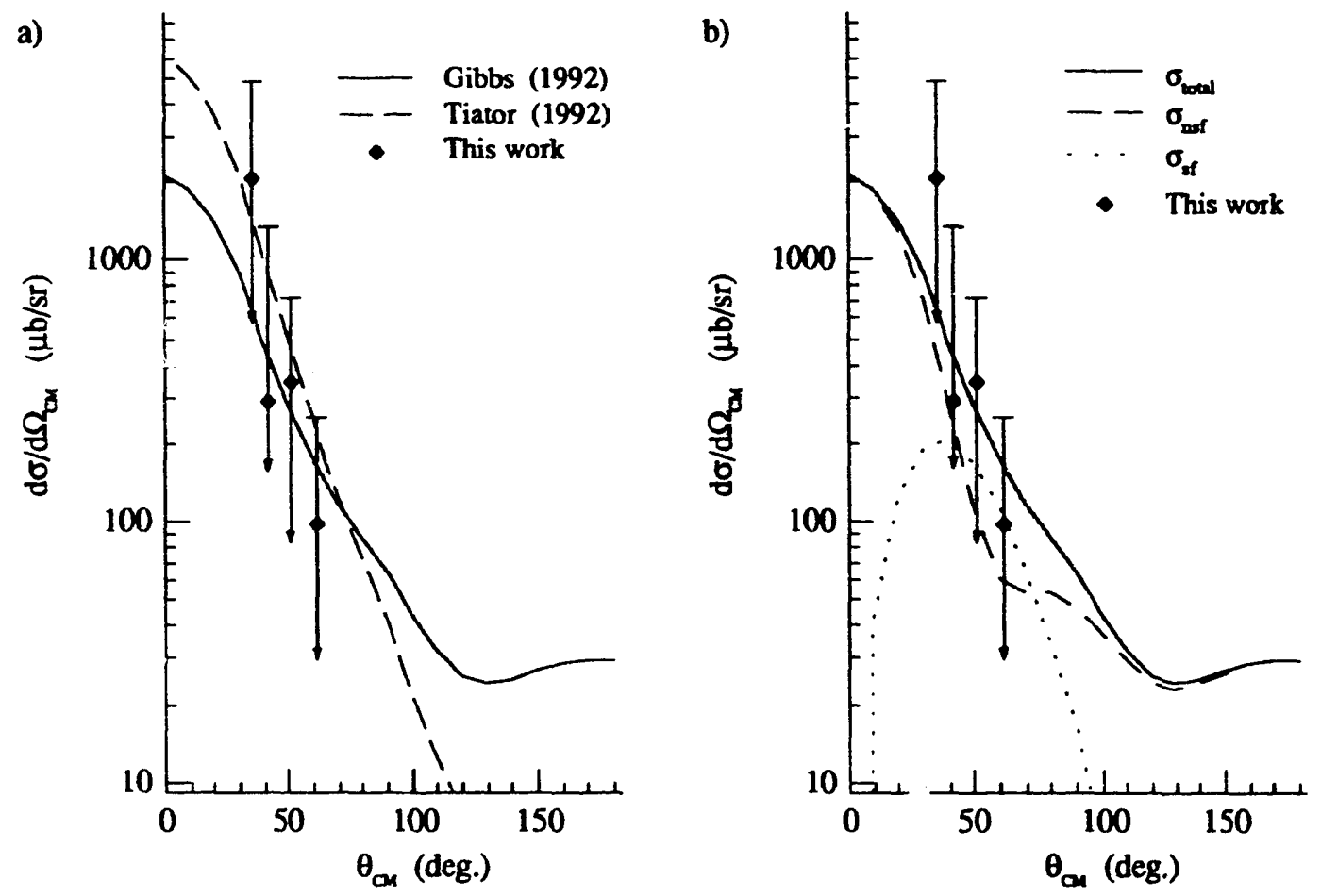

Figure 4.2: Center-of-mass differential cross sections for the ${ }^{3} \mathrm{H}\left(\pi^{+},{ }^{3} \mathrm{He}\right) \pi^{0}$ reaction at $180 \mathrm{MeV}$. a) Solid line corresponds to $: \pi \mathrm{N}$ T-matrix calculation by Gibbs; dashed line corresponds to a coupled channel calculation by Tiator. b) Solid line corresponds to full Gibbs calculation; dashed line corresponds to non-spin flip contribution; dotted line corresponds to spin flip contribution. 


\section{Chapter 5: Experimental Apparatus and Data Acquisition System for Inclusive Pion Single Charge Exchange Measurements on ${ }^{3} \mathrm{He}:{ }^{3} \mathrm{He}\left(\pi^{ \pm}, \pi^{9}\right)$ and ${ }^{3} \mathrm{He}\left(\pi^{ \pm}, \pi^{0} \mathrm{p}\right)$}

The inclusive pion single charge exchange measurements on ${ }^{3} \mathrm{He}$ were performed at the Clinton $\mathrm{P}$. Anderson Meson Physics Facility (LAMPF) at the Los Alamos National Laboratory. These measurements employed the LAMPF $\pi^{0}$ Spectrometer, an array of plastic scintillation counters, and a liquid ${ }^{3} \mathrm{He}$ target. This chapter contains a general description of these experimental components and a brief account of the data acquisition system.

\section{Pion Beam}

This experiment took place in the Low Energy Pion (LEP) experimental hall located in Area A of the LAMPF facility (see Figure 2.1). A schematic diagram of the LEP channel is shown in Figure 5.1 The channel was designed with a short flight path from the pion production target to the experimental hall in order to minimize the fraction of low energy pions which decay in flight. At an incident beam energy of $245 \mathrm{MeV}$, approximately $50 \%$ of the incident pion flux was lost to pion decay. The procedures for determining the magnetic field settings and tuning the pion beam are similar to those for the $\mathrm{P}^{3}$ channel described earlier in Chapter 2.

\section{Calibration of Incident Pion Flux}

During the measurements, the primary proton flux was monitored by a toroid loop located near the A-1 target interaction region (see Figure 2.1). The signal from this toroid was proportional to the 
primary proton beam current. For a specific pion energy, the incident pion flux was proportional to the product of the proton flux and the thickness of the A-1 target. The toroid signals from both the A-1 and A-2 targets were calibrated simultaneously at each incident pion beam energy by means of activation measurements so that the incident pion flux during a measurement could be determined. A thin disk of plastic scintillator $(\mathrm{CH})$, with diameter larger than the transverse dimensions of the beam spot, was exposed to the pion beam and ${ }^{11} \mathrm{C}$ nuclei, which have a half life of 20.3 minutes, were produced by the ${ }^{12} \mathrm{C}\left(\pi^{*},{ }^{11} \mathrm{C}\right) \mathrm{X}$ reactions. The ${ }^{11} \mathrm{C}$ content of the irradiated scintillator disks was determined by counting the $\beta$-decay positrons. The pion beam flux was deduced from the ${ }^{11} \mathrm{C}$ content of the disks since the cross sections for the ${ }^{12} \mathrm{C}\left(\pi^{+},{ }^{11} \mathrm{C}\right) \mathrm{X}$ reactions are known. ${ }^{55}$ Typical beam fluxes during this experiment were $3 \times 10^{6}$ to $5 \times$ $10^{6} \pi / s$.

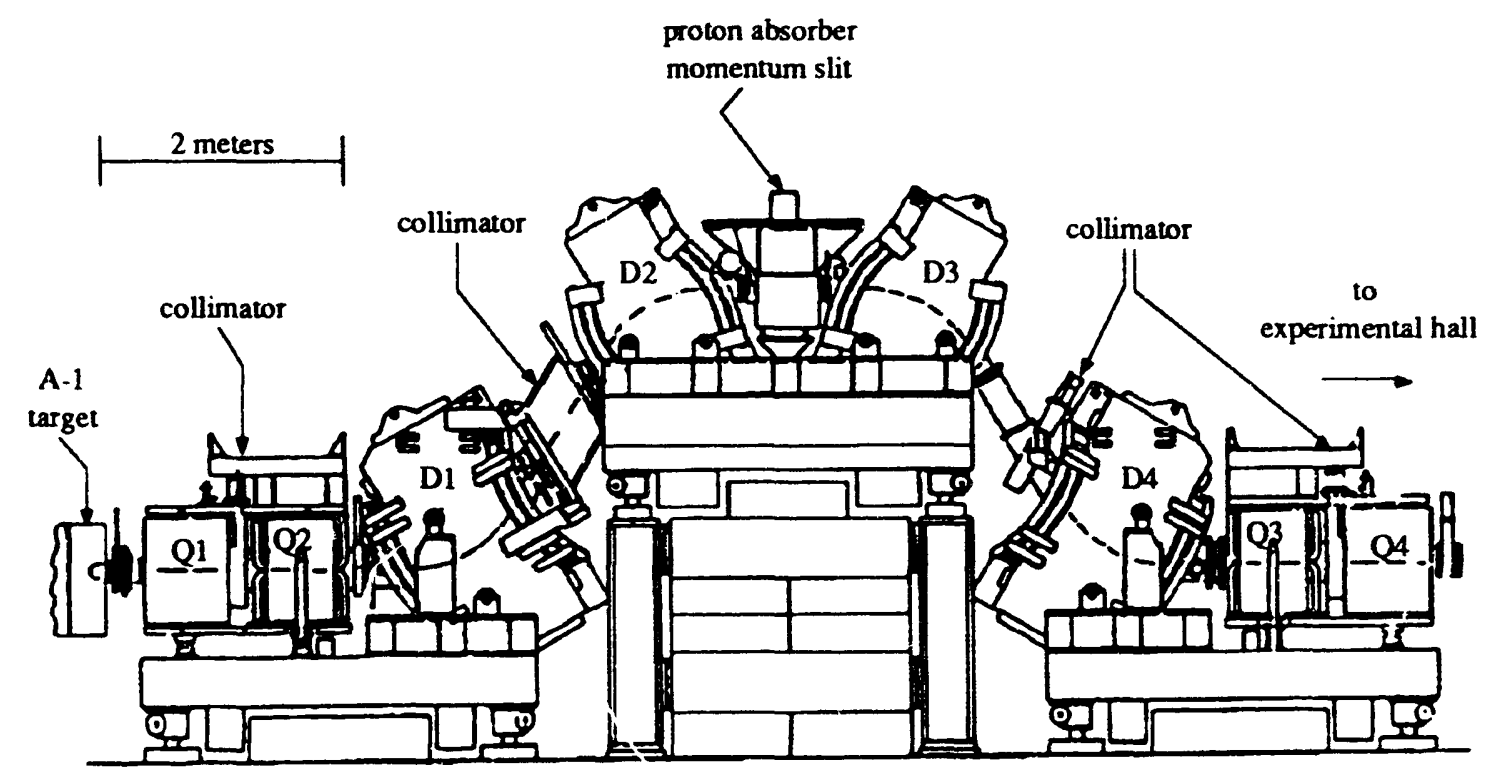

Figure 5.1: Schematic drawing of Low Energy Pion Channel. A series of quadrupole magnets (Q) and collimators define the beam spot size. The momentum and momentum spread of the incident pion beam is determined by a series of dipole magnets (D) as well as the collimators. The proton absorber may be used to separate protons from the pion beam. 


\section{Experimental Apparatus}

The three principal components of the experimental apparatus were the LAMPF $\pi^{0}$ Spectrometer, an array of $\Delta E-E$ plastic scintillation counters, and a liquid ${ }^{3} \mathrm{He}$ target. A top view of this experimental set-up is shown in Figure 5.2. In the ${ }^{3} \mathrm{He}\left(\pi^{*}, \pi^{0} \mathrm{p}\right)$ measurements, protons were detected in coincidence with neutral pions using the scintillation counters. The liquid ${ }^{3} \mathrm{He}$ target was designed and maintained by the University of Virginia members of the experimental collaboration. In addition to the ${ }^{3} \mathrm{He}$ target, a $\mathrm{CH}_{2}$ target was used to measure the conversion efficiency of the $\pi^{\circ}$ Spectrometer and a $C D_{2}$ target was utilized in the proton counter calibration measurements.

\section{The LAMPF $\pi^{\circ}$ Spectrometer}

The neutral pion is a short lived particle $\left(\tau=8.4 \times 10^{-17} \mathrm{~s}\right)$ which decays $98.8 \%$ of the time into two photons: $\pi^{0} \rightarrow 2 \gamma$. It is impossible to detect a neutral pion directly since it will not reach a detector. (A 100 $\mathrm{MeV} \pi^{0}$ will travel approximately $20 \mathrm{~nm}$ before it decays.) Until 1980 , the detection of neutral pions was limited chiefly to experiments which did not require good energy resolution, due to the difficulties inherent in the simultaneous detection of two high energy gamma rays with good resolution and sufficient angular acceptance. The design of the LAMPF $\pi^{0}$ Spectrometer is based on the principle that if two photons of nearly equal energy are detected in coincidence, the energy resolution of the spectrometer will depend primarily on the angular resolution of the opening angle between the two photons. The energy of a $\pi^{0}$ may be determined from the following relationship between the opening angle, $\eta$, and the energy difference between two photons, $X$,

$$
\begin{gathered}
E_{\pi^{0}}^{2}=\gamma^{2} m_{x^{0}}^{2}=\frac{2 m_{x^{1}}^{2}}{(1-\cos \eta)\left(1-X^{2}\right)} \\
\text { where } X=\frac{E_{\gamma_{1}}-E_{\gamma_{2}}}{E_{\gamma_{1}}+E_{\gamma_{2}}} .
\end{gathered}
$$

In the limit as $X$ approaches zero, the energy resolution of the spectrometer depends solely on the opening angle between the two photons and hence on the position resolution for reconstructing a photon event. 


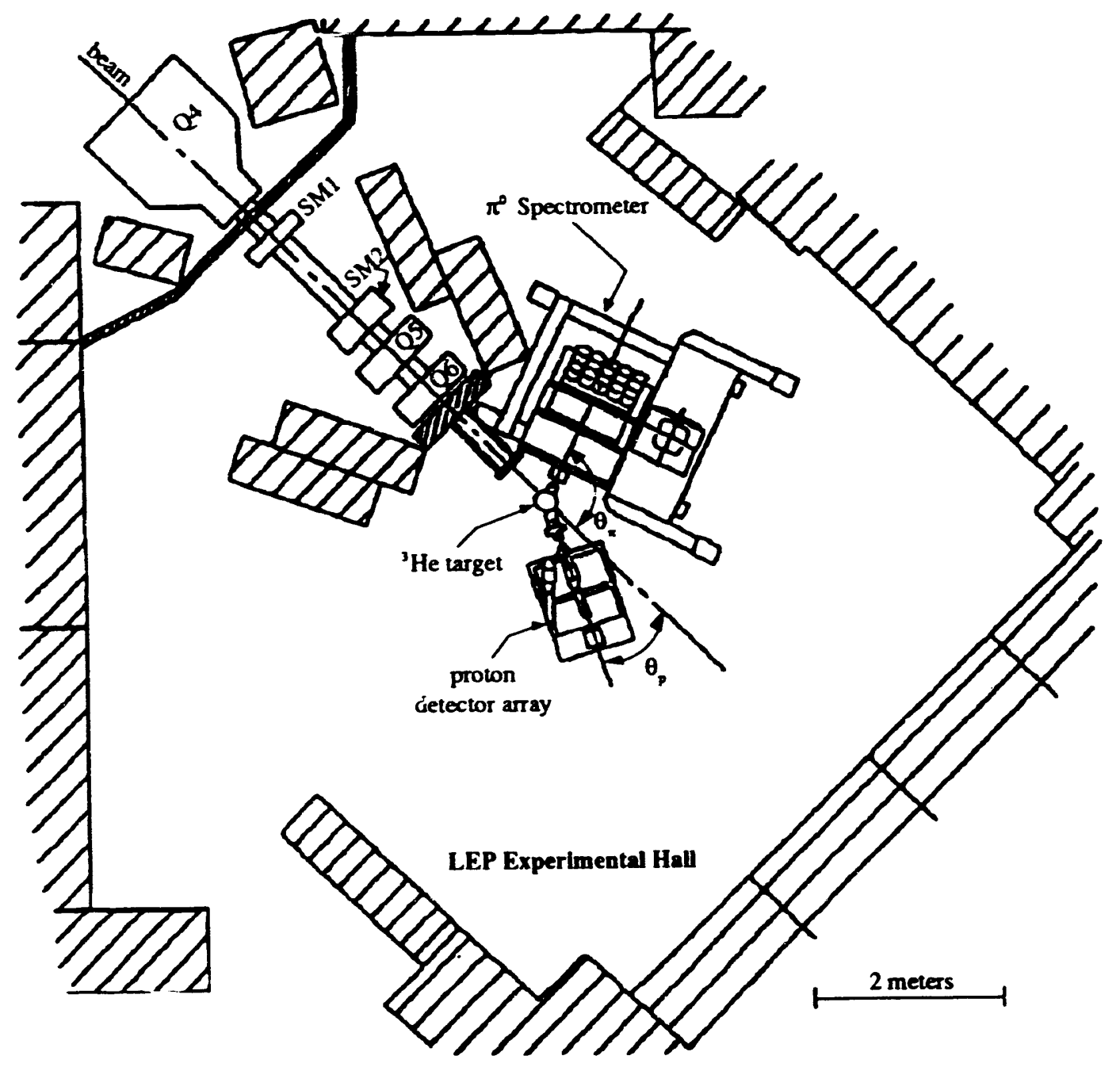

Figure 5.2: Top view of experimental set-up. Two additional quadrupoles (Q5 and Q6) were installed to extend the length of the LEP beam line shown in Figure 5.1. Two steering magnets, designated as SM1 and SM2, were controlled from within the counting house. The principal components of the experimental apparatus included a proton detector array, the LAMPF $\pi^{\circ}$ Spectrometer, and the University of Virginia ${ }^{3} \mathrm{He}$ target. The proton detector array consisted of eight plastic scintillation detoctors. 


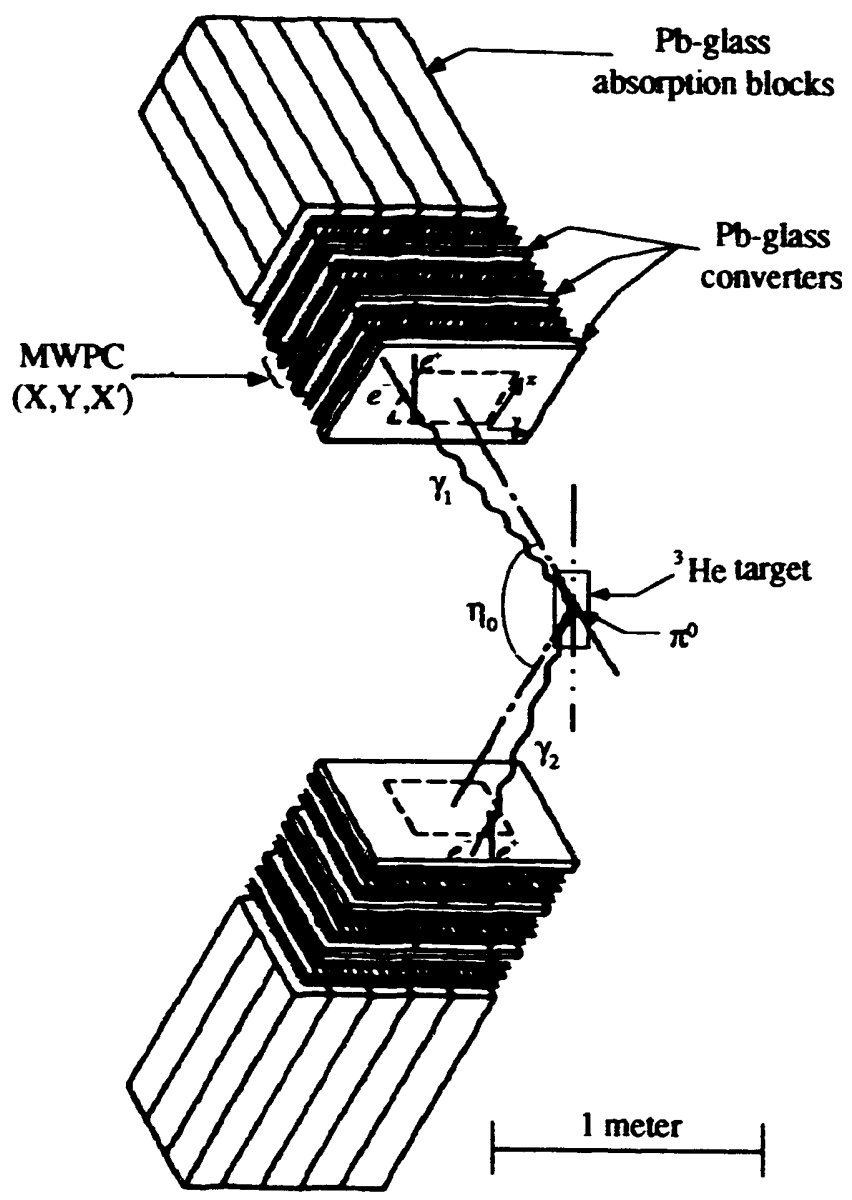

Figure 5.3: Schematic diagram of $\pi^{0}$ Spectrometer photon arms in one-post mode. In this figure, the two photon arms are located out of the scattering plane. The incident beam and scattering plane are perpendicular to the page. The opening angle, $\eta_{0}$, between the two arms may be adjusted to detect neutral pions over a wide range of kinetic energies.

The $\pi^{\circ}$ Spectrometer is made up of two photon detector packages. In this experiment, the spectrometer was placed in the "one-post" configuration with the two detector packages located out of the scattering plane as shown in Figure 5.3. Each package consists of three conversion planes and an array of lead glass total absorption counters. Each conversion plane consists of a lead glass converter and three multiwire proportional chambers (MWPC). Two of these wire chambers (denoted X and $\mathbf{X}$ ' in Figure 5.3) have $1 \mathrm{~mm}$ wire spacing perpendicular to the scattering plane. A third chamber (Y), with a $2 \mathrm{~mm}$ wire spacing in the orthogonal direction, is sandwiched between the $\mathrm{X}$ and $\mathrm{X}^{\prime}$ chambers. 
A photon has a probability of approximately $30 \%$ to convert into charged particles in any one of the lead glass converters. The most probable process is $\mathrm{e}^{+} \mathrm{e}^{-}$production. The positions of the charged leptons from the shower are recorded by the multiwire proporional chambers. The origin of the shower was traced back to the converter using these positions. The algorithm for resolving the origin of the shower is well understood..$^{56}$ Once the position of the two photon conversions was determined, the opening angle of the $\pi^{0}$ decay could be calculated by assuming that the decay originated at the center of the target. The energy of the detected photon was determined from the sum of the energy deposited in the lead glass converters and total absorption counters. The dimensions of the total absorption counters were chosen to minimize the leakage of the lepton shower at the back and sides of each detector package.

Plastic scintillation counters (not shown in Figure 5.3) were located at the front of each photon detector package to veto any events which were initiated by charged particles. Additional plastic scintillation counters were located behind each of the lead glass converters. More detailed descriptions of the design and performance of the LAMPF $\pi^{0}$ Spectrometer may be found in References 57 and 58.

\section{Proton Detector Array}

The proton detector array consisted of eight $\Delta E-E$ plastic scintillation counters. A schematic diagram of an individual proton detector is shown in Figure 5.4. The correlation between the pulse heights from the two scintillation counters, which make up a proton detector, provided very good particle identification. Using this scheme, protons were detected over an energy range of roughly 25 to $200 \mathrm{MeV}$.

Six of these counters were located in the scattering plane and two were located out of the scattering plane. The detectors were mounted on a table which could be rotated through $90^{\circ}$. The two proton detector configurations used in the measurements are shown in Figure 5.5. The table was attached to the pivot post for the $\pi^{0}$ Spectrometer and located on the opposite side of the beam line from the spectrometer. A diagram of the proton detector mounting scheme is shown in Figure 5.6. 


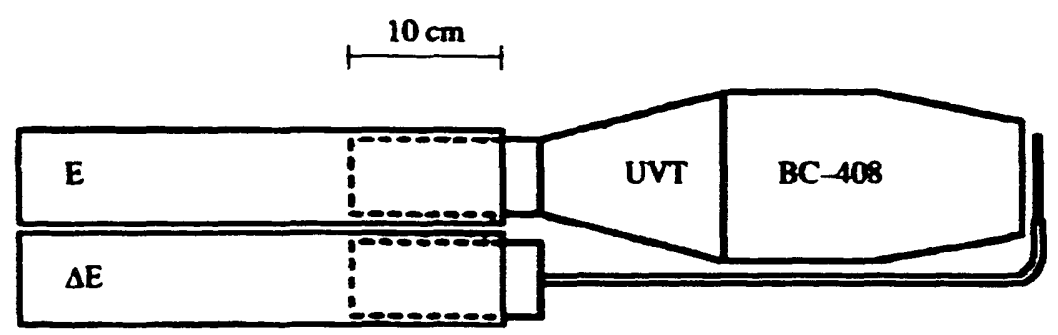

Figure 5.4: Proton telescope. A $3.2 \mathrm{~mm}$ thick plastic scintillator (BC-4C88) connected to a UVT light guide and $2^{n}$ photomultiplier tube formed the $\Delta E$ counter. The thicker $E$ counter, made of the same materials as the $\Delta \mathrm{E}$ counter, stopped protons of up to $\sim 175 \mathrm{MeV}$ kinetic energy. The circular cross section of the $\Delta \mathrm{E}$ counter was slightly smaller than the thicker counter and defined the solid angular acceptance $(8.46 \pm 0.25$ msr) of the telescope. The photomultipliers (EMI 9813B 2" tubes) and bases resided in the $\mu$-metal shields shown at the left.

a)

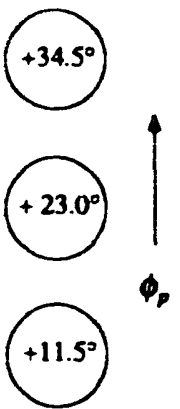

b)
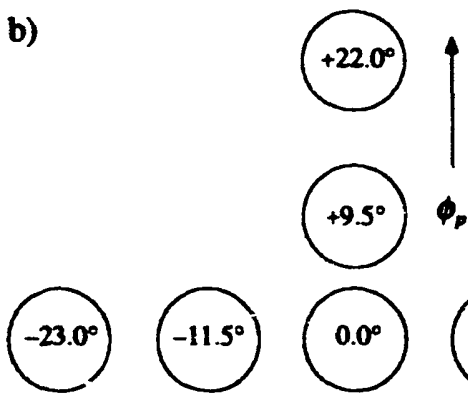

$\boldsymbol{\theta}_{\boldsymbol{p}}$

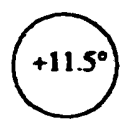

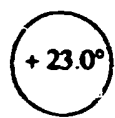
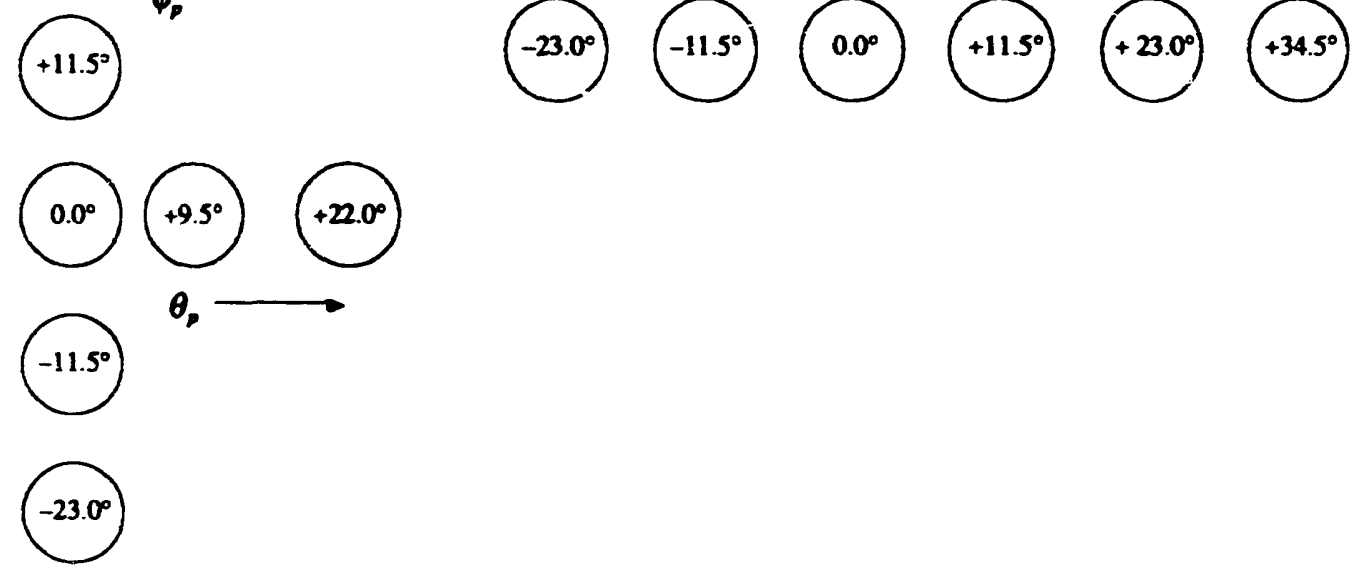

Figure 5.5: Proton detector configurations: a) vertical and b) horizontal. Values for the in-plane $\left(\theta_{p}\right)$ and out-of-plane $\left(\phi_{p}\right)$ angular deflections from the central detector are shown. 

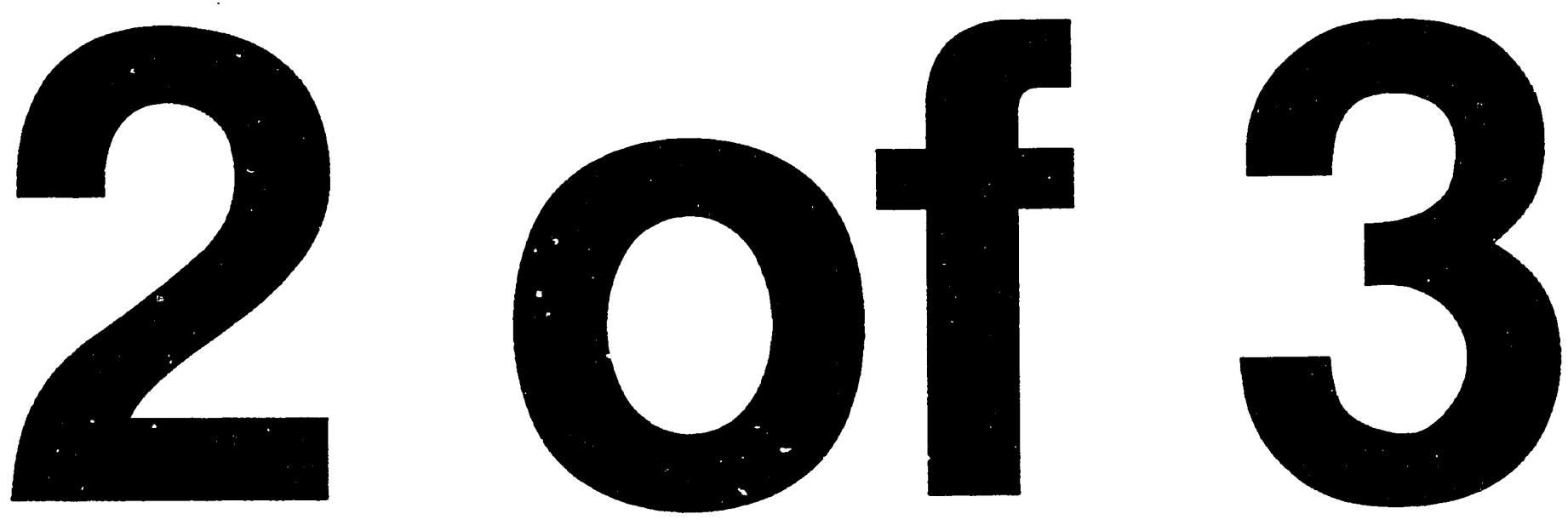


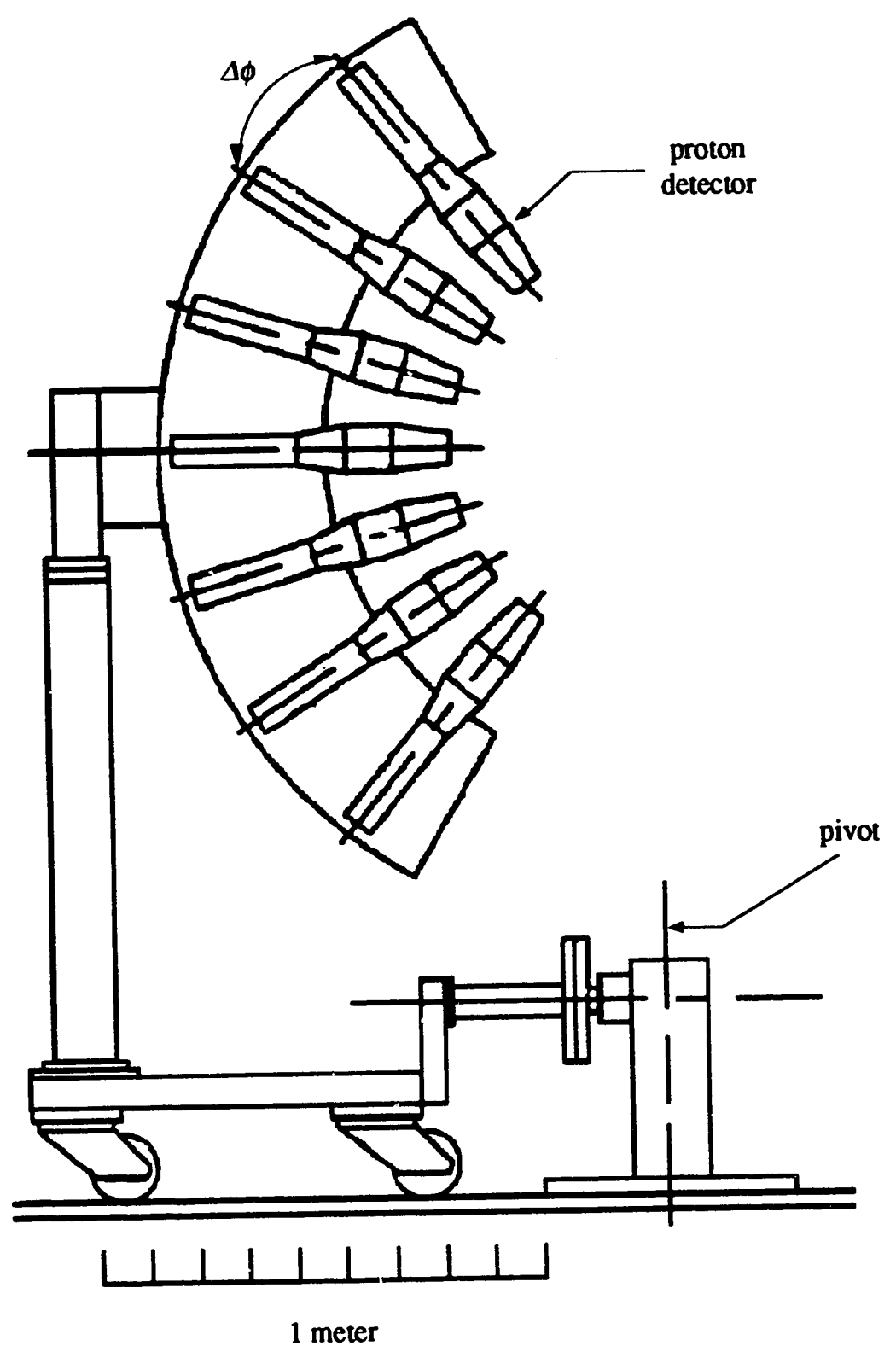

Figure 5.6: Side view of proton detector array in the vertical configuration. The angular separation, $\Delta \phi$, between detectors in this vertical plane was $11.5^{\circ}$. Two additional counters were located at same in-plane positions as the central telescope and correspunding out-of-plane angular positions of $9.5^{\circ}$ and $22^{\circ}$. This table could be rotated $90^{\circ}$ to maximize the angular range over which recoil protons were detected either in or out of the scattering plane. 


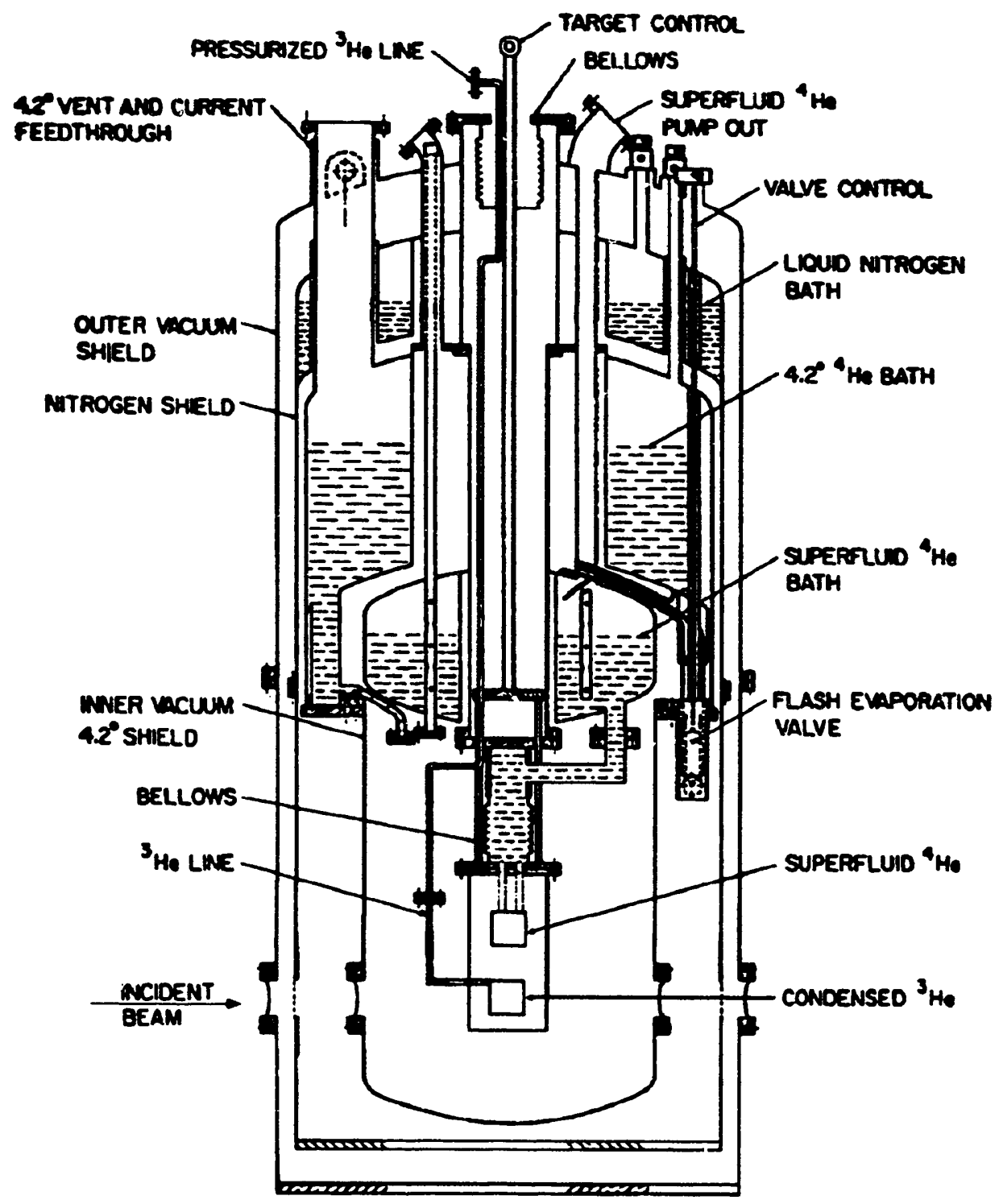

Figure 5.7: Schematic diagram ${ }^{32}$ of the University of Virginia ${ }^{3} \mathrm{He}$ cryostat. The ${ }^{3} \mathrm{He}$ target cell was a 7.62 $\mathrm{cm}$ tall by $5.4 \mathrm{~cm}$ diameter cylinder with $40 \mathrm{mg} / \mathrm{cm}^{2}$ stainless steel walls. The average ${ }^{3} \mathrm{He}$ areal thickness was about $400 \mathrm{mg} / \mathrm{cm}^{2}$. 


\section{University of Virginia ${ }^{3}$ He cryostat}

The University of Virginia ${ }^{3} \mathrm{He}$ cryostat, shown in Figure 5.7, has been successfully employed in a number of experiments. ${ }^{32,5950}$ Liquid ${ }^{3} \mathrm{He}$ was contained in a vertical target cell kept in thermal equilibrium with a superfluid ${ }^{4} \mathrm{He}$ bath. The dimensions of the cylindrical ${ }^{3} \mathrm{He}$ target cell were $7.62 \mathrm{~cm}$ tall and 5.4 $\mathrm{cm}$ in diameter, it had $40 \mathrm{mg} / \mathrm{cm}^{2}$ stainless steel walls. The nominal ${ }^{3} \mathrm{He}$ areal thickness at $1.5 \mathrm{~K}$ was 397 $\mathrm{mg} / \mathrm{cm}^{2}$, determined from measurements of the ${ }^{3} \mathrm{He}$ vapor pressure and $\mathrm{x}$-ray photographs of the pion beam spot on the target cell. The ${ }^{3} \mathrm{He}$ areal thickness was determined from the ${ }^{3} \mathrm{He}$ density and the target thickness which was illuminated by the incident beam. Based on the vapor pressure measurements, the fluctuations in the target thickness during the course of the experiment were not more than $3 \% .^{61}$

\section{Electronics and Data Acquisition System}

The data acquisition procedures in these measurements fell in one of two categories: "single-arm" or "coincidence" mode. In single-arm mode, information from the $\pi^{0}$ Spectrometer was used to trigger an event, while in coincidence mode, information from the $\pi^{0}$ Spectrometer and one of the plastic scintillation counters was required to trigger an event. The master trigger for a $\pi^{0}$ event in single-arm mode required a coincidence betveen a logic pulse from each photon detector of the spectrometer. This was known as a gamma-gamma event. Each of these logic pulses, or gamma pulses, required that the following logical conditions be fulfilled,

$$
\text { gamma }=\left(S_{1}+S_{2}+S_{3}\right) \cdot \bar{V} \cdot \sum_{i, j}\left(C_{i}+B_{j}\right),
$$

where $S_{i}$ are the signals from the plastic scintillators located behind the lead glass converters, $V$ is the veto counter at the front of the detector, and $C$ and $B$ are the lead glass converter and total absorption counters respectively. The sum over the $C$ and $B$ analog sigrals represents the total energy deposited in the photon detector. A threshold of approximately $20 \mathrm{MeV}$ is placed on this sum. Additional requirements on the master event trigger included the conditions that the beam was in the experimental hall and that the computer and electronics were not busy.

In single-arm data acquisition mode, an event was recorded on magnetic tape if any two out of 
three multiwire proportional chambers within a conversion plane fired and a gamma-gamma event was triggered. Constraints on the relative timing between the two photon detectors were used to distinguish between real and accidental coincidences between the two detectors. Digitized pulse heights from the lead glass counters and plastic scintillators were used to reconstruct the total energy of the gamma event. Information from the multiwire proportional chambers provided the location and width of the lepton showers. When data were accumulated in coincidence mode for the ${ }^{3} \mathrm{He}\left(\pi^{+}, \pi^{0} \mathrm{p}\right)$ measurements, a signal from either the $E$ or $\Delta E$ plastic scin:illation counters in the proton detector array was required in coincidence with the gamma-gamma event.

A Microprogrammable Branch Driver (MBD) acted as a buffer for the electronic information in a similar manner as in the elastic charge exchange measurements discussed in Chapter 2. The data acquisition package was based on a modified version of the standard $\pi^{0}$ Spectrometer analyzer which has been described elsewhere. ${ }^{56}$ This modified version included software for recording and displaying pulse height and timing information from the proton detectors.

\section{Experimental Procedures}

Several measurements were performed during this experiment in addition to the ${ }^{3} \mathrm{He}\left(\pi^{+}, \pi^{9}\right)$ and ${ }^{3} \mathrm{He}\left(\pi^{+}, \pi^{0} \mathrm{p}\right)$ measurements. The incident pion beam flux was calibrated through a series of ${ }^{12} \mathrm{C}$ activation measurements mentioned earlier. Measurements of the $\mathrm{p}\left(\pi^{-}, \pi^{0}\right) \mathrm{n}$ reaction were executed to determine the conversion efficiency of the $\pi^{0}$ Spectrometer. The conversion efficiency is the probability that two photons, which are a result of a $\pi^{0}$ decay, will be detected in coincidence by the $\pi^{0}$ Spectrometer. Protons from the $d\left(\pi^{+}, p\right)$ p reaction and from a proton beam were used to calibrate the pulse height spectra for each scintillation counter over a range of proton kinetic energies. (The proton detector energy calibration was not crucial for these measurements since the spectra were integrated over kinetic energy in order to determine coincidence cross sections. The results of the proton detector energy calibration measurements are presented in Appendix D.)

For each experimental configuration at which data were accumulated using the ${ }^{3} \mathrm{He}$ target, 
additional measurements were performed to sample the background contributions from the ${ }^{3} \mathrm{He}$ target cell walls. Since the contribution from the target cell walls was small, evacuating the ${ }^{3} \mathrm{He}$ target cell was deemed too time consuming for these measurements. Instead, the cryostat was raised out of the beam line and data were collected with and without a dummy target in place. The dummy target was made of stainless steel and had identical cylindrical dimensions as those of the ${ }^{3} \mathrm{He}$ target cell except for a wall thickness which was four times as thick as the ${ }^{3} \mathrm{He}$ target cell walls. Measurements without a target in place were used to subtract the contribution from air to the dummy target measurements since the dummy target itself was not evacuated and was not inside an evacuated chamber. The background contribution from the target cell walls for any given measurement was not more than $12 \%$. 


\section{Chapter 6: Data Analysis and Results for Inclusive Pion Single Charge Exchange Measurements on ${ }^{3} \mathrm{He}:{ }^{3} \mathrm{He}\left(\pi^{ \pm}, \pi^{0}\right)$ and ${ }^{3} \mathrm{He}\left(\pi^{ \pm}, \pi^{0} \mathbf{p}\right)$}

The data analysis procedures for extracting cross sections from the inclusive single charge exchange measurements fall into three categories. First, $\pi^{0}$ energy spectra were reconstructed from raw event data using a modified version of the data acquisition software package. To assemble these spectra, a number of constraints were placed on the raw event data to ensure that a two photon event was in fact the result of a $\pi^{0}$ decay event. These constraints included, but were not restricted to, limits on the relative timing between triggers from the two photon detector arms of the $\pi^{0}$ Spectrometer and the measured energy difference between two photons. For the coincidence data analysis, additional two dimensional histograms were constructed from $\triangle E$ and $E$ pulse height spectra from the proton scintillation counters. Polygonal gates were placed on these $\Delta \mathrm{E}-\mathrm{E}$ correlation plots to separate protons from other charged particles. During the second stage of the data analysis, once $\pi^{\circ}$ energy spectra had been computed, a number of parameters related to the experimental apparatus performance were determined. These quantities included such items as the conversion efficiency and solid angular acceptance of the $\pi^{0}$ Spectrometer in addition to the beam flux calibrations mentioned in the previous chapter. In the third and final stage of the data analysis, doubly differential cross sections for the single-arm measurements and triply differential cross sections for the coincidence measurements were computed by combining the calibration information with the $\pi^{0}$ energy spectra. 


\section{Analysis Procedures}

In the preliminary analysis steps, the raw event data were examined and constraints were placed on timing and pulse height spectra. Pulse height information from the various elements of the $\pi^{\circ}$ Spectrometer, such as the veto scintillators, the lead glass converters and absorption counters, must be collated to reconstruct $a \pi^{0}$ event. This is in addition to pulse height information from the proton scintillatic $n$ cerinters required for $a \pi^{0}$ p coincidence event. In this section, the initial requirements on the raw event data for reconstructing $\pi^{0}$ energy spectri for both the coincidence and single-arm measurements will be discussed.

\section{Proton Detectors}

The performance of the proton detector array was monitored through a series of stabilization measurements. Each $\mathrm{E}$ detector in a proton telesccpe had a light-emitting diode (LED) built into the detector. Measurements of the LED pulse height in each detector were performed at regular intervals throughout the experiment. During these measurements, the LEDs were pulsed at a fixed rate. Shifts in LED peak position in a pulse height spectrum could indicate changes in the detector system, such as fluctuations in the photomultiplier tube gains. This information was used as an indicator of the overall detector stability throughout the experiment. The LED peak position as a function of nun number is shown in Figure 6.1.

Polygon tests on $\Delta \mathrm{E}-\mathrm{E}$ pulse height spectra from the proton detectors were used to distinguish between protons and other charged particles, such as pions. The results from these tests could te used to require that a proton be detected in coincidence with a $\pi^{0}$ in order to reconstruct $\pi^{0}$ energy spectra for the ${ }^{3} \mathrm{He}\left(\pi^{2}, \pi^{0} \mathrm{p}\right)$ coincidence measurements. The energy deposited in the scintillator material can be expressed in terms of the charge (z) and velocity $(\beta)$ of the incident particle using the Bethe-Bloch formula,

$$
\frac{d E}{d x}=2 \pi N_{a} r_{a}^{2} m_{a} c^{2} \rho \frac{Z}{A} \frac{z^{2}}{\beta^{2}}\left[\ln \left(\frac{2 m_{a} \gamma^{2} \beta^{2} c^{2} W_{\max }}{I^{2}}\right)-2 \beta^{2}\right] \text {, }
$$

where $N_{a}$ is Avogadro's number, $r$, is the classical electron radius, $m_{e}$ is the mass of the electron, and $\rho, I$, $Z$, and $A$ refer to vie density, mean excitation potential, atomic number, and atomic weight of the scintillator material cespectively. In the energy range of interest, the light output of the individual counters was proportional to the energy deposited by a charged particle. Therefore, light from particles with the same 


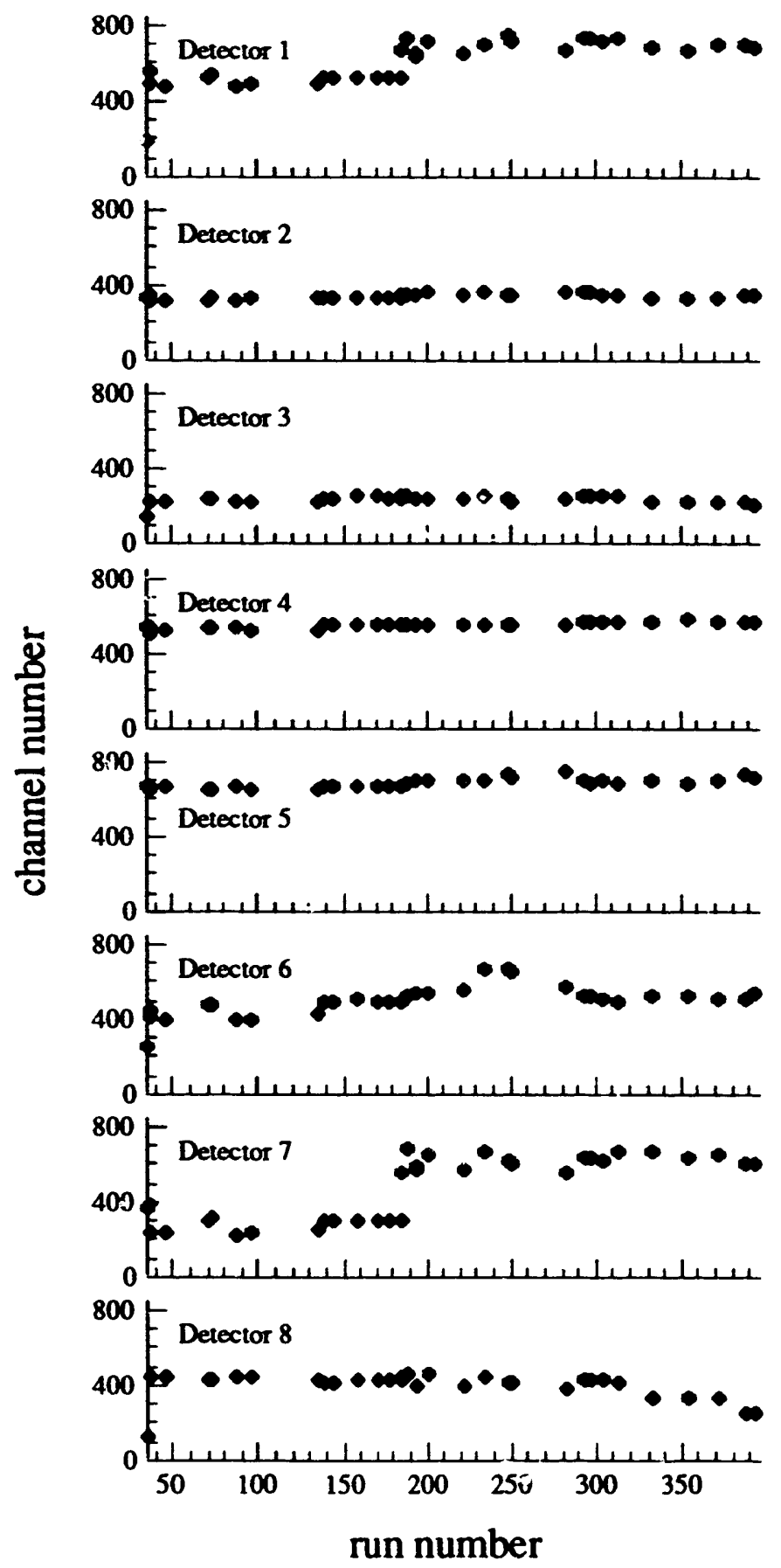

Figure 6.1: Proton stabilization data. A light emitting diode (LED) was atuached to each E counter in the proton detector array to monitor photomultiplier tube gain shifts. In this figure, the LED peak position in pulse height spectra for each of the $E$ counters is shown as a function of run number. The large shift in LED peak position observed near run 180 in some of pulse height spectra may be attributed to a change in the LED pulse rate from $70 \mathrm{kHz}$ to $180 \mathrm{~Hz}$. 


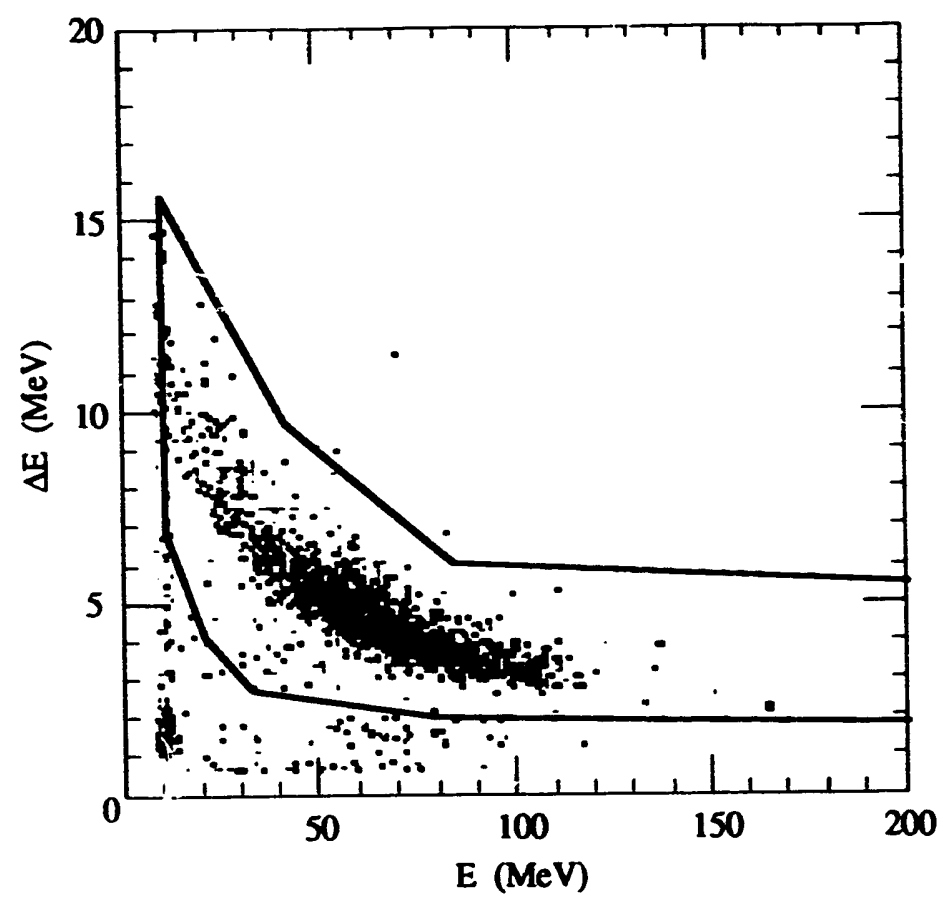

Figure 6.2: Example of a proton polygon gate on a two dimensional plot of energy deposited in the $\Delta E$ and E scintillation counters of the central detector.

mass and charge manifests itself as a distinct band in two dimensional histograms of pulse height spectra from the $\Delta E$ and $E$ scintillation counters. An example of a polygon gate is shown in Figure 6.2 Ideally, these gates should remain fixed throughout the experiment. In fact, only one gate was adjusted because of a significant change in an individual detector's response.

\section{LAMPF $\pi^{\circ}$ Spectrometer}

The $\pi^{\circ}$ Spectrometer subtends an angular range of approximately $30^{\circ}$ in the laboratory system. Additional constraints may be placed on the reconstructed $\pi^{0}$ scattering angle which was determined from the position information of the two photon event. In this manner, the spectrometer acceptance could be divided into several smaller angular bins corresponding to different $\pi^{\circ}$ laboratory scattering angles. The requirements on the $\pi^{0}$ energy resolution and statistical accuracy of the measurements determine the choice of angular bin size. In the coincidence analysis, a single $24^{\circ}$ bin centered at $\theta_{c}$ was used in order to improve the statistical accuracy of the measurements, where $\theta_{c}$ is the central scattering angle of the 
spectrometer. In the single-arm analysis, the $\pi^{\circ}$ Spectron.eter acceptance was divided into three $8^{\circ}$ bins corresponding to angles of $\theta_{c}$ and $\theta_{c} \pm 8^{\circ}$. For the conversion efficiency analysis, the energy spectra were divided into twelve $2^{\circ}$ bins since the value of the differential cross section for the free single charge exchange reaction, ${ }^{3} \mathrm{H}\left(\pi, \pi^{9}\right) \mathrm{n}$, could vary by as much as $15 \%$ over a single $24^{\circ}$ bin. The conversion efficiency analysis will be discussed in greater detail later in this Chapter.

\section{Timing and Pulse Height Constraints}

The relative timing between the triggers from the two photon detector arms of the $\pi^{0}$ Spectrometer was used to determine the ratio of real to accidental events. An example of the limits placed on the timing spectra for a valid $\pi^{0}$ event is shown in Figure 6.3. Thie ratio of accidental to real events was typically not more than 5\%.

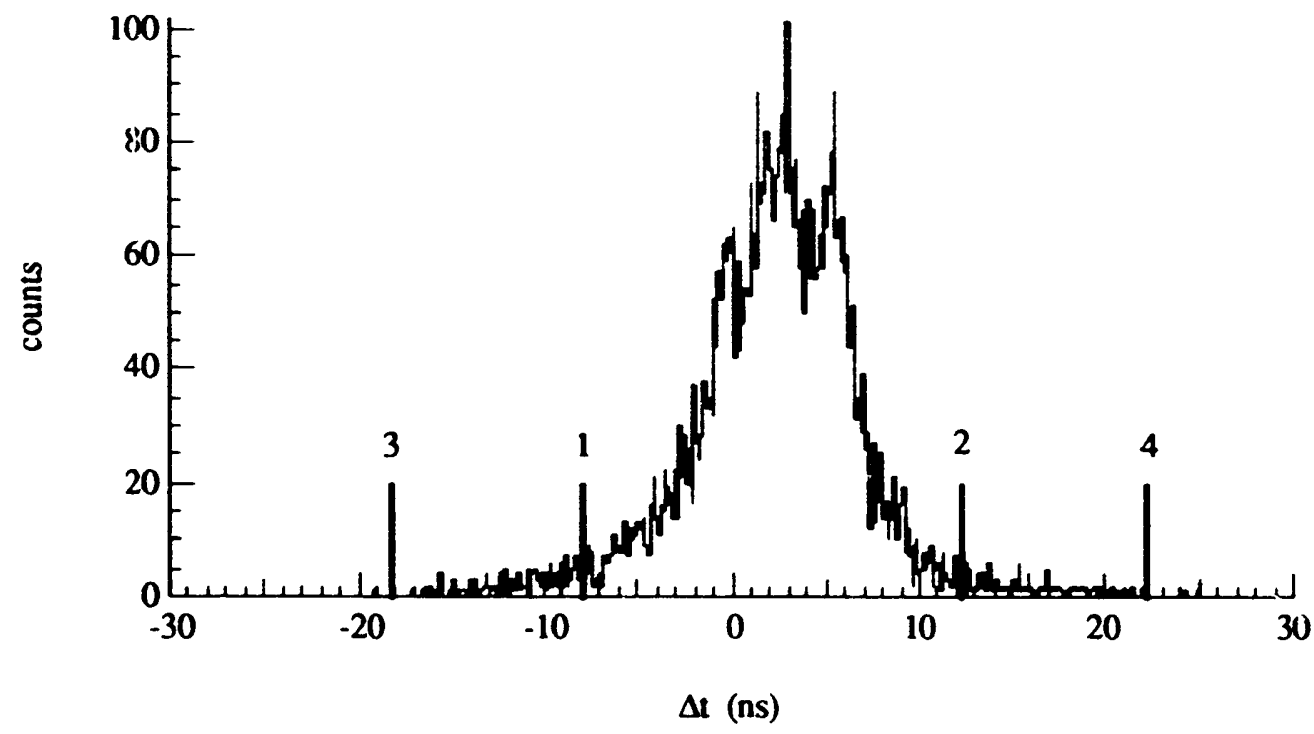

Figure 6.3: Relative timing between the two photon detector arms of the $\pi^{0}$ Spectrometer. The relative timing between the two photon detector arms must lie within markers 1 and 2 for a valid $\pi^{\circ}$ event. Events whose relative timing occurred outside markers 1 and 2 but within markers 3 and 4 were labeled as accidental coincidences between the two photon detector arms. Markers 1 and 2 were placed so as to limit the relative timing of good $\pi^{0}$ events to $\pm 10 \mathrm{~ns}$ about the coincidence peak. The position of the centroid for this distribution is arbitrary. 


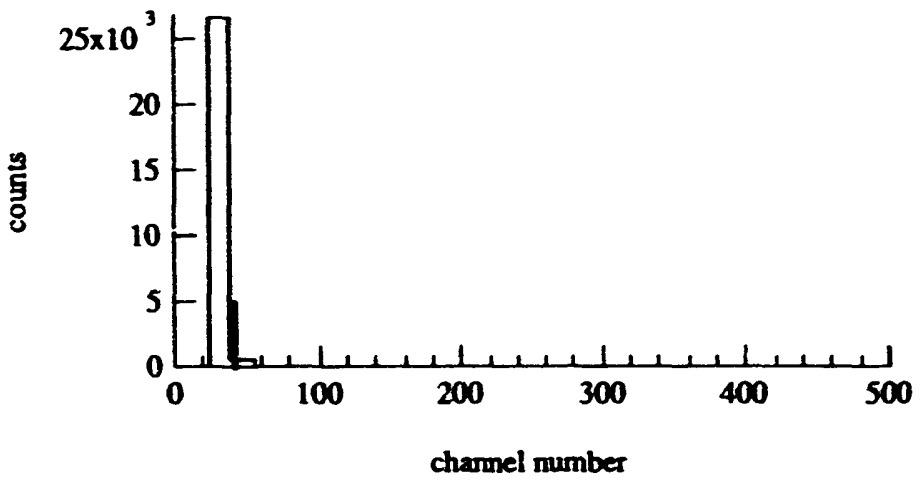

Figure 6.4: Pulse height spectrum from a veto counter. The pulse height in the veto counter resulting from a charged particle is greater than the value indicated by the marker in this figure. This marker was placed so that events which passed other kinematic constraints, such as the relative timing and energy difference between two photon events, generated a pulse height less than the value indicated by this marker. Although the signals from the veto counters were part of the master trigger for a good $\pi^{\circ}$ event, charged particles which generated a signal less than the threshold in the veto counters would not veto an event. The prominent peak in this spectrum is from the ADC pedestal.

Pulse height information from scintillation counters at the front of each photon detector arm were used to veto any events which were initiated by charged particles rather than photons. The probability that a $100 \mathrm{MeV}$ photon will interact with a veto counter is approximately 2\%. An example of the pulse height spectra from one of these veto counters is shown in Figure 6.4.

The total energy of each detected photon was reconstructed from the pulse height information provided by each of the lead glass converters and total absorption counters. The fractional difference may be expressed in terms of the measured photon energies from each photon detector arm, $E_{\gamma_{1}}$ and $E_{\gamma_{2}}$, as

$$
X=\frac{E_{\gamma_{1}}-E_{\gamma_{2}}}{E_{\gamma_{1}}+E_{\gamma_{2}}} .
$$

A distribution of this energy difference is shown in Figure 6.5.

A constraint, XCUT, was placed on the range of acceptable fractionie ifferences between the total energies deposited in each photon detector arm such that.

$$
|X| \leq X C U T \text {. }
$$

The range of acceptable values for $X$ determined the $\pi^{0}$ energy resolution and statistical uncertainties of the measurements. For small values of XCUT, the $\pi^{\circ}$ energy resolution depends primarily on the position resolution of the wire chamber information as can be seen from the following relationship between $\pi^{c}$ 
energy, $E_{x^{*}}$, and the opening angle between the two photons, $\eta$.

$$
E_{x^{0}}^{2}=\frac{2 m_{x^{\circ}}^{2}}{(1-\cos \eta)\left(1-X^{2}\right)} .
$$

However, for larger values of XCUT, the statistical quality of the measurement will improve as the number of accepted events increases. Studies of XCUT demonstrated that the statistical accuracy did not significantly improve while the energy resolution rapidly decreased for XCUT values greater than 0.25 . The results of these studies are shown in Figures 6.6 and 6.7.

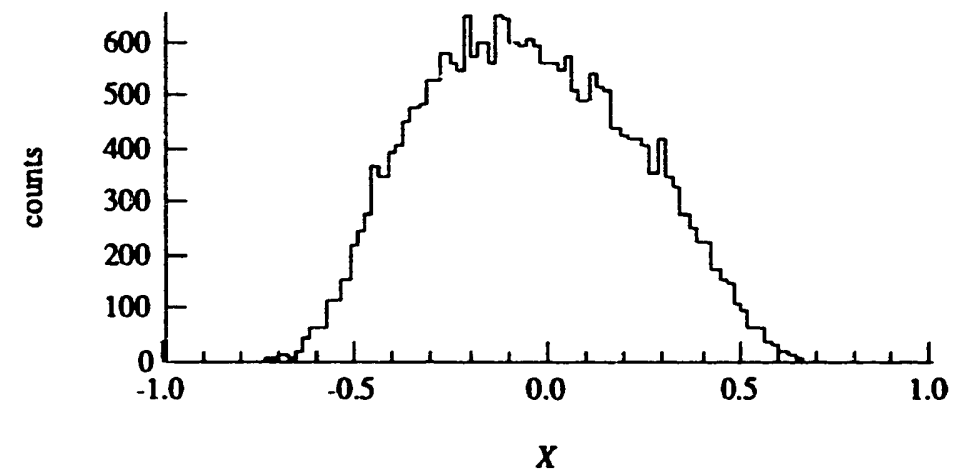

Figure 6.5: Reconstructed distribution of the fractional energy difference between two detected photons, $X$. This example was taken from ${ }^{3} \mathrm{He}\left(\pi^{+}, \pi^{9}\right)$ measurements at $\theta_{\mathrm{x}^{0}}=70^{\circ}$. The asymmetry in this distribution may be attributed to photon attenuation in the ${ }^{3} \mathrm{He}$ cryostat. Photons which are detected by the lower photon arm (arm 2) travel through less material than those detected by the upper arm.

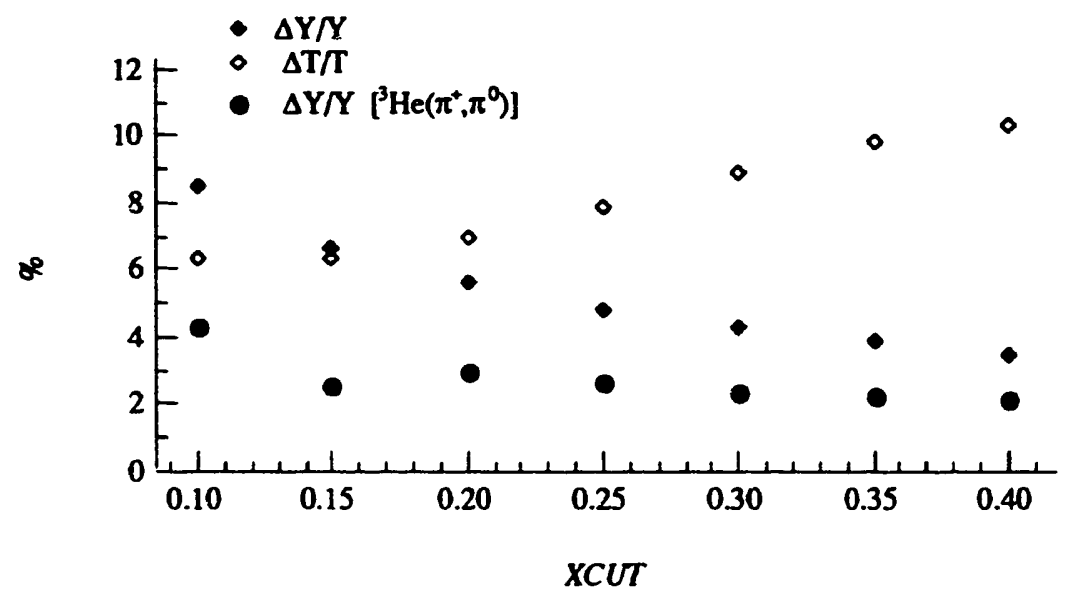

Figure 6.6: Effect of XCUT of on the statistical uncertainty $(\Delta Y / Y)$ and energy resolution $(\Delta T / T)$ of $\pi^{0}$ energy spectra from the $p\left(\pi^{-} \pi^{9}\right) n$ (diamonds) and ${ }^{3} \mathrm{He}\left(\pi^{*} \pi^{9}\right) \times$ (circles) reactions. 


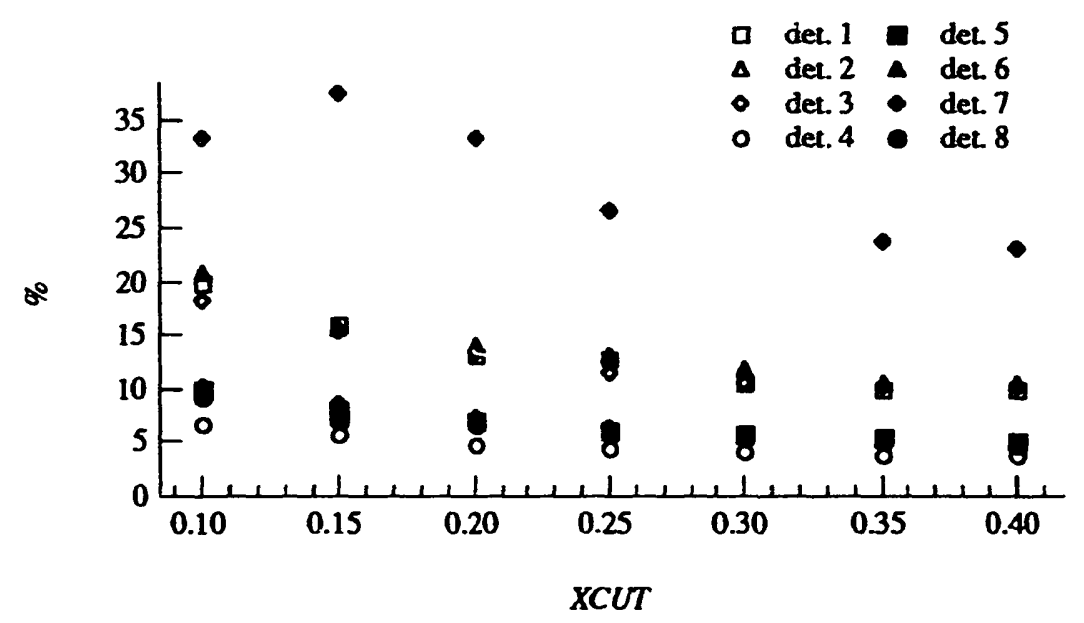

Figure 6.7: Effect of XCUT on the statistical uncertainty for ${ }^{3} \mathrm{He}\left(\pi^{+}, \pi^{\circ} \mathrm{p}\right)$ measurements at $\theta_{x^{\circ}}=90^{\circ}$ and $\theta_{\mathrm{p}}$ $=30.4^{\circ}$. The detector number refers to the proton detector.

\section{Wire Chamber Analysis}

The opening angle between two photons was determined from wire chamber information. The information from individual wires within a wire chamber was encoded into cluster centroids and widths using a Lecroy PCOS readout system. The cluster patterns from the three wire chambers uniquely determined the position of a lepton shower within a conversion plane. The wire chamber tests for a valid photon event in any given conversion plane inclứed the following conditions:

1) at least two wire chambers within a conversion plane must fire,

2) the number of hits in any given wire chamber not exceed a maximum value,

3) that a valid conversion, which had satisfied the previous two conditions, had not already occurred in a previous conversion plane located at a closer distance to the target.

The position information was then processed by the data analysis software to reconstruct the photon trajectory from the target. Three types of efficiencies are associated with the wire chamber performance and track reconstruction procedures: an on-line wire chamber efficiency, a replay wire chamber efficiency, and a tracking efficiency. These efficiencies are plotted versus nun number in Figure 6.8. The overall wire chamber efficiency for the $\pi^{0}$ Spectrometer may be expressed as the product of these efficiencies, 


$$
\varepsilon_{w c}=\varepsilon_{b r} \cdot \varepsilon_{\text {on-Ene }} \cdot \varepsilon_{\text {replay }} \cdot
$$

Since information from a $\pi^{0}$ event was recorded on magnetic tape on the condition that at least two out of three wire chambers in a conversion plane had fired, the on-line wire chamber efficiencies cannot be reconstructed from the raw event data during the off-line data analysis procedures. Instead, these efficiencies were calculated during data acquisition, as will be described below, and recorded on tape.

The efficiency of an individual wire chamber within a conversion plane is the ratio of the threefold coincidences between hits in all three wire chambers to the number of events in which the other two chambers fire with or without a hit in the third chamber. The efficiency of wire chamber $X$ within conversion plane $i$ of arm $k$ can be expressed as,

$$
\varepsilon_{x x}^{k}=\frac{\left(i X \cdot i Y \cdot i X^{\prime}\right)}{\left(i Y \cdot i X^{\prime}\right)}
$$

where $i$ may range from one to three and $X, Y$, and $X^{\prime}$ refer to the three wire chambers within a conversion plane. The condition that all three wire chambers record hits is given by $\left(i X \cdot i Y \cdot i X^{\prime}\right)$. In general, the efficiencies of the individual wire chambers varied between $95 \%$ and $99 \%$.

The efficiency for detecting a charged lepton shower in conversion plane $i$ of arm $k$ may be expressed as the product of the individual wire chamber efficiencies within that conversion plane,

$$
\varepsilon_{i}^{k}=\varepsilon_{i x}^{k} \cdot \varepsilon_{i Y}^{k} \cdot \varepsilon_{i x^{\prime}}^{k} .
$$

The overall wire chamber efficiency of an individual photon detector arm may be expressed in terms of the efficiencies for detecting a shower in any one conversion plane as follows,

$$
\varepsilon^{k}=\frac{\sum_{i=1}^{3} \operatorname{MAJ}\left(i X \cdot i Y \cdot i X^{\prime}\right)}{\sum_{i=1}^{3} \frac{\operatorname{MAJ}\left(i X \cdot i Y \cdot i X^{\prime}\right)}{\varepsilon_{i}^{k}}}
$$

The function $\operatorname{MAJ}\left(i X \cdot i Y \cdot i X^{\prime}\right)$ refers to the majority condition that at least two out of three wire chambers within a conversion plane fire. And finally, the on-line wire chamber efficiency is the product of the two photon detector arm wire chamber efficiencies,

$$
\varepsilon_{\text {on-line }}=\varepsilon^{1} \cdot \varepsilon^{2}
$$

Typical values for the on-line efficiencies during the experiment were about $80 \%$. 


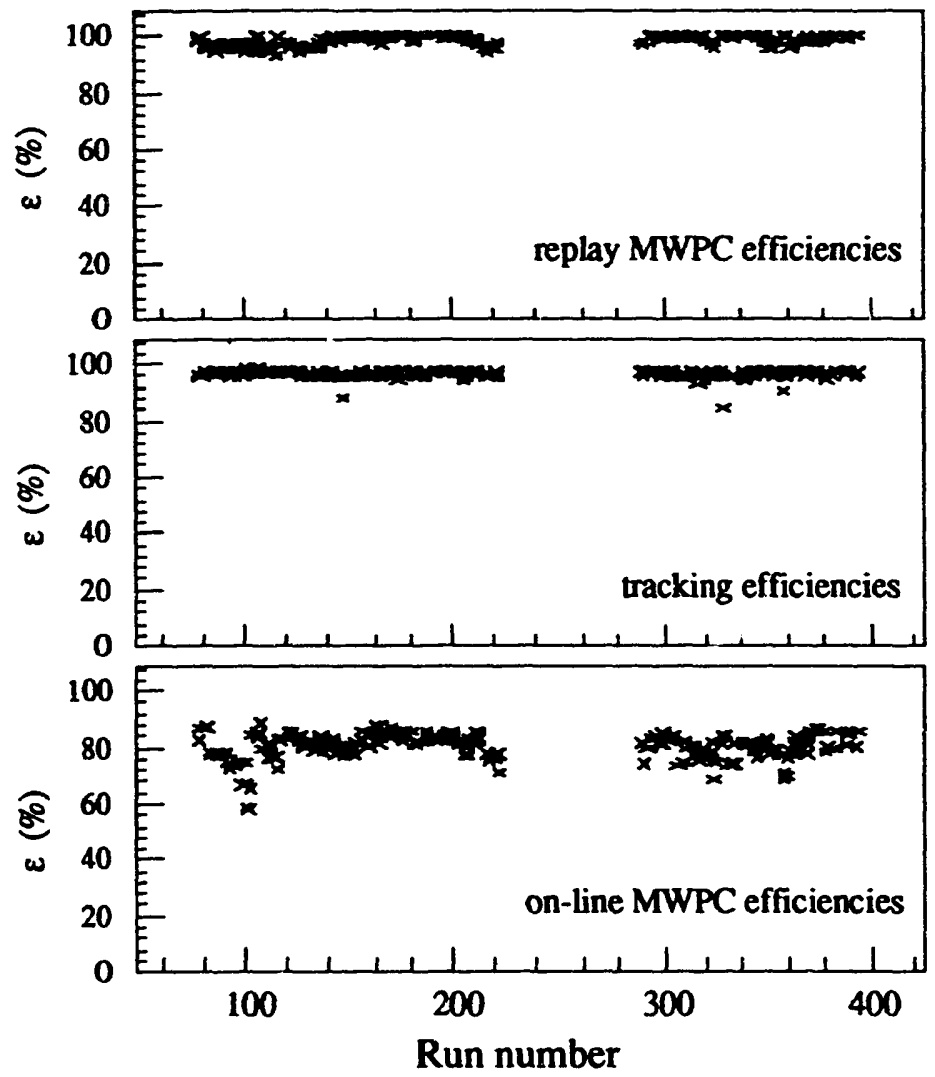

Figure 6.8: Wire chamber efficiencies from replay hot wire tables (top), tracking efficiencies (middle), and on-line MWPC efficiencies (bottom). The large gap observed between runs 240 and 280 coincides with the proton detector calibration measurements

A second set of wire chamber efficiencies, known as replay efficiencies, were calculated during the off-line data analysis process. Defects in an individual wire chamber could cause a chamber to appear less efficient. Occasionally, some wires within an individual wire chamber fired more frequently than cthers due to problems with the wire chamber read out electronics. A list of these wires, also known as "hot" wires, was maintained and monitored throughout the data acquisition process so that any hit patterns which resulted from these hot wires were disregarded. During the data analysis process, additional hot wires which were overlooked during the experiment were added to this list. A replay wire chamber efficiency was computed using the same algorithm as outlined in Equations 6.6 through 6.9. This replay efficiency corrected for events which were recorded on magnetic tape but no longer passed the conditions 
for recoristructing the position of a photon shower because of the additional hot wires. The replay efficiencies for individual wire chambers during this analysis typically varied between $98 \%$ and $100 \%$.

The tracking e ciency represents the efficiency for which a good photon event could be reconstructed from the wire chamber hit patterns. If an event passed the wire chamber conversion tests, a tracking subroutine computed the photon trajectory and conversion point based on the wire chamber information. This routine rejected events if the hit pattern in a wire chamber was outside the fiducial region of a wire chamber or if an event could not be traced back to the target within certain constraints on the slope of its trajectory. The tracking efficiency may be defined as,

$$
\varepsilon_{t r}=1-\frac{F_{1}+F_{2}}{N_{\text {cotal }}-F_{c}}
$$

where $F_{1}$ and $F_{2}$ are the number of events in either photon detector arm which fail the tracking tests, $N_{\text {toote }}$ is the total number of events which were processed by the analyzer and recorded on magnetic tape, and $F_{c}$ is the number of events which fail the three previously mentioned conditions on a valid wire chamber event during the replay analysis process.

\section{$\pi^{0}$ Spectrometer Acceptance and Conversion Efficiency}

Once $\pi^{0}$ energy spectra had been extracted from the raw event data, the solid angular acceptance and conversion efficiency of the $\pi^{0}$ Spectrometer had to be determined before differential cross sections could be computed. The solid angular acceptance of the $\pi^{0}$ Spectrometer was computed using a Monte Carlo simulation. The conversion efficiency was determined from observations of the $e^{1} H\left(\pi^{-}, \pi^{9}\right)$ n reaction.

\section{Solid Angular Acceptance of the $\pi^{0}$ Spectrometer}

The solid angular acceptance of the $\pi^{0}$ Spectrometer depends on the geometrical set-up of the spectrometer and on the kinetic energy of thic scatered $\pi^{\circ}$. Traditionally, this acceptance function has been computed using a Monte Carlo simulation of the $\pi^{0}$ Spectrometer known as PIANG. In this analysis, PIANG was modified to include the probability of photon attenuation in the ${ }^{3} \mathrm{He}$ target. The effects of this photon attenuation on the solid angular acceptance of the spectrometer are illustrated in Figure 6.9. 

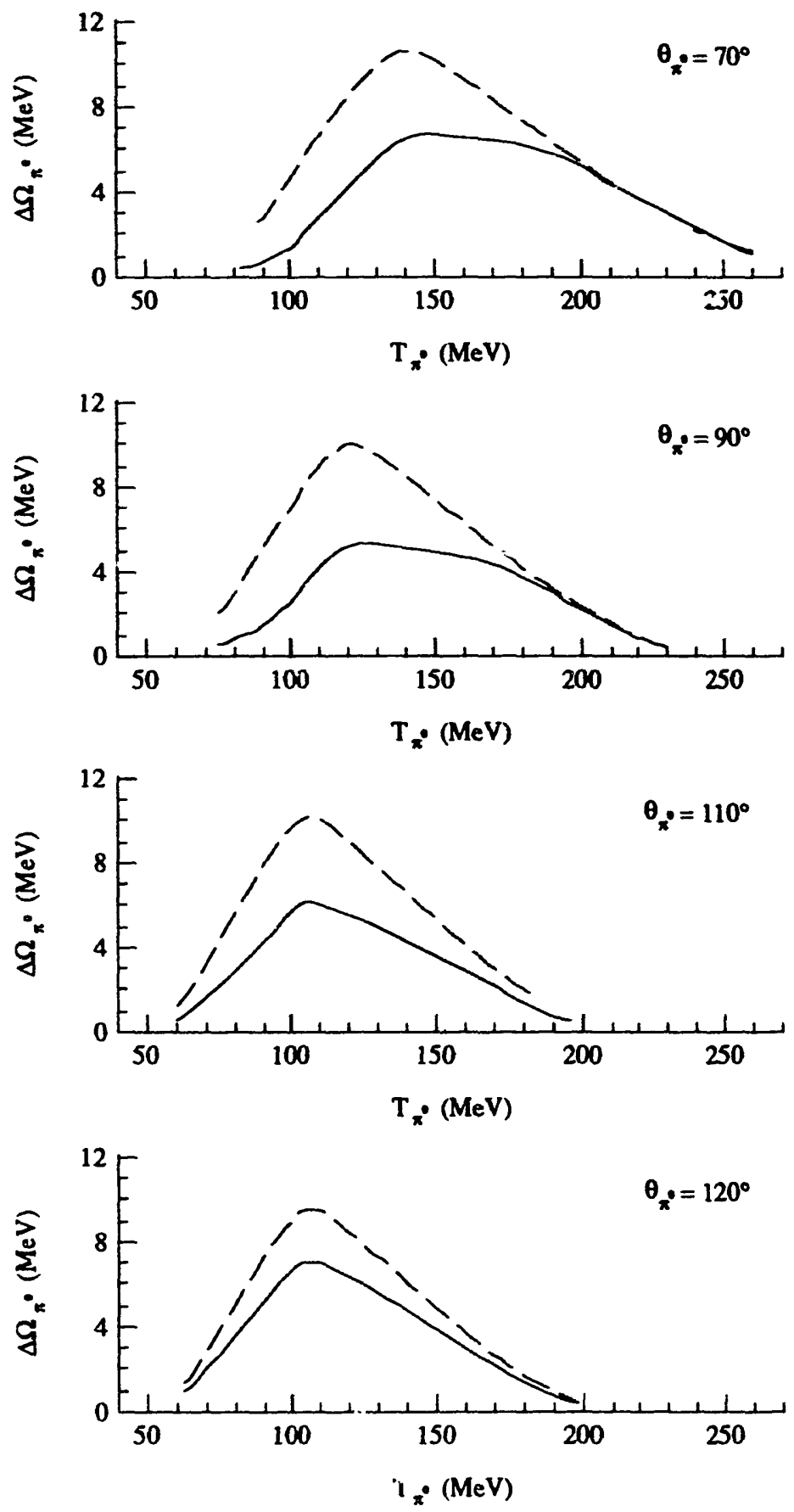

Figure 6.9: Solid angular acceptance of $\pi^{0}$ Spectrometer as a function of $\pi^{0}$ kinetic energy for each laboratory scattering angle $\left(\theta_{\pi}\right)$ obtained from the Monte Carlo simulation PIANG. Solid angular acceptance functions are shown with (solid) and without (dashed) photon attenuation in the ${ }^{3} \mathrm{He}$ target. All calculations assume a value of 0.25 for XCUT as was used in the data analysis. The statistical fluctuations in these results are less than $1 \%$. 
The Monte Carlo simulation included the following assumptions about the experimental conditions: a finite spot size and momentum bite of the incident beam in addition to a specific geometrical orientation of the spectrometer and ${ }^{3} \mathrm{He}$ target. The probability of photon attenuation in the target or spectrometer, which is re'ated to the photon detection efficiency, was affected by the physical specifications of the spectrometer and the ${ }^{3} \mathrm{He}$ cryostat, such as the thickness, type of material of a particular component, and orientation with respect to the photon. A total of sixteen separate sets of acceptance functions were calculated corresponding to the angular bins and $\pi^{0}$ scattering angles for the single-arm and coincidence measurcments.

\section{Conversion Efriciency}

The conversion efficiency is the probability that the $\pi^{0}$ Spectrometer will detect two photons in coincidence which are the result of a $\pi^{0}$ decay. The expected value of the conversion efficiency for a 100 $\mathrm{MeV} \pi^{0}$ is approximately $41 \%^{62}$, based on the amount of converter material present in each photon detector arm. By observing $\pi^{0}$ decays over a range of $\pi^{0}$ kinetic energies from the ${ }^{1} H\left(\pi^{-}, \pi^{9}\right)$ n reaction, one can deduce the conversion efficiency of the spectrometer, $\tilde{C}$, in the following manner,

$$
\tilde{C}=\frac{\left\langle\frac{d \sigma}{d \Omega}\right\rangle_{\text {exp }}}{\left\langle\frac{d \sigma}{d \Omega}\right\rangle_{\text {soapi }}}
$$

where

$$
\left\langle\frac{d \sigma}{d \Omega}\right\rangle_{\exp }=\frac{\tilde{N}_{\mathrm{x}^{0}}}{\left(2 \cdot\langle\rho \tau\rangle_{\mathrm{CH}_{2}} / \cos \theta_{1}\right)} \cdot \frac{1}{\Delta \Omega_{M C}},
$$

and $\left\langle\frac{d \sigma}{d \Omega}\right\rangle_{\text {raspi }}$ is the differential cross section for the ${ }^{1} H\left(\pi^{-}, \pi^{0}\right) n$ reaction from $\pi N$ phase shift calculations. ${ }^{20}$ This phase shift analysis of $\pi$ p elastic scattering data reproduces existing measurements of the differential cross section for the tree charge exchange reaction quite well. The factor of two represents the number of hydrogen atoms per $\mathrm{CH}_{2}$ molecule and $\langle\rho \tau\rangle_{\mathrm{CH}_{2}}$ is the areal thickness of the $\mathrm{CH}_{2}$ target in units of $\mathrm{CH}_{2}$ molecules/mb. The measured $\pi^{0}$ yield from hydrogen, $\tilde{N}_{\pi^{0}}$, was determined as follows,

$$
\tilde{N}_{\pi^{0}}=\frac{N_{C H_{2}}}{N_{\pi} \cdot \ell \cdot \varepsilon_{w c}}-\frac{N_{{ }^{12} c}}{N_{\pi} \cdot \ell \cdot \varepsilon_{w c}} \cdot f_{{ }^{12} c}
$$

where the $\pi^{0}$ yields from the $\mathrm{CH}_{2}$ and ${ }^{12} \mathrm{C}$ targets, $N_{\mathrm{CH}_{2}}$ and $N_{{ }^{12} \mathrm{C}}$ respectively, have been corrected for live 
time, $\ell$, wire chamber efficiency, $\varepsilon_{w c}$, and the number of incident pions on target, $N_{x}$. The ratio of the ${ }^{12} \mathrm{C}$ content of the $\mathrm{CH}_{2}$ and ${ }^{12} \mathrm{C}$ targets is expressed as

$$
f_{a_{C}}=\frac{\langle\rho \tau\rangle_{\mathrm{CH}_{2}}}{\langle\rho \tau\rangle_{\mathrm{a}_{c}}}
$$

The number of incident pions on target was determined from the calibration constant $\boldsymbol{x}_{\text {ed }}$ (in units of pions per toroid), which was derived from the ${ }^{12} \mathrm{C}$ activation measurements, and the number of proton toroid counts accumulated during a given run, $T$,

$$
N_{\mathrm{x}}=\kappa_{\text {ead }} \cdot T \text {. }
$$

During the conversion efficiency analysis, $\pi^{\circ}$ energy spectra from $24^{\circ}$ angular bins were subdivided into twelve spectra corresponding to a $2^{\circ}$ bin size, for two reasons. First, the differential cross section varied by as much as $20 \%$ over a single $24^{\circ}$ bin. Second, the spectrometer angular acceptance, $\Delta \Omega_{\text {MC }}$ was a strong function of the $\pi^{0}$ kinetic energy. The kinetic energy of a $\pi^{0}$ from charge exchange on hydrogen could vary by as much as $15 \mathrm{MeV}$ over a $24^{\circ}$ angular range (see Figure 6.10). The solid angular acceptance of the spectrometer was calculated for each of these smaller bins at a $\pi^{0}$ kinetic energy corresponding to the $\pi^{0}$ energy for the central scattering angle within the $2^{\circ}$ bin. A measured yield was extracted using Equation 6.13. The total measured yield was computed by adding together the yields from the smaller bins weighted by the value of the differential cross section for the central scattering angle corresponding to that particular bin.

A further complication in this analysis was an uncertainty in the actual areal thickness of the $\mathrm{CH}_{2}$ target. The $\mathrm{CH}_{2}$ target was composed of five separate slabs. During the course of this analysis, it became clear from comparison of normalized $\pi^{0}$ yields accumulated under identical experimental conditions that on some occasions one or more of the thinner pieces of $\mathrm{CH}_{2}$ was not in place during the measurements. An example of this discrepancy, where the normalized $\pi^{0}$ yield from two separate runs under identical experimental conditions is not the same, is shown in Figure 6.11. A list of the $\mathrm{CH}_{2}$ thicknesses which were used in these measurements is presented in Table 6.1. The results of the conversion efficiency measurements with and without the $\mathrm{CH}_{2}$ thickness correction are presented in Figures 6.12 and 6.13. The corrected results are in good agreement with previously measured values. ${ }^{62.63}$ 


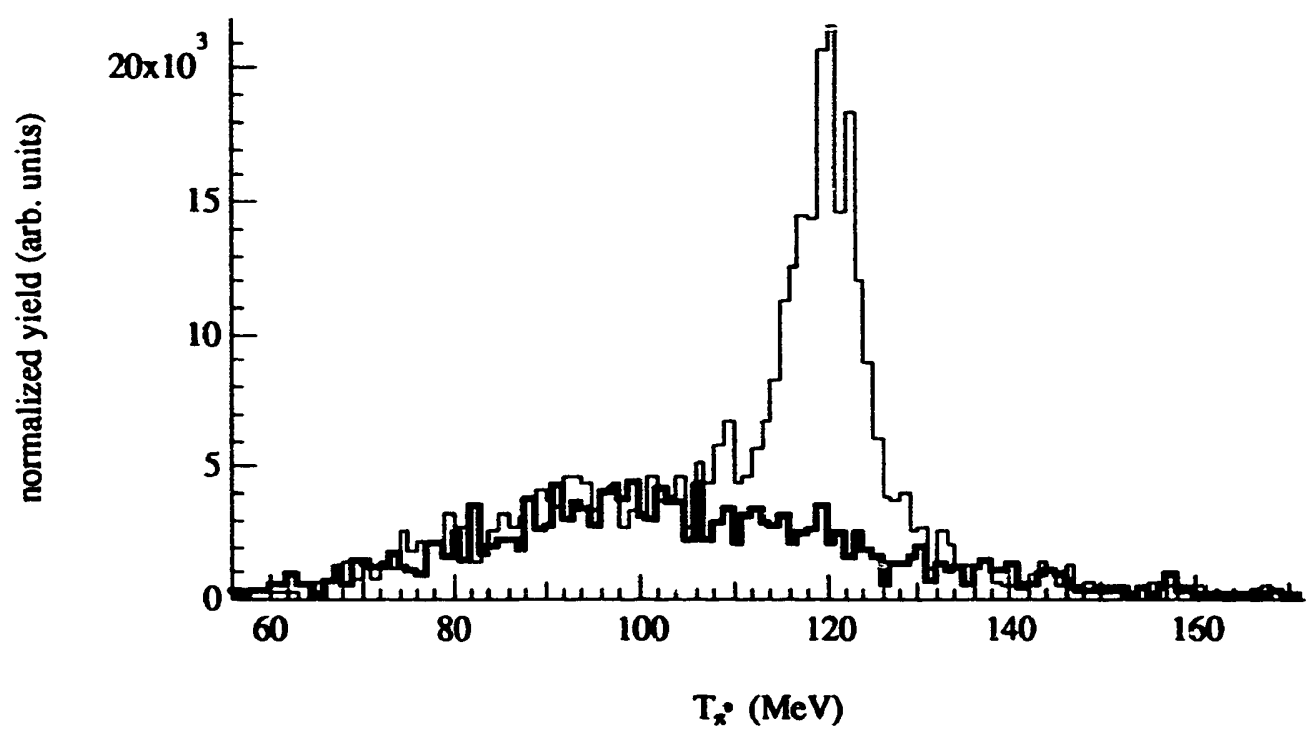

Figure 6.10: Normalized $\pi^{0}$ energy spectra from $\mathrm{CH}_{2}$ (thin line) and ${ }^{12} \mathrm{C}$ (thick line) targets. The yields have been corrected for live time, wire chamber efficiencies, and the relative target thicknesses of the $\mathrm{CH}_{2}$ and ${ }^{12} \mathrm{C}$ targets. The angular bin width in this example is $24^{\circ}$; note the large width of the $\pi^{\circ}$ peak from hydrogen. The $\pi^{0}$ yield from a $\mathrm{CH}_{2}\left({ }^{12} \mathrm{C}\right)$ measurement is the sum of all the counts in the $\mathrm{CH}_{2}\left({ }^{12} \mathrm{C}\right) \pi^{0}$ energy spectrum shown above.

\begin{tabular}{|c|c|c|}
\hline \multicolumn{3}{|c|}{ Thickness of $\mathrm{CH}_{2}$ targets } \\
\hline target no. & $\begin{array}{c}\langle\rho \tau\rangle_{C \mu^{3}} \\
\left(\mathrm{~g} / \mathrm{cm}^{2}\right)\end{array}$ & $f_{t^{\prime}}$ \\
\hline 1 & 0.0674 & 0.121 \\
\hline 2 & 0.0676 & 0.122 \\
\hline 3 & 0.138 & 0.249 \\
\hline 4 & 0.141 & 0.255 \\
\hline$J$ & 0.140 & 0.253 \\
\hline
\end{tabular}

Table 6.1: List of $\mathrm{CH}_{2}$ areal thicknesses in units of $\left(\mathrm{g} / \mathrm{cm}^{2}\right)$ which were employed in the conversion efficiency measurements. The total areal thickness when all five targets were used was $0.554 \mathrm{~g} / \mathrm{cm}^{2} ; f_{t a t}$ represents the relative contribution to the total $\mathrm{CH}_{2}$ thickness of an individual target. 

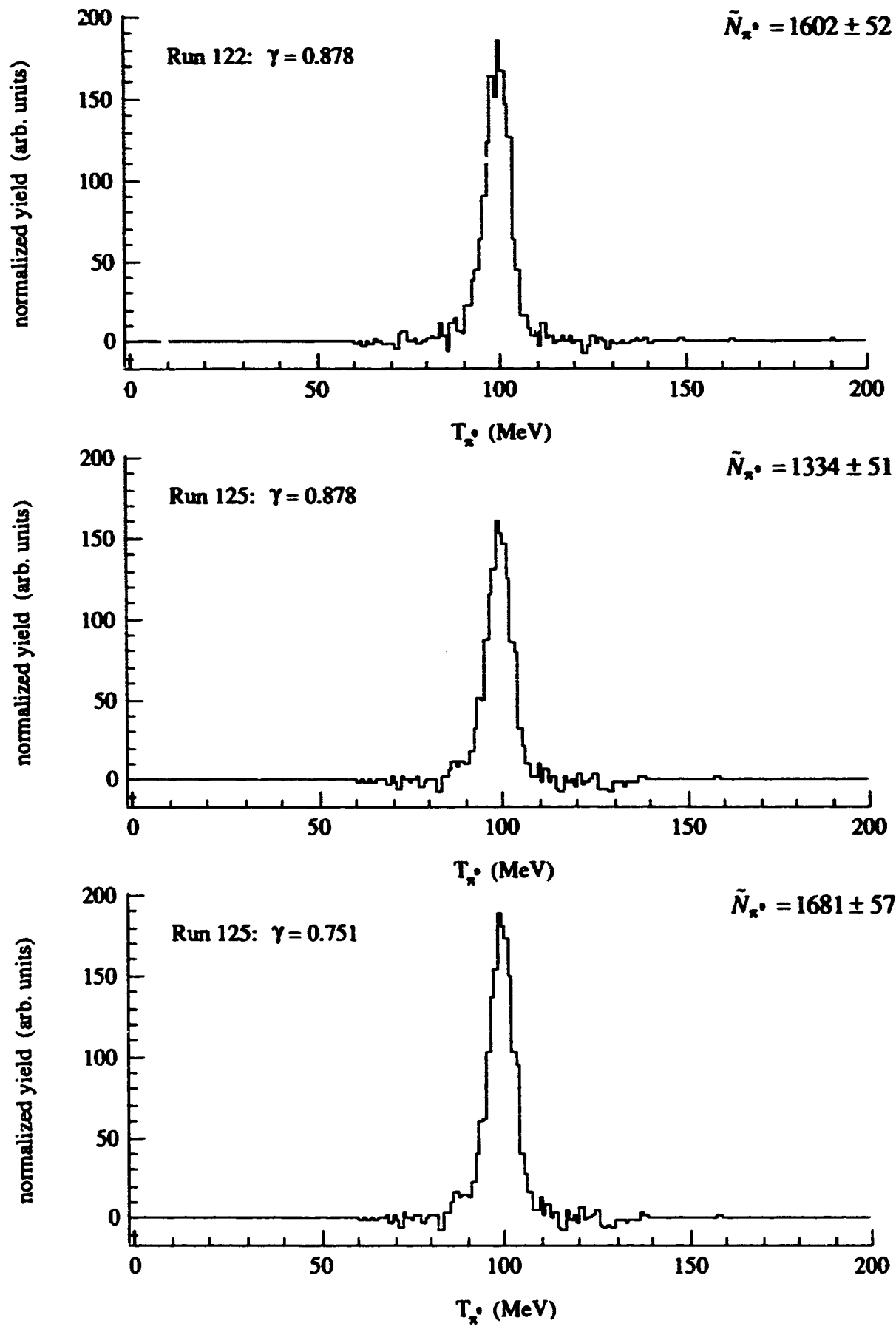

Figure 6.11: Normalized $\pi^{0}$ energy spectra from a $\mathrm{CH}_{2}$ target with ${ }^{12} \mathrm{C}$ background subtracted at $\theta_{\pi^{\circ}}=90^{\circ}$ and $T_{n}=149 \mathrm{MeV}$. Runs 122 and 125 were performed under identical experimental conditions and the net normalized yield, $\bar{N}_{x^{\circ}}$, should be the same for both runs if the same $\mathrm{CH}_{2}$ target was used. The top two plots assume the same $\mathrm{CH}_{2}$ thickness was used $(\gamma=0.878)$. In the bottom spectrum, it is assumed that both of the thinnest slabs of $\mathrm{CH}_{2}$ were not used $(\gamma=0.751)$ during Run 125. The actual thickness of the $\mathrm{CH}_{2}$ target may be expressed as $\gamma \cdot\langle\rho \tau\rangle_{\mathrm{CH}_{2}}$, where $\langle\rho \tau\rangle_{\mathrm{CH}_{2}}$ is the nominal $\mathrm{CH}_{2}$ areal thickness. 


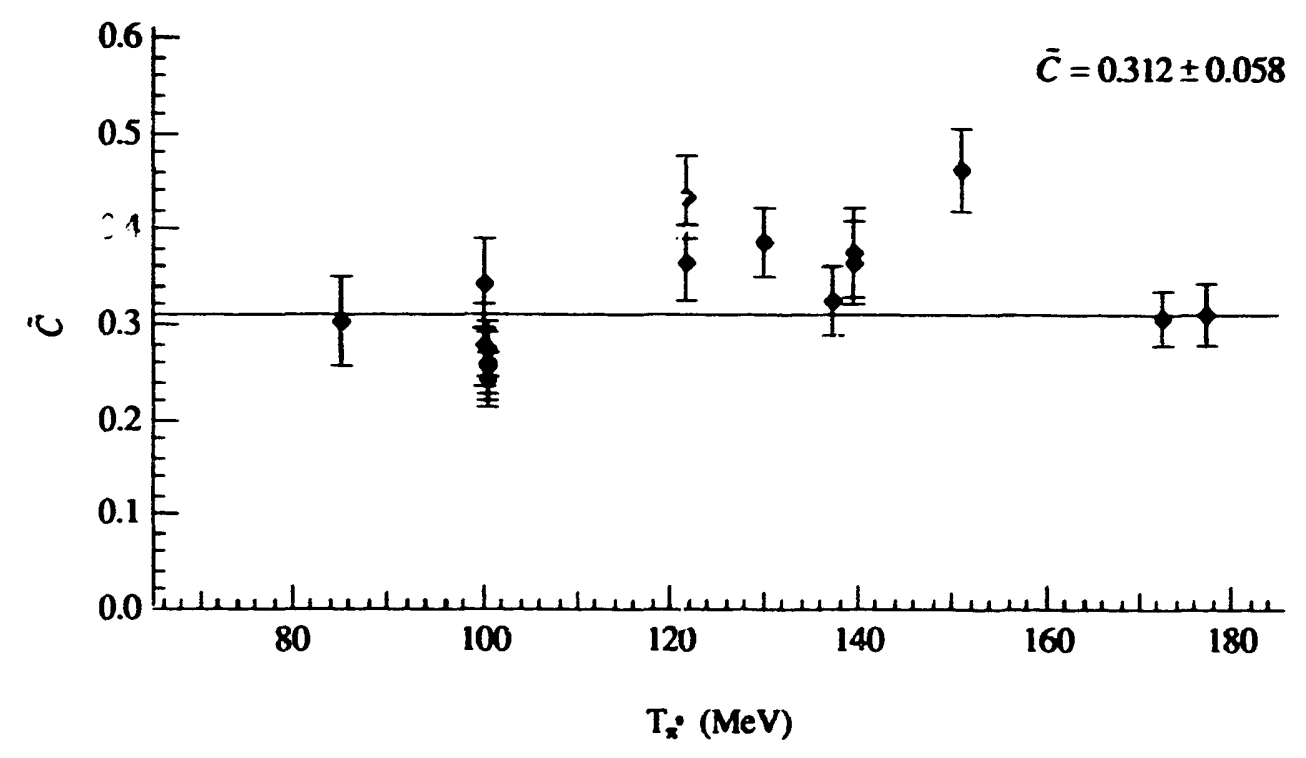

Figure 6.12: Uncorrected conversion efficiencies. The value of the weighted average of the conversion efficiency measurements without the $\mathrm{CH}_{2}$ target correction is $0.312 \pm 0.058$, where the uncertainty represents the standard deviation from the measured values.

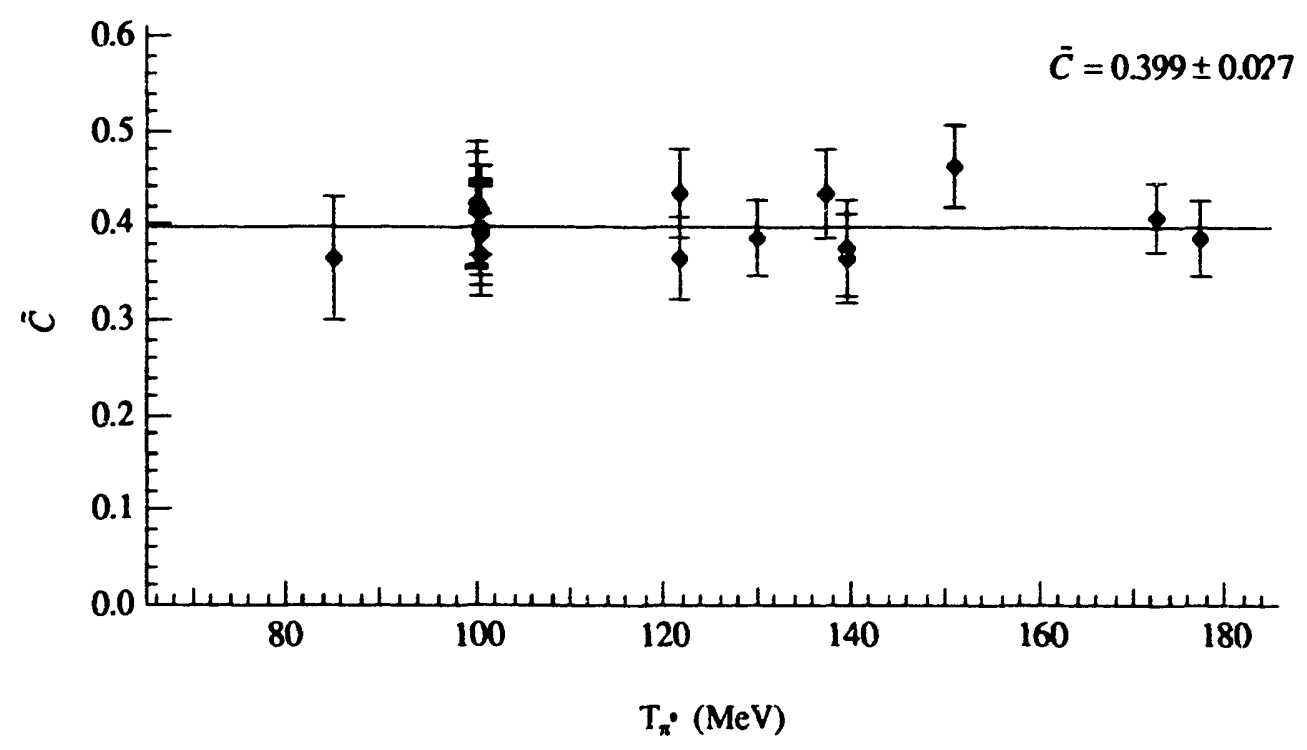

Figure 6.13: Corrected conversion efficiencies. The value of the weighted average of the conversion efficiency measurements with the $\mathrm{CH}_{2}$ target correction is $0.399 \pm 0.027$, where the uncertainty represents the standard deviation from the measured values. 


\section{Determination of ${ }^{3} \mathrm{He}\left(\pi^{ \pm}, \pi^{0}\right)$ and ${ }^{3} \mathrm{He}\left(\pi^{ \pm} \pi^{0} \mathrm{p}\right)$ Differential Cross Sections}

Once $\pi^{\circ}$ energy spectra had been reconstructed and the analysis of the conversion efficiency data had been completed, differential cross sections were computed. The differential cross sections for the single-arm and coincidence measurements may be expressed in terms of the following experimentally measured quantities,

$$
\begin{aligned}
& { }^{3} \mathrm{He}\left(\pi^{+}, \pi^{0}\right): \frac{d^{2} \sigma}{d E_{x^{0}} d \Omega_{x^{0}}}=\frac{\tilde{N}_{x^{0}}}{\langle\rho \tau\rangle} \cdot \frac{1}{\tilde{C}} \\
& { }^{3} \mathrm{He}\left(\pi^{t}, \pi^{0} \mathrm{p}\right): \frac{d^{3} \sigma}{d E_{\pi^{\circ}} d \Omega_{n^{\circ}} d \Omega_{p}}=\frac{\bar{N}_{\pi^{0} p}}{\langle\rho \tau\rangle} \cdot \frac{1}{\bar{C}} \cdot \frac{1}{\Delta \Omega_{p}},
\end{aligned}
$$

where $\bar{N}_{z^{\circ}}$ and $\tilde{N}_{x^{\circ} p}$ are the normalized $\pi^{\circ}$ yields for a particular $\pi^{0}$ kinetic energy from the single-arm and coincidence measurements respectively, and $\tilde{C}$ is the weighted average of the $\pi^{0}$ Spectrometer conversion efficiencies including the $\mathrm{CH}_{2}$ correction factor. The areal thickness, $\langle\rho \tau\rangle$, of the liquid ${ }^{3} \mathrm{He}$ target was 397 $\pm 12 \mathrm{mg} / \mathrm{cm}^{2}$. The solid angular acceptance of an individual proton detector, $\Delta \Omega_{p}$, was $8.46 \pm 0.25 \mathrm{msr}$.

Procedure for Determining $\pi^{\bullet}$ Energy Spectra

To obtain $\pi^{0}$ energy spectra from the raw $\pi^{0}$ spectra, one must first subtract the accidental events from the real events. The accidental events were the result of random coincidences between the two photon detector arms for a $\pi^{\circ}$ event and between the photon detector arms and the proton detector trigger for a $\pi^{\circ} \mathrm{p}$ event. These events were reconstructed by requiring that the relative timing between the two photon detector arms be outside the coincidence gate. (See Figure 6.3.) An example of a raw $\pi^{0}$ energy spectrum from a single-arm measurement with both real and accidental $\pi^{0}$ events is shown in Figure 6.14. In this analysis, it was assumed that the random $\pi^{0}$ events followed the same energy distribution as the real events. The fraction of random events was calculated using the following expression,

$$
f_{\text {rand }}=\frac{\Sigma_{\text {nedd }}}{\Sigma_{\text {nad }}}
$$

where $\Sigma_{\text {mal }}\left(\Sigma_{\text {rand }}\right)$ represents the total yield of real (random) events from integration of the $\pi^{0}$ energy spectra. The same fraction of random events was subtracted from each $\pi^{0}$ energy bin,

$$
\hat{y}_{i}=y_{i} \cdot\left(1-f_{\text {mand }}\right) \text {, }
$$

where $y_{i}$ is the number of counts in bin $i$. The yield in bin $i$ was then corrected for live time, $\ell$, the number 
of incident pions, $N_{x}$ the wire chamber efficiency, $\varepsilon_{m}$, and the solid angular acceptance of the Spa:trometer, $\Delta \mathbf{\Omega}_{\mathrm{wc}}$

$$
\bar{y}_{i}=\frac{\hat{y}_{i}}{N_{x} \cdot \ell \cdot \varepsilon_{m} \cdot \Delta \mathbf{\Omega}_{u c}} .
$$

The sclid angutiar acceptance of the spectrometer is computed for the $x^{0}$ energy corresponding to bin $i$.

At this point, data from separate runs under the same experimental conditions were added together, treating each data set as an independent measurement. These steps, outlined in Equations 6.18 through 6.20, were followed for each of the ${ }^{3} \mathrm{He}$, air, and dummy target measurements. An example of a $\pi^{0}$ energy spectrum is shown in Figure 6.15.

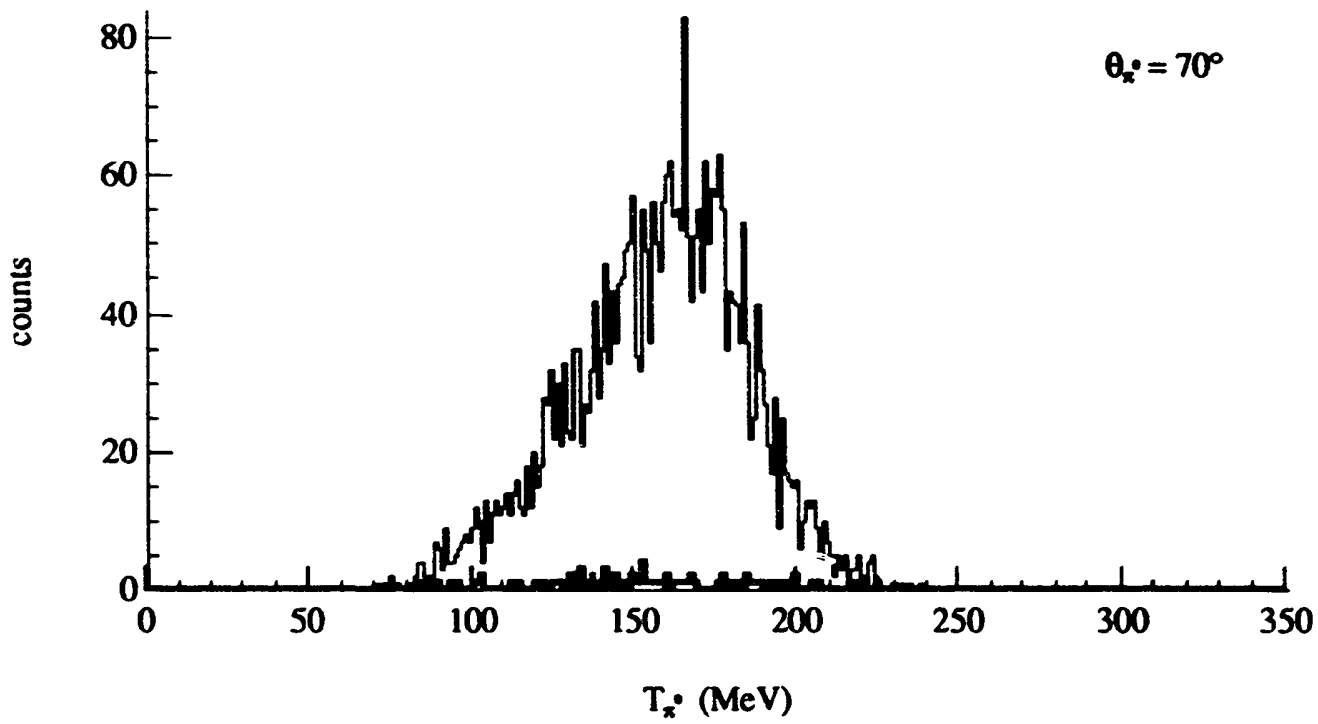

Figure 6.14: Raw $\pi^{0}$ erergy spectra for real (thin line) and accidental (thick line) $\pi^{0}$ events derived from a ${ }^{3} \mathrm{He}\left(\pi, \pi^{9}\right)$ measurement. In this figure, the $\pi^{0}$ energy specitra have not been corrected for any of the variables in Equation 6.20 such as live time, solid angular acceptance of the spectrometer, or the number of incident pions. 


\section{Backgroand Subtraction}

Once $\pi^{\circ}$ energy spectra for both the ${ }^{3} \mathrm{He}$ foreground and background measurements had been computed by following the procedures outlined in the previous section, the background from the target cell walls was subtracted on a bin by bin basis as follows,

$$
\bar{N}_{i}=\bar{y}_{i}-\bar{b}_{i}
$$

where the background for bin $i, \bar{b}_{i}$, is the normalized yield from the dummy target, $\bar{d}_{i}$, less the yield from air, $\tilde{a}_{i}$, within the dummy target,

$$
\tilde{b}_{i}=\frac{1}{4}\left(\tilde{d}_{i}-\tilde{a}_{i}\right) \text {. }
$$

The factor of four accounts for the difference between the thicknesses of the stainless steel walls of the dummy target and ${ }^{3} \mathrm{He}$ target cell. An example of the typical background contribution to a sirgle-arm measurement is shown in Figure 6.15.

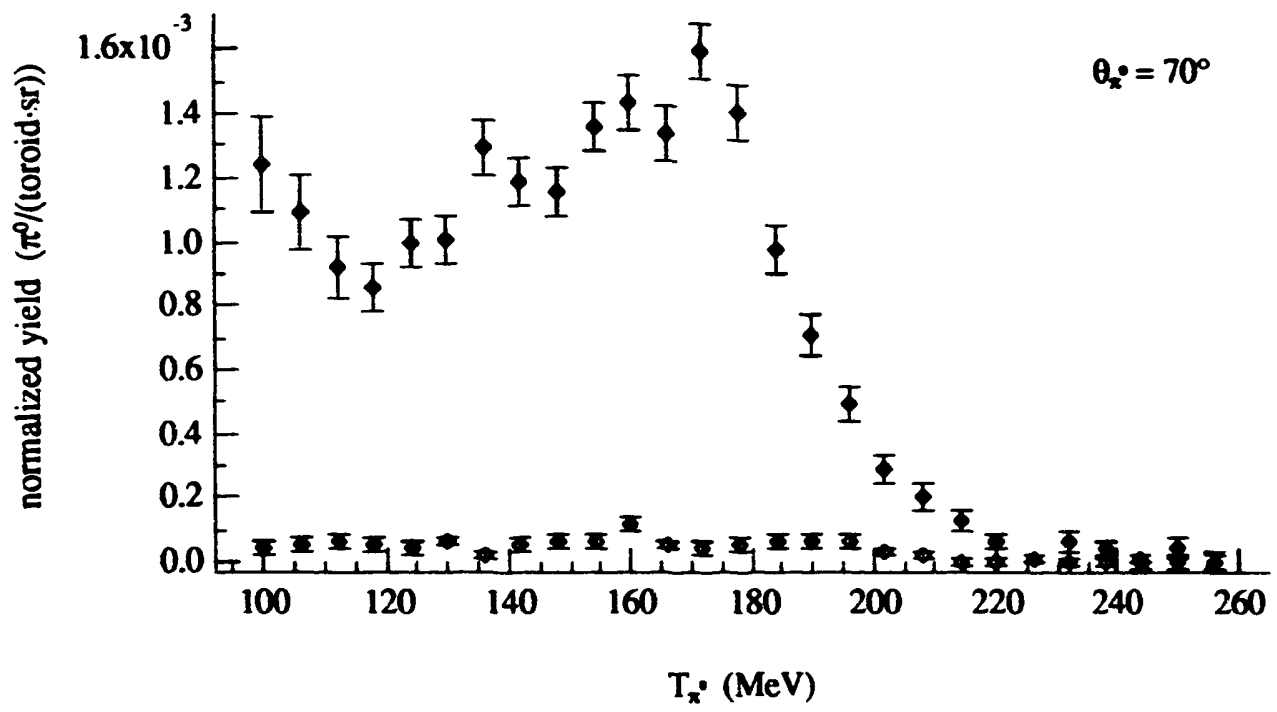

Figure 6.15: Normalized $\pi^{0}$ energy spectrum from ${ }^{3} \mathrm{He}\left(\pi^{-}, \pi^{9}\right)$ measurements (solid points). In this example, the background contribution (open points) has been obtained from the dummy target and air measurements using Equation 6.22. The uncertainties in the ${ }^{3} \mathrm{He}$ and background spectra reflect statistical fluctuations in the raw ev nt data and those associated with the subtraction of the randomi coincidences between the two photon detector arms. 


\section{Statistica! and Systematic Uncertainties}

In the inclusive single charge exchange measurements, the statistical uncenainties derive from the fluctuations of real and accidental $\pi^{0}$ events. These uncertainties were added together in quadrature to determine the overall statistical uncertainty. However, due to the limited data in the random $\pi^{0}$ energy spectra, the accuracy of these events was not believed to be known to better than $15 \%$. Nevertheless, the uncertainty in the accidental events is a negligible contribution to the overall systematic uncertainty.

The three dominant sources of systematic errors in these measurements were:

1) the areal thickness of the ${ }^{3} \mathrm{He}$ target,

2) the incident pion flux calibration constants from the ${ }^{12} \mathrm{C}$ activation measurements,

3) the conversion efficiency of the $\pi^{0}$ Spectrometer.

The uncertainty in the areal thickness of the ${ }^{3} \mathrm{He}$ target was not more than $3 \%$, as discussed previously. The activation constant $x_{\infty \alpha}$, which was determined by computing a weighted average of several activation measurements at each incident beam energy, was known to an accuracy of better than $5 \%$. The conversion efficiency was the dominant source of the normalization uncertainty, primarily due to the $\mathrm{CH}_{2}$ target thickness correction factor and the uncertainty in the value of the differential cross section for the ${ }^{1} \mathrm{H}\left(\pi, \pi^{0}\right) \mathrm{n}$ reaction. Due to the intraduction of the thickness correction factor, it was assumed that the $\mathrm{CH}_{2}$ target thickness was not known to an accuracy of better than $5 \%$. It is believed that the $\pi \mathrm{N}$ phase shift code, used to calculate values of the differential cross section for the ${ }^{1} \mathrm{H}\left(\pi^{-}, \pi^{9}\right)$ n reaction for the conversion efficiency analysis, overestimated the accuracy of previously measured values ${ }^{66,05}$ of the cross section. In the present analysis, the differential cross sections from the phase shift calculations were assigned an uncertainty based on the reported values for the measured cross sections. This corresponded to an $8 \%$ normalization uncerainty for each conversion efficiency r.easurement except for those measurements at incident pion energies of 245 and $290 \mathrm{MeV}$ to which a value of $12 \%$ was assigned. The overall uncertainty assigned to the conversion efficiency constant, $\dot{C}$, was determined by computing the standard deviation of the weighted average from the measured values.

Other sources of systematic uncertainty included the wire chamber efficiency $\left(\varepsilon_{m c}\right)$ and the 
electronics live time $(\ell)$. These contributions, typically less than $1 \%$, were negligible in comparison to other sources. The statistical fluctuations in the Monte Carlo calculations of the solid angular acceptance $\left(\Delta \Omega_{m C}\right)$ were less than $1 \%$. A value of $20 \%$ for the absolute uncertainty in the Monte Carlo results has been previously reported. 


\section{Chapter 7: Presentation of Results from Inclusive Pion Single Charge Exchange Measurements on ${ }^{3} \mathrm{He}:{ }^{3} \mathrm{He}\left(\pi^{ \pm}, \pi^{0}\right)$ and ${ }^{3} \mathrm{He}\left(\pi^{+}, \pi^{0} \mathbf{p}\right)$}

The results of inclusive single charge exchange measurements on ${ }^{3} \mathrm{He}$ will be presented in this Chapter. The presentation and discussion of these results have been divided into two categories: 1) single-arm studies of quasi-free pion single charge exchange on ${ }^{3} \mathrm{He}:{ }^{3} \mathrm{He}\left(\pi^{+}, \pi^{9}\right)$, and 2) coincidence studies of proton knockout from pion single charge exchange on ${ }^{3} \mathrm{He}:{ }^{3} \mathrm{He}\left(\pi^{ \pm}, \pi^{0} \mathrm{p}\right)$. Doubly differential cross sections for the ${ }^{3} \mathrm{He}\left(\pi^{4}, \pi^{9}\right)$ and ${ }^{3} \mathrm{He}\left(\pi^{-}, \pi^{9}\right)$ reactions will be presented. An attempt will be made to extract angular distributions from the single-arm measurements, which are limited to the quasi-free scattering region, by performing an extrapolation of the doubly differential cross sections to the low outgoing $\pi^{0}$ kinetic energies which were not observed. Comparisons of ratios of the doubly differential cross sections for the ${ }^{3} \mathrm{He}\left(\pi^{-}, \pi^{0}\right)$ and ${ }^{3} \mathrm{He}\left(\pi^{+}, \pi^{9}\right)$ reactions to simple isospin scaling predictions for single scattering and double scattering processes will be shown. Comparisons of the single-arm measurements from this work with previous measurements of pion double charge exchange and inelastic scattering on ${ }^{3} \mathrm{He}$ will be examined for indications of multiple scattering. 
Following the discussion of the single-arm measurements, triply differential cross sections for the ${ }^{3} \mathrm{He}\left(\pi^{+}, \pi^{0} \mathrm{p}\right)$ and ${ }^{3} \mathrm{He}\left(\pi^{-}, \pi^{0} \mathrm{p}\right)$ reactions will be presented as a function of outgoing $\pi^{0}$ energy. The triply differential cross sections will be integrated over outgoing $\pi^{0}$ kinetic energy to extract proton angular distributions as a function of $\pi^{0}$ scattering angle. The doubly differential cross sections for the ${ }^{3} \mathrm{He}\left(\pi^{+}, \pi^{0} \mathrm{p}\right)$ reaction will be integrated over proton scattering angle to compute a singly differential cross section. Comparisons of triply, doubly, and singly differential cross sections with results from quasi-elastic proton knockout measurements from Klein et al., ${ }^{42} \mathrm{He}\left(\pi^{+}, \pi^{+} \mathrm{p}\right)$, will be shown.

\section{Quasi-free Pion Single Charge Exchange on ${ }^{3} \mathrm{He}:{ }^{3} \mathrm{He}\left(\pi^{ \pm}, \pi^{0}\right)$}

\section{Doubly Differential Cross Sections for the ${ }^{3} \mathrm{He}\left(\pi^{+}, \pi^{9}\right)$ reactions}

The experimental parameters for the single-arm measurements of quasi-free pion single charge exchange on ${ }^{3} \mathrm{He}$ are listed in Table 7.1. These measurements were performed with the LAMPF $\pi^{0}$ Spectrometer and a liquid ${ }^{3} \mathrm{He}$ target as described in Chapter 5 . The data analysis procedures for extracting doubly differential cross sections were discussed in Chapter 6. Doubly differential cross sections for the ${ }^{3} \mathrm{He}\left(\pi^{+}, \pi^{0}\right)$ reaction are shown in Figures 7.1 through 7.4. Doubly differential cross sections for the ${ }^{3} \mathrm{He}\left(\pi^{-}, \pi^{9}\right)$ reaction are shown in Figures 7.5 and 7.6. Comparisons of $\pi^{0}$ energy spectra from the ${ }^{3} \mathrm{He}\left(\pi^{+}, \pi^{9}\right)$ and ${ }^{3} \mathrm{He}\left(\pi^{-}, \pi^{0}\right)$ reactions are shown in Figures 7.7 and 7.8. Comparisons of $\pi^{0}$ energy spectra, at nearly the same $\pi^{0}$ scattering angle but different geometrical set-ups of the $\pi^{0}$ Spectrometer, were used to check the Monte Carlo simulation of the solid angular acceptance of the spectrometer. An example of this comparison is shown in Figure 7.9.

The arrows in Figures 7.1 through 7.4 correspond to the outgoing $\pi^{0}$ kinetic energy for the free single charge exchange reaction: $\pi^{+} n \rightarrow \pi^{0} p$. The arrows at lower outgoing $\pi^{0}$ kinetic energies in Figures 7.5 and 7.6 correspond to the $\pi^{0}$ kinetic energy for the free single charge exchange reaction: $\pi^{-} p \rightarrow \pi^{0} n$. The second set of arrows at higher $\pi^{0}$ kinetic energies in Figures 7.5 and 7.6 point to the $\pi^{0}$ kinetic energy for the pion-induced isobaric analog transition: ${ }^{3} \mathrm{He}\left(\pi^{-}, \pi^{9}\right)^{3} \mathrm{H}$. The uncertainties shown in these plots represent the statistical fluctuations in the data. The acceptance of the $\pi^{0}$ Spectrometer was divided into 
three angular bins with $a$ bin width of $8^{\circ}$ corresponding to $\pi^{\circ}$ scattering angles of $\theta_{c}$ and $\theta_{c} \pm 8^{\circ}$, where $\theta_{c}$ is the central angle of the $\pi^{0}$ Spectrometer with respect to the incident beam.

\begin{tabular}{|c|c|c|c|}
\hline \multicolumn{4}{|c|}{ Experimental Parameters for ${ }^{3} \mathrm{He}\left(\pi^{t}, \pi^{\circ}\right)$} \\
\hline \multicolumn{3}{|c|}{ Measurements } \\
\hline Reaction & $\begin{array}{c}\theta_{c} \\
\text { (deg.) }\end{array}$ & \multicolumn{1}{|c|}{$\begin{array}{c}\theta_{\pi} \\
\text { (deg.) }\end{array}$} & $\begin{array}{c}\mathrm{T}^{\circ} \\
(\mathrm{MeV})\end{array}$ \\
\hline${ }^{3} \mathrm{He}\left(\pi^{\circ}, \pi^{\circ}\right)$ & $70^{\circ}$ & $62^{\circ}, 70^{\circ}, 78^{\circ}$ & $100-256$ \\
\hline${ }^{3} \mathrm{He}\left(\pi^{\circ}, \pi^{\circ}\right)$ & $90^{\circ}$ & $82^{\circ}, 90^{\circ}, 98^{\circ}$ & $80-218$ \\
\hline${ }^{3} \mathrm{He}\left(\pi^{\circ}, \pi^{\circ}\right)$ & $110^{\circ}$ & $102^{\circ}, 110^{\circ}, 118^{\circ}$ & $66-180$ \\
\hline${ }^{3} \mathrm{He}\left(\pi^{\circ}, \pi^{\circ}\right)$ & $120^{\circ}$ & $112^{\circ}, 120^{\circ}, 128^{\circ}$ & $68-176$ \\
\hline${ }^{3} \mathrm{He}\left(\pi, \pi^{\circ}\right)$ & $70^{\circ}$ & $62^{\circ}, 70^{\circ}, 78^{\circ}$ & $100-256$ \\
\hline${ }^{3} \mathrm{He}\left(\pi, \pi^{\circ}\right)$ & $110^{\circ}$ & $102^{\circ}, 110^{\circ}, 118^{\circ}$ & $66-180$ \\
\hline
\end{tabular}

Table 7.1: Experimental parameters for single-arm inclusive single charge exchange measurements. The central angle of the $\pi^{0}$ Spectrometer is designated as $\theta_{c}$ Cross sections for the ${ }^{3} \mathrm{He}\left(\pi^{t}, \pi^{0}\right)$ reactions were extracted by dividing the acceptance of the spectrometer into three angular bins with an $8^{\circ}$ bin width corresponding to $\theta_{n}$. . The observable range of outgoing $\pi^{0}$ kinetic energies is listed in the column labeled "To". 

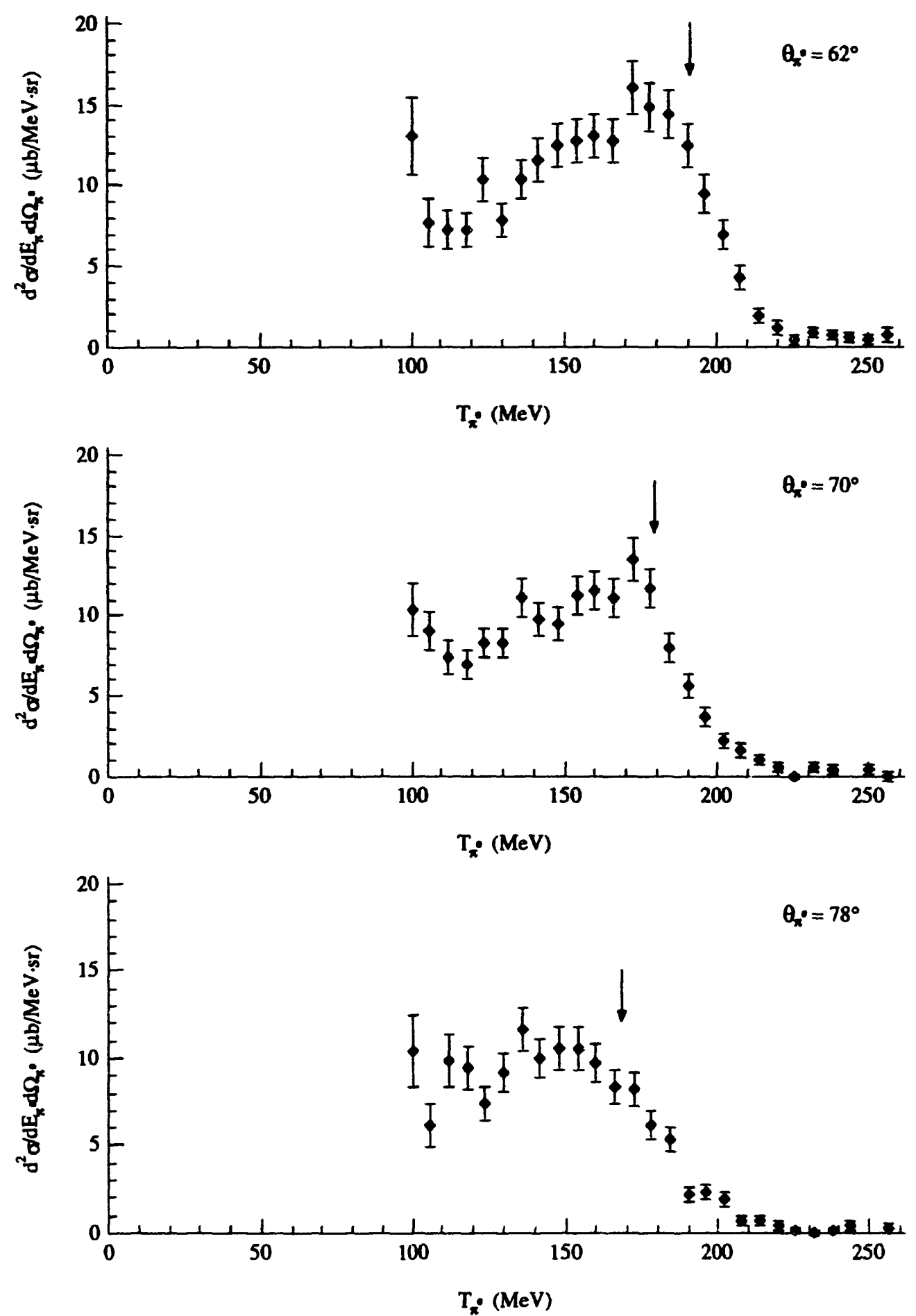

Figure 7.1: Doubly differential cross sections for the ${ }^{3} \mathrm{He}\left(\pi^{+}, \pi^{0}\right)$ reaction at $T_{\pi}=245 \mathrm{MeV}$. An overall systematic uncertainty of $8 \%$ is not included in this figure. These spectra are derived from data accumulated at a central $\pi^{\circ}$ Spectrometer scattering angle of $70^{\circ}$. The spectrometer acceptance was partitioned into three sections each corresponding to an $8^{\circ}$ angular bin. The arrow points to the corresponding $\pi^{\circ}$ kinetic energy for the free single charge exchange reaction: $n\left(\pi^{+}, \pi^{0}\right)$ p. 

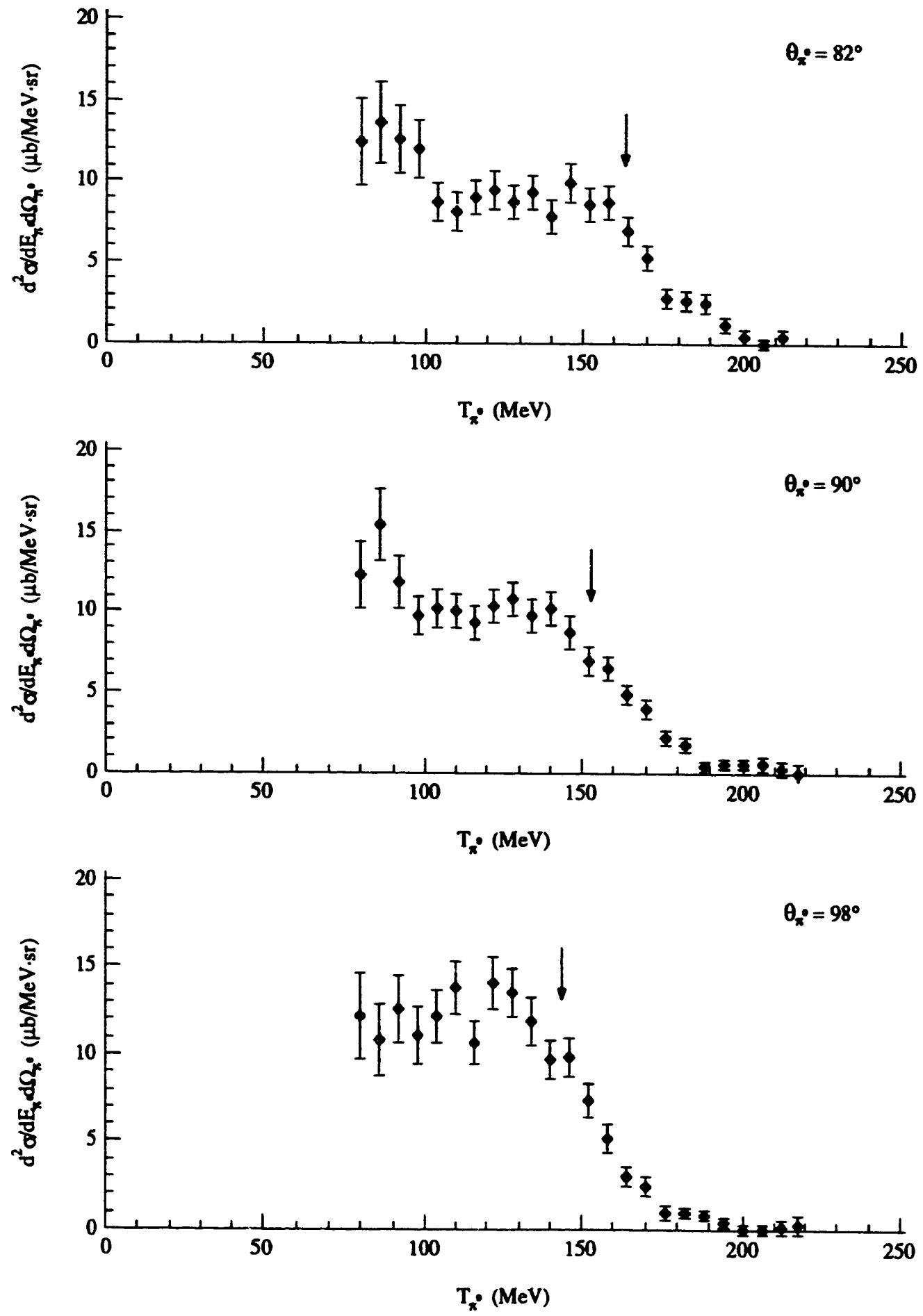

Figure 7.2: Doubly differential cross sections for the ${ }^{3} \mathrm{He}\left(\pi^{+}, \pi^{9}\right)$ reaction at $T_{x}=245 \mathrm{MeV}$. An overall systematic uncertainty of $8 \%$ is not included in this figure. These spectra are derived from data accumulated at a central $\pi^{\circ}$ Spectrometer scattering angle of $90^{\circ}$. The spectrometer acceptance was partitioned into three sections each corresponding to an $8^{\circ}$ angular bin. The arrow points to the corresponding $\pi^{\circ}$ kinetic energy for the free single charge exchange reaction: $n\left(\pi^{+}, \pi^{9}\right)$ p. 

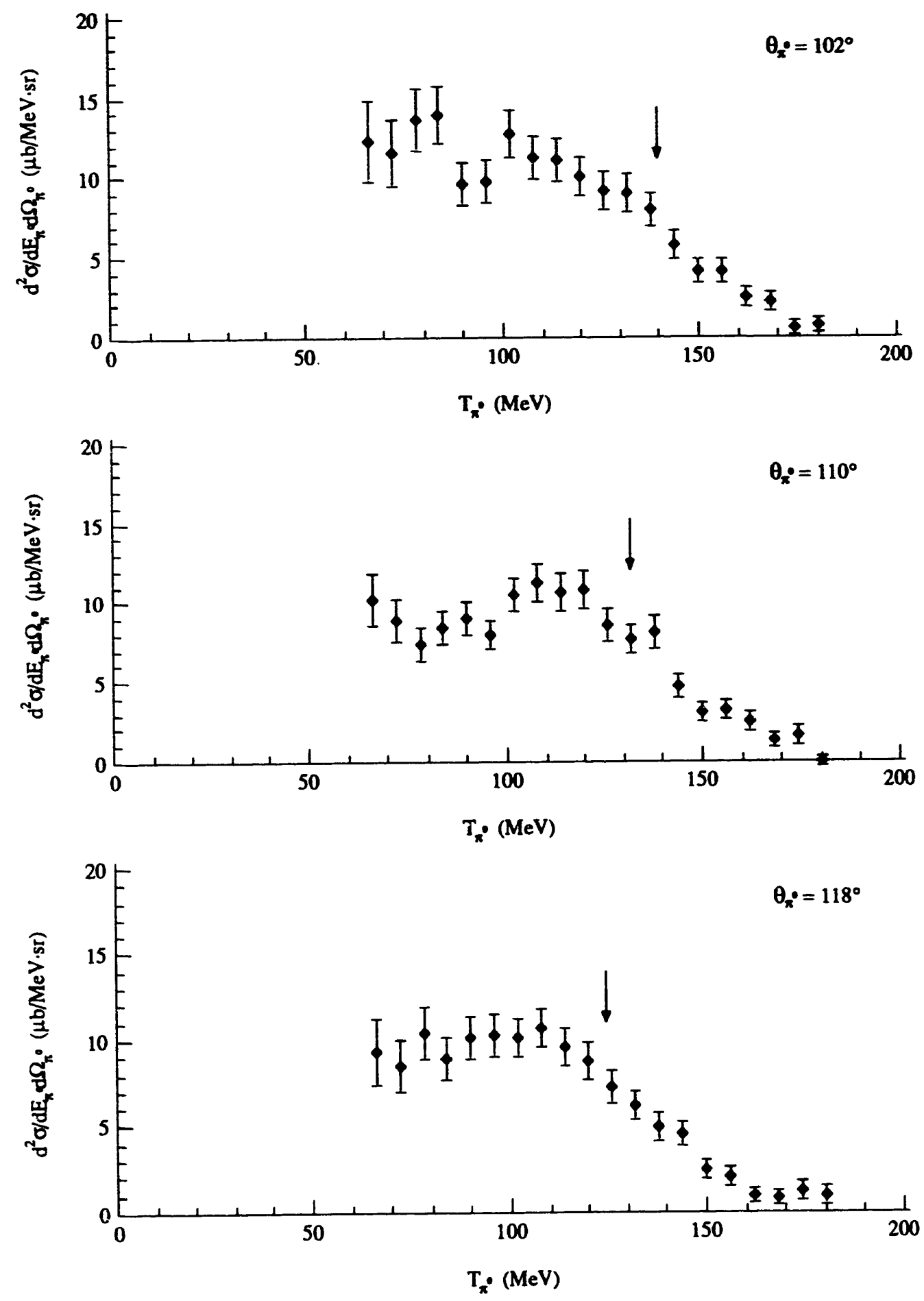

Figure 7.3: Doubly differential cross sections for the ${ }^{3} \mathrm{He}\left(\pi^{+}, \pi^{0}\right)$ reaction at $\mathrm{T}_{\pi}=245 \mathrm{MeV}$. An overall systematic uncertainty of $8 \%$ is not included in this figure. These spectra are derived from data accumulated at a central $\pi^{\circ}$ Spectrometer scattering angle of $110^{\circ}$. The spectrometer acceptance was partitioned into three sections each corresponding to an $8^{\circ}$ angular bin. The arrow points to the corresponding $\pi^{\circ}$ kinetic energy for the free single charge exchange reacti.$n\left(\pi^{+}, \pi^{0}\right) p$. 

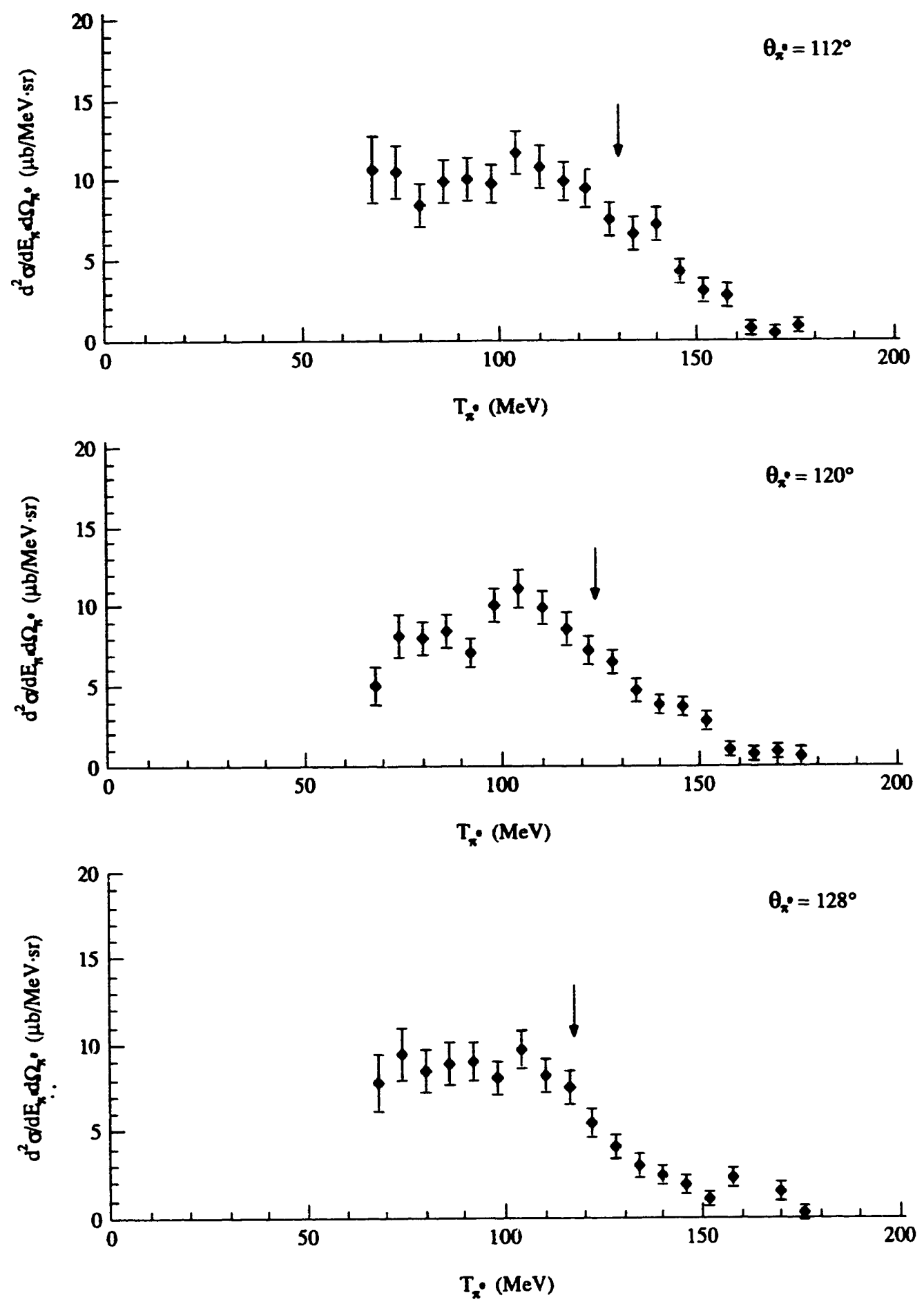

Figure 7.4: Doubly differential cross sections for the ${ }^{3} \mathrm{He}\left(\pi^{+}, \pi^{9}\right)$ reaction at $\mathrm{T}_{x}=245 \mathrm{MeV}$. An overall systematic uncertainty of $8 \%$ is not included. These spectra are derived from data accumulated at a central $\pi^{0}$ Spectrometer scattering angle of $120^{\circ}$. The spectrometer acceptance was partitioned into three sections each corresponding to an $8^{\circ}$ angular bin. The arrow points to the corresponding $\pi^{0}$ kinetic energy for the free single charge exchange reaction: $n\left(\pi^{+}, \pi^{0}\right) p$. 

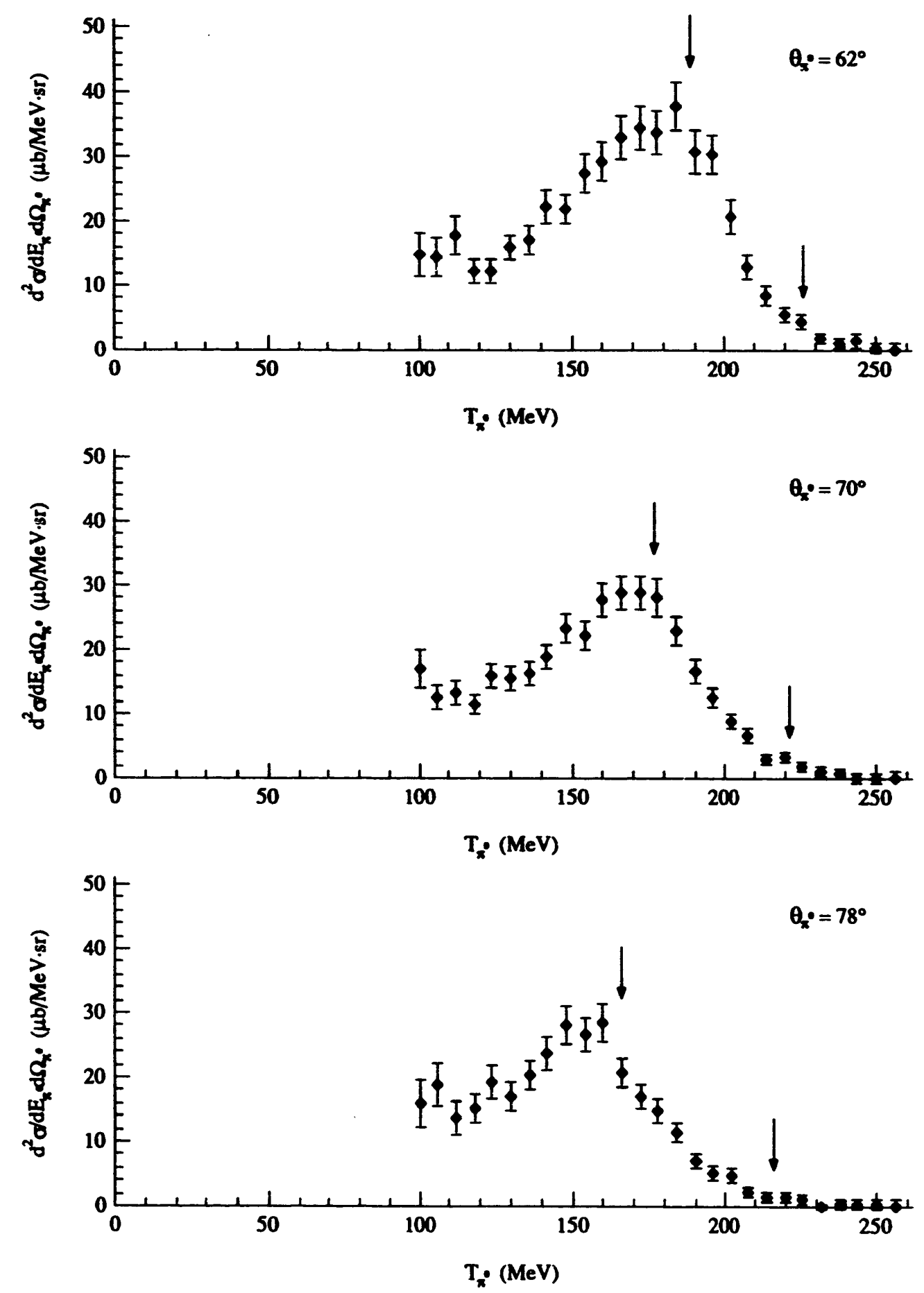

Figure 7.5: Doubly differential cross sections for the ${ }^{3} \mathrm{He}\left(\pi^{-}, \pi^{9}\right)$ reaction at $\mathrm{T}_{\pi}=245 \mathrm{MeV}$. An overall systematic uncertainty of $8 \%$ is not included. These spectra are derived from data accumulated at a central scattering angle of $70^{\circ}$. The spectrometer acceptance was partitioned into three sections each corresponding to an $8^{\circ}$ angular bin. The right and left arrows point to the $\pi^{\circ}$ kinetic energies for the elastic charge exchange $\left({ }^{3} \mathrm{He}\left(\pi^{-}, \pi^{9}\right)^{3} \mathrm{H}\right)$ and free single charge exchange $\left(p\left(\pi^{-}, \pi^{9}\right) \mathrm{n}\right)$ reactions respectively. 

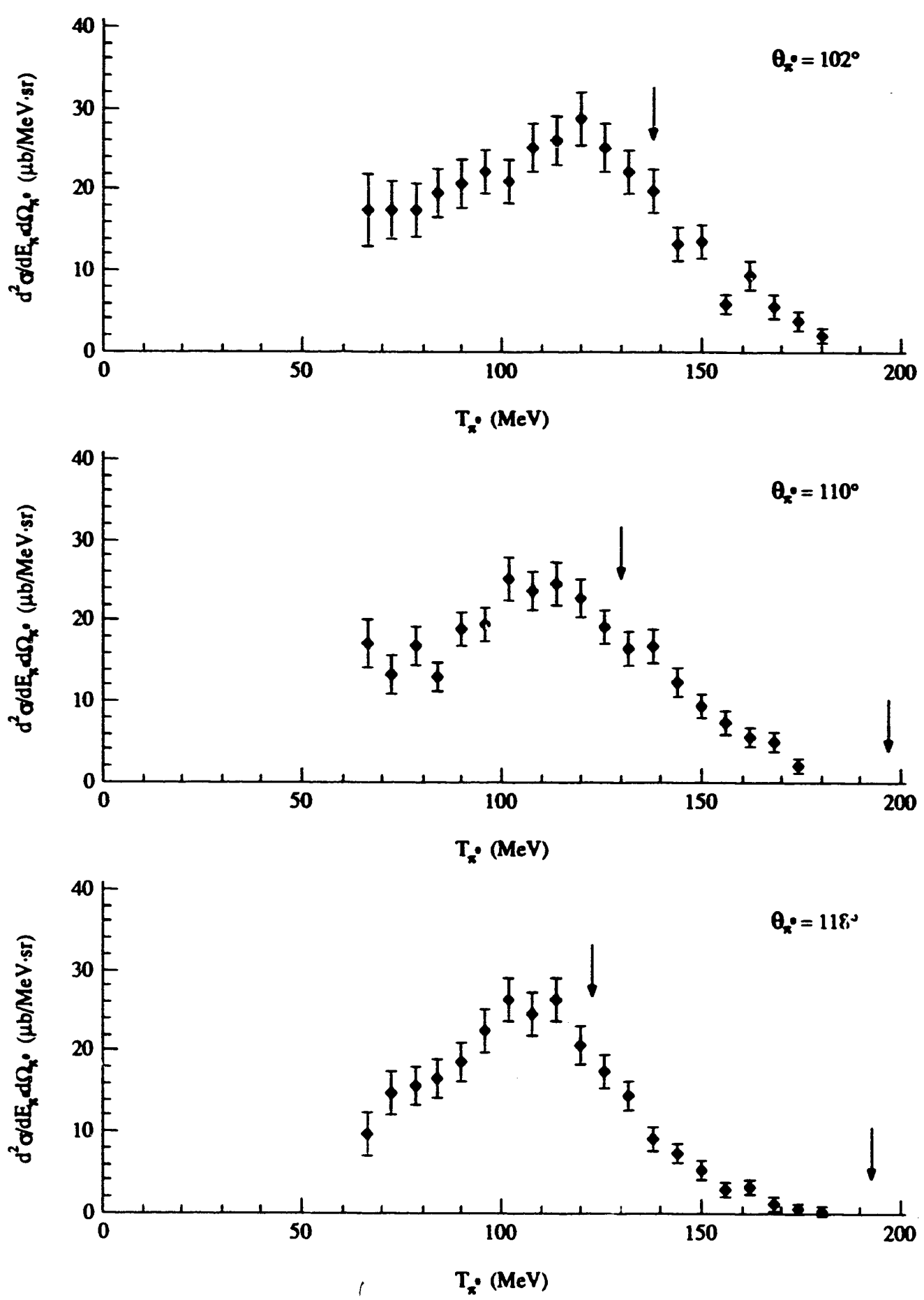

Figuie 7.6: Doubly differential cross sections for the ${ }^{3} \mathrm{He}\left(\pi^{-}, \pi^{9}\right)$ reaction at $\mathrm{T}_{\mathrm{z}}=245 \mathrm{MeV}$. An overall systematic uncertainty of $8 \%$ is not included. These spectra are derived from data accumulated at a central scattering angle of $110^{\circ}$. The spectrometer acceptance was partitioned into three sections each corresponding to an $8^{\circ}$ angular bin. The right and left arrows point to the $\pi^{\circ}$ kinetic energies for the elastic charge exchange $\left({ }^{3} \mathrm{He}\left(\pi^{-}, \pi^{0}\right)^{3} \mathrm{H}\right)$ and free single charge exchange $\left(\mathrm{p}\left(\pi^{-}, \pi^{0}\right) \mathrm{n}\right)$ reactions respectively. 


$$
\sqrt{1}
$$



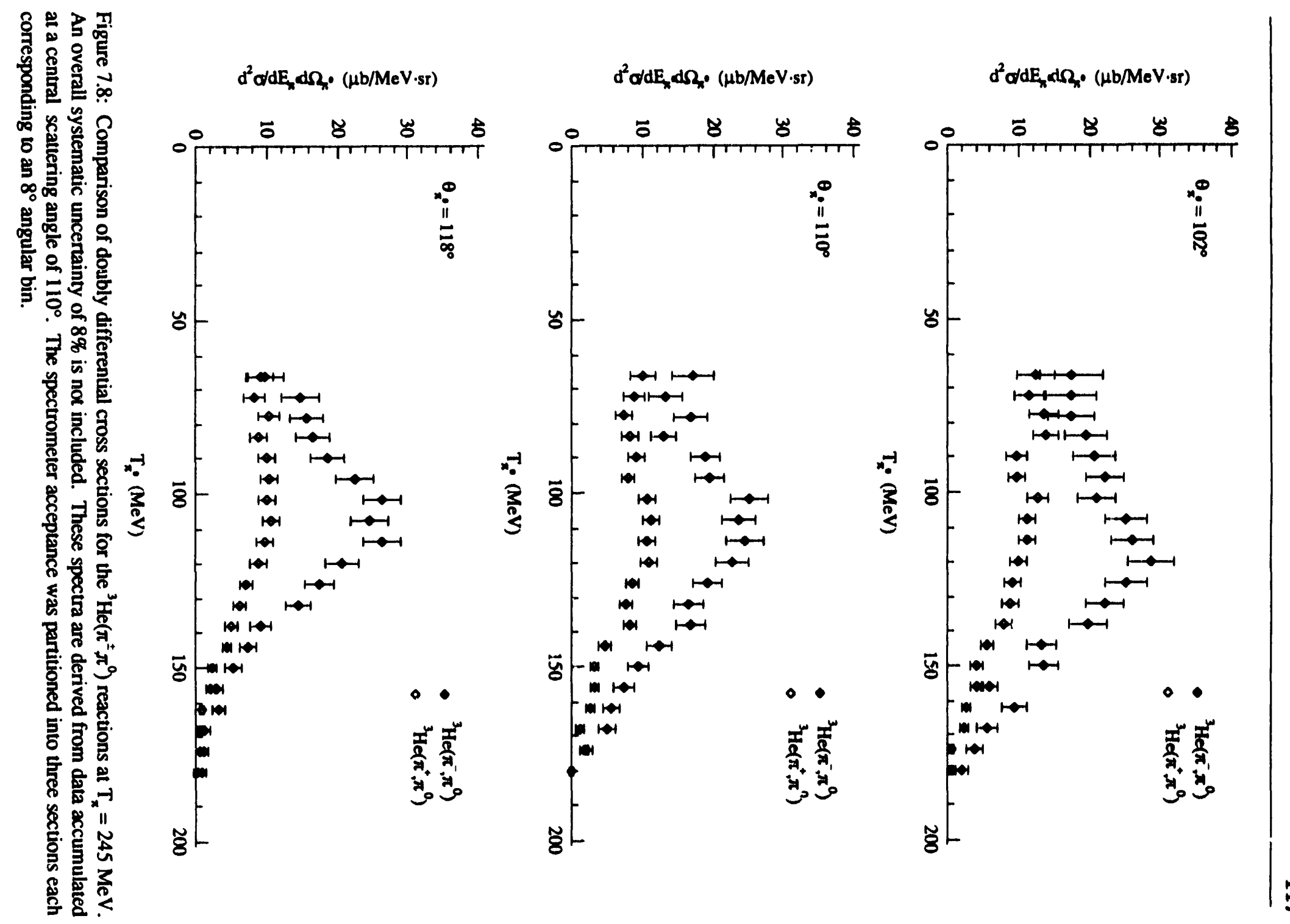


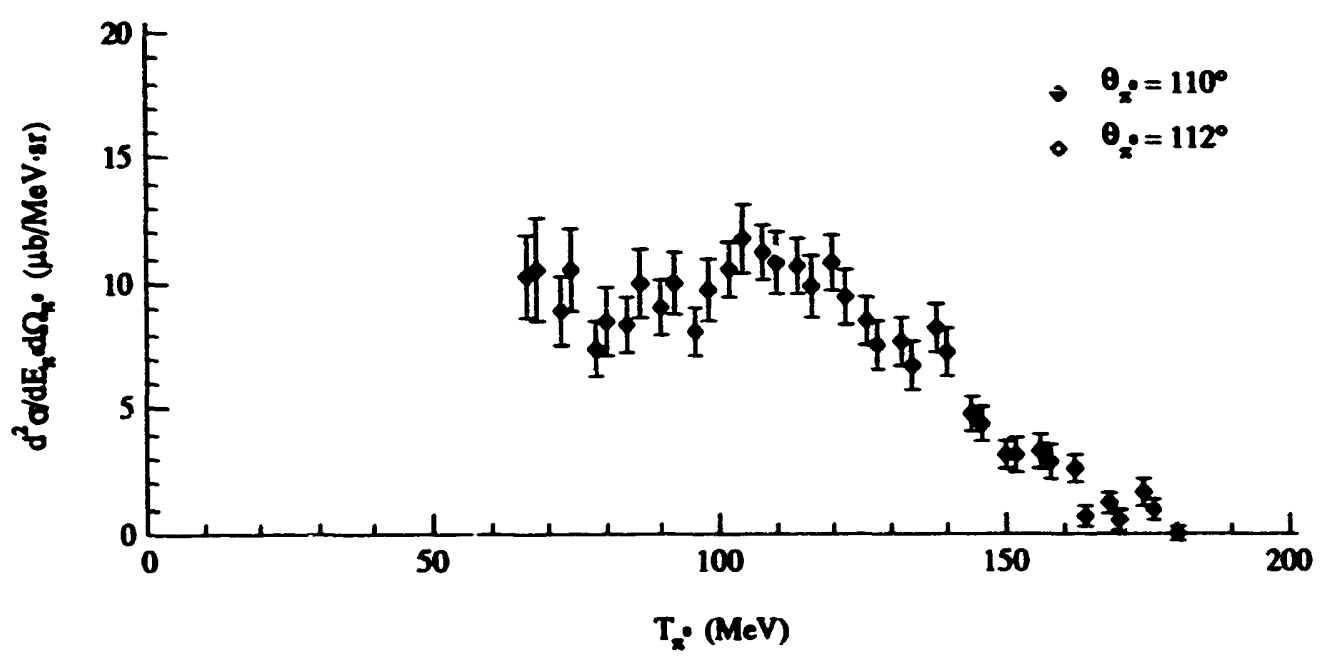

Figure 7.9: Comparison of measurements of $\pi^{0}$ energy spectra from the ${ }^{3} \mathrm{He}\left(\pi^{+}, \pi^{0}\right)$ reaction at $\pi^{0}$ laboratory scautering angles of $110^{\circ}$ and $112^{\circ}$. Different geometrical set-ups for the $x^{\circ}$ Spectrometer were used to accumulate the data for these measurements. Differences between experimental set-ups included the relative orientation of the target and spectrometer and the opening angles between the two photon detectors. The central scattering angles of the $\pi^{\circ}$ Spectrometer for the measurements at $110^{\circ}$ and $112^{\circ}$ were $110^{\circ}$ and $120^{\circ}$ respectively. An overall systematic uncertainty of $8 \%$ is not included in this figure.

\section{Angular Distributions for the ${ }^{3} H e\left(\pi^{t}, \pi^{0}\right)$ Reactions}

Two methods were developed to extract $\pi^{0}$ angular distributions from doubly differential cross sections for the ${ }^{3} \mathrm{He}\left(\pi^{*}, \pi^{0}\right)$ reactions. In the first method, $\pi^{0}$ energy spectra were integrated over the measured energy region and no attempt was made to extrapolate the measurements to $\pi^{0}$ kinetic energies below the lowest measured energy point. In the second method, an estimate of the unmeasured contribution to the differential cross section was made by extrapoiating the doubly differential cross section at the lowest measured energy point to zero for a $\pi^{\circ}$ kinetic energy of zero. An uncertainty of value equal to the correction was assigned to this extrapolation correction. Examples of these integration methods are shown in Figure 7.1C. The uncorrected and corrected $\pi^{0}$ angular distribution for the ${ }^{3} \mathrm{He}\left(\pi^{*}, \pi^{9}\right)$ reactions are shown in Figure 7.11 and presented in Tables 7.2 and 7.3. 


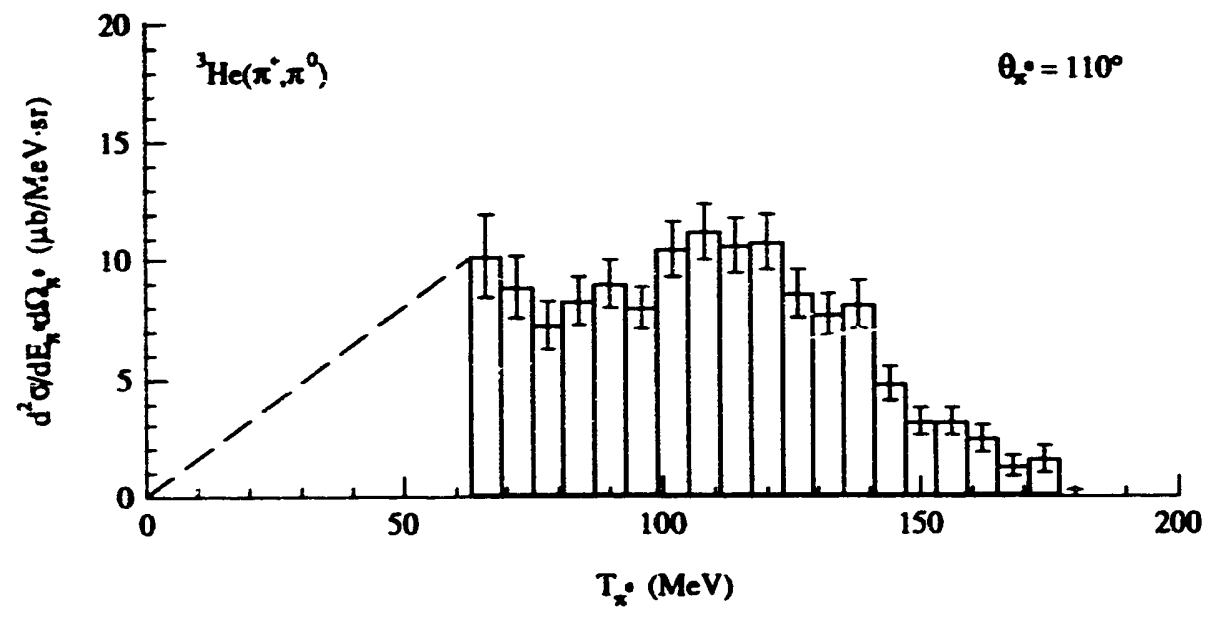

Figure 7.10: Doubly differential cross secticn for ${ }^{3} \mathrm{He}\left(\pi^{*}, \pi^{\circ}\right)$ at $245 \mathrm{MeV}$ and $\theta_{\mathrm{z}} \mathrm{O}=110^{\circ}$. Differential cross sections were extracted from the single-arm charge exchange measurements in one of two ways. In the first method, the $\pi^{\circ}$ energy spectra were integrated over the measured region corresponding to the histogrammed region showi in this figure. Differential cross sections for ${ }^{3} \mathrm{He}\left(\pi^{4}, \pi^{0}\right)$, determined by this method, are shown in Figures 7.11a and 7.11b. In the second method, a crude estimate of the missing strength at low outgoing $\pi^{\circ}$ energies was performed by extrapolating the doubly differential cross section at the lowest outgoing $\pi^{\circ}$ energy to zero. This corresponds to the dashed line shown in the figure. An uncertainty equal to the area under this line was assigned to this correction. Differential cross $\operatorname{sections}$ for ${ }^{3} \mathrm{He}\left(\pi^{t}, \pi^{0}\right)$, as determined using this method, are shown in Figures 7.1 lc and 7.11d.

\begin{tabular}{|c|c|c|c|c|c|}
\hline \multirow{2}{*}{$\begin{array}{c}\theta_{\mathbf{R}^{*}} \\
\text { (deg.) }\end{array}$} & \multirow{2}{*}{$\begin{array}{c}d \sigma / d \Omega_{\alpha} \\
(\mathrm{mb} / \mathrm{sr})\end{array}$} & \multirow{2}{*}{$\underset{(\mathrm{mb} / \mathrm{sr})}{d \sigma / d \boldsymbol{\Omega}_{\mathrm{cor}}}$} & \multicolumn{3}{|c|}{ Uncertainty (mb/sr) } \\
\hline & & & $\overline{U^{\prime b}}$ & Extrapolation & Total \\
\hline 62 & 2.54 & $3.1 ?$ & 0.21 & 0.63 & 0.67 \\
\hline 70 & 1.97 & 2.65 & 0.17 & 0.68 & 0.70 \\
\hline 78 & 1.73 & 2.46 & 0.15 & 0.73 & 0.75 \\
\hline 102 & 2.02 & 2.56 & 0.18 & 0.53 & 0.56 \\
\hline 110 & 1.74 & 2.21 & 0.15 & 0.47 & 0.49 \\
\hline 118 & 1.55 & 1.93 & 0.14 & 0.38 & 0.40 \\
\hline
\end{tabular}

'Includes crude extrapolation of unmeasured $\pi^{\circ}$ energy spectra.

Uncertainty in uncortected differential cross section. Includes statistical and nomalization uncertainties.

Table 7.2: Differential cross sections for the ${ }^{3} \mathrm{He}\left(\pi^{i}, \pi^{9}\right)$ reaction at $245 \mathrm{MeV}$. The cxtrapolation uncertainty is the uncertainty $2.5 s$ sociated with the estimate of the unmeasured portion of the doubly differential cross section. 


\begin{tabular}{|l|l|l|ll|l|}
\hline \multirow{2}{*}{$\begin{array}{c}\theta_{R^{*}} \\
(\mathrm{deg} .)\end{array}$} & $d \sigma / d \Omega_{z^{*}}$ & $d \sigma / d \Omega_{\text {cor }}$ & \multicolumn{4}{|c|}{ Uncertainty (mb/sr) } \\
\cline { 4 - 6 } & $(\mathrm{mb} / \mathrm{sr})$ & $(\mathrm{mb} / \mathrm{sr})$ & $U^{b}$ & Extrapolation & Total \\
\hline 62 & 1.062 & 1.452 & 0.090 & 0.390 & 0.400 \\
\hline 70 & 0.821 & 1.211 & 0.070 & 0.390 & 0.396 \\
\hline 78 & 0.710 & 1.145 & 0.062 & 0.435 & 0.439 \\
\hline 82 & 0.968 & 1.428 & 0.085 & 0.460 & 0.468 \\
\hline 90 & 0.947 & 1.427 & 0.082 & 0.480 & 0.487 \\
\hline 98 & 0.984 & 1.444 & 0.086 & 0.460 & 0.468 \\
\hline 102 & 0.976 & 1.356 & 0.086 & 0.380 & 0.390 \\
\hline 110 & 0.817 & 1.132 & 0.070 & 0.315 & 0.323 \\
\hline 112 & 0.810 & 1.125 & 0.071 & 0.315 & 0.323 \\
\hline 118 & 0.769 & 1.049 & 0.067 & 0.280 & 0.288 \\
\hline 120 & 0.652 & 0.887 & 0.057 & 0.235 & 0.242 \\
\hline 128 & 0.593 & 0.863 & 0.053 & 0.270 & 0.275 \\
\hline
\end{tabular}

Includes crude extrapolation of unmeasured $\pi^{0}$ energy spectra.

'Uncertainty of uncorrected differential cross section. Includes statistical and normalization uncertainties.

Table 7.3: Differential cross sections for the ${ }^{3} \mathrm{He}\left(\pi^{*}, \pi^{9}\right)$ reaction at $245 \mathrm{MeV}$. The extrapolation tainty is the uncertainty associated with the estimate of the unmeasured portion of the doubly differeni.al cross section.

A deviation from the angular distribution for free pion single charge exchange at large $\pi^{0}$ scattering angles is seen in both single-arm measurements. This deviation may be attributed to the missing surength from the unmeasured region of the doubly differential cross section at low $\pi^{0}$ kinetic energies. The deviation at forward angles may be due in part to Pauli blocking. A correction for the unmeasured doubly differential cross section improves the agreement between the angular distribution for single charge exchange on ${ }^{3} \mathrm{He}$ and the free charge exchange reaction somewhat. The slight deviation from the free charge exchange behavior at large angles may be an indication that multiple scatlering processes play an important ro!e in inclusive pion single charge exchange on ${ }^{3} \mathrm{He}$ since an excess of missing strength in the doubly differential cross section at low outgoing pion kinetic energies could account for this deviation. 
Other indications of multiple scattering in the ${ }^{3} \mathrm{He}\left(\pi^{+}, \pi^{3}\right)$ reactions wiil be discussed in a later section of this

Chapter.

a)

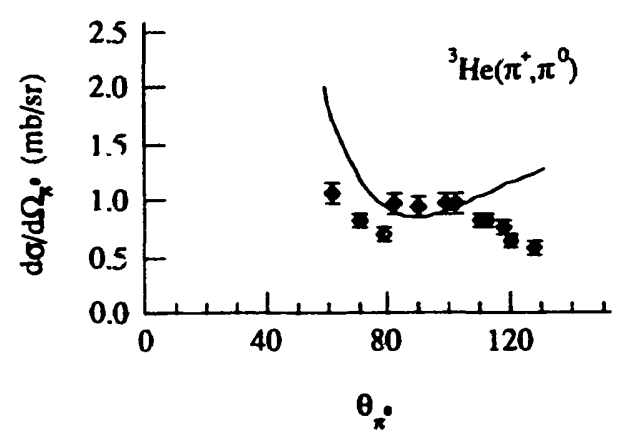

c)

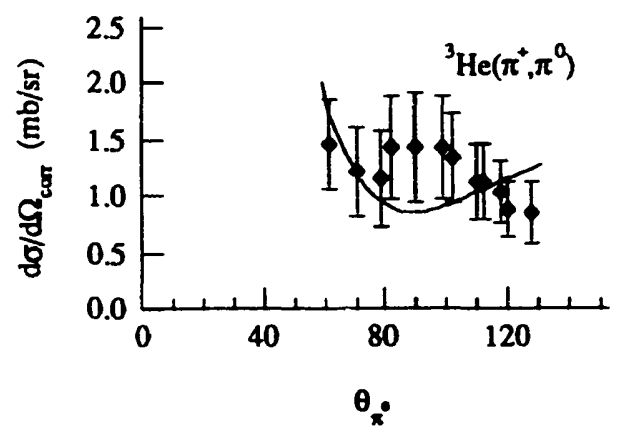

b)

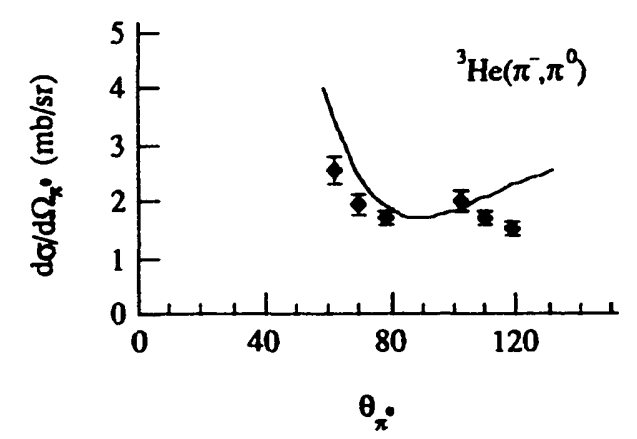

d)

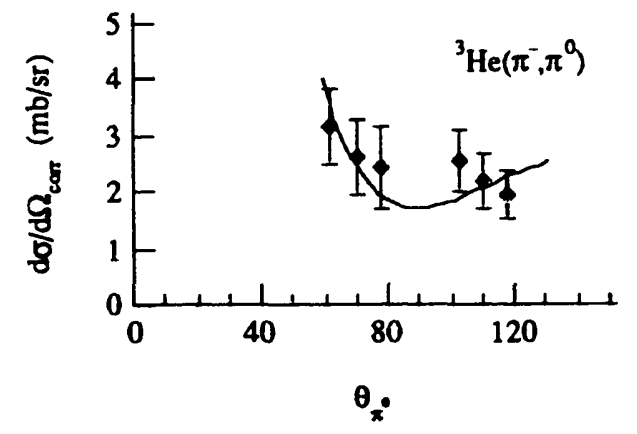

Figure 7.11: Differential cross sections for ${ }^{3} \mathrm{He}\left(\pi^{+}, \pi^{0}\right)$ (right) and ${ }^{3} \mathrm{He}\left(\pi^{-}, \pi^{9}\right)$ (left) at an incident pion energy of $245 \mathrm{MeV}$. The curves indicate the differential cross section for free single charge exchange on a proton from $\pi \mathrm{N}$ phase shift calculations. The curves on the left have been scaled by a factor of two to account for the presence of two protons in ${ }^{3} \mathrm{He}$. No attempt has been made in figures a) or b) to extrapolate the doubly differential cross sections to low $\pi^{0}$ energies. In figures c) and d), a crude estimate of the missing strength at low outgoing $\pi^{0}$ energies has been included. 
Ratios of Doubly Differential Cross Sections from Single-Arm Measurements of Pion Charge

\section{Exchange and Inelastic Scattering}

In the $\Delta$-resonance region, comparisons between cross sections for pion charge exchange and non-charge exchange reaction channels can be examined to determine the relative importance of single scattering and double scattering processes. In a naive picture, the contribution from isospin channels other than the $\mathrm{T}=3 / 2$ channel may be neglected in the $\Delta$-resonance region and simple isospin scaling predictions can be computed for single scattering and double scatering processes.

\section{Isospin Scaling Predictions for Single and Double Scattering Ratios}

In the quasi-free scattering regime, a pion interacts incoherently with individual nucleons within a nucleus. By assuming that a pion interacts with only one nucleon in a nucleus, one can compute a ratio for the ${ }^{3} \mathrm{He}\left(\pi^{-}, \pi^{9}\right)$ to ${ }^{3} \mathrm{He}\left(\pi^{+}, \pi^{9}\right)$ cross sections as follows,

$$
\begin{aligned}
\sigma\left(\pi^{-}, \pi^{0}\right) / \sigma\left(\pi^{+}, \pi^{0}\right) & =\frac{2 \mid\left\langle\left.\pi^{-} p|A| \pi^{0} n\right|^{2}\right.}{\mid\left\langle\left.\pi^{+} n|A| \pi^{0} p\right|^{2}\right.} \\
& =2 .
\end{aligned}
$$

The factor of two accounts for the two protons in the ${ }^{3} \mathrm{He}$ nucleus. This ratio is the same whether or not $T=1 / 2$ scattering amplitudes are neglected. Ratios can also be computed for pion inelastic scattering on ${ }^{3} \mathrm{He}$. If the contributions from the $\mathrm{T}=1 / 2$ channel are neglected, the ratio between the cross sections for inelastic scattering and charge exchange on ${ }^{3} \mathrm{He}$ may be exprcised as

$$
\sigma\left(\pi^{+}, \pi^{+}\right): \sigma\left(\pi^{-}, \pi^{-}\right): \sigma\left(\pi^{-}, \pi^{0}\right): \sigma\left(\pi^{+}, \pi^{0}\right) \approx 19: 11: 4: 2 .
$$

Deviations from this ratio may be evidence of multiple scattering or, in the case of the inelastic scattering channels, non-negligible contributions from $\mathrm{T}=1 / 2$ scattering amplitudes.

A pion which interacts with more than one nucleon in the nucleus will lose more energy than a pion which interacts with only one nucleon. Therefore, the outgoing pion energy distributions below the quasi-free peak can be examined for evidence of multiple scattering. Simple isospin scaling ratios for double scattering processes may be computed in a similar manner as the single scattering ratios. In a model in which double scattering is the dominant process, the relative strength of the $\pi^{-}$and $\pi^{+}$single charge exchange cross sections for ${ }^{3} \mathrm{He}$ can be written as, 


$$
\begin{aligned}
\sigma_{2}\left(\pi^{-}, \pi^{0}\right) & =2\left|\left\langle\pi^{-} n|A| \pi^{-} n\right\rangle\left\langle\pi^{-} p|A| \pi^{0} n\right\rangle^{2}+2\right|\left\langle\pi^{-} p|A| \pi^{0} n\right\rangle\left\langle\pi^{0} n|A| \pi^{0} n\right\rangle^{2} \\
& +2\left|\left\langle\pi^{-} p|A| \pi^{0} n\right\rangle\left\langle\pi^{0} p|A| \pi^{0} p\right\rangle^{2}+2\right|\left\langle\pi^{-} p|A| \pi^{-} p\right)\left\langle\pi^{-} p|A| \pi^{0} n\right\rangle^{2} \\
& \approx 72 / 81\left|A_{3 / 2}\right|^{2}
\end{aligned}
$$

and

$$
\begin{aligned}
\sigma_{2}\left(\pi^{*}, \pi^{0}\right) & =2\left|\left\langle\pi^{+} \mathrm{p}|A| \pi^{+} \mathrm{p}\right\rangle\left\langle\pi^{+} n|A| \pi^{0} \mathrm{p}\right\rangle\right|^{2}+2\left|\left\langle\pi^{+} n|A| \pi^{0} \mathrm{p}\right\rangle\left\langle\pi^{0} \mathrm{p}|A| \pi^{0} \mathrm{p}\right\rangle\right|^{2} \\
& \approx 52 / 81\left|A_{3 / 2}\right|^{2}
\end{aligned}
$$

where the factor of two accounts for the two protons in ${ }^{3} \mathrm{He}$. If contributions from the $\mathrm{T}=1 / 2$ channel are ignored, the ratio of these two charge exchange cross sections is,

$$
\sigma_{2}\left(\pi^{-}, \pi^{0}\right) / \sigma_{2}\left(\pi^{*}, \pi^{0}\right)=1.38
$$

Similar double scattering predictions can be computed for non-charge exchange and double charge exchange on ${ }^{3} \mathrm{He}$. The relative strengths of the non-charge exchange and charge exchange channe!s for the ${ }^{3}$ He system may be written as

$$
\sigma_{2}\left(\pi^{+}, \pi^{+}\right): \sigma_{2}\left(\pi^{-}, \pi^{-}\right): \sigma_{2}\left(\pi^{-}, \pi^{0}\right): \sigma_{2}\left(\pi^{+}, \pi^{0}\right): \sigma_{2}\left(\pi^{-}, \pi^{+}\right) \approx 25.75: 5.75: 4: 2.89: 1 \text {. (7.6) }
$$

\section{Indications of Multiple Scattering}

In Figures 7.12 and 7.13 , the ratios of the doubly differential cross sections for the ${ }^{3} \mathrm{He}\left(\pi, \pi^{9}\right)$ reaction to those for the ${ }^{3} \mathrm{He}\left(\pi^{+}, \pi^{9}\right)$ reaction are shown as a function of $\pi^{0}$ kinetic energy. The arrows near the center of the energy distribution point to the $\pi^{0}$ kinetic energy for the free charge exchange process, $\pi \mathrm{p} \rightarrow \pi^{\circ} \mathrm{n}$. The slight rise at energies below the arrow and slight dip at energies above the arrow can be attributed to the differences in the position of the quasi-free peak for the ${ }^{3} \mathrm{He}\left(\pi^{-}, \pi^{9}\right)$ and ${ }^{3} \mathrm{He}\left(\pi^{+}, \pi^{9}\right)$ reactions. At a $\pi^{0}$ scattering angle of $70^{\circ}$, the corresponding $\pi^{\circ}$ kinetic energies for the free $\pi^{*} \mathrm{n}$ and $\pi^{\mathrm{p}} \mathrm{p}$ single charge exchange channels are 179 and $177 \mathrm{MeV}$ respectively. The rise in the ratio above $\pi^{0}$ kinetic energies of $200 \mathrm{MeV}$ and at $\pi^{\circ}$ scattering angles of $62^{\circ}, 70^{\circ}$, and $78^{\circ}$ can be explained by the presence of the isobaric analog transition, which can only take place for an incident $\pi,{ }^{3} \mathrm{He}\left(\pi^{-}, \pi^{9}\right)^{3} \mathrm{H}$.

The intriguing feature in all of the single charge exchange (SCX) ratios, which are shown in Figures 7.12 and 7.13 , is the trend towards the double scatering predictions at lower $\pi^{0}$ kinetic energies. The measured doubly differential cross sections do not extend to low enough energies to confirm 

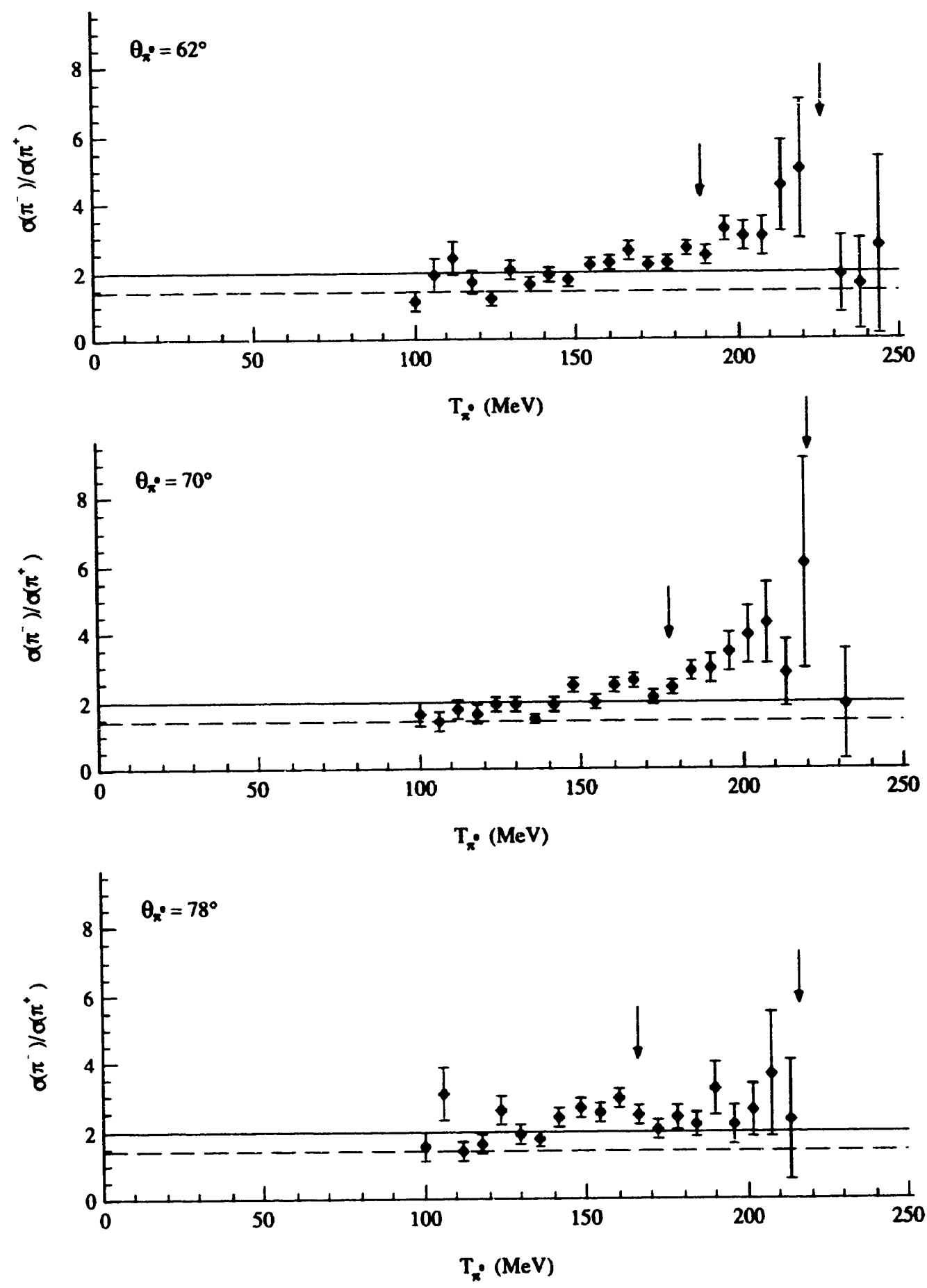

Figure 7.12: Ratio of the doubly differential cross sections for ${ }^{3} \mathrm{He}\left(\pi^{-}, \pi^{\circ}\right)$ to ${ }^{3} \mathrm{He}\left(\pi^{+}, \pi^{\circ}\right)$ at $62^{\circ}, 70^{\circ}$, and $78^{\circ}$ for an incident energy of $245 \mathrm{MeV}$. The solid line is the ratio determined by isospin considerations for scattering from a single nucleon. The dashed line is the isospin ratio assuming a double scatuering process. The arrow at the left corresponds to the $\pi^{0}$ kinetic energy for $p\left(\pi^{0}, \pi^{0}\right)$ n. The arrow at the right corresponds to the $\pi^{0}$ kinetic energy for elastic single charge exchange, ${ }^{3} \mathrm{He}\left(\pi^{-}, \pi^{0}\right)^{3} \mathrm{H}$. 

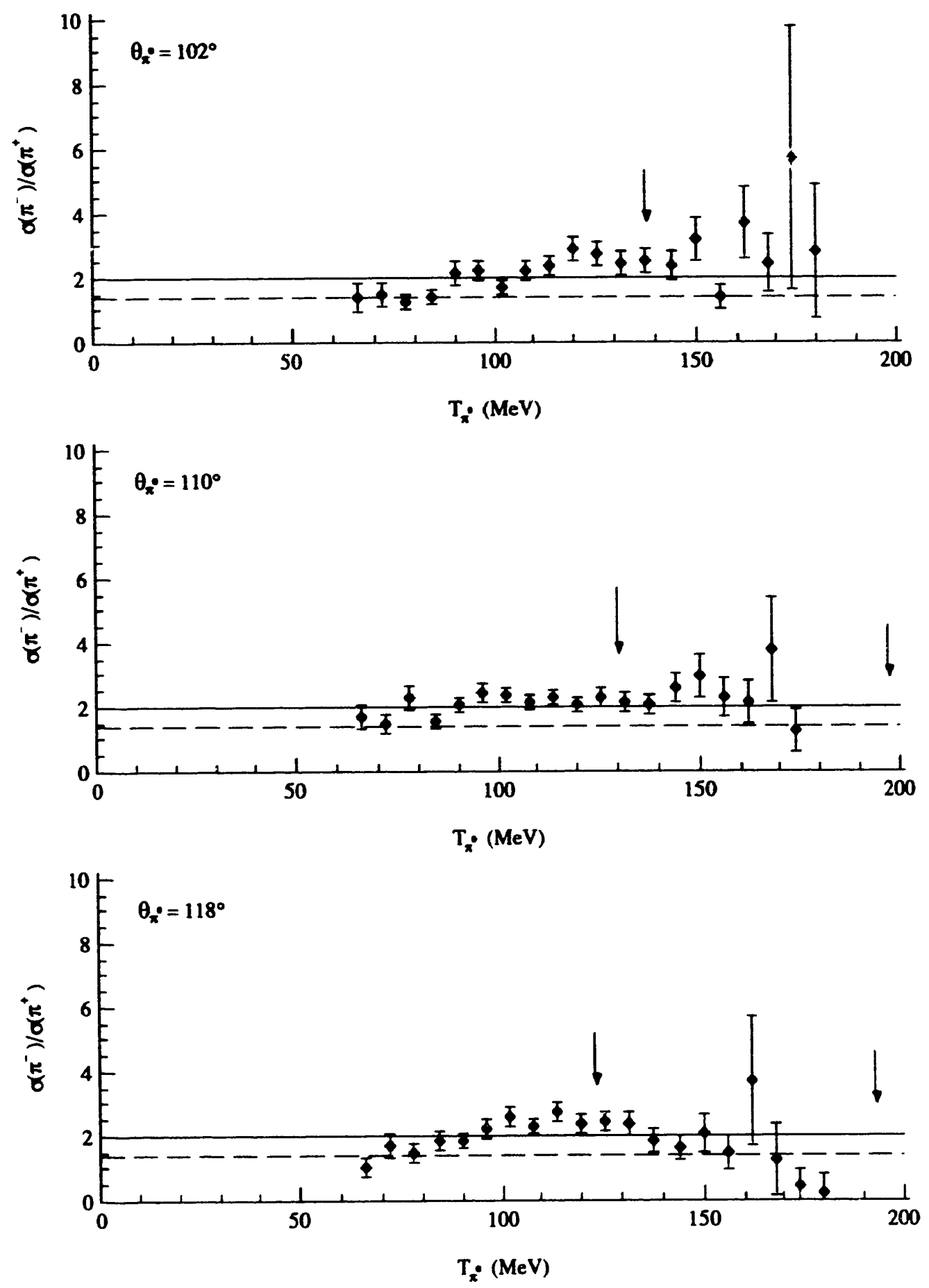

Figure 7.13: Ratio of the doubly differential cross sections for ${ }^{3} \mathrm{He}\left(\pi^{-}, \pi^{\circ}\right)$ to ${ }^{3} \mathrm{He}\left(\pi^{+}, \pi^{\circ}\right)$ at $102^{\circ}, 110^{\circ}$, and $128^{\circ}$ for an incident pion energy of $245 \mathrm{MeV}$. The solid line is the ratio determined by isospin considerations for scattering from a single nucleon. The dashed line is the isospin ratio assuming a double scattering process. The arrow at the left corresponds to the $\pi^{0}$ kinetic energy for $p\left(\pi^{0}, \pi^{0}\right)$ n. The arrow at the right corresponds to the $\pi^{0}$ kinetic energy for elastic single charge exchange, ${ }^{3} \mathrm{He}\left(\pi^{0}, \pi^{0}\right)^{3} \mathrm{H}$. 
a)

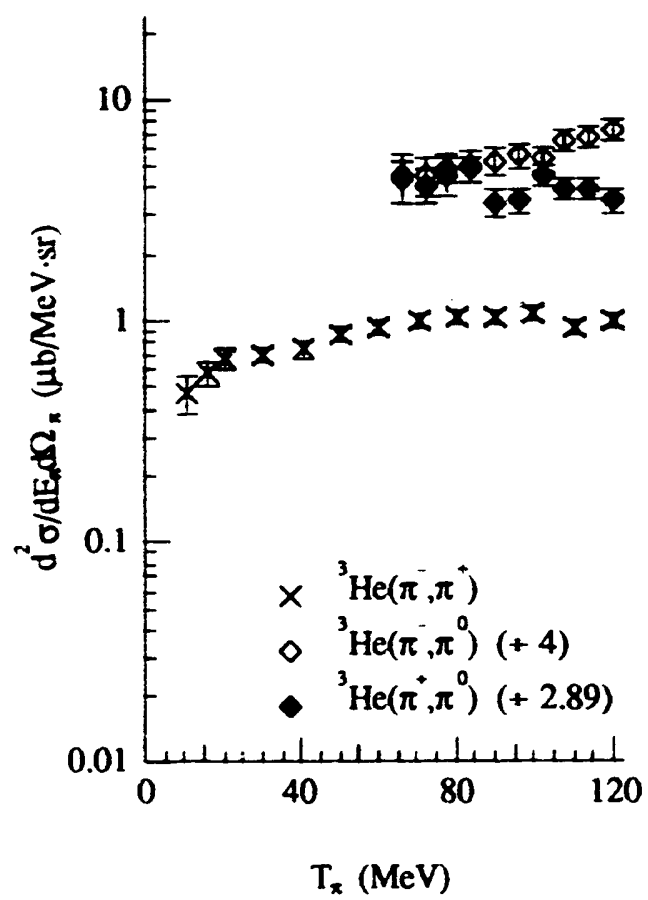

b)

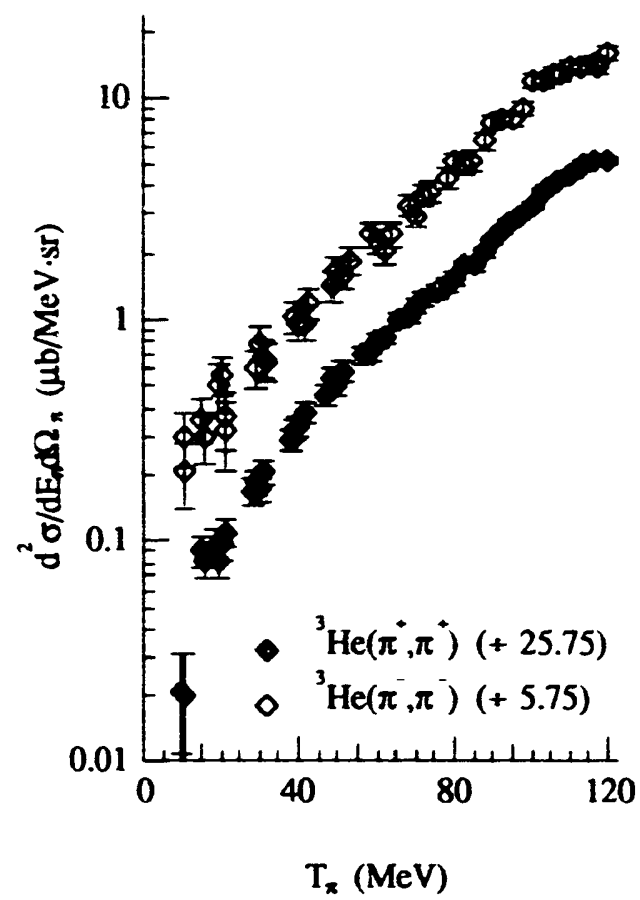

Figure 7.14: Comparisons of doubly differential cross sections for a) pion single charge exchange, double charge exchange, and b) non-charge exchange on ${ }^{3} \mathrm{He}$. The non-charge exchange $\left({ }^{3} \mathrm{He}\left(\pi^{*}, \pi^{*}\right)\right)$ and double charge exchange $\left({ }^{3} \mathrm{He}\left(\pi^{-}, \pi^{\top}\right)\right)$ data at $\mathrm{T}_{x}=240 \mathrm{MeV}$ and $\theta_{x}=105^{\circ}$ have been taken from Reference 6 . The non-charge exchange and single charge results, which derive from measurements at $T_{x}=245 \mathrm{MeV}$ and $\theta_{x}$ $=102^{\circ}$, have been re-scaled by the factors given in the figures. If double scattering alone were responsible for the low energy tail, one would expect these re-scaled cross sections to fall on the same curve.

whether this trend continues or not. Comparisons of the relative strengths of the ${ }^{3} \mathrm{He}\left(\pi^{+}, \pi^{0}\right)$ and ${ }^{3} \mathrm{He}\left(\pi^{-}, \pi^{9}\right)$ cross sections, shown in Figure 7.14a, exhibit a trend towards double scattering while the non-charge exchange data, shown in Figure 7.14b, do not. Moreover, the re-scaled single charge exchange cross sections exhibit nearly the same energy dependence as the double charge exchange (DCX) spectrum. However, these re-scaled cross sections do not fall on the same curve as the DCX spectrum. This deviation may be viewed as an indication of non-negligible contributions from either single scattering processes or more complicated processes. Pion absorption, which is expected to be suppressed in the single charge exchange channels, may account for the deviation of the inelastic scattering measurements from the pure double scattering predictions by removing scattered pions from the reaction channel. Other indications of multiple scattering in the ${ }^{3} \mathrm{He}$ single charge exchange and inelastic scattering measurements will be discussed in Chapter 8. 


\section{Coincidence Measurements of Quasi-free Pion Single Charge Exchange on ${ }^{3} \mathrm{He}:{ }^{3} \mathrm{He}\left(\pi^{ \pm}, \pi^{0} \mathrm{p}\right)$}

\section{Triply Differential Cross Sections for the ${ }^{3} \mathrm{He}\left(\pi^{ \pm}, \pi^{0} \mathrm{p}\right)$ Reactions}

The experimental parameters for the coincidence measurements of quasi-free pion single charge exchange on ${ }^{3} \mathrm{He}$ are presented in Table 7.4. These measurements were performed with the LAMPF $\pi^{0}$ Spectrometer, an array of plastic sciutillation counters, and a liquid ${ }^{3} \mathrm{He}$ target as described in Chapter 5. The data analysis procedures for extracting triply differential cross sections have been previously discussed in Chapter 6. Triply differential cross sections for the ${ }^{3} \mathrm{He}\left(\pi^{*}, \pi^{0} \mathrm{p}\right)$ reaction are shown in Figures 7.15 through 7.23. Triply differential cross sections for the ${ }^{3} \mathrm{He}\left(\pi^{-}, \pi^{0} \mathrm{p}\right)$ reaction are shown in Figures 7.24 through 7.26. The uncertainties shown in these plots are representative of the statistical fluctuations in the data. A single $24^{\circ}$ angular bin, centered on the central scattering angle of $\pi^{\circ}$ Spectrometer, was used to compute the triply differential cross sections. Protons were detected over a range of kinetic energies from approximately 25 to $175 \mathrm{MeV}$. Triply differential cross sections were computed by summing over all proton energies. No attempt was made to correct these triply differential cross sections for low energy protons which were not detected with the experimental apparatus.

\begin{tabular}{|c|c|c|c|}
\hline \multicolumn{4}{|c|}{ Experimental Parameters for ${ }^{3} \mathrm{He}\left(\pi^{ \pm}, \pi^{0} \mathrm{p}\right)$ Measurements } \\
\hline Reaction & $\begin{array}{c}\theta_{x} \\
\text { (deg.) }\end{array}$ & $\begin{array}{c}\theta_{p} \\
\text { (deg.) }\end{array}$ & $\begin{array}{c}\mathrm{T}_{\boldsymbol{x}} \\
(\mathrm{MeV})\end{array}$ \\
\hline${ }^{3} \mathrm{He}\left(\pi^{+}, \pi^{0} \mathrm{p}\right)$ & $70^{\circ}$ & $-37.0^{\circ},-46.5,-51.2^{\circ}$ & $100-256$ \\
\hline${ }^{3} \mathrm{He}\left(\pi^{+}, \pi^{0} \mathrm{p}\right)$ & $90^{\circ}$ & $-30.4^{\circ},-35.4^{\circ}$ & $80-218$ \\
\hline${ }^{3} \mathrm{He}\left(\pi^{+}, \pi^{0} \mathrm{p}\right)$ & $110^{\circ}$ & $-21.3^{\circ},-26.3^{\circ}$ & $66-180$ \\
\hline${ }^{3} \mathrm{He}\left(\pi, \pi^{0} \mathrm{p}\right)$ & $120^{\circ}$ & $-17.2^{\circ},-22.0^{\circ}$ & $68-176$ \\
\hline${ }^{3} \mathrm{He}\left(\pi, \pi^{0} \mathrm{p}\right)$ & $70^{\circ}$ & $-37.0^{\circ},-46.5^{\circ}$ & $100-256$ \\
\hline${ }^{3} \mathrm{He}\left(\pi, \pi^{0} \mathrm{p}\right)$ & $110^{\circ}$ & $-23.0^{\circ}$ & $66-180$ \\
\hline
\end{tabular}

Table 7.4: Experimental parameters for the $\pi^{0} \mathrm{p}$ coincidence measurements. Triply differential cross sections for the ${ }^{3} \mathrm{He}\left(\pi^{*}, \pi^{0}\right)$ reactions were measured at $\pi^{0}$ laboratory scattering angles corresponding to $\theta_{\pi} 0$. The scattering angle of the central proton detector in the proton detector array is designated as $\theta_{p}$. The observable range of outgoing $\pi^{0}$ kinetic energies is listed in the coiumn labeled " $T_{*}$ ". 
The $\pi^{0}$ energy spectra from the ${ }^{3} \mathrm{He}\left(\pi^{+}, \pi^{0} \mathrm{p}\right)$ reaction exhibit a characteristic quasi-free peak at $\pi^{0}$ kinetic energies and proton recoil angles near the corresponding kinematic parameters for the free charge exchange process, $\pi^{+} n \rightarrow \pi^{0} \mathrm{p}$, which are presented in Table 7.5. The triply differential cross sections exhibit a rough symmetry - similar magnitudes and widths for the prominent peak which is associated with the quasi-free mechanism - about the proton recoil angle for free charge exchange. Examples of this symmetry are shown in Figures 7.16 and 7.20 for $\pi^{\circ}$ laboratory scattering angles of $70^{\circ}$ and $110^{\circ}$ respectively. In these figures, the central proton detector was placed at a laboratory angle close to the quasi-free proton angle and the $\pi^{0}$ energy spectra corresponding to proton detectors equidistant from the central proton detector, either in or out of the scattering plane, exhibit the same general shape.

\begin{tabular}{|l|l|l|l|}
\hline \multicolumn{2}{|c|}{$n\left(\pi^{+}, \pi^{0}\right)_{p}$ at } & $T_{x}=245 \mathrm{MeV}$ \\
\hline $\begin{array}{c}\theta_{x^{*}} \\
\text { (deg.) }\end{array}$ & $\begin{array}{c}T_{x^{*}} \\
(\mathrm{MeV})\end{array}$ & $\begin{array}{c}\theta_{p} \\
(\text { deg. })\end{array}$ & $\begin{array}{c}T_{p} \\
(\mathrm{MeV})\end{array}$ \\
\hline 70 & 179.3 & -45.6 & 71.6 \\
\hline 90 & 153.1 & -35.4 & 97.8 \\
\hline 110 & 131.8 & -26.3 & 119.1 \\
\hline 120 & 123.3 & -22.2 & 127.6 \\
\hline
\end{tabular}

Table 7.5: List of kinematic values for the free single charge exchange reaction, $\pi^{*} n \rightarrow \pi^{0} p$. 


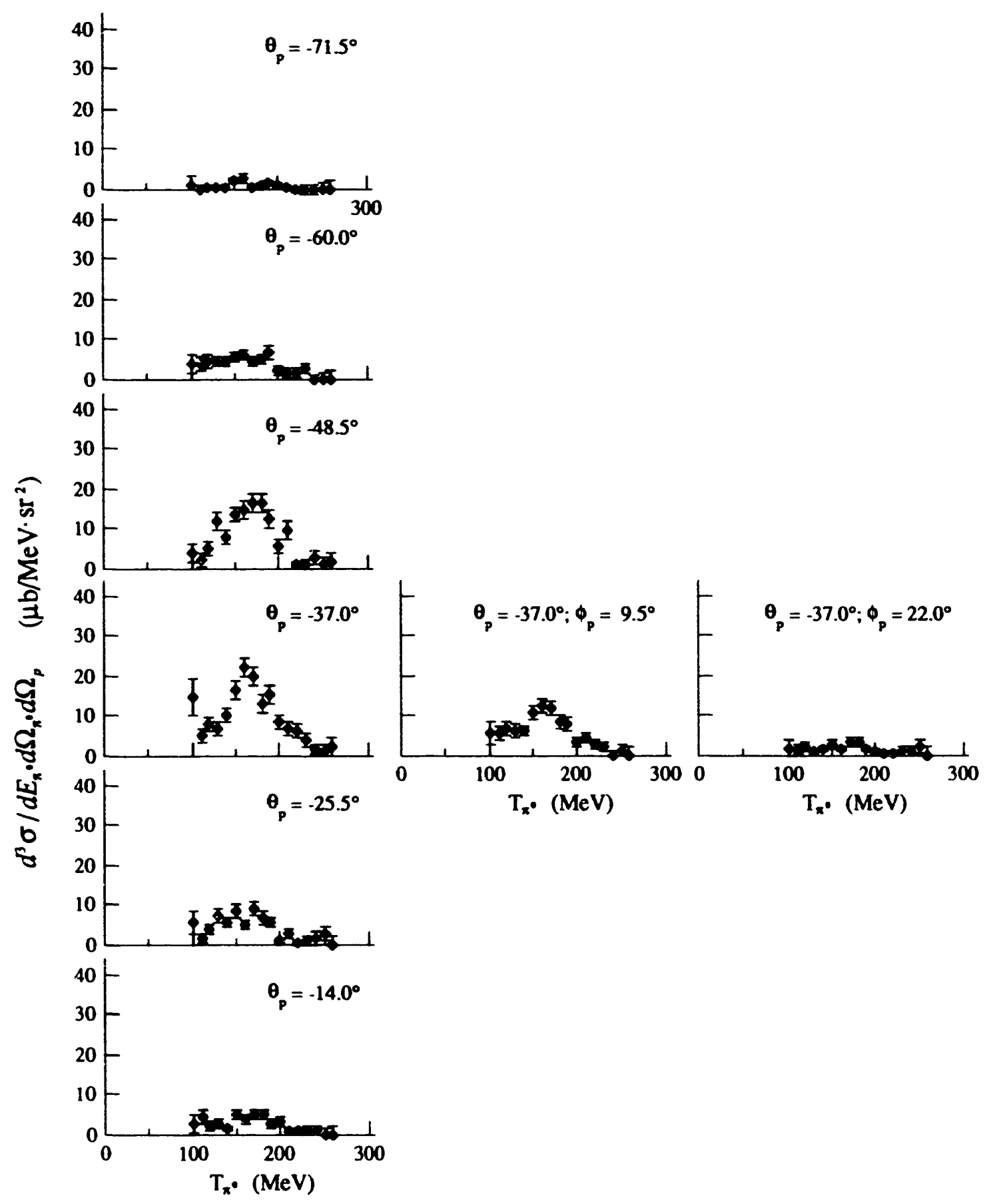

Figure 7.15: Triply differential cross sections for the ${ }^{3} \mathrm{He}\left(\pi^{*}, \pi^{0} \mathrm{p}\right)$ reaction at an incident pion energy of 245 $\mathrm{MeV}$ and $\theta_{x^{\circ}}=70^{\circ}$. An overall systematic uncertainty of $8.5 \%$ is not included in this figure. These data were accumulated with the proton detector array placed in the horizontal configuration at a laboratory angle corresponding to $\theta_{p}=-37.0^{\circ}$ for the central proton detector. The in-plane and out-of-plane proton angles are labeled as $\theta_{p}$ and $\phi_{p}$ respectively. 


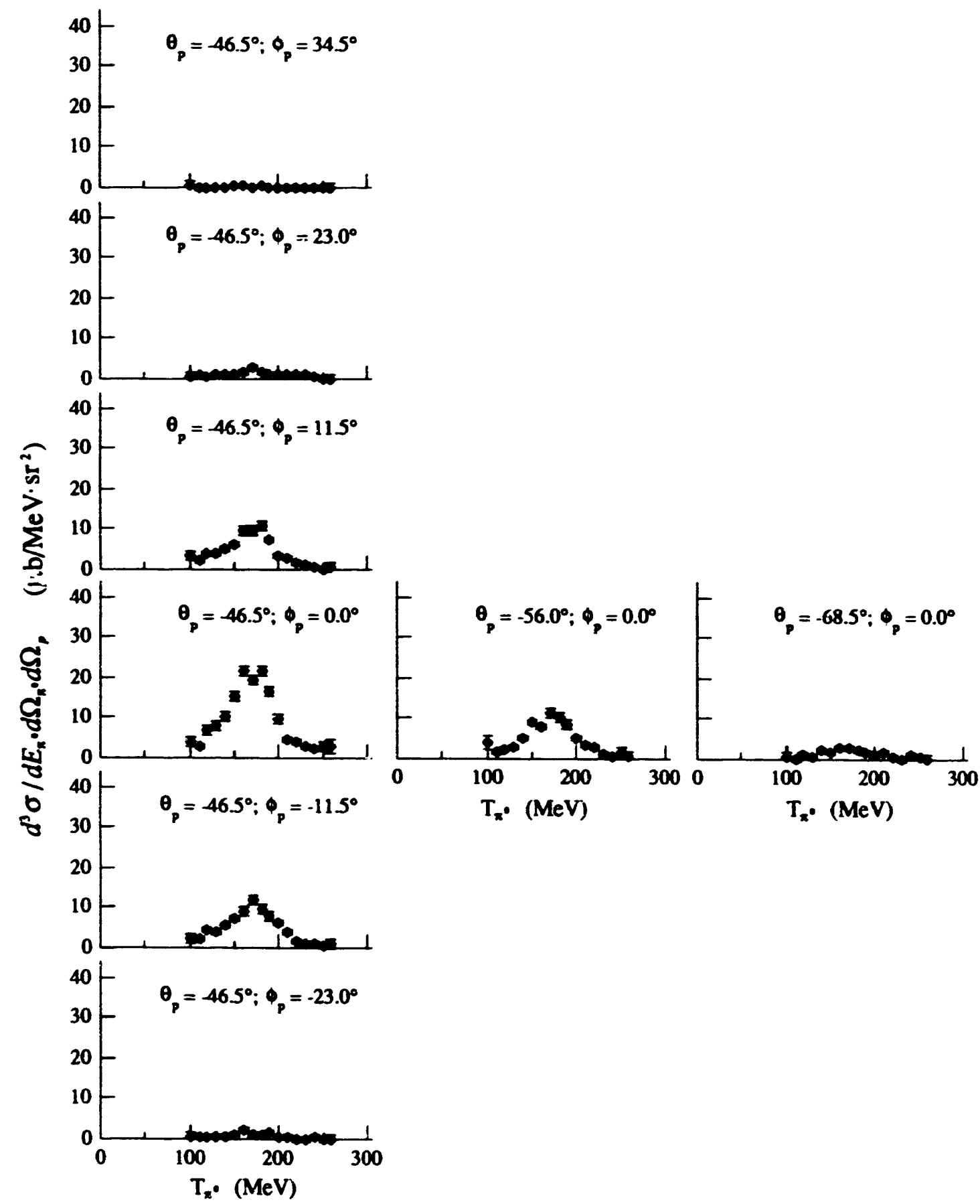

Figure 7.16: Triply differential cross sections for the ${ }^{3} \mathrm{He}\left(\pi^{*}, \pi^{0} \mathrm{p}\right)$ reaction at an incident pion energy of 245 $\mathrm{MeV}$ and $\theta_{x}=70^{\circ}$. An overall systematic uncertainty of $8.5 \%$ is not included in this figure. These data were accumulated with the proton detector array placed in the vertical configuration at a laboratory angle corresponding to $\theta_{p}=-46.5^{\circ}$ for the central proton detector. The in-plane and out-of-plane proton angles are labeled as $\theta_{p}$ and $\phi_{p}$ respectively. 


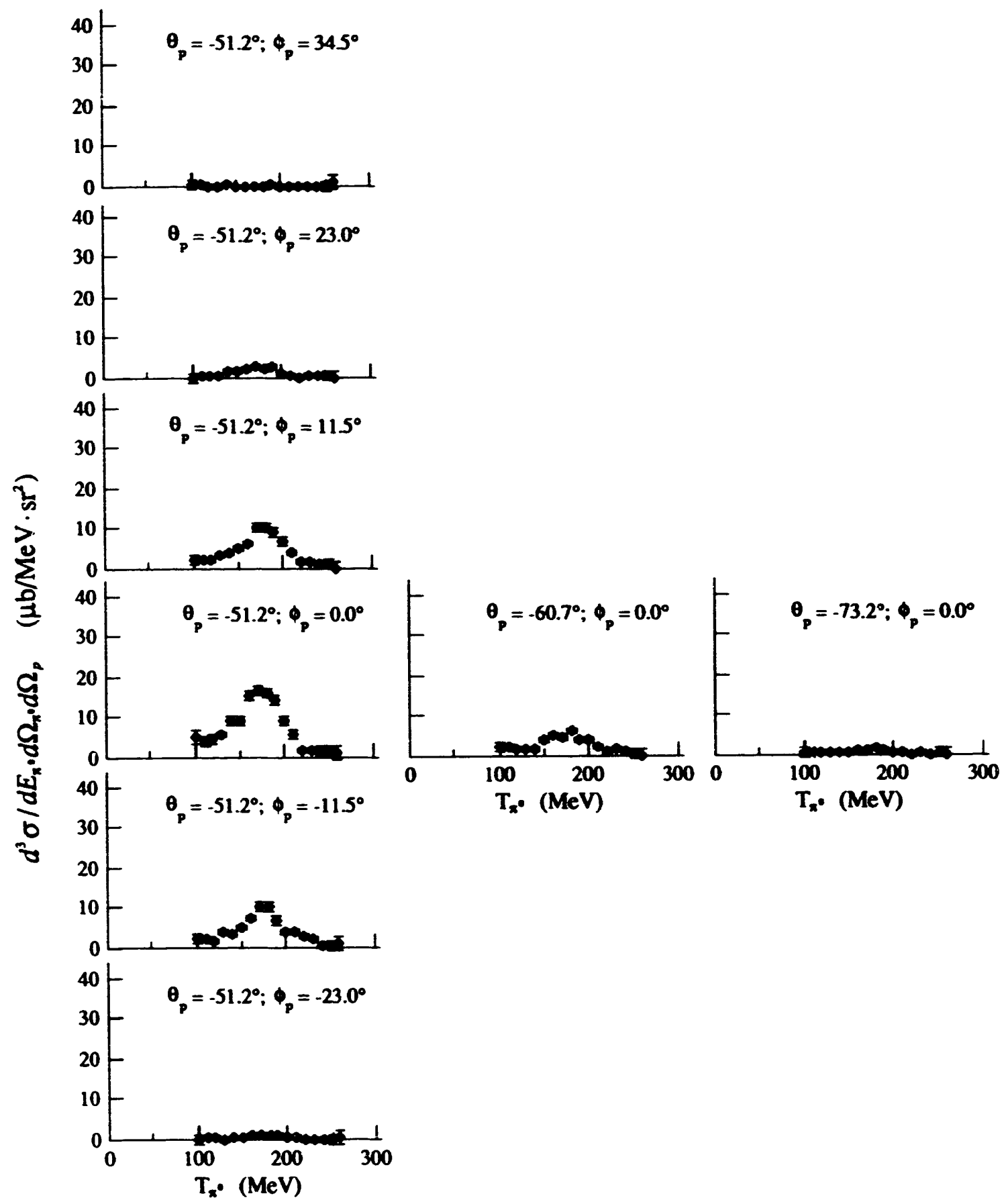

Figure 7.17: Triply differential cross sections for the ${ }^{3} \mathrm{He}\left(\pi^{+}, \pi^{0} \mathrm{p}\right)$ reaction at an incident pion energy of 245 $\mathrm{MeV}$ and $\theta_{x^{\circ}}=70^{\circ}$. An overall systematic uncertainty of $8.5 \%$ is not included in this figure. These data were accumulated with the proton detector array placed in the vertical configuration at a laboratory angle corresponding to $\theta_{p}=-51.2^{\circ}$ for the central proton detector. The in-plane and out-of-plane proton angles are labeled as $\theta_{p}$ and $\phi_{p}$ respectively. 


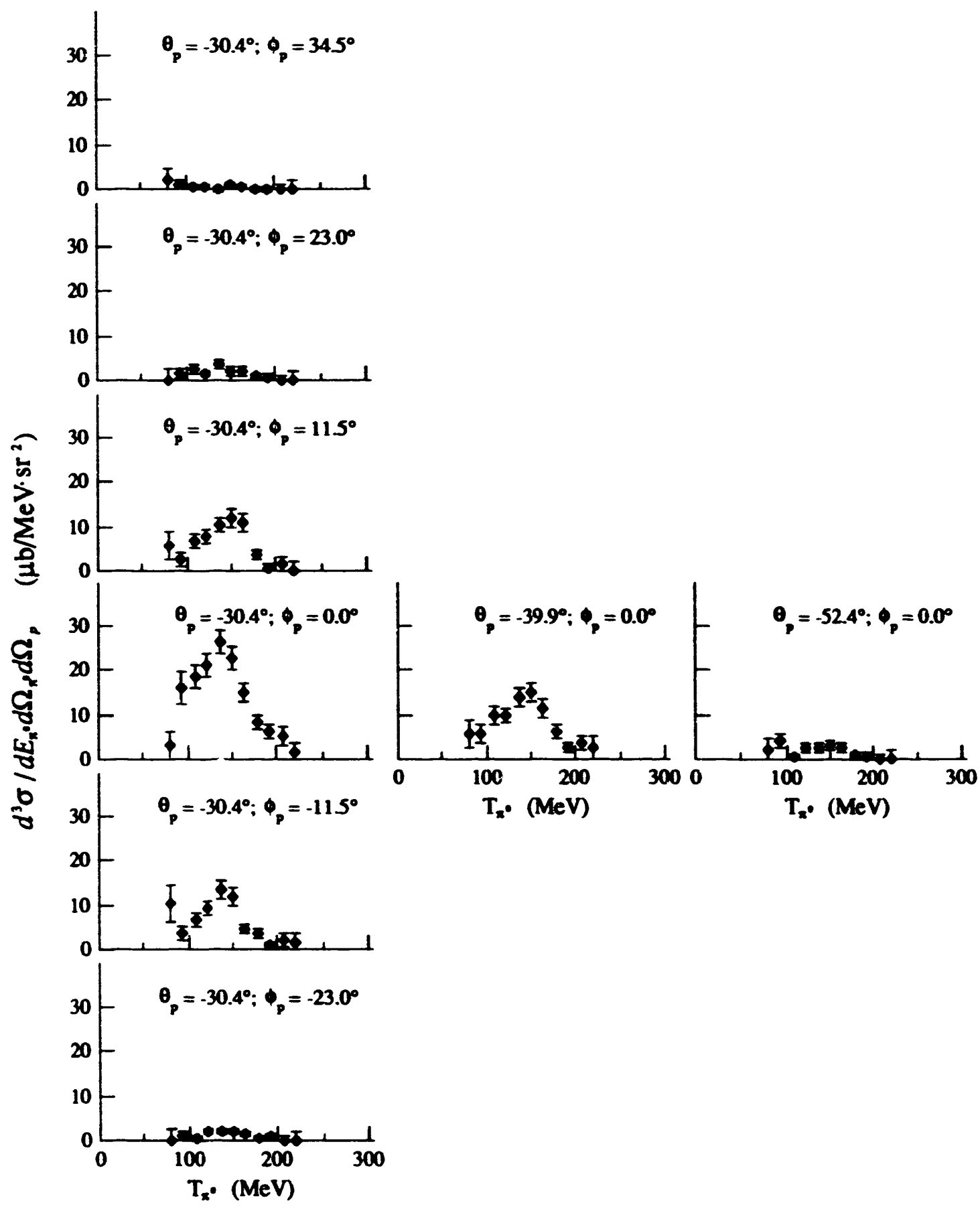

Figure 7.18: Triply differential cross sections for the ${ }^{3} \mathrm{He}\left(\pi^{*}, \pi^{0} \mathrm{p}\right)$ reaction at an incident pion energy of 245 $\mathrm{MeV}$ and $\theta_{z^{\circ}}=90^{\circ}$. An overall systematic uncertainty of $8.1 \%$ is not included in this figure. These data were accumulated with the proton detector array placed in the vertical configuration at a laboratory angle corresponding to $\theta_{p}=-30.4^{\circ}$ for the central proton detector. The in-plane and out-of-plane proton angles are labeled as $\theta_{p}$ and $\phi_{p}$ respectively. 


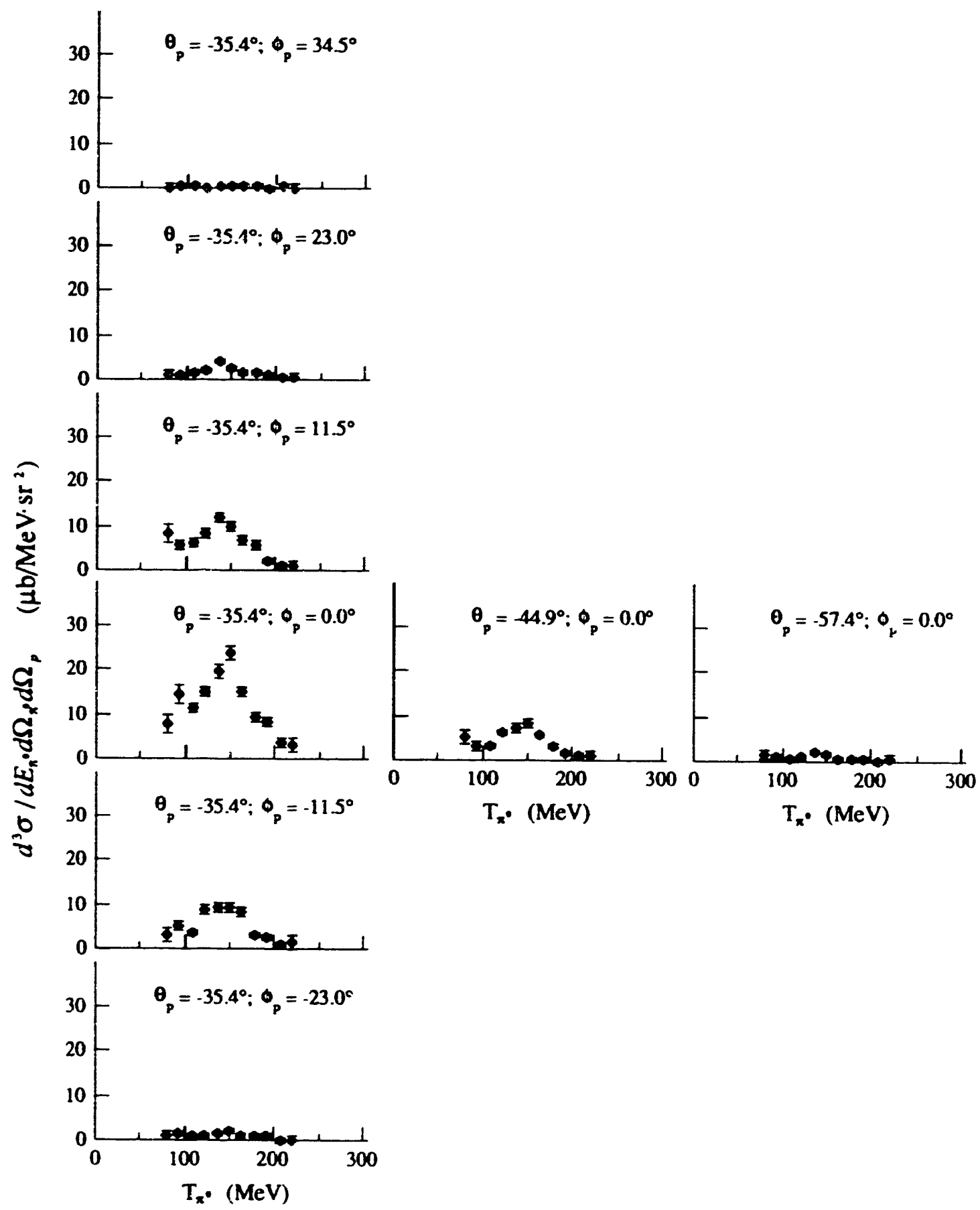

Figure 7.19: Triply differential cross sections for the ${ }^{3} \mathrm{He}\left(\pi^{*}, \pi^{0} \mathrm{p}\right)$ reaction at an incident pion energy of 245 $\mathrm{MeV}$ and $\theta_{x^{0}}=90^{\circ}$. An overall systematic uncertainty of $8.1 \%$ is not included in this figure. These data were accumulated with the proton detector array placed in the vertical configuration at a laboratory angle corresponding to $\theta_{\mathrm{p}}=-35.4^{\circ}$ for the central proton detector. The in-plane and out-of-plane proton angles are labeled as $\theta_{p}$ and $\phi_{p}$ respectively. 


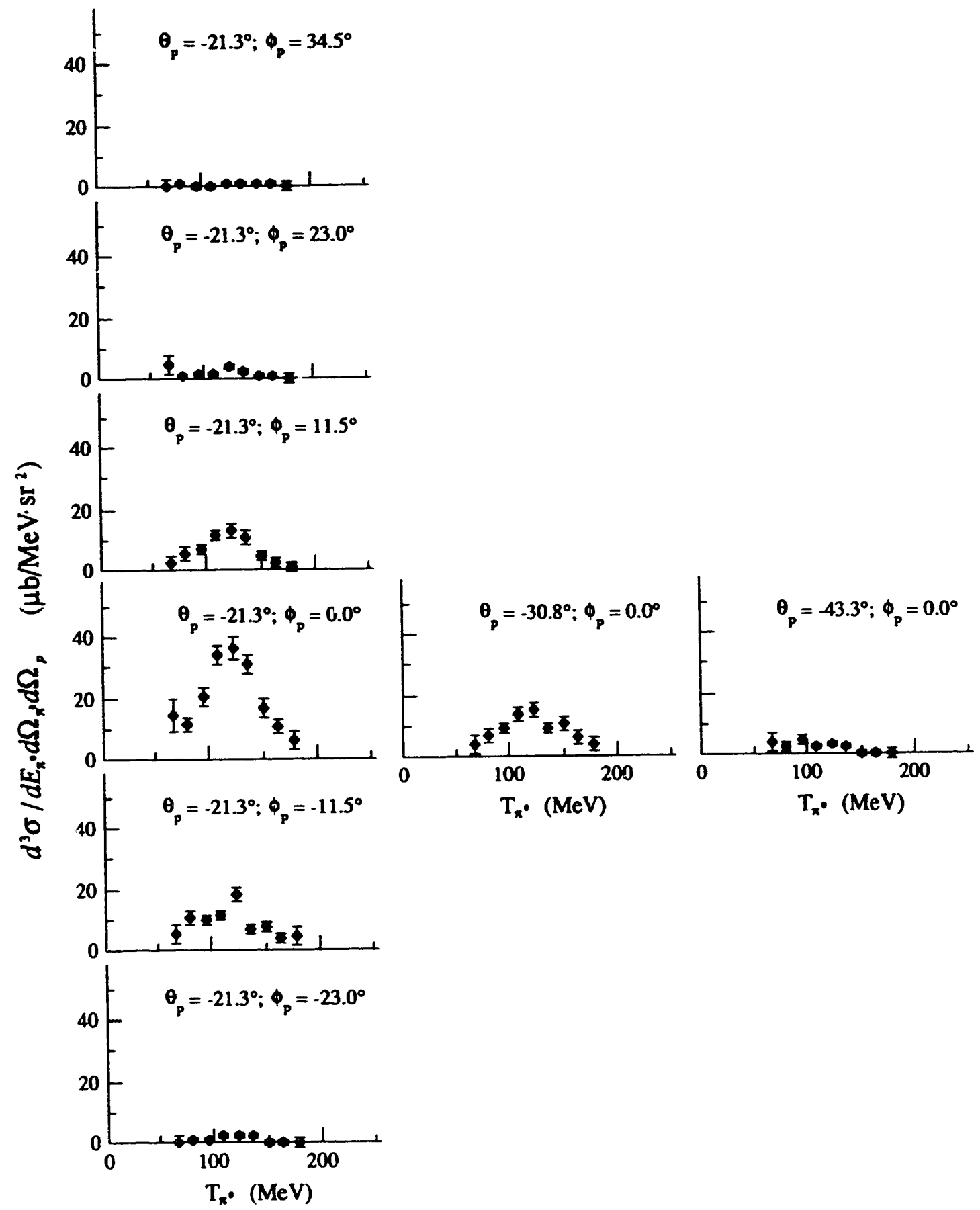

Figure 7.20: T:iply differential cross sections for the ${ }^{3} \mathrm{He}\left(\pi^{*}, \pi^{0} \mathrm{p}\right)$ reaction at an incident pion energy of 245 $\mathrm{MeV}$ and $\theta_{\mathrm{x}^{0}}=110^{\circ}$. An overall systematic uncertainty of $8.3 \%$ is not included in this figure. These data were accumulated with the proton detector array placed in the vertical configuration at a laboratory angle corresponding to $\theta_{p}=-21.3^{\circ}$ for the central proton detector. The in-plane and out-of-plane proton angles are labeled as $\theta_{p}$ and $\phi_{p}$ respectively. 


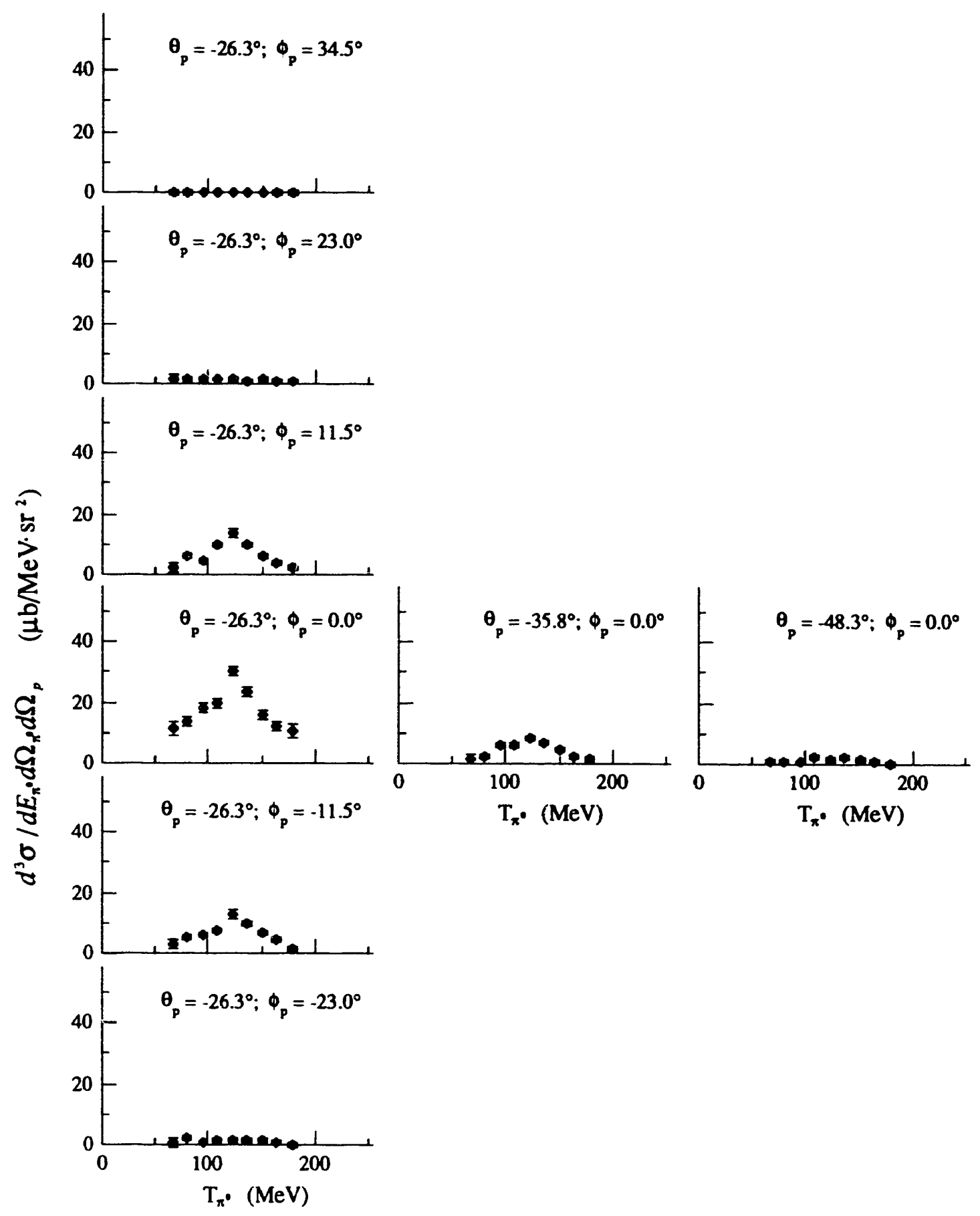

Figure 7.21: Triply differential cross sections for the ${ }^{3} \mathrm{He}\left(\pi^{+}, \pi^{0} \mathrm{p}\right)$ reaction at an incident pion energy of 245 $\mathrm{MeV}$ and $\theta_{\pi}=110^{\circ}$. An overall systematic uncertainty of $8.3 \%$ is not included in this figure. These data were accumulated with the proton detector array placed in the vertical configuration at a laboratory angle corresponding to $\theta_{p}=-26.3^{\circ}$ for the central proton detector. The in-plane and out-of-plane proton angles are labeled as $\theta_{p}$ and $\phi_{p}$ respectively. 


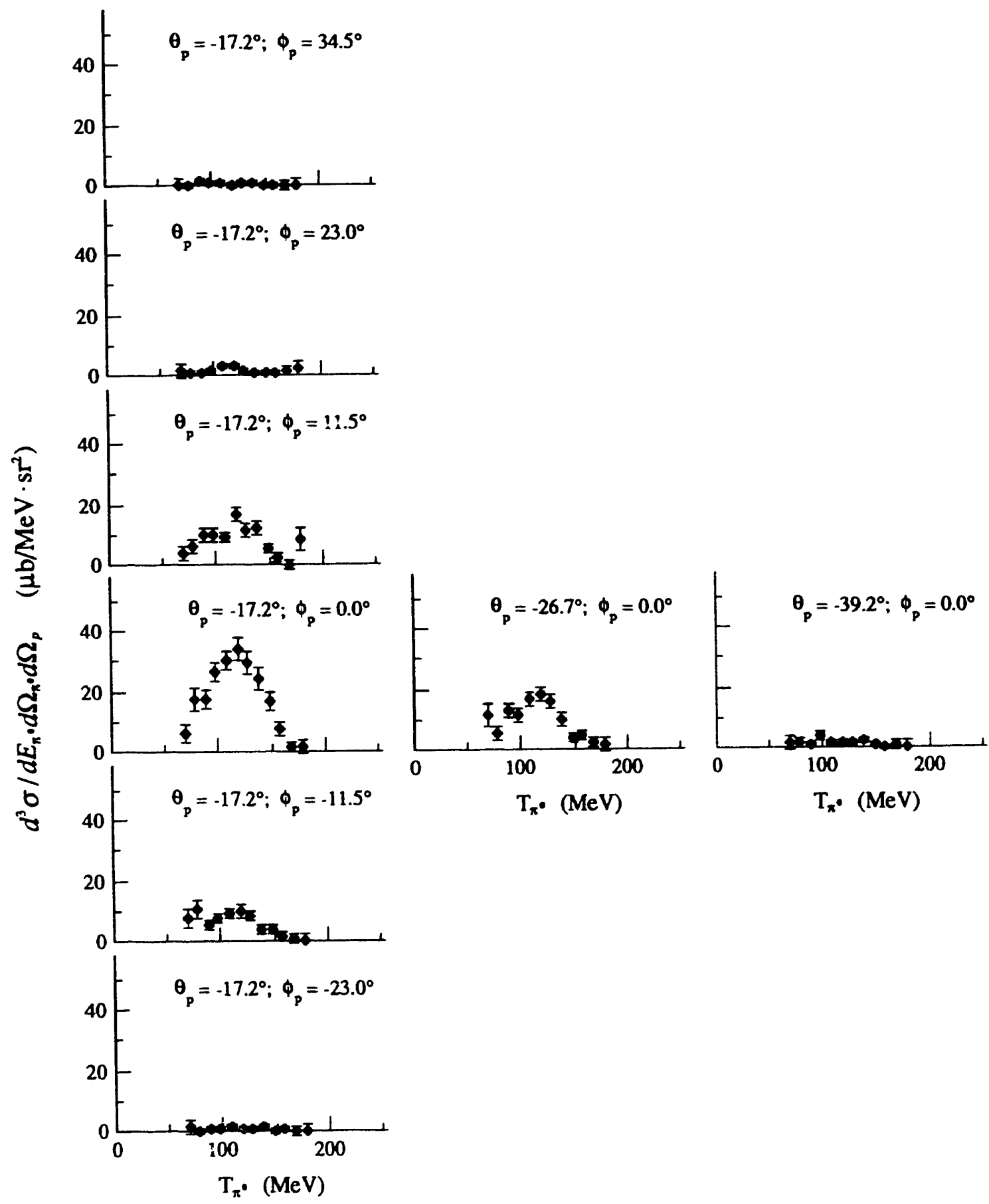

Figure 7.22: Triply differential cross sections for the ${ }^{3} \mathrm{He}\left(\pi^{+}, \pi^{0} \mathrm{p}\right)$ reaction at an incident pion energy of 245 $\mathrm{MeV}$ and $\theta_{\mathrm{x}} \mathrm{O}=120^{\circ}$. An overall systematic uncertainty of $8.4 \%$ is not included in this figure. These data were accumulated with the proton detector array placed in the vertical configuration at a laboratory angle corresponding to $\theta_{p}=-17.2^{\circ}$ for the central proton detector. The in-plane and out-of-plane proton angles are labeled as $\theta_{p}$ and $\phi_{p}$ respectively. 


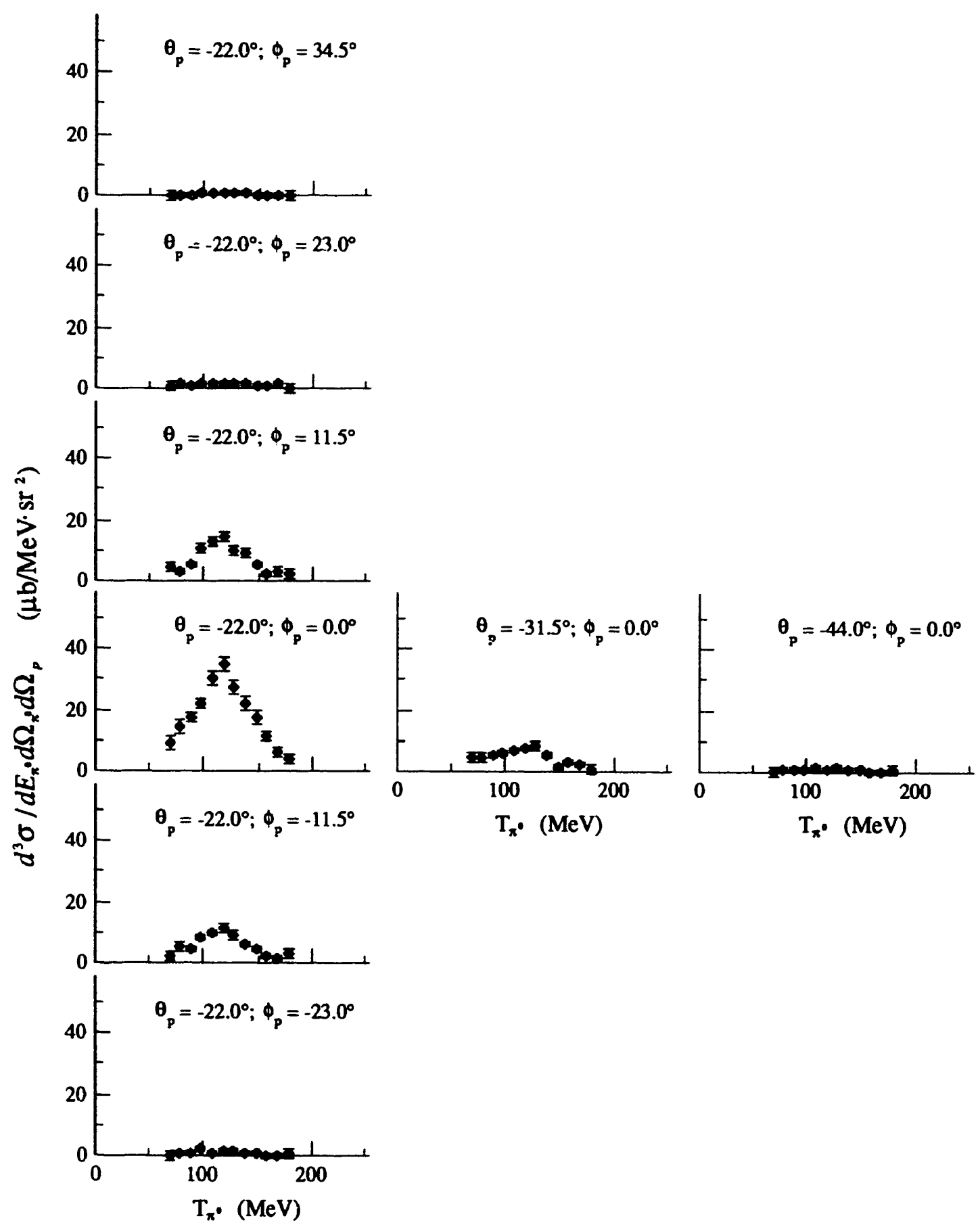

Figure 7.23: Triply differential cross sections for the ${ }^{3} \mathrm{He}\left(\pi^{+}, \pi^{0} \mathrm{p}\right)$ reaction at an incident pion energy of 245 $\mathrm{MeV}$ and $\theta_{\pi^{\circ}}=120^{\circ}$. An overall systematic uncertainty of $8.4 \%$ is not included in this figure. These data were accumulated with the proton detector array placed in the vertical configuration at a laboratory angle corresponding to $\theta_{\mathrm{p}}=-22.0^{\circ}$ for the central proton detector. The in-plane and out-of-plane proton angles are labeled as $\theta_{\mathrm{p}}$ and $\phi_{\mathrm{p}}$ respectively. 

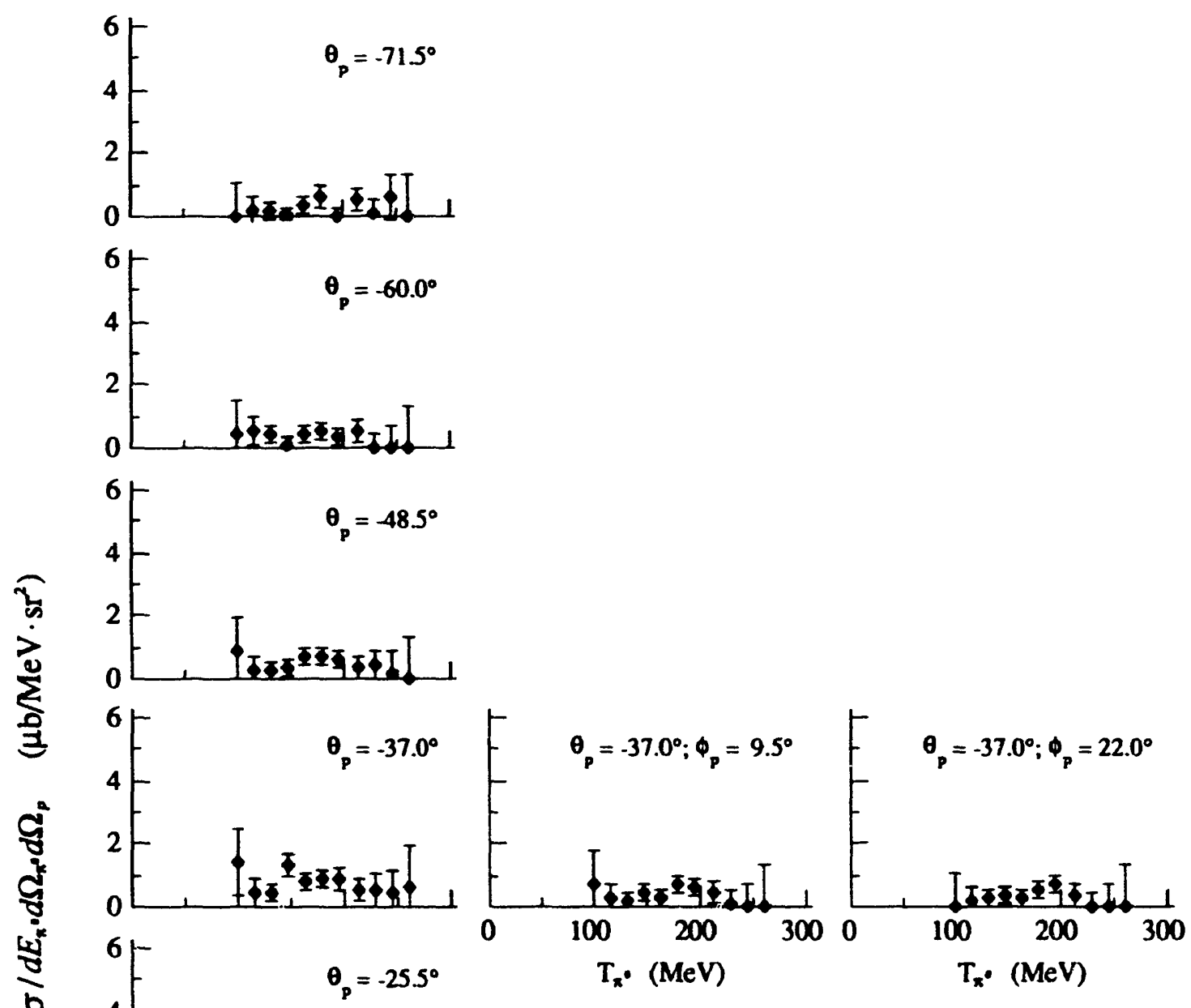

ib
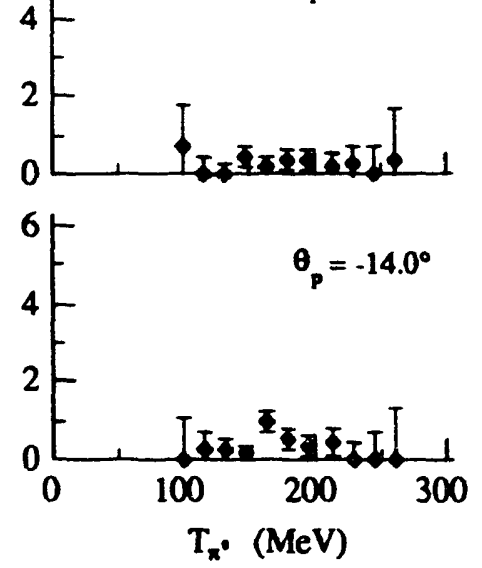

Figure 7.24: Triply differential cross sections for the ${ }^{3} \mathrm{He}\left(\pi^{-}, \pi^{0} \mathrm{p}\right)$ reaction a: an incident pion energy of 245 $\mathrm{MeV}$ and $\theta_{\mathrm{R}^{\circ}}=70^{\circ}$. An overall systematic uncertainty of $8.1 \%$ is not included in this figure. These data were accumulated with the proton detector array placed in the horizontal configuration at a laboratory angle corresponding to $\theta_{p}=-37.0^{\circ}$ for the central proton detector. The in-plane and out-of-plane proton angles are labeled as $\theta_{p}$ and $\phi_{p}$ respectively. 

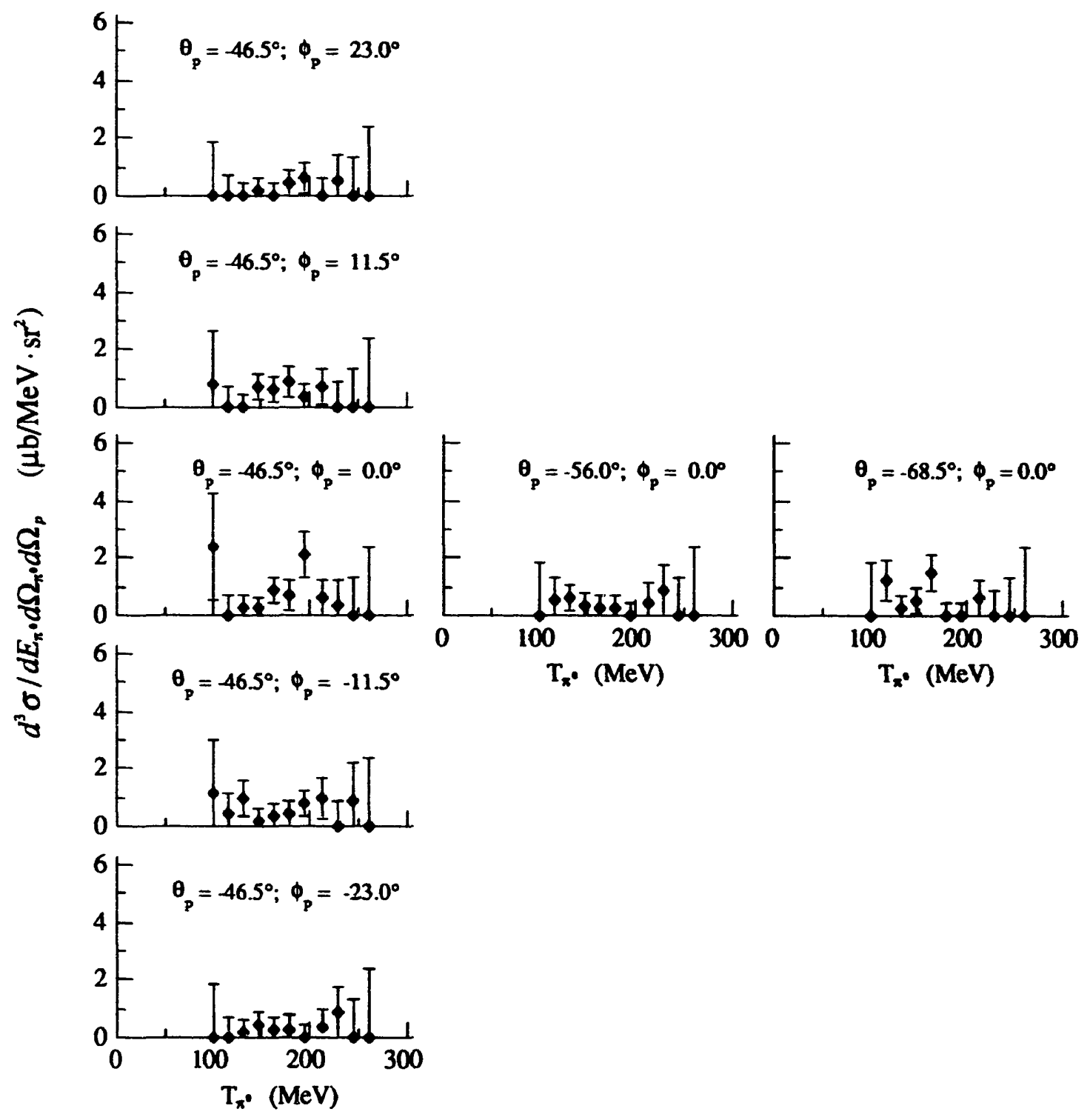

Figure 7.25: Triply differential cross sections for the ${ }^{3} \mathrm{He}\left(\pi^{-}, \pi^{0} \mathrm{p}\right)$ reaction at an incident pion energy of 245 $\mathrm{MeV}$ and $\theta_{\pi^{\circ}}=70^{\circ}$. An overall systematic uncertainty of $8.1 \%$ is not included in this figure. These data were accumulated with the proton detector array placed in the horizontal configuration at a laboratory angle corresponding to $\theta_{p}=-46.5^{\circ}$ for the central proton detector. The in-plane and out-of-plane proton angles are labeled as $\theta_{p}$ and $\phi_{p}$ respectively. 

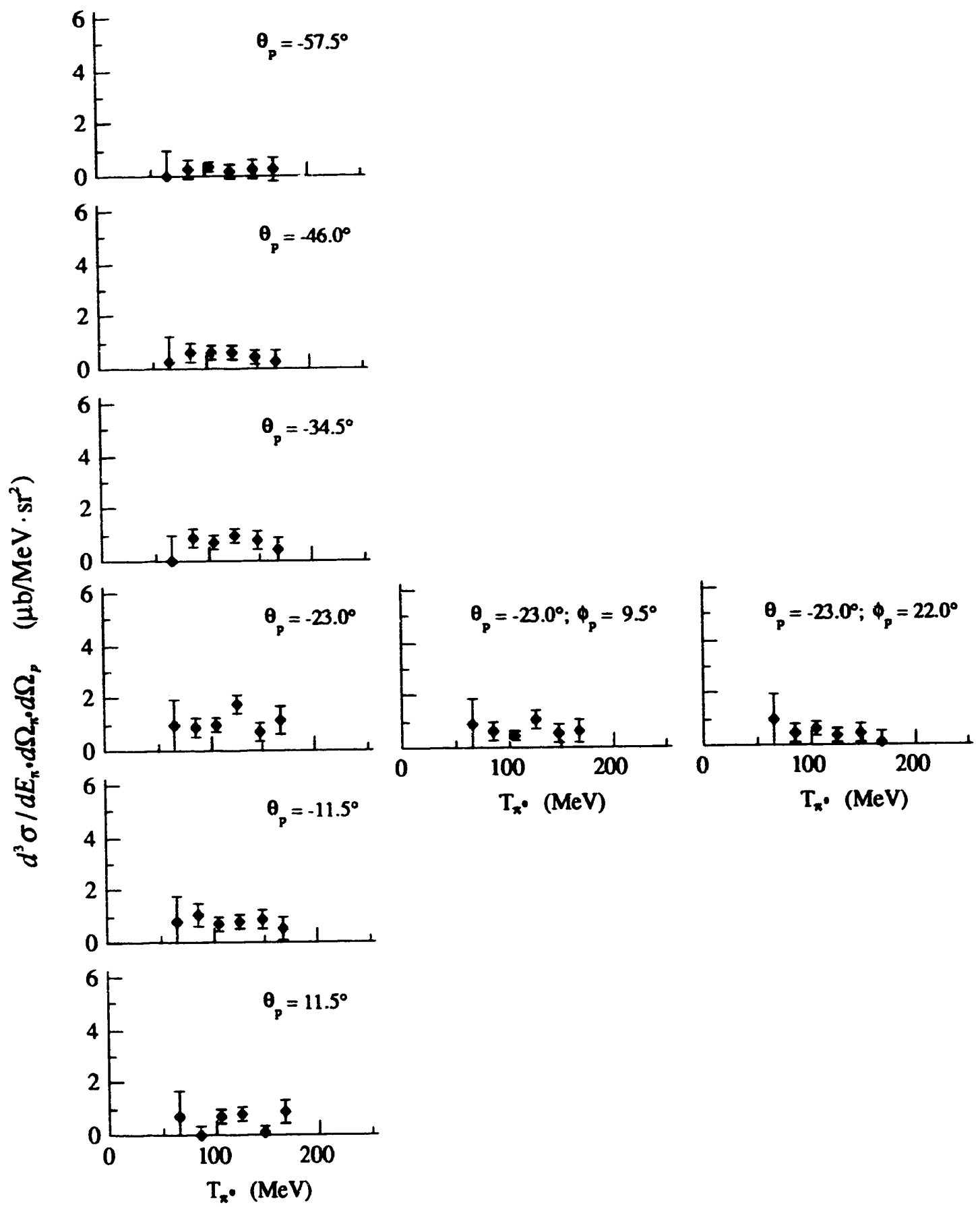

Figure 7.26: Triply differential cross sections for the ${ }^{3} \mathrm{He}\left(\pi^{-}, \pi^{0} \mathrm{p}\right)$ reaction at an incident pion energy of 245 $\mathrm{MeV}$ and $\theta_{z^{\circ}}=110^{\circ}$. An overall systematic uncertainty of $8.1 \%$ is not included in this figure. These data were accumulated with the proton detector array placed in the vertical configuration at a laboratory angle corresponding to $\theta_{p}=-23.0^{\circ}$ for the central proton detector. The in-plane and out-of-plane proton angles are labeled as $\theta_{p}$ and $\phi_{p}$ respectively. 


\section{Proton Angular Distributions for the ${ }^{3} \mathrm{He}\left(\pi^{ \pm}, \pi^{0} \mathrm{p}\right)$ Reactions}

Two methods were developed to extract proton angular distributions from the triply differential cross sections. In one method, the triply differential cross sections were integrated over the measured $\pi^{\circ}$ energy spectra. In the other method, the triply differential cross sections were extrapolated to low outgoing $\pi^{0}$ kinetic energies by fitting the $\pi^{0}$ energy spectra with a function of the form,

$$
\mathrm{F}(z)=a+b \cdot z+c \cdot z^{2}+\frac{A}{\sqrt{2 \pi \sigma^{2}}} \exp \left(-i^{2} / 2\right),
$$

where $z=\left(T_{:^{*}}-\left\langle T_{x^{*}}\right\rangle\right) / \sigma$, and $a, b, c, A, \sigma$, and $\left\langle T_{x^{\circ}}\right\rangle$ are variable parameters of the fit. Since these two methods produced consistent results, cross sections were computed using the first method, which made no assumptions about the functional form of the outgoing $\pi^{0}$ energy spectra.

The doubly differential cross sections for the ${ }^{3} \mathrm{He}\left(\pi^{+}, \pi^{0} \mathrm{p}\right)$ reaction are shown as a function of in-plane $\left(\theta_{p}\right)$ and out-of-plane $\left(\phi_{p}\right)$ proton angle in Figures 7.27 through 7.30. The arrows in these figures point to the proton angle for the free single charge exchange reaction, $\pi^{+} n \rightarrow \pi^{0} \mathrm{p}$. These angular distributions exhibit a common quasi-free peak at slightly smaller in-plane proton angles than the corresponding free proton angles and are symmetric about the nominal quasi-free proton angle. As the quasi-free proton angle shifts towards smaller angles, the proton angular distribution becomes narrower. This effect can be seen in comparison of the full-widths-at-half-maximum for the proton angular distributions which are approximately $20^{\circ}$ and $12^{\circ}$ at $\pi^{\circ}$ scautering angles of $70^{\circ}$ and $120^{\circ}$ respectively. These cross sections are presented in tabular form in Appendix E.

The doubly differential cross sections for the ${ }^{3} \mathrm{He}\left(\pi^{-}, \pi^{0} \mathrm{p}\right)$ reaction, shown in Figures 7.31 and 7.32 appear to be symmetric about the nominal quasi-free proton angle as well. However, the statistical quality of this data is insufficient to draw more meaningful conclusions about the general shape of these angular distributions. Comparisons of the proton angular distributions for the ${ }^{3} \mathrm{He}\left(\pi^{+}, \pi^{0} \mathrm{p}\right)$ and ${ }^{3} \mathrm{He}\left(\pi^{-}, \pi^{0} \mathrm{p}\right)$ measurements are shown in Figures 7.33 and 7.34 . The ratio of the ${ }^{3} \mathrm{He}\left(\pi^{+}, \pi^{0} \mathrm{p}\right)$ to ${ }^{3} \mathrm{He}\left(\pi^{-}, \pi^{0} \mathrm{p}\right)$ doubly differential cross sections near the quasi-free proton angle is roughly 20:1 which agrees with naive predictions for the relative strength of single scattering and double scattering processes. ${ }^{21}$ 

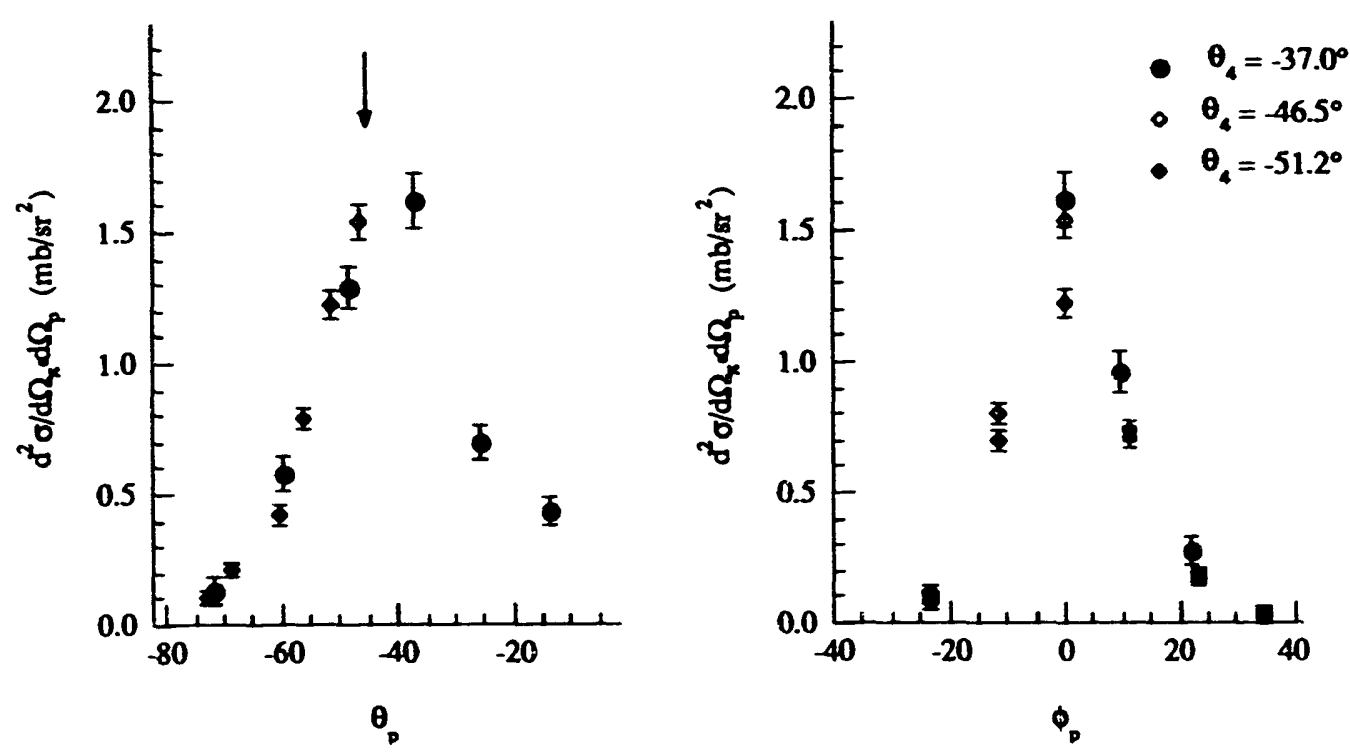

Figure 7.27: Proton angular distributions in the ${ }^{3} \mathrm{He}\left(\pi^{*}, \pi^{0} \mathrm{p}\right)$ reaction at $245 \mathrm{MeV}$ and $\theta_{x^{0}}=70^{\circ}$. An overall systematic uncertainty of $8.5 \%$ is not included in this figure. These data were accumulated with the central detector in the proton detector array at a laboratory angle corresponding to $\theta_{4}$. Except for the measurements at $\theta_{4}=-37.0^{\circ}$, the proton detector array was set up in the vertical configuration. The in-plane and out-of-plane proton angles are labeled as $\theta_{\mathrm{p}}$ and $\phi_{\mathrm{p}}$ respectively. The arrow corresponds to the proton angle for free single charge exchange: $\pi^{+} n \rightarrow \pi^{0} p$.
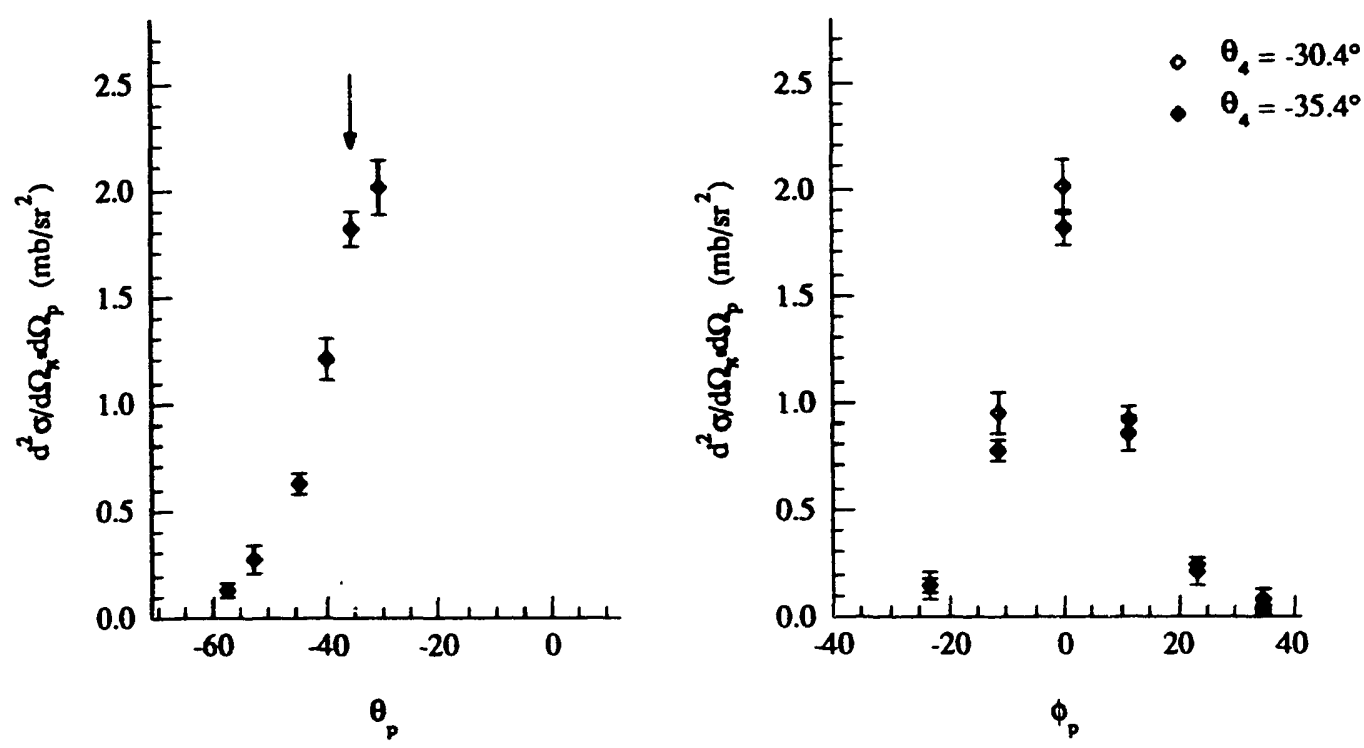

Figure 7.28: Proton angular distributions in the ${ }^{3} \mathrm{He}\left(\pi^{+}, \pi^{0} \mathrm{p}\right)$ reaction at $245 \mathrm{MeV}$ and $\theta_{x^{0}}=90^{\circ}$. An overall systematic uncertainty of $8.1 \%$ is not included in this figure. These data were accumulated with the proton detector array in the vertical configuration and the central detector placed at a laboratory angle corresponding to $\theta_{4}$. The in-plane and out-of-plane proton angles are labeled as $\theta_{p_{0}}$ and $\phi_{p}$ respectively. The arrow corresponds to the proton angle for free single charge exchange: $\pi^{+} n \rightarrow \pi^{0} p$. 

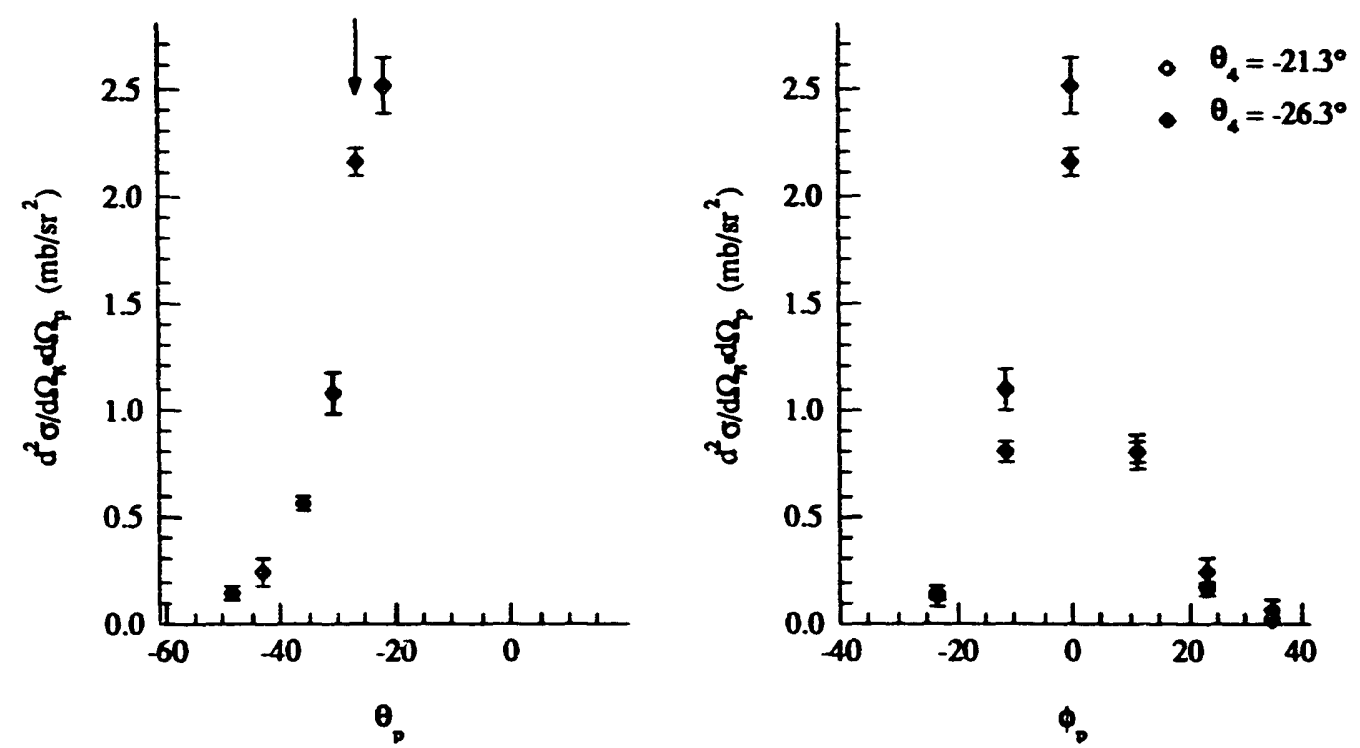

Figure 7.29: Proton angular distributions in the ${ }^{3} \mathrm{He}\left(\pi^{*}, \pi^{\circ} \mathrm{p}\right)$ reaction at $245 \mathrm{MeV}$ and $\theta_{\pi^{\circ}}=110^{\circ}$. An overall systematic uncertainty of $8.3 \%$ is not included in this figure. These data were accumulated with the proton detector array in the vertical configuration and the central detector placed at a laboratory angle corresponding to $\theta_{4}$. The in-plane and out-of-plane proton angles are labeled as $\theta_{p}$ and $\phi_{p}$ respectively. The arrow corresponds to the proton angle for free single charge exchange: $\pi^{+} n \rightarrow \pi^{\circ} \mathrm{p}$.
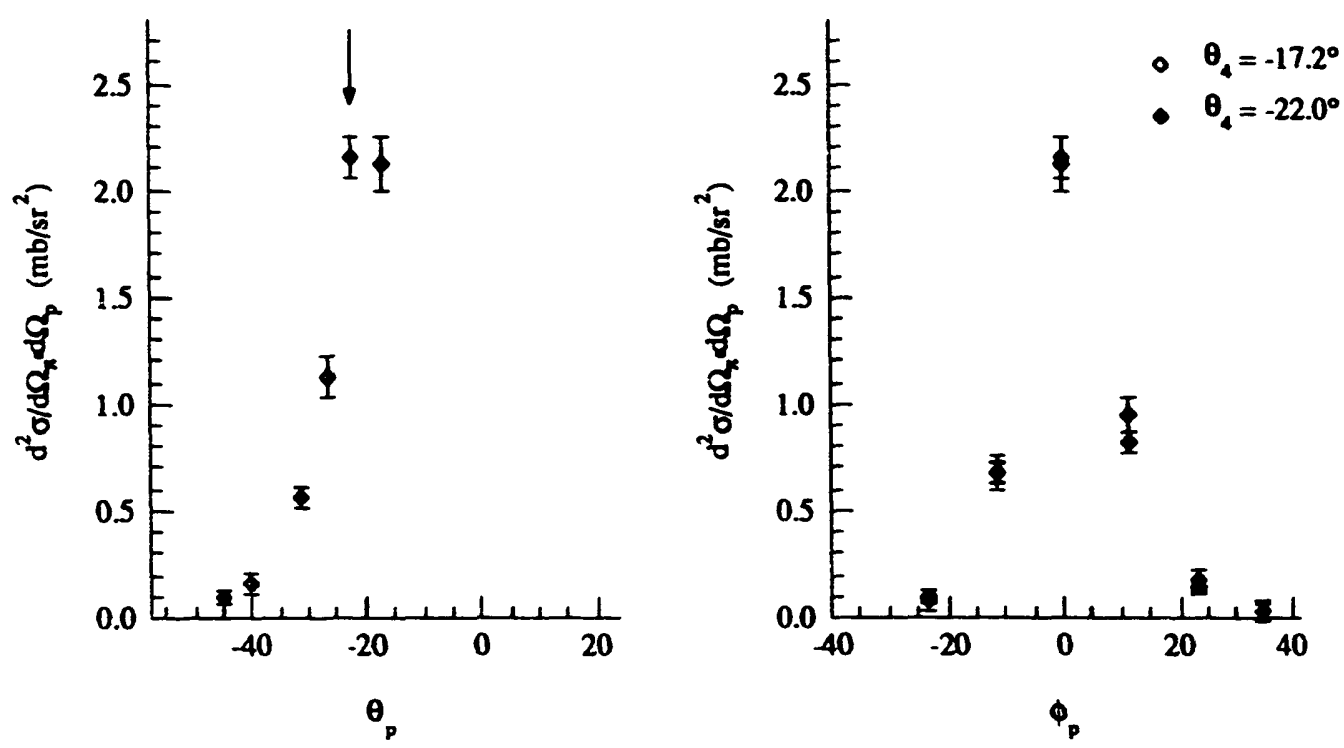

Figure 7.30: Proton angular distributions in the ${ }^{3} \mathrm{He}\left(\pi^{+}, \pi^{0} \mathrm{p}\right)$ reaction at $245 \mathrm{MeV}$ and $\theta_{\varepsilon^{2}}=120^{\circ}$. An overall systematic uncertainty of $8.4 \%$ is not included in this figure. These data were accumulated with the proton detector array in the vertical configuration and the central detector placed at a laboratory angle corresponding to $\theta_{4}$. The in-plane and out-of-plane proton angles are labeled as $\theta_{p}$ and $\phi_{p}$ respectively. The arrow corresponds to the proton angle for free single charge exchange: $\pi^{*} n \rightarrow \pi^{0} \mathrm{p}$. 

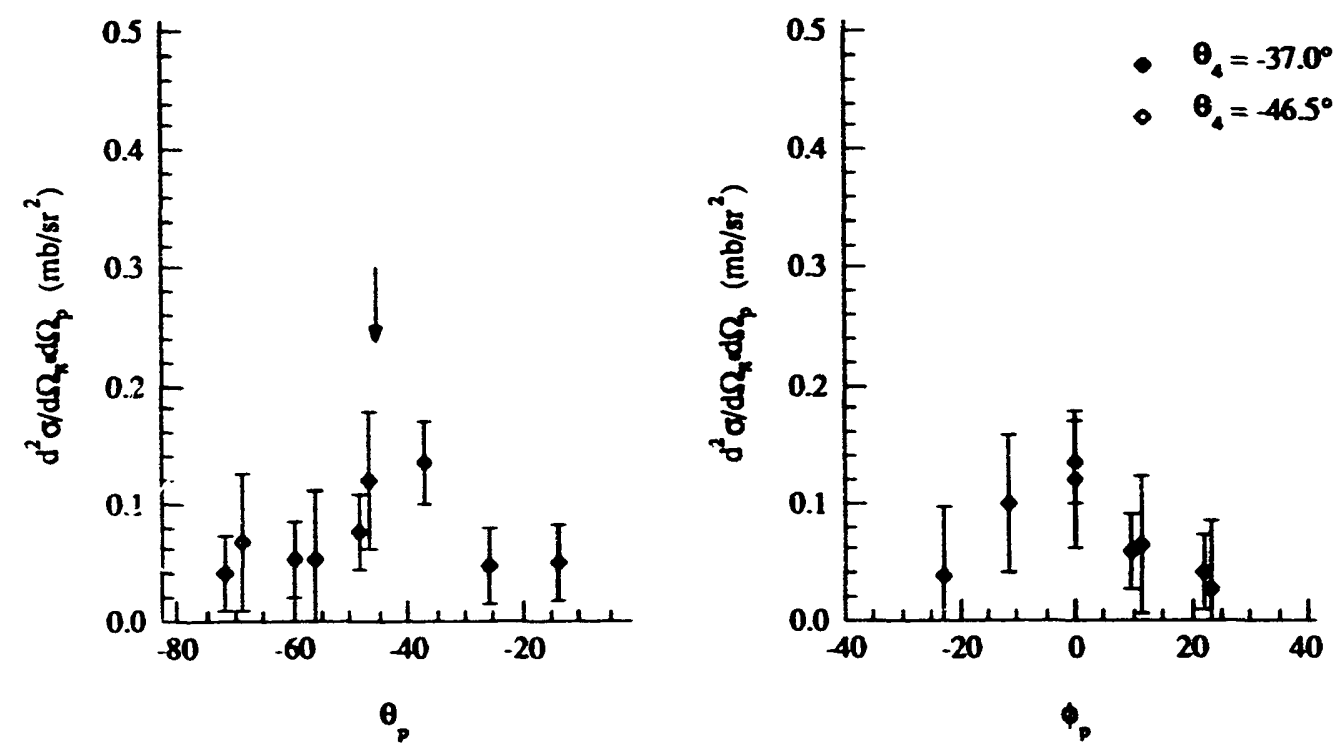

Figure 7.31: Proton angular distributions in the ${ }^{3} \mathrm{He}\left(\pi^{-}, \pi^{\circ} \mathrm{p}\right)$ reaction at $245 \mathrm{MeV}$ and $\theta_{2} 0^{0}=70^{\circ}$. An overall systematic uncertainty of $8.4 \%$ is not included in this figure. These data were accumulated with the proton detector array in the vertical configuration and the central detector placed at a laboratory angle corresponding to $\theta_{4}$. The in-plane and out-of-plane proton angles are labeled as $\theta_{p_{0}}$ and $\phi_{p}$ respectively. The arrow corresponds to the proton angle for free single charge exchange: $\pi^{+} n \rightarrow \pi^{0} \mathrm{p}$.
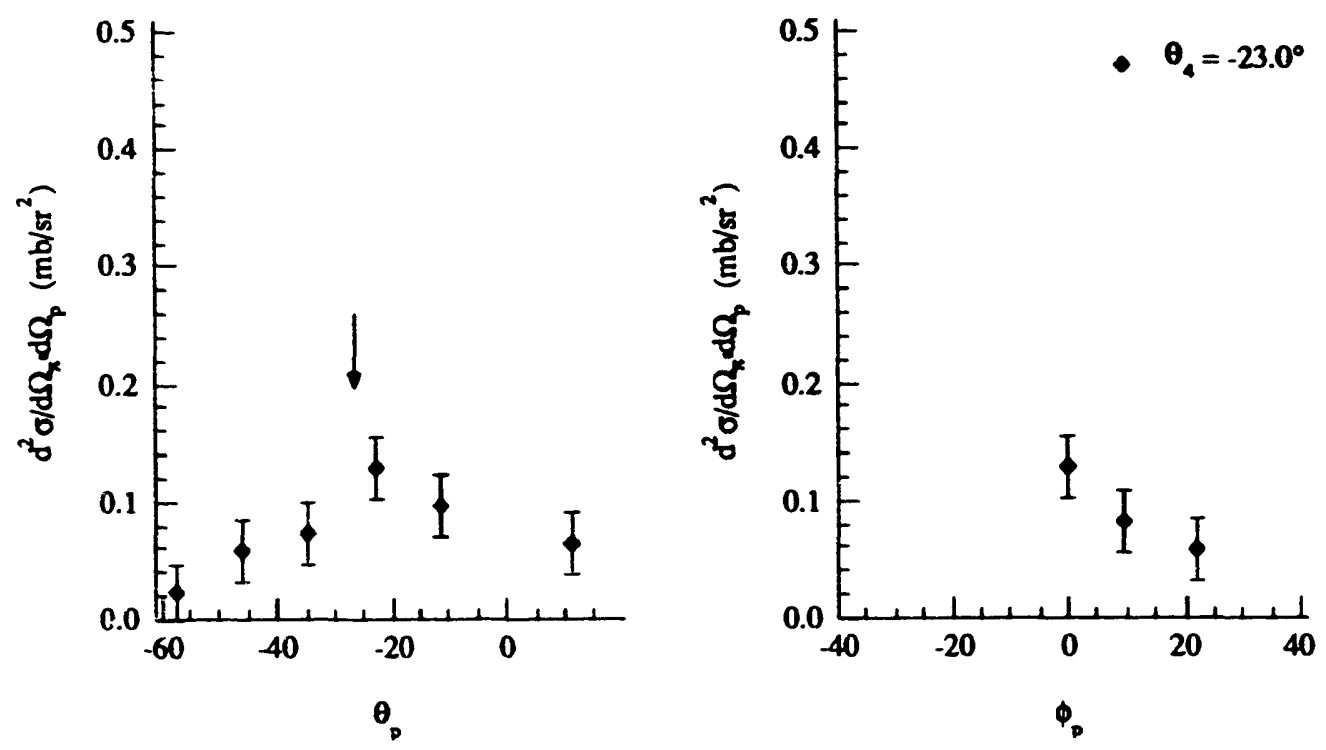

Figure 7.32: Proton angular distributions in the ${ }^{3} \mathrm{He}\left(\pi^{-}, \pi^{\circ} \mathrm{p}\right)$ reaction at $245 \mathrm{MeV}$ and $\theta_{R}=110^{\circ}$. An overall systematic uncertainty of $8.4 \%$ is not included in this figure. These data were accumulated with the proton detector array in the vertical configuration and the central detector placed at a laboratory angle corresponding to $\theta_{4}$. The in-plane and out-of-plane proton angles are labeled as $\theta_{p}$ and $\phi_{p}$ respectively. The arrow corresponds to the proton angle for free single charge exchange: $\pi^{+} \mathbf{n} \rightarrow \pi^{0} \mathbf{p}$. 

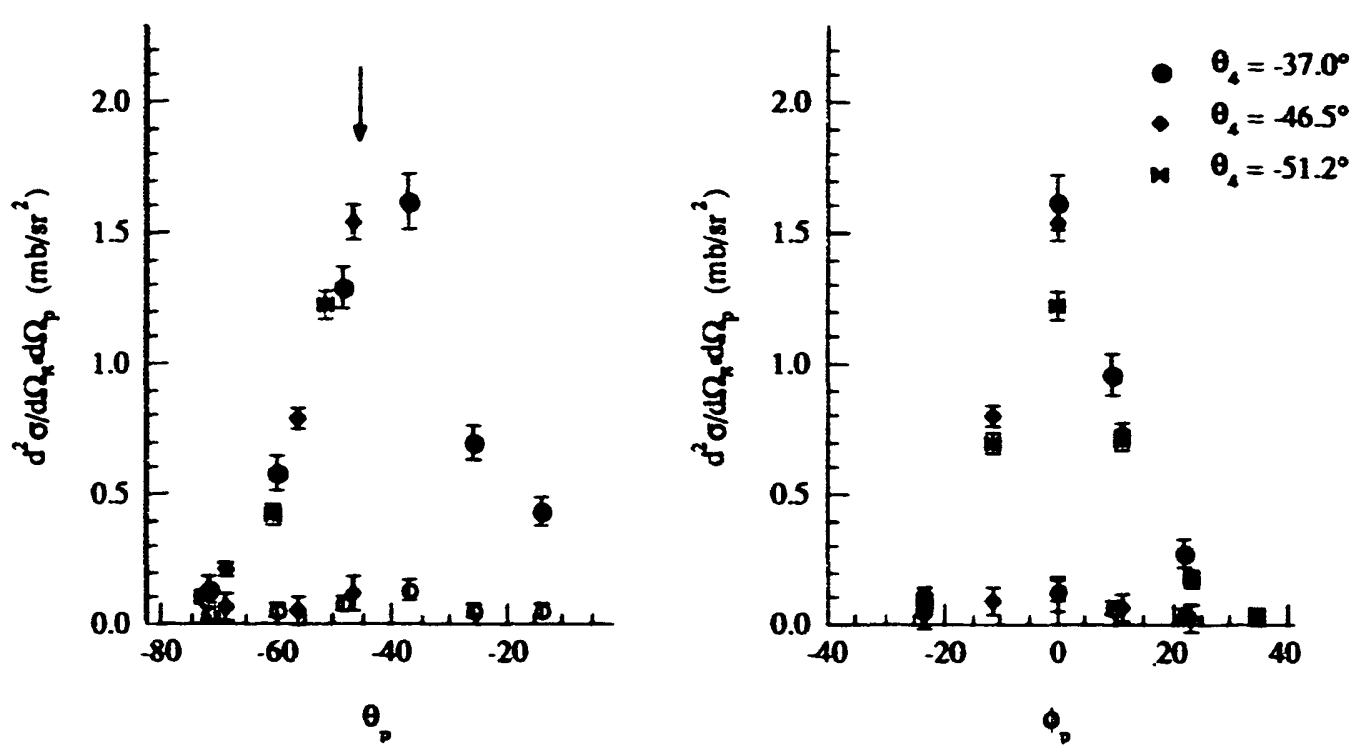

Figure 7.33: Proton angular distributions in the ${ }^{3} H e\left(\pi^{+}, \pi^{\circ} \mathrm{p}\right)$ (solid points) and ${ }^{3} \mathrm{He}\left(\boldsymbol{\pi}^{-}, \pi^{0} \mathrm{p}\right)$ (hollow points) reactions at $245 \mathrm{MeV}$ and $\theta_{x^{\circ}}=70^{\circ}$. Except for the measurements at $\theta_{4}=-37.0^{\circ}$, these data were accumulated with the proton detector array in the vertical configuration and the central detector placed at a laboratory angle corresponding $10 \theta_{4}$. The in-ptane a.d out-of-plane proton angles are labeled as $\theta_{p}$ and $\phi_{p}$ respectively. The arrow corresponds to the proton angle for free single charge exchange: $\pi^{*} n \rightarrow x^{0} p$.
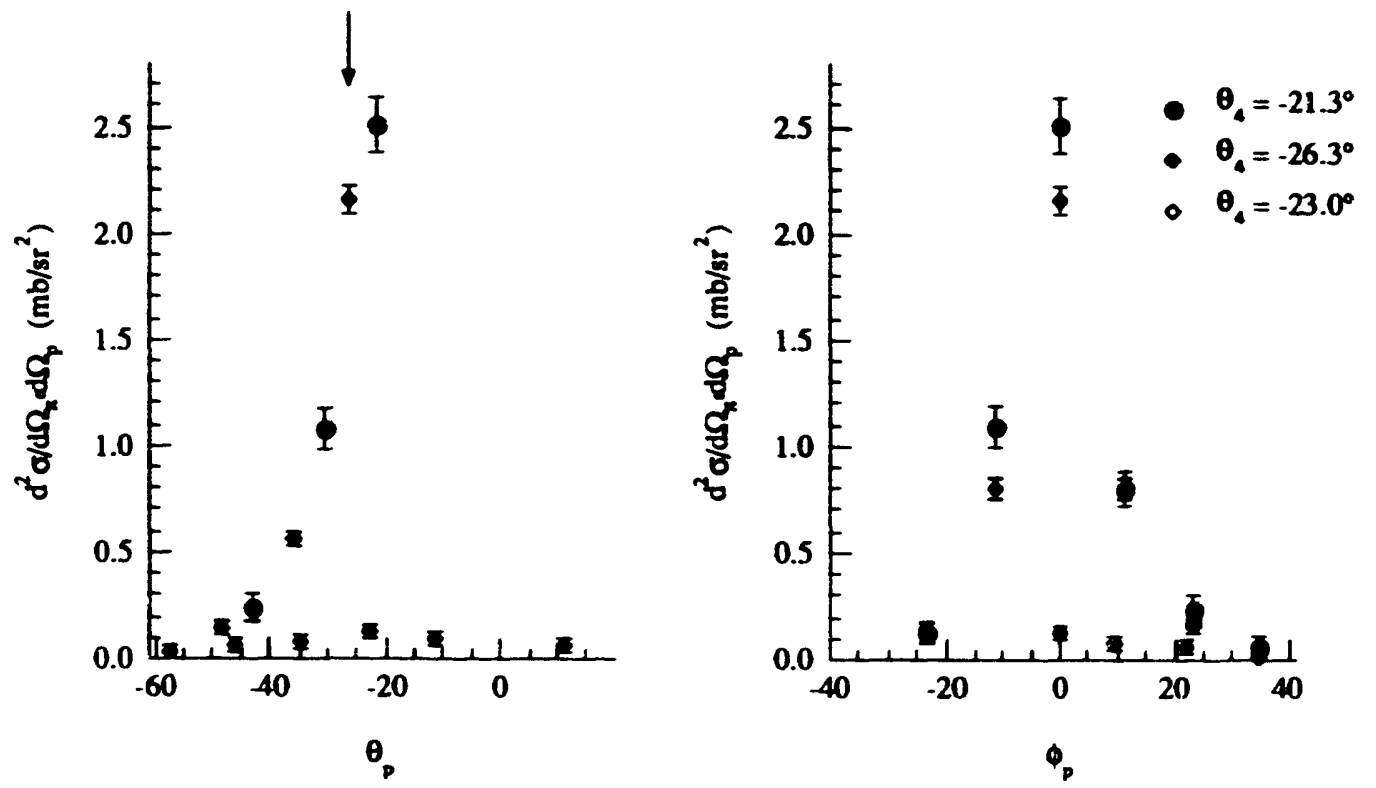

Figure 7.34: FTcion angular distributions in the ${ }^{3} \mathrm{He}\left(\pi^{4}, \pi^{0} \mathrm{p}\right)$ (solid points) and ${ }^{3} \mathrm{He}\left(\pi^{-}, \pi^{0} \mathrm{p}\right)$ (hollow points) reactions at $245 \mathrm{MeV}$ and $\theta_{x^{0}}=110^{\circ}$. These data were accumulated with the proton detector array in the vertical configuration and the central detector placed at a laboratory angle corresponding to $\theta_{4}$. The in-plane and out-of-plane proton angles are labeled as $\theta_{p}$ and $\phi_{p}$ respectively. The arrow corresponds to the proton angle for free single charge exchange: $\pi^{\dagger} n \rightarrow \pi^{\circ} \mathrm{p}$. 


\section{$\pi^{0}$ Angular Distribution in the ${ }^{3} H e\left(\pi^{*}, x^{0} p\right)$ Reaction}

The quasi-free proton angles and singly differential cross sections were determined from fitting the proton angular distributions by a two dimensional gaussian function of the form

$$
\frac{d^{2} \sigma}{d \Omega_{n} \alpha \Omega_{p}}=\frac{A}{2 \pi \sigma^{2}} \cdot \exp \left(-\frac{\left(\theta_{p}-\left\langle\theta_{p}\right\rangle\right)}{2 \sigma^{2}}\right) \cdot \exp \left(-\frac{\phi_{p}}{2 \sigma^{2}}\right)
$$

where the volume of the two dimensional gaussian $A$, its widh $\sigma$, and position of the quasi-free proton angle $\left\langle\theta_{p}\right\rangle$ were variable parameters of the fit and $\theta_{p}$ and $\phi_{p}$ are the in-plane and out-of-plane proton angles respectively.

The best fit to the data was determined by performing a least squares minimization of the gaussian statistic, $\chi^{2}$, using subroutines from the MINUT analysis package." An example of this fil is shown in Figure 7.35. This gaussian was constrained to be symmetric about the nominal quasi-free proton angle. These proton angular distributions were numerically integrated over the proton solid angle to determine the singly differential cross section, $d \sigma / d \mathbf{\Omega}_{\mathbf{x}^{\circ}}$. A list of the quasi-free proton angles and their deviations from the free proton angle are presented in Table 7.6. The $\pi^{0}$ angular distribution for the $\mathrm{He}\left(\pi^{*}, \pi^{\circ} \mathrm{p}\right)$ reaction is shown in Figure 7.36 and presented in Table 7.6.
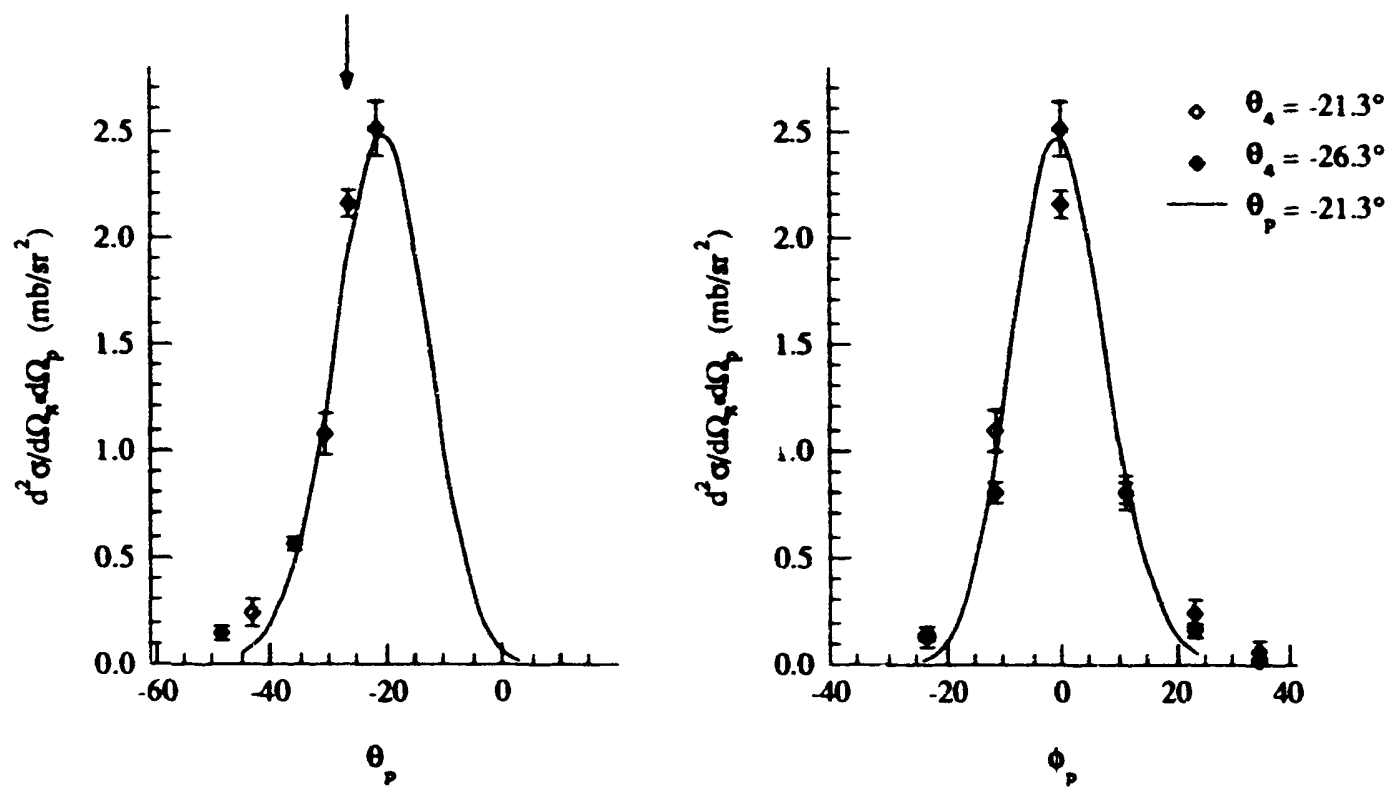

Figure 7.35: Comparison of two dimensional gaussian fit (solid line) with the measured proton angular distribution from ${ }^{3} \mathrm{He}\left(\pi^{\circ}, \pi^{\circ} \mathrm{p}\right)$ at $245 \mathrm{MeV}$ and $\theta_{x^{\circ}}=110^{\circ}$. The in-plane and out-of-plane proton angles are labeled as $\theta_{p}$ and $\phi_{p}$ respectively. 


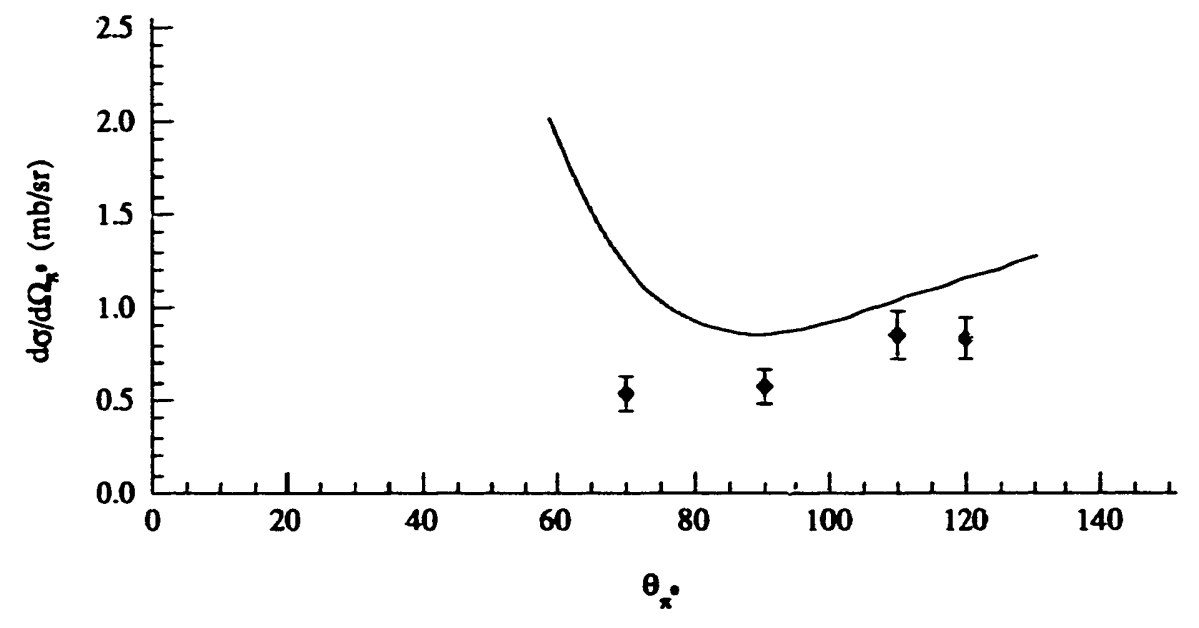

Figure 7.36: Differential cross section for ${ }^{3} \mathrm{He}\left(\pi^{+}, \pi^{0} \mathrm{p}\right)$ at $245 \mathrm{MeV}$. The curve corresponds to a $\pi \mathrm{N}$ phase shift calculation of the differential cross section for the free single charge exchange reaction: $\pi^{-} p \rightarrow \pi^{0} n$.

\begin{tabular}{|l|c|c|c|c|}
\hline \multicolumn{5}{|c|}{${ }^{3} \mathrm{He}\left(\pi^{*}, \pi^{0} \mathrm{p}\right)$ at $\mathrm{T}_{\mathrm{z}}=245 \mathrm{MeV}$} \\
\hline $\begin{array}{c}\theta_{\pi^{\prime}} \\
\text { (deg.) }\end{array}$ & $\begin{array}{c}\theta_{f} \\
(\mathrm{deg} .)\end{array}$ & $\begin{array}{c}\left(\theta_{p}\right) \\
(\mathrm{deg} .)\end{array}$ & $\begin{array}{c}\Delta \theta_{p} \\
(\text { deg.) }\end{array}$ & $\begin{array}{c}d \sigma / d \Omega_{x^{\prime}} \\
(\mathrm{mb} / \mathrm{sr})\end{array}$ \\
\hline 70 & -45.6 & $-42.5 \pm 0.5$ & $-3.1 \pm 0.5$ & $0.53 \pm 0.07 \pm 0.05(0.09)$ \\
\hline 90 & -35.4 & $-31.0 \pm 0.5$ & $-4.4 \pm 0.5$ & $0.58 \pm 0.08 \pm 0.05(0.09)$ \\
\hline 110 & -26.3 & $-21.0 \pm 0.5$ & $-5.3 \pm 0.5$ & $0.86 \pm 0.11 \pm 0.07(0.13)$ \\
\hline 120 & -22.2 & $-17.5 \pm 0.5$ & $-4.7 \pm 0.5$ & $0.84 \pm 0.10 \pm 0.07(0.12)$ \\
\hline
\end{tabular}

Table 7.6: Differential cross sections for the ${ }^{3} \mathrm{He}\left(\pi^{+}, \pi^{0} \mathrm{p}\right)$ reaction at $\mathrm{T}_{\pi}=245 \mathrm{MeV}$. The uncertainties in the differential cross section originate from the uncertainty in the fit (first term) and the overall normalization uncertair ty (second term). The total uncertainty is given in parentheses. The columns labeled " $\theta_{p}$ " and " $\left\langle\theta_{p}\right\rangle$ " represent the proton angles for the free single charge exchange and the quasi-free single charge exchange processes respectively. The proton angles for the quasi-free charge exchange reaction were determined from fitting the proton angular distributions by a two dimensional gaussian. The difference between the free and quasi-free proton angles is presented in the column labeled " $\Delta \theta_{p}$ ". 
Comparisons with Previous Coincidence Measurements of Pion Quasi-elastic Scattering on

${ }^{3} \mathrm{He}:{ }^{3} \mathrm{He}\left(\pi^{+}, \pi^{+} \mathrm{p}\right)$

Apart from this work, only one other experiment to measure quasi-free pion-induced proton knockout on ${ }^{3} \mathrm{He}$ has taken place. In this experiment, which was performed by Klein et al.. ${ }^{41.42}$ quasi-elastic pion scattering on ${ }^{3} \mathrm{He},{ }^{3} \mathrm{He}\left(\pi^{+}, \pi^{+}\right)$and ${ }^{3} \mathrm{He}\left(\pi^{*}, \pi^{+} \mathrm{p}\right)$, was observed over a range of outgoing pion scattering angles .., $\mathrm{m} 45^{\circ}$ to $\mathrm{i} 35^{\circ}$ and incident pion energies from 170 to $320 \mathrm{MeV}$. This experiment employed a magnetic spectrometer and a single scintillation counter, which had a solid angle of $110 \mathrm{msr}$, to detect protons in coincidence with charged pions. The proton detector was positioned at a laboratory angle corresponding to quasi-free kinematics.

Comparisons between the single-arm and coincidence measurements from this work and the quasi-elastic pion scattering measurements by Klein et $a l^{41}$ are shown in Figures 7.37 and 7.38. In Figures $7.37 \mathrm{a}$ and $7.37 \mathrm{~b}$, the quasi-elastic scattering cross sections have been renormalized according to the expected isospin dependence of charge exchange relative to the quasi-elastic scattering processes on ${ }^{3} \mathrm{He}$ in the $\Delta$-resonance region. Accounting for the two protons in ${ }^{3} \mathrm{He}$, the expected isospin dependence for the quasi-elastic knockout measurements relative to quasi-free single charge exchange measurements is,

$$
\begin{array}{r}
\sigma\left(\pi^{+}, \pi^{+} p\right): \sigma\left(\pi^{+}, \pi^{0} p\right)=(2 \cdot 9): 2 . \\
=9: 1
\end{array}
$$

The general shape and overall magnitude of the quasi-free peaks for both of the single-arm measurements are in good agreement from the center of the quasi-free peak through the high energy tail. The enhancement of the charge exchange over the quasi-elastic scattering cross section on the low energy side of the quasi-free peak may be a signature of multiple scattering contributions and other higher order processes, such as $\Delta \mathrm{N}$ interactions. As mentioned previously, multiple scattering is expected to play a more important role in the charge exchange process than in the quasi-elastic scattering process because of the weaker coupling to the $\mathrm{T}=0$ absorption channel for the former channel.

The principal difference between the renormalized triply differential cross sections for quasielastic scattering on ${ }^{3} \mathrm{He}$ and the quasi-free single charge exchange cross sections shown in Figure $7.37 \mathrm{~b}$ is the magnitude of the quasi-free peak. This discrepancy may be attributed in part to the differences in 
proton detector design for the two measurements. In the coincidence measurements by Klein et al. ${ }^{42}$ the single proton detector spanned a larger solid angle (110 msr) than the individual proton detectors $(8.46 \mathrm{msr})$ used in the single charge exchange measurements which have been presented in this work. Therefore, these coincidence measurements from quasi-free single charge exchange are more sensitive to the position of the proton detector than the quasi-elastic scattering measurements. The triply differential cross section for the single charge exchange measurement shown in Figure $7.37 \mathrm{~b}$ corresponds to a proton angle $\left(-46.5^{\circ}\right)$ that is slightly larger than the nominal quasi-free angle $\left(-42.5^{\circ}\right)$. This circumstance may account for some portion of the difference in magnitude between the coincidence cross sections. This angular offset could cause a deviation of the value for the doubly differential cross section of $\sim 15 \%$. This estimate is based on an extrapolation of the proton angular distribution, shown in Figure 7.27, from the actual detector position to the nominal quasi-free angle. If the missing strength is assigned to the region of the peak in the triply differential cross section, this would correspond to a peak height of $\sim 39 \mu \mathrm{b} / \mathrm{MeV} \cdot \mathrm{sr}^{2}$ assuming a peak width of $12 \mathrm{MeV}$. This estimated peak height would be in agreement with the re-scaled quasi-elastic measurement.

Another possible cause of the apparent suppression of the charge exchange relative to the non-char ${ }_{0}$ e exchange coincidence measurement could be Pauli blocking. Since the incident $\pi^{+}$exchanges one unit of charge with the ${ }^{3} \mathrm{He}$ nucleus in single charge exchange, the final state of the single charge exchange coincidence measurement consists of three protons whereas the final state of the inelastic measurement consists of only two protons and a neutron. Therefore, Pauli blocking, which would affect the proton distribution at forward angles, may also contribute to the apparent suppression of the quasi-free single charge exchange peak. Apart from this difference, the widths and peak positions of the quasi-free peaks for both measurements are in very good agreement.

The outgoing pion energy spectra from the single-arm quasi-free single charge exchange and elastic scattering measurements are dominated by a broad peak associated with the quasi-free knockout process. In Figure 7.38, pion energy spectra from the coincidence measurements have been renormalized so that the peak heights of the single-arm and coincidence cross sections are the same. The general shape 
of the coincidence and single-arm spectra for bnth channels are similar apart from the enhancement in the single-arm spectra at low outgoing pion kinetic energies which may be due to multiple interaction processes. Again, the larger deviation at low outgoing pion energies in the single charge exchange spectra may be an indication of a larger contribution from these more complex processes to the charge exchange channel.

a)

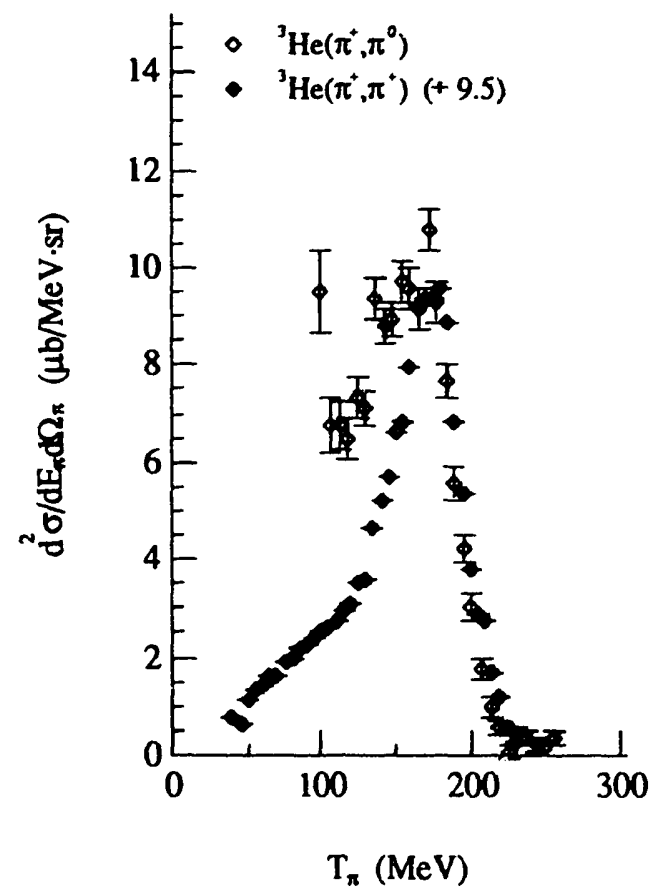

b)

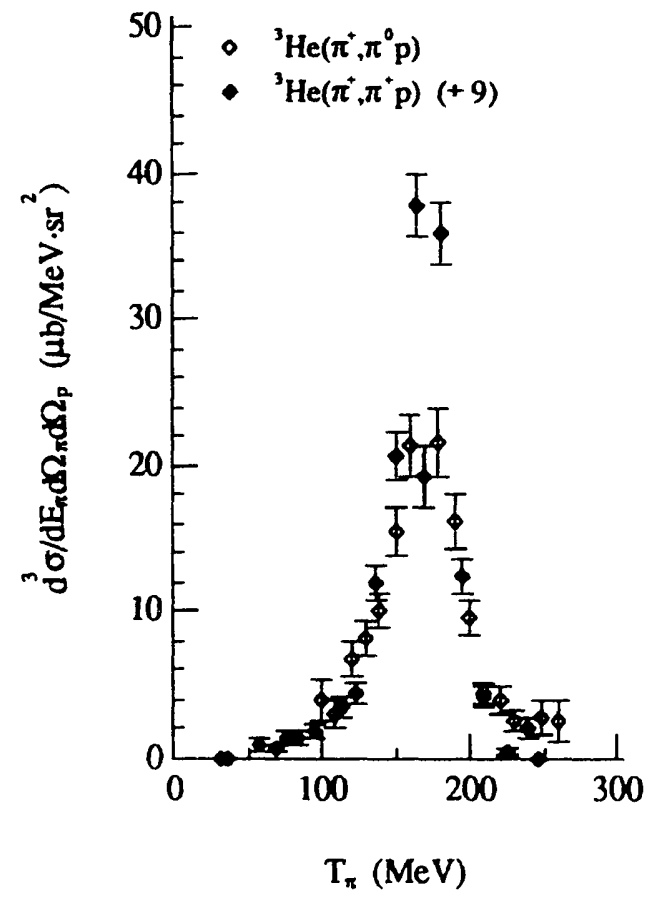

Figure 7.37: Comparisons of quasi-free pion charge exchange and scattering on ${ }^{3} \mathrm{He}$ : a) ${ }^{3} \mathrm{He}\left(\pi^{+}, \pi^{0}\right)$ at 245 $\mathrm{MeV}$ and $\theta_{\pi^{0}}=70^{\circ}$ from this work and ${ }^{3} \mathrm{He}\left(\pi^{*}, \pi^{\dagger}\right)$ at $270 \mathrm{MeV}$ and $\theta_{\pi^{0}}=75^{\circ}$ by Klein et al. and b) ${ }^{3} \mathrm{He}\left(\pi^{+}, \pi^{0} \mathrm{p}\right)$ at $245 \mathrm{MeV}, \theta_{\pi^{0}}=70^{\circ}$, and $\theta_{\mathrm{p}}=-46.5^{\circ}$ from this work and ${ }^{3} \mathrm{He}\left(\pi^{+}, \pi^{+} \mathrm{p}\right)$ at $270 \mathrm{MeV}, \theta_{\pi^{0}}=75^{\circ}$, and $\theta_{p}=-43.0^{\circ}$ by Klein et al. ${ }^{41}$ 
a)

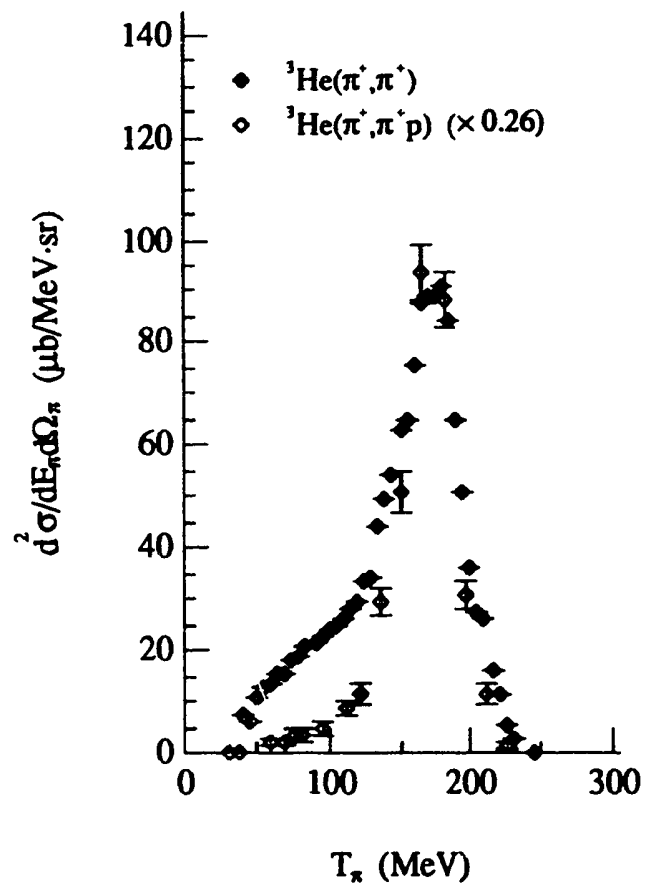

b)

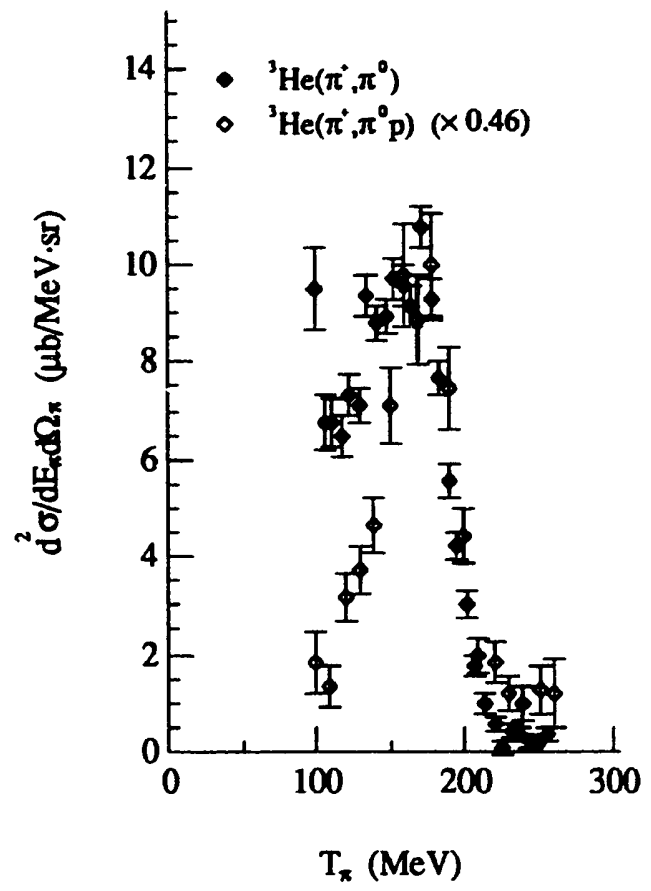

Figure 7.38: Comparisons of single-arm and coincidence measurements of quasi-free pion charge exchange and scattering on ${ }^{3} \mathrm{He}$ : a) ${ }^{3} \mathrm{He}\left(\pi^{+}, \pi^{+}\right)$and ${ }^{3} \mathrm{He}\left(\pi^{+}, \pi^{+} \mathrm{p}\right)$ at $270 \mathrm{MeV}$ and $\theta_{\pi^{0}}=75^{\circ}$ by Klein et al. ${ }^{41}$ and b) ${ }^{3} \mathrm{He}\left(\pi^{+}, \pi^{9}\right)$ and ${ }^{3} \mathrm{He}\left(\pi^{+}, \pi^{0} \mathrm{p}\right)$ at $245 \mathrm{MeV}$ and $\theta_{\pi^{0}}=70^{\circ}$ from this work. The proton angles for the non-charge exchange and charge exchange measurements were $-43.0^{\circ}$ and $-46.5^{\circ}$ respectively. 


\section{Chapter 8: Comparisons of Inclusive Pion Single Charge Exchange Measurements with Theoretical Calculations}

In this Chapter, inclusive pion single charge exchange and inelastic scattering measurements on

${ }^{3} \mathrm{He}$ will be compared with results from impulse approximation calculations. The contribution of quasi-free knockout mechanisms to inclusive charge exchange and inelastic scattering cross sections will be estimated based on these calculations. Comparisons of distorted wave impulse approximation (DWIA) predictions with charge exchange and previous non-charge exchange measurements will be shown. Evidence of enhanced multiple scattering contributions in the inclusive charge exchange measurements relative to the non-charge exchange measurements will be discussed. The isospin scaling behavior for single scattering processes in these measurements will also be investigated.

First, a brief description of the impulse approximation formalism will be provided. Then, plane wave impulse approximation (PWIA) and DWIA predictions for doubly differential cross sections from the single-arm charge exchange measurements at an incident pion energy of $245 \mathrm{MeV}$ and $\pi^{0}$ laboratory scattering angles from $62^{\circ}$ to $128^{\circ}$ will be presented. Finally, comparisons between DWIA predictions for ${ }^{3} \mathrm{He}\left(\pi^{+}, \pi^{9}\right)$ cross sections from this work and predictions for ${ }^{3} \mathrm{He}\left(\pi^{ \pm}, \pi^{+}\right)$cross sections and a recent work by Yuly ${ }^{6}$ will be presented.

\section{Impulse Approximation}

Doubly differential cross sections for inclusive pion charge exchange on ${ }^{3} \mathrm{He}$ were computed using a factorized impulse approximation approach. These calculations were performed using the formalism of Chant and Roos. ${ }^{66,67}$ In this approach, the interaction between a pion and ${ }^{3} \mathrm{He}$ nucleus is described as a 
single pion-nucleon interaction in which a nucleon is knocked out of the nucleus. This mechanism is often referred to as a quasi-free knockout process since multiple scatering as well as other higher order processes are neglected. Therefore, impulse approximation predictions can be used to estimate the contribution of single scattering processes to the single-arm measurements from this work. A diagram of this quasi-free knockout mechanism is shown in Figure 8.1.

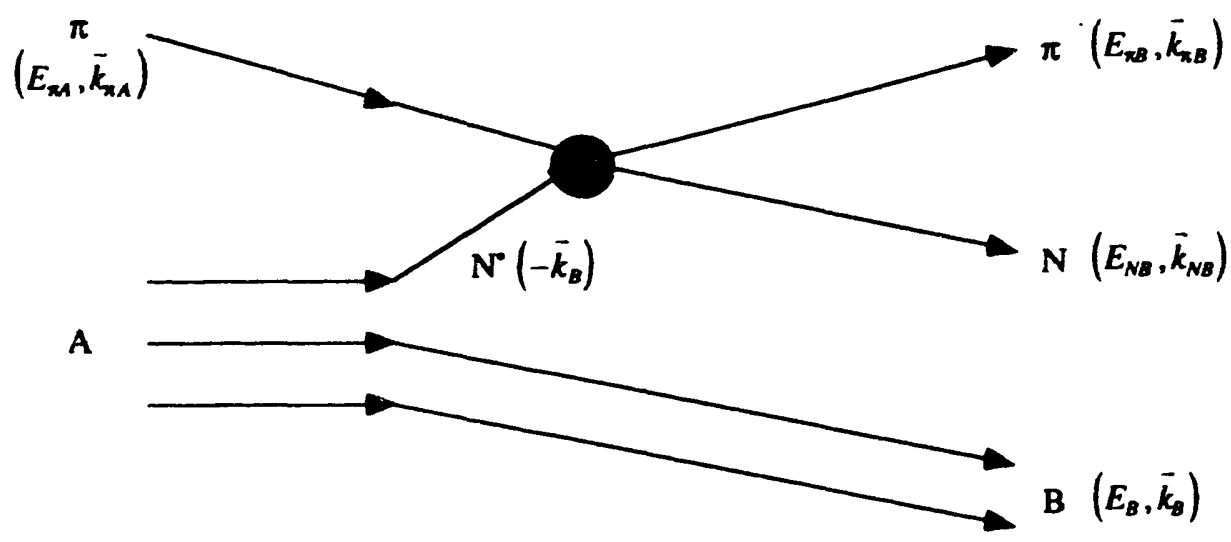

Figure 8.1: Schematic diagram of the quasi-free pion-induced nucleon knockout reaction: $A(\pi, \pi N) \mathbb{R}$. The kinematic variables $\bar{k}_{X A}, \bar{k}_{x B}$, and $\bar{k}_{N B}$ refer to the relative momenta of the incoming pion, the outgoing pion, and the outgoing nucleon respectively with regard to either A or B.

\section{Impulse Approximation Formalism}

The discussion of the impulse approximation formalism employed in this thesis is a summary of more detailed accounts from References 66 and 67 . Briefly, the general three body operator for the $\mathrm{A}(\pi, \pi \mathrm{N}) \mathrm{B}$ reaction is replaced by a two-body $\pi-\mathrm{N}$ transition operator. In addition, it is assumed that the in-medium two-body transition matrix associated with this operator varies slowly enough so that it may be replaced by the free $\pi \mathrm{N}$ transition matrix evaluated at its asymptotic momenta. The validity of this substitution is based on the assumption that the differences between a free pion-nucleon interaction and an in-medium pion-nucleon interaction are small. The binding energy of ${ }^{3} \mathrm{He}(7.7 \mathrm{MeV})$ is sufficiently small 
with respect to the incident pion kinetic energy of these measurements so that this approximation should be acceptable.

The factorized DWIA cross section for the $\mathrm{A}(\pi, \pi \mathrm{N}) \mathrm{B}$ reaction in which a nucleon with angular momentum $(\ell, j)$ is knocked out of nucleus A with ground state spin $J_{i}^{\pi}$ may be formulated as

$$
\frac{d^{3} \sigma}{d \Omega_{x} d \Omega_{N} d E_{x}}=\frac{2 \pi}{\hbar v_{x}} w_{B} C^{2} S_{i j} \mid\left\langle\left. i\right|^{2} \times \sum_{A}\left|T_{B A}^{\alpha a A}\right|^{2}\right.
$$

where $v_{x}$ is the relative velocity between the incoming pion and the target nucleus; $w_{B}$ is the energy density of the final state; $C^{2}$, an isospin Clebsch-Gordan coefficient which connects the initial and final states, is given by $\left\langle T_{N}, t_{N} ; T_{B}, t_{B} \mid T_{A} t_{A}\right\rangle ;$ and $S_{i j}$ is the single nucleon spectroscopic factor originating from the overlap of the initial and final nuclear states. The ground state spin of the residual nucleus B is given by $J_{f}^{x}$. The square of the half-off-the-energy siell $\pi \mathrm{N}$-matrix, summed and averaged over the initial and final nucleon spins, is expressed as $|\langle t\rangle|^{2}$. The distorted wave matrix element, $T_{B A}^{\text {aen }}$, may be written as

$$
T_{B A}^{\alpha e A}=\frac{1}{(2 \ell+1)^{1 / 2}} \int \chi_{\pi B}^{(-)}\left(\bar{k}_{x B}, \bar{r}\right) \chi_{N B}^{(-r}\left(\bar{k}_{N B}, \bar{r}\right) \chi_{M A}^{(+)}\left(\bar{k}_{x A}, \bar{r}\right) \phi_{A A}^{a}(\bar{r}) d^{3} r,
$$

where the distorted waves for the incoming pion, the outgoing pion, and the knocked out nucleon are represented by $\chi_{\pi A}, \chi_{\pi B}$, and $\chi_{N B}$ respectively. The recoil correction, $\gamma_{,}$is given by $\gamma=M_{B} /\left(M_{B}+1\right)$, where $M_{B}$ is the mass of the spectator nucleus in atomic mass units. The wavefunction of the bound single nucleon, $\phi_{\ell A}^{a}$, which arises from the overlap of the target nucleus $A$ and the residual nucleus $B$, has a principal quantum number $\alpha$, orbital angular momentum $\ell$, and third component of angular momentum $A$.

The triply differential cross section, presented in Equation 8.1 , may be rewritten in terms of the relative momenta of the knocked out nucleon $\left(\bar{k}_{N B}\right)$, the incoming pion $\left(\vec{k}_{M A}\right)$ and the scattered pion $\left(\vec{k}_{x B}\right)$ after explicitly including the energy density,

$$
\begin{aligned}
& \frac{d^{3} \sigma}{d \Omega_{\pi} d \Omega_{N} d E_{x}}=C^{2} S_{i j}|\langle\bar{t}\rangle|^{2}\left\{\frac{E_{\pi A} E_{\pi B} E_{N B}}{(2 \pi)^{5}(\hbar c)^{6}} \cdot \frac{k_{\Omega B} k_{N B} c}{k_{n A}}\right\} \\
& \times \frac{1}{1+\left(\frac{E_{N B}}{E_{B}}\right)\left(1-\frac{k_{M A}}{k_{N B}} \cos \theta_{N}+\frac{k_{n B}}{k_{N B}} \cos \left(\theta_{x}+\theta_{N}\right)\right)} \times \sum_{\Lambda}\left|T_{B A}^{\alpha A N}\right|^{2},
\end{aligned}
$$

where $\theta_{\pi}$ and $\theta_{N}$ are the angles between the incoming pion and the outgoing pion or nucleon respectively. The doubly differential cross section for quasi-free nucleon knockout is determined by integrating the triply differential cross section over the nucleon solid angle, 


$$
\frac{d^{2} \sigma}{d \Omega_{\mathrm{x}} d E_{\mathrm{z}}}(\pi, \pi N)=\int d \Omega_{N} \frac{d^{3} \sigma}{d \Omega_{\mathrm{z}} d \Omega_{N} d E_{\mathrm{z}}}
$$

Then, the inclusive doubly differential cross section for quasi-free single charge exchange on ${ }^{3} \mathrm{He}$ may be expressed as the sum of the cross sections for single charge exchange from each nucleon within the nucleus as either,

$$
{ }^{3} \mathrm{He}\left(\pi^{-}, \pi^{0}\right): \quad \frac{d^{2} \sigma}{d \Omega_{\mathrm{x}} d E_{\mathrm{z}}}=2 \cdot \frac{d^{2} \sigma}{d \Omega_{\mathrm{x}} d E_{\mathrm{z}}}\left(\pi^{-}, \pi^{0} n\right)
$$

or

$$
{ }^{3} \mathrm{He}\left(\pi^{+}, \pi^{9}\right): \quad \frac{d^{2} \sigma}{d \Omega_{\pi} d E_{\mathrm{x}}}=1 \cdot \frac{d^{2} \sigma}{d \Omega_{\pi} d E_{\mathrm{\pi}}}\left(\pi^{+}, \pi^{0} p\right)
$$

Initial Energy and Final Energy Prescriptions

The transition matrix element itself is expressed in terms of the free two body operator,

$$
|\langle\vec{b}\rangle|^{2}=\frac{1}{\left(2 s_{a}+1\right)\left(2 s_{b}+1\right)} \sum_{\sigma_{a} \sigma_{b} \sigma_{a} \sigma_{b}}\left\langle\left(\bar{k}_{f}, \sigma_{a^{\prime}}^{\prime}, \sigma_{b}^{\prime}\left|k_{f}^{(+)}\right| \bar{k}_{i}, \sigma_{a}, \sigma_{b}\right\rangle\right\}^{2} .
$$

where $t_{f}^{(+)}$is the free two body operator for $a+b$ scattering and $\bar{k}_{i}\left(\vec{k}_{f}\right)$ is the initial (final) relative momenta of particles $a$ and $b$. In the free scattering case, the momenta of the scattered particle in the initial and final states are the same. However, the initial and final relative momenta of the scattered pions are not the same for the off-energy shell transition. Two prescriptions were used to evaluate the half-off-theenergy shell transition matrix. The first prescription evaluates the on-shell matrix element at the value for the relative momenta between the pion and nucleon in the initial state, e.g.

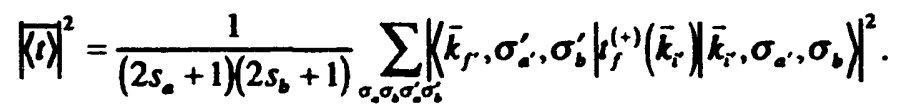

This is referred to as the initial energy prescription. The second prescription evaluates the matrix element at the value for the relative momenta in the final state (final energy prescription). Doubly differential cross sections for inclusive charge exchange on ${ }^{3} \mathrm{He}$ were computed using both of these prescriptions.

\section{Impulse Approximation Calculation}

The impulse approximation calculations presented in this thesis were executed using the three

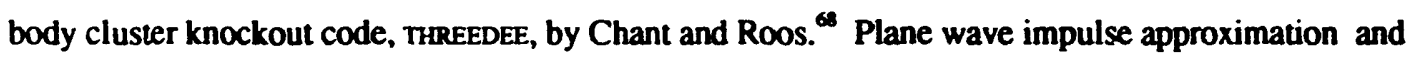
distorted wave impulse approximation calculations were performed. Doubly differential cross sections were determined by integrating the triply differential cross sections (Equations 8.1 and 8.3) using a 
Gauss-Legendre quadrature technique and weighting the integral by the number of the appropriate nucleon species within the nucleus as in Equations 8.5 and 8.6. Differential cross sections were computed by integrating the doubly differential cross sections over outgoing pion kinetic energy.

\section{PWIA Calculation}

In the plane wave approximation, distortions of the incident and scattered pion as well as the final state interactions of the outgoing nucleon are neglected. The wavefunctions of the outgoing nucleon and those for the incoming and outgoing pions are expressed in terms of plane waves of the form,

$$
\psi_{k}(\bar{r})=\frac{1}{(2 \pi)^{3 / 2}} e^{i \bar{i}}
$$

where $\bar{r}$ and $\vec{k}$ are the relative position and momentum of the particle respectively. Using the plane waye approximation, the distorted wave matrix element expressed in Equation 8.2 can be simplified as follows,

$$
T_{B A}^{\alpha A}=\frac{1}{(2 \ell+1)^{3 / 2}} \int e^{\bar{u}_{B} \cdot \bar{r}} \phi_{\ell A}^{a}(\bar{r}) d^{3} r,
$$

where $\bar{k}_{B}$ is the recoil momentum of the residual nucleus. This expression for the matrix element is proportional to the Fourier transform of the wavefunction of the bound single nucleon.

\section{DWIA Calculation}

In the distorted wave approximation, the wavefunctions of the incident and scattered pions are distorted by a phenomenological pion-nucleus optical potential. The wavefunction of the outgoing nucleon is modified by an optical potential which simulates the final state interactions between the knocked out nucleon and the residual nucleus.

The distorted pion wavefunctions are computed using a modified Klein-Gordon equation with a Kisslinger-type potential,

$$
\left(-\nabla^{2}+\mu^{2}\right) \chi_{\pi}=\left[E_{\mathrm{m}}^{2}-E_{\mu} V_{c}-U\right] \chi_{\pi}
$$

where

$$
U(r)=-A b_{0} p_{u}^{2} \rho(r)+A b_{1} \bar{\nabla} \cdot(\rho(r) \bar{\nabla}) .
$$

$A$ is the number of nucleons in the initial nucleus, $V_{c}$ is the Coulomb potential, $\mu$ is given by $\mu=m_{x} c / \hbar$, $\rho(r)$ is the nuclear density, and $b_{0}$ and $b_{1}$ are the complex phenomenological $\pi \mathrm{N} s$ - and $p$-wave parameters respectively. The parametrization of Coutingame and Holtkamp ${ }^{69}$ was used to determine the values for $b_{0}$ 
and $b_{r}$. This parametrization has met with reasonable success in reproducing $\pi^{ \pm}$elastic scattering data on nuclei ranging from ${ }^{9} \mathrm{Be}$ to ${ }^{200} \mathrm{~Pb}$ in the $\Delta$-resonance region, but has not been as successful with $\pi^{t}$ elastic scattering data on ${ }^{3} \mathrm{He} .{ }^{51,70}$ However, Khandake: ${ }^{71}$ has shown that calculations of inelastic $\pi^{+}{ }^{3} \mathrm{He}$ cross sections are much less sensitive to the exact values for these parameters than the elastic scattering cross sections.

In the present calculation, the optical potential parametrization of Nadasen et $a .^{72}$ was used to generate wavefunctions for the knocked out nucleon. This potential was determined from fitting proton elastic scattering data on nuclei ranging from Si to $\mathrm{Pb}$ over an energy range from 40 to $180 \mathrm{MeV}$. Previous DWIA calculations ${ }^{6}$ of pion inelastic scattering on ${ }^{3} \mathrm{He}$, which employed this potential, are in reasonably good agreement with existing measurements at incident pion energies and scattering angles similar to those of the single-arm charge exchange measurements of this work.

\section{Comparisons of Impulse Approximation Calculations with Experimental Results}

First, PWIA and DWIA calculations will be shown in comparison with a selection of the singlearm charge exchange results. Then, DWIA predictions for the $\pi^{0}$ angular distribution will be shown in comparison with a selection of the single-arm and coincidence measurements from this work. Finally, these predictions will be used to examine evidence of multiple scattering behavior in quasi-free pion single charge exchange and inelastic scattering on ${ }^{3} \mathrm{He}$.

\section{Comparisons of PWIA and DWIA Calculations with Inclusive Pion Single Charge Exchange}

\section{Results}

The PWIA and DWIA predictions for the doubly differential cross section, with a selection of the ${ }^{3} \mathrm{He}\left(\pi^{t}, \pi^{9}\right)$ measurements at an incident pion energy of $245 \mathrm{MeV}$ and $\pi^{0}$ laboratory scattering angles ranging from $62^{\circ}$ to $128^{\circ}$, are shown in Figures 8.2 through 8.4. DWIA predictions for the ${ }^{3} \mathrm{He}\left(\pi^{+}, \pi^{\circ}\right)$ differential cross section are shown in Figure 8.5 and presented in Tables 8.1 and 8.2. The DWIA prediction for the ${ }^{3} \mathrm{He}\left(\pi^{+} \pi^{0} \mathrm{p}\right)$ differential cross section is shown in comparison with experimental results in 
Figure 8.6 and listed in Table 8.3.

The PWIA predictions, shown in Figures 8.2 through 8.3, overestimate the value for the $\mathrm{He}^{3}\left(\pi^{*}, \pi^{9}\right)$ doubly differential cross sections at every $\pi^{0}$ scattering angle by nearly a factor of two. This is not surprising since the PWIA calculation ignores distortions of either the incoming or outgoing pion waves. It is these distortions which mimic the processes that remove incident and scattered pions from the charge exchange channel and thus cause a decrease in the value for the computed cross sections.

In general, the agreement between the DWIA calculations and the experimental results for the magnitude of the doubly differential cross sections at the quasi-free peak is quite good. Although the DWIA computations overestimate the magnitude of the quasi-free peak by nearly $30 \%$ at the smallest and largest $\pi^{\circ}$ laboratory scattering angles of $62^{\circ}$ and $128^{\circ}$ respectively. These calculations also reproduce the high energy shoulder of the doubly differential cross section quite well. However, at outgoing $\pi^{0}$ kinetic energies below the quasi free peak, the DWLA predictions and experimental results begin to diverge. At the lowest energy point, the DWIA calculations underestimate the doubly differential cross section by nearly an order of magnitude at all $\pi^{0}$ scattering angles. This is perhaps not surprising since multiple scautering processes, which are not included in the DWIA calculations, are expected to cause an enhancement of the doubly differential cross section at $\pi^{0}$ kinetic energies below the quasi-free peak. However, the limited coverage and poor statistical quality of the single-arm measurements at low outgoing $\pi^{0}$ energies prevent any quantitative conclusions about multiple scattering contributions.

\section{$\pi^{l}$ Angular Distributions from the ${ }^{3} \mathrm{He}\left(\pi^{2}, \pi^{2}\right)$ and ${ }^{3} \mathrm{He}\left(\pi^{2}, \pi^{2} p\right)$ Reactions}

Singly differential cross sections were computed by integrating the DWIII predictions for the doubly differential cross sections over outgoing $\pi^{0}$ kinetic energy. The uncertainty in the DWIA results arise from the extrapolation of the doubly differential cross section to zero at the high energy tail where the two body $\pi \mathrm{N} t$-matrix is not available. These results are presented in Tables 8.1 and 8.2 and shown in Figure 8.5. The agreement between the single-arm experimental results and the DWIA predictions is not very good. It is difficult to speculate on this disagreement without further knowledge of the magnitude of the unmeasured portion of the spectrum below the quasi-free peak. The uncorrected angular distributions 

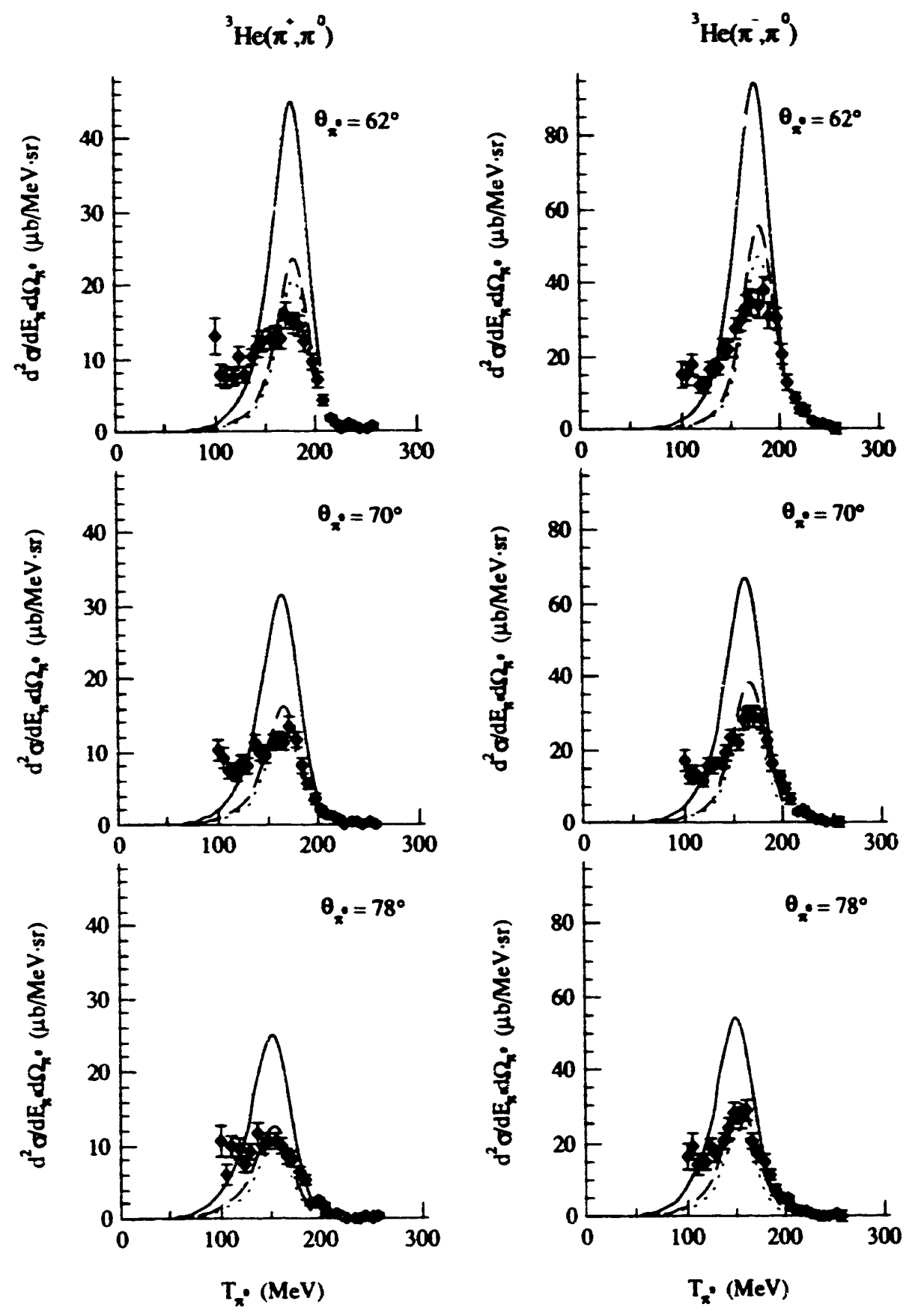

Figure 8.2: Results of PWIA (solid line) and DWIA calculations for $\pi^{\circ}$ energy spectra from the ${ }^{3} \mathrm{He}\left(\pi^{+}, \pi^{9}\right)$ (right column) and ${ }^{3} \mathrm{He}\left(\pi^{-}, \pi^{\circ}\right.$ ) (left column) reactions at $\pi^{\circ}$ laboratory scautering angles of $62^{\circ}, 70^{\circ}$, and $78^{\circ}$ and an incident pion energy of $245 \mathrm{MeV}$. The DWIA calculations were performed using both initial energy (doted line) and final energy (dashed line) prescriptions. 

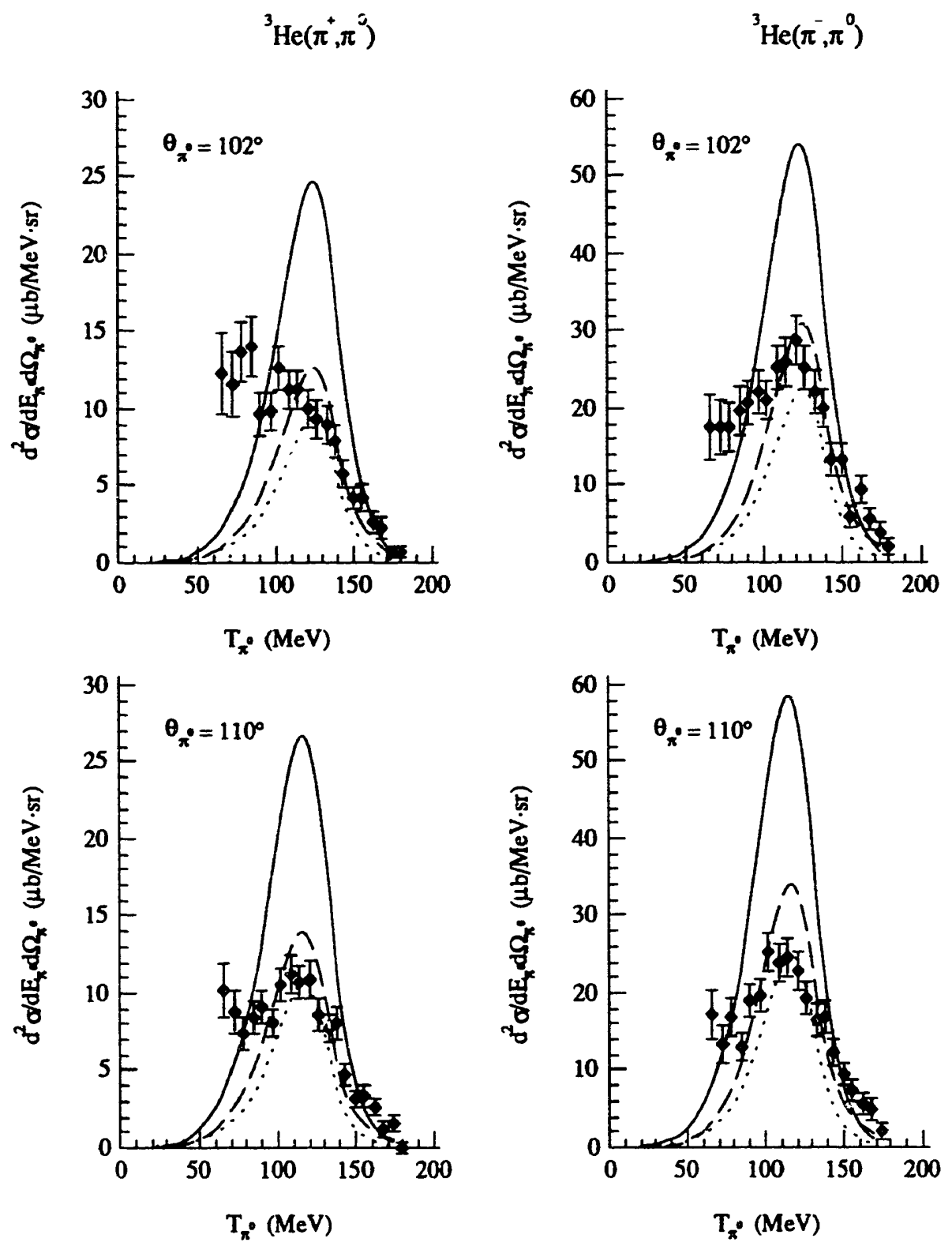

Figure 8.3: Results of PWIA (solid line) and DWIA calculations for $\pi^{0}$ energy spectra from the ${ }^{3} \mathrm{He}\left(\pi^{+}, \pi^{0}\right)$ (right column) and ${ }^{3} \mathrm{He}\left(\pi^{-}, \pi^{\circ}\right)$ (left column) rear a at $\pi^{\circ}$ laboratory scattering angles of $102^{\circ}$ and $110^{\circ}$ and an incident pion energy of $245 \mathrm{MeV}$. The $\mathrm{D}$ IA calculations were performed using both initial energy (dotted line) and final energy (dashed line) presstijaivivis. 

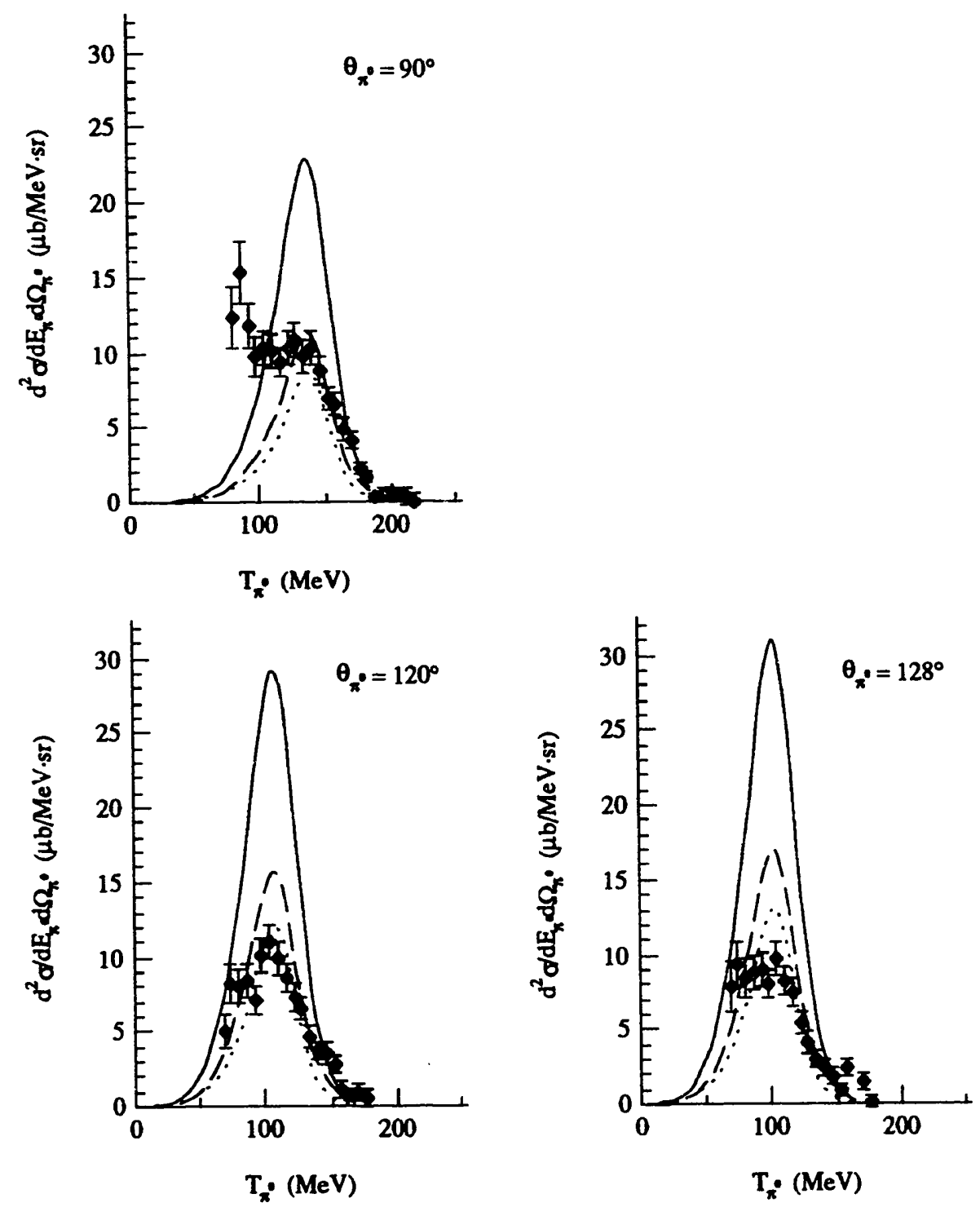

Figure 8.4: Results of PWIA (solid line) and DWIA calculations for $\pi^{\circ}$ energy spectra from the ${ }^{3} \mathrm{He}\left(\pi^{*}, \pi^{9}\right)$ reaction at $\pi^{\circ}$ laboratory scattering angles of $90^{\circ}, 120^{\circ}$ and $128^{\circ}$ and an incident pion energy of $245 \mathrm{MeV}$. The DWIA calculations were performed using both initial energy (dottcd line) and final energy (dashed line) prescription. 
were determined by integrating over the measured energy spectrum. The corrected distributions include an estimate of the unmeasured contribution based on a linear extrapolation from the lowest energy point to zero at an outgoing $\pi^{0}$ kinetic energy of zero.

A comparison of the DWIA result with the differential cross section from the ${ }^{3} \mathrm{He}\left(\pi^{+} ; \pi^{0} \mathrm{p}\right)$ coincidence measurement is presented in Table 8.3 and shown in Figure 8.6 The agreement between the DWIA prediction and this result is quite good. This agreement implies that the ${ }^{3} \mathrm{He}\left(\pi^{+}, \pi^{0} p\right)$ reaction can be described reasonably well by a single nucleon knockout model in the region of the quasi-free peak and that the discrepancies between the DWIA predictions and the single-arm measurements may be autributed to multiple scattering or more complex processes.

\begin{tabular}{|c|c|c|c|c|}
\cline { 2 - 5 } & \multicolumn{4}{|c|}{$d \sigma / d \Omega_{n}(\mathrm{mb} / \mathrm{sr})$} \\
\hline$\theta_{\pi^{\circ}}$ & \multicolumn{2}{|c|}{ Data } & \multicolumn{2}{c|}{ DWIA } \\
\cline { 2 - 5 }$($ deg.) & uncorrected & corrected & IEP & FEP \\
\hline 62 & $1.062 \pm 0.090$ & $1.452 \pm 0.400$ & $0.83 \pm 0.01$ & $0.97 \pm 0.01$ \\
\hline 70 & $0.821 \pm 0.070$ & $1.211 \pm 0.396$ & $0.59 \pm 0.00$ & $0.73 \pm 0.01$ \\
\hline 78 & $0.710 \pm 0.062$ & $1.145 \pm 0.439$ & $0.47 \pm 0.00$ & $0.61 \pm 0.00$ \\
\hline 90 & $0.947 \pm 0.082$ & $1.427 \pm 0.487$ & $0.43 \pm 0.00$ & $0.59 \pm 0.00$ \\
\hline 102 & $0.976 \pm 0.086$ & $1.356 \pm 0.390$ & $0.47 \pm 0.00$ & $0.65 \pm 0.00$ \\
\hline 110 & $0.817 \pm 0.070$ & $1.132 \pm 0.323$ & $0.51 \pm 0.00$ & $0.71 \pm 0.00$ \\
\hline 120 & $0.652 \pm 0.057$ & $0.887 \pm 0.242$ & $0.57 \pm 0.00$ & $0.78 \pm 0.00$ \\
\hline 128 & $0.593 \pm 0.053$ & $0.863 \pm 0.275$ & $0.60 \pm 0.00$ & $0.83 \pm 0.00$ \\
\hline
\end{tabular}

Table 8.1: Differential cross sections for ${ }^{3} \mathrm{He}\left(\pi^{+}, \pi^{0}\right)$ at $245 \mathrm{MeV}$. DWIA calculations were performed using the initial energy (IEP) and final energy prescriptions (FEP). The uncertainties in the calculations are the result of extrapolation of the energy spectra at endpoints where the two-body $t$-matrix is not available. 


\begin{tabular}{|c|c|c|c|c|}
\hline \multirow{3}{*}{$\begin{array}{c}\theta_{R} \\
\text { (deg.) }\end{array}$} & \multicolumn{4}{|c|}{$d \sigma / d \Omega_{n^{\prime}}(\mathrm{mb} / \mathrm{sr})$} \\
\hline & \multicolumn{2}{|c|}{ Data } & \multicolumn{2}{|c|}{ DWIA } \\
\hline & uncorrected & corrected & IEP & FEP \\
\hline 62 & $2.54 \pm 0.21$ & $3.17 \pm 0.67$ & $1.87 \pm 0.03$ & $2.31 \pm 0.09$ \\
\hline 70 & $1.97 \pm 0.17$ & $2.65 \pm 0.70$ & $1.33 \pm 0.01$ & $1.72 \pm 0.03$ \\
\hline 78 & $1.73 \pm 0.15$ & $2.46 \pm 0.75$ & $1.06 \pm 0.00$ & $1.46 \pm 0.01$ \\
\hline 102 & $2.02 \pm 0.18$ & $2.56 \pm 0.56$ & $1.06 \pm 0.00$ & $1.54 \pm 0.01$ \\
\hline 110 & $1.74 \pm 0.15$ & $2.21 \pm 0.49$ & $1.15 \pm 0.00$ & $1.67 \pm 0.01$ \\
\hline
\end{tabular}

Table 8.2: Differential cross sections for ${ }^{3} \mathrm{He}\left(\pi^{-}, \pi^{9}\right)$ at $245 \mathrm{MeV}$. DWIA calculations were performed using the initial energy (IEP) and final energy prescriptions (FEP). The uncertainties in the calculations are the result of extrapolation of the energy spectra at endpoints where the two-body $t$-matrix is not available.

a)

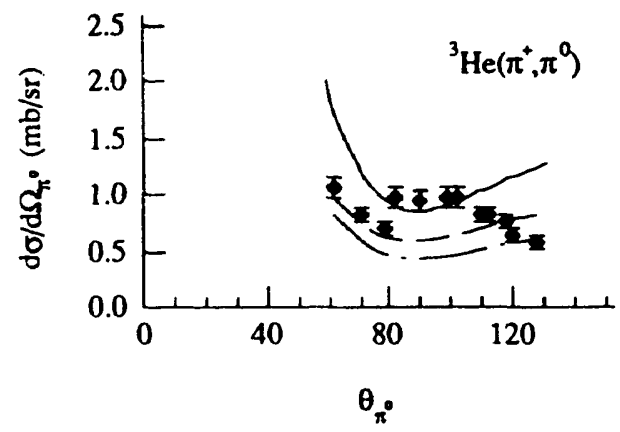

c)

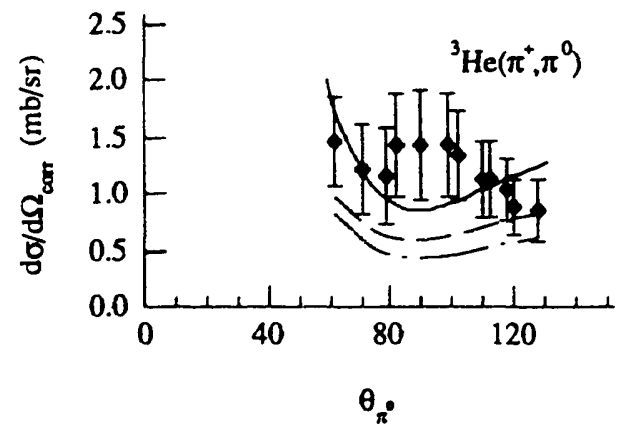

b)

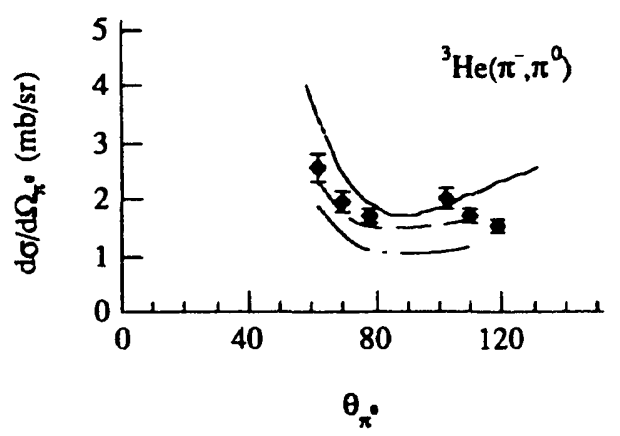

d)

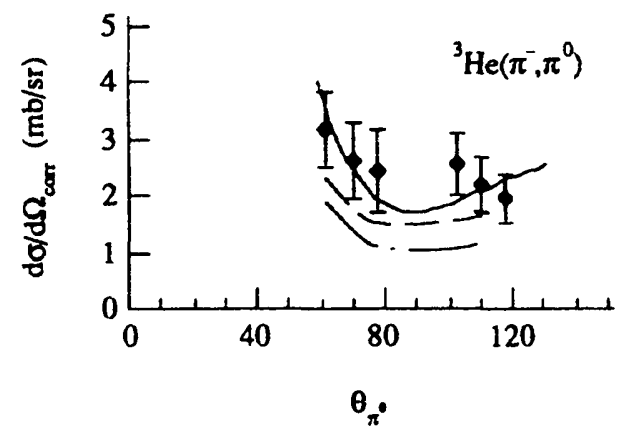

Figure 8.5: Differential cross sections for ${ }^{3} \mathrm{He}\left(\pi^{+}, \pi^{0}\right)$ (right) and ${ }^{3} \mathrm{He}\left(\pi^{+}, \pi^{0}\right)$ (left) at $\mathrm{T}_{\pi}=245 \mathrm{MeV}$. The solid curves represent the differential cross section for free single charge exchange scaled by the number of the appropriate nucleons. The dot-dashed and dashed curves are the results of DWIA calculations using the initial and final energy prescriptions respectively. No attempt has been made in figures a) and b) to correct the experimental results for the unmeasured contribution at low outgoing pion kinetic energies. In figures c) and d), an estimate of the missing strength, based on a linear extrapolation of the doubly differential cross section from the lowest energy point to zero at an outgoing $\pi^{0}$ energy of zero, has been included. 


\begin{tabular}{|c|c|c|c|}
\cline { 2 - 4 } & \multicolumn{3}{|c|}{$d \sigma / d \Omega_{\pi}(\mathrm{mb} / \mathrm{sr})$} \\
\hline$\theta_{R^{2}}$ & Data & \multicolumn{2}{|c|}{ DWIA } \\
\cline { 3 - 4 } (deg.) & & IEP & FEP \\
\hline 70 & $0.53 \pm 0.09$ & $0.59 \pm 0.00$ & $0.73 \pm 0.01$ \\
\hline 90 & $0.58 \pm 0.09$ & $0.43 \pm 0.00$ & $0.59 \pm 0.00$ \\
\hline 110 & $0.86 \pm 0.13$ & $0.51 \pm 0.00$ & $0.71 \pm 0.00$ \\
\hline 120 & $0.84 \pm 0.12$ & $0.57 \pm 0.00$ & $0.78 \pm 0.00$ \\
\hline
\end{tabular}

Table 8.3: Differential cross sections for ${ }^{3} \mathrm{He}\left(\pi^{+}, \pi^{0} \mathrm{p}\right)$ at $245 \mathrm{MeV}$. DWIA calculations were performed using the initial energy (IEP) and final energy prescriptions (FEP). The uncertainties in these calculations are the result of extrapolating the $\pi^{0}$ energy spectra from the high energy endpoints to an energy region where the two-body $t$-matrix is not available.

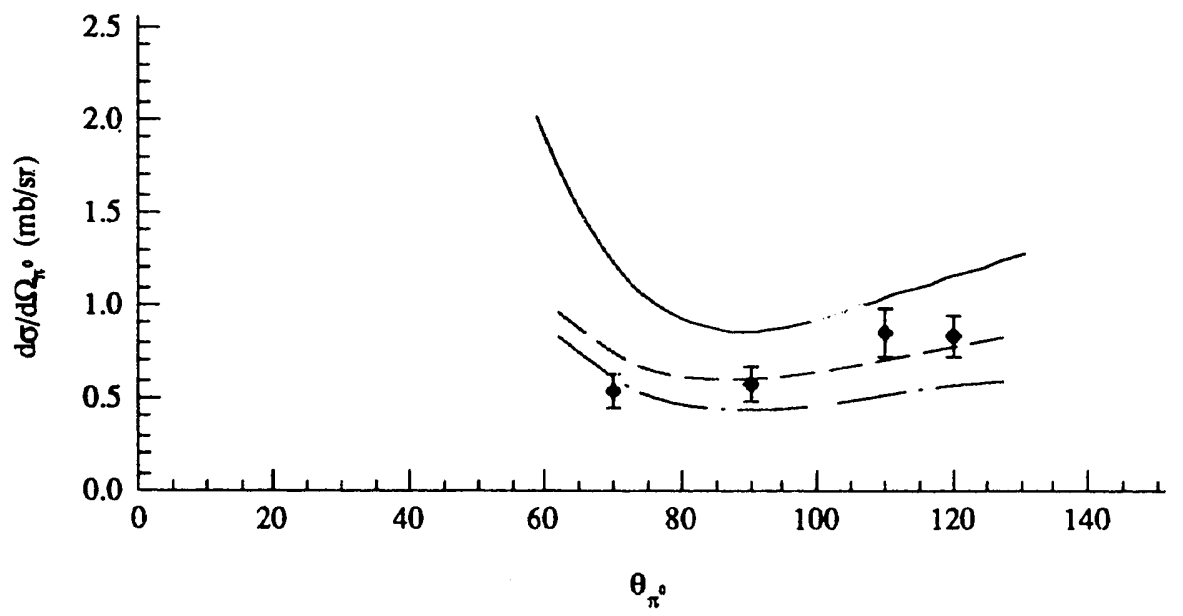

Figure 8.6: Differential cross sections for ${ }^{3} \mathrm{He}\left(\pi^{+}, \pi^{0} \mathrm{p}\right)$ at an incident energy of $245 \mathrm{MeV}$. The solid curve represents the differential cross section for free pion single charge exchange from $\pi \mathrm{N}$ phase shift calculations. The dot-dashed and dashed curves are the results of DWIA calculations using the initial and final energy prescriptions respectively. 


\section{Comparisons of Pion Single Charge Exchange and Inelastic Scattering Measurements on}

\section{${ }^{3}$ He with DWIA Calculations}

The inclusive single charge exchange measurements presented in this work constitute the sole experimental effort on quasi-free pion single charge exchange on ${ }^{3} \mathrm{He}$. The results from this work and a previous pion inelastic scattering measurement on ${ }^{3} \mathrm{He}$ will be shown in comparison with DWIA predictions.

The experimental ${ }^{6}$ study of pion inelastic scattering on ${ }^{3} \mathrm{He}$ took place at LAMPF using the Little Yellow Spectrometer system to detect scattered pions. A systematic measurement of the energy and angular dependence of the ${ }^{3} \mathrm{He}\left(\pi^{ \pm}, \pi^{+}\right)$reactions was performed at incident pion energies of 120,180 and $240 \mathrm{MeV}$ and pion laboratory scattering angles of $50^{\circ}, 80^{\circ}, 105^{\circ}$, and $130^{\circ}$. Doubly differential cross sections for pion inelastic scattering on ${ }^{3} \mathrm{He}$ at $240 \mathrm{MeV}$ and pion laboratory scattering angles of $80^{\circ}, 105^{\circ}$, and $130^{\circ}$ are shown in comparison with the results from this work and recent DWIA calculations in Figures 8.7 through 8.9.

\section{Indications of Multiple Scattering}

In Figures 8.7 through 8.9, the $y$-axes have been scaled according to the expected isospin relationship, which is based on the isospın dependence of the free pion-nucleon cross sections, between the inelastic and single charge exchange channels for the ${ }^{3} \mathrm{He}$ nucleus,

$$
\sigma\left(\pi^{+}, \pi^{+}\right): \sigma\left(\pi^{-}, \pi^{-}\right): \sigma\left(\pi^{-}, \pi^{0}\right): \sigma\left(\pi^{+}, \pi^{0}\right) \approx 19: 11: 4: 2 .
$$

This relationship assumes that the contributions to the cross sections from the $T=1 / 2$ scattering amplitudes are negligible and that a pion interacts with a single nucleon within the nucleus. If the relative heights of the charge exchange and inelastic scattering peaks are equivalent, this can be seen as an indication that the single scattering approximation is valid in the quasi-free scattering region.

Overall, the agreement between the DWIA predictions and the experimental results is quite good from the center of the quasi-free peak through the high energy tail. In the region below the quasi-fre,e peak, the DWIA predictions underestimate the doubly differential cross sections. The enhancement of brsth ${ }^{3} \mathrm{He}\left(\pi^{+}, \pi^{+}\right)$and ${ }^{3} \mathrm{He}\left(\pi^{+}, \pi^{0}\right)$ over the theoretical precictions in the low energy region is an indicaticn that 
either the distortions of the incoming or outgoing pion are not being treated correctly or that more complex interactions are occurring or possibly both. However, the agreement between the DWIA predictions and the coincidence results implies that the discrepancies most likely originate from more complex processes. This disagreement is worse for the single charge exchange measurements than for the inelastic scattering measurements. A similar enhancement has also been seen in comparison of quasi-free charge exchange and inelastic scattering on ${ }^{16} \mathrm{O}$ at $160 \mathrm{MeV} .{ }^{37}$ (See Figure 1.14.) However, the large uncerainties and limited coverage at low outgoing $\pi^{0}$ energies of the single charge exchange measurements presented in this work do not permit one to reach any firm conclusions about this effect. 
$\theta_{\pi}=78^{\circ}$
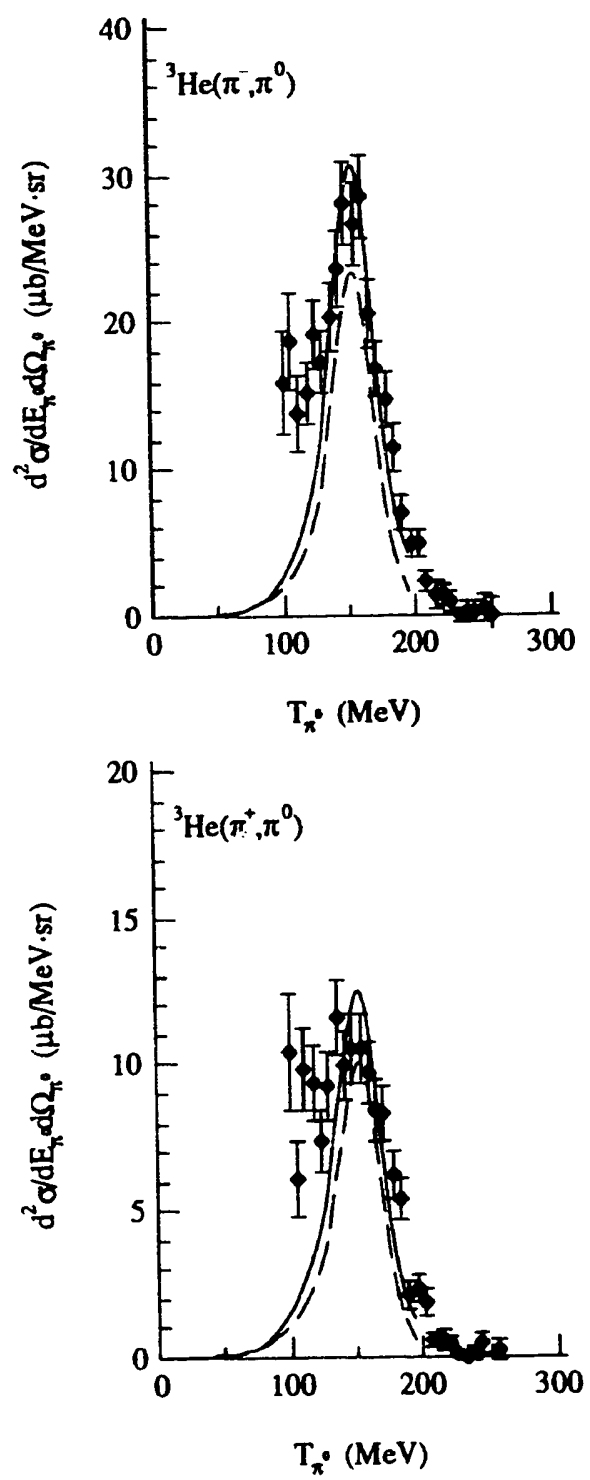

$\theta_{\pi}=80^{\circ}$
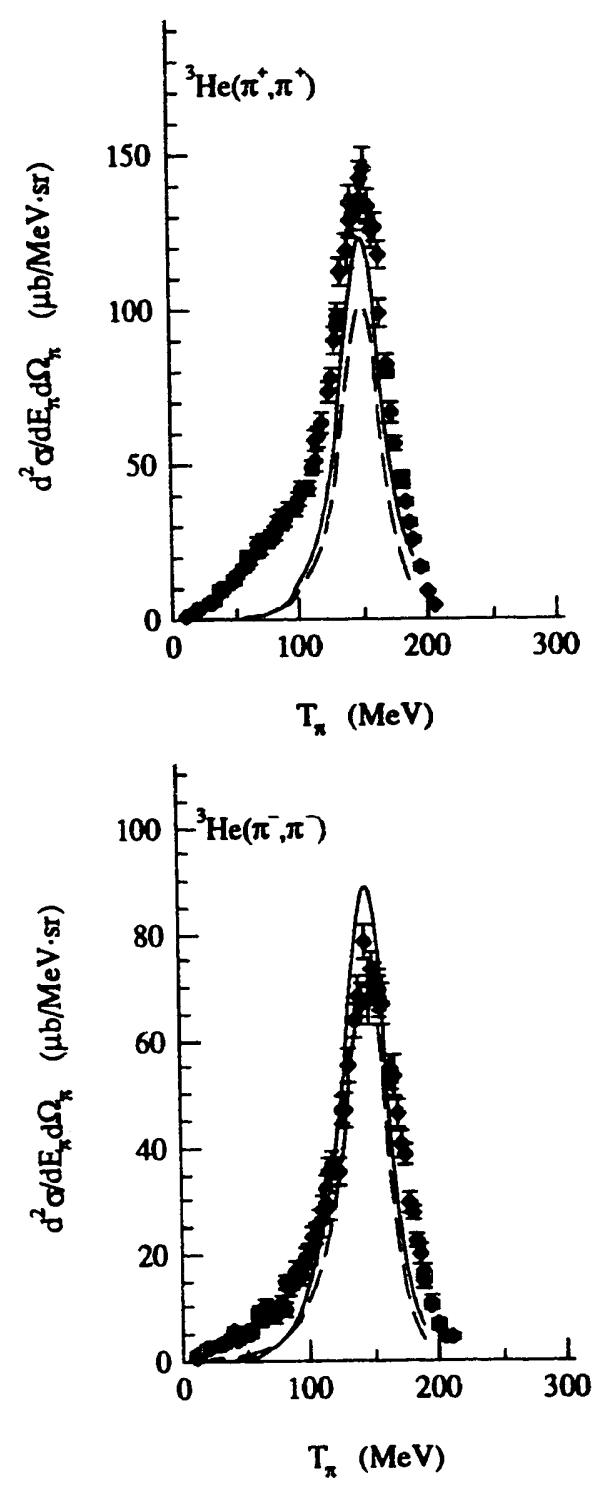

Figure 8.7: Comparisons of doubly differential cross sections for pion inelastic scattering and charge exchange on ${ }^{3} \mathrm{He}$ with DWIA calculations. The solid and dashed lines correspond to DWIA calculations using the final energy and initial energy prescription respectively. The ${ }^{3} \mathrm{He}\left(\pi^{t}, \pi^{t}\right)$ measurements at pion laboratory scattering angle of $80^{\circ}$ and an incident pion energy of $240 \mathrm{MeV}$ are from Reference 6 . The ${ }^{3} \mathrm{He}\left(\pi^{+}, \pi^{0}\right)$ measurements at $78^{\circ}$ and $245 \mathrm{MeV}$ are from this work. The $\mathrm{y}$-axes have been scaled according to the isospin factors from Equation 8.12 so that the relative peak heights of the cross section for each channel should be approximately the same. 


$$
\theta_{\mathbf{x}}=102^{\circ}
$$
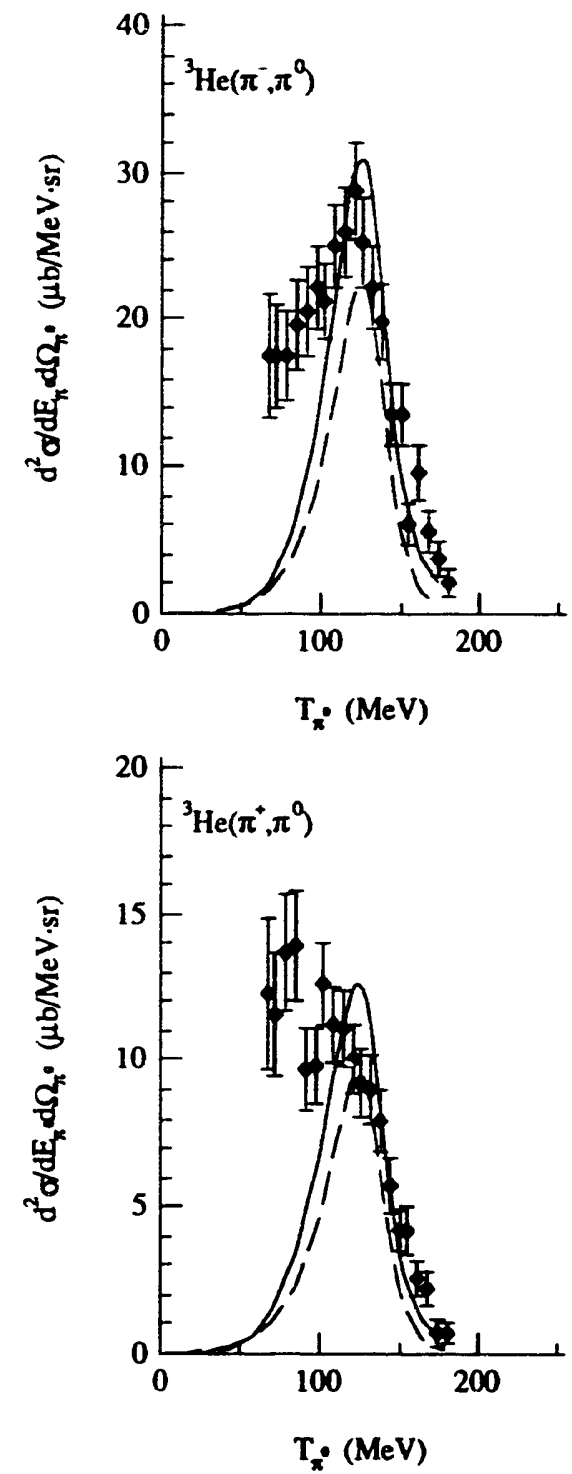

$\theta_{\mathbf{x}}=105^{\circ}$
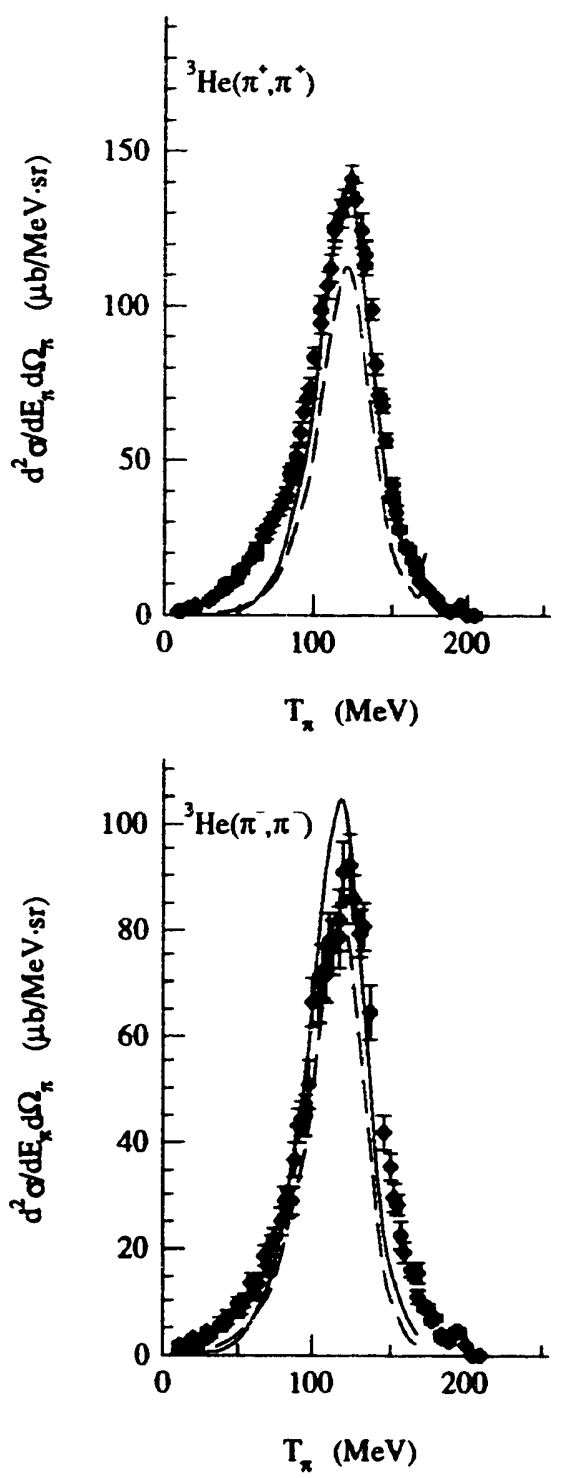

Figure 8.8: Cor aparisons of doubly differential cross sections for pion inelastic scattering and charge exchange on ${ }^{3} \mathrm{He}$ with DWIA calculations. The solid and dashed lines correspond to DWIA calculations using the final energy and initiai energy prescription respectively. The ${ }^{3} \mathrm{He}\left(\pi^{t}, \pi^{ \pm}\right)$measurements at pion laboratory scattering angle of $105^{\circ}$ and an incident pion energy of $240 \mathrm{MeV}$ are from Reference 6 . The ${ }^{3} \mathrm{He}\left(\pi^{ \pm}, \pi^{9}\right)$ measurements at $102^{\circ}$ and $245 \mathrm{MeV}$ are from this work. The y-axes have been scaled according to the isospin factors from Equation 8.12 so that the relative peak heights of the cross section for each channel should be approximately the same. 

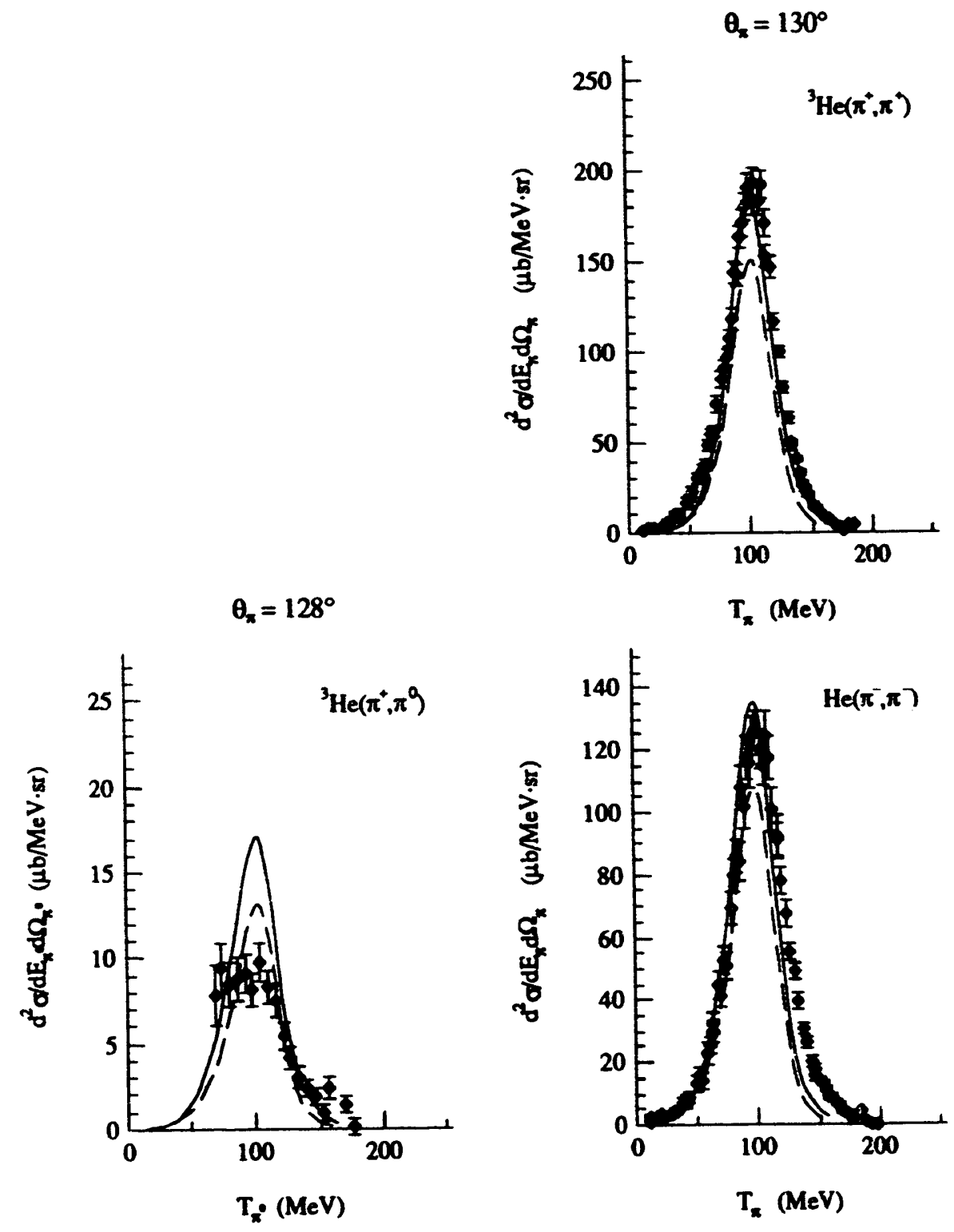

Figure 8.9: Comparisons of doubly differential cross sections for pion inelastic scatuering and charge exchange on ${ }^{3} \mathrm{He}$ with DWIA caiculations. The solid and dashed lines correspond to DWIA calculations using the final energy and initial energy prescription respectively. The ${ }^{3} \mathrm{He}\left(\pi^{ \pm}, \pi^{t}\right)$ measurements at pion laboratory scattering angle of $130^{\circ}$ and an incident pion energy of $240 \mathrm{MeV}$ are from Reference 6 . The ${ }^{3} \mathrm{He}\left(\pi^{*}, \pi^{0}\right)$ measurements at $128^{\circ}$ and $245 \mathrm{MeV}$ are from this work. The $y$-axes have been scaled according to the isospin factors from Equation $\mathbf{8 . 1 2}$ so that the relative peak heights for each channel should be approximately the same. 


\section{Chapter 9: $\quad$ Summary and Conclusions}

To fully understand pion-nucleus interactions, experimental information on several reaction channels is needed. These channels include pion scattering, pion charge exchange, and pion absorption. In special cases of pion scattering and charge exchange interactions with nuclei, the final state consists of a discrete state, e.8. elastic scattering or inelastic scattering to a discrete state, or the pion-induced isobaric analog transition. In quasi-free scattering, a pion interacts incoherently with individual nucleons within a nucleus. Quasi-free scattering can be used as a probe of nuclear medium modifications to the free $\pi \mathrm{N}$ interaction. In this thesis, results from measurements of the pion-induced isobaric analog transition and quasi-free pion single charge exchange on three body nuclei have been presented.

The measurement of the differential cross section for the pion-induced isobaric analog transition,

${ }^{3} \mathrm{H}\left(\pi^{*}, \pi^{9}\right)^{3} \mathrm{He}$, was performed at L.A.MF in 1991 using a magnetic spectrometer, equipped with an array of nineteen silicon surface barrier detectors and a focal plane scintillation counter, to detect recoil ${ }^{3} \mathrm{He}$ nuclei. Thin tritiated titanium foils were used as targets to minimize the energy loss of the recoil nuclei. Differential cross sections were measured at an incident pion energy of $142 \mathrm{MeV}$ and $\pi^{0}$ center-of-mass angles of $42.0^{\circ}, 51.5^{\circ}$, and $71.0^{\circ}$. Upper limits on the differential cross section were determined for an incident pion energy of $180 \mathrm{MeV}$ and $\pi^{\circ}$ center-of-mass angles of $35.4^{\circ}, 42.0^{\circ}, 51.1^{\circ}$, and $60.8^{\circ}$. The original goals of this measurement were to determine the energy dependence of the total cross section and test predictions of the importance of the spin-flip contribution to the differential cross section. The first goal was not met due to the poor statistical quality of the data and limited angular range of the measurement, as a result of a factor of three less tritium in the tritiated titanium foils than originally anticipated and larger than expected backgrounds. Nonetheless, the results of this measurement are in good agreement with recent theoretical 
calculations of the differential cross section for the pion-induced isobaric analog transition in the three nucleon system. In addition, the spin flip contribution to Gibbs' calculation" for the differential cross section is necessary to reproduce the measured angular distribution at an incident energy of $142 \mathrm{MeV}$.

The inclusive measurements of single charge exchange on ${ }^{3} \mathrm{He}$ utilized the LAMPF $\pi^{0}$. Spectrometer, an array of plastic scintillation detectors, and a liquid ${ }^{3} \mathrm{He}$ target in a series of single-arm and coincidence measurements at an incident pion energy of $245 \mathrm{MeV}$. Doubly differential cross sections for the ${ }^{3} \mathrm{He}\left(\pi^{4}, \pi^{9}\right)$ and ${ }^{3} \mathrm{He}\left(\pi^{-}, \pi^{9}\right)$ reactions were measured over ranges of $\pi^{0}$ laboratory scattering angles from $62^{\circ}$ to $128^{\circ}$ and $62^{\circ}$ to $118^{\circ}$ respectively. Neutral pions were detected over an energy range covering the quasi-free peak. An angular distribution was extracted from the doubly differential cross sections although with accuracy limited by the necessity of extrapolating the measurement to the unobserved low $\pi^{0}$ energy region.

Triply differential cross sections for the ${ }^{3} \mathrm{He}\left(\pi^{+}, \pi^{0} \mathrm{p}\right)$ reaction were measured at $\pi^{0}$ laboratory scattering angles of $70^{\circ}, 90^{\circ}, 110^{\circ}$, and $120^{\circ}$ and a range of coincident proton angles. Protons were detected over an energy range from 25 to $175 \mathrm{MeV}$. Triply differential cross sections for the ${ }^{3} \mathrm{He}\left(\pi^{-}, \pi^{0} \mathrm{p}\right)$ reaction were measured at $\pi^{\circ}$ laboratory scattering angles of $70^{\circ}$ and $110^{\circ}$ and a selection of proton angles. The position of the proton detector array and observable $\pi^{0}$ energy range were chosen in order to overlap the corresponding kinematical parameters for the free charge exchange reaction, $\pi^{+} n \rightarrow \pi^{0} p$. These triply differential cross sections were integrated over $\pi^{0}$ kinetic energy to determine proton angular distributions as a function of $\pi^{0}$ scattering angle for the ${ }^{3} \mathrm{He}\left(\pi^{ \pm}, \pi^{0} \mathrm{p}\right)$ reactions. These anguiar ciistributions for the ${ }^{3} \mathrm{He}\left(\pi^{+}, \pi^{0} \mathrm{p}\right)$ reaction were integrated over proton solid angle to obtain a $\pi^{0}$ angular distribution.

The results of the single-arm measurements, as well as previous measurements of pion inelastic scattering on ${ }^{3} \mathrm{He}$, exhibit the strong influence of the $\Delta$-resonance on this simple nuclear system. Simple isospin scaling predictions for the relative strength of the charge exchange and non-charge exchange channels, based on the dominance of the $\Delta$-resonance and a single scattering approximation, are consistent with ratios of the doubly differential cross sections near the quasi-free peak. A trend towards a prediction for the relative strengths of the single charge exchange cross sections, based on the isospin dependence of 
the free $\pi \mathrm{N}$ cross sections and assuming a double scattering mechanism, is observed in the single charge exchange data. This effect is not observed in comparisons of the relative strengths for the non-charge exchange data with double scattering predictions. However, the single charge exchange measurements do not extend to as low outgoing pion kinetic energies as the non-charge exchange measurenents. The answer as to whether these double scattering predictions will be substantiated at low outgoing $\pi^{0}$ energies must wait for a future experiment in which the entire $\pi^{0}$ energy spectrum is measured for a range of $\pi^{0}$ scattering angles. A proposal for an inclusive measurement of the single charge exchange reaction on ${ }^{3} \mathrm{He}$ and ${ }^{4} \mathrm{He}$ has been approved at LAMPF. This experiment might furnish the answers to some of the questions raised by the inclusive measurements from this work.

The doubly diffeicintial cross sections for both single-arm measurements, ${ }^{3} \mathrm{He}\left(\pi^{+} \pi^{9}\right)$ and ${ }^{3} \mathrm{He}\left(\pi^{-}, \pi^{0}\right)$, were integrated over the measured $\pi^{0}$ kinetic energy range to determine a singly differential cross section. The results of this integration are not in good agreement with the angular distribution for the free single charge exchange reaction. A crude attempt to extrapolate the doubly differential cross sections to low outgoing $\pi^{0}$ kinetic energies improves the overall agroement somewhat. At large angles, the magnitude, but not the shape, of the free charge exchange distribution is reproduced. The uncertainties in the corrected angular distributions are too large to comment on the differences between the charge exchange and non-charge exchange results. ${ }^{6}$

DWIA calculations accurately reproduce the magnitude and high energy shoulder of the quasi-free peak of the doubly differential cross sections for the ${ }^{3} \mathrm{He}\left(\pi^{2}, \pi^{0}\right)$ reactions over a range of $\pi^{0}$ scattering angles from $62^{\circ}$ to $128^{\circ}$. Comparisons between the single-arm measurements and results from pion inelastic scattering on ${ }^{3} \mathrm{He}$ with DWIA calculations show an enhancement of the doubly differential cross section for the single charge exchange over the non-charge exchange reactions at low outgoing pion kinetis energies. This enhancement may be an indication that multiple scattering plays a more important role in the single charge exchange than the non-charge exchange reactions on ${ }^{3} \mathrm{He}$. It has been speculated that the single charge exchange reaction is weakly coupled to the two-nucleon absorption channel. ${ }^{34}$ Therefore, a microscopic treatment of the ${ }^{3} \mathrm{He}\left(\pi^{ \pm}, \pi^{9}\right)$ processes may lead to information about the $\mathrm{NN}$ or $\Delta \mathrm{N}$ interaction. 
A detailed treatment, along the lines of the $\Delta$-hole caiculation by Takaki and Thies" for pion inelastic scattering on ${ }^{16} \mathrm{O}$, may elicit information about the $\Delta$-nucleus interaction in ${ }^{3} \mathrm{He}$ that a factorized DWIA approach cannot. Since the factorized DWIA calculation models the interaction as a single nucleon knockout process, it ignores contributions from processes which involve more than one nucleon, e.g. a pion may interact with a nucleon, excite a $\Delta$ which in tum may interact with oher nucleons as it propagates through the nucleus, $\Delta \mathrm{N} \rightarrow \Delta \mathrm{N}$. The ${ }^{3} \mathrm{He}$ calculation should be simpler than the ${ }^{16} \mathrm{O}$ calculation since the ${ }^{3} \mathrm{He}$ nuclear wavefunctions are well understood.

A similar microscopic treatment of the ${ }^{3} \mathrm{He}\left(\pi^{2} \pi^{0} \mathrm{p}\right)$ reactions might also yield interesting information about $N N$ or $\Delta N$ interactions. Calculations" which have failed to reproduce $\pi^{0}$ energy spectra ${ }^{20}$ in the ${ }^{16} \mathrm{O}\left(\pi^{*}, \pi^{0} \mathrm{p}\right)$ reaction may be easier to perform for the simpler ${ }^{3} \mathrm{He}$ system. The measured $\pi^{0}$ energy spectra from the coincidence measurements, ${ }^{3} \mathrm{He}\left(\pi^{*} \pi^{0} \mathrm{p}\right)$, exhibit a rough symmetry about the nominal quasi-free proton angle. The general shapes of these spectra are also in agreement with measurements of the ${ }^{3} \mathrm{He}\left(\pi^{+}, \pi^{+} \mathrm{p}\right)$ reaction by Klein et al. ${ }^{42}$ The proton angular distributions also appear symmetric about the quasi-free recoil angle. The $\pi^{0}$ angular distribution from the ${ }^{3} \mathrm{He}\left(\pi^{+}, \pi^{0} \mathrm{p}\right)$ reaction is in reasonable agreement with DWIA predictions. These points imply that the ${ }^{3} \mathrm{He}\left(\pi^{*} \pi^{0} \mathrm{p}\right)$ reaction is predominarity a quasi-free process. The ${ }^{3} \mathrm{He}\left(\pi^{-}, \pi^{0} \mathrm{p}\right)$ results are more difficult to interpret. A $\pi^{0}$ angular distribution cannot be extracted for the ${ }^{3} \mathrm{He}\left(\pi^{-}, \pi^{0} p\right)$ reaction due to the poor statistical quality of these measurements. The ratios of proton angular distributions for the ${ }^{3} \mathrm{He}\left(\pi^{+}, \pi^{0} \mathrm{p}\right)$ and ${ }^{3} \mathrm{He}\left(\pi^{-}, \pi^{0} \mathrm{p}\right)$ reactions near the quasi-free proton angle are roughly 20:1 which agree with naive predictions for the relative strength of single scattering and double scattering processes. This is perhaps not surprising since at least two nucleons must be involved in the ${ }^{3} \mathrm{He}\left(\pi^{-}, \pi^{0} \mathrm{p}\right)$ reaction for a proton with sufficient kinetic energy to be detected in coincidence.

This work presents the first experimental results for inclusive pion single charge exchange reactions in three body nuclei. In addition, it also presents the first results for the pion-induced isobaric analog transition at forward $\pi^{\circ}$ center-of-mass angles and incident pion energies below the $\Delta$-resonance. The exclusive and single-arm measurements are in reasonable agreement with theoretical predictions. At 


$$
\begin{aligned}
& \omega \\
& \stackrel{\rho}{\stackrel{\omega}{\omega}}
\end{aligned}
$$


low outgoing pion kinetic energies, there is a slight enhancement of the doubly differential cross sections for the ${ }^{3} \mathrm{He}\left(\pi^{5}, \pi^{9}\right)$ reactions relative to inelastic scattering measurements on ${ }^{3} \mathrm{He}$. This enhancement may be seen as evidence of a weaker coupling to the absorption channel for the charge exchange reactions. Future experimental efforts to measure the single charge exchange cross sections over the entire $\pi^{0}$ kinematic energy range are needed to determine whether this trend continues or not.

The ${ }^{3} \mathrm{He}\left(\pi^{+}, \pi^{0} \mathrm{p}\right)$ measurements reproduce the expected behavior for a process which is dominated by a quasi-free interaction. A factorized DWIA calculation reproduces the $\pi^{0}$ angular distribution for the ${ }^{3} \mathrm{He}\left(\pi^{+}, \pi^{0} \mathrm{p}\right)$ reaction. This implies that the factorized DWIA approach of Chant and Roos ${ }^{\sigma / 2}$ correctly calculates the differential cross section for the single nucleon knockout process in ${ }^{3} \mathrm{He}$. Therefore, deviations between the DWIA predictions and the doubly differential cross sections for ${ }^{3} \operatorname{IIe}\left(\pi^{+}, \pi^{0}\right)$ may be attributed to multiple scattering or nther more complex processes. The ${ }^{3} \mathrm{He}\left(\pi^{-}, \pi^{0} \mathrm{p}\right)$ measurements await a more detailed theoretical treatment to fully understand the mechanisms for this reaction. These measurements could also use further experimental study to improve their statistical accuracy. 


\section{Appendix A: Outline of Elastic Single Charge Exchange Analysis}

This Appendix contains an outline of the methods for extracting a cross section from the raw data for the exclusive single charge exchange measurement, ${ }^{3} \mathrm{H}\left(\pi^{+},{ }^{3} \mathrm{He}\right) \pi^{0}$. This outline assumes that the reader is familiar with the LAMPF $Q$ data acquisition package.

1. Replay the data using the E1026 Q data acquisition package and save the silicon surface barrier detector pulse height spectra to a IISV file. A normalization information file, RU0xxx.inf, where xxx is the nun number, containing the live time, spectrometer angle, target angle, spectrometer momentum setting, and digitized ionization counts, is created during the replay process.

2. Set the particle gates on the $\mathrm{CH}_{2}$ calibration pulse height spectra following the convention outlined in Table C.1.

\begin{tabular}{|l|l|l|l|}
\hline Particle & Lower Gate \# & Upper Gate \# & Peak Index \\
\hline$p$ & 1 & 2 & 1 \\
\hline$d$ & 3 & 4 & 2 \\
\hline$\alpha$ & 5 & 6 & 3 \\
\hline $3 \mathrm{He}$ & 7 & 8 & 4 \\
\hline $3 \mathrm{H}$ & 9 & 0 & 5 \\
\hline
\end{tabular}

Table A.1: HPL markers for particle identification.

3. Run the program, GET_CUTS, to generate a ASCII file of the gate positions for different particles form the $\mathrm{CH}_{2}$ calibration spectra, called RU0xxx.CUT.

4. Next, run the program, HSV_TO_DCL, to create ASCII files of channel number versus pulse height for each silicon detector pulse height spectrum. The pulse height spectra without the scintillator coincidence are labeled, RUOxxx_RFPy.MIN, where $y$ is the detector number and $x \mathbf{x x}$ is the run number. Pulse height spectra with the scintillator coincidence requirement are labeled RU0xxx_SFPy.MIN. 
After steps 1 through 4 have been completed, all of the necessary data files are in place to run the analysis programs. The raw yields for each particle type may be extracted from the raw data using either of two different methods - a MINUT driven fitting program and a Brute analysis program. The details of these analysis procedures are discussed in Chapter 2 . The $\pi^{+} p$ and $\pi^{+} t$ elastic scattering data were analyzed using the MINUIT driven programs. The exclusive single charge exchange data were analyzed using the Brute analysis method. The following instructions describe how to use these analysis programs.

5. Extract yields from the pulse height spectra for the particular particle of interest.

\section{MINUIT Method:}

Automatic method: In most cases where peaks were clearly distinguishable from one another, an automatic MINUTT fitting program, MULTIFIT, could be used. Two DCL command files were created to assign logical names to the appropriate files for a specific particle type, run number, and detector number. The first DCL command file could be used to extract a ynild from an individual detector, by typing,

'sem_setup $\langle\mathrm{P} 1\rangle\langle\mathrm{P} 2\rangle\langle\mathrm{P} 3\rangle$ ',

where $\left\langle\mathrm{P} 1>\right.$ is the run number, $<\mathrm{P} 2>$ is the detector \#, and $\left\langle\mathrm{P} 3>\right.$ is the $\mathrm{CH}_{2}$ fiducial run number. Leave $<\mathrm{P} 3>$ blank if $<\mathrm{P} 1>$ is a fiducial run.

To extract a yield from all of the silicon focal plane detectors for a given run, type

$$
\text { 'semulti_fit }\langle\mathrm{P} 1\rangle\langle\mathrm{P} 2\rangle \text { ', }
$$

where $<\mathrm{P} 1>$ is the run number, $<\mathrm{P} 2>$ is the $\mathrm{CH}_{2}$ fiducial run number. Leave $<\overrightarrow{\mathrm{P}} 2>$ blank if $\langle\mathrm{P} 1>$ is a fiducial run.

Card driven method: In cases of overlapping pulse heights from different types of particles or when the automatic MINUIT fitting method failed to converge to a reasonable result, a card driven version of the MINUIT fitting program, ALT_MULTIFIT, could be used. This method required that the user supply a MINUIT fitting card with the appropriate limits on each parameter. The name of the card should be RU0xxx_RFPy.INP. As for the automatic method, a DCL command file was created to assign logical names to the appropriate files. To extract a yield from an individual detector using the card driven method, type

'\$@domult $\langle\mathrm{P} 1\rangle\langle\mathrm{P} 2\rangle\langle\mathrm{P} 3\rangle$ ',

where $<\mathrm{P} 1>$ is the run number, $<\mathrm{P} 2>$ is the detector \#, and $<\mathrm{P} 3>$ is the $\mathrm{CH}_{2}$ fiducial run number.

Leave $<\mathrm{P} 3>$ blank if $<\mathrm{P} 1>$ is a fiducial run.

The MINUIT fitting programs generate the following files:

1) A MINUIT fituing coefficient file, RUOxxx_RFPy.CF which contains the raw yields of each peak in a pulse height spectrum.

2) A Topdrawer file, RU0xxx_RFPy.TOP which may be used to display a pulse height spectrum and the MINUIT fit to the spectrum.

3) A MINUIT output filc, RU0xxx_RFPy.OUT, which records the progression of the MINUIT fit and summarizes the fitting results. 


\section{Brute Method:}

This method sums up the number of counts between two gates in a pulse height spectra. Equal-sized regions on either side of the software gate are sampled to determine the average number of background counts per channel. Two DCL command files were created to extract the net yield of a specific type of particle. To extract a yield from an individual detector, type

$$
\text { 'serunbrute }\langle\mathrm{P} 1\rangle\langle\mathrm{P} 2\rangle\langle\mathrm{P} 3\rangle\langle\mathrm{P} 4\rangle \text { ', }
$$

where $\langle\mathrm{P} 1\rangle$ is the run number, $\langle\mathrm{P} 2\rangle$ :s the detector number, $\langle\mathrm{P} 3\rangle$ is the peak index from Table $\mathrm{C}-1$, and $\langle\mathrm{P} 4\rangle$ is the $\mathrm{CH}_{2}$ fiducial run number.

To extract a yield from all of the detectors for a particular run, type

$$
\text { '\$Qb_all }\langle\mathrm{P} 1\rangle\langle\mathrm{P} 2\rangle\langle\mathrm{P} 3\rangle \text { ', }
$$

where $\left\langle\mathrm{P} 1>\right.$ is the run number, $<\mathrm{P} 2>$ is the peak index from Table $\mathrm{C}-1$, and $\left\langle\mathrm{P} 3>\right.$ is the $\mathrm{CH}_{2}$ fiducial run number.

6. Once the raw yields from each detector for a particular run had been extracted, the data were corrected for live time, relative detector efficiency, momentum bite of the detector, and target angle. After the data from several different momentum settings had been analyzed through step 5, these data were added together, treating each run as an independent measurement, and re-binned into equal spaced momentum bins.

MINUTT Method: To normalize the data, type

$$
\text { 'seyield }\langle\mathrm{P} 1\rangle\langle\mathrm{P} 2\rangle \text { ', }
$$

where $<\mathrm{Pl}>$ is the run number, and $<\mathrm{P} 2>$ is the peak index from Table $\mathrm{C}-1$. To add data from several runs together, type

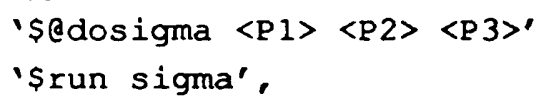

where $\langle\mathrm{Fl}\rangle$ is the incident beam energy, $\langle\mathrm{P} 2\rangle$ is the spectrometer angle, and $\langle\mathrm{P} 3\rangle$ is the reaction channel (PIP, PIT, or SCX). The program, SIGMA, prompts the user for the foreground and background run numbers and check to ascertain that they contain data taken under the same experimental conditions.

Brute Method: To normalize and re-bin the charge exchange data, type

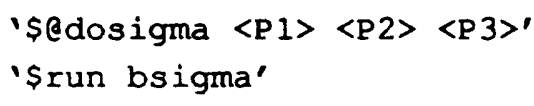

where $\langle\mathrm{P} 1\rangle$ is the incident beam energy, $\langle\mathrm{P} 2\rangle$ is the spectrometer angle, and $\langle\mathrm{P} 3\rangle$ is the reaction channel (PIP, PIT, or SCX). The program, BSIGMA, prompts the user for the foreground and background run numbers and check to ascertain that they contain data taken under the same experimental conditions.

Both of these methods, MINUIT and Brute, create a Topdrawer file of the re-binned data labelled $\left.\left.\langle\mathrm{P} 3\rangle_{-}<\mathrm{P} 1\right\rangle_{-} \mathrm{P} 2\right\rangle_{\text {. TOP }}$ and a file containing the net normalized yield, $\left.\langle\mathrm{P} 3\rangle_{-}\langle\mathrm{P} 1\rangle_{-} \mathrm{P} 2\right\rangle_{\text {.INF, for a }}$ particular experimental set up. The information from this second file, the net yield of recoil particles from charge exchange, $\pi^{+} p$, or $\pi^{+} t$ elastic scattering, is used as input for the differential cross section calculations. 


\title{
Appendix B: Monte Carlo Simulations of the Little Yellow Spectrometer
}

\author{
A brief description of the Little Yellow Spectrometer Monte Carlo simulations is contained in this \\ Appendix. These simulations, developed for experiment E1026, were used to evaluate the performance of \\ the spectrometer and to extract a meanigful result from the ${ }^{3} \mathrm{H}\left(\pi^{+}, \mathrm{He}\right) \pi^{0}$ data. Comparisons with measured \\ results are shown and a sample calculation of the correction factor for the ${ }^{3} \mathrm{He}$ yield from ${ }^{3} \mathrm{H}\left(\pi^{+},{ }^{3} \mathrm{He}\right) \pi^{0}$ is \\ periormed.
}

\section{Overview of Monte Carlo Simulation}

A good Monte Carlo event required that the detected particle did not stray outside the physical limits of the spectrometer vacuum box and that it struck one of the silicon surface barrier detectors. The net yield of particles in each detector bin for a particular momentum setting, $p_{c}$, was corrected for the detector efficiency, $\varepsilon_{i}$, and momentum bin , $\Delta p$, in the following manner,

$$
\begin{gathered}
\bar{Y}_{i}=\frac{Y_{i}}{\varepsilon_{i} \cdot \Delta p}, \\
\text { where } \Delta p=p_{c} \delta p_{M C}
\end{gathered}
$$

Finally, the normalized Monte Carlo yields from several momentum settings were added together to complete the momentum spectrum for a particular reaction channel. The procedures for adding together and re-binning the Monte Carlo data were identical to those outlined in Chapter 3 for combining experimental data from different spectrometer momentum setuings. A flow chart diagram of the Litule Yellow Spectrometer Monte Carlo simulation is shown in Figure B.1 


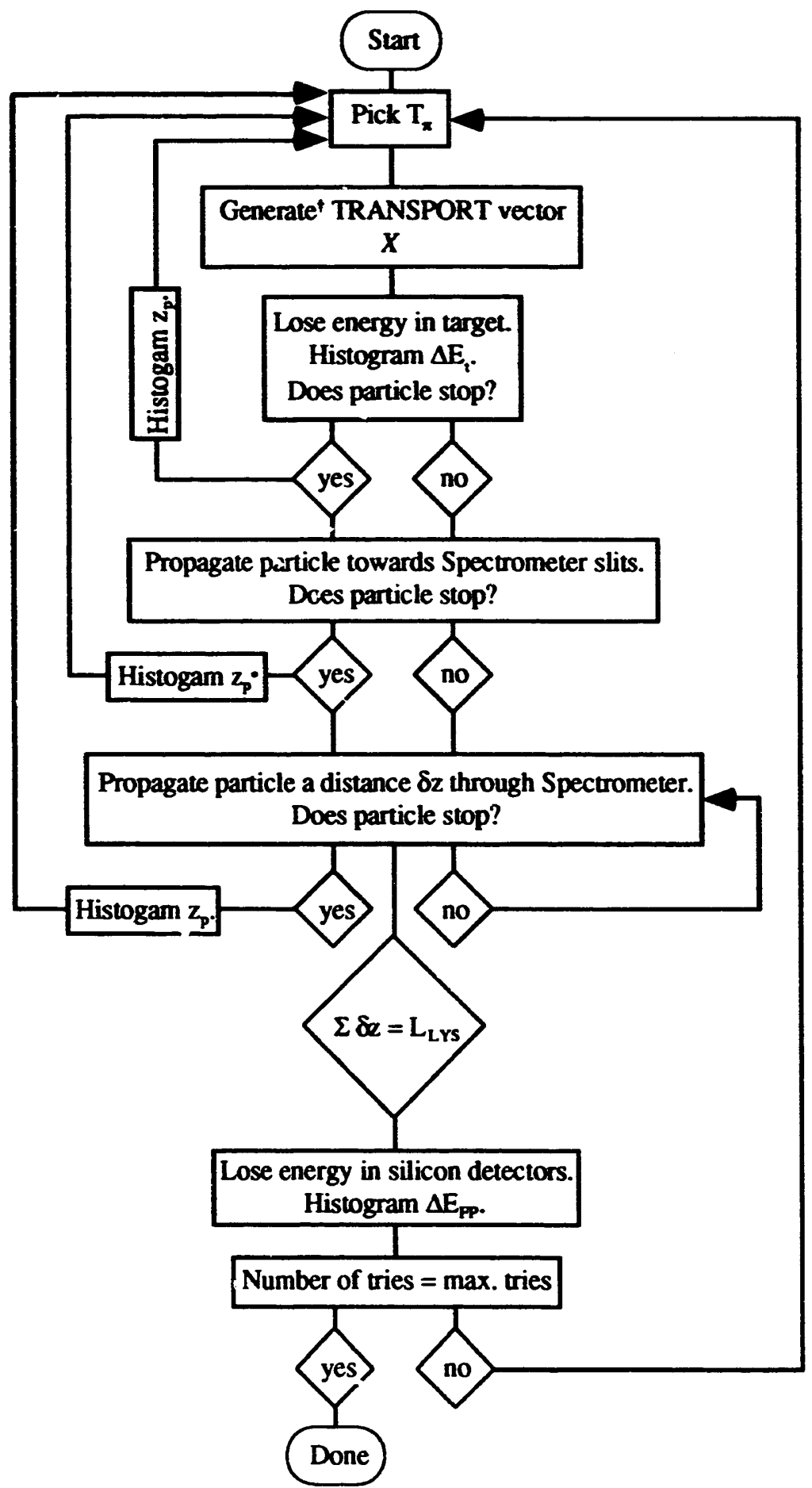

TTe angular and energy distribution, chosen for generating Monte Carlo events, depended on the chcice of reaction channel, exclusive single charge exchange or elastic scattering.

${ }^{\prime} z_{p}$ is the distance irom the target.

Figure B.1: Little Yellow Spectrometer Monte Carlo Flow Chart 


\section{Monte Carlo Peak Positions}

The Monte Carlo simulation was developed for extracting ${ }^{3} \mathrm{He}$ yields when only a portion of the total distribution of ${ }^{3} \mathrm{He}$ perticles from the ${ }^{3} \mathrm{H}\left(\pi^{+},{ }^{3} \mathrm{He}\right) \pi^{0}$ reaction had been measured. Compariscns of Monte Carlo momentum spectra with elastic scattering measurements were made to verify that the simulation could reproduce peak positions and shapes. Monte Carlo and measured peak positions for $\pi^{\dagger} p$ and $\pi^{+}{ }^{3} \mathrm{H}$ elastic scattering are presented in Table B.1.

\begin{tabular}{|c|c|c|c|l|}
\cline { 3 - 5 } \multicolumn{2}{c|}{} & \multicolumn{2}{|c|}{$\bar{p}(\mathrm{MeV} / \mathrm{c})$} \\
\hline Reaction & $\begin{array}{c}\mathrm{T}_{\pi} \\
(\mathrm{MeV})\end{array}$ & $\theta_{\mathrm{L}}$ & \multicolumn{1}{c|}{ E1026 } & LYS Monte Carlo \\
\hline $\mathrm{p}\left(\pi^{+}, \mathrm{p}\right) \pi^{+}$ & 142 & $40^{\circ}$ & $295.0 \pm 1.5$ & $293.2 \pm 4.8$ \\
\hline $\mathrm{p}\left(\pi^{+}, \mathrm{p}\right) \pi^{+}$ & 180 & $60^{\circ}$ & $213.72 \pm 0.82$ & $212.7 \pm 2.8$ \\
\hline${ }^{3} \mathrm{H}\left(\pi^{+},{ }^{3} \mathrm{H}\right) \pi^{+}$ & 180 & $65^{\circ}$ & $214.7 \pm 6.3$ & $213.9 \pm 2.9$ \\
\hline
\end{tabular}

Table B.1: Comp.rison of measured peak positions (E1026), $\bar{p}$, with Monte Carlo results for elastic scattering measure :ents.

\section{Monte Carlo Dispersion Constant and Acceptance Function}

The Monte Carlo simulation of the spectrometer acceptance was performed as follows. The "detector" efficiencies, relative to the central detector, were determined by throwing a flat distribution of particles. This distribution filled the phase space of the spectrometer and the net yields in each detector were compared to the yield from the central detector to determine the relative efficiency of each detector in the same manner as proton distributions from the ${ }^{12} \mathrm{C}\left(\pi^{+}, \mathrm{p}\right) \mathrm{X}$ reaction were used during the experiment. A comparison of the Monte Carlo acceptance function with the measured acceptance is shown in Figure B.2.

The Monte Carlo dispersion constant, $\delta p_{M C}$, was derived from the relationship between the 
momentum deviation of a monoenergetic beam of particles from the central momentum setting of the spectrometer and the peak position of this beam at the focal plane. This relationship is depicted in Figure

B.3. Differences between the Monte Carlo dispersion constant and previous measured values with the identical silicon detector instrumentation are less than 5\% and may be attributed to differences in the experimental set-up and the Monte Carlo description of the spectrometer and its instrumentation As previously reported by Kinney, small deviations in the placement of the detector package with respect to the true focal plane of the Litule Yellow Spectrometer can cause a few percent change in the dispersion constant.

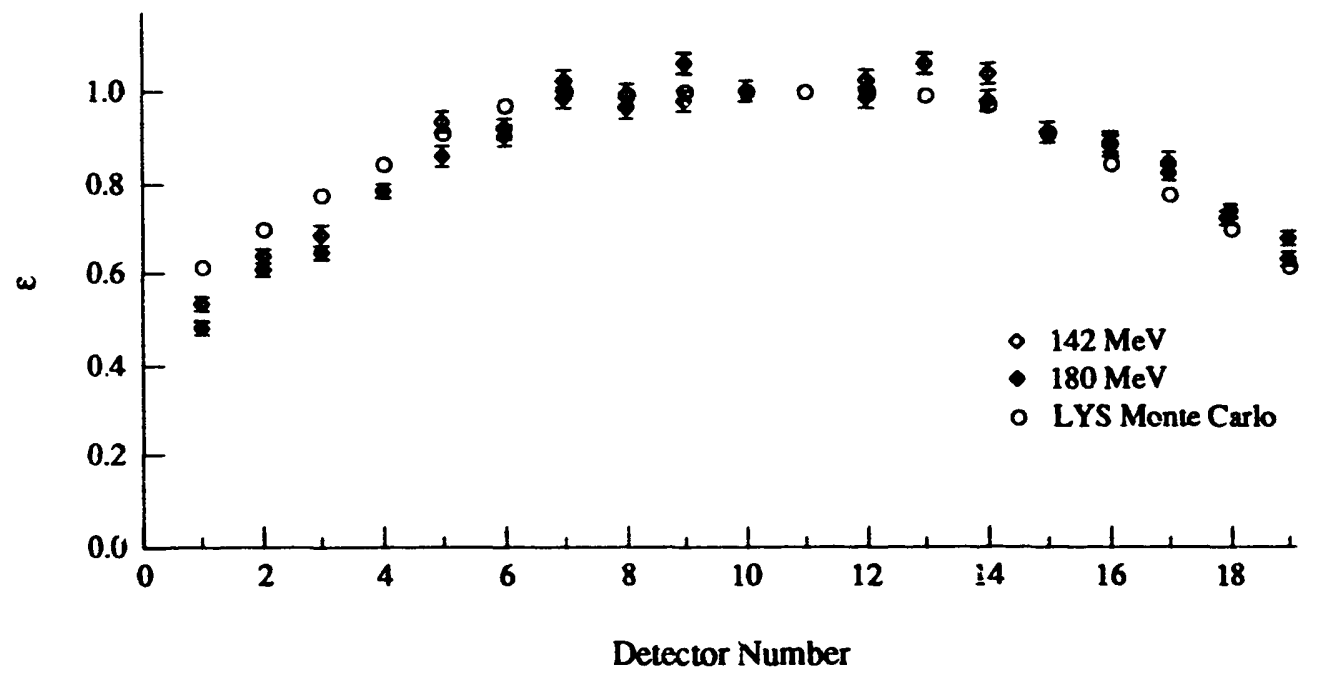

Figure B.2: Comparison of Monte Carlo results with measured acceptance function of the Litule Yellow Spectrometer. 


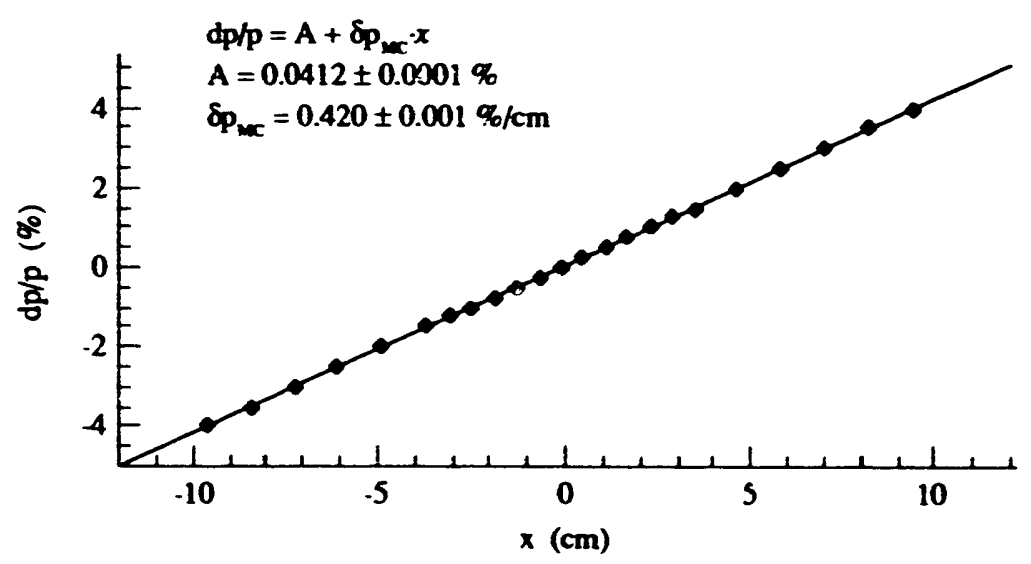

Figure B.3: Deviation from the central momentum setting of the spectrometer of a monoenergetic point-like source of particles as a function of Monte Carlo peak position (solid points) in the dispersive direction at the focal plane. The curve represents a linear least-squares fit to the Monte Carlo data; the dispersion constant is the slope of this line. The previously reported value ${ }^{48}$ of the dispersion constant is $0.438 \pm 0.002$ $\% / \mathrm{cm}$ for the Little Yellow Spectrometer configured with the same detector package as was used in the present measurement.

\section{Monte Carlo Angular Distributions for Elastic Scattering and Charge Exchange Reactions}

The passage of charged particles from the interaction region to the focal plane of the Little Yellow Spectrometer was simulated by a set of TRANSPORT ${ }^{33}$ matrices. A TRANSPORT coordinate, defined as the spatial and momentum displacement from the central trajectory of the spectrometer,

$$
X=\left[\begin{array}{l}
x \\
\theta \\
v \\
\phi \\
z \\
\delta p
\end{array}\right],
$$

was generated on an event by event basis according to the particular reaction channel of interest and its kinematical constraints.

The initial angular distribution has an important effect on the shape of the Monte Carlo 
momentum spectra from elastic charge exchange or elastic scattering reactions. A comparison of the measured momentum spectrum of recoil ${ }^{3} \mathrm{H}$ particles from $\pi^{+3} \mathrm{H}$ elastic scattering with Monte Carlo simulations using two types of angular distributions is shown in Figure B.4. For $\pi^{+}$p elastic scattering s mulations, SCATPI ${ }^{20}$ calculations of the differential cross sections were used to parametrize the energy and angular dependence of the recoil proton distribution as a function of incident beam energy. An empirical parametrization of the triton angular distribution for $\pi^{+3} \mathrm{H}$ elastic scattering at $180 \mathrm{MeV}$ was determined from measurements by Pillai et. al. ${ }^{\text {so }}$ Recent calculations by Gibbs ${ }^{11}$ were used to parametrize the energy and angular distributions of ${ }^{3} \mathrm{He}$ particles from elastic single charge exchange, ${ }^{3} \mathrm{H}\left(\pi^{+}, \mathrm{He}\right) \pi^{0}$. A list of the Monte Carlo parameters for the recoil angular distributions is presented in Table B.2. An example of a typical fit to the angular distribution for $\pi^{+} \mathrm{p}$ elastic scattering at $142 \mathrm{MeV}$ is shown in Figure B.5.

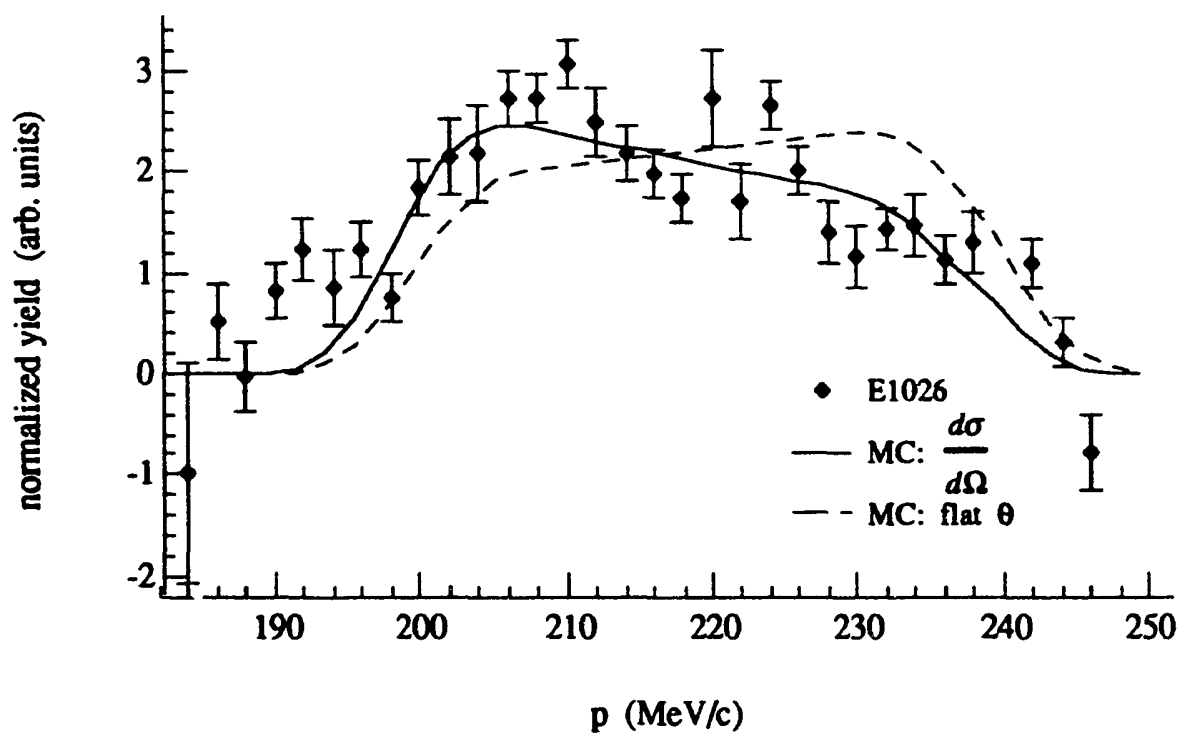

Figure B.4: Recoil ${ }^{3} \mathrm{H}$ momentum distribution (solid points) from $\pi^{+3} \mathrm{H}$ elastic scautering at $180 \mathrm{MeV}$ and $\theta_{3}=60^{\circ}$ in comparison with Monte Carlo predictions assuming a flat angular dependence (dashed line) and the actual angular dependence (solid line) for the recoil angular distribution. It is clear from this comparison that the initial angular distribution plays an important role in the shape of the measured momentum spectra. 


\begin{tabular}{|c|c|c|c|c|c|c|}
\hline \multicolumn{7}{|c|}{$\begin{array}{c}\text { Monte Carlo Parameters for Generating Recoil Angular } \\
\text { Distributions }\end{array}$} \\
\hline Reaction & $\begin{array}{c}\mathrm{T}_{\mathrm{z}} \\
(\mathrm{MeV})\end{array}$ & KO & $\mathbf{K} \mathbf{1}$ & $\mathrm{K} 2$ & K3 & K4 \\
\hline$\pi^{+} \mathbf{p}$ & 142 & 6.4 & 0 & 483 & -1218 & 808 \\
\hline$\pi+p$ & 180 & 0.674 & 2.32 & -3.35 & 0 & 0 \\
\hline$\pi+3 \mathrm{H}$ & 180 & 52.8 & 0 & -587.8 & 1071 & -546 \\
\hline SCX & 142 & 2113 & 0 & -8391 & 13644 & -7057 \\
\hline SCX & 180 & 2279 & 0 & -18348 & 31588 & -15679 \\
\hline
\end{tabular}

Table B.2: Monte Carlo parameters for recoil angular distributions. The functions, used to describe the laboratory differential cross section, were of the form $K 0+K 1 \cdot y+K 2 \cdot y^{2}+K 3 \cdot y^{3}+K 4 \cdot y^{4}$, where $y$ is the cosine of the recoil laboratory scattering angle.

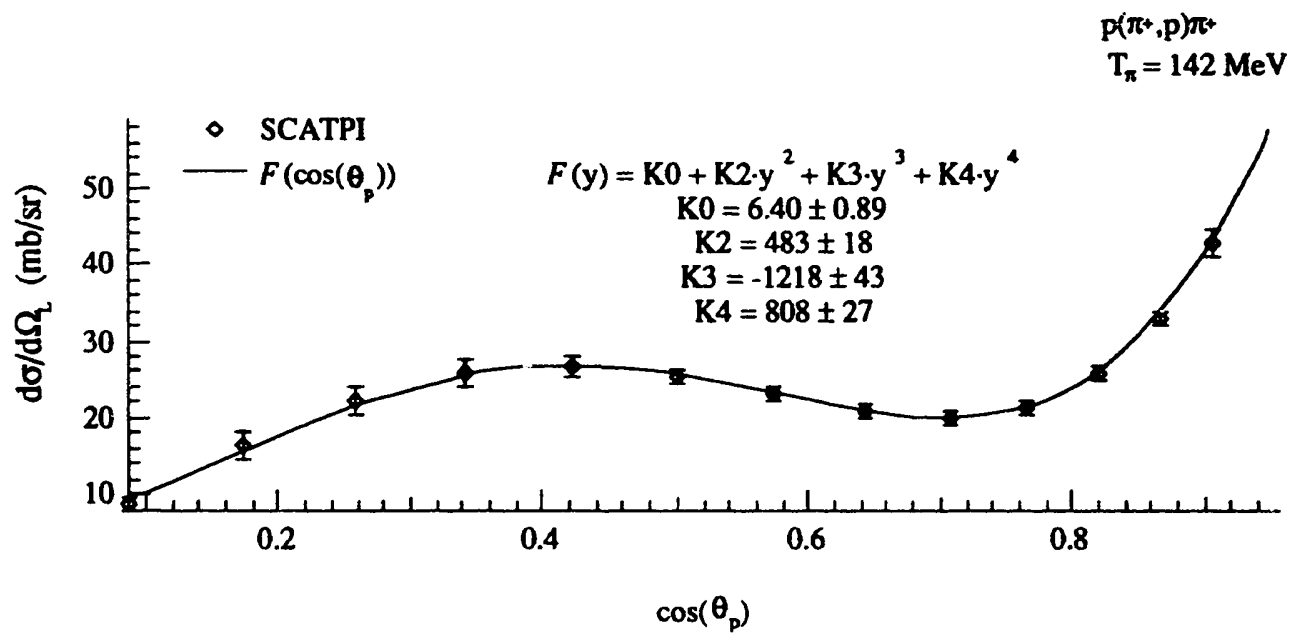

Figure B.5: Laboratory differential cross sections (open points) for $p\left(\pi^{+}, p\right) \pi^{+}$elastic scattering at $142 \mathrm{MeV}$ from $\pi \mathrm{N}$ phase shift calculations ${ }^{20} ; \theta_{\mathrm{p}}$ is the laboratory angle of the proton and $y$ is the cosine of thisg angle. The curve is a fourth order polynomial fit to the differential cross section. 
Monte Carlo Event Generator for Complicated Angular Distributions

A Monte Carlo rejection method ${ }^{74}$ was used to generate the angular coordinate for the TRANSPORT ray according to the fourth order polynomials which described the angular distribution for the particle of interest. The rejection method zenerates Monte Carlo events according to a simple distribution, compares the event to the more complicated distribution, and rejects the event if it does not follow the appropriate distribution. This is an easier methoo of generating Monte Carlo events than solving for the inverse function of the fourth order polynomial. A simple single value function, for which each $x$ value has a corresponding unique $y$ value, is chosen for the initial distribution such that it is always greater than the function which describes the desired distribution of Monte Carlo events, $F(y)$. For this simulation, linear functions of the form,

$$
G(y)=A+B \cdot y
$$

were used, where $y$ is the cosine of the laboratory scattering angle. Then, a random number, $\lambda$, is generated between 0 and $A$, where

$$
A=\int_{y=\infty}^{y} G(y) d y,
$$

and $y_{\max }$ and $y_{\min }$ are the upper and lower limits on the interval over which Monte Carlo events will be generated. Next, the value for $y$ corresponding to $\lambda$, is calculated by solving the following quadratic equation for $y_{\infty}$

$$
\lambda=\int_{y_{-}}^{y_{2}} G(y) d y=\left[A \cdot y+\frac{1}{2} B \cdot y^{2}\right]_{y_{-}}^{y_{0}} .
$$

Finally, a second random number is generated, $\Lambda$, between 0 and $G\left(y_{0}\right)$. This event is accepted if

$\Lambda \leq F\left(y_{0}\right)$. The success rate for generating good events using the rejection method may be expressed as,

$$
\text { success rate }=\frac{\int_{y=\infty}^{y / T} F(y) d y}{\int_{y=\infty}^{y_{y}} G(y) d y} .
$$

An example of this method is shown in Figure B.6 which displays a fit to the laboratory differential cross section for $\pi^{+}$p elastic scattering at $142 \mathrm{MeV}, F\left(\cos \left(\theta_{\mathrm{p}}\right)\right)$, along with the chosen function for the rejection method, $G\left(\cos \left(\theta_{p}\right)\right)$. A comparison between the Monte Carlo results and the initial distribution function, $F\left(\cos \left(\theta_{p}\right)\right)$, is shown in Figure B.7. 


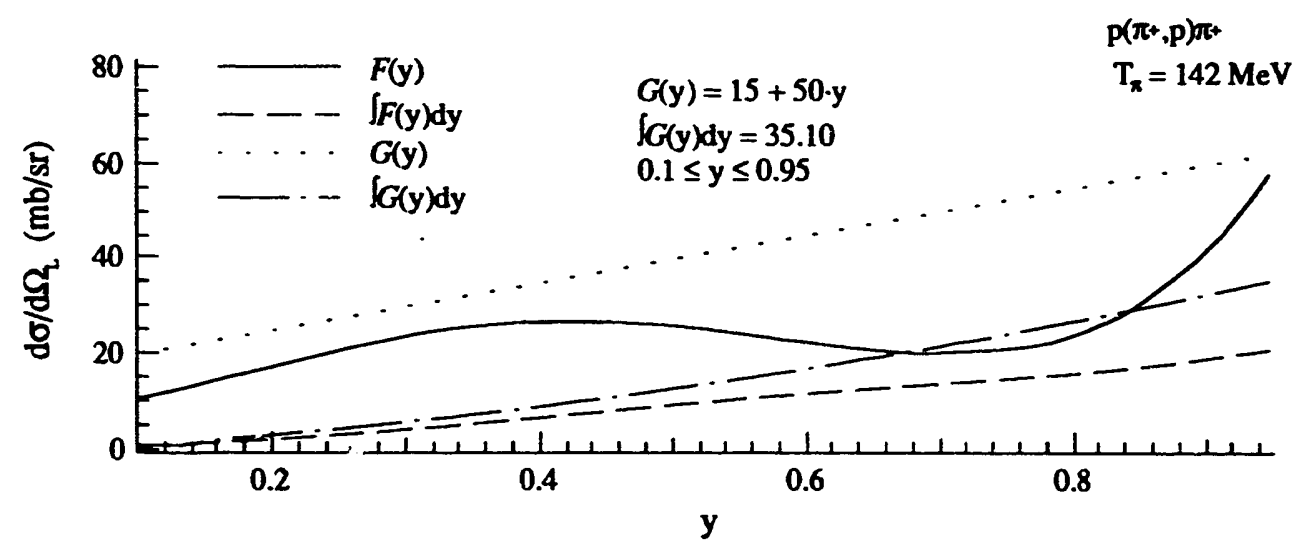

Figure B.6: Empirical functions for generating recoil angles using the rejection method for Monte Carlo simulations of the $\mathrm{p}\left(\pi^{+}, \mathrm{p}\right) \pi^{+}$reaction at $142 \mathrm{MeV}$. The solid line corresponds to the function, $F(y)$, which describes the differential cross section; the dashed line corresponds to the linear function, $G(y) ; y$ is the cosine of the proton laboratory angle.

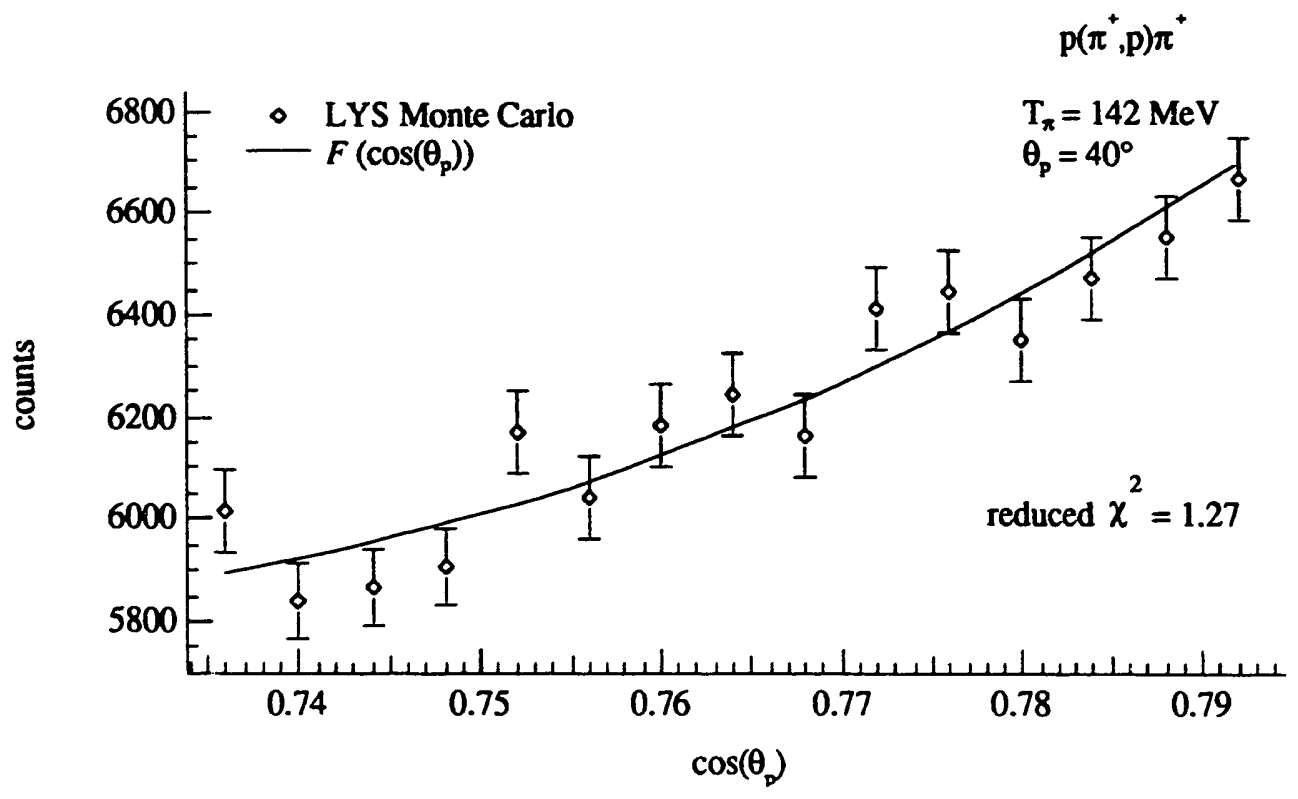

Figure B.7: Monte Carlo distribution of proton laboratory scattering angles (open points) in comparison with the polynomial fit to the laboratory differential cross section for $\pi^{+} \mathrm{p}$ elastic scattering at $142 \mathrm{MeV}$, $F\left(\cos \left(\theta_{\mathrm{p}}\right)\right)$. In this example, the spectrometer is placed at a laboratory scattering angle of $40^{\circ}$. 


\section{The Monte Carlo Correction Factor: $\gamma$}

Only a fraction of the recoil ${ }^{3} \mathrm{He}$ yield from ${ }^{3} \mathrm{H}\left(\pi^{+}, \mathrm{He}\right) \pi^{0}$ was observed for most of the ${ }^{3} \mathrm{H}\left(\pi^{+},{ }^{3} \mathrm{He}\right) \pi^{0}$ measurements. The Litule Yellow Spectrometer Monte Carlo simulation could be used to calculate a correction factor, $\gamma$, so that a differential cross section could be extracted from these measurements. In Figure B.8, a measurement of the ${ }^{3} \mathrm{He}$ yield from ${ }^{3} \mathrm{H}\left(\pi^{+},{ }^{3} \mathrm{He}\right) \pi^{0}$ at $142 \mathrm{MeV}$ and a laboratory angle of $65^{\circ}$ is shown in comparison with the Monte Carlo prediction for the ${ }^{3} \mathrm{He}$ peak shape. Once this function was calculated, it was integrated and normalized so that the Monte Carlo peak area is unity. The correction factor was determined by integrating the normalized function over the limits of the momentum region of the measured ${ }^{3} \mathrm{He}$ peak. In Figure B.9, the normalized integral of the Monte Carlo peak shape from Figure B.8 is shown as a function of momentum. The correction factor for the example in Figure B.8 may be determined by finding the value for $\gamma$ which corresponds to the upper limit on the measured momentum region in Figure B.9.

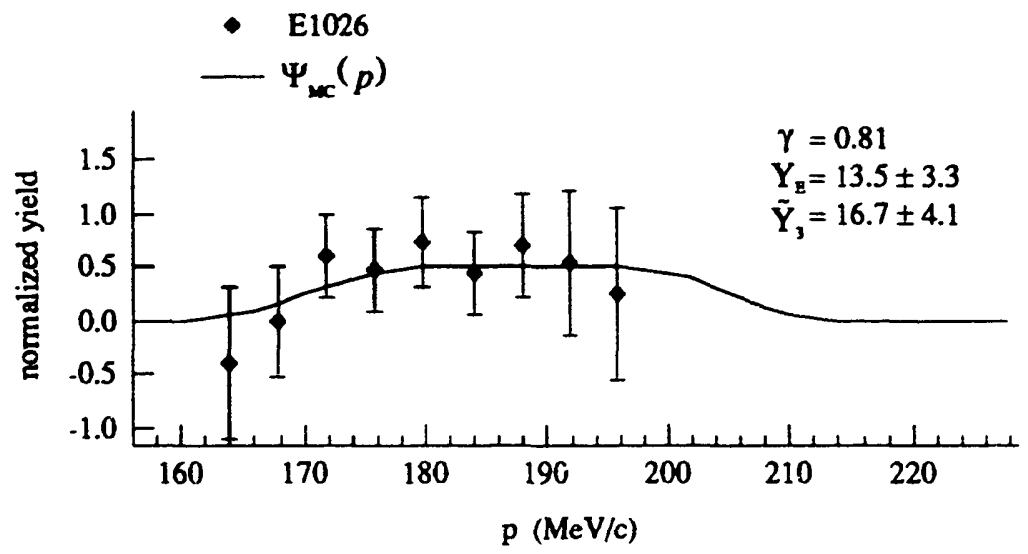

Figure B.8: Measured ${ }^{3} \mathrm{He}$ yield from ${ }^{3} \mathrm{H}\left(\pi^{+},{ }^{3} \mathrm{He}\right) \pi^{0}$ at $142 \mathrm{MeV}$ and $\theta_{3}=65^{\circ}$. In this figure, the Monte Carlo function, $\Psi_{M C}$, has been scaled so that the integral of the function is equal to the net expected yield of ${ }^{3} \mathrm{He}$ particles from ${ }^{3} \mathrm{H}\left(\pi^{+}, \mathrm{He}\right) \pi^{0}, \bar{Y}_{3}$. 


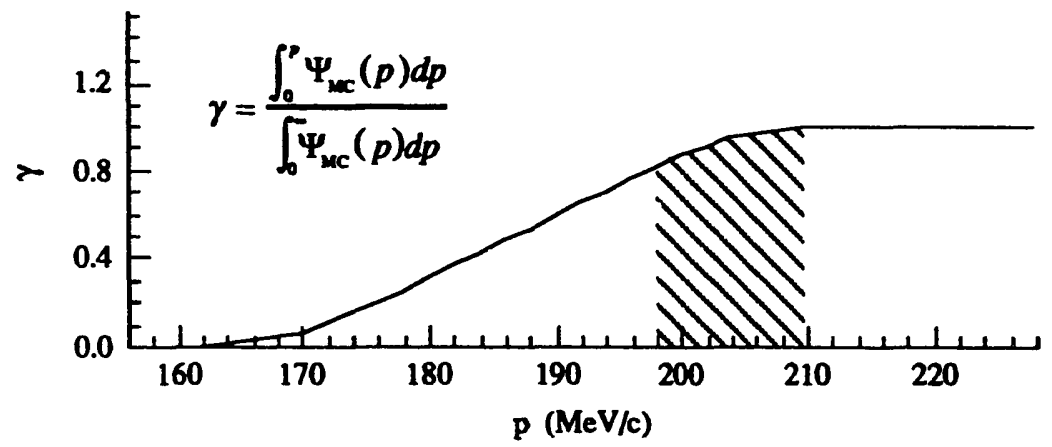

Figure B.9: Calculation of the correction factor, $\gamma$, for the previous example shown in Figure B.8. The shaded region corresponds to the unmeasured region of the ${ }^{3} \mathrm{He}$ peak. 


\section{Appendix C: Energy Loss Parametrization}

This appendix includes information about the energy loss parametrisations and Monie Carlo generators for the Little Yellow Spectrometer simulation. The energy losses of heavy charged particles in the targets and silicon surface barricr detectors were calculatej using the residual range method, as follows. First, the initial range of a particle, $R_{\text {inisiar }}$ is calculated for the material of interest. The range of a particle can be expressed in terms of the proton range in the same material, $R_{p}$, as follows,

$$
\mathrm{R}(T)=\frac{\mathrm{M}}{\mathbf{Z}^{2}} \cdot \mathrm{R}_{\mathbf{p}}\left(\frac{T}{\mathrm{M}}\right) \text {, }
$$

where $\mathrm{M}, \mathrm{Z}$, and $T$ are the mass (in proton mass units), charge, and kinetic energy of the particle. The proton range can be described by a power law function of the form,

$$
R_{p}(\tau)=A+B \cdot \tau^{c} \text {, }
$$

where $\tau$ is the kinetic energy of the proton and the coefficients, $A, B$, and $C$, are determined from fitting proton range data ${ }^{75}$ for the material of interest. Table C.1 lists the range parametrizations for each material included in the Monte Carlo simulation. A typical fit to proton range data from $\mathrm{CH}_{2}$ is shown in

\begin{tabular}{|c|c|c|c|}
\hline \multicolumn{4}{|c|}{$\begin{array}{c}\text { Monte Carlo Range Parameters for Generating Energy Loss } \\
\text { Distributions }\end{array}$} \\
\hline material & $\begin{array}{r}A\left(10^{-1}\right) \\
\left(\mathrm{g} / \mathrm{cm}^{2}\right)\end{array}$ & $\underset{\left(\mathrm{g} / \mathrm{MeV} \cdot \mathrm{cm}^{2}\right)}{\mathrm{B}\left(10^{-3}\right)}$ & $\mathbf{C}$ \\
\hline $\mathrm{CH}_{2}$ & $1.16 \pm 0.20$ & $1.85 \pm 0.04$ & $1.788 \pm 0.007$ \\
\hline${ }^{12} \mathrm{C}$ & $1.64 \pm 0.15$ & $2.50 \pm 0.05$ & $1.750 \pm 0.008$ \\
\hline Silicon & $2.54 \pm 0.27$ & $3.16 \pm 0.07$ & $1.730 \pm 0.008$ \\
\hline Titanium & $4.39 \pm .020$ & $3.62 \pm 0.07$ & $1.733 \pm 0.006$ \\
\hline
\end{tabular}
Figure C.1.

Table C.1: Monte Carlo range parameters for energy loss calculations. These parameters were determined fits to tables of proton range data. ${ }^{75}$ 
The final range of the particle, $\boldsymbol{R}_{\text {frel }}$, is calculated by subtracting the amount of the material that the particle travels through from the initial range of the particle,

$$
R_{\text {fur }}=R_{-}-\rho \tau \text {, }
$$

where $\rho \tau$ is the thickness of the material in $g / \mathrm{cm}^{2}$. If $R_{\text {find }}$ is greater than zero the remaining kinetic energy of the particle is determined from Equations C.1 and C.2. If $R_{f}$ is less than or equal to zero, th: particle stops in the material. The difference between the initial and final kinetic energies of the particle is the mean energy loss, $\bar{\Delta}$. If the particle does not stop in the material, the energy loss of the particle, $\Delta$, is smeared according to a gaussian distribution of the form,

$$
f(\tau, \Delta) \propto \exp \left(-\frac{(\Delta-\bar{\Delta})^{2}}{2 \sigma^{2}}\right)
$$

For non-relativistic heavy particles, the energy spread of this gaussian, $\sigma$, may be expressed as,

$$
\sigma^{3}=4 \pi N_{e} r_{0}^{2}\left(m_{\bullet} c^{2}\right)^{2} \frac{Z}{A} \rho x \quad\left(M e V^{2}\right)
$$

where $N_{a}$ is Avogadro's number, $r_{0}$ and $m_{a}$ are the classical electron radius and mass, and $Z, A, \rho$, and $x$ are the atomic number, atomic weight, density (in $\mathrm{g} / \mathrm{cm}^{3}$ ) and thickness (in $\mathrm{cm}$ ) of the material. One may assume that the energy loss of heavy charged particles in a material follows a gaussian distribution when the mean energy loss of a particle is much greater than the maximum allowed energy loss resulting from a single collision in the material. This condition was satisfied during the course of these measurements.

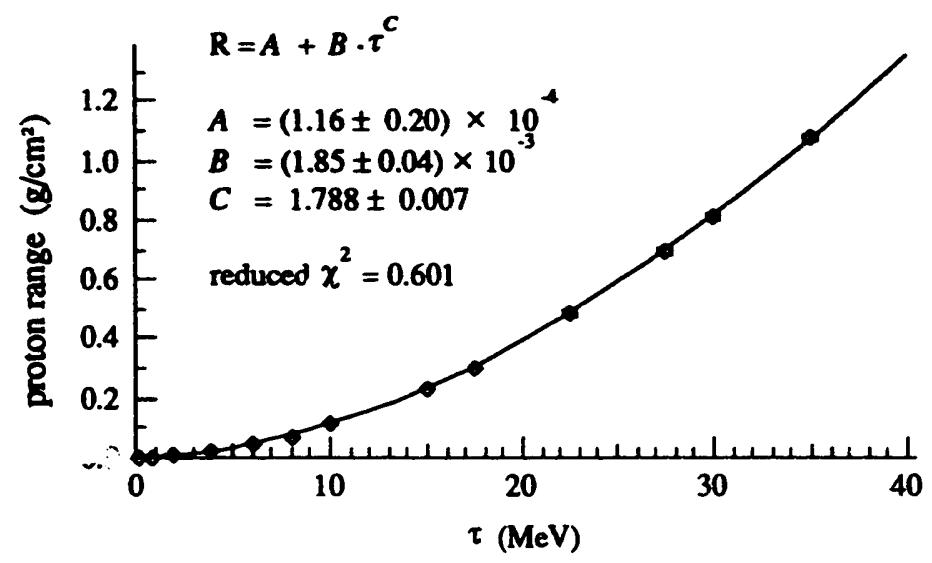

Figure C.1: Typical power law fit to data from proton range tables. In this example, the material is $\mathrm{CH}_{2}$. 


\section{Appendix D: Proton Detector Calibration}

Two methods of obtaining monoenergetic protons were employed in the energy calibration measurements of the proton scintillation counters. In the first method, the proton absorbing slit wirt: it the LEP cha.inel (shown in Figure 5.1) was removed and the detectors were calibrated using monoenergetic protons which have the same magnetic rigidity, p/q, as the incident pions. In the second method, two detectors were calibrated simultaneously by observing protons from pion absorption on deuterium using a $\mathrm{CD}_{2}$ target: $\pi^{+} \mathrm{d} \rightarrow \mathrm{pp}$. The range of observable proton kinetic energies using either of these two methods was extended by placing a variable amount of aluminum degrader in front of the proton detector of interest. A list of the calibration methods and corresponding proton kinetic energies is presented in Table D.1.

\begin{tabular}{|c|l|l|l|l|l|}
\hline method & $\begin{array}{c}\mathrm{T}_{\mathrm{x}} \\
(\mathrm{MeV})\end{array}$ & $\begin{array}{c}\mathrm{T}_{\mathrm{p}} \\
(\mathrm{MeV})\end{array}$ & $\begin{array}{c}\mathrm{Al} \text { degrader } \\
(\mathrm{in})\end{array}$ & $\begin{array}{c}\Delta \mathrm{E} \\
(\mathrm{MeV})\end{array}$ & $\begin{array}{c}\mathrm{E} \\
(\mathrm{MeV})\end{array}$ \\
\hline $\mathrm{d}\left(\pi^{+}, \mathrm{p}\right) \mathrm{p}$ & 149 & 141.0 & 0.0 & 1.9 & 136.3 \\
\hline $\mathrm{d}\left(\pi^{+}, \mathrm{p}\right) \mathrm{p}$ & 149 & 141.0 & 0.5 & 2.1 & 120.0 \\
\hline $\mathrm{d}\left(\pi^{+}, \mathrm{p}\right) \mathrm{p}$ & 149 & 141.0 & 1.0 & 2.3 & 101.9 \\
\hline $\mathrm{d}\left(\pi^{+}, \mathrm{p}\right) \mathrm{p}$ & 149 & 167.2 & 0.0 & 1.7 & 163.3 \\
\hline $\mathrm{d}\left(\pi^{+}, \mathrm{p}\right) \mathrm{p}$ & 149 & 167.2 & 0.5 & 1.8 & 149.1 \\
\hline $\mathrm{d}\left(\pi^{+}, \mathrm{p}\right) \mathrm{p}$ & 149 & 167.2 & 1.0 & 1.9 & 133.8 \\
\hline proton beam & 230 & 60.5 & 0.0 & 3.7 & 55.5 \\
\hline proton beam & 270 & 76.9 & 0.0 & 3.0 & 72.9 \\
\hline proton beam & 270 & 76.9 & 0.5 & 4.4 & 43.5 \\
\hline
\end{tabular}

Table D.1: Proton detector calibration measurements. The initial proton kinetic energy from these calibration procedures is listed in column " $T$ ". The amount of energy deposited in the $\Delta E$ and $E$ scintillation counters is presented in the columns labeled " $\Delta E$ " and " $E$ ". The amount of energy deposited in each of the detector elements was derived from energy loss calculations. These energy loss calculations included assumptions about the amount of air, target, and scintillator wrappirig material that a proton traveled through before depositing energy in a scintillation counter. 


\section{Calibration Procedure}

During the calibration measurements using the proton beam method, each proton detector was placed directly in the beam line one at a time. In the pion absorption studies, the prown detector array was positioned uch that protons were observed by two proton detectors sin: altaneously at laboratory angles of $56^{\circ}$ and $79^{\circ}$. Examples of the pulse height spectra from the $\Delta E$ and $E$ scintillation counters using these methods are shown in Figures D.1 and D.2. The proton peak positions were determined from fitting pulse height spectra by a skewed gaussian plus a second order polynomial to describe the background.

The nominal proton kinetic enargies from each of these methods, presented in Table D.1, was corrected for pruton energy losses in various materials to determine the energy deposited in the scintillation counters. These energy losses were calculated using the Bethe-Bloch formula for the stopping power, or the energy loss per unit lergth, of heavy charged particles in various materials. These materials included the $\mathrm{CD}_{2}$ target, the distance in air which protons traveled from the target to the proton detector, and the scinillator wrapping material (aluminized mylar and black tape).

The energy calibration of each scintillation counter was determined by fitting the corrected proton energy losses, $E$, in the scintillation counters and the peak positions, $x$, for each scintillation counter by the following relationship,

$$
E=B \cdot(x-A),
$$

where $A$ and $B$ are in units of (channel number) and (MeV/channel number) respectively. Examples of typical fits to $\triangle E$ and E scintillation counter calibration data are shown in Figures D.3 and D.4. The results of these fits are presented in Tables D.3 and D.4. In these fitting procedures, uncertainties of 2 and 0.25 $\mathrm{MeV}$ were assigned to the calculated proton energy losses in the $\mathrm{E}$ and $\Delta \mathrm{E}$ scintillation counters respectively. These uncertainties arise from uncertainties in the proton energy losses in other materials, e.g. scintillator wrapping material and the absorber thicknesses. Small discrepancies in the description of the experimental set-up during the calibration measurements, such as the distance from the end of the beam line to the target stand for calculating energy losses of beam protons in air, also contributed to the uncertainty in the actual proton energy that was deposited in a scintillation counter. The proton energy calibration 
information was sufficiently accurate for the purpose of this experiment in which proton energy spectra were integrated from 25 to $175 \mathrm{MeV}$ to determine triply differential cross sections from the ${ }^{3} \mathrm{He}\left(\pi^{ \pm}, \pi^{\circ} \mathrm{p}\right)$ data.

a)

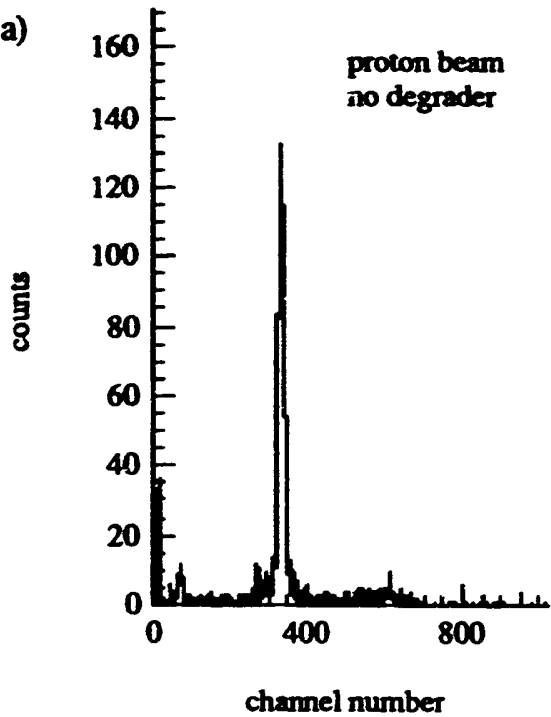

c)

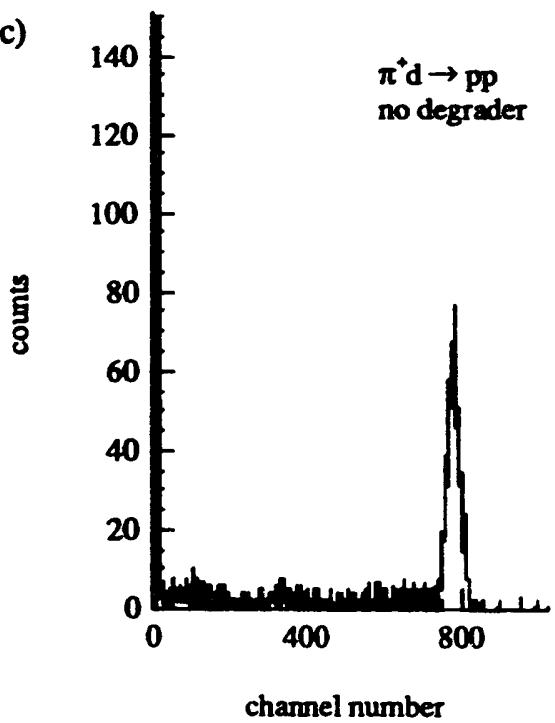

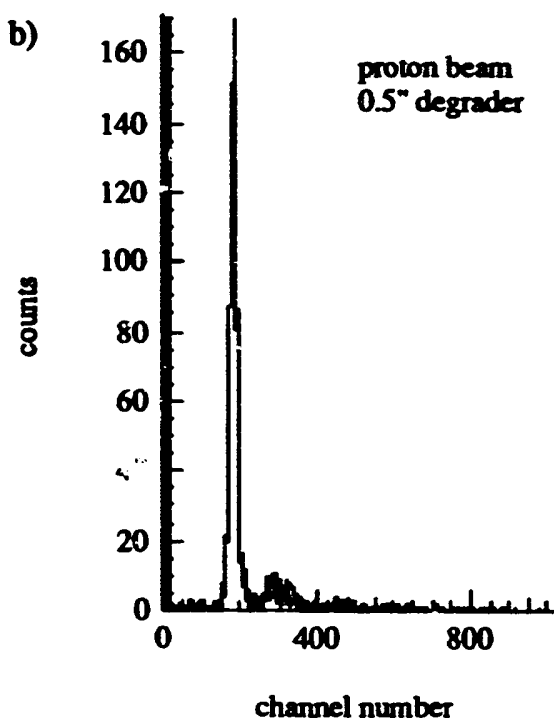

d)

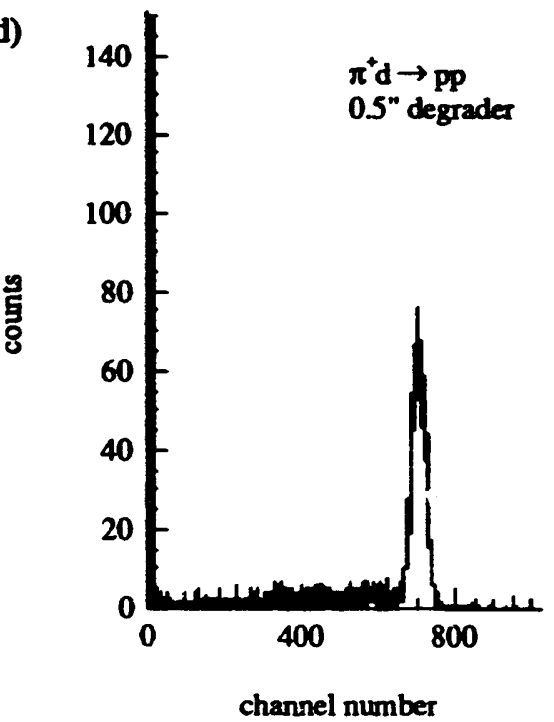

Figure D.1: Pulse height spectra from an E scintillation counter. These spectra were accumulated during calibration measurements using beam protons a) with and b) without an aluminum degrader and protons from the $d\left(\pi^{*}, p\right) p$ reaction $c$ ) with and $d$ ) without an aluminum degrader. These spectra are from measurements using the central proton detector (detector 4 ). 

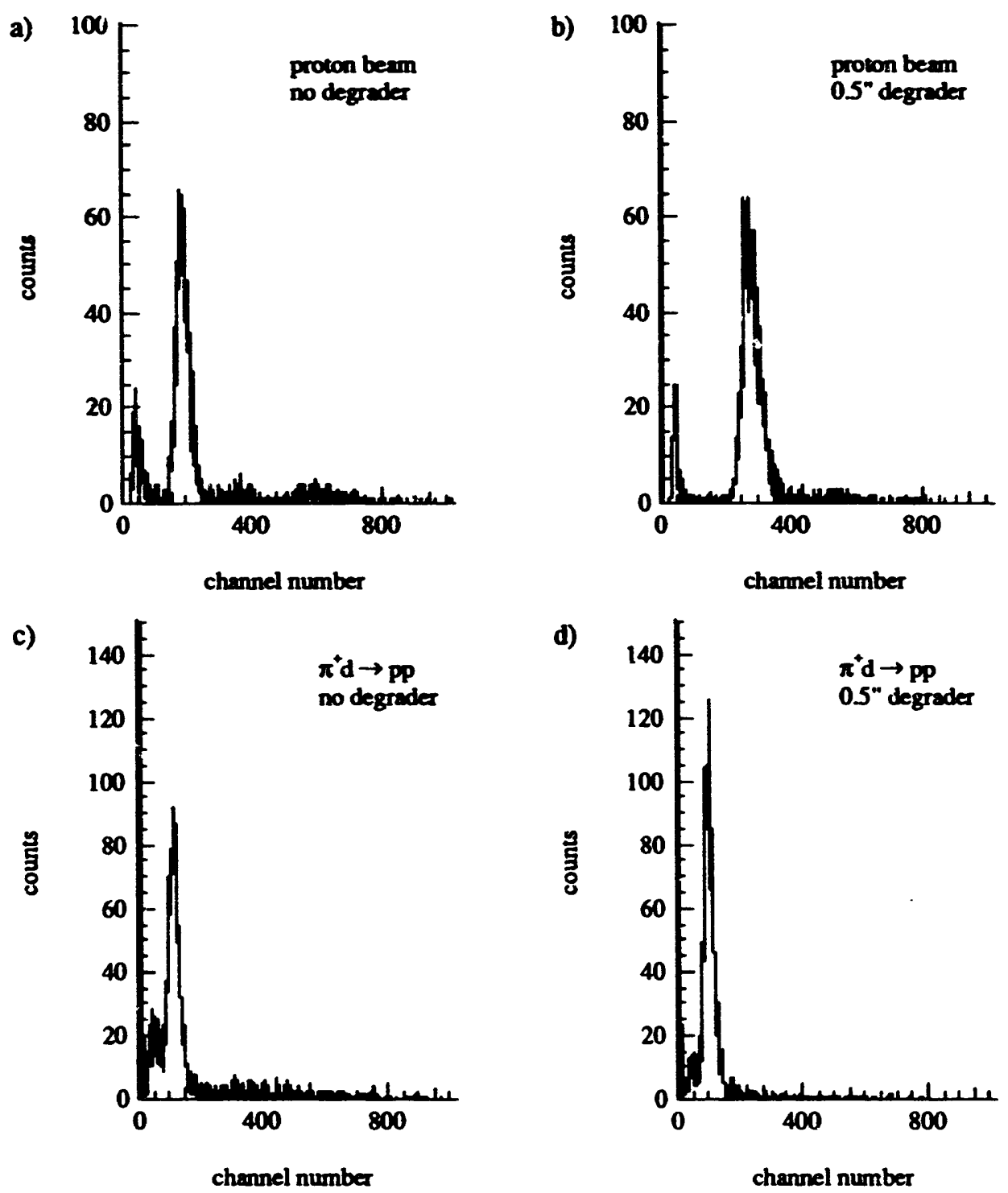

Figure D.2: Pulse height spectra from a $\Delta E$ scintillation counter. These spectra were accumulated during calibration measurements using beam protons a) with and b) without an aluminum degrader and protons from the $d\left(\pi^{+}, p\right) p$ reaction $c$ ) with and d) without an aluminum degrader. These spectra are from measurements using the central proton detector (detector 4). 


\begin{tabular}{|c|c|c|}
\hline \multicolumn{3}{|c|}{ Calibration Parameters for E counters } \\
\hline detector & $A$ & $B$ \\
\hline 1 & $-244 \pm 15$ & $0.1268 \pm 0.0025$ \\
\hline 2 & $-86 \pm 12$ & $0.1903 \pm 0.0037$ \\
\hline 3 & $-45.9 \pm 6.2$ & $0.3619 \pm 0.0064$ \\
\hline 4 & $-j 3 \pm 12$ & $0.2009 \pm 0.0038$ \\
\hline 5 & $-63 \pm 11$ & $0.2110 \pm 0.0041$ \\
\hline 6 & $-125 \pm 11$ & $0.1585 \pm 0.0026$ \\
\hline 7 & $-76.2 \pm 5.5$ & $0.1691 \pm 0.0017$ \\
\hline 8 & $-41.3 \pm 2.9$ & $0.3024 \pm 0.0029$ \\
\hline
\end{tabular}

Table D.2: Energy calibration parameters for $E$ scintillation counters. These parameters were derived from fitting the calibration data for each E scintillation by a function of the form of Equation D.1.

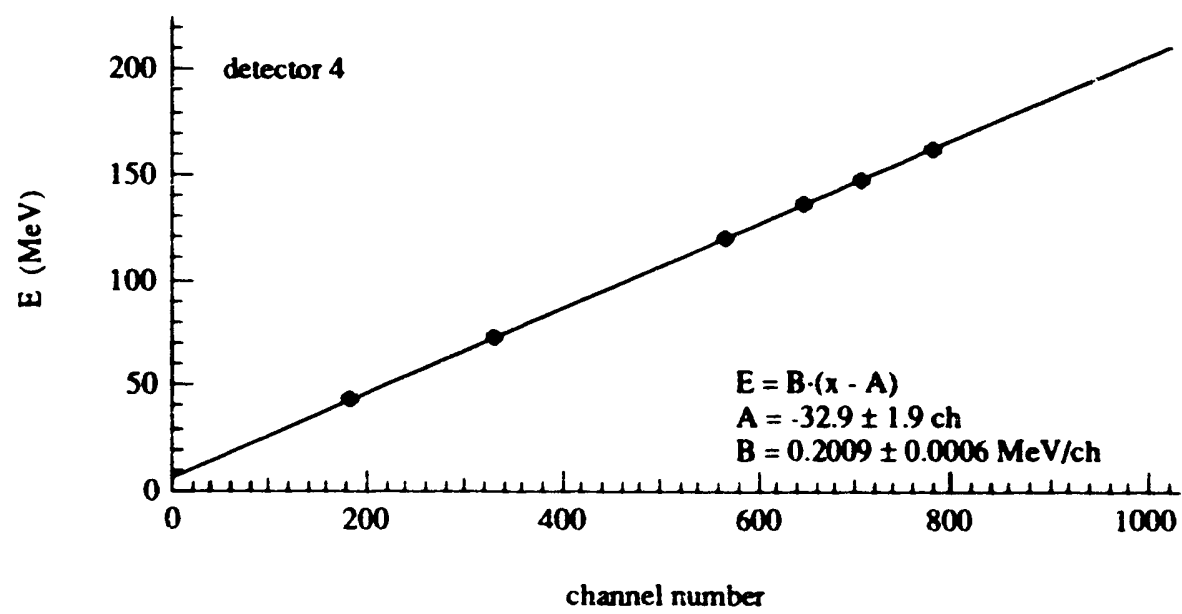

Figure D.3: E scintillation counter calibration data. This example displays the calibration data from the E scintillation counter of the central proton detaxtor (detector 4). 


\begin{tabular}{|c|c|c|}
\hline \multicolumn{3}{|c|}{$\begin{array}{c}\text { Calibration Parameters for } \Delta E \\
\text { counters }\end{array}$} \\
\hline detectar & $A$ & $\begin{array}{c}B \\
\left(10^{-2}\right)\end{array}$ \\
\hline 1 & $-3 \pm 14$ & $1.085 \pm 0.054$ \\
\hline 2 & $-25 \pm 14$ & $1.147 \pm 0.069$ \\
\hline 3 & $-5 \pm 12$ & $1.145 \pm 0.065$ \\
\hline 4 & $-18 \pm 11$ & $1.519 \pm 0.092$ \\
\hline 5 & $-42 \pm 13$ & $1.359 \pm 0.094$ \\
\hline 6 & $-13 \pm 10$ & $1.490 \pm 0.074$ \\
\hline 7 & $7.4 \pm 5.8$ & $1.198 \pm 0.033$ \\
\hline 8 & $-3 \pm 10$ & $0.988 \pm 0.036$ \\
\hline
\end{tabular}

Table D.3: Energy calibration parameters for $\Delta E$ scintillation counters. These parameters were derived from fiting the calibration data for each $\Delta E$ scintillation by a function of the form of Equation $D .1$.

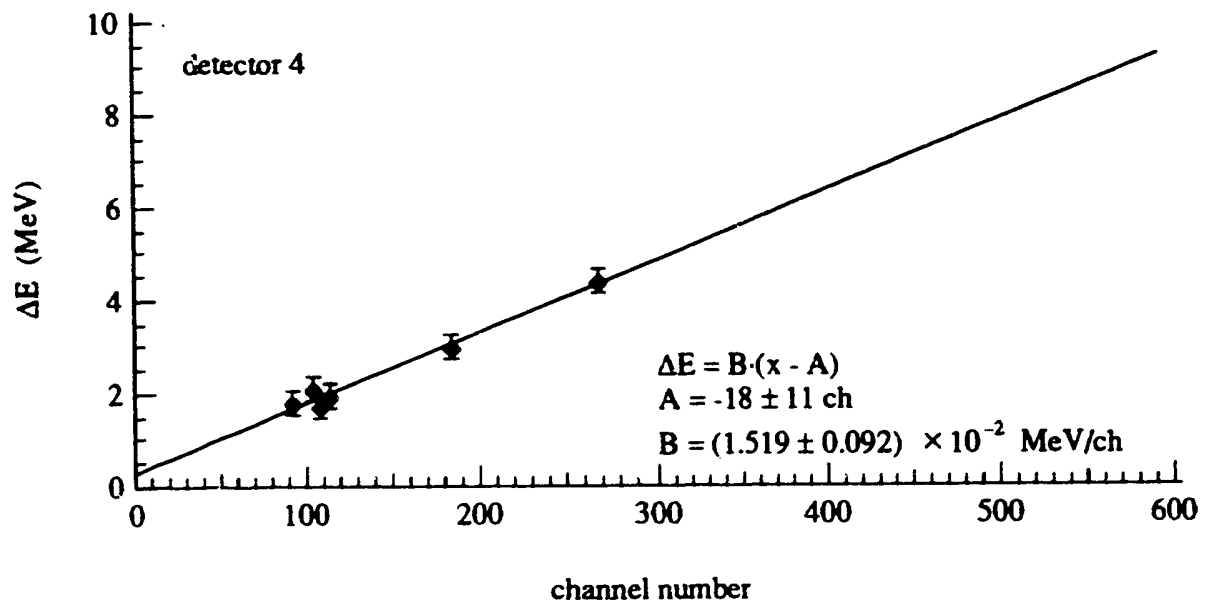

Figure D.4: $\Delta E$ scintillation counter calibration data. This example displays the calibration data from the $\triangle \mathrm{E}$ scintillation counter of the central proton detector (detector 4 ). 


\section{Appendix E: Tables of Inclusive Pion Single Charge Exchange Cross Sections}

The tables in this appendix present the cross sections from the single-arm and coincidence measurements of pion single charge exchange on ${ }^{3} \mathrm{He}$. Doubly differential cross sections for the single-arm ${ }^{3} \mathrm{He}\left(\pi^{+}, \pi^{9}\right)$ measurements at $\mathrm{T}_{x}=245 \mathrm{MeV}$ and central $\pi^{\circ}$ Spectrometer scattering angles of $70^{\circ}, 90^{\circ}, 110^{\circ}$, and $120^{\circ}$ are presented in separate tables. Each of these tables presents the doubly differential cross sections for the three angular bins at a specific central scattering angle of the $\pi^{0}$ Spectrometer. Triply differential cross sections for the ${ }^{3} \mathrm{He}\left(\pi^{+}, \pi^{0} \mathrm{p}\right)$ measurements follow the single-arm data. These cross sections are presented with the corresponding in-plane and out-of-plane proton angles as a function of outgoing $\pi^{0}$ kinetic energy and grouped together according to the proton detector array configuration which was used to acquire the data. Doubly differential cross sections for the ${ }^{3} \mathrm{He}\left(\pi^{+}, \pi^{0} \mathrm{p}\right)$ measurements are tabulated as a function of proton angle at $\pi^{\circ}$ laboratory scattering angles of $70^{\circ}, 90^{\circ}, 110^{\circ}$, and $120^{\circ}$. 


\begin{tabular}{|c|c|c|c|}
\hline \multicolumn{4}{|c|}{${ }^{3} \mathrm{He}\left(\pi^{+}, \pi^{0}\right)$ at $\mathrm{T}_{x}=245 \mathrm{MeV}$} \\
\hline \multirow{2}{*}{$\begin{array}{c}\mathrm{T}_{\boldsymbol{x}^{*}} \\
(\mathrm{MeV})\end{array}$} & \multicolumn{3}{|c|}{$\mathrm{d}^{2} \sigma / \mathrm{d} \mathrm{E}_{x^{\prime}} \mathrm{d} \Omega_{\pi}(\mu \mathrm{b} / \mathrm{MeV} \cdot \mathrm{sr})$} \\
\hline & $\theta_{x^{\circ}}=62^{\circ}$ & $\theta_{x}=70^{\circ}$ & $\theta_{x^{\circ}}=78^{\circ}$ \\
\hline $\mathrm{i} 00.0$ & $13.01 \pm 2.06$ & $10.37 \pm 1.33$ & $10.44 \pm 1.85$ \\
\hline 106.0 & $7.74 \pm 1.31$ & $9.05 \pm 1.02$ & $6.11 \pm 1.17$ \\
\hline 112.0 & $7.29 \pm 1.09$ & $7.41 \pm 0.82$ & $9.83 \pm 1.22$ \\
\hline 118.0 & $7.25 \pm 0.94$ & $7.01 \pm 0.70$ & $9.39 \pm 1.04$ \\
\hline 124.0 & $10.34 \pm 0.98$ & $8.29 \pm 0.70$ & $7.35 \pm 0.84$ \\
\hline 130.0 & $7.87 \pm 0.83$ & $8.22 \pm 0.66$ & $9.22 \pm 0.84$ \\
\hline 136.0 & $10.35 \pm 0.86$ & $11.14 \pm 0.71$ & $11.61 \pm 0.88$ \\
\hline 142.0 & $11.60 \pm 0.88$ & $9.81 \pm 0.67$ & $9.94 \pm 0.79$ \\
\hline 148.0 & $12.41 \pm 0.91$ & $9.51 \pm 0.66$ & $10.57 \pm 0.82$ \\
\hline 154.0 & $12.77 \pm 0.90$ & $11.24 \pm 0.72$ & $10.58 \pm 0.81$ \\
\hline 160.0 & $12.97 \pm 0.91$ & $11.49 \pm 0.75$ & $9.69 \pm 0.79$ \\
\hline 166.0 & $12.81 \pm 0.93$ & $11.18 \pm 0.72$ & $8.38 \pm 0.74$ \\
\hline 172.0 & $15.97 \pm 1.01$ & $13.47 \pm 0.80$ & $8.25 \pm 0.74$ \\
\hline 178.0 & $14.83 \pm 0.98$ & $11.72 \pm 0.77$ & $6.20 \pm 0.63$ \\
\hline 184.0 & $14.36 \pm 0.98$ & $7.93 \pm 0.66$ & $5.38 \pm 0.60$ \\
\hline 190.0 & $12.49 \pm 0.96$ & $5.61 \pm 0.58$ & $2.15 \pm 0.38$ \\
\hline 196.0 & $9.44 \pm 0.82$ & $3.69 \pm 0.50$ & $2.31 \pm 0.42$ \\
\hline 202.0 & $6.89 \pm 0.73$ & $2.24 \pm 0.41$ & $1.88 \pm 0.41$ \\
\hline 208.0 & $4.33 \pm 0.66$ & $1.57 \pm 0.36$ & $0.63 \pm 0.25$ \\
\hline 214.0 & $1.85 \pm 0.48$ & $1.09 \pm 0.30$ & $0.61 \pm 0.31$ \\
\hline 220.0 & $1.12 \pm 0.39$ & $0.54 \pm 0.24$ & $0.42 \pm 0.22$ \\
\hline 226.0 & $0.36 \pm 0.34$ & $-0.02 \pm 0.15$ & $0.06 \pm 0.14$ \\
\hline 232.0 & $0.94 \pm 0.35$ & $0.52 \pm 0.27$ & $0.02 \pm 0.15$ \\
\hline 238.0 & $0.71 \pm 0.32$ & $0.40 \pm 0.26$ & $0.13 \pm 0.18$ \\
\hline 244.0 & $0.51 \pm 0.35$ & $-0.14 \pm 0.22$ & $0.46 \pm 0.30$ \\
\hline 250.0 & $0.38 \pm 0.32$ & $0.40 \pm 0.34$ & $-0.15 \pm 0.25$ \\
\hline 256.0 & $0.71 \pm 0.46$ & $0.00 \pm 0.36$ & $0.23 \pm 0.32$ \\
\hline
\end{tabular}

Table E.1: Doubly differential cross sections for the ${ }^{3} \mathrm{He}\left(\pi^{+}, \pi^{0}\right)$ reaction at $\mathrm{T}_{n}=245 \mathrm{MeV}$. These spectra are derived from data which were accumulated at a central $\pi^{\circ}$ Spectrometer s( attering angle of $70^{\circ}$. The spectrometer acceptance was partitioned into three sections each corresponding to an $8^{\circ}$ angular bin. An overall systematic uncertainty of $8 \%$ is not included. 


\begin{tabular}{|c|c|c|c|}
\hline \multicolumn{4}{|c|}{${ }^{3} \mathrm{He}\left(\pi^{2}, \pi^{0}\right)$ at $\mathrm{T}_{x}=245 \mathrm{MeV}$} \\
\hline \multirow{2}{*}{$\begin{array}{c}\mathrm{T}_{\mathbf{x}} \\
(\mathrm{MeV})\end{array}$} & \multicolumn{3}{|c|}{$\mathrm{d}^{2} \sigma / \mathrm{dE} \mathrm{E}_{\mathrm{x}} \mathrm{d} \Omega_{\mathrm{r}}(\mu \mathrm{b} / \mathrm{MeV} \cdot \mathrm{sr})$} \\
\hline & $\theta_{x^{\prime}}=82^{\circ}$ & $\theta_{x^{\circ}}=90^{\circ}$ & $\theta_{x}=98^{\circ}$ \\
\hline 80.0 & $12.52 \pm 2.55$ & $12.29 \pm 1.89$ & $12.16 \pm 2.31$ \\
\hline 86.0 & $13.66 \pm 2.32$ & $15.38 \pm 1.81$ & $10.86 \pm 1.89$ \\
\hline 92.0 & $12.64 \pm 1.87$ & $11.80 \pm 1.31$ & $12.55 \pm 1.69$ \\
\hline 98.0 & $12.00 \pm 1.48$ & $9.82 \pm 1.00$ & $11.15 \pm 1.37$ \\
\hline 104.0 & $8.68 \pm 1.06$ & $10.20 \pm 0.88$ & $12.24 \pm 1.20$ \\
\hline 110.0 & $8.19 \pm 0.93$ & $10.10 \pm 0.79$ & $13.85 \pm 1.15$ \\
\hline 116.0 & $8.99 \pm 0.87$ & $9.33 \pm 0.72$ & $10.65 \pm 0.91$ \\
\hline 122.0 & $9.47 \pm 0.85$ & $10.37 \pm 0.74$ & $14.06 \pm 1.02$ \\
\hline 128.0 & $8.71 \pm 0.79$ & $10.80 \pm 0.73$ & $13.52 \pm 0.98$ \\
\hline 134.0 & $9.29 \pm 0.84$ & $9.73 \pm 0.72$ & $11.93 \pm 0.94$ \\
\hline 140.0 & $7.86 \pm 0.77$ & $10.25 \pm 0.74$ & $9.69 \pm 0.84$ \\
\hline 146.0 & $9.86 \pm 0.86$ & $8.74 \pm 0.70$ & $9.92 \pm 0.86$ \\
\hline 152.0 & $8.58 \pm 0.83$ & $6.95 \pm 0.63$ & $7.36 \pm 0.76$ \\
\hline 158.0 & $8.79 \pm 0.82$ & $6.55 \pm 0.63$ & $5.18 \pm 0.65$ \\
\hline 164.0 & $6.94 \pm 0.74$ & $4.90 \pm 0.55$ & $2.97 \pm 0.51$ \\
\hline 170.0 & $5.38 \pm 0.68$ & $4.05 \pm 0.51$ & $2.46 \pm 0.47$ \\
\hline 176.0 & $2.74 \pm 0.53$ & $2.23 \pm 0.42$ & $0.93 \pm 0.34$ \\
\hline 182.0 & $2.64 \pm 0.53$ & $1.77 \pm 0.40$ & $0.98 \pm 0.32$ \\
\hline 188.0 & $2.49 \pm 0.52$ & $0.38 \pm 0.24$ & $0.75 \pm 0.30$ \\
\hline 194.0 & $1.15 \pm 0.40$ & $0.59 \pm 0.31$ & $0.44 \pm 0.22$ \\
\hline 200.0 & $0.46 \pm 0.38$ & $0.62 \pm 0.35$ & $0.03 \pm 0.26$ \\
\hline 206.0 & $-0.02 \pm 0.30$ & $0.60 \pm 0.39$ & $-0.08 \pm 0.30$ \\
\hline 212.0 & $0.39 \pm 0.37$ & $0.32 \pm 0.50$ & $0.14 \pm 0.38$ \\
\hline 218.0 & $-0.11 \pm 0.50$ & $0.00 \pm 0.59$ & $0.19 \pm 0.51$ \\
\hline
\end{tabular}

Table E.2: Doubly differential cross sections for the ${ }^{3} \mathrm{He}\left(\pi^{+}, \pi^{0}\right)$ reaction at $\mathrm{T}_{\mathrm{x}}=245 \mathrm{MeV}$. These spectra are derived from data which were accumulated at a central $\pi^{\circ}$ Spectrometer scattering angle of $90^{\circ}$. The spectrometer acceptance was partitioned into three sections each corresponding to an $8^{\circ}$ angular bin. An overall systematic uncertainty of $8 \%$ is not included. 


\begin{tabular}{|c|c|c|c|}
\hline \multicolumn{4}{|c|}{${ }^{3} \mathrm{He}\left(\pi^{*}, \pi^{0}\right)$ at $\mathrm{T}_{x}=245 \mathrm{MEV}$} \\
\hline \multirow{2}{*}{$\begin{array}{c}\mathrm{T}_{\boldsymbol{x}} \\
(\mathrm{MeV})\end{array}$} & \multicolumn{3}{|c|}{$d^{2} \sigma / d \mathrm{E}_{\mathrm{x}} \cdot d \Omega_{\mathrm{x}} \cdot \quad(\mu \mathrm{b} / \mathrm{MeV} \cdot \mathrm{sr})$} \\
\hline & $\theta_{x^{\circ}}=102^{\circ}$ & $\theta_{x}=110^{\circ}$ & $\theta_{x^{\circ}}=118^{\circ}$ \\
\hline 66.0 & $12.30 \pm 2.38$ & $10.22 \pm 1.51$ & $9.33 \pm 1.73$ \\
\hline 72.0 & $11.62 \pm 1.87$ & $8.86 \pm 1.16$ & $8.44 \pm 1.31$ \\
\hline 78.0 & $13.67 \pm 1.65$ & $7.33 \pm 0.90$ & $10.45 \pm 1.22$ \\
\hline 84.0 & $13.96 \pm 1.51$ & $8.37 \pm 0.86$ & $8.90 \pm 0.99$ \\
\hline 90.0 & $9.69 \pm 1.18$ & $9.05 \pm 0.80$ & $10.14 \pm 0.96$ \\
\hline 96.0 & $9.84 \pm 1.01$ & $8.04 \pm 0.67$ & $10.23 \pm 0.90$ \\
\hline 102.0 & $12.70 \pm 1.04$ & $10.56 \pm 0.74$ & $10.14 \pm 0.84$ \\
\hline 108.0 & $11.22 \pm 0.96$ & $11.20 \pm 0.76$ & $10.68 \pm 0.80$ \\
\hline 114.0 & $11.14 \pm 0.99$ & $10.70 \pm 0.77$ & $9.64 \pm 0.79$ \\
\hline 120.0 & $10.02 \pm 0.93$ & $10.86 \pm 0.79$ & $8.75 \pm 0.79$ \\
\hline 126.0 & $9.23 \pm 0.93$ & $8.55 \pm 0.73$ & $7.23 \pm 0.73$ \\
\hline 132.0 & $8.97 \pm 0.91$ & $7.66 \pm 0.71$ & $6.21 \pm 0.70$ \\
\hline 138.0 & $7.93 \pm 0.91$ & $8.15 \pm 0.77$ & $4.93 \pm 0.68$ \\
\hline 144.0 & $5.73 \pm 0.83$ & $4.78 \pm 0.64$ & $4.52 \pm 0.65$ \\
\hline 150.0 & $4.20 \pm 0.68$ & $3.14 \pm 0.56$ & $2.51 \pm 0.53$ \\
\hline 156.0 & $4.19 \pm 0.72$ & $3.25 \pm 0.59$ & $2.08 \pm 0.50$ \\
\hline 162.0 & $2.57 \pm 0.61$ & $2.55 \pm 0.54$ & $0.91 \pm 0.41$ \\
\hline 168.0 & $2.25 \pm 0.62$ & $1.29 \pm 0.47$ & $0.80 \pm 0.35$ \\
\hline 174.0 & $0.66 \pm 0.42$ & $1.63 \pm 0.59$ & $1.26 \pm 0.53$ \\
\hline 180.0 & $0.73 \pm 0.41$ & $-0.04 \pm 0.29$ & $1.00 \pm 0.51$ \\
\hline
\end{tabular}

Table E.3: Doubly differential cross sections for the ${ }^{3} \mathrm{He}\left(\pi^{+}, \pi^{0}\right)$ reaction at $\mathrm{T}_{x}=245 \mathrm{MeV}$. These spectra are derived from data which were accumulated at a central $\pi^{\circ}$ Spectrometer scauering angle of $110^{\circ}$. The spectrometer acceptance was partitioned into three sections each corresponding to an $8^{\circ}$ angular bin. An overall systematic uncertainty of $8 \%$ is not included. 


\begin{tabular}{|c|c|c|c|}
\hline \multicolumn{4}{|c|}{${ }^{3} \mathrm{He}\left(\pi^{+}, \pi^{0}\right)$ at $\mathrm{T}_{x}=245 \mathrm{MeV}$} \\
\hline \multirow{2}{*}{$\begin{array}{c}\mathrm{T}_{\boldsymbol{x}} \\
(\mathrm{MeV})\end{array}$} & \multicolumn{3}{|c|}{$d \sigma / d \mathrm{E}_{x} \cdot d \Omega_{\mathrm{x}} \cdot \quad(\mu \mathrm{b} / \mathrm{MeV} \cdot \mathrm{sr})$} \\
\hline & $\theta_{N^{\circ}}=112^{\circ}$ & $\theta_{x^{\circ}}=120^{\circ}$ & $\theta_{R}=128^{\circ}$ \\
\hline 68.0 & $10.60 \pm 1.86$ & $5.02 \pm 1.13$ & $7.85 \pm 1.58$ \\
\hline 74.0 & $10.49 \pm 1.45$ & $8.21 \pm 1.08$ & $9.42 \pm 1.30$ \\
\hline 80.0 & $8.43 \pm 1.21$ & $8.00 \pm 0.88$ & $8.44 \pm 1.10$ \\
\hline 86.0 & $9.94 \pm 1.16$ & $8.49 \pm 0.81$ & $8.84 \pm 0.99$ \\
\hline 92.0 & $10.07 \pm 1.02$ & $7.17 \pm 0.71$ & $9.07 \pm 0.92$ \\
\hline 98.0 & $9.72 \pm 0.90$ & $10.09 \pm 0.76$ & $8.07 \pm 0.81$ \\
\hline 104.0 & $11.73 \pm 0.95$ & $11.05 \pm 0.75$ & $9.72 \pm 0.82$ \\
\hline 110.0 & $10.81 \pm 0.93$ & $9.95 \pm 0.73$ & $8.26 \pm 0.77$ \\
\hline 116.0 & $9.88 \pm 0.89$ & $8.62 \pm 0.69$ & $7.52 \pm 0.76$ \\
\hline 122.0 & $9.49 \pm 0.87$ & $7.30 \pm 0.64$ & $5.47 \pm 0.65$ \\
\hline 128.0 & $7.49 \pm 0.81$ & $6.49 \pm 0.63$ & $4.13 \pm 0.58$ \\
\hline 134.0 & $6.69 \pm 0.81$ & $4.69 \pm 0.60$ & $3.01 \pm 0.59$ \\
\hline 140.0 & $7.24 \pm 0.85$ & $3.83 \pm 0.53$ & $2.46 \pm 0.48$ \\
\hline 146.0 & $4.34 \pm 0.66$ & $3.64 \pm 0.60$ & $1.83 \pm 0.48$ \\
\hline 152.0 & $3.13 \pm 0.65$ & $2.78 \pm 0.57$ & $1.02 \pm 0.44$ \\
\hline 158.0 & $2.81 \pm 0.66$ & $1.07 \pm 0.47$ & $2.34 \pm 0.54$ \\
\hline 164.0 & $0.68 \pm 0.46$ & $0.79 \pm 0.43$ & $-0.30 \pm 0.35$ \\
\hline 170.0 & $0.49 \pm 0.47$ & $0.92 \pm 0.49$ & $1.44 \pm 0.53$ \\
\hline 176.0 & $0.94 \pm 0.47$ & $0.59 \pm 0.55$ & $0.21 \pm 0.47$ \\
\hline
\end{tabular}

Table E.4: Doubly differential cross sections for the ${ }^{3} \mathrm{He}\left(\pi^{*}, \pi^{0}\right)$ reaction at $\mathrm{T}_{x}=245 \mathrm{MeV}$. These spectra are derived from data which were accumulated at a central $\pi^{\circ}$ Spectrometer scattering angle of $120^{\circ}$. The spectrometer acceptance was partitioned into three sections each corresponding to an $8^{\circ}$ angular bin. An overall systematic uncertainty of $8 \%$ is not included. 


\begin{tabular}{|c|c|c|c|}
\hline \multicolumn{4}{|c|}{${ }^{3} \mathrm{He}\left(\pi, \pi^{0}\right)$ at $\mathrm{T}_{\pi}=245 \mathrm{MeV}$} \\
\hline \multirow{2}{*}{$\begin{array}{c}\mathrm{T}_{\mathbf{x}} \\
(\mathrm{MeV})\end{array}$} & \multicolumn{3}{|c|}{$\mathrm{d}^{2} \sigma / \mathrm{dE} \mathrm{x}_{\mathrm{x}} \mathrm{d} \Omega_{\mathrm{x}}(\mu \mathrm{b} / \mathrm{MeV} \cdot \mathrm{sr})$} \\
\hline & $\theta_{x^{\circ}}=62^{\circ}$ & $\theta_{x^{\circ}}=70^{\circ}$ & $\theta_{x^{\circ}}=78^{\circ}$ \\
\hline 100.0 & $14.87 \pm 3.17$ & $16.95 \pm 2.53$ & $15.88 \pm 3.35$ \\
\hline 106.0 & $14.59 \pm 2.62$ & $12.65 \pm 1.78$ & $18.81 \pm 2.88$ \\
\hline 112.0 & $17.65 \pm 2.44$ & $13.22 \pm 1.56$ & $13.81 \pm 2.23$ \\
\hline 118.0 & $12.23 \pm 1.79$ & $11.40 \pm 1.32$ & $15.10 \pm 1.90$ \\
\hline 124.0 & $12.10 \pm 1.62$ & $15.73 \pm 1.37$ & $19.11 \pm 1.98$ \\
\hline 130.0 & $15.94 \pm 1.62$ & $15.63 \pm 1.33$ & $17.19 \pm 1.76$ \\
\hline 136.0 & $17.01 \pm 1.62$ & $16.25 \pm 1.24$ & $20.39 \pm 1.75$ \\
\hline 142.0 & $22.13 \pm 1.81$ & $18.96 \pm 1.34$ & $23.73 \pm 1.81$ \\
\hline 148.0 & $21.67 \pm 1.69$ & $23.36 \pm 1.48$ & $28.03 \pm 1.93$ \\
\hline 154.0 & $27.52 \pm 1.96$ & $22.13 \pm 1.44$ & $26.70 \pm 1.88$ \\
\hline 160.0 & $29.23 \pm 1.99$ & $27.91 \pm 1.62$ & $28.60 \pm 1.91$ \\
\hline 166.0 & $33.09 \pm 2.16$ & $28.73 \pm 1.70$ & $20.59 \pm 1.68$ \\
\hline 172.0 & $34.40 \pm 2.18$ & $28.77 \pm 1.69$ & $16.93 \pm 1.48$ \\
\hline 178.0 & $33.57 \pm 2.19$ & $28.34 \pm 1.76$ & $14.74 \pm 1.46$ \\
\hline 184.0 & $37.88 \pm 2.36$ & $22.87 \pm 1.59$ & $11.53 \pm 1.27$ \\
\hline 190.0 & $30.89 \pm 2.15$ & $16.51 \pm 1.38$ & $6.98 \pm 1.12$ \\
\hline 196.0 & $30.28 \pm 2.13$ & $12.63 \pm 1.21$ & $4.98 \pm 0.97$ \\
\hline 202.0 & $20.72 \pm 1.91$ & $8.83 \pm 1.10$ & $4.87 \pm 0.91$ \\
\hline 208.0 & $12.98 \pm 1.53$ & $6.68 \pm 1.05$ & $2.27 \pm 0.67$ \\
\hline 214.0 & $8.33 \pm 1.27$ & $3.04 \pm 0.73$ & $1.42 \pm 0.81$ \\
\hline 220.0 & $5.61 \pm 1.11$ & $3.25 \pm 0.83$ & $1.47 \pm 0.69$ \\
\hline 226.0 & $4.60 \pm 1.07$ & $1.85 \pm 0.69$ & $0.88 \pm 0.59$ \\
\hline 232.0 & $1.78 \pm 0.81$ & $0.99 \pm 0.66$ & $0.00 \pm 0.51$ \\
\hline 238.0 & $1.15 \pm 0.77$ & $0.58 \pm 0.67$ & $0.28 \pm 0.59$ \\
\hline 244.0 & $1.40 \pm 0.94$ & $0.00 \pm 0.72$ & $0.33 \pm 0.69$ \\
\hline 250.0 & $0.41 \pm 0.85$ & $0.00 \pm 0.90$ & $0.41 \pm 0.83$ \\
\hline 256.0 & $0.00 \pm 1.07$ & $0.00 \pm 1.20$ & $0.00 \pm 1.07$ \\
\hline
\end{tabular}

Table E.5: Doubly differential cross sections for the ${ }^{3} \mathrm{He}\left(\pi^{-}, \pi^{0}\right)$ reaction at $\mathrm{T}_{\pi}=245 \mathrm{MeV}$. These spectra are derived from data which were accumulated at a central $\pi^{\circ}$ Spectrometer scattering angle of $70^{\circ}$. The spectrometer acceptance was partitioned into three sections each corresponding to an $8^{\circ}$ angular bin. An overall systematic uncertainty of $8 \%$ is not included. 


\begin{tabular}{|c|c|c|c|}
\hline \multicolumn{4}{|c|}{${ }^{3} \mathrm{He}\left(\pi, \pi^{0}\right)$ at $\mathrm{T}_{n}=245 \mathrm{MeV}$} \\
\hline \multirow{2}{*}{$\underset{(\mathrm{MeV})}{\mathrm{T}_{\mathbf{0}}}$} & \multicolumn{3}{|c|}{$\mathrm{d}^{2} \sigma / \mathrm{dE}_{\mathrm{x}} \mathrm{d} \Omega_{\mathrm{x}}(\mu \mathrm{b} / \mathrm{MeV} \cdot \mathrm{sr})$} \\
\hline & $\theta_{x^{\circ}}=102^{\circ}$ & $\theta_{x^{\circ}}=110^{\circ}$ & $\theta_{\alpha^{\circ}}=118^{\circ}$ \\
\hline 66.0 & $17.52 \pm 4.09$ & $17.31 \pm 2.75$ & $9.74 \pm 2.43$ \\
\hline 72.0 & $17.56 \pm 3.18$ & $13.37 \pm 2.03$ & $14.68 \pm 2.41$ \\
\hline 78.0 & $17.45 \pm 2.82$ & $16.96 \pm 1.94$ & $15.65 \pm 2.05$ \\
\hline 84.0 & $19.57 \pm 2.55$ & $13.12 \pm 1.50$ & $16.47 \pm 1.89$ \\
\hline 90.0 & $20.60 \pm 2.45$ & $19.00 \pm 1.60$ & $18.76 \pm 1.79$ \\
\hline 96.0 & $22.12 \pm 2.16$ & $19.57 \pm 1.46$ & $22.47 \pm 1.84$ \\
\hline 102.0 & $21.19 \pm 1.94$ & $25.32 \pm 1.63$ & $26.35 \pm 1.81$ \\
\hline 108.0 & $25.13 \pm 2.03$ & $23.79 \pm 1.55$ & $24.74 \pm 1.72$ \\
\hline 114.0 & $26.07 \pm 2.15$ & $24.50 \pm 1.61$ & $26.26 \pm 1.80$ \\
\hline 120.0 & $28.74 \pm 2.22$ & $22.78 \pm 1.62$ & $20.88 \pm 1.69$ \\
\hline 126.0 & $25.19 \pm 2.16$ & $19.35 \pm 1.53$ & $17.52 \pm 1.56$ \\
\hline 132.0 & $2.2 .18 \pm 2.08$ & $16.63 \pm 1.48$ & $14.57 \pm 1.49$ \\
\hline 138.0 & $19.90 \pm 2.04$ & $16.90 \pm 1.57$ & $9.05 \pm 1.25$ \\
\hline 144.0 & $13.47 \pm 1.75$ & $12.40 \pm 1.39$ & $7.52 \pm 1.18$ \\
\hline 150.0 & $13.47 \pm 1.73$ & $9.39 \pm 1.29$ & $5.18 \pm 1.01$ \\
\hline 156.0 & $5.97 \pm 1.21$ & $7.49 \pm 1.25$ & $3.04 \pm 0.89$ \\
\hline 162.0 & $9.53 \pm 1.68$ & $5.48 \pm 1.18$ & $3.38 \pm 0.93$ \\
\hline 168.0 & $5.56 \pm 1.34$ & $4.87 \pm 1.17$ & $1.03 \pm 0.76$ \\
\hline 174.0 & $3.74 \pm 1.20$ & $2.01 \pm 0.87$ & $0.56 \pm 0.61$ \\
\hline 180.0 & $2.04 \pm 0.98$ & $-0.82 \pm 0.73$ & $0.24 \pm 0.60$ \\
\hline
\end{tabular}

Table E.6: Doubly differential cross sh ions for the ${ }^{3} \mathrm{He}\left(\pi^{-} \pi^{9}\right)$ reaction at $T_{x}=245 \mathrm{MeV}$. These spectra are derived from data which were accumulated at a central $\pi^{\circ}$ Spectrometer scattering angle of $110^{\circ}$. The spectrometer acceptance was partitioned into three sections each corresponding to an $8^{\circ}$ angular bin. An overall systematic uncertainty of $8 \%$ is not included. 


\begin{tabular}{|c|c|c|c|c|c|c|c|c|}
\hline \multicolumn{9}{|c|}{${ }^{3} \mathrm{He}\left(\pi^{+}, \pi^{0} \mathrm{p}\right)$ at $\mathrm{T}_{\mathrm{k}}=245 \mathrm{MeV}$ and $\theta_{x}=70^{\circ}$} \\
\hline \multirow{2}{*}{$\begin{array}{c}\mathrm{T}_{\mathrm{n}} \\
(\mathrm{MeV})\end{array}$} & \multicolumn{8}{|c|}{$\mathrm{d}^{3} \sigma / \mathrm{dE} \mathrm{E}_{\mathrm{N}} \mathrm{d} \Omega_{\mathrm{N}} \mathrm{d} \Omega_{\mathrm{p}}\left(\mu \mathrm{b} / \mathrm{MeV} \cdot \mathrm{sr}^{2}\right)$} \\
\hline & $\left(-37.0^{\circ}, 22.0^{\circ}\right)$ & $\left(-37.0^{\circ}, 9.5^{\circ}\right)$ & $\left(-71.5^{\circ}, 0.0^{\circ}\right)$ & $\left(-60.0^{\circ}, 0.0^{\circ}\right)$ & $\left(-48.5^{\circ}, 0.0^{\circ}\right)$ & $\left(-37.0^{\circ}, 0.0^{\circ}\right)$ & $\left(-25.5^{\circ}, 0.0^{\circ}\right)$ & $\left(-14.0^{\circ}, 0.0^{\circ}\right)$ \\
\hline 100 & $1.63 \pm 2.01$ & $5.48 \pm 2.82$ & $1.15 \pm 2.01$ & $4.14 \pm 2.45$ & $3.90 \pm 2.28$ & $14.55 \pm 4.57$ & $5.47 \pm 2.82$ & $2.78 \pm 2.01$ \\
\hline 110 & $1.57 \pm 1.05$ & $5.59 \pm 1.97$ & $0.00 \pm 1.04$ & $3.95 \pm 1.66$ & $2.28 \pm 1.60$ & $5.18 \pm 1.90$ & $1.92 \pm 1.20$ & $4.57 \pm 1.86$ \\
\hline 120 & $2.08 \pm 1.01$ & $6.82 \pm 1.75$ & $0.82 \pm 0.83$ & $4.77 \pm 1.53$ & $5.26 \pm 1.61$ & $8.11 \pm 1.92$ & $3.92 \pm 1.33$ & $2.26 \pm 1.01$ \\
\hline 130 & $1.01 \pm 0.75$ & $6.04 \pm 1.44$ & $0.62 \pm 0.63$ & $4.53 \pm 1.25$ & $11.66 \pm 2.01$ & $7.02 \pm 1.62$ & $7.41 \pm 1.60$ & $2.62 \pm 0.99$ \\
\hline 140 & $1.54 \pm 0.67$ & $6.12 \pm 1.34$ & $0.39 \pm 0.51$ & $4.53 \pm 1.15$ & $8.01 \pm 1.59$ & $10.07 \pm 1.72$ & $5.66 \pm 1.28$ & $1.66 \pm 0.70$ \\
\hline 150 & $2.67 \pm 0.90$ & $10.89 \pm 1.73$ & $2.44 \pm 0.83$ & $5.48 \pm 1.23$ & $13.85 \pm 1.95$ & $16.60 \pm 2.14$ & $8.30 \pm 1.51$ & $5.15 \pm 1.20$ \\
\hline 160 & $1.48 \pm 0.67$ & $12.58 \pm 1.88$ & $2.80 \pm 0.90$ & $6.27 \pm 1.33$ & $14.93 \pm 2.05$ & $22.02 \pm 2.49$ & $5.22 \pm 1.22$ & $3.81 \pm 1.04$ \\
\hline 170 & $3.57 \pm 1.02$ & $11.80 \pm 1.85$ & $0.69 \pm 0.61$ & $4.48 \pm 1.14$ & $16.45 \pm 2.17$ & $19.89 \pm 2.40$ & $8.80 \pm 1.60$ & $5.06 \pm 1.21$ \\
\hline 180 & $3.60 \pm 1.05$ & $8.79 \pm 1.63$ & $1.13 \pm 0.61$ & $4.93 \pm 1.28$ & $16.31 \pm 2.24$ & $13.03 \pm 2.00$ & $6.72 \pm 1.50$ & $4.93 \pm 1.23$ \\
\hline 190 & $1.76 \pm 0.76$ & $8.14 \pm 1.63$ & $1.49 \pm 0.73$ & $6.60 \pm 1.46$ & $12.67 \pm 2.02$ & $15.35 \pm 2.23$ & $5.86 \pm 1.38$ & $2.58 \pm 0.95$ \\
\hline 200 & $1.03 \pm 0.78$ & $3.21 \pm 1.13$ & $1.36 \pm 0.74$ & $2.43 \pm 0.95$ & $5.57 \pm 1.43$ & $8.47 \pm 1.77$ & $1.14 \pm 0.68$ & $3.51 \pm 1.19$ \\
\hline 210 & $0.52 \pm 0.64$ & $4.44 \pm 1.38$ & $0.37 \pm 0.64$ & $1.58 \pm 0.86$ & $9.67 \pm 2.04$ & $6.58 \pm 1.75$ & $3.12 \pm 1.16$ & $1.33 \pm 0.79$ \\
\hline 220 & $0.44 \pm 0.77$ & $2.73 \pm 1.18$ & $0.00 \pm 0.77$ & $1.59 \pm 0.94$ & $1.28 \pm 0.84$ & $6.08 \pm 1.77$ & $0.44 \pm 0.77$ & $1.07 \pm 0.77$ \\
\hline 230 & $1.15 \pm 1.16$ & $2.34 \pm 1.27$ & $0.00 \pm 0.95$ & $2.59 \pm 1.33$ & $1.32 \pm 0.95$ & $4.17 \pm 1.62$ & $1.37 \pm 1.27$ & $1.15 \pm 1.16$ \\
\hline 240 & $0.99 \pm 1.23$ & $0.00 \pm 1.23$ & $0.00 \pm 1.23$ & $0.16 \pm 1.23$ & $3.02 \pm 1.64$ & $1.70 \pm 1.23$ & $1.51 \pm 1.50$ & $0.99 \pm 1.23$ \\
\hline 250 & $2.29 \pm 1.66$ & $1.14 \pm 1.81$ & $0.00 \pm 1.66$ & $0.00 \pm 1.66$ & $1.34 \pm 1.66$ & $0.95 \pm 1.66$ & $2.74 \pm 1.81$ & $0.00 \pm 1.66$ \\
\hline 260 & $0.00 \pm 2.55$ & $0.00 \pm 2.55$ & $0.00 \pm 2.55$ & $0.00 \pm 2.55$ & $1.46 \pm 2.55$ & $2.06 \pm 2.55$ & $0.00 \pm 2.55$ & $0.00 \pm 2.55$ \\
\hline
\end{tabular}

Table E.7: Triply differential cross sections for the ${ }^{3} \mathrm{He}\left(\pi^{*}, \pi^{0} \mathrm{p}\right)$ reaction at $\mathrm{T}_{\mathrm{z}}=245 \mathrm{MN}$ and $\theta_{\mathrm{p}}=70^{\circ}$. The in-plane $\left(\theta_{\mathrm{p}}\right)$ and out-ofplane $\left(\phi_{p}\right)$ proton laboratory angles are designated as $\left(\theta_{p}, \phi_{p}\right)$. An overall systematic uncertainty of $8.5 \%$ is not included. 


\begin{tabular}{|c|c|c|c|c|c|c|c|c|}
\hline \multicolumn{9}{|c|}{${ }^{3} \mathrm{He}\left(\pi^{\circ}, \pi^{0} \mathrm{p}\right)$ at $\mathrm{T}_{x}=245 \mathrm{MeV}$ and $\theta_{x^{\circ}}=70^{\circ}$} \\
\hline \multirow{2}{*}{$\begin{array}{c}T_{n^{0}} \\
(\mathrm{MeV})\end{array}$} & \multicolumn{8}{|c|}{$d^{3} \sigma / d E_{x} d \Omega_{\kappa} d \Omega_{p}\left(\mu b / M e V \cdot s r^{2}\right)$} \\
\hline & $\left(-68.5^{\circ}, 0.0^{\circ}\right)$ & $\left(-56.0^{\circ}, 0.0^{\circ}\right)$ & $\left(-46.5^{\circ}, 34.5^{\circ}\right)$ & $\left(-46.5^{\circ}, 23.0^{\circ}\right)$ & $\left(-46.5^{\circ}, 11.5^{\circ}\right)$ & $\left(-46.5^{\circ}, 0.0^{\circ}\right)$ & $\left(-46.5^{\circ},-11.5^{\circ}\right)$ & $\left(-46.5^{\circ},-23.0^{\circ}\right)$ \\
\hline 100 & $0.61 \pm 1.02$ & $3.71 \pm 1.50$ & $0.45 \pm 1.02$ & $0.76 \pm 1.02$ & $3.58 \pm 1.20$ & $4.01 \pm 1.39$ & $2.09 \pm 1.18$ & $0.76 \pm 1.02$ \\
\hline 110 & $0.24 \pm 0.53$ & $1.52 \pm 0.64$ & $0.26 \pm 0.53$ & $1.25 \pm 0.61$ & $2.29 \pm 0.73$ & $2.97 \pm 0.83$ & $2.07 \pm 0.69$ & $0.43 \pm 0.53$ \\
\hline 120 & $0.95 \pm 0.40$ & $2.15 \pm 0.53$ & $0.00 \pm 0.35$ & $0.38 \pm 0.38$ & $3.99 \pm 0.72$ & $6.85 \pm 0.93$ & $4.46 \pm 0.77$ & $0.32 \pm 0.36$ \\
\hline 130 & $0.71 \pm 0.32$ & $3.11 \pm 0.55$ & $0.00 \pm 0.26$ & $0.90 \pm 0.30$ & $4.09 \pm 0.63$ & $8.12 \pm 0.89$ & $3.82 \pm 0.61$ & $0.48 \pm 0.31$ \\
\hline 140 & $2.04 \pm 0.41$ & $4.93 \pm 0.64$ & $0.11 \pm 0.22$ & $0.96 \pm 0.29$ & $4.97 \pm 0.64$ & $10.08 \pm 0.91$ & $5.69 \pm 0.68$ & $0.62 \pm 0.25$ \\
\hline 150 & $1.51 \pm 0.34$ & $9.02 \pm 0.84$ & $0.45 \pm 0.23$ & $0.93 \pm 0.31$ & $6.47 \pm 0.71$ & $15.42 \pm 1.10$ & $7.16 \pm 0.75$ & $1.10 \pm 0.32$ \\
\hline 160 & $3.01 \pm 0.49$ & $7.87 \pm 0.79$ & $0.58 \pm 0.25$ & $1.64 \pm 0.38$ & $9.78 \pm 0.84$ & $21.34 \pm 1.30$ & $9.24 \pm 0.86$ & $2.05 \pm 0.41$ \\
\hline 170 & $2.94 \pm 0.49$ & $11.48 \pm 0.97$ & $0.22 \pm 0.23$ & $2.60 \pm 0.47$ & $9.75 \pm 0.89$ & $19.22 \pm 1.25$ & $12.02 \pm 0.99$ & $1.11 \pm 0.32$ \\
\hline 180 & $2.44 \pm 0.46$ & $10.30 \pm 0.94$ & $0.73 \pm 0.28$ & $1.81 \pm 0.41$ & $11.01 \pm 0.97$ & $21.69 \pm 1.37$ & $9.56+0.82$ & $1.10 \pm 0.33$ \\
\hline 190 & $1.73 \pm 0.41$ & $8.73 \pm 0.89$ & $0.16 \pm 0.25$ & $1.38 \pm 0.35$ & $7.54 \pm 0.83$ & $15.20 \pm 1.22$ & $8.17 \pm 0.87$ & $1.68 \pm 0.41$ \\
\hline 200 & $1.33 \pm 0.39$ & $4.90 \pm 0.71$ & $0.00 \pm 0.28$ & $1.00 \pm 0.39$ & $3.60 \pm 0.64$ & $9.63 \pm 1.00$ & $6.29 \pm 0.81$ & $0.66 \pm 0.31$ \\
\hline 210 & $1.43 \pm 0.43$ & $3.63 \pm 0.67$ & $0.07 \pm 0.33$ & $1.26 \pm 0.42$ & $3.02 \pm 0.60$ & $4.31 \pm 0.72$ & $3.91 \pm 0.69$ & $0.60 \pm 0.35$ \\
\hline 220 & $0.41 \pm 0.39$ & $2.69 \pm 0.64$ & $0.00 \pm 0.39$ & $0.93 \pm 0.42$ & $1.58 \pm 0.58$ & $4.02 \pm 0.77$ & $1.49 \pm 0.48$ & $0.12 \pm 0.39$ \\
\hline 230 & $0.21 \pm 0.48$ & $1.42 \pm 0.58$ & $0.10 \pm 0.49$ & $1.12 \pm 0.56$ & $0.86 \pm 0.52$ & $2.56 \pm 0.70$ & $1.40 \pm 0.55$ & $0.00 \pm 0.48$ \\
\hline 240 & $0.89 \pm 0.63$ & $0.46 \pm 0.63$ & $0.00 \pm 0.62$ & $0.74 \pm 0.62$ & $0.71 \pm 0.69$ & $2.08 \pm 0.75$ & $1.02 \pm 0.68$ & $0.60 \pm 0.66$ \\
\hline 250 & $0.38 \pm 0.84$ & $1.72 \pm 0.87$ & $0.00 \pm 0.84$ & $0.00 \pm 0.84$ & $0.22 \pm 0.84$ & $2.72 \pm 1.10$ & $0.36 \pm 0.84$ & $0.00 \pm 0.84$ \\
\hline 260 & $0.00 \pm 1.29$ & $0.80 \pm 1.36$ & $0.00 \pm 1.29$ & $0.00 \pm 1.29$ & $0.43 \pm 1.33$ & $2.60 \pm 1.50$ & $0.87 \pm 1.29$ & $0.00 \pm 1.29$ \\
\hline
\end{tabular}

Table E.8: Triply differential cross sections for the ${ }^{3} \mathrm{He}\left(\pi^{2}, \pi^{0} \mathrm{p}\right)$ reaction at $\mathrm{T}_{8}=245 \mathrm{MeV}$ and $\theta_{p}=70^{\circ}$. The in-plane $\left(\theta_{p}\right)$ and out-ofplane $\left(\phi_{p}\right)$ proton laboratory angles are designated as $\left(\theta_{p}, \phi_{p}\right)$. An overall systematic uncertainty of $8.5 \%$ is not included. 


\begin{tabular}{|c|c|c|c|c|c|c|c|c|}
\hline \multicolumn{9}{|c|}{${ }^{3} \mathrm{He}\left(\pi, \pi^{\circ} \mathrm{p}\right)$ at $\mathrm{T}_{x}=245 \mathrm{MeV}$ and $\theta_{x}=70^{\circ}$} \\
\hline \multirow{2}{*}{$\underset{(\mathrm{MeV})}{\mathrm{T}_{\mathrm{R}}}$} & \multicolumn{8}{|c|}{$d^{3} \sigma / d E_{R} d \Omega_{n} d \Omega_{p}\left(\mu b / M e V \cdot s r^{2}\right)$} \\
\hline & $\left(-73.2^{\circ}, 0.0^{\circ}\right)$ & $\left(-60.7^{\circ}, 0.0^{\circ}\right)$ & $\left(-51.2^{\circ}, 34.5^{\circ}\right)$ & $\left(-51.2^{\circ}, 23.0^{\circ}\right)$ & $\left(-51.2^{\circ}, 11.5^{\circ}\right)$ & $\left(-51.2^{\circ}, 0.0^{\circ}\right)$ & $\left(-51.2^{\circ},-11.5^{\circ}\right)$ & $\left(-51.2^{\circ},-23.0^{\circ}\right)$ \\
\hline 100 & $0.73 \pm 1.25$ & $1.99 \pm 1.33$ & $0.49 \pm 1.28$ & $0.00 \pm 1.25$ & $2.15 \pm 1.25$ & $5.31 \pm 1.68$ & $2.48 \pm 1.33$ & $0.00 \pm 1.25$ \\
\hline 110 & $0.30 \pm 0.65$ & $2.09 \pm 0.80$ & $0.30 \pm 0.65$ & $0.31 \pm 0.65$ & $2.21 \pm 0.80$ & $3.84 \pm 1.02$ & $2.14 \pm 0.84$ & $0.40 \pm 0.65$ \\
\hline 120 & $0.39 \pm 0.45$ & $1.67 \pm 0.52$ & $0.10 \pm 0.42$ & $0.70 \pm 0.44$ & $2.49 \pm 0.64$ & $4.73 \pm 0.85$ & $1.91 \pm 0.62$ & $0.70 \pm 0.42$ \\
\hline 130 & $0.47 \pm 0.32$ & $1.51 \pm 0.44$ & $0.15 \pm 0.32$ & $0.68 \pm 0.36$ & $3.46 \pm 0.64$ & $5.64 \pm 0.81$ & $4.14 \pm 0.70$ & $0.15 \pm 0.32$ \\
\hline 140 & $0.41 \pm 0.32$ & $1.61 \pm 0.42$ & $0.34 \pm 0.29$ & $1.58 \pm 0.42$ & $3.71 \pm 0.61$ & $8.95 \pm 0.94$ & $3.27 \pm 0.57$ & $0.35 \pm 0.27$ \\
\hline 150 & $0.73 \pm 0.30$ & $3.74 \pm 0.59$ & $0.19 \pm 0.26$ & $1.79 \pm 0.41$ & $5.26 \pm 0.70$ & $9.36 \pm 0.94$ & $5.00 \pm 0.69$ & $0.39 \pm 0.27$ \\
\hline 160 & $1.27 \pm 0.36$ & $4.94 \pm 0.69$ & $0.19 \pm 0.28$ & $2.48 \pm 0.51$ & $6.40 \pm 0.78$ & $15.28 \pm 1.21$ & $7.52 \pm 0.85$ & $0.91 \pm 0.32$ \\
\hline 170 & $1.04 \pm 0.37$ & $4.56 \pm 0.67$ & $0.11 \pm 0.27$ & $2.59 \pm 0.51$ & $10.01 \pm 0.99$ & $16.43 \pm 1.27$ & $10.49 \pm 1.02$ & $1.03 \pm 0.35$ \\
\hline 180 & $1.70 \pm 0.44$ & $6.27 \pm 0.81$ & $0.00 \pm 0.29$ & $2.00 \pm 0.46$ & $10.00 \pm 1.02$ & $15.77 \pm 1.28$ & $10.48 \pm 1.05$ & $1.17 \pm 0.37$ \\
\hline 190 & $1.19 \pm 0.42$ & $3.93 \pm 0.68$ & $0.32 \pm 0.33$ & $3.01 \pm 0.60$ & $8.91 \pm 0.99$ & $14.22 \pm 1.25$ & $6.62 \pm 0.85$ & $1.02 \pm 0.38$ \\
\hline 200 & $0.84 \pm 0.38$ & $4.24 \pm 0.73$ & $0.27 \pm 0.35$ & $1.26 \pm 0.44$ & $6.58 \pm 0.91$ & $8.98 \pm 1.06$ & $4.21 \pm 0.75$ & $0.44 \pm 0.35$ \\
\hline 210 & $0.57 \pm 0.40$ & $2.16 \pm 0.56$ & $0.00 \pm 0.40$ & $0.68 \pm 0.47$ & $3.74 \pm 0.74$ & $5.82 \pm 0.94$ & $4.00 \pm 0.76$ & $0.68 \pm 0.42$ \\
\hline 220 & $0.09 \pm 0.48$ & $0.95 \pm 0.57$ & $0.00 \pm 0.48$ & $0.22 \pm 0.48$ & $1.48 \pm 0.56$ & $1.98 \pm 0.59$ & $2.61 \pm 0.75$ & $0.00 \pm 0.48$ \\
\hline 230 & $0.31 \pm 0.63$ & $1.58 \pm 0.63$ & $0.14 \pm 0.59$ & $0.56 \pm 0.59$ & $1.73 \pm 0.76$ & $1.66 \pm 0.70$ & $2.10 \pm 0.75$ & $0.20 \pm 0.59$ \\
\hline 240 & $0.26 \pm 0.77$ & $0.88 \pm 0.77$ & $0.00 \pm 0.77$ & $0.47 \pm 0.77$ & $1.21 \pm 0.77$ & $1.59 \pm 0.87$ & $0.78 \pm 0.78$ & $0.11 \pm 0.77$ \\
\hline 250 & $0.49 \pm 1.03$ & $0.49 \pm 1.03$ & $0.00 \pm 1.03$ & $0.36 \pm 1.03$ & $1.04 \pm 1.03$ & $1.84 \pm 1.12$ & $0.84 \pm 1.06$ & $0.00 \pm 1.03$ \\
\hline 260 & $0.00 \pm 1.59$ & $0.00 \pm 1.59$ & $1.37 \pm 1.64$ & $0.00 \pm 1.59$ & $0.24 \pm 1.59$ & $0.90 \pm 1.63$ & $1.14 \pm 1.59$ & $0.75 \pm 1.59$ \\
\hline
\end{tabular}

Table E.9: Triply differential cross sections for the ${ }^{3} \mathrm{He}\left(\pi^{+}, \pi^{\circ} \mathrm{p}\right)$ reaction at $\mathrm{T}_{\mathrm{n}}=245 \mathrm{MU}$ and $\theta_{\pi^{\circ}}=70^{\circ}$. The in-plane $\left(\theta_{\mathrm{p}}\right)$ and out-ofplane $\left(\phi_{p}\right)$ proton laboratory angles are designated as $\left(\theta_{p}, \phi_{p}\right)$. An overall systematic uncertainty of $8.5 \%$ is not included. 


\begin{tabular}{|c|c|c|c|c|c|c|c|c|}
\hline \multicolumn{9}{|c|}{${ }^{3} \mathrm{He}\left(\pi, \pi^{0} \mathrm{p}\right)$ at $\mathrm{T}_{n}=245 \mathrm{MeV}$ and $\theta_{n}=90^{\circ}$} \\
\hline$\underset{(\mathrm{MeV})}{\mathrm{T}^{*}}$ & \multicolumn{8}{|c|}{$d^{3} \sigma / d E_{n} d \Omega_{n} d \Omega_{p}\left(\mu b / M e V \cdot s r^{2}\right)$} \\
\hline 80 & $1.82 \pm 2.39$ & $5.49 \pm 3.05$ & $1.80 \pm 2.40$ & $0.00 \pm 2.38$ & $5.49 \pm 3.05$ & $3.23 \pm 3.18$ & $10.08 \pm 4.13$ & $0.00 \pm 2.38$ \\
\hline 108 & $0.63 \pm 0.65$ & $9.65 \pm 1.87$ & $0.53 \pm 0.61$ & $2.41 \pm 0.94$ & $6.52 \pm 1.54$ & $18.30 \pm 2.58$ & $6.52 \pm 1.54$ & $0.72 \pm 0.51$ \\
\hline 122 & $2.44 \pm 0.82$ & $9.92 \pm 1.64$ & $0.54 \pm 0.39$ & $1.65 \pm 0.67$ & $7.55 \pm 1.43$ & $21.11 \pm 2.40$ & $9.17 \pm 1.58$ & $1.80 \pm 0.70$ \\
\hline 136 & $2.72 \pm 0.86$ & $13.78 \pm 1.94$ & $0.00 \pm 0.38$ & $3.43 \pm 0.97$ & $10.11 \pm 1.66$ & $26.28 \pm 2.68$ & $13.23 \pm 1.90$ & $2.04 \pm 0.75$ \\
\hline 150 & $3.19 \pm 0.96$ & $15.04 \pm 2.09$ & $1.15 \pm 0.59$ & $2.30 \pm 0.82$ & $11.79 \pm 1.85$ & $22.53 \pm 2.56$ & $11.97 \pm 1.87$ & $1.93 \pm 0.75$ \\
\hline 192 & $0.63 \pm 0.83$ & $2.66 \pm 1.15$ & $0.00 \pm 0.71$ & $0.63 \pm 0.83$ & $0.58 \pm 0.79$ & $5.97 \pm 1.73$ & $0.99 \pm 0.71$ & $0.99 \pm 0.71$ \\
\hline 206 & $0.00 \pm 1.12$ & $3.68 \pm 1.71$ & $0.00 \pm 1.12$ & $0.00 \pm 1.12$ & $1.40 \pm 1.43$ & $5.17 \pm 2.03$ & $2.23 \pm 1.33$ & $0.00 \pm 1.12$ \\
\hline 220 & $0.00 \pm 2.23$ & $2.40 \pm 2.65$ & $0.00 \pm 2.23$ & $0.00 \pm 2.23$ & $0.00 \pm 2.23$ & $1.70 \pm 2.23$ & $1.70 \pm 2.23$ & $0.00 \pm 2.23$ \\
\hline
\end{tabular}

Table E.10: Triply differential cross sections for the ${ }^{3} \mathrm{He}\left(\pi^{+}, \pi^{\circ} \mathrm{p}\right)$ reaction at $\mathrm{T}_{\mathrm{n}}=245 \mathrm{MeV}$ and $\theta_{\mathrm{n}}=90^{\circ}$. The in -plane $\left(\theta_{\mathrm{p}}\right)$ and outof-plane $\left(\phi_{p}\right)$ proton laboratory angles are designated as $\left(\theta_{p}, \phi_{p}\right)$. An overall systematic uncertainty of $8.1 \%$ is not included. 


\begin{tabular}{|c|c|c|c|c|c|c|c|c|}
\hline \multicolumn{9}{|c|}{${ }^{3} \mathrm{He}\left(\pi, \pi^{\circ} \mathrm{p}\right)$ at $\mathrm{T}_{\mathrm{x}}=245 \mathrm{MeV}$ and $\theta_{x}=90^{\circ}$} \\
\hline \multirow{2}{*}{$\begin{array}{c}T^{\infty} \\
(\mathrm{MeV})\end{array}$} & \multicolumn{8}{|c|}{$d^{3} \sigma / d E_{R} d \Omega_{\alpha} d \Omega_{p}\left(\mu b / M e V \cdot s r^{2}\right)$} \\
\hline & $\left(-57.4^{\circ}, 0.0^{\circ}\right)$ & $\left(-44.9^{\circ}, 0.0^{\circ}\right)$ & $\left(-35.4^{\circ}, 34.5^{\circ}\right)$ & $\left(-35.4^{\circ}, 23.0^{\circ}\right)$ & $\left(-35.4^{\circ}, 11.5^{\circ}\right)$ & $\left(-35.4^{\circ}, 0.0^{\circ}\right)$ & $\left(-35.4^{\circ},-11.5^{\circ}\right)$ & $\left(-35.4^{\circ},-23.0^{\circ}\right)$ \\
\hline 80 & $1.62 \pm 1.26$ & $5.00 \pm 1.76$ & $0.00 \pm 1.26$ & $1.27 \pm 1.26$ & $8.18 \pm 2.21$ & $7.57 \pm 2.05$ & $3.07 \pm 1.49$ & $0.77 \pm 1.26$ \\
\hline 94 & $1.08 \pm 0.71$ & $3.22 \pm 0.95$ & $0.42 \pm 0.63$ & $1.13 \pm 0.62$ & $5.86 \pm 1.21$ & $14.18 \pm 1.88$ & $4.94 \pm 1.17$ & $1.35 \pm 0.60$ \\
\hline 108 & $0.67 \pm 0.35$ & $3.33 \pm 0.65$ & $0.39 \pm 0.34$ & $1.32 \pm 0.41$ & $6.02 \pm 0.84$ & $11.29 \pm 1.16$ & $3.80 \pm 0.67$ & $1.22 \pm 0.40$ \\
\hline 122 & $1.02 \pm 0.30$ & $6.07 \pm 0.74$ & $0.20 \pm 0.22$ & $1.87 \pm 0.41$ & $8.07 \pm 0.85$ & $14.80 \pm 1.15$ & $8.57 \pm 0.87$ & $0.94 \pm 0.33$ \\
\hline 136 & $1.89 \pm 0.41$ & $7.30 \pm 0.81$ & $0.29 \pm 0.23$ & $4.17 \pm 0.61$ & $11.63 \pm 1.02$ & $19.75 \pm 1.33$ & $9.13 \pm 0.90$ & $1.44 \pm 0.37$ \\
\hline 150 & $1.48 \pm 0.38$ & $8.05 \pm 0.87$ & $0.67 \pm 0.27$ & $2.48 \pm 0.49$ & $9.74 \pm 0.96$ & $23.85 \pm 1.51$ & $9.46 \pm 0.95$ & $1.80 \pm 0.42$ \\
\hline 164 & $0.74 \pm 0.27$ & $5.54 \pm 0.74$ & $0.58 \pm 0.29$ & $1.37 \pm 0.38$ & $6.58 \pm 0.81$ & $14.85 \pm 1.22$ & $8.14 \pm 0.91$ & $0.90 \pm 0.34$ \\
\hline 178 & $0.43 \pm 0.29$ & $3.25 \pm 0.62$ & $0.33 \pm 0.27$ & $1.59 \pm 0.43$ & $5.40 \pm 0.80$ & $9.04 \pm 1.03$ & $2.92 \pm 0.61$ & $1.02 \pm 0.35$ \\
\hline 192 & $0.28 \pm 0.41$ & $1.52 \pm 0.53$ & $0.16 \pm 0.37$ & $1.09 \pm 0.42$ & $1.89 \pm 0.56$ & $8.22 \pm 1.16$ & $2.48 \pm 0.66$ & $0.87 \pm 0.42$ \\
\hline 206 & $0.00 \pm 0.59$ & $0.86 \pm 0.72$ & $0.27 \pm 0.59$ & $0.27 \pm 0.59$ & $1.22 \pm 0.66$ & $3.58 \pm 0.97$ & $1.09 \pm 0.60$ & $0.14 \pm 0.59$ \\
\hline 220 & $0.28 \pm 1.18$ & $1.09 \pm 1.24$ & $0.00 \pm 1.18$ & $0.72 \pm 1.18$ & $1.24 \pm 1.18$ & $3.11 \pm 1.40$ & $1.28 \pm 1.35$ & $0.00 \pm 1.18$ \\
\hline
\end{tabular}

Table E.11: Triply differential cross sections for the ${ }^{3} \mathrm{He}\left(\pi, \pi^{\circ} \mathrm{p}\right)$ reaction at $\mathrm{T}_{n}=245 \mathrm{MeV}$ and $\theta_{p}=90^{\circ}$. The in-plane $\left(\theta_{p}\right)$ and oulof-plane $\left(\phi_{p}\right)$ proton laboratory angles are designated as $\left(\theta_{p}, \phi_{p}\right)$. An overall systematic uncertainty of $8.1 \%$ is not included. 


\begin{tabular}{|c|c|c|c|c|c|c|c|c|}
\hline \multicolumn{9}{|c|}{${ }^{3} \mathrm{He}\left(\pi, \pi^{0} \mathrm{p}\right)$ at $\mathrm{T}_{\mathrm{n}}=245 \mathrm{MeV}$ and $\theta_{n}=110^{\circ}$} \\
\hline \multirow{2}{*}{$\begin{array}{c}\mathrm{T}_{*} \\
(\mathrm{MeV})\end{array}$} & \multicolumn{8}{|c|}{$\mathrm{d}^{3} \sigma / \mathrm{dE} \mathrm{E}_{\mathrm{N}} \mathrm{d} \Omega_{\mathrm{R}} \mathrm{d} \Omega_{\mathrm{p}}\left(\mu \mathrm{b} / \mathrm{MeV} \cdot \mathrm{sr}^{2}\right)$} \\
\hline & $\left(-43.3^{\circ}, 0.0^{\circ}\right)$ & $\left(-30.8^{\circ}, 0.0^{\circ}\right)$ & $\left(-21.3^{\circ}, 34.5^{\circ}\right)$ & $\left(-21.3^{\circ}, 23.0^{\circ}\right)$ & $\left(-21.3^{\circ}, 11.5^{\circ}\right)$ & $\left(-21.3^{\circ}, 0.0^{\circ}\right)$ & $\left(-21.3^{\circ},-11.5^{\circ}\right)$ & $\left(-21.3^{\circ},-11.5^{\circ}\right)$ \\
\hline 66 & $3.61 \pm 2.89$ & $3.62 \pm 2.88$ & $0.00 \pm 2.16$ & $4.54 \pm 2.89$ & $2.02 \pm 2.16$ & $14.23 \pm 5.05$ & $5.12 \pm 2.94$ & $0.00 \pm 2.16$ \\
\hline 80 & $2.05 \pm 1.20$ & $6.84 \pm 2.01$ & $0.84 \pm 0.91$ & $0.84 \pm 0.90$ & $5.46 \pm 2.13$ & $10.96 \pm 2.54$ & $10.67 \pm 2.49$ & $0.38 \pm 0.90$ \\
\hline 94 & $4.23 \pm 1.23$ & $9.23 \pm 1.80$ & $0.06 \pm 0.53$ & $1.52 \pm 0.85$ & $6.53 \pm 1.51$ & $20.28 \pm 2.67$ & $9.64 \pm 1.84$ & $0.83 \pm 0.54$ \\
\hline 108 & $2.27 \pm 0.80$ & $13.31 \pm 1.91$ & $-0.01 \pm 0.42$ & $1.83 \pm 0.77$ & $11.23 \pm 1.76$ & $34.06 \pm 3.06$ & $11.25 \pm 1.77$ & $2.58 \pm 0.86$ \\
\hline 122 & $2.85 \pm 1.02$ & $14.93 \pm 2.21$ & $0.82 \pm 0.67$ & $3.43 \pm 1.12$ & $13.24 \pm 2.08$ & $36.55 \pm 3.43$ & $17.82 \pm 2.39$ & $2.49 \pm 0.91$ \\
\hline 136 & $2.23 \pm 0.99$ & $9.27 \pm 1.86$ & $1.08 \pm 0.78$ & $2.47 \pm 1.06$ & $10.92 \pm 2.03$ & $30.74 \pm 3.38$ & $6.92 \pm 1.62$ & $2.32 \pm 0.99$ \\
\hline 150 & $0.27 \pm 0.72$ & $10.44 \pm 2.20$ & $0.75 \pm 0.72$ & $0.84 \pm 0.71$ & $4.67 \pm 1.57$ & $16.24 \pm 2.76$ & $7.60 \pm 1.89$ & $0.00 \pm 0.71$ \\
\hline 164 & $0.00 \pm 0.98$ & $6.07 \pm 1.97$ & $0.91 \pm 0.99$ & $1.10 \pm 0.98$ & $2.32 \pm 1.31$ & $10.19 \pm 2.56$ & $3.89 \pm 1.68$ & $0.20 \pm 0.98$ \\
\hline 178 & $0.00 \pm 1.67$ & $3.79 \pm 2.60$ & $0.00 \pm 1.67$ & $0.00 \pm 1.67$ & $0.42 \pm 1.70$ & $6.30 \pm 2.87$ & $4.61 \pm 2.87$ & $0.00 \pm 1.67$ \\
\hline
\end{tabular}

Table E.12: Triply differential cross sections for the ${ }^{3} \mathrm{He}\left(\pi^{+}, \pi^{\circ} \mathrm{p}\right)$ reaction at $\mathrm{T}_{\mathrm{s}}=245 \mathrm{MeV}$ and $\theta_{\mathrm{p}}=110^{\circ}$. The in-plane $\left(\theta_{p}\right)$ and outof-plane $\left(\phi_{p}\right)$ proton laboratory angles are designated as $\left(\theta_{p}, \phi_{p}\right)$. An overall systematic uncertainty of $8.3 \%$ is not included. 


\begin{tabular}{|c|c|c|c|c|c|c|c|c|}
\hline \multicolumn{9}{|c|}{${ }^{3} \mathrm{He}\left(\pi^{\prime}, \pi^{0} \mathrm{p}\right)$ at $\mathrm{T}_{\mathrm{n}}=245 \mathrm{MeV}$ and $\theta_{n}=110^{\circ}$} \\
\hline \multirow{2}{*}{$\begin{array}{c}\mathrm{T}_{\mathrm{N}} \\
(\mathrm{MeV})\end{array}$} & \multicolumn{8}{|c|}{$\mathrm{d}^{3} \sigma / \mathrm{dE} \mathrm{E}_{\mathrm{d}} \mathrm{d} \Omega_{\mathrm{N}} \mathrm{d} \Omega_{\mathrm{p}}\left(\mu \mathrm{b} / \mathrm{MeV} \cdot \mathrm{sr}^{2}\right)$} \\
\hline & $\left(-48.3^{\circ}, 0.0^{\circ}\right)$ & $\left(-35.8^{\circ}, 0.0^{\circ}\right)$ & $\left(-26.3^{\circ}, 34.5^{\circ}\right)$ & $\left(-26.3^{\circ}, 23.0^{\circ}\right)$ & $\left(-26.3^{\circ}, 11.5^{\circ}\right)$ & $\left(-26.3^{\circ}, 0.0^{\circ}\right)$ & $\left(-26.3^{\circ},-11.5^{\circ}\right)$ & $\left(-26.3^{\circ},-11.5^{\circ}\right)$ \\
\hline 66 & $0.42 \pm 1.13$ & $1.82 \pm 1.19$ & $0.19 \pm 1.11$ & $1.52 \pm 1.19$ & $2.27 \pm 1.36$ & $11.00 \pm 2.30$ & $3.10 \pm 1.37$ & $1.0 s \pm 1.14$ \\
\hline 80 & $0.94 \pm 0.46$ & $2.30 \pm 0.73$ & $0.02 \pm 0.46$ & $1.22 \pm 0.57$ & $5.95 \pm 1.06$ & $13.27 \pm 1.56$ & $5.19 \pm 0.98$ & $2.13 \pm 0.70$ \\
\hline 94 & $0.94 \pm 0.35$ & $5.71 \pm 0.80$ & $0.21 \pm 0.30$ & $1.46 \pm 0.44$ & $4.45 \pm 0.70$ & $18.12 \pm 1.41$ & $5.88 \pm 0.81$ & $0.42 \pm 0.30$ \\
\hline 108 & $1.95 \pm 0.43$ & $5.83 \pm 0.71$ & $0.10 \pm 0.22$ & $1.25 \pm 0.34$ & $9.47 \pm 0.90$ & $19.48 \pm 1.30$ & $7.46 \pm 0.80$ & $1.47 \pm 0.40$ \\
\hline$: 22$ & $1.57 \pm 0.41$ & $8.59 \pm 0.93$ & $0.12 \pm 0.26$ & $1.79 \pm 0.46$ & $13.33 \pm 1.16$ & $30.01 \pm 1.75$ & $13.05 \pm 1.15$ & $1.66 \pm 0.48$ \\
\hline 136 & $1.90 \pm 0.49$ & $6.74 \pm 0.91$ & $0.16 \pm 0.29$ & $1.05 \pm 0.40$ & $9.89 \pm 1.08$ & $23.38 \pm 1.66$ & $9.62 \pm 1.06$ & $170 \pm 0.47$ \\
\hline 150 & $1.62 \pm 0.52$ & $4.84 \pm 0.84$ & $0.00 \pm 0.37$ & $1.53 \pm 0.48$ & $6.30 \pm 0.96$ & $15.67 \pm 1.51$ & $6.86 \pm 1.00$ & $.24 \pm 0.45$ \\
\hline 164 & $0.58 \pm 0.50$ & $2.58 \pm 0.73$ & $0.08 \pm 0.50$ & $0.90 \pm 0.54$ & $3.44 \pm 0.86$ & $12.33 \pm 1.57$ & $4.79 \pm 0.98$ & $0.97 \pm 0.56$ \\
\hline 178 & $0.15 \pm 0.86$ & $1.69 \pm 1.01$ & $0.07 \pm 0.86$ & $0.43 \pm 0.86$ & $2.58 \pm 1.04$ & $10.32 \pm 1.91$ & $1.63 \pm 0.96$ & $0.14 \pm 0.86$ \\
\hline
\end{tabular}

Table E.13: Triply differential cross sections for the ${ }^{3} \mathrm{He}\left(\pi^{*}, \pi^{0} \mathrm{p}\right)$ reaction at $T_{x}=245 \mathrm{MeV}$ and $\theta_{\mathrm{p}}=110^{\circ}$. The in-plane $\left(\theta_{\mathrm{p}}\right)$ and outof-plane $\left(\phi_{p}\right)$ proton laboratory angles are designated as $\left(\theta_{p}, \phi_{p}\right)$. An overall systematic uncertainty of $8.3 \%$ is not included. 


\begin{tabular}{|c|c|c|c|c|c|c|c|c|}
\hline \multicolumn{9}{|c|}{${ }^{3} \mathrm{He}\left(\pi^{+}, \pi^{0} \mathrm{p}\right)$ at $\mathrm{T}_{n}=245 \mathrm{MeV}$ and $\theta_{\pi^{\circ}}=120^{\circ}$} \\
\hline \multirow{2}{*}{$\begin{array}{c}\mathrm{T}_{\pi^{0}} \\
(\mathrm{MeV})\end{array}$} & \multicolumn{8}{|c|}{$\mathrm{d}^{3} \sigma / d \mathrm{E}_{n} \mathrm{~d} \Omega_{\pi} \mathrm{d} \Omega_{\mathrm{p}}\left(\mu \mathrm{b} / \mathrm{MeV} \cdot \mathrm{sr}^{2}\right)$} \\
\hline & $\left(-39.2^{\circ}, 0.0^{\circ}\right)$ & $\left(-26.7^{\circ}, 0.0^{\circ}\right)$ & $\left(-17.2^{\circ}, 34.5^{\circ}\right)$ & $\left(-17.2^{\circ}, 23.0^{\circ}\right)$ & $\left(-17.2^{\circ}, 11.5^{\circ}\right)$ & $\left(-17.2^{\circ}, 0.0^{\circ}\right)$ & $\left(-17.2^{\circ},-11.5^{\circ}\right)$ & $\left(-17.2^{\circ},-11.5^{\circ}\right)$ \\
\hline 68 & $1.53 \pm 2.03$ & $11.11 \pm 4.00$ & $0.00 \pm 2.03$ & $1.33 \pm 2.03$ & $4.01 \pm 2.40$ & $5.92 \pm 2.92$ & $7.29 \pm 3.24$ & $1.53 \pm 2.03$ \\
\hline 78 & $1.40 \pm 1.41$ & $5.07 \pm 1.96$ & $0.00 \pm 1.07$ & $0.70 \pm 1.07$ & $5.76 \pm 2.09$ & $17.20 \pm 3.61$ & $10.93 \pm 2.88$ & $0.00 \pm 1.07$ \\
\hline 88 & $0.66 \pm 0.85$ & $12.72 \pm 2.55$ & $1.20 \pm 0.85$ & $0.60 \pm 0.81$ & $9.57 \pm 2.21$ & $17.37 \pm 2.98$ & $4.96 \pm 1.59$ & $0.86 \pm 0.91$ \\
\hline 98 & $3.87 \pm 1.23$ & $11.67 \pm 2.13$ & $0.69 \pm 0.73$ & $1.42 \pm 0.74$ & $10.09 \pm 1.98$ & $26.53 \pm 3.22$ & $7.32 \pm 1.69$ & $0.57 \pm 0.65$ \\
\hline 108 & $1.72 \pm 0.78$ & $16.46 \pm 2.41$ & $0.51 \pm 0.59$ & $3.24 \pm 1.07$ & $9.10 \pm 1.79$ & $30.52 \pm 3.28$ & $9.05 \pm 1.78$ & $1.61 \pm 0.77$ \\
\hline 118 & $1.33 \pm 0.72$ & $18.09 \pm 2.64$ & $0.00 \pm 0.54$ & $2.68 \pm 1.02$ & $16.68 \pm 2.54$ & $33.85 \pm 3.62$ & $9.83 \pm 1.95$ & $0.65 \pm 0.69$ \\
\hline 128 & $1.27 \pm 0.74$ & $16.11 \pm 2.65$ & $0.46 \pm 0.61$ & $1.72 \pm 0.87$ & $11.67 \pm 2.25$ & $29.66 \pm 3.59$ & $8.18 \pm 1.89$ & $0.46 \pm 0.61$ \\
\hline 138 & $2.00 \pm 1.00$ & $9.75 \pm 2.21$ & $0.53 \pm 0.71$ & $0.46 \pm 0.71$ & $12.31 \pm 2.49$ & $23.94 \pm 3.47$ & $3.99 \pm 1.42$ & $1.62 \pm 0.91$ \\
\hline 148 & $0.66 \pm 0.88$ & $4.16 \pm 1.61$ & $0.00 \pm 0.87$ & $0.57 \pm 0.88$ & $4.93 \pm 1.75$ & $16.72 \pm 3.22$ & $3.70 \pm 1.52$ & $0.00 \pm 0.87$ \\
\hline 158 & $0.00 \pm 1.11$ & $4.39 \pm 1.86$ & $0.00 \pm 1.11$ & $0.73 \pm 1.11$ & $2.53 \pm 1.41$ & $7.70 \pm 2.47$ & $1.35 \pm 1.41$ & $0.71 \pm 1.12$ \\
\hline 168 & $1.01 \pm 1.54$ & $2.17 \pm 1.54$ & $0.00 \pm 1.54$ & $1.62 \pm 1.82$ & $0.00 \pm 1.54$ & $1.62 \pm 1.82$ & $1.01 \pm 1.54$ & $0.00 \pm 1.54$ \\
\hline 178 & $0.00 \pm 2.31$ & $1.51 \pm 2.31$ & $0.00 \pm 2.31$ & $1.92 \pm 2.61$ & $8.03 \pm 3.63$ & $1.74 \pm 2.31$ & $0.00 \pm 2.31$ & $0.00 \pm 2.31$ \\
\hline
\end{tabular}

Table E. 14: Triply differential cross sections for the ${ }^{3} \mathrm{He}\left(\pi^{+}, \pi^{0} \mathrm{p}\right)$ reaction at $\mathrm{T}_{\mathrm{x}}=245 \mathrm{MeV}$ and $\theta_{\mathrm{p}}=120^{\circ}$. The in-plane $\left(\theta_{p}\right)$ and outof-plane $\left(\phi_{p}\right)$ proton laboratory angles are designated as $\left(\theta_{p}, \phi_{p}\right)$. An overall systematic uncertainty of $8.4 \%$ is not included. 
${ }^{3} \mathrm{He}\left(\pi^{+}, \pi^{0} \mathrm{p}\right)$ at $\mathrm{T}_{\pi}=245 \mathrm{MeV}$ and $\theta_{\pi}=120^{\circ}$

\begin{tabular}{|c|c|c|c|c|c|c|c|c|}
\hline \multirow{2}{*}{$\begin{array}{c}T_{\pi^{0}} \\
(\mathrm{MeV})\end{array}$} & \multicolumn{8}{|c|}{$\mathrm{d}^{3} \sigma / \mathrm{d} \mathrm{E}_{\pi} \mathrm{d} \Omega_{x} \mathrm{~d} \Omega_{\mathrm{p}}\left(\mu \mathrm{b} / \mathrm{MeV} \cdot \mathrm{sr}^{2}\right)$} \\
\hline & $\left(-44.0^{\circ}, 0.0^{\circ}\right)$ & $\left(-31.5^{\circ}, 0.0^{\circ}\right)$ & $\left(-22.0^{\circ}, 34.5^{\circ}\right)$ & $\left(-22.0^{\circ}, 23.0^{\circ}\right)$ & $\left(-22.0^{\circ}, 11.5^{\circ}\right)$ & $\left(-22.0^{\circ}, 0.0^{\circ}\right)$ & $\left(-22.0^{\circ},-11.5^{\circ}\right)$ & $\left(-22.0^{\circ},-11.5^{\circ}\right)$ \\
\hline 68 & $0.00 \pm 1.33$ & $4.87 \pm 1.79$ & $0.00 \pm 1.33$ & $1.13 \pm 1.36$ & $4.41 \pm 1.63$ & $8.90 \pm 2.25$ & $2.59 \pm 1.43$ & $0.00 \pm 1.33$ \\
\hline 78 & $1.10 \pm 0.70$ & $4.60 \pm 1.23$ & $0.21 \pm 0.70$ & $1.70 \pm 0.77$ & $2.96 \pm 1.00$ & $14.11 \pm 1.95$ & $5.04 \pm 1.20$ & $1.09 \pm 0.78$ \\
\hline 88 & $0.51 \pm 0.47$ & $5.48 \pm 1.00$ & $0.12 \pm 0.47$ & $0.74 \pm 0.48$ & $5.09 \pm 1.00$ & $17.45 \pm 1.78$ & $4.75 \pm 0.93$ & $0.91 \pm 0.47$ \\
\hline 98 & $0.97 \pm 0.41$ & $5.99 \pm 0.91$ & $0.49 \pm 0.39$ & $1.41 \pm 0.46$ & $10.55 \pm 1.21$ & $22.25 \pm 1.76$ & $8.06 \pm 1.06$ & $1.93 \pm 0.54$ \\
\hline 108 & $1.21 \pm 0.47$ & $6.59 \pm 0.91$ & $0.44 \pm 0.33$ & $1.69 \pm 0.49$ & $13.21 \pm 1.29$ & $30.52 \pm 1.96$ & $9.63 \pm 1.10$ & $0.48 \pm 0.39$ \\
\hline 118 & $0.82 \pm 0.38$ & $7.82 \pm 1.04$ & $0.79 \pm 0.40$ & $1.68 \pm 0.48$ & $14.27 \pm 1.40$ & $35.07 \pm 2.20$ & $11.36 \pm 1.25$ & $1.57 \pm 0.47$ \\
\hline 128 & $1.20 \pm 0.52$ & $8.46 \pm 1.15$ & $0.56 \pm 0.41$ & $1.57 \pm 0.50$ & $9.50 \pm 1.21$ & $26.97 \pm 2.05$ & $9.03 \pm 1.18$ & $1.14 \pm 0.50$ \\
\hline 138 & $1.13 \pm 0.53$ & $5.02 \pm 0.97$ & $0.78 \pm 0.53$ & $1.55 \pm 0.57$ & $9.24 \pm 1.29$ & $22.18 \pm 2.00$ & $5.95 \pm 1.03$ & $0.85 \pm 0.47$ \\
\hline 148 & $0.61 \pm 0.57$ & $1.86 \pm 0.71$ & $0.17 \pm 0.57$ & $1.07 \pm 0.65$ & $5.66 \pm 1.12$ & $17.53 \pm 1.97$ & $4.69 \pm 1.02$ & $0.60 \pm 0.58$ \\
\hline 158 & $0.27 \pm 0.73$ & $2.98 \pm 1.07$ & $0.00 \pm 0.73$ & $0.54 \pm 0.77$ & $2.12 \pm 0.85$ & $11.26 \pm 1.78$ & $2.61 \pm 1.00$ & $0.00 \pm 0.73$ \\
\hline 168 & $0.26 \pm 1.01$ & $2.08 \pm 1.11$ & $0.37 \pm 1.01$ & $1.25 \pm 1.03$ & $2.73 \pm 1.32$ & $5.67 \pm 1.52$ & $1.52 \pm 1.03$ & $0.00 \pm 1.01$ \\
\hline 178 & $0.82 \pm 1.52$ & $0.68 \pm 1.51$ & $0.00 \pm 1.51$ & $0.00 \pm 1.51$ & $2.09 \pm 1.54$ & $3.88 \pm 1.73$ & $3.11 \pm 1.66$ & $0.77 \pm 1.51$ \\
\hline
\end{tabular}

Table E.15: Triply differential cross sections for the ${ }^{3} \mathrm{He}\left(\pi^{*}, \pi^{0} \mathrm{p}\right)$ reaction at $\mathrm{T}_{\mathrm{s}}=245 \mathrm{MeV}$ and $\theta_{n_{0}}=120^{\circ}$. The in-plane $\left(\theta_{\mathrm{p}}\right)$ and outof-plane $\left(\phi_{p}\right)$ proton laboratory angles are designated as $\left(\theta_{p}, \phi_{p}\right)$. An overall systematic uncertainty of $8.4 \%$ is not included. 


\begin{tabular}{|c|c|c|c|c|c|c|c|c|}
\hline \multicolumn{9}{|c|}{${ }^{3} \mathrm{He}\left(\pi, \pi^{0} \mathrm{p}\right)$ at $\mathrm{T}_{\pi}=245 \mathrm{MeV}$ and $\theta_{\pi^{\circ}}=70^{\circ}$} \\
\hline \multirow{2}{*}{$\begin{array}{c}\mathrm{T}_{\pi^{0}} \\
(\mathrm{MeV})\end{array}$} & \multicolumn{8}{|c|}{$d^{3} \sigma / d E_{k} d \Omega_{x} d \Omega_{p}\left(\mu b / M e V \cdot s r^{2}\right)$} \\
\hline & $\left(-37.0^{\circ}, 22.0^{\circ}\right)$ & $\left(-37.0^{\circ}, 9.5^{\circ}\right)$ & $\left(-71.5^{\circ}, 0.0^{\circ}\right)$ & $\left(-60.0^{\circ}, 0.0^{\circ}\right)$ & $\left(-48.5^{\circ}, 0.0^{\circ}\right)$ & $\left(-37.0^{\circ}, 0.0^{\circ}\right)$ & $\left(-25.5^{\circ}, 0.0^{\circ}\right)$ & $\left(-14.0^{\circ}, 0.0^{\circ}\right)$ \\
\hline 100 & $0.00 \pm 1.03$ & $0.73 \pm 1.04$ & $0.00 \pm 1.03$ & $0.48 \pm 1.04$ & $0.86 \pm 1.04$ & $1.44 \pm 1.09$ & $0.73 \pm 1.06$ & $0.00 \pm 1.03$ \\
\hline 116 & $0.19 \pm 0.40$ & $0.28 \pm 0.41$ & $0.18 \pm 0.42$ & $0.54 \pm 0.46$ & $0.26 \pm 0.40$ & $0.43 \pm 0.43$ & $0.00 \pm 0.40$ & $0.29 \pm 0.41$ \\
\hline 132 & $0.23 \pm 0.27$ & $0.18 \pm 0.26$ & $0.18 \pm 0.26$ & $0.41 \pm 0.27$ & $0.29 \pm 0.26$ & $0.45 \pm 0.27$ & $0.00 \pm 0.25$ & $0.30 \pm 0.26$ \\
\hline 148 & $0.35 \pm 0.22$ & $0.48 \pm 0.24$ & $0.06 \pm 0.22$ & $0.11 \pm 0.22$ & $0.34 \pm 0.23$ & $1.28 \pm 0.33$ & $0.41 \pm 0.26$ & $0.18 \pm 0.22$ \\
\hline 164 & $0.28 \pm 0.23$ & $0.29 \pm 0.24$ & $0.34 \pm 0.25$ & $0.42 \pm 0.25$ & $0.69 \pm 0.26$ & $0.82 \pm 0.29$ & $0.20 \pm 0.23$ & $1.01 \pm 0.30$ \\
\hline 180 & $0.57 \pm 0.27$ & $0.66 \pm 0.28$ & $0.62 \pm 0.31$ & $0.53 \pm 0.26$ & $0.72 \pm 0.28$ & $0.90 \pm 0.31$ & $0.36 \pm 0.27$ & $0.50 \pm 0.25$ \\
\hline 196 & $0.67 \pm 0.29$ & $0.57 \pm 0.29$ & $0.00 \pm 0.27$ & $0.33 \pm 0.28$ & $0.59 \pm 0.28$ & $0.92 \pm 0.31$ & $0.37 \pm 0.31$ & $0.31 \pm 0.27$ \\
\hline 212 & $0.34 \pm 0.35$ & $0.46 \pm 0.36$ & $0.56 \pm 0.36$ & $0.50 \pm 0.37$ & $0.36 \pm 0.35$ & $0.53 \pm 0.36$ & $0.16 \pm 0.34$ & $0.43 \pm 0.36$ \\
\hline 228 & $0.00 \pm 0.47$ & $0.04 \pm 0.47$ & $0.08 \pm 0.47$ & $0.00 \pm 0.47$ & $0.41 \pm 0.47$ & $0.56 \pm 0.49$ & $0.31 \pm 0.47$ & $0.00 \pm 0.47$ \\
\hline 244 & $0.00 \pm 0.71$ & $0.00 \pm 0.71$ & $0.62 \pm 0.72$ & $0.00 \pm 0.71$ & $0.20 \pm 0.71$ & $0.46 \pm 0.71$ & $0.00 \pm 0.71$ & $0.00 \pm 0.71$ \\
\hline 260 & $0.00 \pm 1.31$ & $0.00 \pm 1.31$ & $0.00 \pm 1.31$ & $0.00 \pm 1.31$ & $0.00 \pm 1.31$ & $0.58 \pm 1.31$ & $0.36 \pm 1.31$ & $0.00 \pm 1.31$ \\
\hline
\end{tabular}

Table E.16: Triply differential cross sections for the ${ }^{3} \mathrm{He}\left(\pi, \pi^{0} \mathrm{p}\right)$ reaction at $\mathrm{T}_{\mathrm{s}}=245 \mathrm{MeV}$ and $\theta_{\alpha}=70^{\circ}$. The in-plane $\left(\theta_{\mathrm{p}}\right)$ and outof-plane $\left(\phi_{\mathrm{p}}\right)$ proton laboratory angles are designated as $\left(\theta_{\mathrm{p}}, \phi_{\mathrm{p}}\right)$. An overall systematic uncertainty of $8.1 \%$ is not included. 


\begin{tabular}{|c|c|c|c|c|c|c|c|}
\hline \multicolumn{8}{|c|}{${ }^{3} \mathrm{He}\left(\pi, \pi^{0} \mathrm{p}\right)$ at $\mathrm{T}_{\pi}=245 \mathrm{MeV}$ and $\theta_{x_{0}}=70^{\circ}$} \\
\hline \multirow{2}{*}{$\underset{(\mathrm{MeV})}{\mathrm{T}_{\boldsymbol{0}}}$} & \multicolumn{7}{|c|}{$\mathrm{d}^{3} \sigma / \mathrm{dE} \mathrm{K}_{\mathrm{d}} \mathrm{d} \Omega_{\mathrm{R}} \mathrm{d} \Omega_{\mathrm{p}}\left(\mu \mathrm{b} / \mathrm{MeV} \cdot \mathrm{sr^{2 }}\right)$} \\
\hline & $\left(-68.5^{\circ}, 0.0^{\circ}\right)$ & $\left(-56.0^{\circ}, 0.0^{\circ}\right)$ & $\left(-46.5^{\circ}, 23.0^{\circ}\right)$ & $\left(-46.5^{\circ}, 11.5^{\circ}\right)$ & $\left(-46.5^{\circ}, 0.0^{\circ}\right)$ & $\left(-46.5^{\circ},-11.5^{\circ}\right)$ & $\left(-46.5^{\circ},-23.0^{\circ}\right)$ \\
\hline 100 & $0.00 \pm 1.86$ & $0.00 \pm 1.86$ & $0.00 \pm 1.86$ & $0.81 \pm 1.87$ & $2.35 \pm 1.87$ & $1.11 \pm 1.87$ & $0.00 \pm 1.86$ \\
\hline 116 & $1.24 \pm 0.74$ & $0.52 \pm 0.81$ & $0.00 \pm 0.73$ & $0.00 \pm 0.73$ & $0.00 \pm 0.73$ & $0.48 \pm 0.73$ & $0.00 \pm 0.73$ \\
\hline 132 & $0.27 \pm 0.46$ & $0.58 \pm 0.46$ & $0.00 \pm 0.46$ & $0.00 \pm 0.46$ & $0.27 \pm 0.46$ & $0.96 \pm 0.60$ & $0.19 \pm 0.46$ \\
\hline 148 & $0.50 \pm 0.40$ & $0.34 \pm 0.47$ & $0.21 \pm 0.41$ & $0.75 \pm 0.43$ & $0.26 \pm 0.40$ & $0.17 \pm 0.42$ & $0.48 \pm 0.45$ \\
\hline 164 & $1.46 \pm 0.61$ & $0.26 \pm 0.40$ & $0.00 \pm 0.40$ & $0.61 \pm 0.48$ & $0.87 \pm 0.46$ & $0.33 \pm 0.45$ & $0.24 \pm 0.40$ \\
\hline 180 & $0.00 \pm 0.43$ & $0.25 \pm 0.43$ & $0.41 \pm 0.46$ & $0.87 \pm 0.57$ & $0.63 \pm 0.49$ & $0.44 \pm 0.43$ & $0.27 \pm 0.51$ \\
\hline 196 & $0.00 \pm 0.48$ & $0.00 \pm 0.48$ & $0.58 \pm 0.51$ & $0.31 \pm 0.48$ & $2.09 \pm 0.78$ & $0.82 \pm 0.48$ & $0.00 \pm 0.48$ \\
\hline 212 & $0.64 \pm 0.62$ & $0.44 \pm 0.69$ & $0.00 \pm 0.62$ & $0.74 \pm 0.66$ & $0.64 \pm 0.62$ & $1.00 \pm 0.71$ & $0.36 \pm 0.62$ \\
\hline 228 & $0.00 \pm 0.85$ & $0.84 \pm 0.87$ & $0.53 \pm 0.86$ & $0.00 \pm 0.85$ & $0.37 \pm 0.85$ & $0.00 \pm 0.85$ & $0.86 \pm 0.86$ \\
\hline 244 & $0.00 \pm 1.28$ & $0.00 \pm 1.28$ & $0.00 \pm 1.28$ & $0.00 \pm 1.28$ & $0.00 \pm i .28$ & $0.84 \pm 1.29$ & $0.00 \pm 1.28$ \\
\hline 260 & $0.00 \pm 2.36$ & $0.00 \pm 2.36$ & $0.00 \pm 2.36$ & $0.00 \pm 2.36$ & $0.00 \pm 2.36$ & $0.00 \pm 2.36$ & $0.00 \pm 2.36$ \\
\hline
\end{tabular}

Table E.17: Triply differential cross sections for the ${ }^{3} \mathrm{He}\left(\pi, \pi^{\circ} \mathrm{p}\right)$ reaction at $\mathrm{T}_{\mathrm{x}}=245 \mathrm{MeV}$ and $\theta_{x^{\circ}}=70^{\circ}$. The in-plane $\left(\theta_{\mathrm{p}}\right)$ and outof-plane $\left(\phi_{p}\right)$ proton laboratory angles are designated as $\left(\theta_{p}, \phi_{p}\right)$. An overall systematic uncertainty of $8.1 \%$ is not included. 


\begin{tabular}{|c|c|c|c|c|c|c|c|c|}
\hline \multicolumn{9}{|c|}{${ }^{3} \mathrm{He}\left(\pi, \pi^{\circ} \mathrm{P}\right)$ at $\mathrm{T}_{n}=245 \mathrm{MeV}$ and $\theta_{n}=110^{\circ}$} \\
\hline \multirow{2}{*}{$\begin{array}{c}\mathrm{T}_{\pi^{0}} \\
(\mathrm{MeV})\end{array}$} & \multicolumn{8}{|c|}{$d^{3} \sigma / d E_{R} d \Omega_{n} d \Omega_{p}\left(\mu b / M e V \cdot s r^{2}\right)$} \\
\hline & $\left(-23.0^{\circ}, 22.0^{\circ}\right)$ & $\left(-23.0^{\circ}, 9.5^{\circ}\right)$ & $\left(-57.5^{\circ}, 0.0^{\circ}\right)$ & $\left(-46.0^{\circ}, 0.0^{\circ}\right)$ & $\left(-34.5^{\circ}, 0.0^{\circ}\right)$ & $\left(-23.0^{\circ}, 0.0^{\circ}\right)$ & $\left(-11.5^{\circ}, 0.0^{\circ}\right)$ & $\left(11.5^{\circ}, 0.0^{\circ}\right)$ \\
\hline 66 & $0.98 \pm 0.99$ & $0.89 \pm 0.97$ & $0.00 \pm 0.97$ & $0.25 \pm 0.97$ & $0.00 \pm 0.97$ & $0.93 \pm 0.97$ & $0.32 \pm 0.97$ & $0.69 \pm 0.97$ \\
\hline 86 & $0.45 \pm 0.34$ & $0.58 \pm 0.36$ & $0.24 \pm 0.33$ & $0.62 \pm 0.36$ & $0.84 \pm 0.38$ & $0.89 \pm 0.38$ & $0.60 \pm 0.36$ & $0.00 \pm 0.32$ \\
\hline 106 & $0.63 \pm 0.23$ & $0.40 \pm 0.21$ & $0.32 \pm 0.22$ & $0.63 \pm 0.24$ & $0.68 \pm 0.26$ & $1.00 \pm 0.28$ & $0.45 \pm 0.20$ & $0.71 \pm 0.25$ \\
\hline 126 & $0.33 \pm 0.24$ & $1.09 \pm 0.32$ & $0.15 \pm 0.23$ & $0.66 \pm 0.28$ & $0.93 \pm 0.30$ & $1.76 \pm 0.39$ & $0.36 \pm 0.23$ & $0.78 \pm 0.31$ \\
\hline 146 & $0.40 \div 0.32$ & $0.54 \pm 0.37$ & $0.27 \pm 0.32$ & $0.46 \pm 0.31$ & $0.80 \pm 0.34$ & $0.69 \pm 0.34$ & $0.54 \pm 0.34$ & $0.10 \pm 0.30$ \\
\hline 166 & $0.13 \pm 0.48$ & $0.58 \pm 0.48$ & $0.22 \pm 0.48$ & $0.28 \pm 0.48$ & $0.40 \pm 0.48$ & $1.17 \pm 0.54$ & $0.28 \pm 0.48$ & $0.87 \pm 0.48$ \\
\hline
\end{tabular}

Table E.18: Triply differential cross sections for the ${ }^{3} \mathrm{He}\left(\pi^{+}, \pi^{\circ} \mathrm{p}\right)$ reaction at $\mathrm{T}_{\mathrm{n}}=245 \mathrm{MeV}$ and $\theta_{\mathrm{r}}=110^{\circ}$. The in-plane $\left(\theta_{\mathrm{p}}\right)$ and outof-plane $\left(\phi_{p}\right)$ proton laboratory angles are designated as $\left(\theta_{p}, \phi_{p}\right)$. An overall systematic uncertainty of $8.1 \%$ is not included. 


\begin{tabular}{|c|c|c|}
\hline \multicolumn{3}{|c|}{${ }^{3} \mathrm{He}\left(\pi^{+}, \pi^{0} p\right)$ at $\mathrm{T}_{x}=245 \mathrm{MeV}$} \\
\hline \multicolumn{3}{|c|}{$\theta_{R}=70^{\circ} ; \theta_{4}=-37.0^{\circ}$} \\
\hline $\begin{array}{c}\theta_{\mathrm{p}} \\
(\mathrm{deg} .)\end{array}$ & $\begin{array}{c}\phi_{\mathrm{p}} \\
(\operatorname{deg} .)\end{array}$ & $\begin{array}{c}\mathrm{d}^{2} \sigma / \mathrm{d} \Omega_{\mathrm{N}} \mathrm{d} \Omega_{x} \\
\left(\mathrm{mb} / \mathrm{sr}^{2}\right)\end{array}$ \\
\hline-37.0 & 22.0 & $0.27 \pm 0.05$ \\
\hline-37.0 & 9.5 & $0.96 \pm 0.08$ \\
\hline-60.0 & 0.0 & $0.58 \pm 0.06$ \\
\hline-48.5 & 0.0 & $1.29 \pm 0.08$ \\
\hline-37.0 & 0.0 & $1.62 \pm 0.10$ \\
\hline-25.5 & 0.0 & $0.70 \pm 0.07$ \\
\hline-14.0 & 0.0 & $0.43 \pm 0.06$ \\
\hline-71.5 & 0.0 & $0.13 \pm 0.05$ \\
\hline
\end{tabular}

\begin{tabular}{|c|c|c|}
\hline \multicolumn{3}{|c|}{${ }^{3} \mathrm{He}\left(\pi^{+}, \pi^{0} \mathrm{p}\right)$ at $\mathrm{T}_{n}=245 \mathrm{MeV}$} \\
\hline \multicolumn{3}{|c|}{$\theta_{\alpha^{\circ}}=70^{\circ} ; \theta_{4}=-46.5^{\circ}$} \\
\hline $\left.\begin{array}{c}\theta_{p} \\
(\text { deg. }\end{array}\right)$ & $\begin{array}{c}\phi_{p} \\
(\text { deg. })\end{array}$ & $\begin{array}{c}\left.d^{2} \sigma / d \Omega_{\alpha} d \Omega_{x} / s r^{2}\right) \\
x\end{array}$ \\
\hline-68.5 & 0.0 & $0.21 \pm 0.03$ \\
\hline-56.0 & 0.0 & $0.78 \pm 0.04$ \\
\hline-46.5 & 34.5 & $0.03 \pm 0.02$ \\
\hline-46.5 & 23.0 & $0.18 \pm 0.02$ \\
\hline-46.5 & 11.5 & $0.74 \pm 0.04$ \\
\hline-46.5 & 0.0 & $1.54 \pm 0.06$ \\
\hline-46.5 & -11.5 & $0.80 \pm 0.04$ \\
\hline-46.5 & -23.0 & $0.12 \pm 0.02$ \\
\hline
\end{tabular}

\begin{tabular}{|c|c|c|}
\hline \multicolumn{3}{|c|}{${ }^{3} \mathrm{He}\left(\pi^{2}, \pi^{0} \mathrm{p}\right)$ at $\mathrm{T}_{n}=245 \mathrm{MeV}$} \\
\hline \multicolumn{3}{|c|}{$\theta_{x^{\circ}}=70^{\circ} ; \theta_{4}=-51.2^{\circ}$} \\
\hline $\begin{array}{c}\theta_{p} \\
\text { (deg.) }\end{array}$ & $\begin{array}{c}\phi_{\mathrm{p}} \\
(\mathrm{deg} .)\end{array}$ & $\begin{array}{c}\mathrm{d}^{2} \sigma / \mathrm{d} \Omega_{\mathrm{x}} \mathrm{d} \Omega_{x} \\
\left(\mathrm{mb} / \mathrm{sr}^{2}\right)\end{array}$ \\
\hline-73.2 & 0.0 & $0.11 \pm 0.03$ \\
\hline-60.7 & 0.0 & $0.43 \pm 0.04$ \\
\hline-51.2 & 34.5 & $0.04 \pm 0.03$ \\
\hline-51.2 & 23.0 & $0.19 \pm 0.03$ \\
\hline-51.2 & 11.5 & $0.71 \pm 0.04$ \\
\hline-51.2 & 0.0 & $1.22 \pm 0.06$ \\
\hline-51.2 & -11.5 & $0.70 \pm 0.04$ \\
\hline-51.2 & -23.0 & $0.08 \pm 0.03$ \\
\hline
\end{tabular}

Table E.19: Doubly differential cross sections for the ${ }^{3} \mathrm{He}\left(\pi^{+}, \pi^{0} \mathrm{p}\right)$ reaction at $\mathrm{T}_{x}=245 \mathrm{MeV}$ and $\theta_{x^{0}}=70^{\circ}$. The cross sections have been tabulated according to the proton detector configuration which was used to accumulate the data. The proton angle of the central proton detector for a particular proton detector configuration is designated as $\theta_{4}$. The labels $\theta_{p}$ and $\phi_{p}$ refer to the in-plane and out-of-plane proton angles respectively. An overall systematic uncertainty of $8.5 \%$ is not included. 


\begin{tabular}{|c|c|c|}
\hline \multicolumn{3}{|c|}{${ }^{3} \mathrm{He}\left(\pi, \pi^{0} \mathrm{p}\right)$ at $\mathrm{T}_{\mathrm{x}}=245 \mathrm{MeV}$} \\
\hline \multicolumn{3}{|c|}{$\theta_{x}=90^{\circ} ; \theta_{4}=-30.4^{\circ}$} \\
\hline $\begin{array}{c}\theta_{\mathrm{p}} \\
(\mathrm{deg} .)\end{array}$ & $\begin{array}{c}\phi_{p} \\
(\text { deg. })\end{array}$ & $\begin{array}{c}\mathrm{d}^{2} \sigma / \mathrm{d} \Omega_{\mathrm{N}} \mathrm{d} \Omega_{\mathrm{p}} \\
\left(\mathrm{mb} / \mathrm{sr}^{2}\right)\end{array}$ \\
\hline-52.4 & 0.0 & $0.27 \pm 0.06$ \\
\hline-39.9 & 0.0 & $1.20 \pm 0.10$ \\
\hline-30.4 & 23.0 & $0.21 \pm 0.06$ \\
\hline-30.4 & 11.5 & $0.85 \pm 0.09$ \\
\hline-30.4 & 0.0 & $2.00 \pm 0.13$ \\
\hline-30.4 & -11.5 & $0.95 \pm 0.09$ \\
\hline-30.4 & -23.0 & $0.14 \pm 0.06$ \\
\hline-30.4 & 34.5 & $0.07 \pm 0.06$ \\
\hline
\end{tabular}

\begin{tabular}{|c|c|c|}
\hline \multicolumn{2}{|c|}{$\mathrm{He}\left(\pi^{2}, \pi^{\circ} \mathrm{p}\right)$ at $\mathrm{T}_{\mathrm{z}}=245 \mathrm{MeV}$} \\
\hline \multicolumn{3}{|c|}{$\theta_{x^{\circ}}=90^{\circ} ; \theta_{\mathrm{a}}=-35.4^{\circ}$} \\
\hline $\begin{array}{c}\theta_{\mathrm{p}} \\
(\text { deg. })\end{array}$ & $\begin{array}{c}\phi_{\mathrm{p}} \\
\left(\text { deg. }^{3}\right.\end{array}$ & $\begin{array}{c}\mathrm{d}^{2} \sigma / \mathrm{d} \Omega_{\mathrm{d}} \mathrm{d} \Omega_{\mathrm{p}} \\
\left(\mathrm{mb} / \mathrm{sr}^{2}\right)\end{array}$ \\
\hline-57.4 & 0.0 & $0.13 \pm 0.03$ \\
\hline-44.9 & 0.0 & $0.63 \pm 0.05$ \\
\hline-35.4 & 23.0 & $0.24 \pm 0.03$ \\
\hline-35.4 & 11.5 & $0.92 \pm 0.06$ \\
\hline-35.4 & 0.0 & $1.82 \pm 0.08$ \\
\hline-35.4 & -11.5 & $0.77 \pm 0.05$ \\
\hline-35.4 & -23.0 & $0.15 \pm 0.03$ \\
\hline-35.4 & 34.5 & $0.05 \pm 0.03$ \\
\hline
\end{tabular}

Table E.20: Doubly differential cross sections for the ${ }^{3} \mathrm{He}\left(\pi^{*}, \pi^{0} \mathrm{p}\right)$ reaction at $T_{\pi}=245 \mathrm{MeV}$ and $\theta_{\pi^{0}}=90^{\circ}$. The cross sections have been tabulated according to the proton detector configuration which was used to accumulate the data. The prcton angle of the central proton detector for a particular proton detector configuration is designated as $\theta_{\varepsilon}$. The labels $\theta_{p}$ and $\phi_{p}$ refer to the in-plane and out-of-plane proton angles respectively. An overall systematic uncertainty of $8.1 \%$ is not included.

\begin{tabular}{|c|c|c|}
\hline \multicolumn{3}{|c|}{${ }^{3} \mathrm{He}\left(\pi^{\circ}, \pi^{0} \mathrm{p}\right)$ at $\mathrm{T}_{\mathrm{x}}=245 \mathrm{MeV}$} \\
\hline \multicolumn{3}{|c|}{$\theta_{\pi^{\circ}}=110^{\circ} ; \theta_{4}=-21.3^{\circ}$} \\
\hline $\begin{array}{c}\theta_{\mathrm{p}} \\
(\mathrm{deg} .)\end{array}$ & $\begin{array}{c}\phi_{p} \\
\text { (deg.) }\end{array}$ & $\begin{array}{c}\mathrm{d}^{2} \sigma / \mathrm{d} \Omega_{\mathrm{r}} \mathrm{d} \Omega_{\mathrm{p}} \\
\left(\mathrm{mb} / \mathrm{sr}^{2}\right)\end{array}$ \\
\hline-43.3 & 0.0 & $0.25 \pm 0.06$ \\
\hline-30.8 & 0.0 & $1.09 \pm 0.09$ \\
\hline-21.3 & 34.5 & $0.06 \pm 0.05$ \\
\hline-21.3 & 23.0 & $0.23 \pm 0.06$ \\
\hline-21.3 & 11.5 & $0.80 \pm 0.08$ \\
\hline-21.3 & 0.0 & $2.51 \pm 0.14$ \\
\hline-21.3 & -11.5 & $1.09 \pm 0.09$ \\
\hline-21.3 & -23.0 & $0.12 \pm 0.05$ \\
\hline
\end{tabular}

\begin{tabular}{|c|c|c|}
\hline \multicolumn{3}{|c|}{${ }^{3} \mathrm{He}\left(\pi^{+}, \pi^{0} p\right)$ at $\mathrm{T}_{\mathrm{x}}=245 \mathrm{MeV}$} \\
\hline \multicolumn{3}{|c|}{$\theta_{n}=110^{\circ} ; \theta_{4}=-26.3^{\circ}$} \\
\hline $\begin{array}{c}\theta_{\mathrm{p}} \\
\text { (deg.) }\end{array}$ & $\begin{array}{c}\phi_{\mathrm{p}} \\
(\mathrm{deg} .)\end{array}$ & $\begin{array}{c}\mathrm{d}^{2} \sigma / \mathrm{d} \Omega_{\mathrm{N}} \mathrm{d} \Omega_{\mathrm{p}} \\
\left(\mathrm{mb} / \mathrm{sr}^{2}\right)\end{array}$ \\
\hline-48.3 & 0.0 & $0.14 \pm 0.03$ \\
\hline-35.8 & 0.0 & $0.56 \pm 0.04$ \\
\hline-26.3 & 34.5 & $0.01 \pm 0.02$ \\
\hline-26.3 & 23.0 & $0.16 \pm 0.03$ \\
\hline-26.3 & 11.5 & $0.81 \pm 0.04$ \\
\hline-26.3 & 0.0 & $2.15 \pm 0.07$ \\
\hline-26.3 & -11.5 & $0.81 \pm 0.04$ \\
\hline-26.3 & -23.0 & $0.15 \pm 0.03$ \\
\hline
\end{tabular}

Table E.21: Doubly differential cross sections for the ${ }^{3} \mathrm{He}\left(\pi^{*}, \pi^{\circ} \mathrm{p}\right)$ reaction at $\mathrm{T}_{\pi}=245 \mathrm{MeV}$ and $\theta_{\pi^{0}}=110^{\circ}$ The cross sections have been tabulated according to the proton detector configuration which was used to accumulate the data. The proton angle of the central proton detector for a particular proton detector configuration is designated as $\theta_{4}$. The labels $\theta_{p}$ and $\phi_{p}$ refer to the in-plane and out-of-plane proton angles respectively. An overall systematic uncertainty of $8.3 \%$ is not included. 


\begin{tabular}{|c|c|c|}
\hline \multicolumn{3}{|c|}{${ }^{3} \mathrm{He}\left(\pi+\pi^{0} \mathrm{p}\right)$ at $\mathrm{T}_{x}=245 \mathrm{MeV}$} \\
\hline \multicolumn{3}{|c|}{$\theta_{x^{\circ}}=120^{\circ} ; \theta_{4}=-17.2^{\circ}$} \\
\hline $\begin{array}{c}\theta_{\mathrm{p}} \\
(\mathrm{deg})\end{array}$ & $\begin{array}{c}\phi_{p} \\
\text { (deg.) }\end{array}$ & $\begin{array}{c}\mathrm{d}^{2} \sigma / \mathrm{d} \Omega \mathrm{d} \mathrm{d}_{\mathrm{p}} \\
\left(\mathrm{mb} / \mathrm{sr}^{2}\right)\end{array}$ \\
\hline-40.2 & 0.0 & $0.15 \pm 0.05$ \\
\hline-26.7 & 0.0 & $1.13 \pm 0.09$ \\
\hline-17.2 & 34.5 & $0.03 \pm 0.04$ \\
\hline-17.2 & 23.0 & $0.17 \pm 0.05$ \\
\hline-17.2 & 11.5 & $0.95 \pm 0.08$ \\
\hline-17.2 & 0.0 & $2.13 \pm 0.12$ \\
\hline-17.2 & -11.5 & $0.68 \pm 0.07$ \\
\hline-17.2 & -23.0 & $0.08 \pm 0.04$ \\
\hline
\end{tabular}

\begin{tabular}{|c|c|c|}
\hline \multicolumn{3}{|c|}{${ }^{3} \mathrm{He}\left(\pi^{+}, \pi^{0} p\right)$ at $\mathrm{T}_{x}=245 \mathrm{MeV}$} \\
\hline \multicolumn{3}{|c|}{$\theta_{*}=120^{\circ} ; \theta_{4}=-22.0^{\circ}$} \\
\hline $\begin{array}{c}\theta_{\mathrm{p}} \\
\text { (deg.) }\end{array}$ & $\begin{array}{c}\phi_{\mathrm{p}} \\
\text { (deg.) }\end{array}$ & $\underset{\left(\mathrm{mb} / \mathrm{sr}^{2}\right)}{\mathrm{d}^{2} \sigma / \mathrm{A} \Omega_{\mathrm{p}}}$ \\
\hline-45.0 & 0.0 & $0.09 \pm 0.03$ \\
\hline-31.5 & 0.0 & $0.56 \pm 0.04$ \\
\hline-22.0 & 34.5 & $0.04 \pm 0.03$ \\
\hline-22.0 & 23.0 & $0.14 \pm 0.03$ \\
\hline-22.0 & 11.5 & $0.82 \pm 0.05$ \\
\hline-22.0 & 0.0 & $2.16 \pm 0.09$ \\
\hline-22.0 & -11.5 & $0.68 \pm 0.04$ \\
\hline-22.0 & -23.0 & $0.09 \pm 0.03$ \\
\hline
\end{tabular}

Table E.22: Doubly differential cross sections for the ${ }^{3} \mathrm{He}\left(\pi^{*}, \pi^{\circ} \mathrm{p}\right)$ reaction at $\mathrm{T}_{\mathrm{x}}=245 \mathrm{MeV}$ and $\theta_{x^{0}}=120^{\circ}$. The cross sections have been tabulated according to the proton detector configuration which was used to accumulate the data. The proton angle of the central proton detector for a particular proton detector configuration is designated as $\theta_{4}$. The labels $\theta_{p}$ and $\phi_{p}$ refer to the in-plane and out-of-plane proton angles respectively. An overall systematic uncertainty of $8.4 \%$ is not included.

\begin{tabular}{|c|c|c|}
\hline \multicolumn{3}{|c|}{${ }^{3} \mathrm{He}\left(\pi, \pi^{0} \mathrm{p}\right)$ at $\mathrm{T}_{x}=245 \mathrm{MeV}$} \\
\hline \multicolumn{3}{|c|}{$\theta_{x^{\circ}}=70^{\circ} ; \theta_{4}=-37.0^{\circ}$} \\
\hline $\begin{array}{c}\theta_{p} \\
\left(\text { deg. }^{\prime}\right)\end{array}$ & $\begin{array}{c}\phi_{p} \\
(\text { deg. })\end{array}$ & 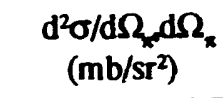 \\
\hline-37.0 & 22.0 & $0.04 \pm 0.03$ \\
\hline-37.0 & 9.5 & $0.06 \pm 0.03$ \\
\hline-71.5 & 0.0 & $0.04 \pm 0.03$ \\
\hline-60.0 & 0.0 & $0.05 \pm 0.03$ \\
\hline-48.5 & 0.0 & $0.08 \pm 0.03$ \\
\hline-37.0 & 0.0 & $0.13 \pm 0.03$ \\
\hline-25.5 & 0.0 & $0.05 \pm 0.03$ \\
\hline-14.0 & 0.0 & $0.05 \pm 0.03$ \\
\hline
\end{tabular}

\begin{tabular}{|c|c|c|}
\hline \multicolumn{3}{|c|}{${ }^{3} \mathrm{He}\left(\pi, \pi^{\circ} \mathrm{p}\right)$ at $\mathrm{T}_{\mathrm{x}}=245 \mathrm{MeV}$} \\
\hline \multicolumn{3}{|c|}{$\theta_{x_{0}}=70^{\circ} ; \theta_{4}=-46.5^{\circ}$} \\
\hline $\begin{array}{c}\theta_{\mathrm{p}} \\
\text { (deg.) }\end{array}$ & $\begin{array}{c}\phi_{\mathrm{p}} \\
(\mathrm{deg} .)\end{array}$ & $\begin{array}{c}\mathrm{d}^{2} \sigma / \mathrm{d} \Omega_{\mathrm{N}} \mathrm{d} \Omega_{\mathrm{x}} \\
\left(\mathrm{mb} / \mathrm{sr}^{2}\right)\end{array}$ \\
\hline-68.5 & 0.0 & $0.07 \pm 0.06$ \\
\hline-56.0 & 0.0 & $0.05 \pm 0.06$ \\
\hline-46.5 & 23.0 & $0.03 \pm 0.06$ \\
\hline-46.5 & 11.5 & $0.07 \pm 0.06$ \\
\hline-46.5 & 0.0 & $0.12 \pm 0.06$ \\
\hline-46.5 & -11.5 & $0.10 \pm 0.06$ \\
\hline-46.5 & -23.0 & $0.04 \pm 0.06$ \\
\hline
\end{tabular}

Table E 23: Doubly differential cross sections for the ${ }^{3} \mathrm{He}\left(\pi^{\circ}, \pi^{0} \mathrm{p}\right)$ reaction at $\mathrm{T}_{\mathrm{x}}=245 \mathrm{MeV}$ and $\theta_{\pi^{0}}=70^{\circ}$. The cross sections have been tabulated according to the proton detector configuration which was used to accumulate the data. The proton angle of the central proton detector for a particular proton detector configuration is designated as $\theta_{4}$. The labels $\theta_{p}$ and $\phi_{p}$ refer to the in-plane and out-of-plane proton angles respectively. An overall systematic uncertainty of $8.5 \%$ is not included. 


\begin{tabular}{|c|c|c|}
\hline \multicolumn{3}{|c|}{${ }^{3} \mathrm{He}\left(\pi, \pi^{0} \mathrm{p}\right)$ at $\mathrm{T}_{x}=245 \mathrm{MeV}$} \\
\hline \multicolumn{3}{|c|}{$\theta_{x}=110^{\circ} ; \theta_{4}=-23.0^{\circ}$} \\
\hline $\begin{array}{c}\theta_{\mathrm{p}} \\
\text { (deg.) }\end{array}$ & $\begin{array}{c}\phi_{p} \\
(\text { deg. })\end{array}$ & $\underset{(\mathrm{mb} / \mathrm{s} / 2)}{\mathrm{d}^{2} \sigma / \mathrm{d} \mathbf{p}_{\mathrm{p}} \mathrm{d} \Omega_{\mathrm{p}}}$ \\
\hline-23.0 & 22.0 & $0.06 \pm 0.02$ \\
\hline-23.0 & 9.5 & $0.08 \pm 0.03$ \\
\hline-57.5 & 0.0 & $0.02 \pm 0.02$ \\
\hline-46.0 & 0.0 & $0.06 \pm 0.02$ \\
\hline-34.5 & 0.0 & $0.07 \pm 0.03$ \\
\hline-23.0 & 0.0 & $0.13 \pm 0.03$ \\
\hline-11.5 & 0.0 & $0.10 \pm 0.03$ \\
\hline 11.5 & 0.0 & $0.06 \pm 0.02$ \\
\hline
\end{tabular}

Table E.24: Doubly differential cross sections for the ${ }^{3} \mathrm{He}\left(\pi^{-}, \pi^{\circ} \mathrm{p}\right)$ reaction at $\mathrm{T}_{\mathrm{x}}=245 \mathrm{MeV}$ and $\theta_{\mathrm{x}} 0=110^{\circ}$. The proton angle of the central proton detector, which was used to accumulate this data, is designated as $\theta_{4}$. The labels $\theta_{p}$ and $\phi_{p}$ refer to the in-plane and out-of-plane proton angles respectively. An overall systematic uncertainty of $8.4 \%$ is not included. 
$\rightarrow$ 


\section{References}

[1] B. M. K. Nefkens, Nucl. Phys. A416 (1984) 193c.

[2] T. Ikehashi and K. Ohta, Nucl. Phys. A536 (1992) 521.

[3] W. Lin and B. D. Serot, Nucl. Phys. A524 (1991) 601.

[4] S. S. Kamalov, L. Tiator, and C. Bennhold, Universitat Mainz Technical Report, MKPH-T-92-13 (1992).

[5] W. R. Gibbs and B. F. Gibson, Phys. Rev. C43 (1991) 1012.

[6] M. E. Yuly, Ph.D. thesis, M.I.T., Los Alamos Scientific Laboratory Report LA-12559-T (1993).

[7] M. D. Cooper et al., Phys. Rev. C25 (1982) 438.

[8] P. Glodis et al., Phys. Rev. Let. 44 (1980) 234.

[9] J. Kallne et al., Phys. Rev. Lett. 42 (1979) 159.

[10] L. Tiator, private communication (1992).

[11] W. R. Gibbs, private communication (1992).

[12] S. S. M. Wong, Introductory Nuclear Physics, Prentice Hall, Englewood Cliffs, NJ (1990).

[13] M. Aguilar-Benitez et al., Particle Properties Data Booklet, North-Holland, Amsterdam (1988).

[14] R. A. Amdt, Scattering Analysis Interaction Dial-In (SAID), an interactive program for $\pi N$ phase shift calculations (1992).

[15] E. Pedroni et al. , Nucl. Phys. A300 (1978) 321.

[16] A. A. Carter et al., Nucl. Phys. B26(1971) 445.

[17] G. Bizard et al., Phys. Let. 31B (1970) 481.

[18] D. V. Bugg et al., Nucl. Phys. B26 (1971) 588.

[19] F. Bulos et al., Phys. Rev. 187 (1969) 1827.

[20] J. B. Walters and G. A. Rebka, Jr., SCATPI, A Subroutine for Calculating $\pi N$ Cross Sections and Polarizations for Incident Pion Kinetic Energies Between 90 and $300 \mathrm{MeV}$, Los Alamos Technical Report LA-7731-MS (1979).

[21] T. Ericson and W. Weise, Pions and Nuclei, Clarendon Press, Oxford (1988).

[22] E. Jans et al., Nucl. Phys. A475 (1987) 687.

[23] C. Marchand et al., Phys. Rev. Lett. 60 (1988) 1703.

[24] K. Dow et al., Phys. Rev. Lett. 61 (1988) 1706.

[25] J. S. McCarthy et al., Phys. Rev. C13 (1976) 712. 
[26] D. A. Sparrow, Phys. Lett. 58B (1975) 309.

[27] M. Waicamatsu, Nucl. Phys. A340 (1980) 289.

[28] A. T. Hess and B. F. Gibson, Phys. Rev. C13 (1976) 749.

[29] R. H. Landau, Phys. Rev. C15 (1977) 2127.

[30] K. P. Lohs and V. B. Mandelsweig, Z. Phys. A283 (1977) 51.

[31] J. M. Eisenberg and V. B. Mandelzweig, Phys. Let. B53 (1975) 405.

[32] L. Orphanos et al., Phys. Rev. C26 (1982) 2111.

[33] W. J. Gerace et al., Phys. Rev. C22 (1980) 1197.

[34] M. Thies, Nucl. Phys. A382 (1982) 434.

[35] D. Ashery et al., Phys. Rev. Let. 47 (1981) 895.

[36] G. Backenstoss et al., Phys. Leth. B137 (1984) 329.

[37] D. Ashery et al., Phys. Rev. Let. 50 (1983) 482.

[38] D. S. Kolun, in Advances in Nuclear Physics, Vol. 3, M. Baranger and E. Vogt, Eds., Plenum Press, New York (1969).

[39] T. Takaki and M. Thies, Phys. Rev. C38 (1988) 2230.

[40] S. Gilad et al., Phys. Rev. Leth 57 (1986) 2637.

[41] A. Klein et al., Phys. Let. B187 (1987) 253; A. Klein, private communication (1993).

[42] A. Klein et al., Nucl. Phys. A472 (1987) 605.

[43] Los Alamos Scientific Laboratory Report, MP-DO-3UHB, 1974 (Revised 1984).

[44] G. J. Krausse and P.A.M. Gram, Nucl. Inst. and Meth. 156 (1978) 365.

[45] E. R. Kinney, Ph.D. thesis, M.I.T., Los Alamos Scientific Laboratory Report LA-11417-T (1988).

[46! S. A. Wood, Ph.D. thesis, M.I.T., Los Alamos Scientific Laboratory Report LA-9932-T (1984).

[47] D. M. Manley, Ph.D. thesis, University of Wyoming, Los Alamos Scientific Laboratory Repor LA-9101-T (1981).

[48] J. B. Walter, Ph.D. thesis, University of Wyoming, Los Alamos Scientific Laboratory Report LA-8377-T (1979).

[49] A. T. Oyer, Ph.D. thesis, University of Wyoming, Los Alamos Scientific Laboratory Report LA-6599-T (1976).

[50] Introdution to Q, Los Alamos Technical Document MP-1-3401-3 (1985); A Guide To Writing An RSX-11M Or VMS Q Analyzer, Los Alamos Technical Document MP-1-3417-1 (1985);

Q-Programmer's Information Manual, Los Alamos Technical Document MP-1-3401-5 (1986).

[51] C. Pillai et al., Phys Rev. C43 (1991) 1838.

[52] F. James and M. Roos, MINUIT, Function Minimization and Error Analysis, CERN Program Library Entry DS06, CERN, Geneva (1989); F. James and M. Roos, Comput. Phys. Commun. 10 (1975) 343. 
[53] S. B. Kaufman et al., Phy. Rev. C32 (1985) 1977.

[54] R. H. Landau, Ann. Phys. 92 (1975) 205.

[55] B. J. Dropesky et al., Phys. Rev. Let. 34 (1975) 821; Phys. Rev. C20 (1979) 1844.

[56] User Information Manual, The PIO Spectrometer Data Acquisition System, Los Alamos Technical Document MP-1-3408-2 (1986).

[57] S. Gilad, Ph.D. thesis, Tel Aviv University (1979).

[58] H. W. Baer et al., Nucl. Inst. Meth. 180 (1981) 445.

[59] J. S. McCarthy et al., Phys. Rev. C15 (1977) 1396.

[60] L. C. Smith et al., Phys. Rev. C40 (1989) 1347.

[61] Handbook of Chemistry and Physics, $72^{\text {sd }}$ ed., Chemical Rubber Publishing Company (1991).

[62] S. Høibraten, Ph.D. thesis, M.I.T., Los Alamos Scientific Laboratory Repor LA-11582-T (1989).

[63] D. Ashery et al., Phys. Rev. C30 (1984) 946.

[64] R. F. Jenefsky et al., Nucl. Phys. A290 (1977) 407.

[65] J. C. Comiso et al., Phys. Rev. D12 (1975) 738.

[66] N. S. Chant and P. G. Roos, Phys. Rev. C15 (1977) 57.

[67] L. Rees, N. S. Chant, and P. G. Roos, Phys. Rev. C26 (1982) 1580.

[68] N. S. Chant and P. G. Roos, Phys. Rev. C27 (1983) 1060.

[69] W. B. Coutingame and D. B. Holtkamp, Phys. Rev. C24 (1981) 2647.

[70] B. Nefkens et al., Phys. Rev. C41 (1991) 1012.

[71] M. A. Khandaker, Ph.D. thesis, University of Washington (1987).

[72] A. Nadasen et al., Phys. Rev. C23 (1981) 1023.

[73] K. L. Brown et al., TRANSPORT, A Computer Program For Designing Charge Particle Beam Transport Systems, SLAC-91 (1974).

[74] W. H. Press et al., Numerical Recipes-The Art of Scientific Computing, Cambridge University Press, New York (1986).

[75] J. F. Janni, At. Dat. Nucl. Dat. Tables 27 (1982) 1; J. F. Janni, At. Dat. Nucl. Dat. Tables 27 (1982) 341 . 

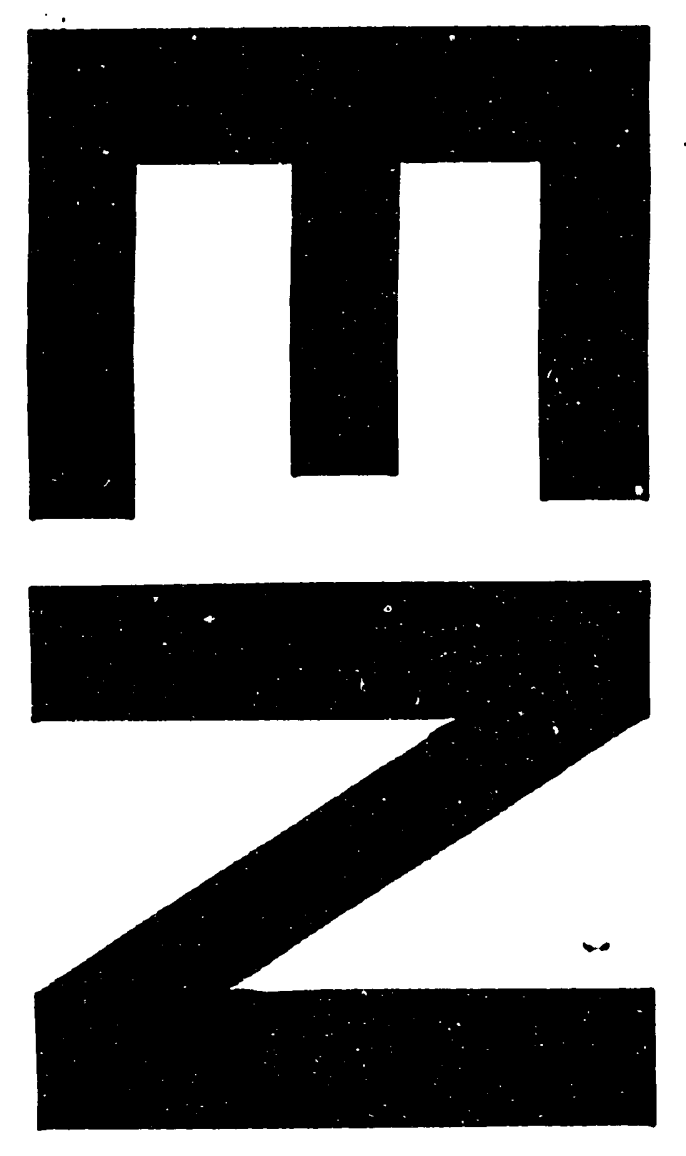

l
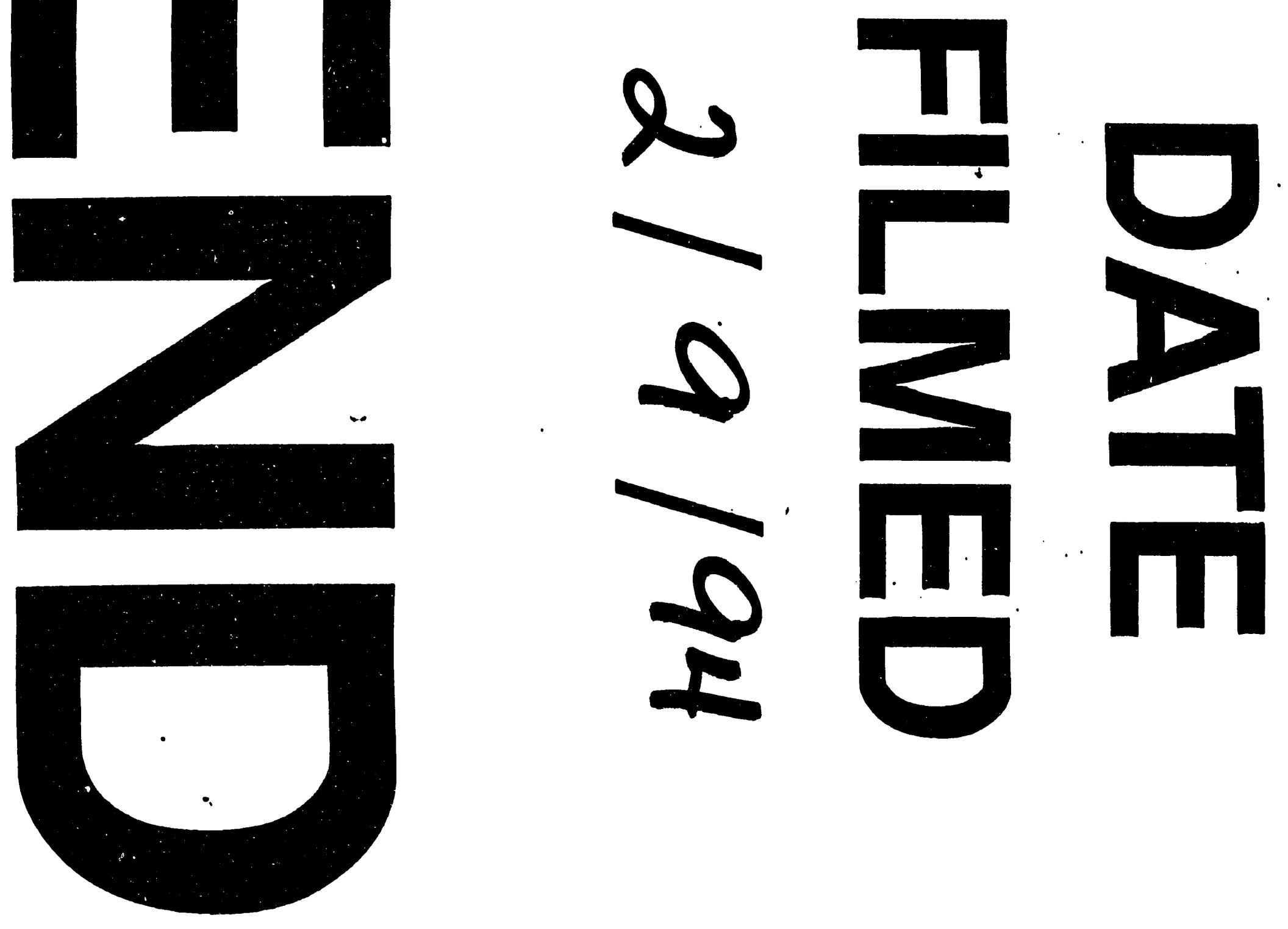